Lehrstuhl für Raumfahrttechnik

Technische Universität München

\title{
Data Handling and Evaluation for Autonomous Experiments in Interplanetary Missions
}

\author{
Robert Senger
}

Vollständiger Abdruck der von der Fakultät für Maschinenwesen der Technischen Universität München zur Erlangung des akademischen Grades eines

Doktor Ingenieurs

genehmigten Dissertation.

Vorsitzender:

Prüfer der Dissertation:
Univ.-Prof. Dr. rer. nat. Ulrich Walter

1. Univ.-Prof. Dr.-Ing. Eduard Igenbergs, i. R.

2. Univ.-Prof. Dr.-Ing. Horst Baier

3. apl. Prof. Dr. rer. nat. Eberhard Grün, Ruprecht-Karls-Universität Heidelberg

Die Dissertation wurde am 16.10.2006 bei der Technischen Universität München eingereicht und durch die Fakultät für Maschinenwesen am 26.06.2007 angenommen. 



\section{Data Handling and Evaluation for Autonomous Experiments in Interplanetary Missions}

Robert Senger 

Für meinen Vater Heinz $\uparrow 30.12 .2004$ 



\section{Vorwort}

An dieser Stelle möchte ich allen danken die mich während der Anfertigung dieser Arbeit über all die Jahre begleitet haben. An erster Stelle meinem Doktorvater Prof. Eduard Igenbergs für seine immerwährende Unterstützung und Geduld. Seinem Nachfolger als Leiter des Lehrstuhls, Prof. Ulrich Walter, für die Freiheiten die ich am Lehrstuhl genießen durfte. Allen Kollegen, deren namentliche Nennung den Rahmen dieser wenigen Zeilen sprengen würde, für die stets gute Atmosphäre am Lehrstuhl.

Mein ganz besonderer Dank gilt meiner Mutter Ursula und meiner Lebensgefährtin Dagmar. Ohne ihr unerschütterliches Vertrauen und ihre fortwährende bedingungslose Unterstützung auch in schwierigsten Zeiten wäre die Fertigstellung dieser Arbeit nicht möglich gewesen.

München, den 2.11.2006 



\section{Table of Contents}









2.1.2 Interplanetary and interstellar dust in the heliosphere ................................... 7













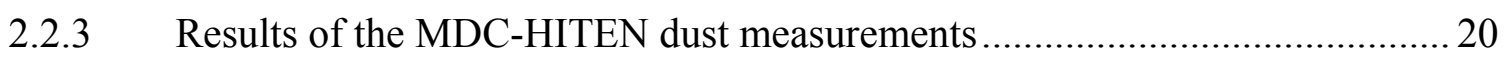

2.3 The MDC mission NOZOMI, (formerly PLANET-B) ........................................... 21







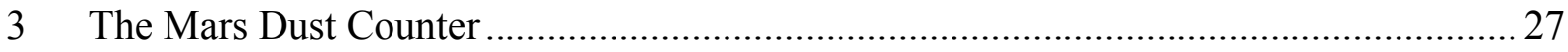



3.1.1 Physical background of the impact ionization process .................................. 28









3.2.3 MDC structural integration and effective solid angle .................................... 36









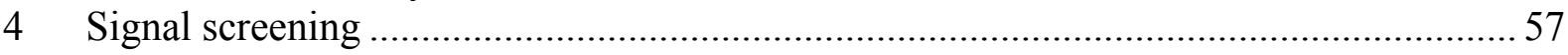













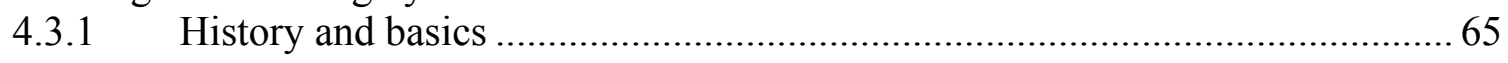

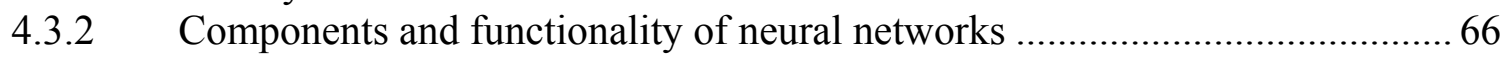





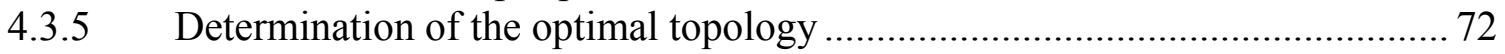





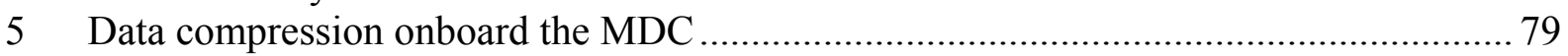



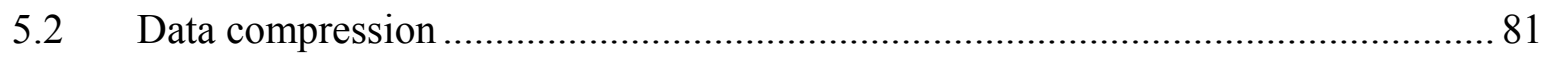

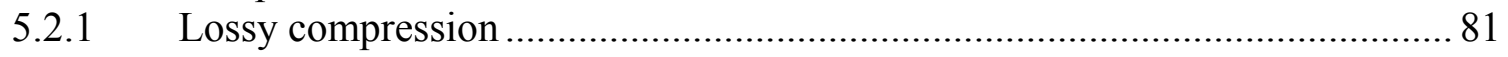








5.3 Implementation and integration of the MDC onboard software upgrade ...............89



5.3.2 Integration of the upgrade into the existing software..................................... 90

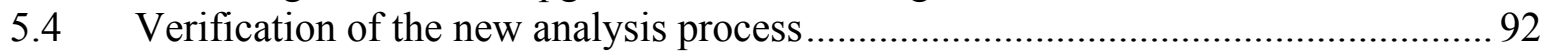

5.4.1 Analysis of MDC-NOZOMI data after compression/decompression............... 94

5.4.2 Analysis of MDC-HITEN data after compression/decompression................... 94







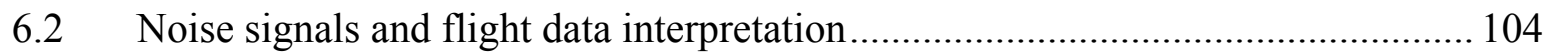



6.2.2 MDC view-axis determination from NOZOMI attitude .............................. 123

6.2.3 NOZOMI flight data and sensor pointing directions .................................... 131



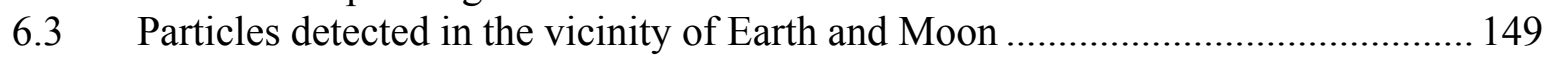

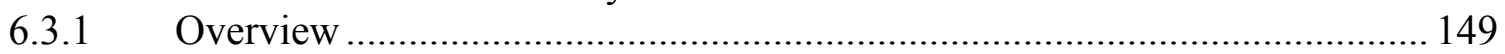



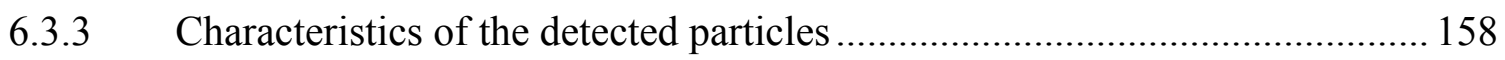





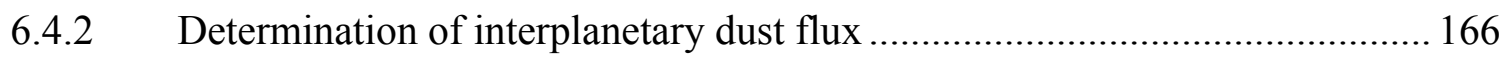













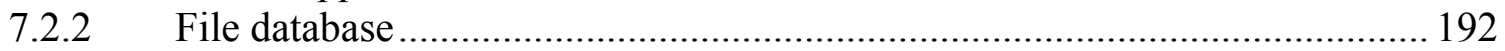

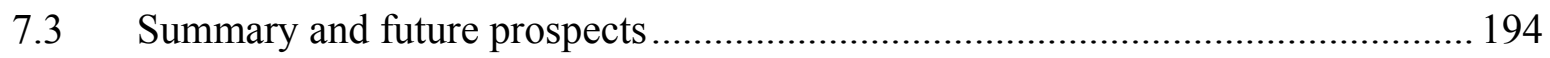

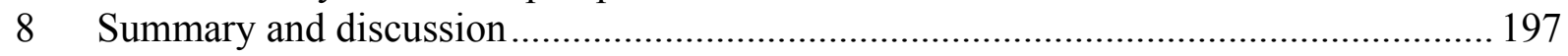





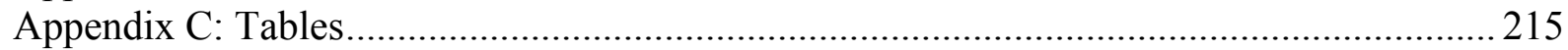



Appendix E: MDC-NOZOMI Publications ................................................................. 245








\section{Figures}

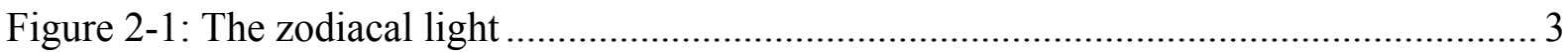

Figure 2-2: A micrometer sized dust particle ................................................................. 4

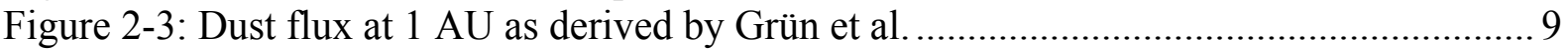

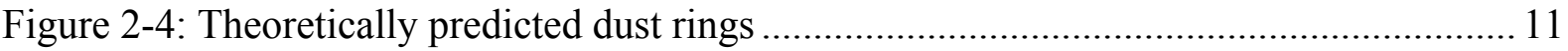

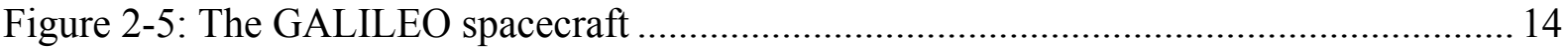

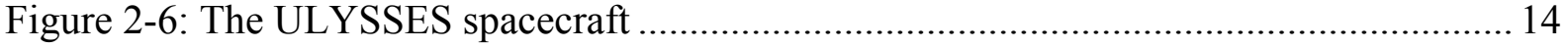

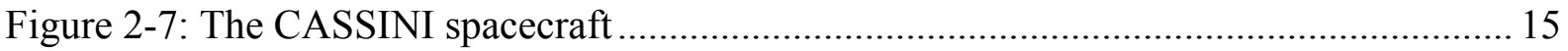

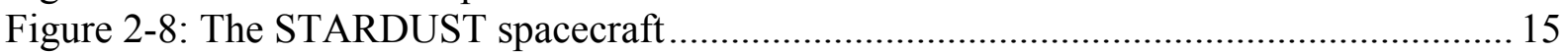

Figure 2-9: The CASSINI Cosmic Dust Analyzer............................................................. 16

Figure 2-10: The HITEN spacecraft............................................................................... 17

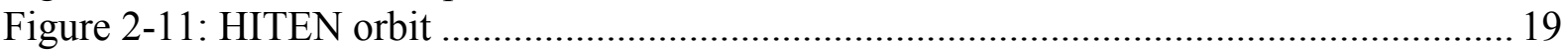

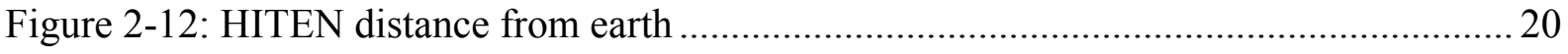

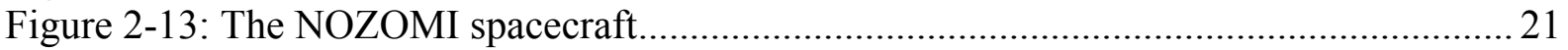







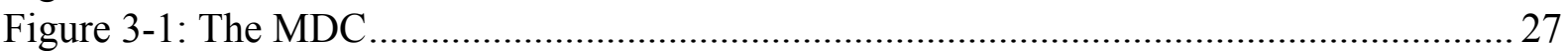

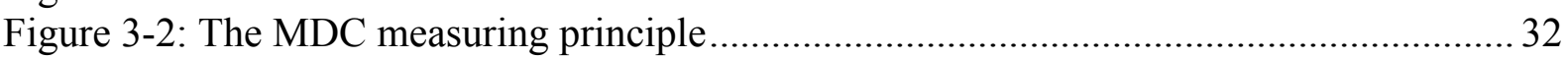

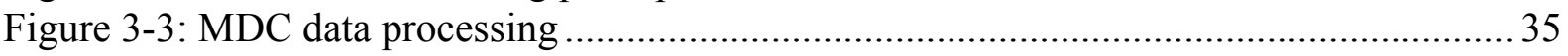

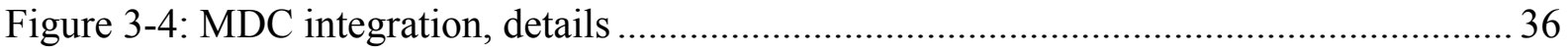

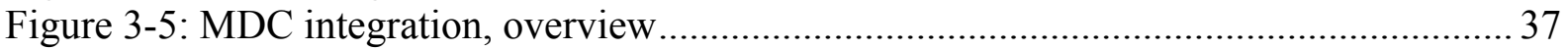

Figure 3-6: Latitude $\beta$ and longitude $\lambda$ of an impacting particle ......................................... 38

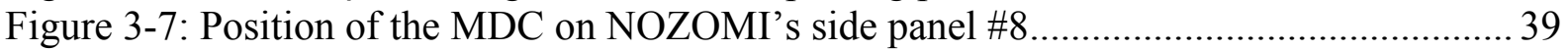



Figure 3-9: Penetrability of the MDC entrance grid over particle size.................................. 41

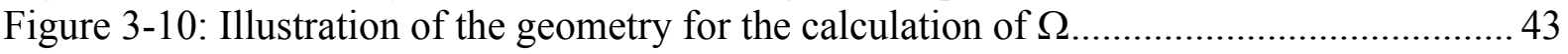





Figure 3-13: Particle size dependence of the effective solid angle $\Omega_{\mathrm{MDC}}(D)$......................... 47

Figure 3-14: Geometry of the calculation of the spin-averaged sensitivity ........................... 48

Figure 3-15: Spin-averaged sensitivity of the MDC on NOZOMI ...................................... 49

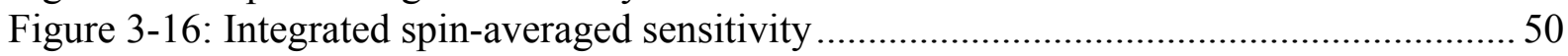



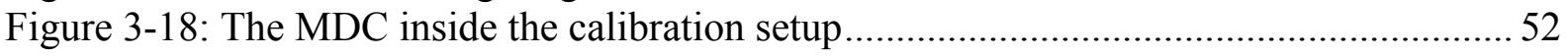

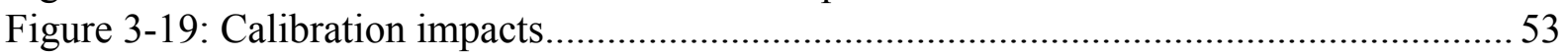

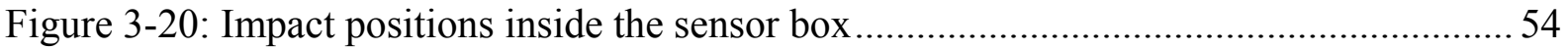

Figure 3-21: Typical calibration signal with characteristic points …..................................... 55

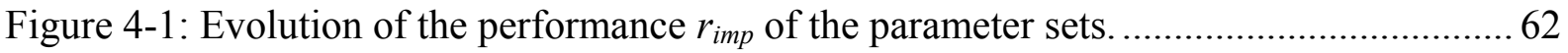

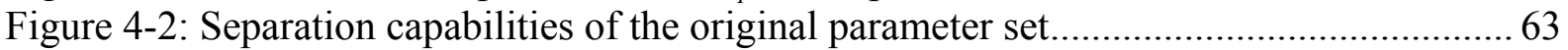

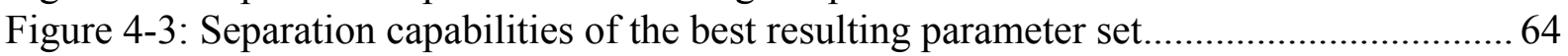



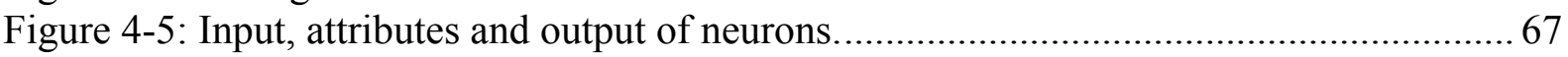

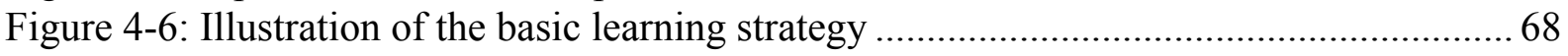

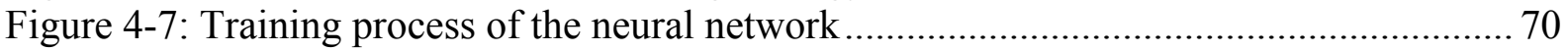

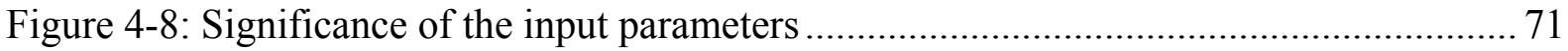

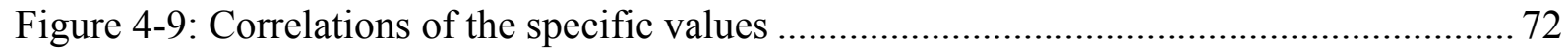


Figure 4-10: Final topology of the neural network with one hidden layer ........................... 75

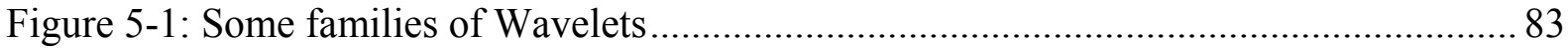

Figure 5-2: Decomposition tree of an MDC neutral channel signal ....................................... 85

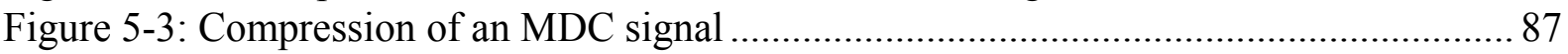

Figure 5-4: Performance of the data compression ........................................................ 88

Figure 5-5: Flow chart of data processing of the new memory management ......................... 91

Figure 5-6: A typical MDC signal before and after compression ..........................................93

Figure 5-7: Impact speed ratio new/original analysis, MDC-HITEN …................................ 96

Figure 5-8: Particle mass ratio new/original analysis, MDC-HITEN ...................................... 97

Figure 5-9: Impact speed ratio new/original analysis, MDC-NOZOMI …............................. 97

Figure 5-10: Particle mass ratio new/original analysis, MDC-NOZOMI ............................... 98

Figure 6-1: Angle of the NOZOMI spin-axis to the Sun over mission time......................... 105

Figure 6-2: A series of noise signals with period $\approx$ NOZOMI spin-period .......................... 107

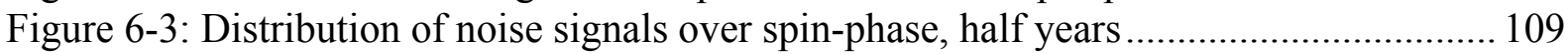

Figure 6-4: Distribution of noise signals over spin-phase, spin-dependency ...................... 110

Figure 6-5: 2-Dimensional illustration of the principles of cluster analysis .......................... 111

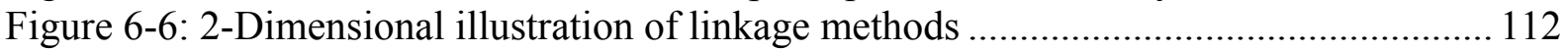

Figure 6-7: Results of the evaluation of metric and linkage method .................................... 114

Figure 6-8: Cluster analysis result of MDC eval. noise data and control data.......................... 116

Figure 6-9: Hierarchy tree of MDC evaluation noise data and control data .......................... 117

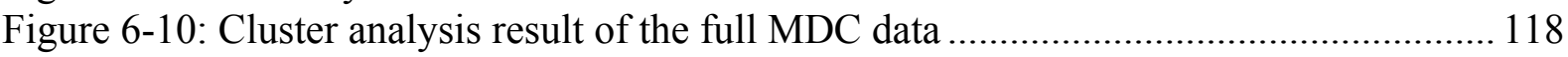

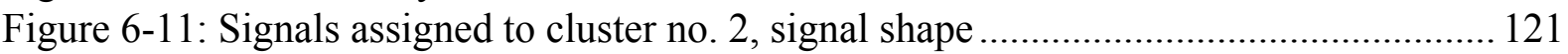

Figure 6-12: Illustration of NOZOMI attitude $(\lambda, \beta)$ and spin-axis vector $\mathrm{s}_{\text {eec }} \ldots \ldots \ldots \ldots \ldots \ldots \ldots \ldots . . . . . . . . .123$

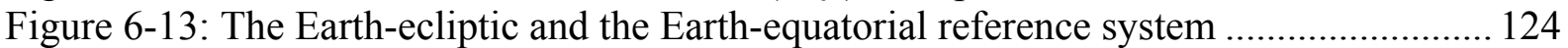

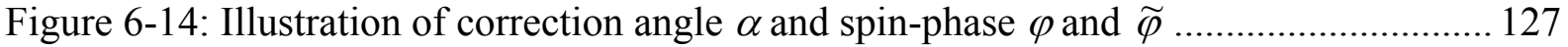

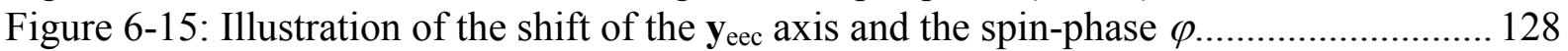

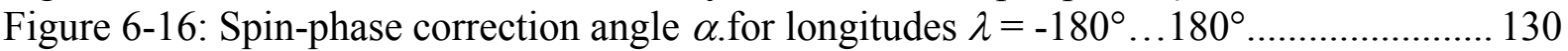

Figure 6-17: NOZOMI spin-axis latitude $\beta$ over mission time, 1998-2002 …...................... 131

Figure 6-18: NOZOMI spin-axis longitude $\lambda$ over mission time, 1998-2002 …................. 132

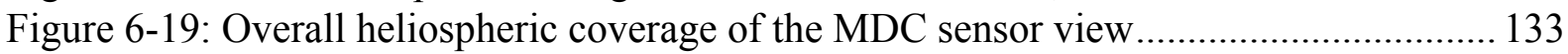

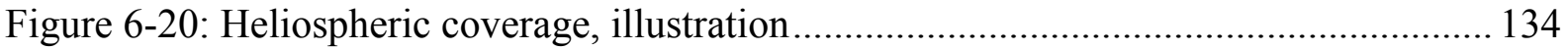

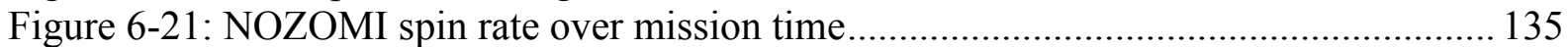

Figure 6-22: Angle of NOZOMI spin-axis to NOZOMI radius vector................................. 136

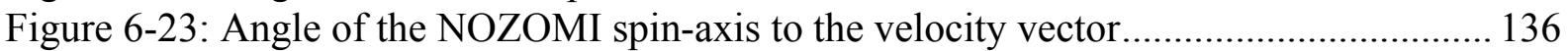

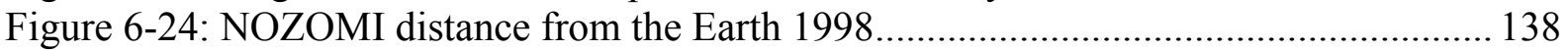

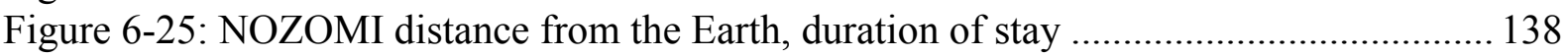

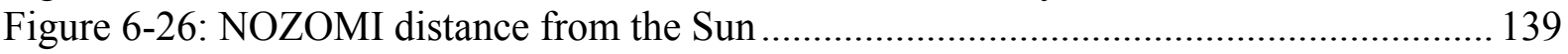

Figure 6-27: NOZOMI distance from the Sun, duration of stay ........................................ 139

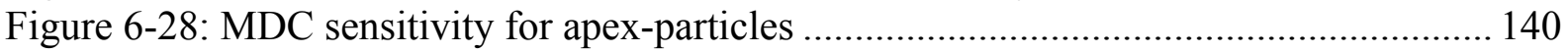

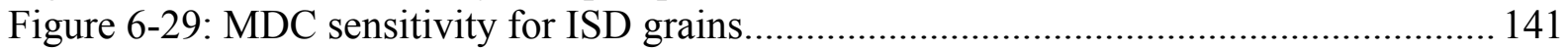

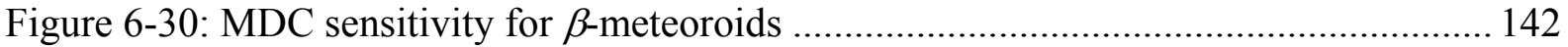

Figure 6-31: Simulation of inclination of a model particle population.................................. 143

Figure 6-32: Simulation of eccentricity of a model particle population ................................ 144

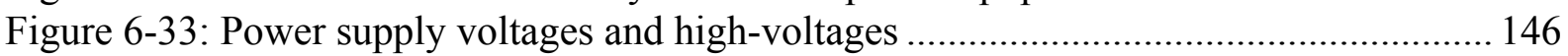

Figure 6-34: Temperatures of the MDC electronics- and sensor-box.................................. 147

Figure 6-35: Spatial distribution of the particles in the Earth-Moon system ......................... 149

Figure 6-36: Mass vs. imp. speed of the particles in the Earth-Moon system ....................... 150

Figure 6-37: Mass vs. abs. speed of the particles in the Earth-Moon system ....................... 152

Figure 6-38: Mass vs. abs. speed of the particles in the eec system .................................... 152

Figure 6-39: Orbital radius vs. abs. speed of the particles in the eeq system ....................... 153 
Figure 6-40: Orbital radius vs. abs. speed of the HITEN particles ...................................... 153

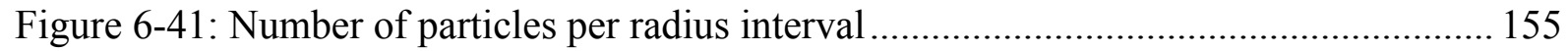

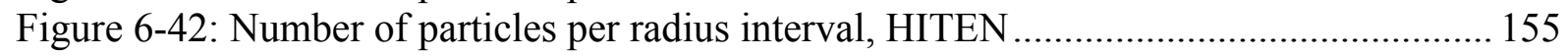

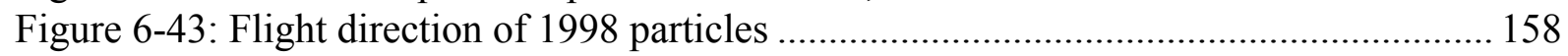

Figure 6-44: Spatial distribution of the particles in the interplanetary space........................ 161

Figure 6-45: Mass vs. impact speed of the particles in the int. space .................................. 162

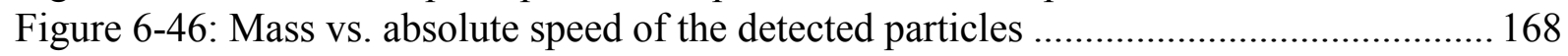

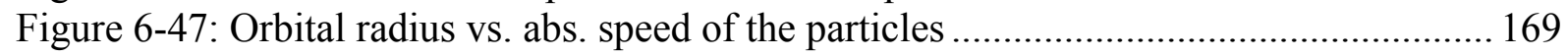

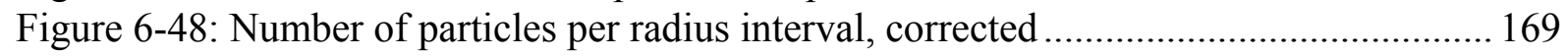

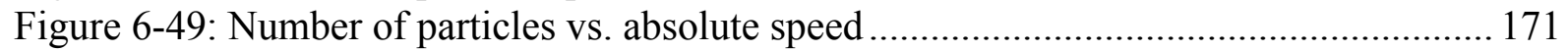

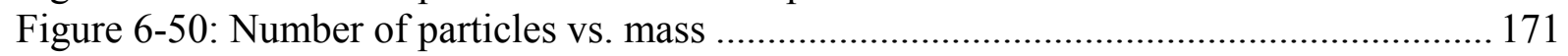

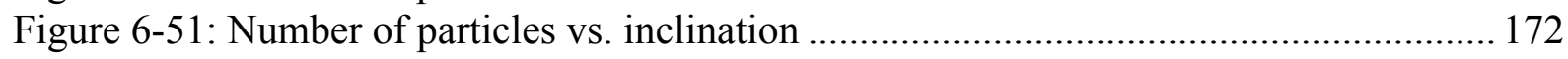

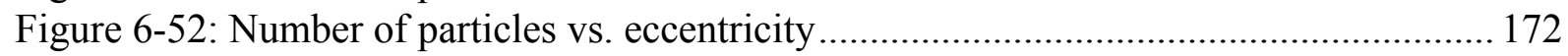

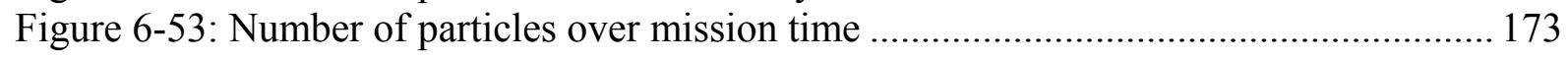

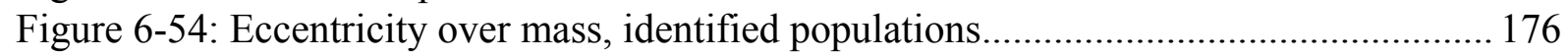

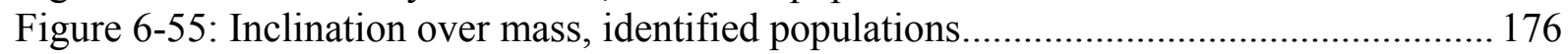

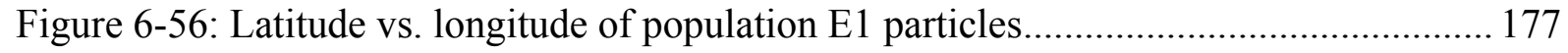

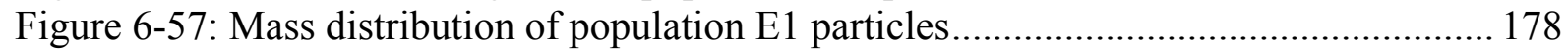

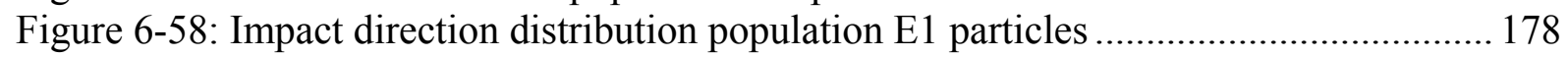

Figure 6-59: Eccentricity distribution population E1 particles ........................................ 179

Figure 6-60: Inclination distribution population E1 particles ........................................... 179

Figure 6-61: Latitude vs. longitude of population E2 particles.......................................... 180

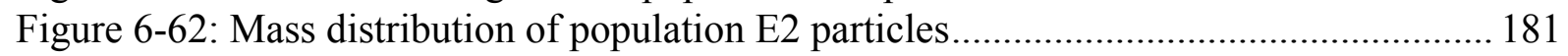

Figure 6-63: Impact direction distribution population E2 particles .................................... 181

Figure 6-64: Eccentricity distribution population E2 particles ......................................... 182

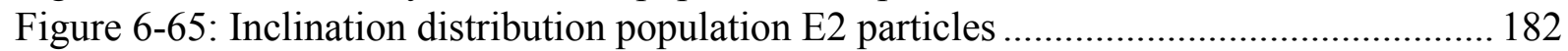

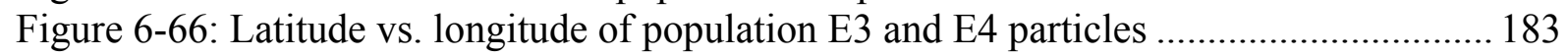

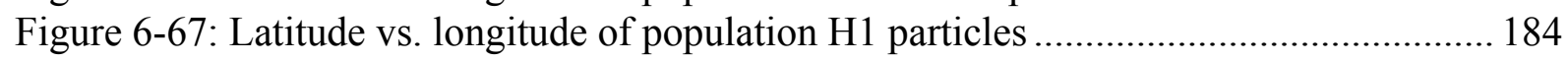

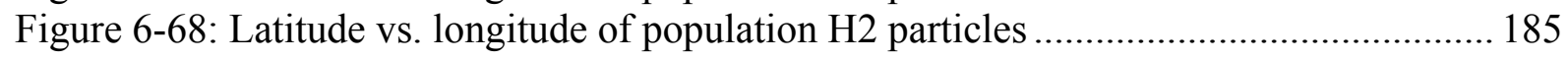

Figure 7-1: Table structure of the MDC online database ................................................ 191 



\section{Tables}

Table 2-1: Interplanetary missions carrying in situ dust experiments ................................... 13

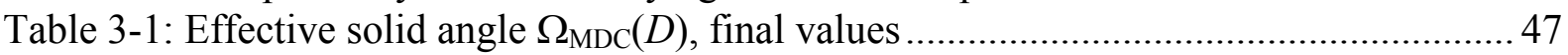

Table 4-1: Results of the optimization of the quality routine parameter set ............................. 64

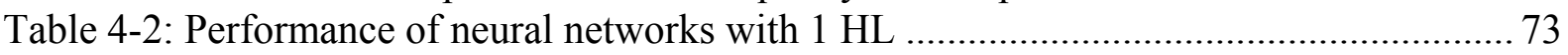

Table 4-3: Performance of neural networks with 2 HL, best 15 topologies ........................... 74

Table 5-1: Validation of the compression on the MDC-HITEN data ..................................... 99

Table 5-2: Validation of the compression on the MDC-NOZOMI data ................................. 99

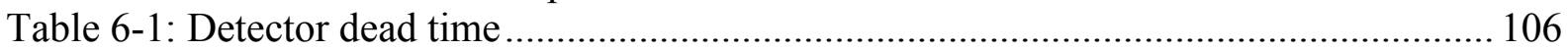

Table 6-2: Results of the evaluation of metric and linkage method...................................... 114

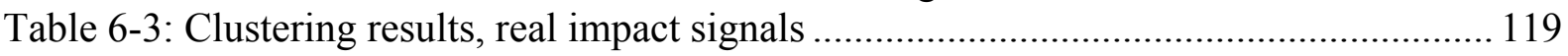

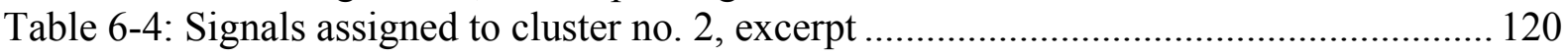

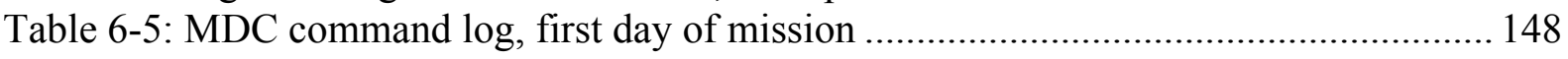

Table 6-6: Detected particles in the vicinity of Earth and Moon ......................................... 151

Table 6-7: Dust flux measurements by MDC-HITEN and MDC-NOZOMI....................... 157

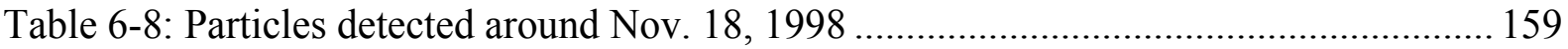

Table 6-9: Detected particles in the interplanetary space, Part 1 ......................................... 163

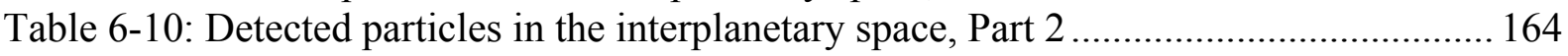

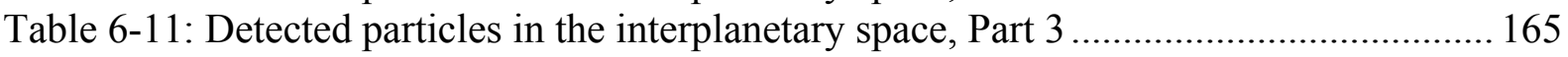

Table 6-12: Comparison of the MDC results and the Grün model..................................... 166

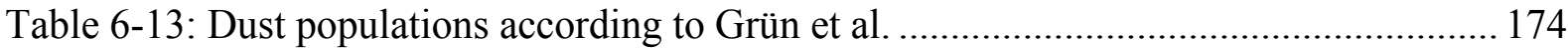

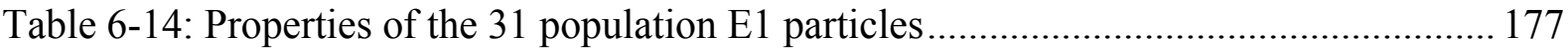

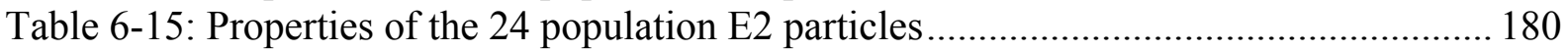

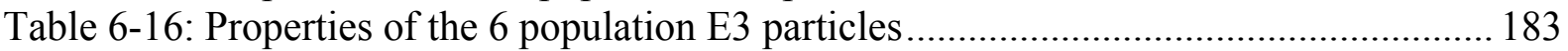

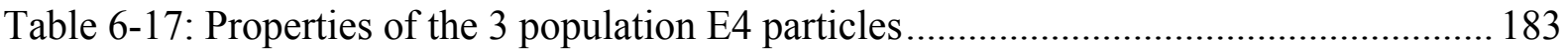

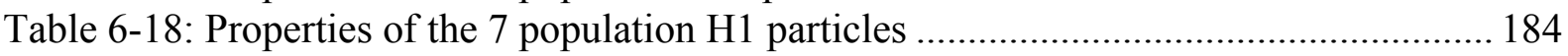

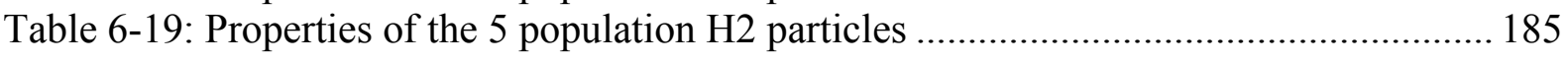





\section{Acronyms and Symbols}

\begin{tabular}{|c|c|}
\hline $\mathrm{A} / \mathrm{D}$ & analog to digital \\
\hline AOCS & Attitude and Orbit Control System \\
\hline CI-PSU & Common Interface Power Supply Unit \\
\hline CSS & cascaded style sheet \\
\hline $\mathrm{EC}$ & electron channel \\
\hline $\mathrm{EC}$ & event count \\
\hline eec & Earth-ecliptic reference system \\
\hline eeq & Earth-equatorial reference system \\
\hline FIFO & first in first out \\
\hline GEO & geostationary Earth orbit \\
\hline HGA & high gain antenna \\
\hline HGA & high gain antenna \\
\hline IC & ion channel \\
\hline $\mathrm{IC}$ & impact count \\
\hline IDP & Interplanetary Dust Particles \\
\hline IMF & Interplanetary Magnetic Field \\
\hline ISAS & Institute of Space and Astronautical Science \\
\hline ISD & Interstellar Dust \\
\hline ISM & Interstellar Medium \\
\hline $\mathrm{J}-\mathrm{KSC}$ & Kagoshima Space Center \\
\hline JPL & Johnson Propulsion Laboratory \\
\hline JSP & java server pages \\
\hline LDEF & Long Duration Exposure Facility \\
\hline LEO & low Earth orbit \\
\hline LGA & low gain antenna \\
\hline LIC & Local Interstellar Cloud \\
\hline LRT & Lehrstuhl für Raumfahrttechnik \\
\hline LRZ & Leibniz Rechenzentrum der Bayerischen Akademie der Wissenschaften \\
\hline MDC & Mars Dust Counter on NOZOMI \\
\hline MDC-BremSat & Munich Dust Counter on BremSat \\
\hline MDC-HITEN & Munich Dust Counter on HITEN \\
\hline MDC-NOZOMI & Mars Dust Counter on NOZOMI \\
\hline MLI & Multi Layer Insulation \\
\hline MMR & Mean Motion Resonance \\
\hline MUSES-A & Former name of the HITEN spacecraft \\
\hline NASA & National Aeronautics and Space Administration \\
\hline $\mathrm{NC}$ & neutral channel \\
\hline $\mathrm{NC}$ & Noise count \\
\hline noz & NOZOMI fixed reference system \\
\hline $\operatorname{noz} \varphi$ & NOZOMI fixed reference system, rotated by the angle $\varphi$ \\
\hline PLANET-B & Former name of the NOZOMI spacecraft \\
\hline PVDF & polyvinylidene fluorid \\
\hline RAM & random access memory \\
\hline ROM & read only memory \\
\hline SSAS & Spin-type Sun Aspect Sensor \\
\hline SSOC & Sagamihara Spacecraft Operation Center \\
\hline TUM & Technische Universität München \\
\hline
\end{tabular}


UDSC

VBA

XML

A

$\mathrm{mA}$

AU

bit

bps

kbps

Byte

kByte, kB

C

deg

g

$\mathrm{kg}$

$\mathrm{h}$

$\mathrm{Hz}$

$\mathrm{kHz}$

$\mathrm{m}$

$\mathrm{mm}$

$\mathrm{km}$

$\mathrm{m}, \min$

$\mathrm{rad}$

rpm

$\mathrm{s}, \mathrm{sec}$

$\mathrm{ms}$

$\mu \mathrm{s}$

$\mathrm{Sr}$

$\mathrm{V}$

W
Usuda Deep Space Center

Visual Basic ${ }^{\circledR}$ for Applications

eXtensible Markup Language

Ampere

milliampere

Astronomical Unit

binary digit

bits per second

kilobits per second

Byte

kilobyte

Coulomb

degree

gram

kilogram

hour

Hertz

kilohertz

meter

millimeter

kilometer

minute

radian

revolutions per minute

second

millisecond

microsecond

sterradian

Volt

Watt 


\section{Introduction}

The main subject of this work is the operation and data analysis of the space science experiment "Mars Dust Counter", abbreviated as MDC (or in some cases, MDC-NOZOMI to avoid confusion of names, since the predecessors are named "Munich Dust Counter", MDC) in the following. The MDC was designed and developed by the Lehrstuhl für Raumfahrttechnik (LRT) at the Technische Universität München (TUM) from 1995 to 1998. Its deep space mission began on July 4, 1998, when it was launched by a Japanese M-V launcher from Kagoshima/Japan onboard Japan's first interplanetary mission NOZOMI (formerly PLANET-B), and was completed by the turn of the year 2003/2004 when the NOZOMI space probe was lost due to technical failure. An overview about the whole mission is given in paragraph 2.3 .

The MDC's primary scientific mission was the detection of interplanetary, interstellar and circumplanetary cosmic dust particles. A brief overview on origin and properties of cosmic dust particles is given in paragraph 2.1. Although the MDC could not fulfill its main mission goal, which was the detection of theoretically predicted dust rings around Mars, the experiment was able to collect valuable scientific data about cosmic dust in the Earth-Moon system and in the interplanetary space between the orbits of Earth and Mars. These scientific results are described in chapter 6.

Despite its name, which can be translated as "hope", the satellite NOZOMI was a quite unlucky guy. Several technical problems that occurred one after the other since mission launch in 1998 not only challenged the operators at the Japanese Institute of Space and Astronautical Science (ISAS), but also the operators of the MDC who had to think about workarounds that were supposed to secure the MDC operation and data transmission despite the aggravated environmental conditions. These considerations and solutions are described in chapters 4 and 5 .

The technical properties of the MDC, its onboard computing and data processing capabilities and the final data analysis process, all which set the boundaries to the upgrades and workarounds mentioned above, are described in chapter 3. The actual data analysis software and the MDC data formats are based on proprietary single user software solutions and local data storage, which can cause problematic redundancies in data storage, software version conflicts and even incompatibilities in a worldwide-distributed scientific community. A solution of this problem that is also a proposal for future projects, based on a database system with worldwide access via standard software and XML-based data formats, is presented in chapter 7.

Finally, the results of this work are summarized and discussed in chapter 8 .

The Mars Dust Counter Project was supported by the Bundesministerium für Bildung und Forschung (BMBF) and the Deutsches Zentrum für Luft- und Raumfahrt (DLR) under the grants 50QM9501 (1995 to 1998) and 50QM9801 (1998 to 2004). The author of this work was in charge of the MDC operations and data analysis from July 2001 until December 2004. 



\section{Exploration of cosmic dust}

The existence of cosmic dust particles was known long before the scientific exploration could take place, due to phenomena that can be viewed without technical utilities like meteors and the zodiacal light, a phenomenon that is caused by the scattering of sunlight on small particles in space. It was first described and correctly interpreted in 1683 by Giovanni Domenico Cassini [Cas 1699] (Cassini also proposed that the zodiacal light may have been be the biblical phenomenon known as the "Star of Bethlehem"). The scientific exploration of cosmic dust began in the early 1950s with the beginning of human space exploration, when the first acoustic dust detection devices were flown onboard A-4 launchers. Main intention of these first dust detection devices and later devices in the low Earth orbit was to determine the danger that future spacecrafts are exposed to due to the existence of micrometeoroides. Today, spacecrafts in the low Earth orbit (LEO) and also in geostationary Earth orbit (GEO) are exposed to another, man-made hazard caused by waste products of the exploration of space itself, the so-called space debris. The properties of cosmic dust and space debris are discussed in paragraph 2.1.

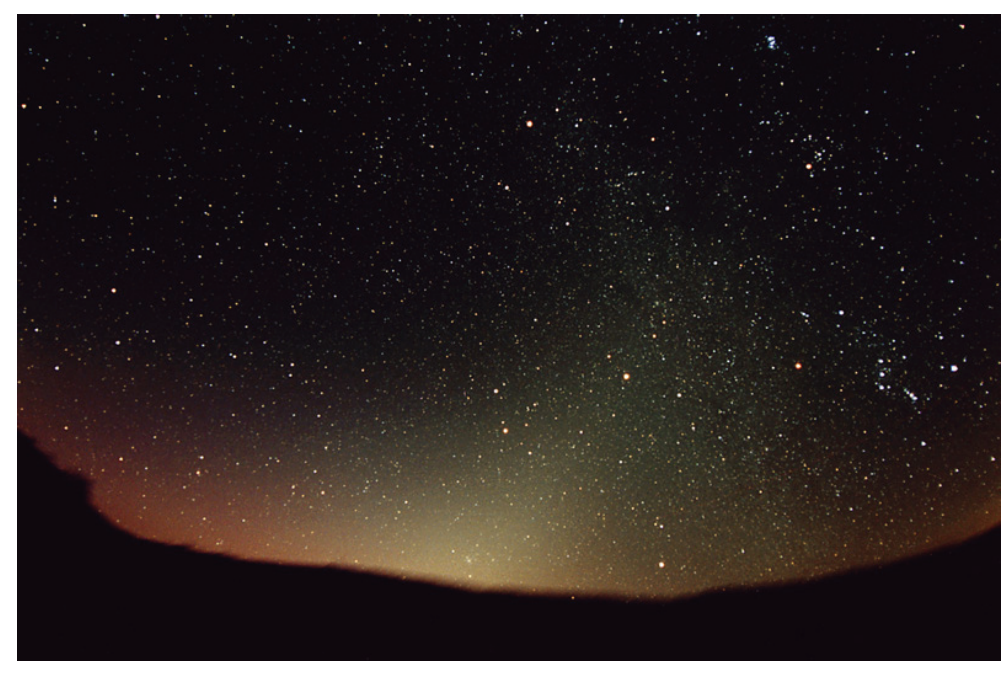

Figure 2-1: The zodiacal light

Photograph of the zodiacal light, taken facing east just before the beginning of the morning twilight.

The MDC-NOZOMI and its predecessors were flown on three missions until now. While the BremSat mission was only a short term mission in the low Earth orbit and suffered from a troublesome data analysis due to massive interferences, the two missions on the satellites HITEN and NOZOMI were a great success for the MDC and the Lehrstuhl für Raumfahrttechnik. The first mission HITEN was launched in 1990 and was cruising in the vicinity of Earth and Moon for about three years. The actual mission NOZOMI was launched in 1998 and was cruising between Earth orbit and Mars orbit for nearly 4 years. In both missions, the MDC was working properly and yielded valuable data from the beginning to the end of the missions. The missions HITEN and NOZOMI are described in paragraph 2.2 and 2.3 , respectively. 


\subsection{Dust in the solar system}

Within the scope of this work, only a brief introduction on the actual knowledge about cosmic dust can be given. Cosmic dust research covers a wide field of topics related to each other, including e.g. solar and planetary formation and evolution, formation of comets and asteroids, interstellar dust clouds, and exobiology. In the following, the known properties of cosmic dust derived from observations and theoretical considerations in the solar system as it is today are discussed. The term interplanetary dust (or interplanetary dust particles, IDPs) is used for dust grains that are orbiting on heliocentric trajectories and are either gravitationally bound to the heliosphere or originate directly from solar system objects. In paragraph 2.1.1, a short and only qualitative introduction into the physical properties and the complex orbital dynamics of dust particles in the heliosphere is given. Interplanetary dust (also named zodiacal dust) and interstellar dust are discussed in paragraph 2.1.2. The term interstellar dust (ISD) is used for particles that do not originate from the solar system, but are part of the interstellar medium (ISM) or, more precisely, of the local interstellar cloud (LIC) that surrounds the sun. As the properties of the ISM are not discussed in this work, the term interstellar dust is limited to interstellar dust particles that penetrate into the solar system due to the relative motion of the sun through the LIC. Dust surrounding the planets (circumplanetary dust), with a special respect to the proposed dust rings around Mars, is discussed in paragraph 2.1.3. For the sake of completeness, a special, man-made population of particles from macroscopic to microscopic sizes orbiting the Earth and known as space debris is discussed in paragraph 2.1.4. In paragraph 2.1.5, a short description of recent missions of dust experiments is given.

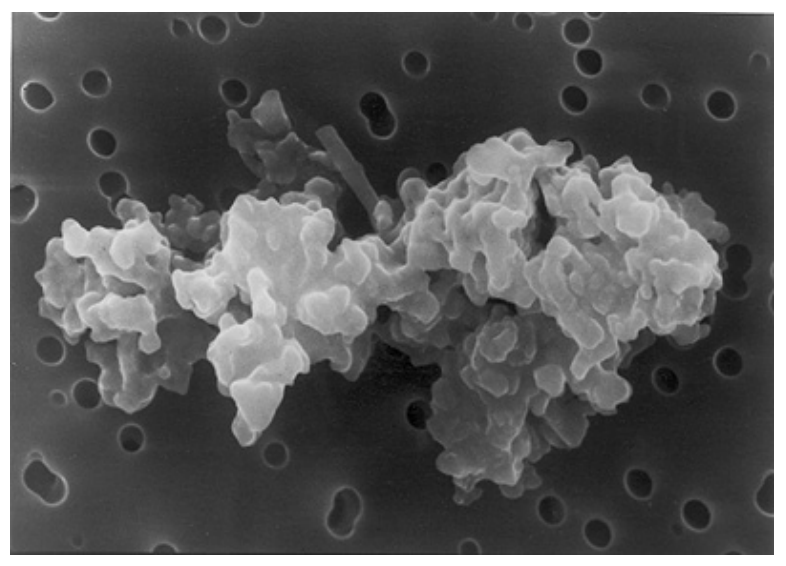

Figure 2-2: A micrometer sized dust particle

Photograph of a micrometer sized dust particle like it can be detected by the MDC experiment. The shown particle is $0.01 \mathrm{~mm}$ in size, and has a mass of $10^{-9} \mathrm{~g}$. 


\subsubsection{Physical properties and orbital dynamics}

Most information about structure, chemical composition and optical properties of cosmic dust particles is gained from collected samples. Sample collection is not limited to in-situ collection with sample return space experiments like LDEF or on the STARDUST mission, but was carried out even before space flight became possible by collection of micrograins in suitable environments on Earth, e.g. from the antarctic peninsula (see e.g. Schmidt [Sch1964]) or the ground sediments of the oceans (see e.g. Grjebine [Grj1964]). Microparticles were also collected from the stratosphere by experiments on high altitude aeroplanes, see Melton [Mel1964]. Typically, dust particles have chondritic elemental composition, dark surface and are porous or fluffy. Densities vary between 1 and $3 \mathrm{~g} / \mathrm{cm}^{3}$ for most investigated particles, with a mean value of $2.0 \mathrm{~g} / \mathrm{cm}^{3}$, see Love et al. [Lov1994].

Unlike the largest celestial bodies, the planets, the orbital dynamics of small bodies and dust are not only subject to the gravitational force, but are also affected or even dominated by a variety of minor forces that originate from solar radiation, solar wind, and interplanetary and planetary magnetic fields. In the following, the various forces acting on cosmic dust particles and their basic influences on the orbital evolution of the particles are discussed.

Gravitation itself exploits different perturbations on a dust particle's orbit. Particles orbiting the sun on a typical low inclination orbit may encounter one of the inner planets and experience significant changes of the orbital elements inclination $i$, eccentricity $e$ and longitude of the ascending node $\Omega$, as Gustafson \& Misconi [Gus1986] have demonstrated by model calculations of the trajectories of sample particles between $10 \mu \mathrm{m}$ and $100 \mu \mathrm{m}$ in size encountering Earth and Venus during $\mathrm{a} \approx 6000$ year journey from $1.1 \mathrm{AU}$ down to $0.3 \mathrm{AU}$. Particles may even be trapped in mean-motion-resonances (MMRs) of the Sun-Planet system (which means, that the particle's evolution in orbital elements is then primarily controlled by the trapping planet, while it is still in heliocentric orbit), and (with consideration of further disturbances caused by radiation pressure and the planets magnetosphere) finally form a circumsolar dust ring, see Liou et al. [Lio1995a], [Lio1997]. In a similar study on trapping of dust in the Earth's 1/1 resonance, Marzari \& Vanzani [Mar1994] found long-lasting, up to $10^{5}$ years trapping of particles in Trojan-type orbits. Retrograde dust particles originating from retrograde sources nearly always get trapped in MMRs mainly with Jupiter, and even can evolve to prograde orbits when trapped in long-lasting resonances until they are ejected from the resonance, as Liou et al. [Lio1999] showed by theroretical modeling. Circumplanetary dust particles orbiting planets are even exposed to further gravitational disturbances caused by the oblateness of the specific planet, or a possibly irregular shape of the planet's moons, see Davis et al. [Dav1981] for a discussion of the gravitational environment in the Mars-PhobosDeimos system.

The radiation of the Sun has strong effect on the orbital evolution of smaller bodies in the solar system. Macroscopic bodies in a size range of meters to some 100 meters like asteroids are affected by the Yarkovski drag that was discovered by I.O. Yarkovski in 1900, a force that results as a consequence of the time a rotating bodies surface needs to warm up when exposed to sunlight and cool down subsequently. The radiation pressure resulting from the thermal emission of the heated surface that has rotated away from direct sunlight produces a drag that leads to an increase or decrease of the semi-major axis $a$ of a prograde or retrograde rotating body, respectively. The Yarkovski effect can be observed directly by radar observation of 
asteroids, see Chesley et al. [Che2003]. In contrary, microscopic bodies are always in thermal equilibrium, and the therefore isotropic thermal emission does not produce a net radiation force. For particles lighter than $10^{-10} \mathrm{~g}$, the radial pressure originating from the Sun's radiation becomes evident. The resulting force is strongly dependent on the particle's albedo and density and is usually described by the ratio $\beta$ of the radiation force to the gravitational force, which is independent from the heliocentric distance and defined as $\beta=F_{\mathrm{rad}} / F_{\text {grav }}$, see Burns et al. [Bur1979]. Typical values of $\beta$ were obtained by Gustafson [Gus1994] by Mie-theory for homogenous spheres with optical constants of the astronomical silicate model by Draine \& Lee [Dra1984], and show that radiation pressure is negligible $(\beta=0)$ for particles with mass greater than $10^{-10} \mathrm{~g}$, has a maximum for particles with mass $10^{-13} \mathrm{~g}(\beta=1.5)$ and decreases for smaller particles when particle size falls below the wavelengths of the solar radiation. Wilck $\&$ Mann [Wil1996] also calculated $\beta$-values from Mie-theory but used models of "typical" dust grains (asteroidal, young cometary, old cometary and interstellar) instead of solid spheres that represent the known albedo and density values of the grains. For all dust particle models, except the fluffy young cometary particles, the calculated $\beta$-values had a maximum of around 0.9 at $m=10^{-13} \mathrm{~g}$. Particles with $\beta>1$ will spiral outwards from the Sun due to the Sun's radiation pressure. Particles that show this behavior are called $\beta$-meteoroids. Another weak, but highly important effect of radiation on a particle's orbit was first described (and misinterpreted) by Poynting [Poy 1903] and discussed later by Robertson [Rob1937], named the Poynting-Robertson effect after its discoverers. The Poynting-Robertson drag causes a loss in orbital angular momentum of particles orbiting the Sun, therefore the particles spiral inwards into the Sun in $400 r^{2} / \beta$ years ( $r$ given in astronomical units) with decreasing eccentricity. A descriptive explanation of the effect is that the radial traveling photons of the Sun's radiation hit a particle on a circular orbit in an angle lower than $90^{\circ}$ to its motion vector as seen from the particle's own inertia system, and thus produce a small braking force. An exact physical interpretation of the Poynting-Robertson effect requires covariant calculations and is given by Srikanth [Sri1999]. Although the Poynting-Robertson drag force is very low, it has significant effect on dust particles' orbital evolution. Further, non-radial drag forces caused by solar radiation that may originate from irregular particle shape are discussed but not well established. Ion impact drag force and the plasma Poynting-Robertson effect caused by solar wind particles are very low (around $10^{4}$ times weaker than the radiation pressure), but due to the non-isotropic nature of the mass flow (decrease by about $50 \%$ from the ecliptic plane to the poles) the plasma PR drag effects orbital elements and increases orbital lifetime of particles on high inclined orbits by 5-10\% (Banaszkiewicz et al. [Ban1994]). Unlike this, planetary perturbations on a particle's eccentricity can reduce the orbital lifetime by a few percent up to $50 \%$ due to altered PR drag on high eccentric orbits, see Gustafson et al. [Gus1987a].

Particles inside the heliosphere are exposed to ultraviolet radiation and a flow of electrons and ions known as solar wind. In laboratory simulation experiments, Cermák et al. [Cer1995] demonstrated that the equilibrium electrostatic potential of dust particles bombarded by ion and electron streams is around $+5 \mathrm{~V}$. Several theoretical studies about charging of dust grains inside plasmas of planetary magnetospheres (reviewed by Horányi [Hor1996b]) showed that the effective equilibrium potential of dust grains depends strongly on the individual characteristics of the plasma and varies between $-30 \mathrm{~V}$ and $+10 \mathrm{~V}$. Due to this usually nonzero electrostatic potential of dust grains, they couple to the interplanetary magnetic field (IMF) or planetary magnetic fields. In the heliosphere, the electrostatic potential of dust grains is around a few volts positive due to the dominating effect of photo-ionization by UV radiation. The IMF is mainly dominated by the solar magnetic field that is carried outside from the Sun by the solar wind plasma. Theoretical considerations by Consolmagno 
[Con1980] showed that the altering polarity of the field can have a random scattering effect on the orbital elements of small dust grains $(<3 \mu \mathrm{m})$ in the order of the Poynting-Robertson effect. Hamilton [Ham1994] showed by theoretical modeling that Lorentz forces acting on a particle within a planet's magnetosphere can induce orbital resonances similar to those of the gravitational force.

\subsubsection{Interplanetary and interstellar dust in the heliosphere}

Dust in the solar system is mainly produced by mutual collisions of larger objects. Most important sources of interplanetary dust particles are asteroids and comets (see e.g. Mann et al. [Man1996a], Liou \& Zook [Lio1996b], Liou et al. [Lio1995b]). Also, Kuiper-belt objects contribute to the zodiacal cloud in the inner solar system, as it was shown by Liou et al. [Lio1996a] and Landgraf et al. [Lan2002], although most of the dust produced in the Kuiperbelt is ejected from the solar system by the giant planets.

For the determination of the overall flux of interplanetary dust, several sources of information are available and were used for developing models. Besides specialized in-situ dust detection experiments that are discussed in paragraph 2.1.5, lunar microcraters were an important source of information about spatial density and mass distribution of cosmic dust particles, see Hartung [Har1976]. Also, the surfaces of returned spacecraft, e.g. periscopic lenses of the early Mercury missions (see Hemenway et al. [Hem1964]) or the replaced and returned Hubble Space Telescope solar arrays (see Moussi et al. [Mou2005]) give information about flux and mass distribution of micrometeoroids.

The dust population in the solar system is not static, but undergoes a permanent dynamical evolution that is discussed by Grün et al. [Grü1985]. For particle masses $>10^{-3} \mathrm{~g}$, many more particles are destroyed by collisions than are generated, while for particle masses $<10^{-6} \mathrm{~g}$ more new particles are generated than lost. Average lifetime until destruction by collision for particles with mass between $10^{-4} \mathrm{~g}$ to $1 \mathrm{~g}$ is about $10^{4}$ years at $1 \mathrm{AU}$, which is short in comparison to the age of the solar system. It was estimated that about 9 tons/sec of particles with masses $>10^{-5} \mathrm{~g}$ are lost inside 1 AU due to collisions. For particle mass between $10^{-5} \mathrm{~g}$ and $10^{-10} \mathrm{~g}$, production rate is 16 times larger than the net loss by Poynting-Robertson drag, which is estimated to 0.26 tons/sec. The main loss mechanism of cosmic dust in the solar system is caused by solar radiation pressure, as discussed above. Particles with mass $<10^{-10} \mathrm{~g}$ are affected by this force due to their small size, are largely injected into hyperbolic orbits and leave the solar system as so called $\beta$-meteoroides.

Most information about the three-dimensional shape of the interplanetary dust cloud is obtained by remote observation of the intensity of the zodiacal light. Polarization of the scattered sunlight also gives information about the surface structure of the scattering particles. Observation of the thermal emission of the dust particles can also be used to determine the three-dimensional structure of the dust cloud, see e.g. Levasseur-Regourd \& Dumont [Lev1990]. At $1 \mathrm{AU}$, temperatures of 130 to $190 \mathrm{~K}$ and 380 to $390 \mathrm{~K}$ for particle sizes of $10^{-4}$ and $10^{-6} \mathrm{~m}$ in size were obtained by Staubach et al. [Sta1993]. Observations by the IRAS spacecraft ("Infrared Astronomical Satellite", see Neugebauer et al. [Neu1984]) revealed even local dust bands that were soon assigned to cometary dust trails, see Sykes \& Walker [Syk1992] for a survey. 
To gather information about the orbital dynamics of interplanetary dust particles, in-situ measurements of the particles' trajectory are required. Impact-ionization dust detectors provide the most efficient facilities for the measurement of dust particles' orbital speed and flight direction. Most in-situ detectors were on low inclined orbits. The observed isotropic distribution of the solar F-corona gives evidence to a small population of dust on isotropic inclined orbits. Although it is possible to determine the dust distribution outside the ecliptic plane from in-ecliptic measurements, since all possible orbits cross the ecliptic plane (Mann \& Kneißel [Man1991]), this is limited due to the low detection rates of high inclined particles, which is caused by the short ecliptic encounter of these particles. The dust detector on the ULYSSES spacecraft is the first experiment on a high inclined orbit.

Several models of the distribution of cosmic dust in the solar system were developed from experimental data obtained by optical observations and in-situ measurements. One of the most popular is that of Divine [Div1993]. Five populations of dust particles were described, namely asteroidal, core, halo, inclined and eccentric that are distinguished by their distribution in size and orbital characteristics. Based on the model of Divine, a new model of the interplanetary dust flux which includes new in-situ measurements by the dust detectors on ULYSSES and GALILEO at orbital distances from 0.7 to $5.4 \mathrm{AU}$, and also incorporates radiation pressure forces acting on small dust particles as described in paragraph 2.1.1., was developed (Staubach \& Grün [Sta1995], Grün et. al. [Grü1997]). While the asteroidal and core populations of Divine's model are left unaltered since the mass ranges of the particles in these populations are not covered by the ULYSSES and GALILEO dust experiments, the populations of the lighter particles $\left(m<10^{-9} \mathrm{~g}\right)$ were altered to fit the new dust data, and to include the new population of interstellar dust grains detected by ULYSSES and GALILEO. Three new populations A, B and C of dust grains on bound orbits were defined with different dominant, but overlapping mass range of $10^{-10} \mathrm{~g}$ to $10^{-12} \mathrm{~g}, 10^{-11} \mathrm{~g}$ to $10^{-14} \mathrm{~g}$, and $10^{-12} \mathrm{~g}$ to $10^{-15} \mathrm{~g}$, respectively. Each of the three new populations shows individual characteristics in the distribution of orbital elements eccentricity $e$ and inclination $i$ of its appropriate particles. While population A mainly consists of low inclination, low eccentricity particles, populations $\mathrm{B}$ and $\mathrm{C}$ show a wider distribution of eccentricity and inclination with higher mean values in inclination. All three populations have a peak at low eccentricities, but also a significant component at high eccentricities. In chapter 6 , this model is used to validate the MDC dust data. 


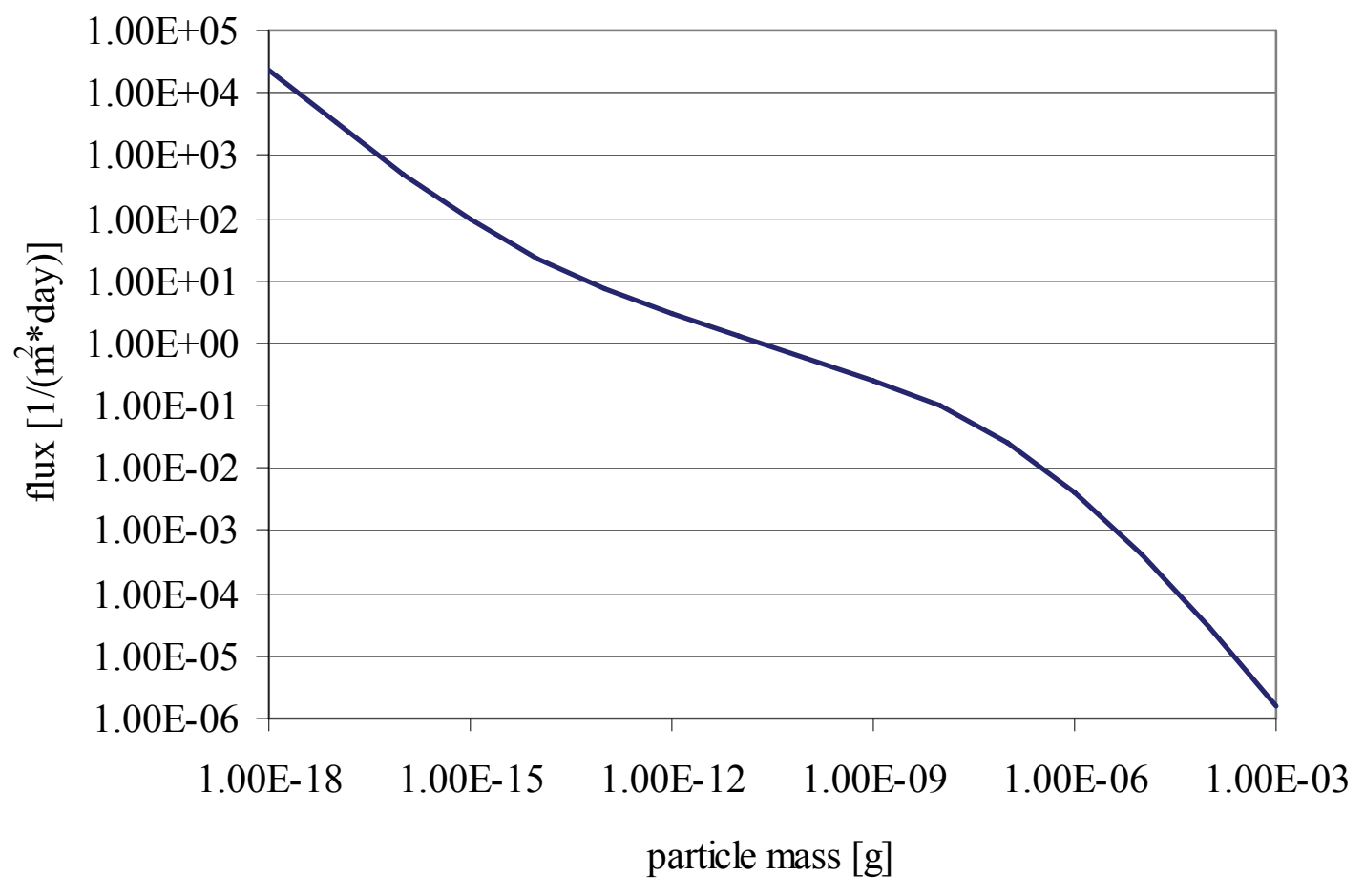

Figure 2-3: Dust flux at $1 \mathrm{AU}$ as derived by Grün et al.

Cumulative flux of cosmic dust particles at $1 \mathrm{AU}$ far away from the Earth as derived by Grün et al. [Grü1985].

Main points of criticism at the models described above are that they do not include any theoretical models of particle dynamics, and that there is no known fundament based on particle dynamics or dust source distribution (e.g. comets) for the empirical definition of the different particle populations. The development of models that include even newer data derived from in-situ measurements (e.g. again GALILEO, ULYSSES, CASSINI) and optical observations (e.g. the DIRBE instrument on COBE), and involve theoretical models of the long-term particle dynamics, are discussed by Dikarev et al. [Dik2002], [Dik2005].

While moving through the interstellar medium, the solar system encounters the interstellar gas and dust cloud, whose existence is known from optical and ultraviolet observations. Currently, the solar system is surrounded by a substructure called the Local Interstellar Cloud (LIC), relative speed of the solar system to the LIC was determined to $26 \mathrm{~km} / \mathrm{sec}$. Dust particles that belong to the interstellar medium penetrate into the inner solar system and contribute to the interplanetary dust flux, although small particles are deflected by the hot plasma in the heliopause, see Linde et al. [Lin2000]. ISD particles that penetrate into the heliosphere interact with the interplanetary magnetic field and are deflected away from the Sun, see Grün et al. [Grü2001]. Interstellar dust particles were detected by the dust detectors on the GALILEO, CASSINI and HELIOS missions in the inner solar system at orbital radii $\leq 1 \mathrm{AU}$, see Altobelli et al. [Alt2005], [Alt2003], [Alt2006], and by the GALILEO and ULYSSES missions outside 1 AU, see Baguhl et al. [Bag1995], [Bag1996]. ISD grains were separated from the interplanetary dust flux by their speed and flight direction. ULYSSES measurements showed that the flight direction of the interstellar dust flux is well aligned with the direction of the interstellar gas flow, which arrives from longitude $252^{\circ}$ and latitude $2^{\circ}$, and never deviates more than $30^{\circ}$ from this direction, see Grün et al. [Grü1997]. ISD grains show a 
constant flux that is independent from orbital radius and ecliptic latitude. Mean mass of the ISD grains detected by ULYSSES at an orbital radius of 5 AU was $10^{-13} \mathrm{~g}$. See also Grün et al. [Grü1994] for a discussion. The in-situ measurements also showed a dependence of the flux of ISD grains from the solar activity due to the filtering effects caused by the interplanetary magnetic field. As Grün et al. [Grü1997] showed, ISD particles with $\beta$-values $>1.5$ will never reach 1 AU. It was shown by theoretical considerations that ISD grains may be captured by the sun by electromagnetic forces (see Kocifaj \& Klačka [Koc2003]), by the Lorentz force (see Kocifaj \& Klačka [Koc2004]) and by Poynting-Robertson drag (see Jackson [Jac2001]). ISD grains are supposed to consist of old matter that originated directly from supernovae explosions, but it is suggested that they undergo significant changes when entering the heliosphere due to solar radiation, see Strazulla [Str1986].

\subsubsection{Circumplanetary dust}

Dust rings around Mars were predicted first by Soter [Sot1971] based on MARINER 6 and MARINER 7 photographs of the Mars surface. They are supposed to reside around the orbits of the two Martian moons Phobos and Deimos. The theoretical considerations are based on the assumption that the two satellites are exposed to the same flux of meteoroides as the crater-covered Mars itself; impacts of these particles should eject material from the surfaces into circum-Martian orbit at velocities of 5 to $15 \mathrm{~km} / \mathrm{sec}$. Since the inner moon Phobos resides inside the Roche limit, particle ejection becomes even easier (see Dobrovolskis et al. [Dob1980]). This material is supposed to form dynamic tori that are probably self-sustained by collisions between 20-50 $\mu \mathrm{m}$ ring particles and the moons Phobos and Deimos.

Unlike the Saturnian ring system, which consists of large bodies and shows a high grade of symmetry (except of the faint E-ring that consists of small dust particles and is currently investigated with the CASSINI dust experiment), the Martian dust rings are subject to a wide range of perturbations and show complex dynamics that lead to spatial and temporal asymmetries. Most significant disturbances of the dust particles besides the strong gravitational forces by the Sun and the moons themselves originate from the solar radiation pressure, electromagnetic forces and the oblate shape of Mars. All these forces show local variations and thus the faint rings show a wide variety of structure over time and space.

Many studies were carried out on the formation and modeling of dust rings around Mars and their supplement by dust grains that originate from impacts on the two moons' surfaces, see Ishimoto \& Mukai [Ish1994], Ishimoto [Ish1996], Banaszkiewicz \& Ip [Ban1991], Kholshevnikov et al. [Kho1993], Krivov \& Hamilton [Kri1997], Krivov \& Jurewicz [Kri1999], Nazzario \& Hyde [Naz1997], and their interaction with the Martian magnetic field and the solar wind, see Dubinin et al. [Dub1991] and Krymskii et al. [Kry1992]. A detailed analysis and numerical simulation of the dynamics and structure of the rings was performed by Hamilton [Ham1996]. The principle spatial distribution calculated by this model is summarized in Figure 2-4 as seen perpendicular to the Mars system. Although this picture gives only a simplified view, it shows one of the most interesting results; the dust ring around the orbit of the inner moon Phobos is shifted towards the Sun, while the ring around the orbit of the outer moon Deimos is shifted away from the Sun. Not visible in this picture is that the inner ring is even tilted out of the equatorial plane when viewed from the Martian vernal 
equinox (the intersection of the Martian equatorial and orbital planes). A seasonal dependence of the dust rings' spatial distribution is obvious.

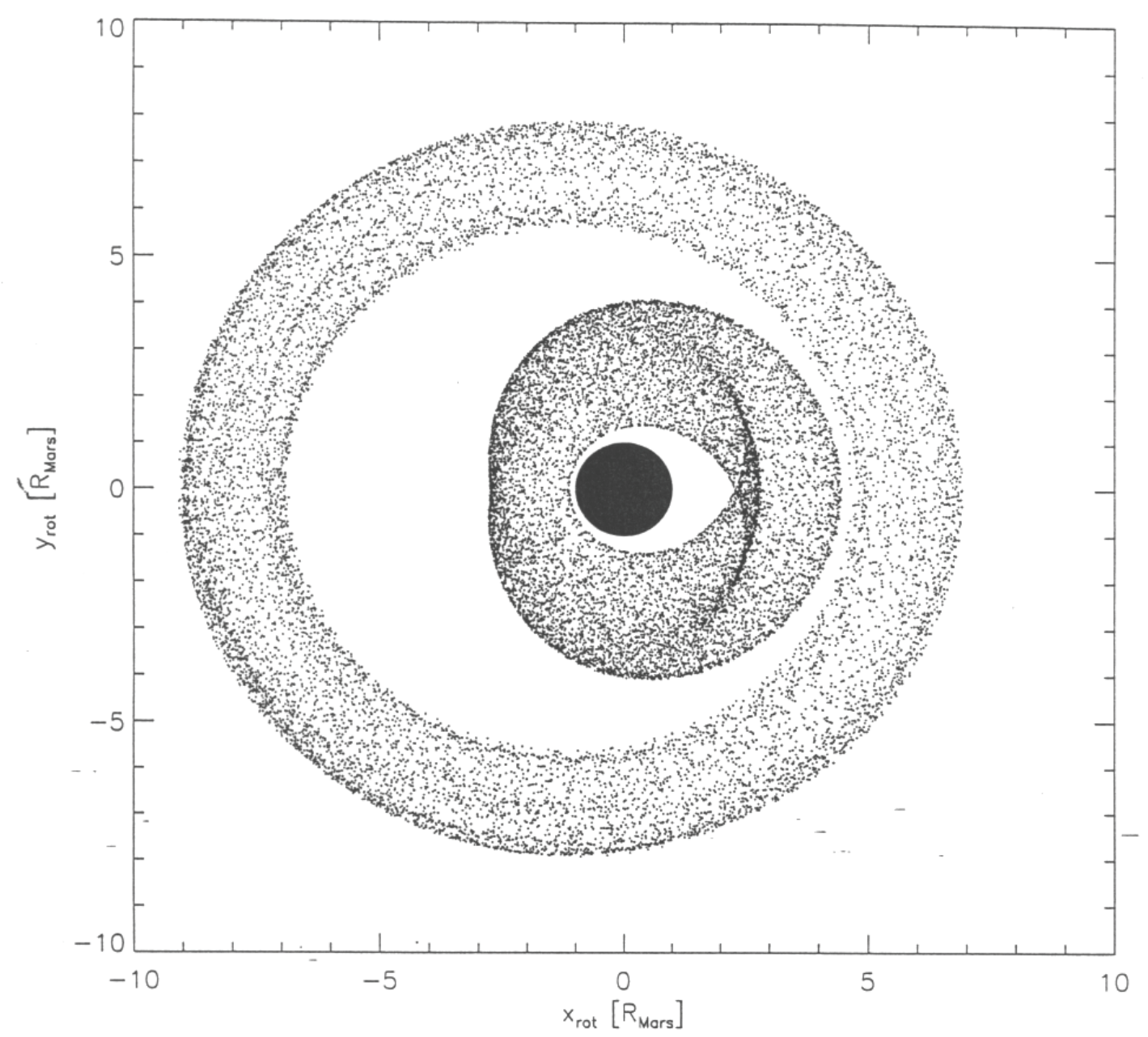

Figure 2-4: Theoretically predicted dust rings

The dust rings around the Martian moons Phobos (inner moon) and Deimos (outer moon) show a complex structure that was determined by Hamilton. The positive $\mathrm{x}$-axis is pointing towards the sun.

Very little experimental data is available to validate the theoretical predictions and models. An analysis of images taken by the Viking spacecraft gave that the optical depth of the rings must be supposed to be lower than $3 \cdot 10^{-5}$. Solar wind disturbances in plasma and magnetic field observations observed by the Phobos- 2 mission's instruments in the orbit of Phobos may correspond to a ring of dust or gas and give a first experimental but indirect evidence of the existence of the predicted dust rings, see Baumgärtel et al. [Bau1996]. The Mars Dust Counter would have been the first dust science experiment in Mars orbit. Although it was determined by Ishimoto et al. [Ish1997a] from the present models that during the two year stay in Mars orbit only a few ten dust ring particles would be detected by the MDC, a direct prove of the existence would have been possible. Observation from Earth comes out to be very difficult due to the very low optical depth of the rings, however, the brightness of the rings varies with the viewing geometry and thus some constellations are conceivable where they may be visible with terrestrial telescopes in future. 


\subsubsection{Space debris}

Since the beginning of human space exploration, a large and still increasing part of particles of all sizes that populate the vicinity of Earth are not of natural origin, but man-made so called "space debris". One can say that a large number of meteors that can be observed in clear nights are of artificial origin. Apart from $\approx 0.1 \mathrm{~mm}$ particles, space debris today dominates in all particle sizes in low Earth orbits, see ESA [ESA1999]. These artificial objects are classified into two groups, so called "primary objects" that were brought directly into orbit like burnt out rocket stages, disused satellites etc., and "secondary objects" that originate from the primary objects by collisions and explosions, or are generated by high velocity impacts of small particles on heavier objects as so called ejecta.

Sample return experiments like LDEF have shown further sources of sub-millimeter particles, like residues of solid rocket boosters that mainly consist of $\mathrm{Al}_{2} \mathrm{O}_{3}$ particles on high elliptic orbits, see Schobert [Sch1996], small particles that erode from the surfaces of spacecrafts by radiation effects or contact with atomic oxygen, see Stark et al. [Sta1997], and fluid metal beads that leaked from Russian RORSAT satellites, see Grinberg et al. [Gri1997]. Solid rocket boosters may even contribute to the debris population in the centimeter range, see Jackson et al. [Jac1997].

Most of the secondary objects are generated on high elliptic or even hyperbolic orbits and either re-enter the Earth's atmosphere or leave Earth orbit. Atmospheric friction and thus caused re-entering of the atmosphere is also a natural drain for particles on stable orbits.

The known population of particles in less than $2000 \mathrm{~km}$ height and in stable orbits or orbits having not negligible half-life periods is composed in the ESA-MASTER-Model, see Klinkrad et al. [Kli1997]. Main purpose of the compilation of space debris catalogs and models is to get reliable information about the exposure of future spacecrafts. Micrometeoroids and sub-millimeter particles, that are the main objects of interest in dust science, do not bring out serious hazards for spacecrafts and are therefore accounted only secondarily. Nevertheless, especially the dynamics of aluminum-oxide particles generated by solid rocket boosters on their way to GEO is of interest, since the achieved results not only can be used for the validation of space debris models, but also the generation of high mass particles in the range of centimeters was noticed by radar observations and engine test bench experiments.

The long-term application of dust detectors like those of the MDC type series in LEO is therefore an interesting research field, and is a precondition to provide future spacecrafts and space stations with some kind of "space weather forecast". The mission of the MDC-BremSat showed that a sophisticated screening of signals will be necessary for future MDC missions in LEO due to presumably massive atmospheric interferences that occurred in the MDCBremSat data stream. 


\subsubsection{Exemplary instruments and missions}

Several past and recent interplanetary missions carried dust experiments, which were designed to detect cosmic dust of different magnitudes. A short list of these missions is shown in Table 2-1 (content taken from [Grü1992a] and supplemented by CASSINI and STARDUST), the list also includes Earth orbiting missions outside LEO.

Table 2-1: Interplanetary missions carrying in situ dust experiments

\begin{tabular}{cccc} 
Spacecraft & Heliocen. dist. [AU] & Detector type & Spin- or 3-Ax. stab. \\
\hline Pioneer 8 & $0.97-1.09$ & impact ionization & in-ecl. spinning \\
Pioneer 9 & $0.75-0.99$ & impact ionization & in-ecl. spinning \\
HEOS 2 & 1 & impact ionization & 3-axis \\
Pioneer 10 & $1-18$ & penetration & out-of-ecl. spinning \\
Pioneer 11 & $1-10$ & penetration & out-of-ecl. spinning \\
Helios 1, 2 & $0.3-1$ & impact ionization & in-ecl. spinning \\
VEGA 1, 2 & $0.73-1.1$ & PVDF & 3-axis \\
Galileo & $0.7-5.2$ & impact ionization & out-of-ecl. spinning \\
Ulysses & $1-5.4$ & impact ionization & out-of-ecl. Spinning \\
Cassini & $0.7-9.5$ & impact ionization & 3-axis \\
Stardust & $1-2.6$ & various & 3-axis \\
\hline
\end{tabular}

In the following, the four missions GALILEO, ULYSSES, CASSINI and STARDUST are described briefly. These are not only the actual missions, but also the dust experiments onboard the GALILEO and ULYSSES spacecrafts (see Grün et al. [Grü1992a], [Grü1992b], [Grü1995a]) are in some aspects similar to the MDC, while the CASSINI dust experiment (see Srama et al. [Sra2004], Auer et al. [Aue2002]) is an advanced version of these experiments . The LRT is project partner for the CASSINI dust experiment. In contrary to GALILEO, ULYSSES and CASSINI, that also carry a number of scientific instruments of other purpose, the STARDUST spacecraft is specially designed for dust measurements, see McDonnell et al. [McD2000] for a description of the STARDUST flux monitor, Brownlee at al. [Bro1996] for a description of the STARDUST mission.

Results of the dust experiments on the missions GALILEO and ULYSSES were published regularly. See Grün et al. [Grü1995b], Krüger et al. [Krü1999b] and Krüger et al. [Krü2001a] for GALILEO results, Grün et al. [Grü1995c], Krüger et al. [Krü1999c] and Krüger et al. [Krü2001b] for ULYSSES results. The results of these four missions are discussed regularly at the annual GUCS (GALILEO, ULYSSES, CASSINI, STARDUST) meetings, also status and results of the MDC-HITEN and MDC-NOZOMI missions were presented there. 


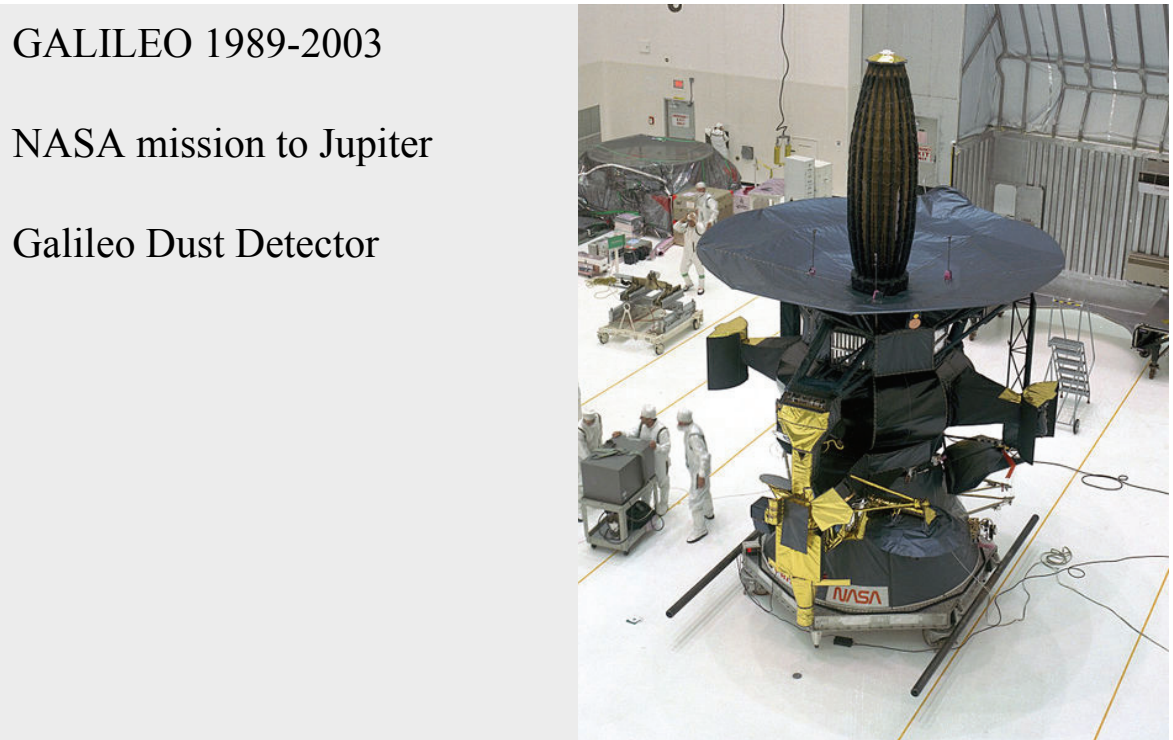

Figure 2-5: The GALILEO spacecraft

The GALILEO mission was launched on October 18, 1989. After a sixyear journey, it arrived at Jupiter in September 1995. On September 21, 2003 it crashed onto Jupiter's surface. Besides a number of other experiments, it carried the Galileo Dust Detector, a descendant from the HEOS detector that was nearly identical to the ULYSSES dust experiment.

\section{ULYSSES 1990-}

NASA/ESA mission to polar solar orbit

Ulysses Dust Experiment

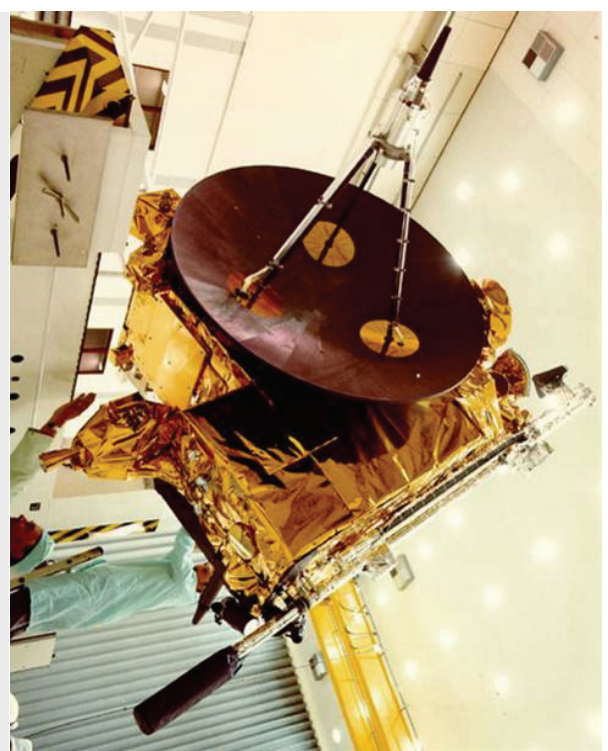

Figure 2-6: The ULYSSES spacecraft

Launched on October 6, 1990, the ULYSSES spacecraft encountered Jupiter on February 8, 1992. After a swing-by at Jupiter, it travels on a high inclined orbit around the Sun. Like GALILEO, its dust detector is a descendant from the HEOS dust detector. See Wenzel et al. [Wen1989] for a description of the ULYSSES mission. 


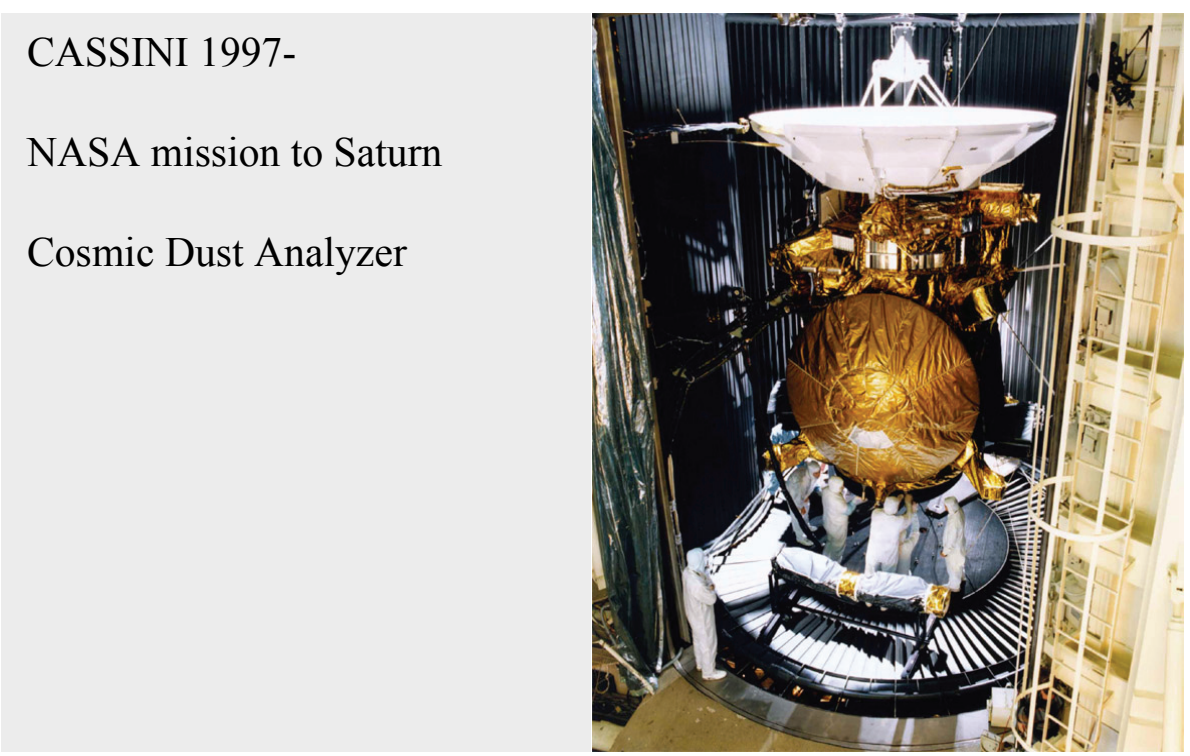

Figure 2-7: The CASSINI spacecraft

Launched on October 15, 1997, CASSINI reached Saturn orbit on July 1, 2004 after fly-bys at Venus, Earth and Jupiter. It carries the CASSINI Cosmic Dust Analyzer, a descendant from the ULYSSES and GALILEO dust detectors with enhanced measuring capabilities.

STARDUST 1999-2006

NASA mission to comet Wild-2

Several dust experiments

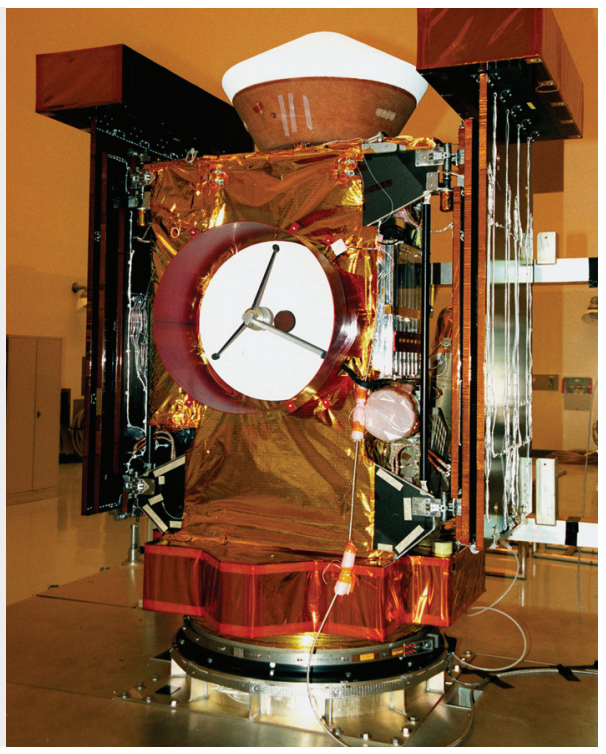

Figure 2-8: The STARDUST spacecraft

Unlike most other missions, the STARDUST spacecraft was designed especially for dust science and carries a number of dust related experiments including a sample return experiment. It was launched on February 7, 1999, and flew by the comet Wild-2 to collect cometary dust particles. On January 15,2006 , a capsule containing these cometary dust particles was delivered back to the Earth. 


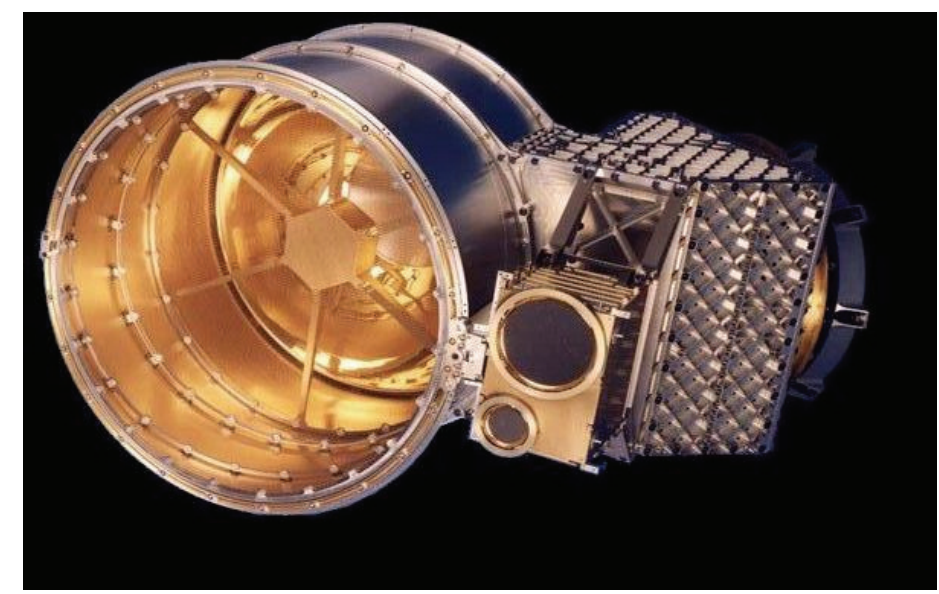

Figure 2-9: The CASSINI Cosmic Dust Analyzer

The instrument consists of two independent experiments, the Dust Analyzer DA (cylindrical box on the left) and the High Rate Detection system HRD attached to the cylinder, which itself consists of two polyvinylidene fluorid (PVDF) sensors. The HRD is designed for measurements during Saturnian ring plane crossings.

Figure 2-9 shows the CASSINI Cosmic Dust Analyzer CDA, which was designed and developed by the Max Planck Institut für Kernphysik, Heidelberg (MPI-K), see Srama et al. [Sra1997], [Sra2004]. It is a descendant from the GALILEO and ULYSSES dust experiments, but with extended measuring capabilities. Like its predecessors, the CDA is primarily an impact ionization detector with a cylindrical shaped sensor box. Additionally, the CDA carries a time-of-flight mass spectrometer inside the sensor box that enables determination of the chemical composition of the impacting particles. A similar experiment setup based on the much smaller, lighter and cheaper MDC experiment is intended by the LRT, this is discussed in chapter 8. Calibration of the CDA was carried out in the dust accelerator facility at the MPI-K using various projectile materials; see Göller [Göl1988], Göller \& Grün [Gö11989] and Goldsworthy et al. [Gol2002]. 


\subsection{The MDC mission HITEN, (formerly MUSES-A)}

HITEN, formerly named MUSES-A, was Japan's first mission outside the low Earth orbit. It was designed and developed by ISAS from 1985 to 1990. Main objective of the mission was the demonstration and testing of Japan's ability to navigate a spacecraft in the Earth-Moon system on complex trajectories, including orbit control, insertion of a sub satellite into lunar orbit, optical navigation experiments and cis-lunar aero brake demonstrations. Several maneuvers were conducted, including Moon swing-by and maneuvering of the spacecraft into the Lagrange points L4 and L5. HITEN carried a small piggyback Moon orbiter named HAGAROMO that was separated from the spacecraft during the mission. The MDC-HITEN was the only scientific experiment onboard. A description of the HITEN mission is given by Uesugi et al. [Ues1991].

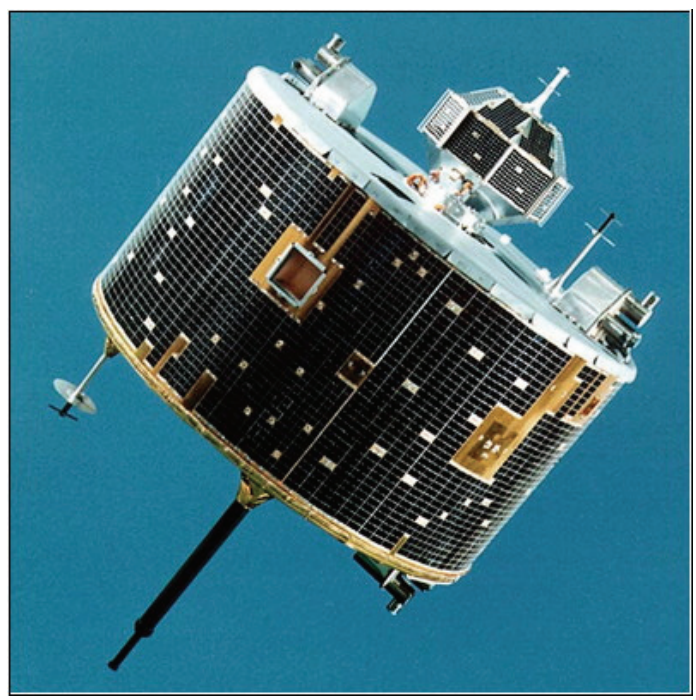

Figure 2-10: The HITEN spacecraft

Photograph of the fully integrated HITEN spacecraft. Mounted on top of the cylindrical body, the small octagonal Moon orbiter HAGAROMO can be seen. The MDC is mounted into the wall of the cylindrical body, the rectangular MDC sensor box and its gold plated inner walls can be seen here.

\subsubsection{Spacecraft properties}

The spacecraft HITEN was spin stabilized with a rotation period of $20 \mathrm{rpm}$. Its shape was cylindrical with a diameter of $1.4 \mathrm{~m}$ and a height of $0.8 \mathrm{~m}$. The top of the spacecraft was coated with a special thermal blanket to cope with the heat that was generated during aero brake maneuvers when HITEN passes the upper limits of the Earth's atmosphere at a speed of about $11 \mathrm{~km} / \mathrm{sec}$. Also on top of the spacecraft, the polyhedral shaped small lunar orbiter HAGAROMO was fixed. Around the cylindrical body, solar cells were mounted that produced a power of about $110 \mathrm{~W}$. HITEN's communication system consisted of two lowgain antenna (LGA) at the top and the bottom of the cylinder, respectively, and one mediumgain antenna (MGA) at the bottom. Both X-band and S-band downlink were available; up- 
link was carried out on S-band. The propulsion system employs eight $23 \mathrm{~N}$ and four $3 \mathrm{~N}$ thrusters that are used for orbital control. Total weight of the spacecraft was $197 \mathrm{~kg}$, where $12 \mathrm{~kg}$ account for the lunar orbiter HAGAROMO and $42 \mathrm{~kg}$ on hydrazine fuel. The MDCHITEN was mounted into the side panels of the satellite, $25 \mathrm{~mm}$ counter-sunk. No other instruments did obscure the field of view of the MDC-HITEN; the aperture angle was 145 degrees.

\subsubsection{Mission}

On January 24, 1990, the spacecraft HITEN was launched by a Japanese Mu-3SII-5 rocket from Kagoshima Space Center, Japan, and was injected directly into a high eccentric translunar orbit. After four orbits around Earth, the first lunar swing-by was conducted on March 18, 1990, when the orbiter HAGAROMO was released and injected into a lunar orbit. The following orbits no. 5 to 7 had a much higher apogee and the orbit describes a precession around Earth until the apogee was on night side. Several Moon swing-by's were conducted during the orbits no. 9 to 14 to keep the spacecrafts apogee on the Earth's night side, which was an essential requirement for the planned ISAS mission GEOTAIL and was demonstrated successfully by the HITEN mission. Aero brake experiments were conducted during the perigee of orbits no. 16 to 19 and were completed successfully. After the $10^{\text {th }}$ lunar swing-by on October 2, 1992, the spacecraft was injected into a trajectory that brought it to two of the Lagrange points of the Earth-Moon system, L4 in October 1992 and L5 in January 1993. The MDC-HITEN was the first dust experiment that could conduct measurements of dust particles that may reside in the vicinity of these stable points. On February 15, 1993, the spacecraft was injected into a polar lunar orbit where it stayed for about one month. On March 10, 1993, it finally crashed onto the lunar surface. The overall results of the HITEN mission are summarized by Uesugi [Ues1996]. 

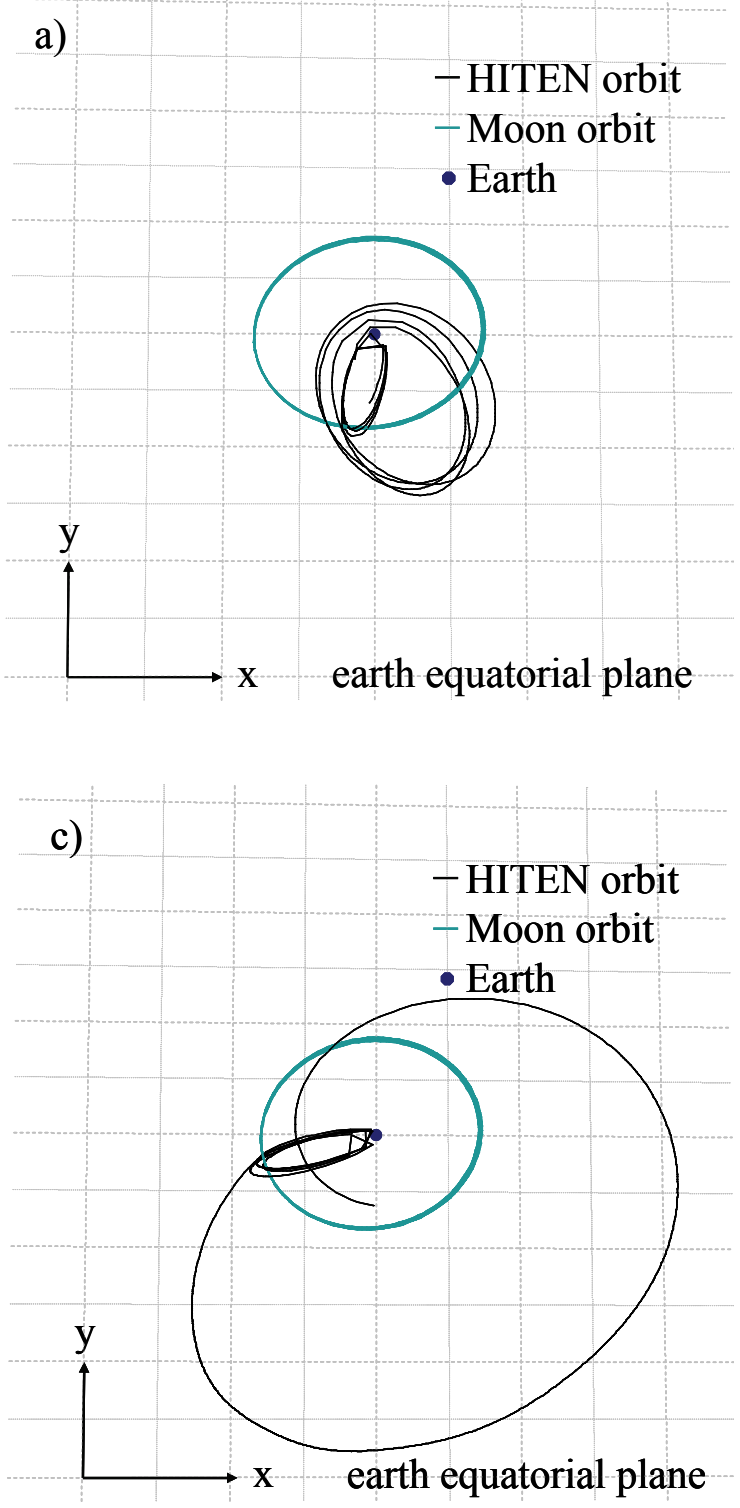
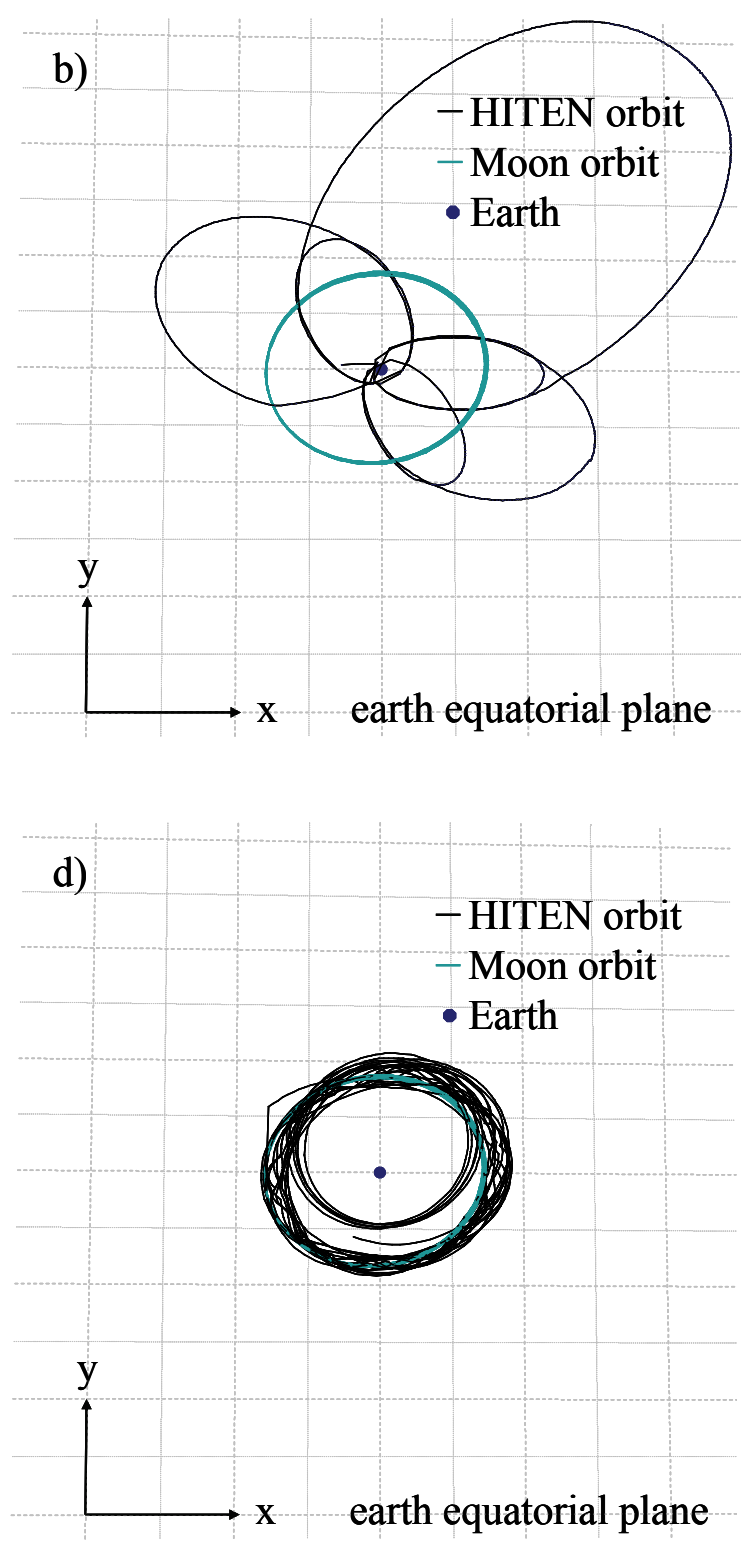

Figure 2-11: HITEN orbit

The complex trajectory of the HITEN spacecraft originated from the main purpose of the mission, which was to demonstrate the ability of the Japanese space organization ISAS to maneuver an interplanetary spacecraft. During the mission, HITEN performed several maneuvers including aero braking, several Moon swing-by's and orbits around the Lagrange points L4 and L5. a) Orbits 1-4 (initial), 5 to 8 (apogee to night side), b) Orbits 9 to 14 (keep apogee at night side), c) Orbits No. 15 to 19 (aero-brake experiments and transfer orbit), d) Orbits 20 to 24 (transfer), Lagrange points, Moon orbit. Note: Kinks in the HITEN orbit shown in this figure are caused by incomplete orbital data or small resolution (one data per day). 


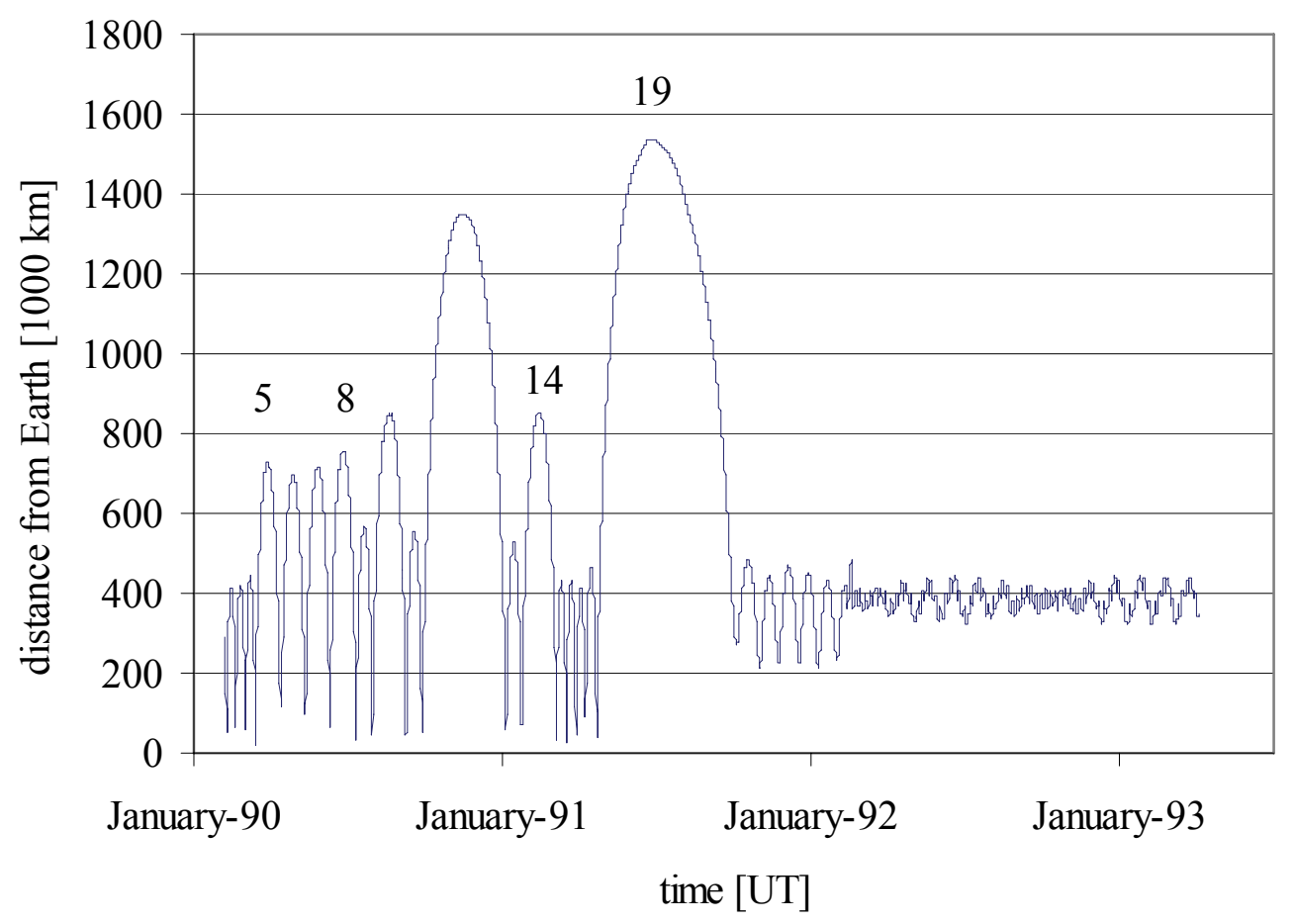

Figure 2-12: HITEN distance from earth

A more intuitive view on the complex HITEN trajectory. Orbit numbers are given for selected orbits, see text for details.

\subsubsection{Results of the MDC-HITEN dust measurements}

Calibration measurements for the MDC-HITEN were carried out at the Max-Planck Institut für Kernphysik in Heidelberg, Germany. Around 1000 particles were shot on locations inside the sensor box, and around 700 on locations outside the sensor box. The MDC-HITEN calibration is described by Münzenmayer et al. [Mün1997].

The MDC-HITEN operation started 38 days after launch. During the first two years of operation, the MDC-HITEN detected an average of slightly more than 0.5 particles per day, which corresponds to a dust flux of 2.2 to $5.5 \cdot 10^{-4}$ particles $/\left(\mathrm{m}^{2} \cdot \mathrm{s}\right)$. Mass range of the detected particles was $10^{-16}$ to $10^{-7} \mathrm{~g}$. The particles detected by the MDC-HITEN can be separated into two populations, apex-particles with low speed around $30 \mathrm{~km} / \mathrm{sec}$, and $\beta$-meteoroides with high speed $>45 \mathrm{~km} / \mathrm{sec}$ and radial flight direction, see Iglseder et al. [Igl1993a]. A later look at the MDC-HITEN data gave evidence that interstellar dust particles were detected by the instrument, see Grün et al. [Grü2001b].

During the following 14 months in Moon orbit, the MDC-HITEN detected around 150 particles with a mass range of $10^{-16}$ to $10^{-7} \mathrm{~g}$, which corresponds to a dust flux of 1.3 to $4.1 \cdot 10^{-4}$ particles $/\left(\mathrm{m}^{2} \cdot \mathrm{s}\right)$. The particles observed in lunar orbit have the same properties in flight direction as during the first two years of the mission, see Iglseder et al. [Igl1996]. 


\subsection{The MDC mission NOZOMI, (formerly PLANET-B)}

NOZOMI, formerly named PLANET-B, was Japan's first interplanetary mission. It was designed and developed from 1992 to 1998 by the Institute of Space and Astronautic Science (ISAS), a department of the University of Tokyo. The objectives of the project are twofold, in addition to the scientific objective, that is the study of the structure and dynamics of the upper Martian atmosphere and its interaction with the solar wind, the engineering objective is to develop basic key technologies for future ISAS missions. The scientific objectives of the NOZOMI mission are described by Nakatani et al. [Nak1995], the engineering objectives by Tsuruda et al. [Tsu1996]. Since the launch capabilities of the designated launcher, the new $\mathrm{M}-\mathrm{V}$ vehicle also developed by ISAS, are moderate, special emphasis was put on extremely lightweight construction of the satellite. NOZOMI was the second mission launched with the $\mathrm{M}-\mathrm{V}$.

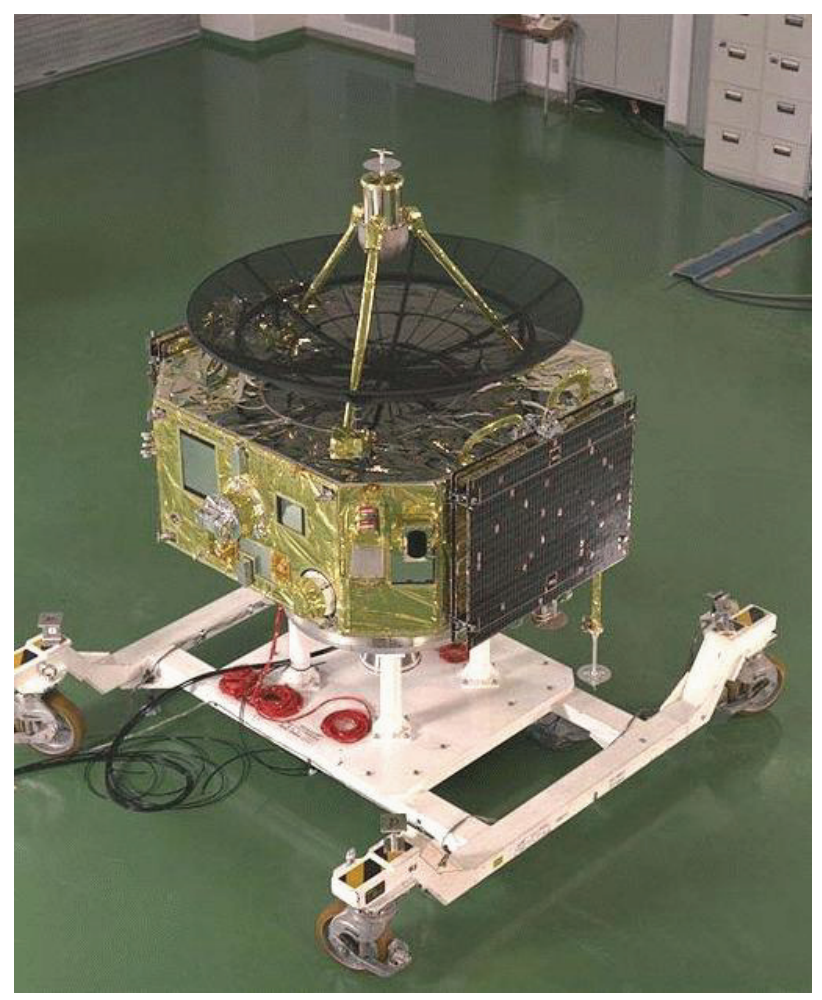

Figure 2-13: The NOZOMI spacecraft

Photograph of the fully integrated NOZOMI spacecraft. The MDC is mounted into the small side panel left of the folded solar panel. Its light grey outer wall and a small part of the gold plated inner wall can be seen here.

\subsubsection{Spacecraft properties and operations}

The NOZOMI spacecraft properties are described in the NOZOMI Prelaunch Report by ISAS [ISA1998]. The dry mass of the satellite without propulsion system is only $186 \mathrm{~kg}$, whereof $35 \mathrm{~kg}$ account for the 14 scientific instruments; the complete wet mass was $580 \mathrm{~kg}$. NOZOMI 
was equipped with two high efficiency solar panels that produce more than $200 \mathrm{~W}$ of power through the mission period. The satellite is spin stabilized with a rotation period of $\approx 6$ to $10 \mathrm{rpm}$. The telemetry system consists of a $1.6 \mathrm{~m}$ parabolic mesh X-band high gain antenna that requires pointing of the antennas bore sight direction and thus the satellites spin axis to Earth, and two omni directional S-band low gain antennas. Both the solar panels and the HGA are fixed to the body, neither Earth-pointing gimbals for the HGA nor a Sun-tracking mechanism for the solar panels were necessary because the angle between Earth and Sun seen from NOZOMI's orbit is within $\pm 45^{\circ}$, which secures power generation by the solar panels. The X-band downlink has bit rates from 2048 bps to 32768 bps, the S-band downlink from $64 \mathrm{bps}$ to $1024 \mathrm{bps}$. Uplink is established via S-band at bit rates of $125 \mathrm{bps}$ or $15625 \mathrm{bps}$. The design lifetime of the satellite was two years (one Martian year), and mainly limited by the amount of fuel that is needed to maneuver the spacecrafts attitude to direct the HGA dish to Earth for data transmission. Fuel became a critical factor after a problem during the first Earth fly-by occurred.

All data that are created by the scientific instruments can be stored by the data handling system in the onboard data recorder during periods when no telemetry via X-band downlink is possible or the instruments data stream exceeds the telemetry capacity. Main tracking of the spacecraft is carried out at Usuda Deep Space Center (UDSC) and Kagoshima Space Center (J-KSC), primary data processing, orbit and attitude determination and control is carried out at the Sagamihara Spacecraft Operation Center (SSOC). At the SSOC, the data acquired from NOZOMI is archived and distributed to the experimenters of the different scientific instruments. All MDC data was uploaded to the LRT via internet right after download from NOZOMI and extraction from the raw telemetry data stream.

\subsubsection{Mission}

Mission launch was scheduled to early July 1998. On July 4, 1998, the mission PLANET-B was launched at Kagoshima Space Center and renamed to NOZOMI following a Japanese tradition. The design of the NOZOMI orbit is described by Kawaguchi et al. [Kaw1995]. Five minutes after launch the spacecraft was inserted into a nearly circular orbit, another 13 minutes later it was kicked to a highly elliptic orbit with periapsis beyond the Moon orbit. Soon after launch, the MDC was set into operation and provided its first data. As the available energy was not sufficient for a direct injection into a Mars transfer orbit, some gravitational maneuvers were necessary. After $6 \frac{1}{2}$ elliptic orbits, the first Moon swing-by took place on September 24, 1998, and brought NOZOMI to a maximum distance of 1.7 million $\mathrm{km}$ from Earth. It returned on a retrograde orbit, and after a second Moon swing-by, a powered Earth swing-by should bring the spacecraft to the final Mars transfer trajectory.

During this powered swing by which took place in $1000 \mathrm{~km}$ height above the South Pacific Ocean, no orbital control was possible. Unfortunately, one of the oxidator vents of the propulsion system was not fully opened and the spacecraft's speed after the swing-by was $100 \mathrm{~m} / \mathrm{sec}$ too low, the missing $\Delta v$ increased to $350 \mathrm{~m} / \mathrm{sec}$ until connection could be established again. Although it would have been possible to correct the trajectory by orbital maneuvers, this would consume too much fuel, endanger the later injection into Mars orbit and prohibit the maneuvers necessary during the mission time in Mars orbit. Hence, the operators at ISAS designed a new orbit strategy that enabled the spacecraft to reach Mars and 
save the fuel needed for orbital operations around Mars, see Kawaguchi et al. [Kaw2003]. Initially the spacecraft remained on the orbit it reached after the Earth swing-by, an elliptic orbit around the Sun that crosses both Mars and Earth orbit. After three orbits around the Sun, NOZOMI reached the Earth again and a swing by maneuver was conducted on December 20, 2002, that brought NOZOMI to a high inclination Earth synchronic orbit. On June 19, 2003, NOZOMI approached Earth again and after a second Earth swing-by, it was brought on the final transfer trajectory to Mars. The Mars orbit insertion was scheduled to December 31, 2003 , and required a $\Delta v$ of $840 \mathrm{~m} / \mathrm{sec}$ instead of $1257 \mathrm{~m} / \mathrm{sec}$ with the original transfer strategy, which secures that enough fuel is left for future orbital maneuvers.

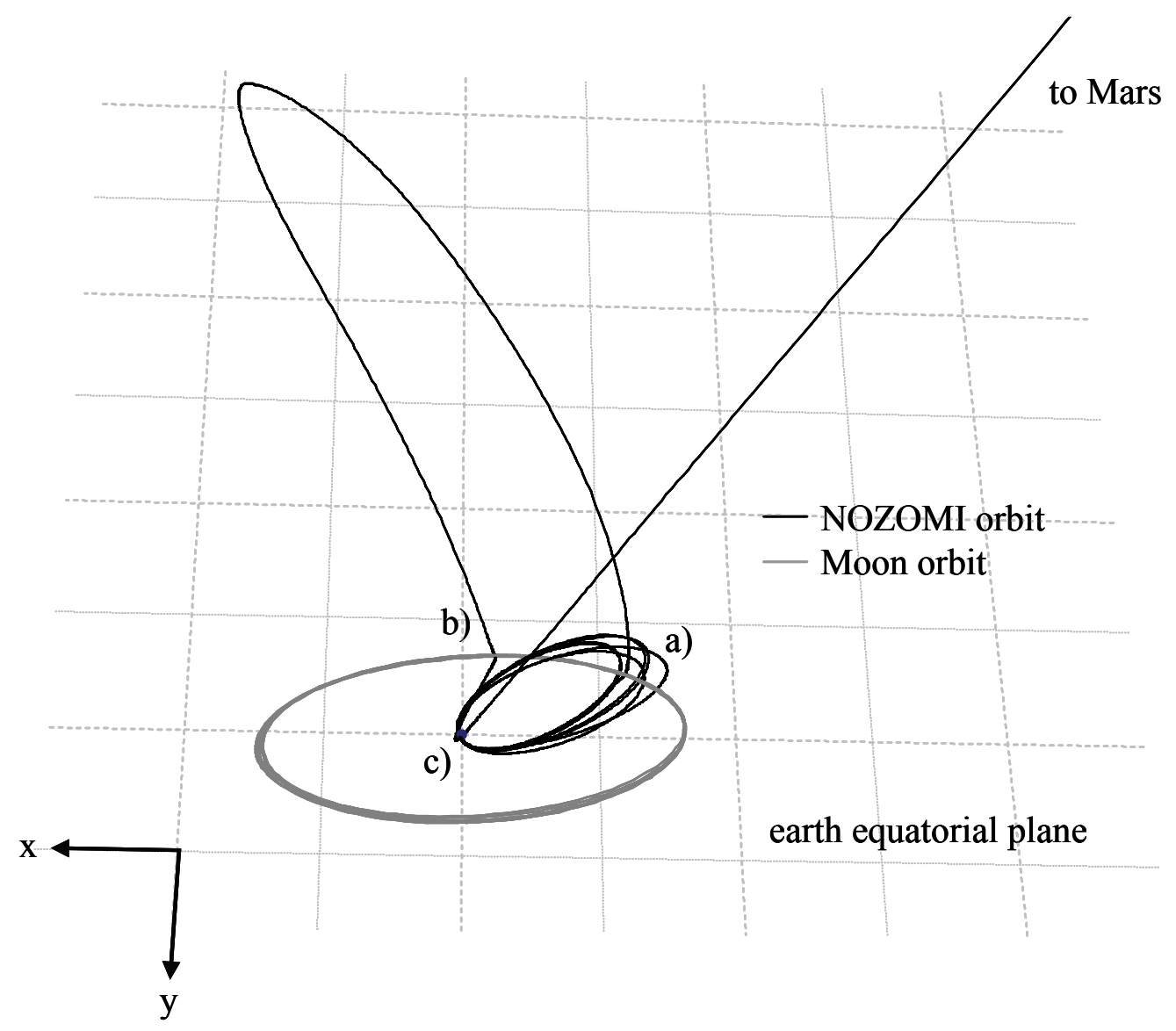

Figure 2-14: NOZOMI orbit in the Earth-Moon system

a) First Moon swing-by, September 24, 1998 b) Second Moon swing-by, December 18, 1998 c) Earth swing-by, December 20, 1998.

The prolonged transfer to Mars gave the MDC the possibility to measure dust in the interplanetary space for long period and thus need not to be seen as a drawback for the MDC project. Since the MDC-HITEN proved to work stable and reliable on a long-term mission, it was expected that the MDC-NOZOMI would do a good job as well. Data acquisition and data transfer during the interplanetary cruising phase were possible and were conducted regularly. 


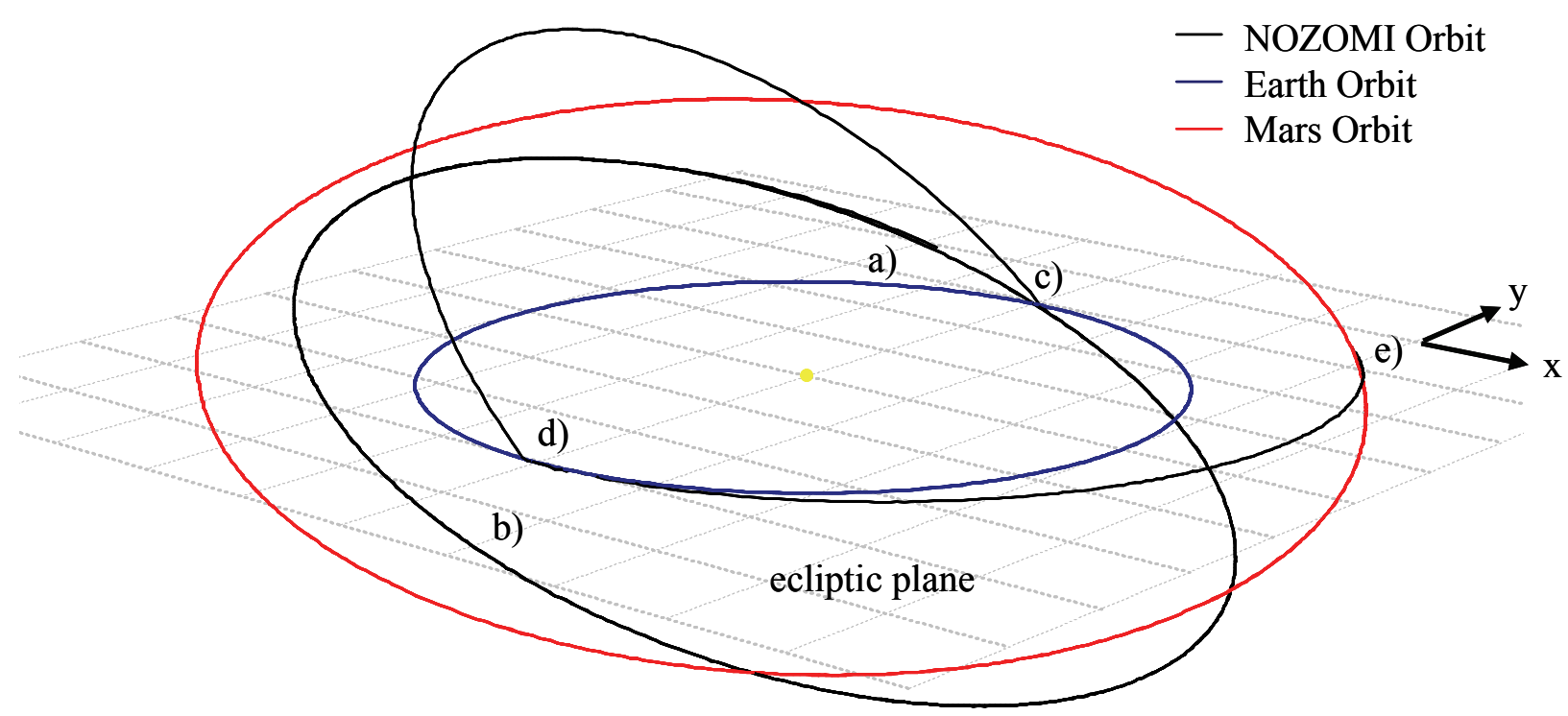

Figure 2-15: NOZOMI orbit in the interplanetary space

a) Insertion into Sun centered orbit January 1998 b) System failure, April 26, 2002 c) First Earth swing-by, December 21, 2002 d) Second Earth swing-by, June 19, 2003 e) Mars fly-by, December 14, 2003.

Insertion into Mars orbit was scheduled in December 2003. The planned orbit was highly elliptic with periapsis at $150 \mathrm{~km}$ and apoapsis at 15 Mars radii, inclination was $170^{\circ}$, which defines a retrograde orbit. The orbit crosses the orbits of the Martian satellites Phobos and Deimos, which both are subject of scientific interest as well. The MDC was expected to detect dust rings around Mars in the orbits of the two moons as they were predicted by theoretical considerations earlier (see paragraph 2.1.3).

On April 24, 2002, a system failure onboard NOZOMI happened that was probably caused by a solar eruption which occurred a few days earlier. The damage onboard NOZOMI was soon identified as a failure of the CI-PSU (Common Interface Power Supply Unit), a power supply unit that was responsible for a couple of satellite subcomponents including the data recorder, thermal control and a control unit that handles the telemetry modes. This failure severely endangered the continuation of the mission. E.g., the satellites telemetry system remained in ranging mode after the failure occurred which made it impossible to transfer MDC data or other data back to Earth. However, the most severe problem was the failure of the thermal control system, since without the heaters the fuel for the main propulsion system gets frozen at larger distances from the Sun. As a first workaround, all satellite systems including the scientific instruments were powered on to produce as much heat as possible, but this could not provide enough energy to heat the fuel. Without the main propulsion system, Mars orbit insertion was not possible.

Detailed analyses of the problem at ISAS lead to the assumption that a short circuit prevents the CI-PSU from working properly. As a solution strategy, a series of "CI-PSU power on" commands sent to the spacecraft was thought to eliminate the short circuit. During the first try in September 2002, the link to NOZOMI was completely lost and could be re-established a couple of weeks later. Because of this incident, further tries to solve the problem and re- 
establish NOZOMI's full functionality were postponed for safety reasons until the last Earth swing by has happened.

These last tries to correct the problem were carried out from September 2003. A series of 10000 "CI-PSU power on" commands were sent to the spacecraft in a high frequency, telemetry was lost again like during the first try, but the failure of the CI-PSU could not be corrected. After re-establishing the telemetry link, a series of small orbital corrections were conducted using the small maneuvering thrusters to conduct NOZOMI on a secure trajectory that minimized the chance that NOZOMI crashes onto Mars' surface. Unlike landing devices, NOZOMI as an orbiter was not biologically clean and could possibly pollute the Mars surface with biological materials from Earth.

On December 12, 2003, NOZOMI passed Mars on the shadow side in a presumed height of $850 \mathrm{~km}$. After the fly-by, ISAS tried to re-establish the telemetry link to NOZOMI. As the exact position and speed of NOZOMI after the fly-by were unknown, it was necessary to scan the potential space segment where it should reside as well as the frequency band due to the Doppler shift. After a couple of days, these tries were given up without any result and on January 10, 2004, the mission was finally declared as lost.

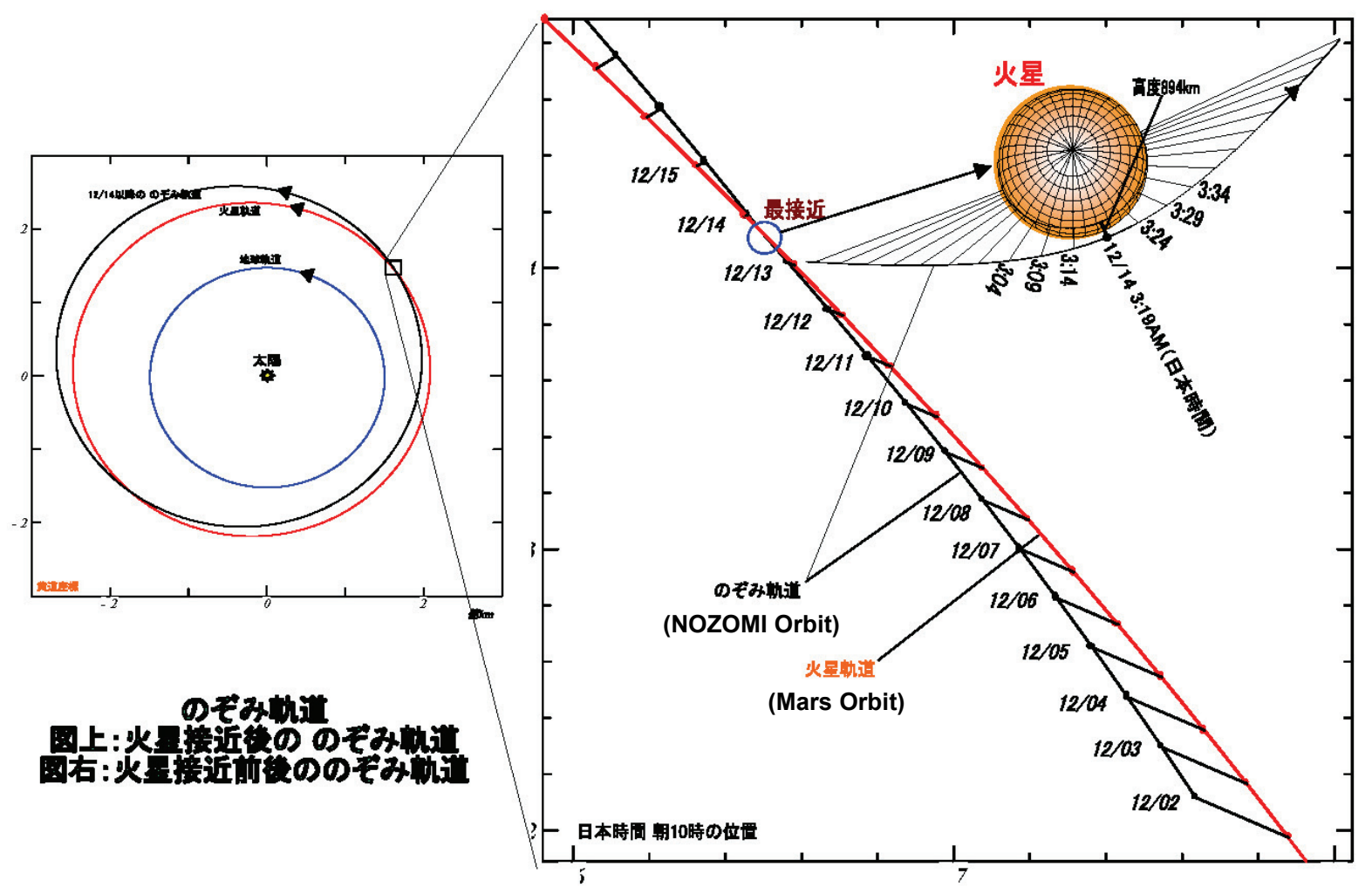

Figure 2-16: NOZOMI end of mission

On December 14, 2003, NOZOMI passed Mars in $894 \mathrm{~km}$ height above the surface. After the fly-by, the telemetry link to NOZOMI could not be reestablished. (Image: ISAS, no English version available) 


\subsubsection{The scientific mission of the MDC}

While the scientific missions of most other experiments onboard NOZOMI are limited to the Mars orbiting phase, the mission of the MDC can be separated into three mission phases with different scientific intentions. These MDC mission phases correspond to the different cruising phases of the spacecraft NOZOMI as follows:

1. The Earth-Moon phase: In this phase, dust particles in the Earth-Moon system were detected.

2. The interplanetary cruising phase: During this transfer phase to Mars, interplanetary and interstellar dust particles were detected. The results of these measurements are compared to the actual models of the interplanetary dust distribution.

3. The mission phase in Mars orbit: The main mission goal of the MDC was the detection of dust rings around Mars. These dust rings were predicted earlier as dust tori that should reside in the orbits of the two Martian moons Phobos and Deimos.

While the regions 1 . and 2. were already explored by other experiments, the MDC would have been the first dust experiment in Mars orbit. The dust rings that first were theoretically predicted by Soter [Sot1971] are considered to consist of ejecta that are thrown from the surface of the moons after impacts of heavier particles. The basic assumption is that the two moons are exposed to the same stream of heavier asteroids as the Mars itself, whose surface is covered with impact craters. Those of these ejecta whose throw-out speed is sufficient to leave the gravitational field of the moon but not sufficient to leave Mars orbit are supposed to build a spatial distribution that at best can be described by a torus in the orbit of the particular moon. While the particle density of these dust rings is too low to be viewed from Earth with the available instruments, some disturbances of the Martian magnetic field recorded by the Phobos-2 spacecraft while passing the Phobos orbit (see Baumgärtel et al. [Bau1996]) give evidence of the existence of the predicted dust rings. The measurements of the MDC in Mars orbit would have been of great scientific interest, see Ishimoto et al. [Ish1997a], Sasaki [Sas1999] and Krivov \& Hamilton [Kri1999]. The moons Phobos and Deimos themselves were also subjects of the NOZOMI scientific objectives, see Inada et al. [Ina1999] for a description of the planned observations by the Mars Imaging Camera (MIC). 


\section{The Mars Dust Counter}

The Mars Dust Counter (MDC or MDC-NOZOMI) is the third in a series of dust experiments designed and developed by the Lehrstuhl für Raumfahrttechnik (LRT) at the Technische Universität München (TUM). In this chapter, the properties of the MDC-NOZOMI are described (see Igenbergs et al. [Ige1998] and ISAS [ISA1998]) since its predecessors, the Munich Dust Counters MDC-HITEN and MDC-BremSat are designed nearly identical and the basic properties and the functionality of the three experiments differ only in some details like size of the sensor box and the number of channels that are recorded by the electronics box.

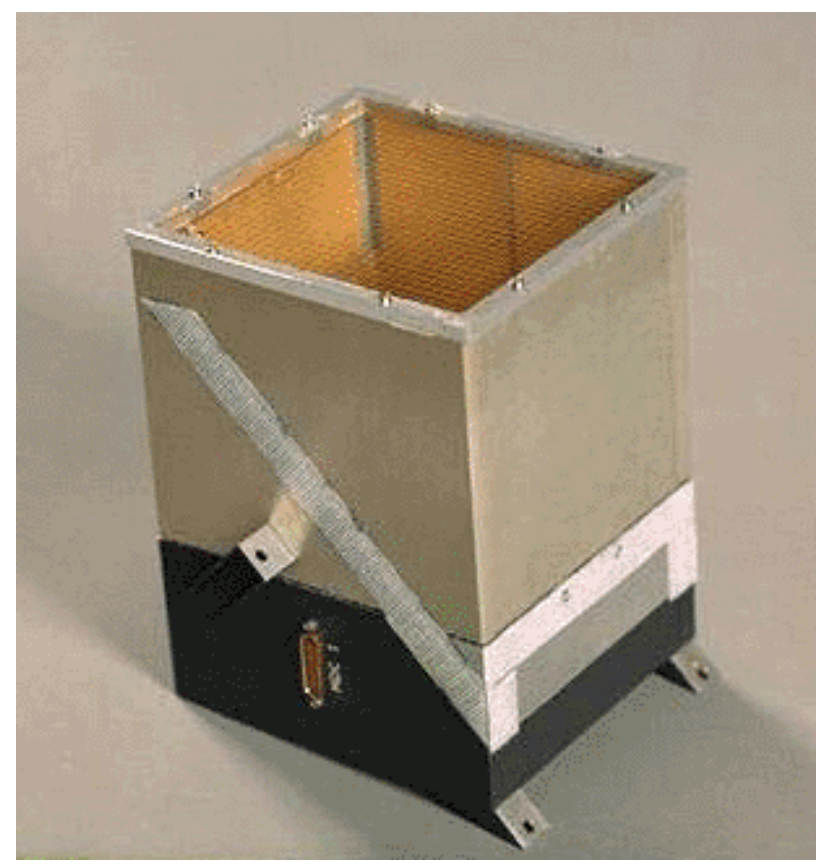

Figure 3-1: The MDC

Photograph of the MDC experiment. Below the sensor box with its goldplated inner walls, the black electronics box is attached.

Paragraph 3.1 gives an overview of the physical principles that are used by the MDC for the detection of cosmic dust particles; paragraph 3.2 describes the technical properties of the MDC. Finally, paragraph 3.3 describes the calibration process of the MDC experiment. 


\subsection{The MDC measuring principle}

The MDC measuring principle is based on the physical effects that are caused by impacts of dust particles on the solid target inside the sensor box. Impacts of particles at velocities greater than $1 \mathrm{~km} / \mathrm{sec}$ cause rather complex phenomena. In principle, the kinetic energy of the impacting particle is transformed into heat that is high enough not only to break the molecular and atomic bindings but also to ionize the melted constituents of the particle and the target. Parts of this ionized matter are thrown out from the impact site at high velocity as so-called ejecta. The characteristics of the hypervelocity impacts, the ejecta and the charge generation process are described in the following paragraphs.

\subsubsection{Physical background of the impact ionization process}

The comprehensive stress that occurs during the first moments of a hypervelocity impact exceeds the rigidity of the solid materials of both the impacting particle and the target material by far, and the matter behaves like a compressible, in-viscid fluid. The shockwaves that run through the projectile and the target compress the material locally to more than $10 \mathrm{Mbar}$, and lead to a fast expanding crater in the surface of the target. Due to complex processes of interference of the shockwaves with their reflections from surfaces, the temperature rises up to $10^{5} \mathrm{~K}$ in the immediate vicinity of the contact zone of projectile and target, while pressure falls. Either gaseous, fluid, or even solid material is then ejected from the impact site. The solid constituencies of these ejecta can reach as high velocities that are sufficient to cause subsequent hypervelocity impacts when these ejecta hit the target again.

The most important effect for the investigation of cosmic dust particles that occurs during this process is that the ejecta are ionized at least partially due to the high temperatures. This was first proven experimentally by Friichtenicht and Slattery [Fri1963]. Different theoretical models of the process of charge generation, depending on the particle's impact speed, were developed. As a basic assumption, the degree of ionization of the material is dependent on the specific intrinsic energy of the material, which can be described as (see Drapatz \& Michel [Dra1974])

$$
\Delta E=\frac{1}{2}\left(\frac{v}{\sqrt{\rho_{\mathrm{P}} / \rho_{\mathrm{T}}}+1}\right)^{2},
$$

whereas $v$ is the impact speed of the projectile, $\rho_{\mathrm{P}}$ the particle's density and $\rho_{\mathrm{T}}$ the target's density. According to this equation, a maximum specific intrinsic energy and therefore charge gain can be achieved by the use of high-density target materials, for this reason, the MDC target area was gold plated. Experimental measurements of the temperature of the plasma gave that the temperature is strongly affected by a wide range of environmental and local material conditions rather than showing a causal and reproducible relation to the total kinetic impact energy.

Although many studies on ion formation and plasma production during the hypervelocity impact process were made (see e.g. Hornung \& Drapatz [Hor1981], Hornung et al. 
[Hor1996a], Kissel \& Krüger [Kis1987], Ratcliff et al. [Rat1996], [Rat1997], Dalmann et al. [Dal1977], Drapatz \& Michel [Dra1974]), the complex process and all its different aspects cannot be explained satisfactory by one single model, not to mention a quantitative determination of the charge gain. For this reason, high effort must be put on experimental calibration of each individual sensor device with respect to its characteristics and operational area in order to allow reliable measurements of mass and impact speed of dust particles. For reliable measurements of particle mass and impact speed, the materials used in the laboratory as substitutes for natural cosmic dust particles must reproduce the properties of the natural particles as good as possible. New materials are developed and tested until today, see e.g. Burchell et al. [Bur2002] and Stübig et al. [Stü2001]. MDC-NOZOMI calibration is described in paragraph 3.3.1.

The determination of impact speed and particle mass from the produced plasma by impact ionization detectors like the MDC is based on two empiric equations that are already described by Friichtenicht \& Slattery [Fri1963],

$$
\begin{aligned}
& t=C_{t} m^{\varepsilon} v^{\eta} \\
& Q=C_{\rho} m^{\alpha} v^{\beta},
\end{aligned}
$$

whereas $t$ is the rise time of the charge signal, $Q$ the charge amplitude, $m$ the particle mass and $v$ the impact speed. The parameters $C_{t}, C_{\rho}, \eta$ and $\beta$ must be determined by calibration experiments. The values of the mass exponents $\varepsilon$ and $\alpha$ are set to 1 and 0 , respectively, in the MDC-HITEN and MDC-NOZOMI calibration. Now, particle mass and impact speed can be determined from rise time and amplitude of a detector's charge signal.

Other studies on hypervelocity impacts showed, that the maximum intensity and total energy of the light flash emitted by a hypervelocity impact is a unique function of mass and impact speed of the impacting particle. Such measurements can also be used for particle properties determination, see e.g. Eichhorn [Eic1975], [Eic1976].

\subsubsection{Material, geometrical and other artifacts}

Experimental examination of hypervelocity impacts with different projectile and target materials gave that the charge gain is highly dependent on both the melting point and the density of the used materials. Using glass and carbon projectiles, the glass projectiles lead to a fourfold higher charge gain than the carbon projectiles despite their nearly same density (glass $2.5 \mathrm{~g} / \mathrm{cm}^{3}$, carbon $2.2 \mathrm{~g} / \mathrm{cm}^{3}$ ), this was explained by the lower melting point of glass that is responsible for the higher production of ejecta.

The effects of impacts with incident angles lower than $90^{\circ}$ were thoroughly examined by Hoffmann [Hof1971], Dietzel et al. [Die1972] and Svedhem \& Pedersen [Sve1992]. In summary, the rise time of the charge signals shows little to no dependence on the incident angle, while the charge gain varies by factors up to 6 .

Also, the influence of the electric field strength inside impact ionization detectors was examined by Dalmann [Dal1977] in an experimental setup, and by Knabe [Kna1980] on the 
GALILEO dust detector. In summary, the charge gain rises rapidly with the electric field, but the rise stops at an electric field strength of $1 \mathrm{~V} / \mathrm{mm}$ (GALILEO Dust Detector) to $20 \mathrm{~V} / \mathrm{mm}$ depending on the detector geometry. Inside the MDC, an electric field of about $4 \mathrm{~V} / \mathrm{mm}$ or higher is given.

Willis et al. [Wil2004] carried out a study about the influence of a number of artifacts (e.g. natural particle charge, incident angles) on the derived mass and speed distribution of cosmic dust particles detected by typical impact ionization detectors. It was shown that the examined artifacts could cause shifts in mass distribution by an order of magnitude, and by a factor of two or three in velocity distribution. 


\subsection{Technical properties}

The technical properties described here are valid for the MDC-NOZOMI. The design of the other members of the dust counter family developed at the LRT is nearly identical, most significant differences are the number of recorded channels (MDC-HITEN \& MDC-BremSat: two, MDC-NOZOMI: three), and the size of the sensor box, which is marginally larger for the MDC-NOZOMI.

The MDC series of experiments are designed as impact ionization detectors. Particles that enter the box through the grid at high velocity will hit the target (the inner walls of the box build the target), vaporize together with a small amount of target material and build plasma. The negative and positive charges of this plasma are then separated by an electrical field inside the sensor box and move to the particular electrode where the charges are transformed into a voltage signal by one charge amplifier for each channel. This signal is digitized by a transient recorder and recorded by the MDC electronics if the amplitude exceeds a certain trigger level.

\subsubsection{Experiment design}

The MDC consists of a sensor box and an electronics box that are fixed by four small screws to each other. The overall dimensions of the experiment are $13.6 \cdot 12.7 \cdot 18.1 \mathrm{~cm}$, its total weight is $730 \mathrm{~g}$. The extremely lightweight outer structure is build from a Nomex ${ }^{\circledR}$ honeycomb structure that is plated with $0.1 \mathrm{~mm}$ aluminum. The inner walls of the sensor box are plated with gold to maximize the charge gain of hypervelocity impacts (see paragraph 3.1). Two electrodes are located at the top half of two opposite sides and are set to a potential of $+220 \mathrm{~V}$ and $-220 \mathrm{~V}$, respectively. All other walls of the sensor box have a potential of $0 \mathrm{~V}$. The open side of the sensor box is covered with a thin grid that is also set to a potential of $0 \mathrm{~V}$, to shield the sensor box from outer electromagnetic fields. Due to the rectangular shape of the sensor box, the electrical field inside the box is highly inhomogeneous.

The electrodes and the remaining inner walls are connected to a particular charge amplifier each, this configuration builds the three channels that are recorded and finally build a full MDC-NOZOMI impact signal. The channels are named electron channel EC, ion channel IC and neutral channel NC, respectively (the MDC-HITEN and MDC-BremSat devices only possess two channels, EC and IC). While the EC mainly records negative charge and the IC positive, the NC records all the charge (positive and negative) that is not accelerated to the particular electrode. The characteristic of the charge amplifiers is logarithmic and the maximum charge that can be processed without overload is $10^{-10} \mathrm{C}$, which limits the measuring range of the MDC (see paragraph 6.2.1 for further discussion). The three signals are continuously digitized by two A/D converters, where the IC and NC share one of the A/D converters in multiplexing mode (in single mode, the $\mathrm{NC}$ is not recorded and the full sampling rate is available for the IC). The continuous digital data stream is initially recorded by a transient recorder that consists of a 1 kByte FIFO buffer. Further digital data processing is described in the following paragraph. 


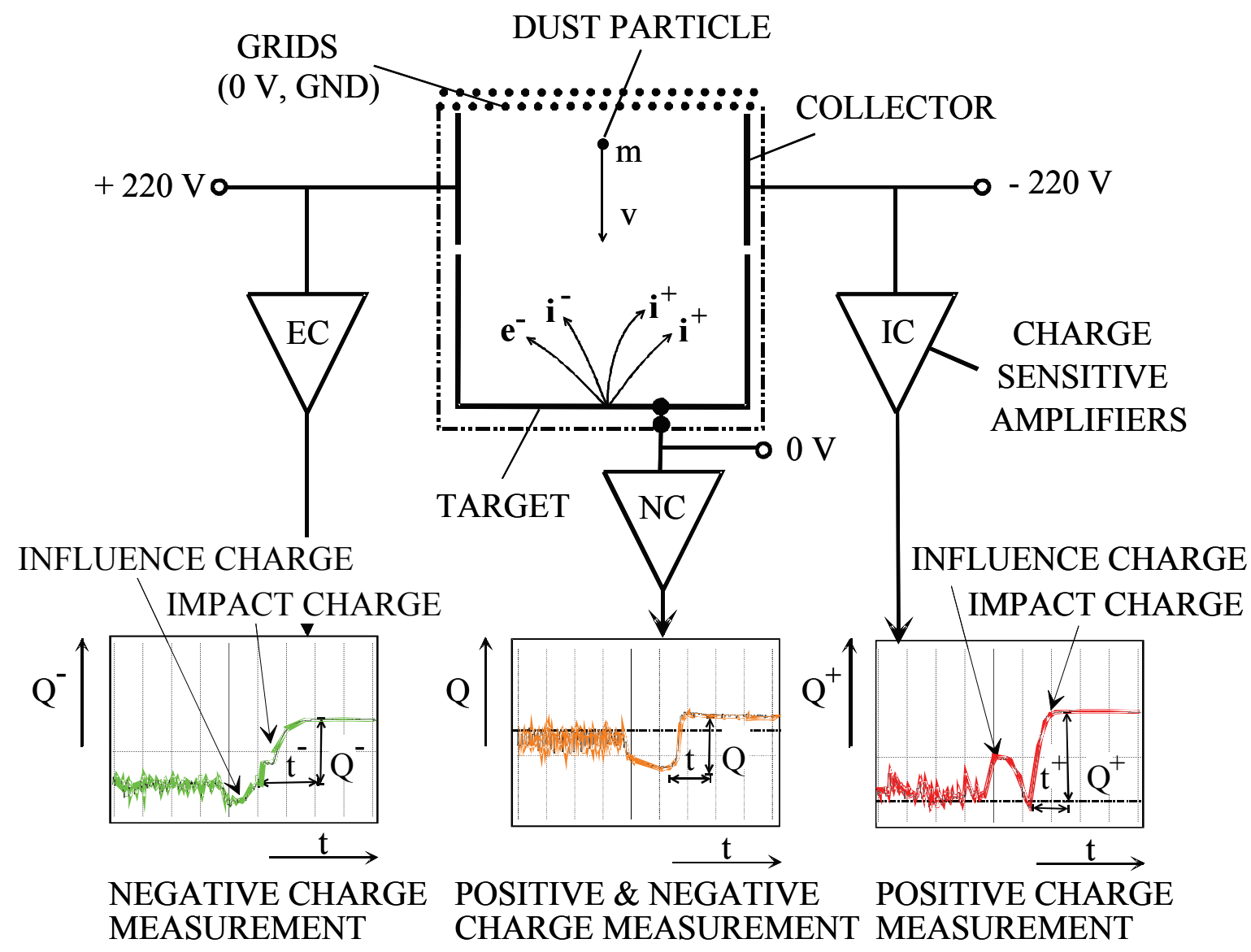

Figure 3-2: The MDC measuring principle

Impacting dust particles vaporize when hitting the target and produce plasma around the impact position. The charges of this plasma (negative free electrons and positive ions) are separated by the electric field inside the sensor box. Three charge sensitive amplifiers continuously convert the charge that hits the specific sensor areas into a voltage signal that is digitized and processed further as described in paragraph 3.2.2.

\subsubsection{Onboard data processing}

The MDC onboard software was developed by the Laboratory for Process Control and RealTime Systems at the Technische Universität München, and is described by Fischer [Fis1998].

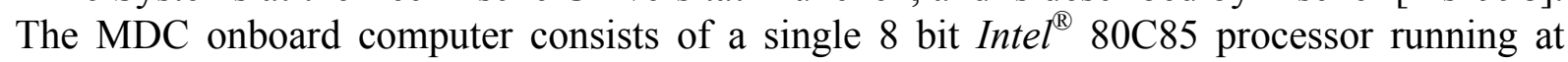
$1.5 \mathrm{MHz}$ clock rate as central processing unit, 4 kByte read only memory (ROM) and 56 kByte of random access memory (RAM) that is used both for calculations and for data storage.

The $56 \mathrm{kByte}$ random access memory is split into 56 single 1 kByte segments, 51 of these are used for impact data storage. One segment is required for actual HSK values and parameters needed for the calculation routines, another one contains a test pulse that is triggered and 
recorded right after a new event is triggered to document the state of the charge amplifiers. The remaining four segments are needed by the qualification routine for calculations.

Each memory segment contains an eight-bit quality key that indicates the status of the segment. The intrinsic quality keys are calculated by a qualification routine and indicate the quality of the signal stored in the memory segment as a numerical value with a range from 2 to 245 (a detailed description of the qualification routine is given in paragraph 4.2.1). A quality key of zero indicates an empty or already read out segment, a quality key of 1 indicates a segment that contains a new recorded signal that is not qualified yet. Quality keys greater than 245 are reserved for special memory segments required for calculations.

A complete flow chart of the onboard data handling process is shown in Figure 3-3. Digital signal data is read from the $1 \mathrm{kByte}$ FIFO buffer if one of the channels exceeds a certain trigger level. This trigger level and the trigger mode that determines which channels are triggered at all can be adjusted by telemetry commands to the desired value. If a signal is triggered, the content of the FIFO buffer is copied to the MDC memory with a temporal adjustment that the final signal contains $80 \mu$ s pre trigger and $120 \mu$ s post trigger data. A rough analysis of the new signal is carried out to check if the signal passes a set of criteria that have to be met by any signal that potentially represents a real impact signal at all, the socalled KO-criteria. If the signal passes these criteria, it is copied to a memory segment that is designated to hold the new-recorded signal. This memory segment can be an empty segment with quality key zero, or, if no empty segments are available, the segment that contains the signal with the lowest quality key which will be overwritten. During this process, some counters are set depending on the results of the KO-criteria check and the memory usage. Every signal that is discarded by the KO-criteria is documented by incrementing the so-called noise count NC. The NC therefore is an indicator of how noisy the MDC environment is at a given period of time. If the signal passes the KO-criteria and is stored in memory, either the so called impact count IC is incremented if an empty memory segment was available, or the so called event count EC is incremented if a previously recorded and qualified signal had to be overwritten. The EC therefore is an indicator of how much recorded and qualified signals were overwritten over time.

During this data handling process, the MDC electronics is busy and therefore cannot process eventually triggered further signals, which therefore get lost. The total dead time for processing events that are discarded by the KO-criteria check is $35 \mathrm{~ms}$, the dead time for processing events that pass the KO-criteria is $65 \mathrm{~ms}$. The total instrument dead time over longer periods can be appreciated by analyzing the progression of the noise count $\mathrm{NC}$ and the event count EC over time. A detailed analysis of instrument dead time during noisy mission phases was carried out and is described in paragraph 6.2.1

Every new signal that is stored in the MDC memory gets an initial quality key of 1 . The qualification of new signals is carried out by the qualification routine that is running as a background task that can be interrupted if new signals are triggered and have to be processed. This is necessary because the qualification routine requires about 3 seconds processing time and would cause a much too high instrument dead time otherwise. Every signal that could not be qualified right after recording is being qualified later.

A memory segment of 1024 bytes contains 926 bytes of signal data, 463 bytes account for the electron channel EC, 232 bytes for the ion channel IC and 231 bytes for the neutral channel NC. The actual state of the MDC (voltages, temperatures) is stored in 7 bytes. 64 bytes each 
contain the shape of a test pulse that is triggered right after the signal was recorded to give evidence about the state of the charge amplifiers. The remainder is used for quality key, impact time, phase count (the satellite's rotation angle) and some more information needed for later impact analysis.

Memory readout is performed at one go by transmission of the whole 56 kBytes of memory to the NOZOMI onboard computer, beginning with the lowest address.

The onboard software that handles all the data processing described above was developed prior to mission launch and is stored in the already mentioned 4 kByte read-only-memory. During the initialization process of the MDC onboard computer, the branch table that contains the memory addresses of the single routines of the software is copied to the random access memory, and this copy is used by the software to determine the address of each routine. This architecture allows the substitution of existing routines or the insertion of additional routines by uploading the new code into a certain memory segment and changing the branch address in the branch table to the memory address of the new routine. This feature was used in the year 2001 when a software upgrade was uploaded that should dynamically adjust the trigger level depending on the gradient of the noise counter, see Fischer [Fis1999]. The parameters of this upgrade were not adjusted properly to the environmental conditions in space, finally it was discarded a couple of months later. The software upgrade that should provide powerful data compression and was developed in 2003 also makes use of this feature, see chapter 5. 


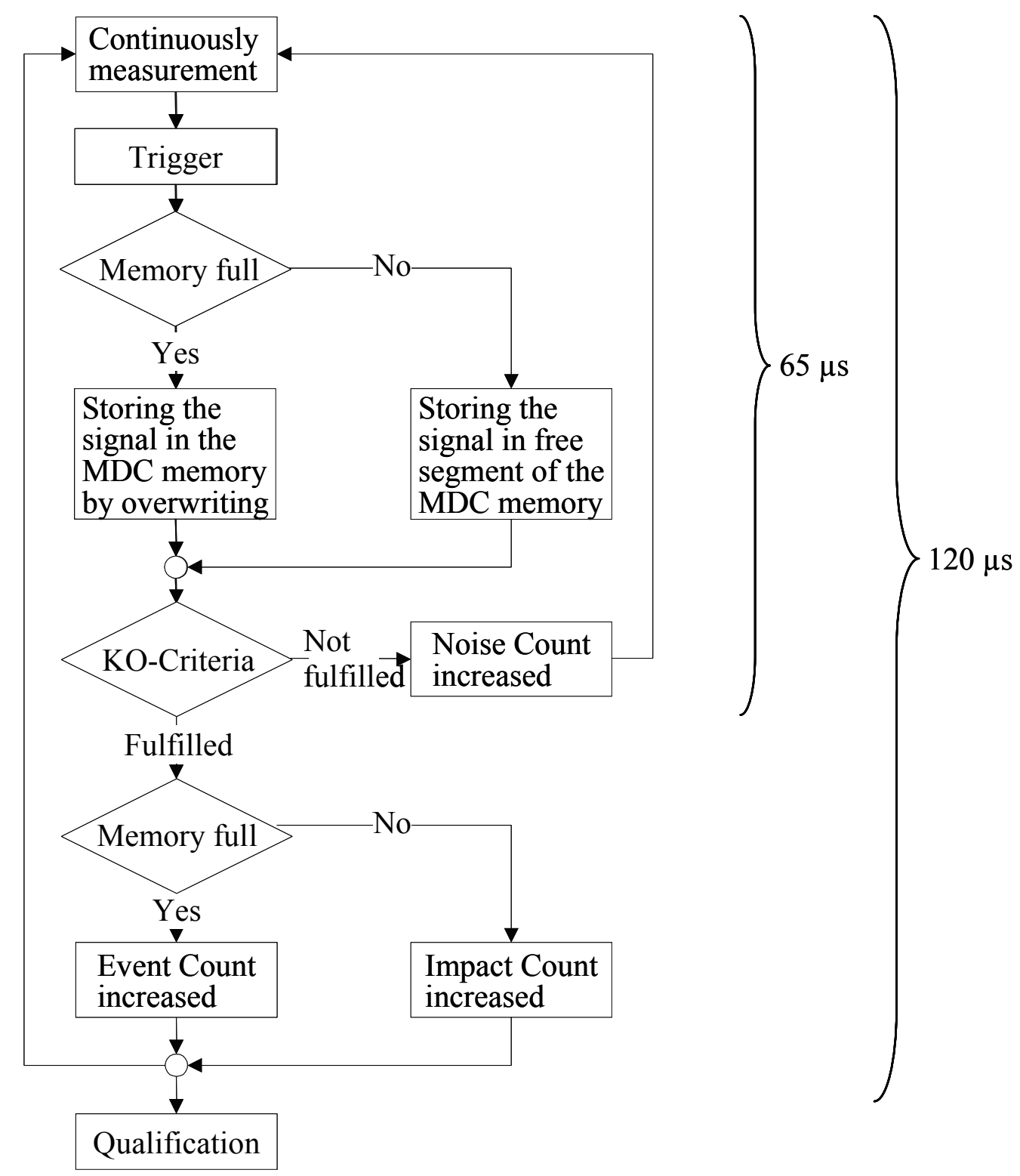

Figure 3-3: MDC data processing

Flow-chart of the data processing routine onboard the MDC. The time values indicate the instrument dead time that occurs during processing of newly triggered signals. 


\subsubsection{MDC structural integration and effective solid angle}

For an exact determination of dust flux (particles per time and area) measured by the MDC, a detailed examination of the geometric conditions of the MDC and NOZOMI is required. The number of particles detected by a dust experiment like the MDC must be referred to a standardized field-of-view and sensor area to give comparable quantitative results. In this paragraph, the geometries of the MDC and of parts of the NOZOMI body that interfere the MDC's field-of-view are examined, and the final individual MDC/NOZOMI system parameter $\Omega_{\mathrm{MDC}}$ (effective solid angle) required for dust flux analysis (see chapter 6) is determined. The structural integration of the MDC into the NOZOMI body is shown in Figure 3-4 and Figure 3-5. All dimensions of parts of the NOZOMI body and information about the location of the MDC were derived from these technical drawings and from ISAS [ISA1998].

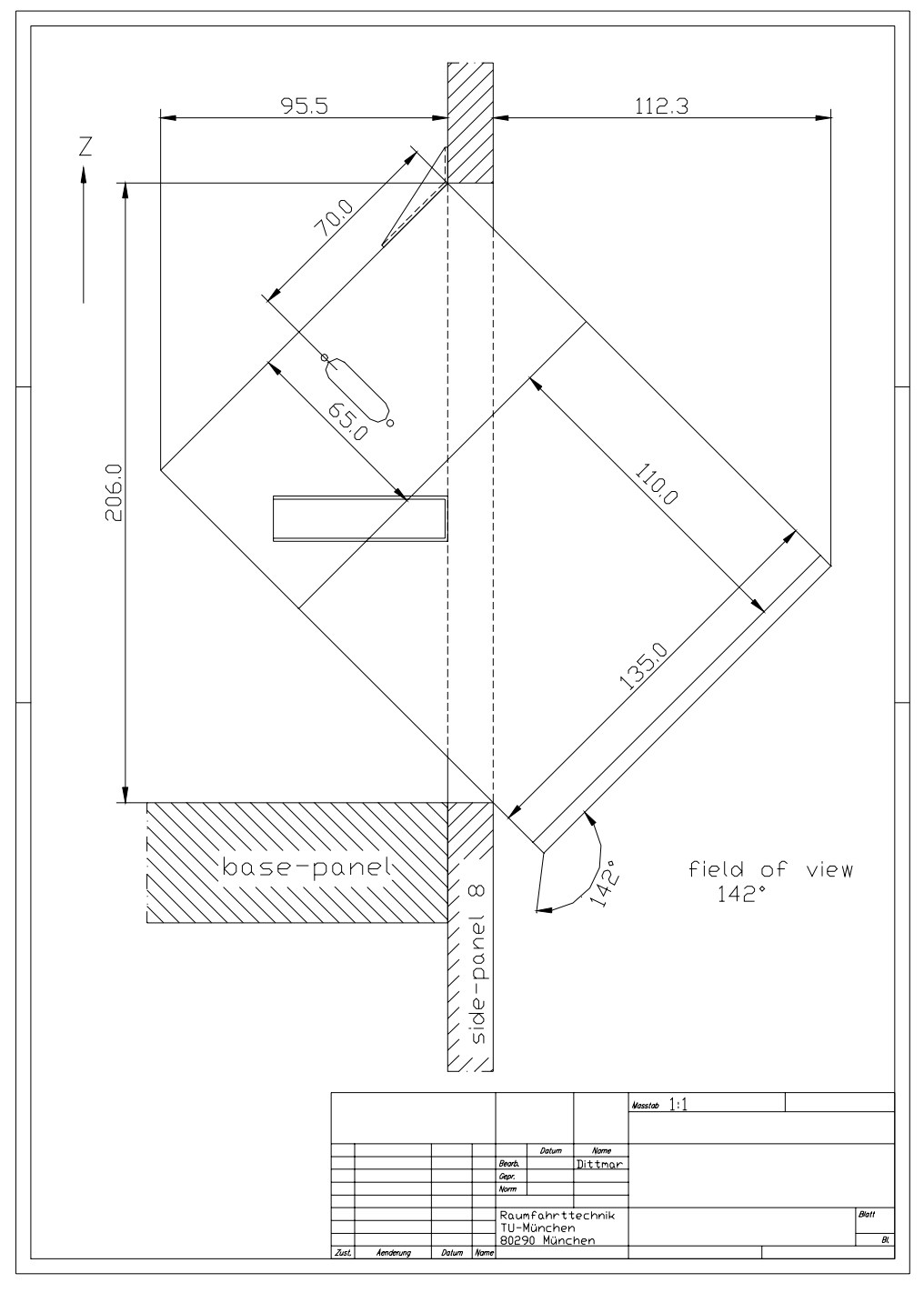

Figure 3-4: MDC integration, details

The MDC is mounted into NOZOMI side-panel \#8, rotated $45^{\circ}$ against the side-panel plane. The side panel restricts the MDC aperture angle to $142^{\circ}$ in the z-direction. 


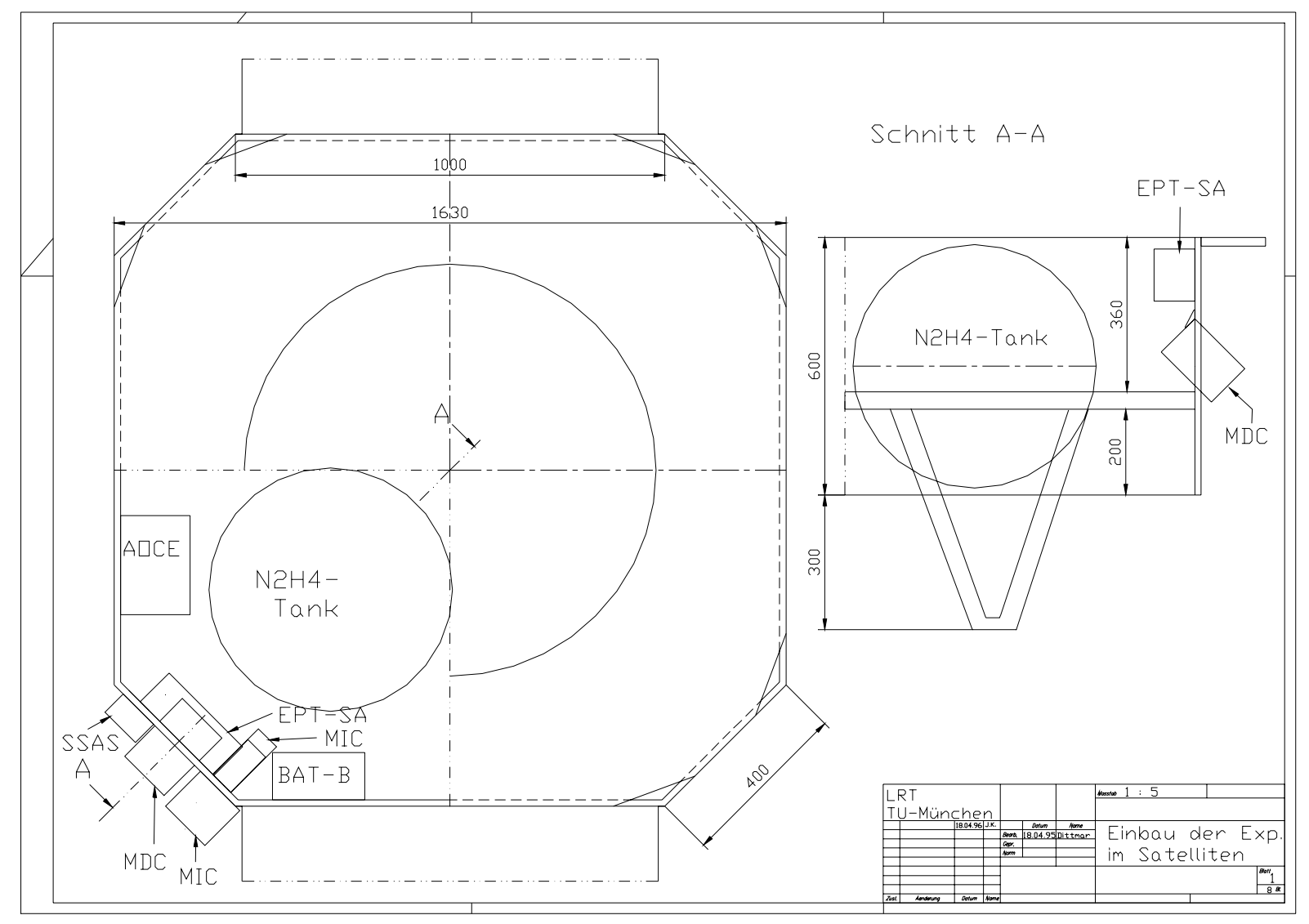

Figure 3-5: MDC integration, overview

The MDC is located on the NOZOMI side panel \#8, next to the SSAS (Sun sensor). Unlike it is shown in this image, the MDC was later shifted towards side panel \#1 (the neighboring panel, clockwise direction).

The field of view of the MDC can be described by the aperture angle and the effective solid angle, both depending on the instrument's and NOZOMI's geometric characteristics. While the aperture angle only gives a simple description of the field of view of the instrument itself without any consideration of possibly complex geometric characteristics, the effective solid angle is an exact value of the instruments detection capabilities. Determination of the effective solid angle requires detailed modeling of the geometric characteristics of the instrument and NOZOMI, and for complex configurations even numerical simulations. As an example, the effective solid angle of the Cosmic Dust Analyzer (CDA) on CASSINI was determined by a Monte-Carlo simulation of virtual impacting particles. For the MDC, this method is not practicable since the geometric conditions of the CDA and the MDC differ significantly. While the CDA target area is obscured only by parts of the instrument body that are located inside the sensor box, the MDC target area is obscured only by parts of the NOZOMI body that are located outside the sensor box at distances of several centimeters to meters. 


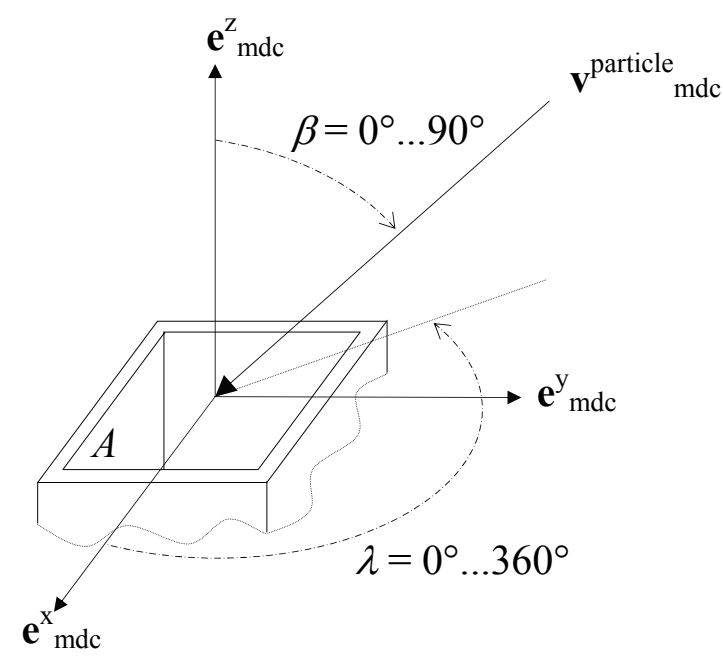

Figure 3-6: Latitude $\beta$ and longitude $\lambda$ of an impacting particle

Longitude $\lambda$ of an impacting particle is measured in mathematically positive direction from the $\mathrm{x}$-axis; latitude $\beta$ is the angle between the $\mathrm{z}$-axis and the particle direction.

Fortunately, the geometric conditions of the MDC instrument and the NOZOMI body are quite simple, analytical modeling and numerical integration is therefore feasible. Since the whole inner walls of the box build the instrument's sensitive area, the effective sensitive area or target area can be simply modeled as the aperture of the MDC's sensor box. Impacts with incident angles up to $90^{\circ}$ can be detected by the MDC, the field of view of the instrument therefore is $180^{\circ}$ and the aperture angle $\beta_{\text {ap }}=90^{\circ}$ (if the target would be restricted to some smaller area inside the box, the aperture angle would be lower). These simple geometric conditions ease the further examinations.

Assuming a simple plain surface as sensitive area of the instrument, the effective sensitive area depending on the incident angle $\beta$ is given by

$$
A(\beta)=A_{\text {total }} \cos (\beta)
$$

whereas $A_{\text {total }}$ is the total surface area $\left(A_{\text {total }}=116 \mathrm{~mm} \cdot 125 \mathrm{~mm}=0.0145 \mathrm{~m}^{2}\right.$ for the MDC). Until now, the effects of the entrance grid are not considered. For a not obscured field of view, the solid angle element $d \Omega$ is given as

$$
d \Omega=\int_{0}^{2 \pi} d \lambda \sin (\beta) d \beta=2 \pi \sin (\beta) d \beta
$$

in the case of axial symmetry (the rectangular shape of the MDC's sensor area does not affect this), and the effective solid angle $\Omega$ calculates to

$$
\Omega=\int_{0}^{\beta_{a p}} 2 \pi \sin (\beta) \cos (\beta) d \beta=2 \pi\left[-\frac{1}{2} \cos ^{2}\left(\beta_{\text {ap }}\right)+\frac{1}{2}\right] .
$$


This evaluates to $\Omega=\pi$. Thus, a detector like the MDC consisting (in principle) of a flat surface which detects particles passing through the surface in one defined fly-through direction covers $1 / 4^{\text {th }}$ of the whole hemisphere (a hemisphere's solid angle is given as $4 \pi \mathrm{sr}$ ) and will detect $1 / 4^{\text {th }}$ of all particles (presumed an isotropic particle field).

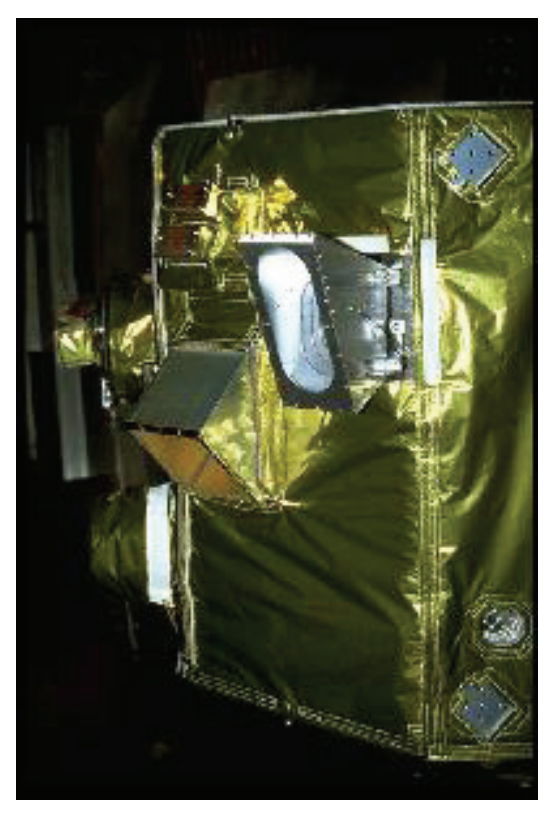

Figure 3-7: Position of the MDC on NOZOMI's side panel \#8

In this picture, the final position of the MDC on the NOZOMI side-panel \#8 can be seen. Next to the MDC, the MIC instrument and the SSAS are located. The golden MLI (Multi-Layer-Insulation) was replaced by black Kapton $^{\circledR}$ prior to launch.

This is only valid if the whole field-of-view of the MDC would not be obscured, which is not the case as can be seen in Figure 3-7. Parts of NOZOMI's body, of other instruments and the solar paddle mounted next to the MDC's side panel obscure the MDC's field of view. Also, the penetrability of the entrance grid must be considered. The determination of the effective solid angle of the MDC must be performed with respect to these obscuring parts of NOZOMI's body. In the following, the calculation method used by the author for effective solid angle determination is described. In principle, all obscuring parts of the NOZOMI body in the field of view were analytically modeled, except of the entrance grid whose obscuring effect was modeled as an averaged clouding if the MDC view. 


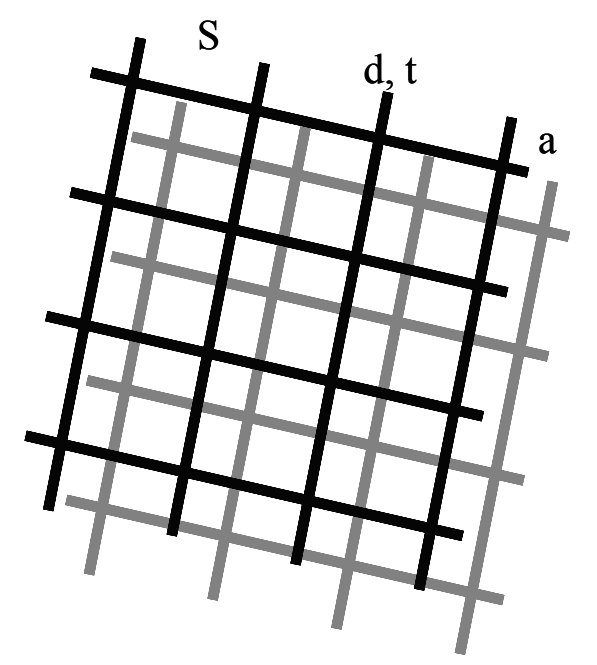

Figure 3-8: Illustration of the MDC entrance grid geometry.

The MDC sensor box is shielded by two identical grids (black and grey in this illustration) with a distance $a \approx 5 \mathrm{~mm}$. Distance between the bars is $S=$ $4 \mathrm{~mm}$, width of the bars is $d=0.1 \mathrm{~mm}$ and thickness of each grid is $t=0.05$ $\mathrm{mm}$.

The MDC entrance grid that shields the inner of the sensor box from outer electromagnetic disturbances had the following dimensions: distance between the bars $S=4 \mathrm{~mm}$, width $d=$ $0.1 \mathrm{~mm}$, thickness $t=0.05 \mathrm{~mm}$, symmetrically in $\mathrm{x}$ - and $\mathrm{y}$ - direction. According to Münzenmayer [Mün1995], the double entrance grid's penetrability $\eta$ calculates to

$$
\eta=\frac{(S-(d+t \cos \beta+D / \sin \beta))^{2}}{S^{2}}
$$

whereas $D$ is the size of the impacting particle and $\beta=0 \ldots 90^{\circ}$ the impact angle. The shape of the penetrability $\eta(\beta, D)$ is shown in Figure 3-9. Assuming a mean particle density of $2.0 \mathrm{~g} / \mathrm{cm}^{3}$ [Lov1994], the particle sizes $(0.1 \mu \mathrm{m}, 1 \mu \mathrm{m}, 10 \mu \mathrm{m}, 100 \mu \mathrm{m}, 1000 \mu \mathrm{m})$ correspond to the masses $\left(10^{-15} \mathrm{~g}, 10^{-12} \mathrm{~g}, 10^{-9} \mathrm{~g}, 10^{-6} \mathrm{~g}, 10^{-3} \mathrm{~g}\right)$. Since the MDC sensor box is shielded by two grids in series with a distance that is much higher than the grids' bar width and thickness, the effect of the grid can be considered by altering the solid angle element $\mathrm{d} \Omega$ given by equation (3-4) to

$$
d \Omega(D)=2 \pi \sin (\beta) \eta^{2}(\beta, D) d \beta
$$

which gives a particle diameter (or particle mass) dependent solid angle element $\mathrm{d} \Omega(D)$ and an effective solid angle $\Omega(D)$. This is resulting from the fact that the order of magnitude of the grid parameters (millimeters and fractions of millimeters) and particle sizes (some micrometers to several 10-100 micrometers) are close to each other. This comes into charge especially for high latitudes $\beta$ (for the other obscuring areas, whose sizes are in the order of magnitude of centimeters to meters, the sizes of the particles are not relevant). 


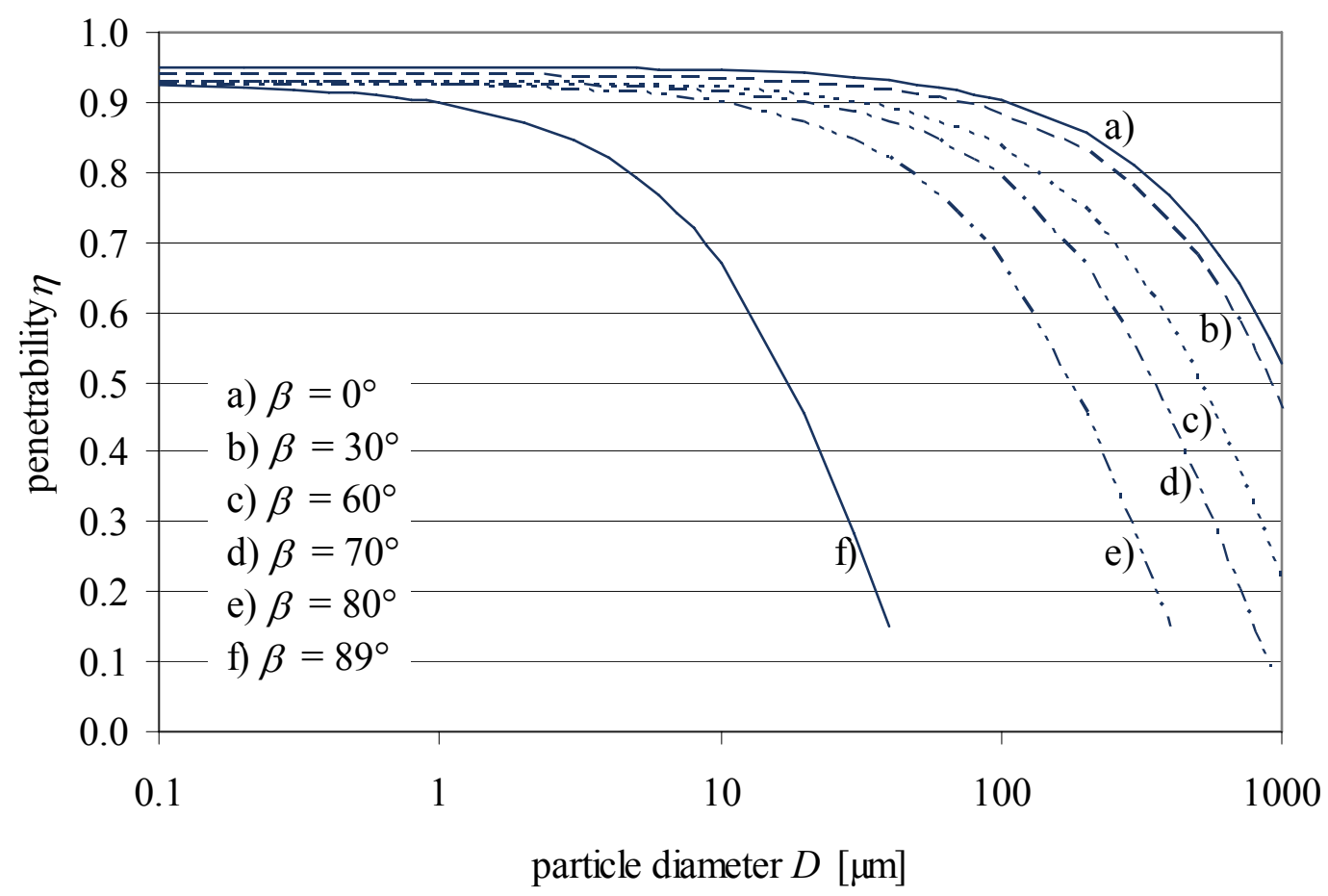

Figure 3-9: Penetrability of the MDC entrance grid over particle size.

For impact angles $\beta$ greater than $10^{\circ}$, the penetrability of the grid is nearly constant for particle sizes from $0.1 \mu \mathrm{m}$ to $10 \mu \mathrm{m}$ at around $95 \%$ to $90 \%$. For impact angles $\beta$ lower than $10^{\circ}$ or particle sizes greater than some tens of micrometers, the penetrability decreases fast. The curves d), e) and f) stop where the bracketed, not squared numerator of equation (3-6) gets negative for the next discrete value $D_{\max }$, hence $\eta\left(D \geq D_{\max }\right)=0$.

All calculations were performed using Microsoft ${ }^{\circledR}$ Excel $^{\circledR}$. First, it must be considered that the dimensions of the MDC sensor box and the obscuring areas as well as the distance of the obscuring areas from the MDC sensor area are all in the order of magnitude of decimeters. Therefore, the effective solid angle is significantly different for every position on the MDC sensor area. While the NOZOMI solar paddle PDL-B is located sufficiently far away from the sensor box (in the magnitude of meters, the resulting differences of view-angles for different positions on the MDC sensor area are therefore very small), the MDC side panel \#8 is located very near the sensor box and thus produces different rates of obscuration for different positions on the sensor area. Hence, the MDC sensor area was divided into 81 smaller sensor areas $(n=-4 \ldots 4, m=-4 \ldots 4)$ and the effective solid angle was calculated for each of the different positions. The results were then averaged to calculate the final effective solid angle of the MDC on NOZOMI.

For each position $(n, m)$, a $90 \times 360$ matrix $\mathbf{O}_{n m i j}$ was created that represents all possible view directions from the center of the MDC effective sensor area, described by latitude $\beta_{i}=$ $0^{\circ} \ldots 89^{\circ}(i=0 \ldots 89)$ and longitude $\lambda_{j}=0^{\circ} \ldots 359^{\circ}(j=0 \ldots 359)$ in steps of $1^{\circ}$ each, see also Figure 3-6. Each cell of this matrix contained either the value +1 (free view direction) or 0 (obscured view direction). The solid angle element for a given latitude $\beta_{i}$ was then calculated by 


$$
\Delta \Omega_{n m i}(D)=2 \pi \sin \left(\beta_{i}\right) \eta\left(\beta_{i}, D\right)\left(\frac{1}{360} \sum_{j=0}^{359} \mathbf{O}_{n m i j}\right) \Delta \beta
$$

whereas $\Delta \beta=1^{\circ}$ is the step-width used. From this, the final effective solid angle $\Omega$ was then calculated by

$$
\Omega_{n m}(D)=\sum_{i=0}^{89} \Delta \Omega_{n m i}(D) \cos \beta_{i}
$$

For a completely free field of view $\left(\mathbf{O}_{n m i j}=+1\right.$ for all $\left.i, j, n, m ; \eta=1\right)$ this evaluates to an effective solid angle of $\Omega=0.99989 \pi \mathrm{sr}$ rather than the exact analytical value of $1 \pi \mathrm{sr}$. The accuracy of this method can therefore be seen as proven to be sufficient for the calculation of $\Omega$, presumed correct modeling of the NOZOMI parts.

In the next step, the cells of the matrix $\mathbf{O}_{\text {nmij }}$ must be filled with the appropriate values +1 or 0 , respectively. To perform this, the obscuring areas had to be modeled and each individual line of view described by $\left(\lambda_{j}, \beta_{i}\right)$ had to be tested whether it crosses at least one of these areas or not. First, the center of the MDC effective sensor area was placed in the origin of a Cartesian coordinate system with the positive z-axis perpendicular to the sensor area. The obscuring areas or planes, respectively, were modeled as planes using the vectorial representation for the $k^{\text {th }}$ plane $(k=1 \ldots N)$

$$
\mathbf{q}_{k}=\mathbf{q}_{k, 0}+\mu \mathbf{x}_{k}+\mathbf{y}_{k},
$$

where $\mathbf{q}_{k, 0}$ is the center point of the $k^{\text {th }}$ area, and the linear independent unit vectors $\mathbf{x}_{k}$ and $\mathbf{y}_{k}$ span the plane $\left(\mathbf{x}_{k} \perp \mathbf{y}_{k},\left|\mathbf{x}_{k}\right|=\left|\mathbf{y}_{k}\right|=1\right)$. The dimensions of the $k^{\text {th }}$ rectangular area are given by $d x_{k}$ and $d y_{k}$ for rectangular areas, or $d r_{k}$ for circular areas (and spheres modeled as circles), respectively. 


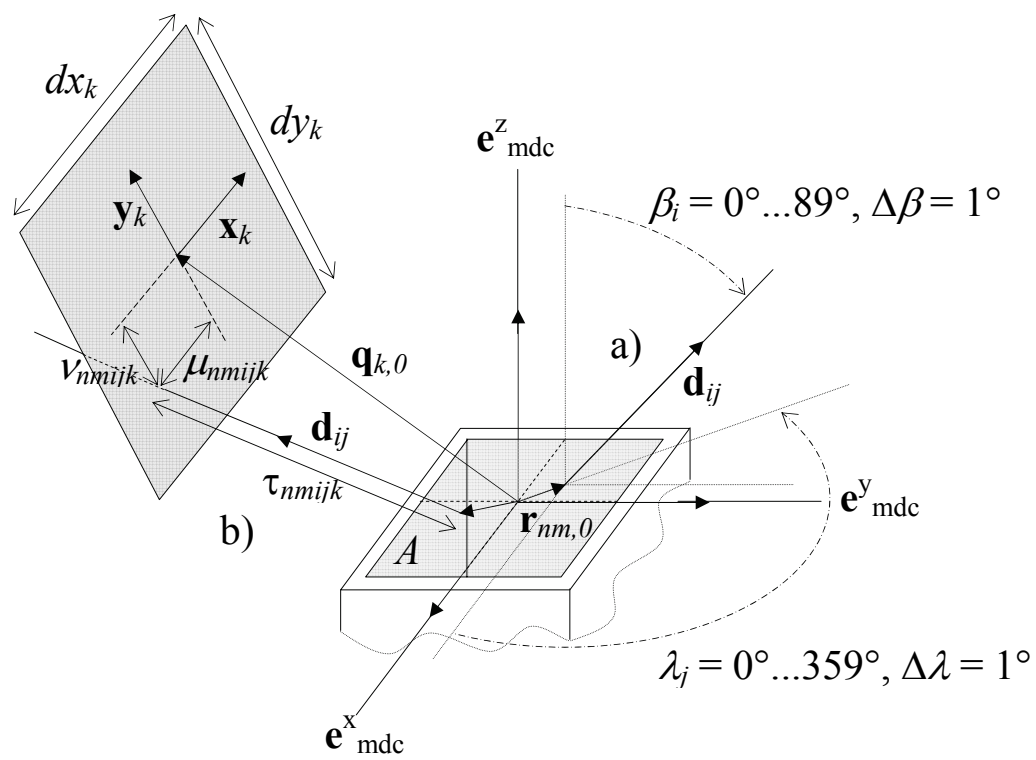

Figure 3-10: Illustration of the geometry for the calculation of $\Omega$

The geometry of the models of the MDC sensor area, the view directions and the obscuring areas is shown here. In case a) the line of view is not obscured, the angles $\beta$ and $\lambda$ are illustrated. In case $b$ ), the line of view crosses the obscuring area, the values $\mu_{n m i j k}, v_{n m i j k}$ and $\tau_{n m i j k}$ are illustrated that are used to determine if there is a point of intersection and if it's located within the area's boundaries. All vectors but $\mathbf{q}_{k, 0}$ and $\mathbf{r}_{n m, 0}$ are unit vectors.

The NOZOMI parts that obscure the MDC field-of-view were modeled from the available technical drawings as follows (all dimensions in millimeters):

$k=1$, NOZOMI side-panel \#8, rectangular area ${ }^{1}$

$$
\mathbf{q}_{1,0}=\left(\begin{array}{c}
25.1 \\
100 \\
-66.2
\end{array}\right), \mathbf{x}_{1}=\left(\begin{array}{c}
0.707 \\
0 \\
0.707
\end{array}\right), \mathbf{y}_{1}=\left(\begin{array}{l}
0 \\
1 \\
0
\end{array}\right), d x_{1}=600, d y_{l}=400
$$

$k=2$, NOZOMI solar paddle PDL-B, rectangular area

$$
\mathbf{q}_{2,0}=\left(\begin{array}{c}
-497 \\
1446 \\
31.7
\end{array}\right), \mathbf{x}_{2}=\left(\begin{array}{c}
-0.5 \\
0.707 \\
0.5
\end{array}\right), \mathbf{y}_{2}=\left(\begin{array}{c}
0.5 \\
0.707 \\
-0.5
\end{array}\right), d x_{2}=2240, d y_{2}=1000
$$

\footnotetext{
${ }^{1}$ Unlike it is shown in the technical drawings (see Figure 3-4 and Figure 3-5), the MDC was finally not mounted in the middle of the side panel \#8, but shifted towards side panel \#1. Since no drawings were available that could give more information, the value $q_{1,0}^{\mathrm{y}}=100 \mathrm{~mm}$ was estimated from photographs (see Figure 3-7) of the final NOZOMI configuration where the MDC seems to be mounted in or near the middle of one half of the side panel \#8.
} 
$k=3$, NMS instrument on side-panel \#1, circular area, not modeled due to lack of data

$$
\mathbf{q}_{3,0}=\left(\begin{array}{l}
\mathrm{N} / \mathrm{A} \\
\mathrm{N} / \mathrm{A} \\
\mathrm{N} / \mathrm{A}
\end{array}\right), \quad \mathbf{x}_{3}=\left(\begin{array}{l}
\mathrm{N} / \mathrm{A} \\
\mathrm{N} / \mathrm{A} \\
\mathrm{N} / \mathrm{A}
\end{array}\right), \quad \mathbf{y}_{3}=\left(\begin{array}{l}
\mathrm{N} / \mathrm{A} \\
\mathrm{N} / \mathrm{A} \\
\mathrm{N} / \mathrm{A}
\end{array}\right), \quad d r_{3}=\mathrm{N} / \mathrm{A}
$$

The line of sight defined by $\left(\lambda_{j}, \beta_{i}\right)$ was also modeled using the vectorial representation

$$
\mathbf{r}_{n m i j}=\mathbf{r}_{n m, 0}+\tau \mathbf{d}_{i j}
$$

whereas $\mathbf{r}_{n m, 0}$ is the center of the $(n, m)^{\text {th }}$ part of the MDC sensor area as described above and $\mathbf{d}_{i j}=\left(\cos \lambda_{j} \sin \beta_{i}, \sin \lambda_{j} \sin \beta_{i}, \cos \beta_{i}\right)^{\mathrm{T}}$ is the current view direction. The point of intersection of the $(i, j)^{\text {th }}$ line of view and the $k^{\text {th }}$ plane is then given by the equation

$$
\begin{aligned}
\mathbf{q}_{k} & =\mathbf{r}_{n m i j} \\
\mathbf{q}_{k, 0}+\mu \mathbf{x}_{k}+\gamma \mathbf{y}_{k} & =\mathbf{r}_{n m, 0}+\tau \mathbf{d}_{i j} \\
\mu \mathbf{x}_{k}+v \mathbf{y}_{k}-\tau \mathbf{d}_{i j} & =\mathbf{r}_{n m, 0}-\mathbf{q}_{k, 0}
\end{aligned}
$$

which represents an inhomogeneous $3 \times 3$ system of linear equations with three independent variables that can be solved directly (there is always exactly one real solution for linear independent vectors $\mathbf{x}_{k}, \mathbf{y}_{k}$ and $\mathbf{d}_{i j}$, the case of linear dependence (the line of view is parallel to the given $k^{\text {th }}$ area) is rather unlikely in this approximation and even would have been trapped by the Excel $^{\circledR}$ engine). For the calculation of $\mu_{n m i j k}, v_{n m i j k}$ and $\tau_{n m i j k}$, the rule of Cramer was used. For the given system of linear equations,

$$
\left(\begin{array}{lll}
x_{k}^{1} & y_{k}^{1} & -d_{i j}^{1} \\
x_{k}^{2} & y_{k}^{2} & -d_{i j}^{2} \\
x_{k}^{3} & y_{k}^{3} & -d_{i j}^{3}
\end{array}\right) \cdot\left(\begin{array}{c}
\mu_{n m i j k} \\
v_{n m i j k} \\
\tau_{n m i j k}
\end{array}\right)=\left(\begin{array}{l}
r_{n m, 0}^{1}-q_{k, 0}^{1} \\
r_{n m, 0}^{2}-q_{k, 0}^{2} \\
r_{n m, 0}^{3}-q_{k, 0}^{3}
\end{array}\right), \text { or } \mathbf{A}_{i j k} \cdot \boldsymbol{\rho}_{n m i j k}=\mathbf{i}_{n m k},
$$

the values $\mu_{n m i j k}, v_{n m i j k}$ and $\tau_{n m i j k}$ are given by

$$
\mu_{n m i j k}=\frac{\operatorname{det}\left(\mathbf{A}_{n m i j k}^{\mu}\right)}{\operatorname{det}\left(\mathbf{A}_{i j k}\right)}\left(v_{n m i j k} \text { and } \tau_{n m i j k} \text { alike }\right),
$$

where the matrix $\mathbf{A}_{n m i j k}^{\mu}$ is derived from the matrix $\mathbf{A}_{i j k}$ by replacing the accordant column by the right side vector $\mathbf{i}_{n m k}$. Given $\mu_{n m i j k}, v_{n m i j k}$ and $\tau_{n m i j k}$, the point of intersection could be easily calculated from the linear or planar equations (3-10) and (3-14) described above, but this is not required here. It must only be determined whether the point of intersection is located in the positive view direction and within the boundaries of the given area $k$. This can be checked by applying the following conditions: 
$\tau_{n m i j k}>0$ (the point of intersection is located in positive direction of the line of view), AND

for rectangular areas:

$\mu_{n m i j k}<1 / 2 d x_{k}$, (the point of intersection is located within the x-dimension of the area), AND

$v_{n m i j k}<1 / 2 d y_{k}$, (the point of intersection is located within the y-dimension of the area),

for circular areas:

$\sqrt{\left(\mu_{n m i j k}\right)^{2}+\left(v_{n m i j k}\right)^{2}}<d r_{k}$ (the point of intersection is located within the circle's dimensions).

If all three (or two for circular areas) conditions are fulfilled, the correspondent cell in the $90 \times 360$ matrix $\mathbf{O}_{n m i j k}$ is set to 0 (obscured by the $k^{\text {th }}$ plane), otherwise to +1 (not obscured by the $k^{\text {th }}$ plane). The final matrix $\mathbf{O}_{n m i j}$ is then build by a logical AND operation over all $\mathbf{O}_{n m i j k}$, $k=1 \ldots N$.

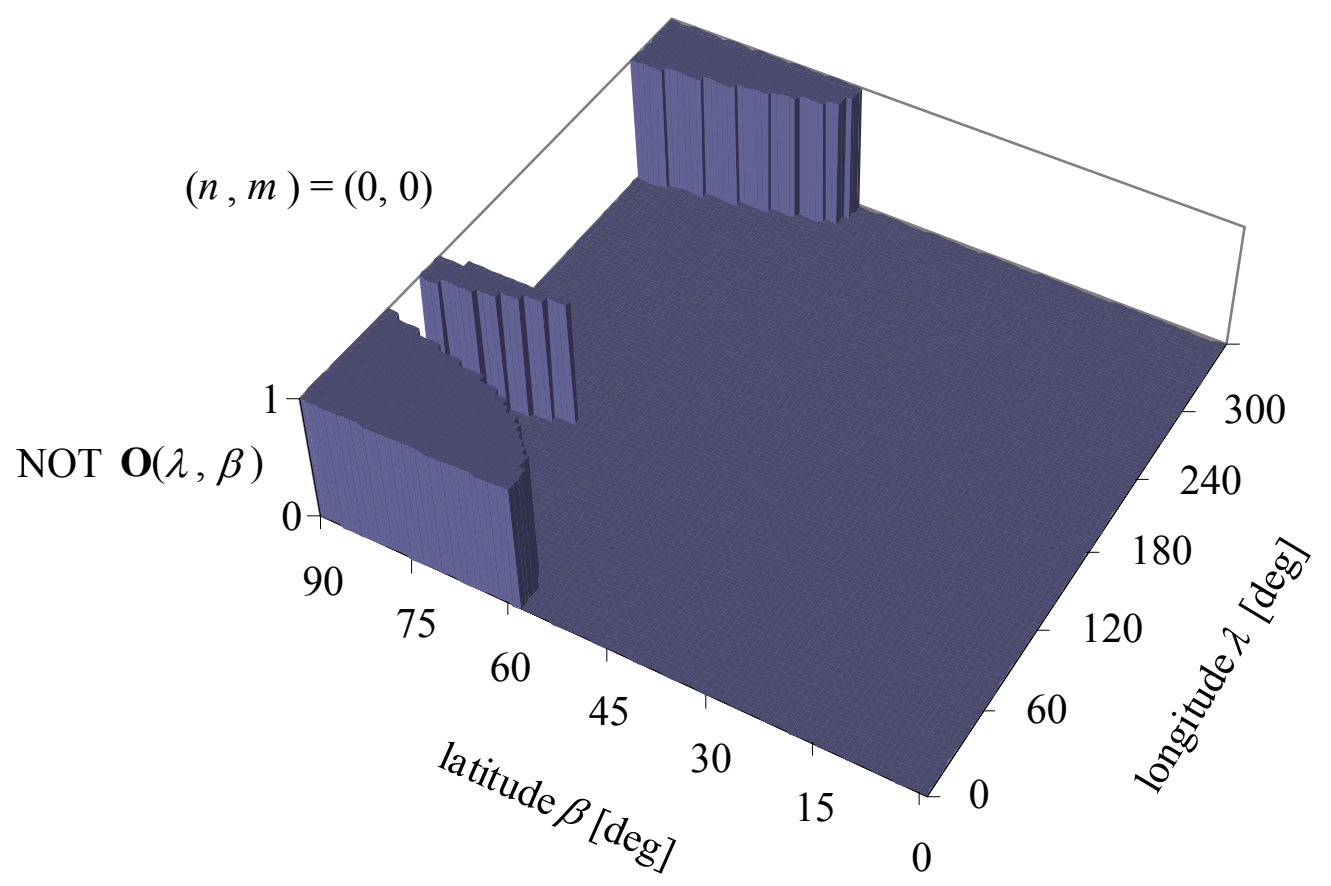

Figure 3-11: Visualization of the MDC view area

In this picture, the inverse of the matrix $\mathbf{O}_{0 o i j}\left(\mathrm{NOT} \mathbf{O}_{00 i j}\right)$ is visualized, $(n$, $m)=(0,0)$. From left to right, the impact angle $\beta=0^{\circ} \ldots 90^{\circ}$ is displayed where $0^{\circ}$ means vertical impact and $90^{\circ}$ striped impact, from bottom to top the longitude angle $\lambda=0^{\circ} \ldots 360^{\circ}$ is displayed. The bottom area with NOT $\mathbf{O}_{00 i j}=0$ represents directions of free view. The exalted area with NOT $\mathbf{O}_{00 i j}$ $=1$ and $\beta=59^{\circ} \ldots 90^{\circ}, \lambda=313^{\circ} \ldots 73^{\circ}$ represents the NOZOMI side panel $\# 8$, the area with NOT $\mathbf{O}_{00 i j}=1$ and $\beta=71^{\circ} \ldots 90^{\circ}, \lambda=103^{\circ} \ldots 124^{\circ}$ represents the NOZOMI solar paddle PDL-B attached to side panel \#7.

Using the method described above with the orientations and best guess dimensions of the two obscuring areas, the effective solid angle of the MDC evaluates to $\Omega_{00}=0.9398 \pi \mathrm{sr}$ as seen 
from the center point of the MDC sensor area, $(n, m)=(0,0)$ without considering the grid $(\eta=1)$.

The final effective solid angle $\Omega_{\mathrm{MDC}}(D)$ is then averaged from the 81 values $\Omega_{n m}(D)$ by

$$
\Omega_{\mathrm{MDC}}(D)=\frac{1}{81} \sum_{n, m=-4}^{4} \Omega_{n m}(D)
$$

Figure 3-12 shows the effective solid angle $\Omega_{n m}(D)$ for $D=5 \mu \mathrm{m}$, Figure 3-13 shows the final effective solid angle $\Omega_{\mathrm{MDC}}(D)$. The discrete values are shown in Table 3-1. For latter calculations of dust flux measured by the MDC, these values were used.

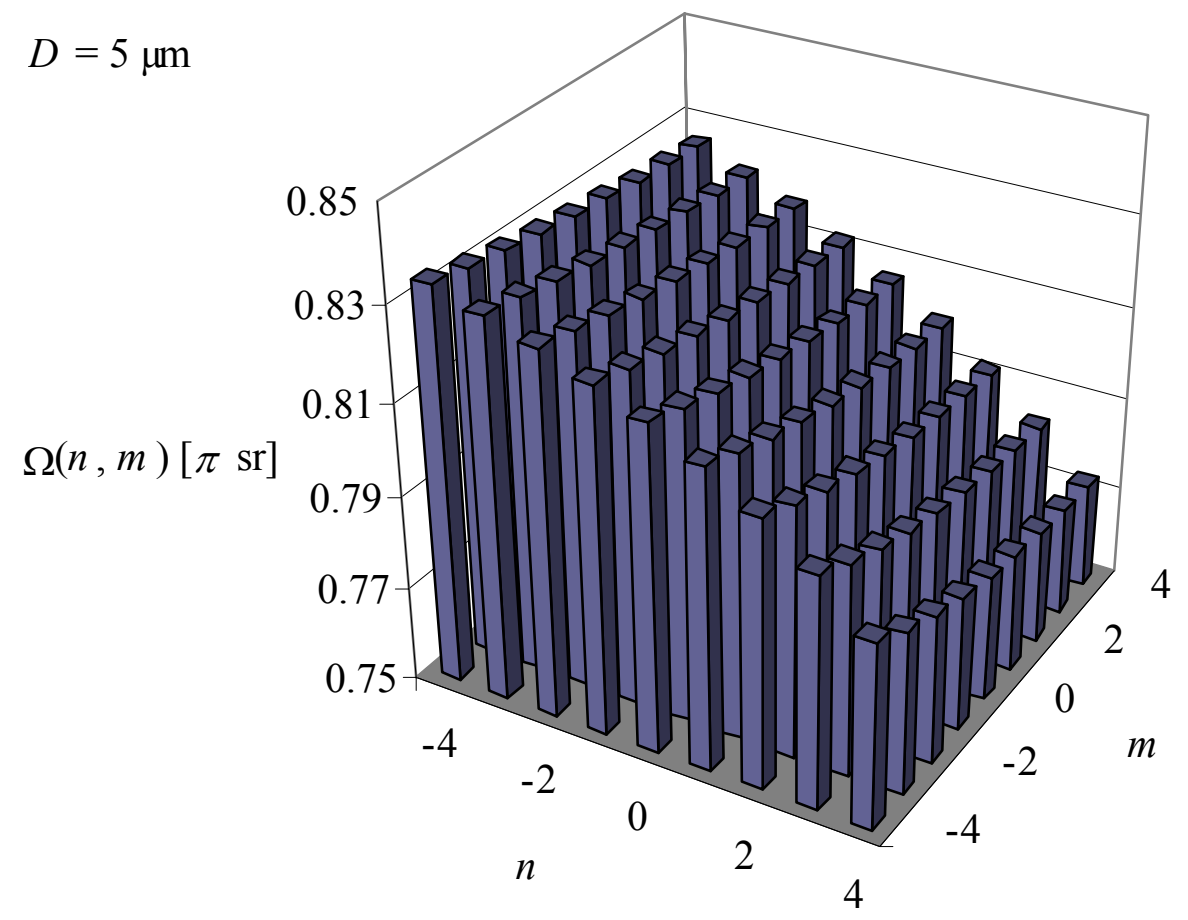

Figure 3-12: Effective solid angle $\Omega_{n m}(D)$ for a particle size of $D=5 \mu \mathrm{m}$

The 81 values $\Omega_{n m}(D)$ are displayed as bars over the MDC sensor area (grey area). Maximum value is $0.834 \pi \mathrm{sr}$ at $(n, m)=(-4,-4)$, minimum value is $0.773 \pi \mathrm{sr}$ at $(n, m)=(4,4)$. Mean value is $0.808 \pi \mathrm{sr}$.

The calculation of the final values shown in Table 3-1 and Figure 3-13 took about 25 minutes on an $800 \mathrm{MHz}$ Intel $^{\circledR}$ Pentium $^{\circledR}$ III desktop PC, due to the large number of $3 \times 3$ determinants that had to be evaluated and the limited computing efficiency of Microsoft ${ }^{\circledR}$ Excel $^{\circledR}$ and VBA scripting. 
Table 3-1: Effective solid angle $\Omega_{\mathrm{MDC}}(D)$, final values

\begin{tabular}{cccccccccc}
\hline$D[\mu \mathrm{m}]$ & 0.1 & 0.2 & 0.3 & 0.4 & 0.5 & 0.6 & 0.7 & 0.8 & 0.9 \\
$\Omega[\pi \mathrm{sr}]$ & 0.817 & 0.817 & 0.816 & 0.816 & 0.816 & 0.816 & 0.816 & 0.816 & 0.815 \\
\hline$D[\mu \mathrm{m}]$ & 1 & 2 & 3 & 4 & 5 & 6 & 7 & 8 & 9 \\
$\Omega[\pi \mathrm{sr}]$ & 0.815 & 0.814 & 0.812 & 0.810 & 0.808 & 0.807 & 0.805 & 0.803 & 0.802 \\
\hline$D[\mu \mathrm{m}]$ & 10 & 20 & 30 & 40 & 50 & 60 & 70 & 80 & 90 \\
$\Omega[\pi \mathrm{sr}]$ & 0.800 & 0.784 & 0.768 & 0.753 & 0.738 & 0.723 & 0.709 & 0.695 & 0.682 \\
\hline$D[\mu \mathrm{m}]$ & 100 & 200 & 300 & 400 & 500 & 600 & 700 & 800 & 900 \\
$\Omega[\pi \mathrm{sr}]$ & 0.669 & 0.552 & 0.457 & 0.379 & 0.314 & 0.259 & 0.214 & 0.176 & 0.144 \\
\hline
\end{tabular}

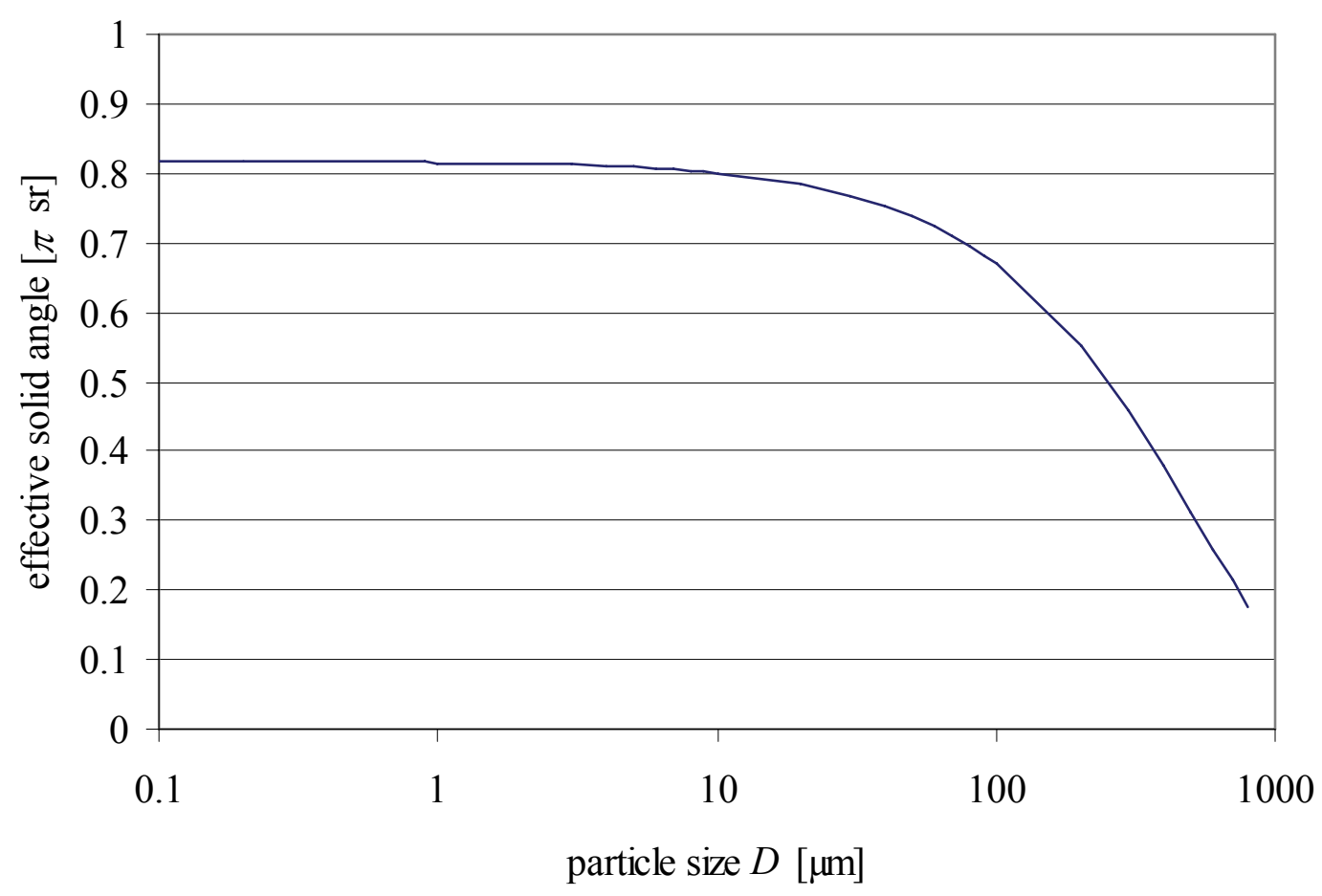

Figure 3-13: Particle size dependence of the effective solid angle $\Omega_{\mathrm{MDC}}(D)$ For particle sizes from $0.1 \mu \mathrm{m}$ to $10 \mu \mathrm{m}$, the effective solid angle is nearly constant around 0.82 to $0.80 \pi \mathrm{sr}$, while it decreases fast for particle sizes greater than some tens of micrometers.

Another interesting characteristic of the MDC/NOZOMI system is the spin-averaged sensitivity of the instrument, or average area of the instrument depending on the angle to the spin-axis, respectively (see Figure 3-14). Based on the method described above, the spinaveraged sensitivity was calculated as described in the following. 


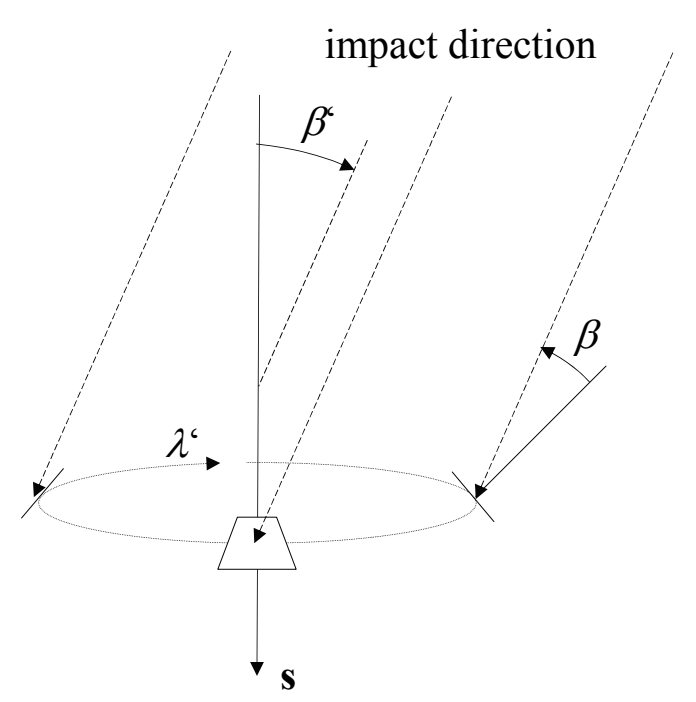

Figure 3-14: Geometry of the calculation of the spin-averaged sensitivity

The effective detector area of the MDC varies with the spin angle for particles that arrive at a certain angle $\beta$ to the NOZOMI spin axis $\mathbf{s}$.

First, the matrices $\mathbf{O}_{n m i j k}$ were enlarged to $180 \times 360$ matrices as required by the range of $\left(\lambda_{i}\right.$, $\beta_{j}{ }_{j}$ ). Instead of rotating the satellite or the MDC, respectively, the view directions were rotated around the negative NOZOMI spin-axis. The view directions were calculated from the angles $\left(\lambda_{i}, \beta_{j}^{\prime}\right)$ as $\mathbf{d}_{i j}^{\prime}=\left(\cos \lambda_{j}^{\prime} \sin \beta_{i i}^{\prime}, \sin \lambda_{j}^{\prime} \sin \beta_{i}^{\prime}, \cos \beta_{i}^{\prime}\right)^{\mathrm{T}}$. The resulting view directions were then transformed from the NOZOMI reference system $\left(\lambda^{\prime}{ }_{i}, \beta_{j}{ }_{j}\right)$ to the MDC reference system $\left(\lambda_{i}, \beta_{j}\right)$ by the transformation

$$
\mathbf{d}_{i j}=\left(\begin{array}{ccc}
\cos \delta^{\prime} & 0 & -\sin \delta^{\prime} \\
0 & 1 & 0 \\
\sin \delta^{\prime} & 0 & \cos \delta^{\prime}
\end{array}\right) \mathbf{d}_{i j}^{\prime},
$$

whereas $\delta=-45^{\circ}$ is the angle of the MDC z-axis to the negative NOZOMI spin-axis. From these view directions, the lines of sight were modeled as described in equation (3-14). Now, the calculation of the matrices $\mathbf{O}_{n m i j k}$ was performed exactly as described above. From these matrices, the final matrix $\mathbf{O}_{n m i j}$ was again built by a logical AND operation over all matrices $\mathbf{O}_{n m i j k}, k=1 \ldots N$ and the matrix $\mathbf{O}_{n m i j 0}$ that contains information about whether the given view direction is pointing to the outside of the sensor box $\left(d_{i j}^{z}>0\right)$. Then, the average area $a_{n m i}$ for a given latitude $\beta_{j}$ and position $(n, m)$ was calculated by

$$
a_{n m i}=\frac{A}{81} \sum_{j=0}^{359} \mathbf{O}_{n m i j} d_{i j}^{z} \Delta \beta,
$$

whereas $A$ is the total MDC sensor area, $\Delta \beta=1^{\circ}$ is the step width and the factor $d_{i j}^{z}$ is the sine of the angle between the view direction and the MDC z-axis. The total average area for a given latitude $\beta_{i}$ was summed up as

$$
a_{i}=\sum_{n, m=-4}^{4} a_{n m i} .
$$


These values are visualized in Figure 3-15. The effect of the grid was not considered here since the quantitative values are not used for any further calculations. The integral of the spinaveraged sensitivity is shown in Figure 3-16. On an $800 \mathrm{MHz}$ Intel $^{\mathbb{R}}$ Pentium $^{\mathbb{R}}$ III desktop PC, the calculation of the final values $a_{i}$ took about 1 hour with Microsoft $^{\circledR}$ Excel $^{\circledR}$ and VBA scripting.

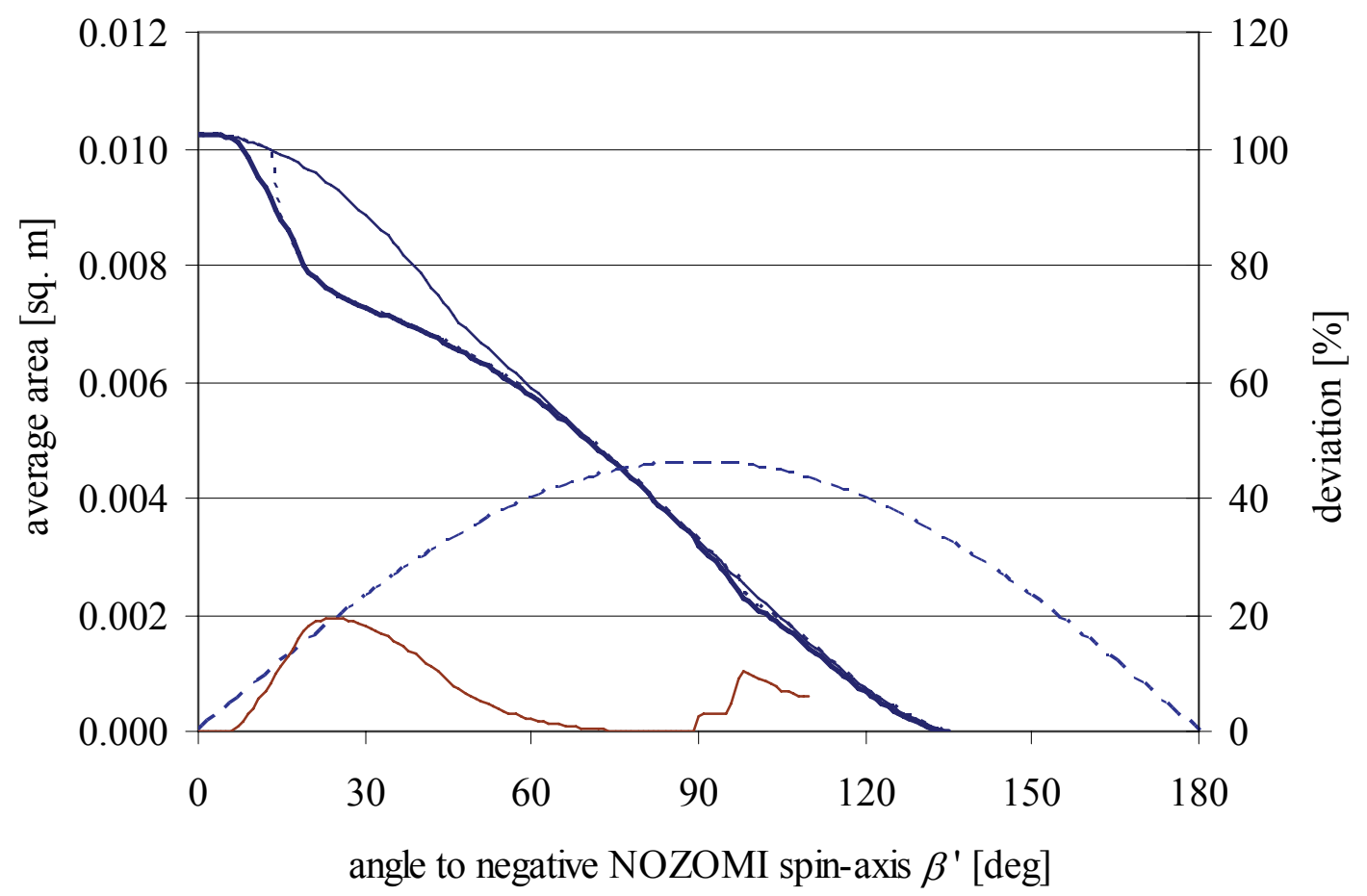

Figure 3-15: Spin-averaged sensitivity of the MDC on NOZOMI

The thick blue line represents the spin-averaged sensitivity of the MDC/NOZOMI system. See text for details.

The final spin averaged sensitivity of the MDC/NOZOMI system is shown in Figure 3-15. The thin blue solid line represents the spin-averaged sensitivity of the MDC without considering the obscuration by the side-panel and the solar-paddle, while the thick blue solid line represents the final spin-averaged sensitivity with consideration of the obscuration. The thin red solid line represents the effects of the obscuring areas as percentage (from $\approx 110^{\circ}$ onwards, the percentage values become invalid due to arithmetic errors caused by very small operands). The side panel affects the average area from $7^{\circ}$ to around $65^{\circ}$ (deviance $>1 \%$ ), maximum deviance is $19.3 \%$ at $24^{\circ}$. The solar panel has only little effect at around $98^{\circ} \pm 10^{\circ}$, maximum is $10.3 \%$. From $135^{\circ}$ to $180^{\circ}$, the average area is exactly zero. For illustration, the dotted blue line shows the average area for the single position $(n, m)=(0,0)$. As can be seen, the effect of the side panel starts later at $14^{\circ}$, but with a much sharper edge. Also for illustration purpose, the dashed blue line represents the spin-averaged sensitivity of a fictitious detector mounted at an angle of $90^{\circ}$ to the spin axis with completely free view area. 


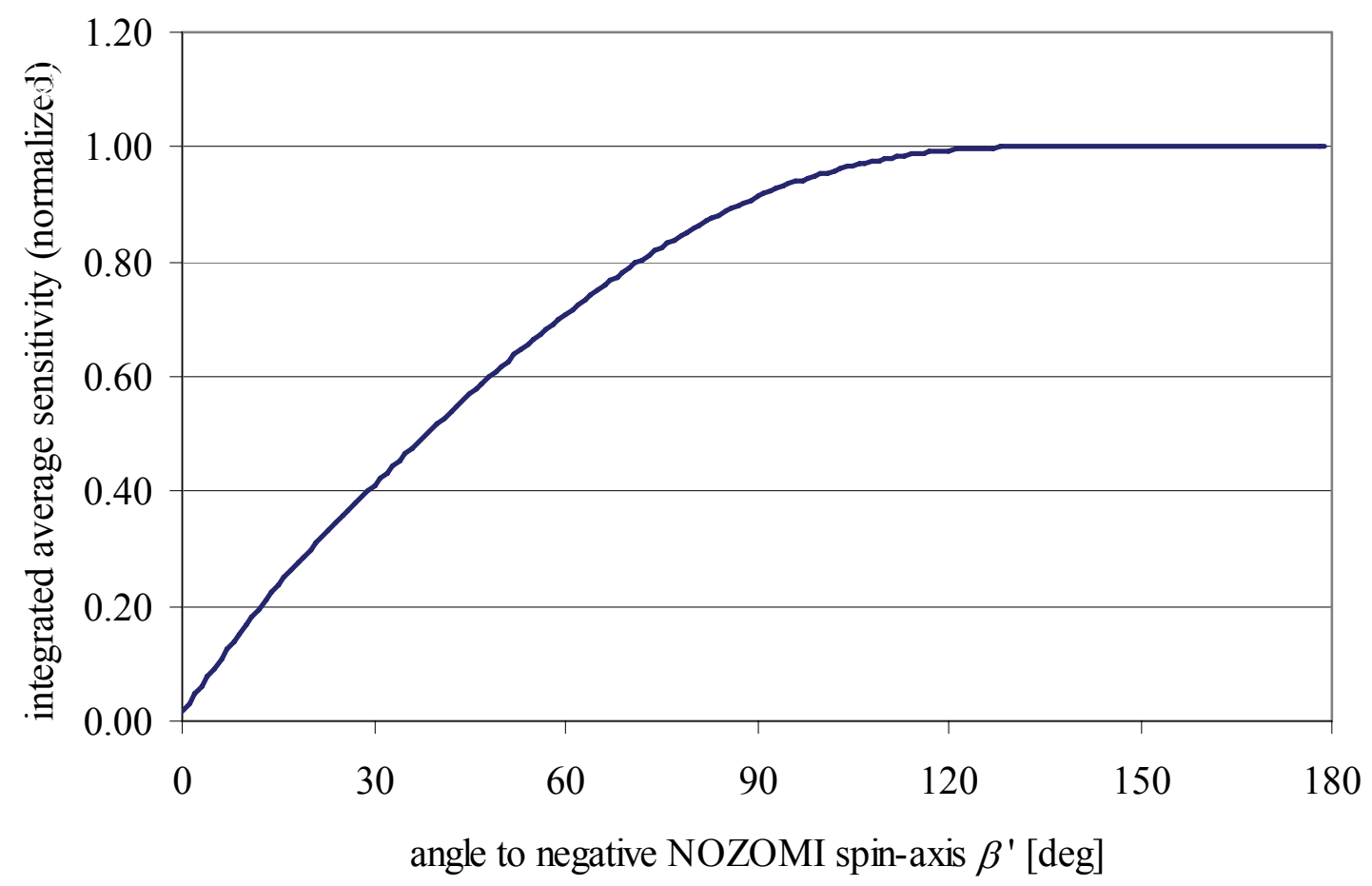

Figure 3-16: Integrated spin-averaged sensitivity

$50 \%$ of all detected particles have impact directions within a cone with $39^{\circ}$ aperture angle, $66 \%$ within $55^{\circ}, 75 \%$ within $65^{\circ}$ and $90 \%$ within $88^{\circ}$.

\subsubsection{Instrument detection capabilities}

To understand and interpret the results of the MDC, the characteristics and dynamics of high velocity impacts and the instrument's sensitivity must be taken into account. The MDC charge amplifiers and $\mathrm{A} / \mathrm{D}$ converters are designed to measure and process charges up to $10^{-10} \mathrm{C}$. Impacts that produce higher charge in either channel will overload the charge amplifiers and important parts of the signal are lost. In this case, rise time and amplitude cannot be determined, and a further analysis is not possible anymore. This usually happens for particles with high mass and high impact velocity. On the other hand, impacts must produce enough charge to be separated from the instruments background noise. As estimation, it can be said that impacts that produce less than $10^{-15} \mathrm{C}$ will not be recognized as impacts any more. Furthermore, any signal has to exceed a certain trigger level to be recorded by the MDC electronics at all. This trigger level must be chosen at a level, which is high enough to discard most noise events prior to processing in order to protect the MDC onboard computer from overload. On the other hand, the trigger level must not be chosen too high, otherwise signals that represent real impacts of low mass and low velocity particles will get lost.

During the whole interplanetary cruising phase (except for the phase where the "autotrigger" software patch was enabled), the instruments trigger level was set to a binary value of 143, which corresponds to a charge of $\approx 10^{-13} \mathrm{C}$, both for the ion channel and the electron channel. Impacts that produce less than $10^{-13} \mathrm{C}$ of positive and/or negative charge are not even triggered and therefore lost. 
Summarized, the instruments detection capabilities are limited to impacts that produce charge between $10^{-13}$ and $10^{-10} \mathrm{C}$. The charge gain of high velocity impacts is discussed in paragraph 3.1 and depends on the particle's mass as well as on its impact velocity. Figure 3-17 shows the calculated measuring range of the MDC for a given trigger level of 143 . It can be estimated that the best detection capabilities of the instrument are at particle masses between $10^{-12}$ and $10^{-11} \mathrm{~g}$. Above and below this interval, dust flux determinations do not give any reliable results.

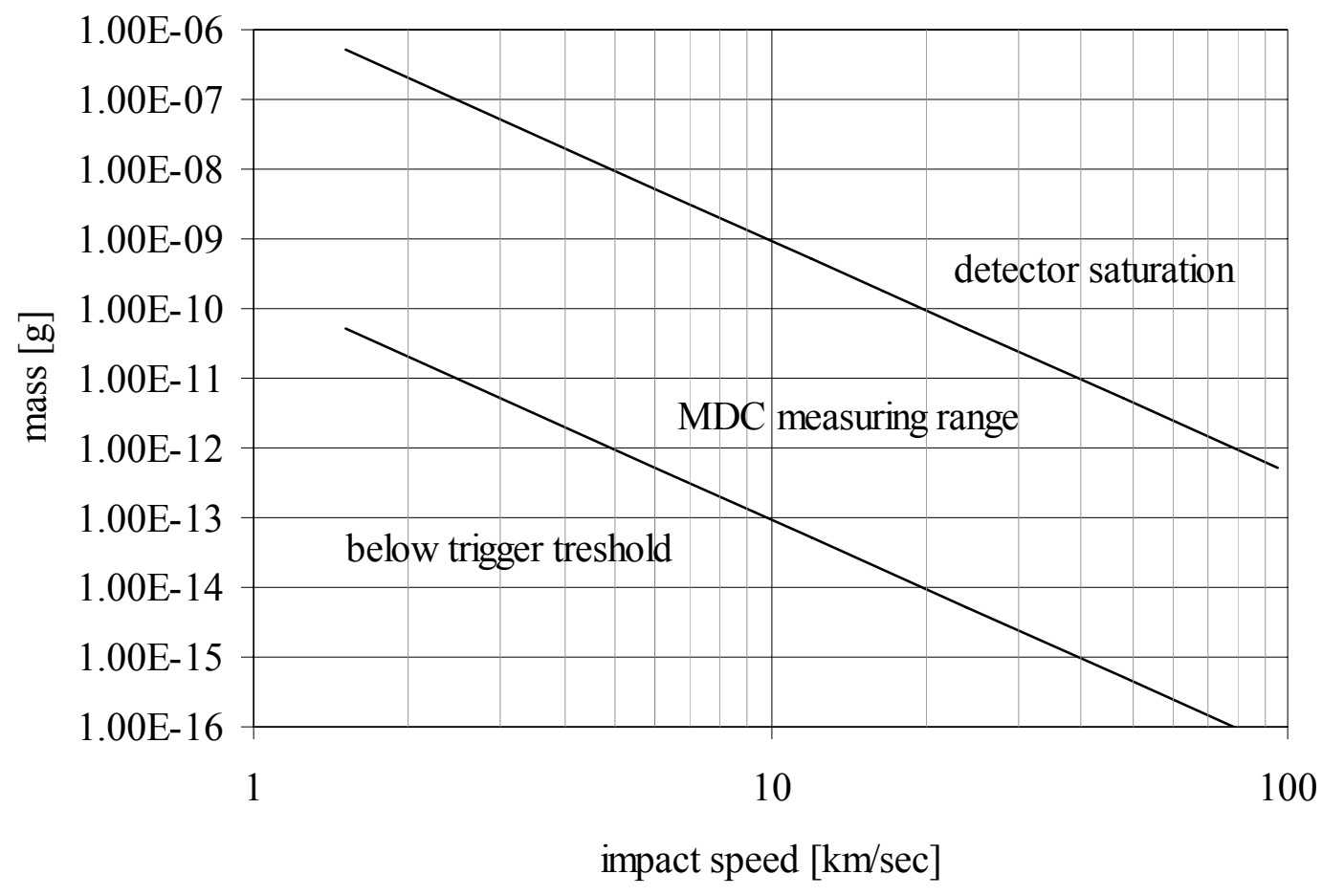

Figure 3-17: MDC measuring range

The MDC measuring range is limited towards low particle mass and low impact speed by the trigger threshold and towards high particle mass and high impact speed by the saturation of the detector electronics. 


\subsection{Data processing on ground}

In this paragraph, MDC-NOZOMI calibration and data analysis are described briefly. For a detailed description of the calibration process, the determination of the required parameters for the data analysis process, and the data analysis itself, see Naumann [Nau2000].

\subsubsection{Calibration}

As already mentioned in paragraph 3.1, no quantitative mathematical model of the charge generation of hypervelocity impacts is available until today. Thus, every detector based on the physical effects of hypervelocity impacts has to be calibrated individually.

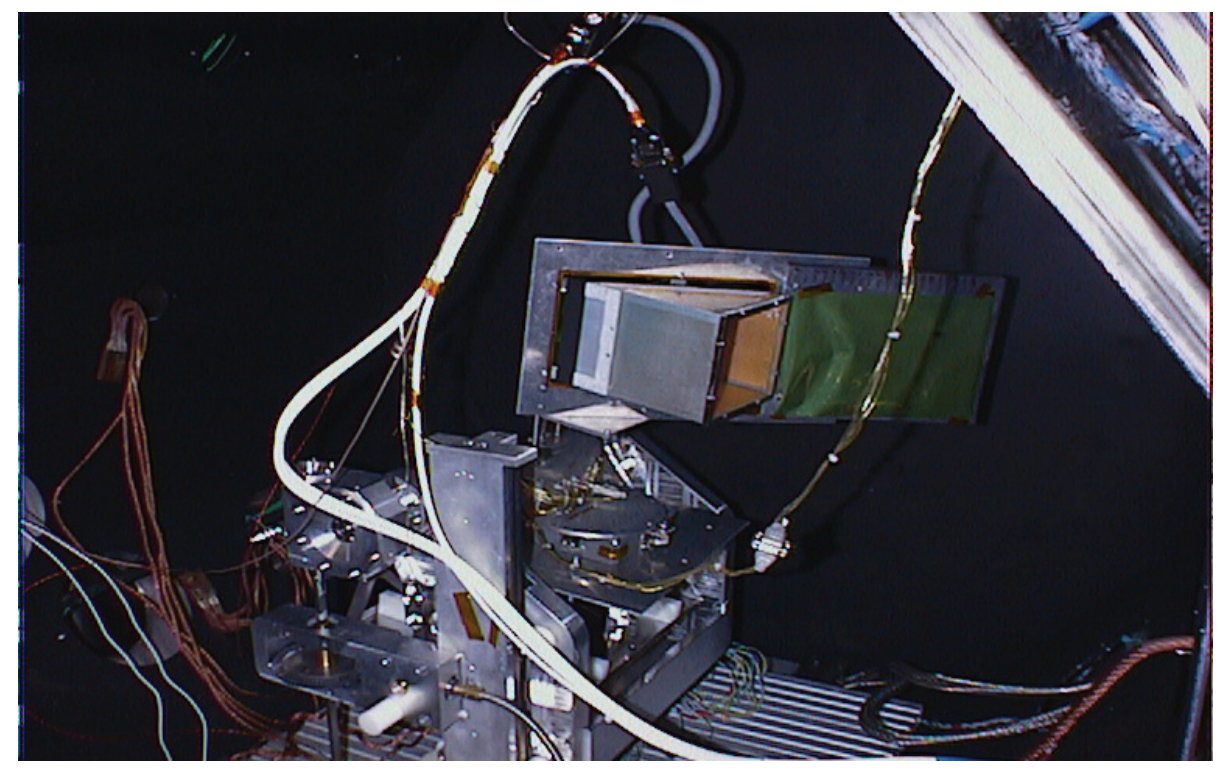

Figure 3-18: The MDC inside the calibration setup

For calibration experiments, particles of known mass and speed were shot into the MDC's target area.

Calibration of the MDC-NOZOMI was carried out in the electrostatic van-de-Graaf dust accelerator of the Max-Planck Institut für Kernphysik (MPI-K) at Heidelberg, Germany. Only ferrous dust particles of a size between $10^{-9} \mathrm{~g}$ and $10^{-15} \mathrm{~g}$ were used as projectiles, and shot onto different positions of the sensor box of the three available units of the MDC (Prototype, Flight Model 1 and Flight Model 2) at a speed range of $1.2 \mathrm{~km} / \mathrm{sec}$ to $60 \mathrm{~km} / \mathrm{sec}$. Altogether 3713 good impact signals were used for the MDC calibration. Mass and speed range of the calibration particles is shown in Figure 3-19. Calibration of the MDC-NOZOMI in the low speed and high mass range in the plasma-dynamic accelerator at the LRT was not possible due to the strong light flash produced by this accelerator, which caused massive interference in the MDC sensor box and prevents the MDC from recording a good impact signal. 


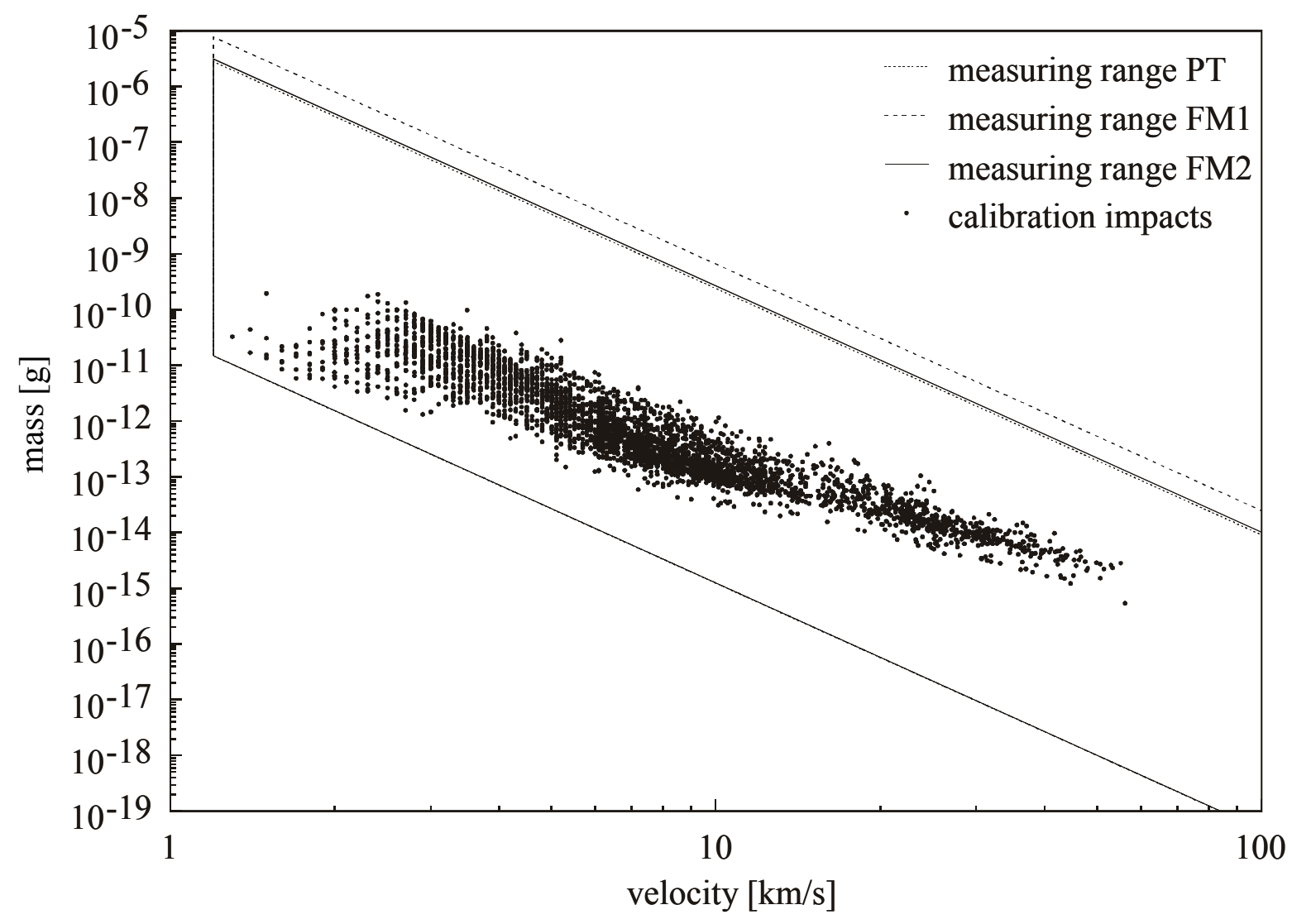

Figure 3-19: Calibration impacts

Particle mass vs. impact speed of the about 3713 calibration shots. All calibration particles have mass and speed within the boundaries of the measuring range of the three MDC models. PT = Prototype, FM1 = Flight Model 1, FM2 = Flight Model 2.

During calibration, particles were shot onto 51 positions inside the sensor box and outside the box on the satellite's MLI (Multi Layer Insulation). These positions are shown in Figure 3-20. The MDC sensor box was divided into 6 areas inside and 2 areas outside the box. From the 3713 calibration signals, the parameter sets needed for the determination of particle mass and impact speed were determined for each of these areas individually (see next paragraph for a description of the parameter sets and the determination of particle mass and impact speed).

MDC-HITEN calibration was also carried out at the Max-Planck Institut für Kernphysik (MPI-K) at Heidelberg, Germany, and is described by Münzenmayer [Mün1997]. 


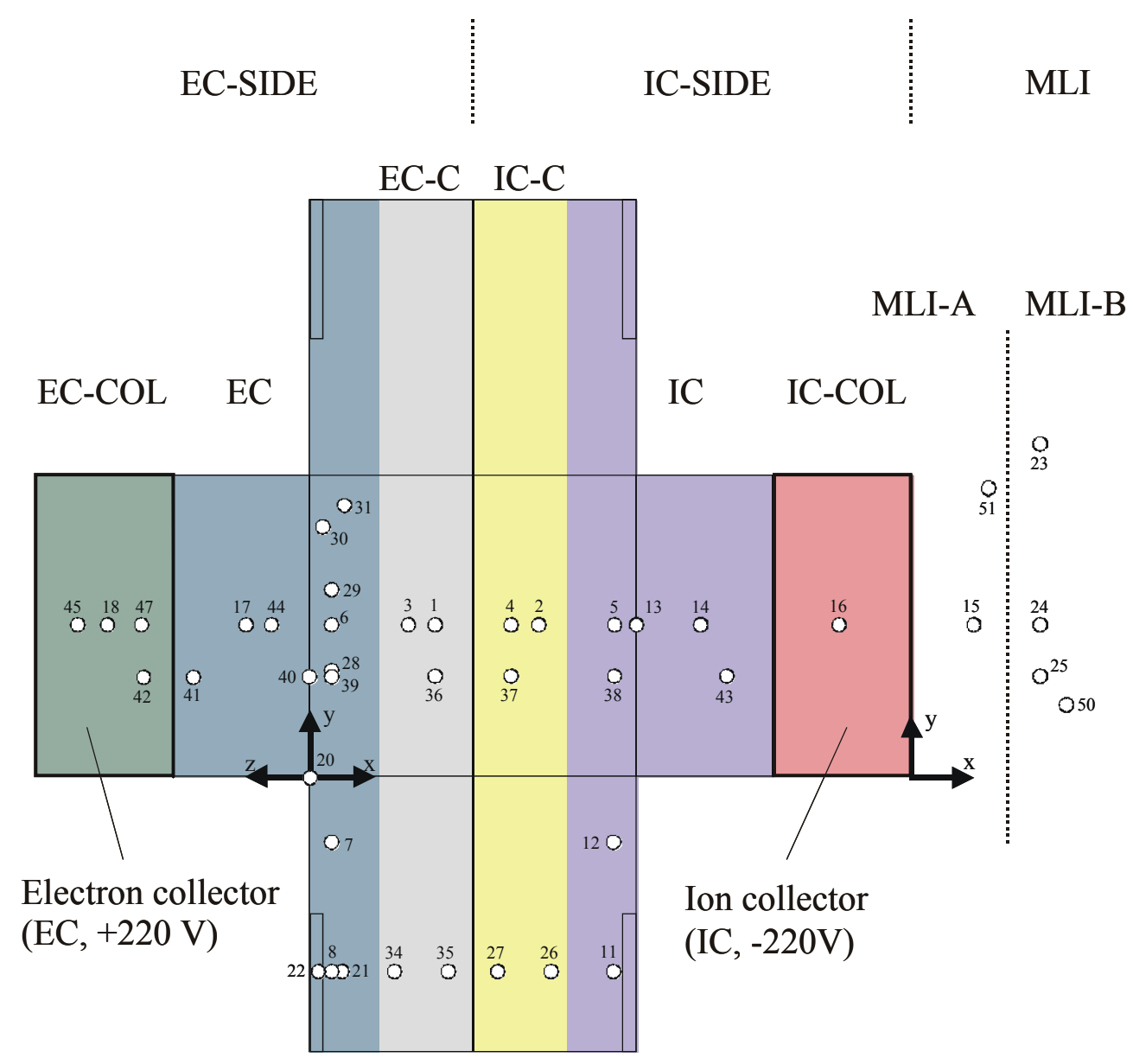

Figure 3-20: Impact positions inside the sensor box

During calibration, particles were shot onto 51 positions inside the sensor box (positions 48 and 49 (MLI-B) not shown in this graph). The sensor box was divided into 6 different areas, EC-COL, EC-C, EC, IC, IC-C and ICCOL, impacts outside the sensor on the areas MLI-A and MLI-B box were also calibrated.

\subsubsection{Data analysis}

The analysis of the downloaded impact signals was carried out on ground with the MDC data analysis software as described by Naumann. Data analysis is carried out in four subsequent steps:

1. Qualification, the selection of impact signals from the total amount of MDC signals

2. Classification, determination of the impact position inside or outside the sensor box

3. Curve analysis, determination of rise time and amplitude of the impact signal

4. Determination of particle mass and impact speed from the signal's rise time, charge amplitude and impact position

Step one is carried out manually by the experimenter (see chapter 4 for a discussion of methods that can be used for automation). Once a signal is qualified as an impact signal rather 
than noise, its impact position must be determined in step two of the analysis process. This is currently done by a routine that compares the new signal that has to be classified with all 3713 calibration signals, whose impact position is known. The impact position of the calibration signal with the highest similarity to the new signal is then taken as the impact position of the new signal. Knowledge of the impact position is required for the later determination of particle mass and impact speed, as the correct parameter set has to be chosen.

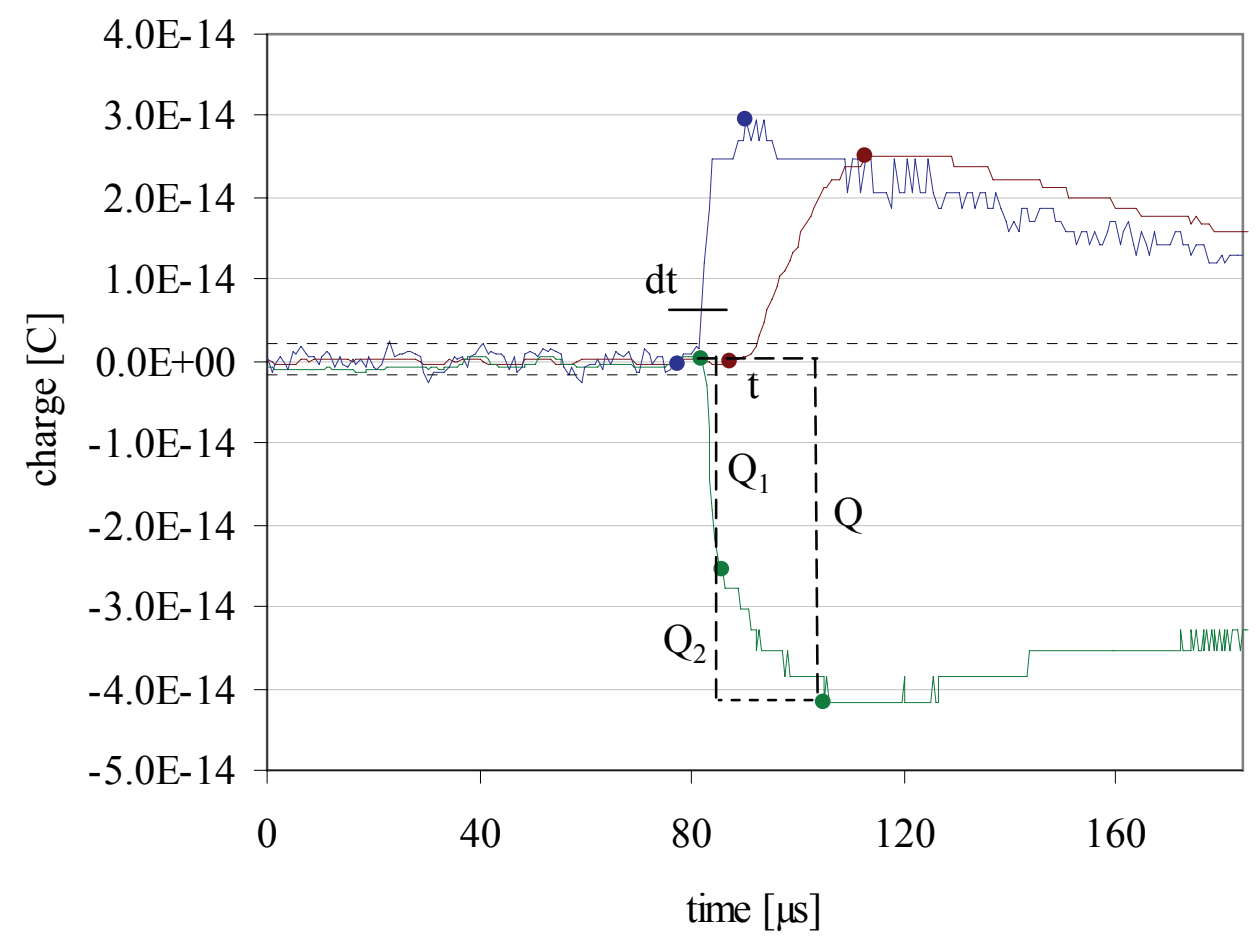

Figure 3-21: Typical calibration signal with characteristic points

In this figure a typical impact signal and its characteristic values rise time $t$, charge $Q$, primary and secondary charge $Q_{1}$ and $Q_{2}$ (all from the electron channel, green curve) and time delay $\mathrm{d} t$ between neutral channel and ion channel (red and blue curves) are displayed.

In step three of the analysis process, rise time and the charge amplitudes of primary and secondary charge (if available) are determined from the signal shape for each individual channel. From the characteristics of hypervelocity impacts and the MDC sensor box characteristics, impact speed $v$ and particle mass $m$ are determined in step four by the following equations:

$$
\left.\begin{array}{l}
v^{c h}=C_{t}^{c h} t^{\eta^{c h}} \\
v^{c h 1, c h 2}=C_{d t}^{c h 1, c h 2} d t^{\kappa^{c h 1, c h 2}} \\
v^{E C}=C_{12}^{E C}\left(Q_{1} / Q_{2}\right)^{\delta^{c h}}
\end{array}\right\} v_{\text {mean }} ; c h=\mathrm{IC}, \mathrm{EC}, \mathrm{NC}
$$


whereas $v=$ impact speed and $m=$ particle mass are the results of the calculation, $Q_{(1,2)}=$ charge, $t=$ rise time, $\mathrm{d} t=$ time delay are the characteristic values determined from the signal shape, and $C_{\mathrm{t}}, \eta, C_{\mathrm{dt}}, \kappa, C_{12}, \delta, C_{\rho}, \beta$ are the parameters which are determined during calibration for each impact position (EC, EC_C, EC_COL, IC_COL, IC_C, IC, MLI-A, MLI$\mathrm{B})$. First, the impact speed is determined from the rise time $t$ of each channel (top equation for impact speed $v$ ). Either all three values derived from the three channels can be used for the calculation of the mean value, or the experimenter can neglect uncertain values. For MLI impacts, the delay time of the beginning of signal rise of two different channels can be used for impact speed determination (middle equation for impact speed $v$ ). For impacts inside the sensor box, the ratio of primary to secondary charge of the electron channel EC can be used for impact speed determination (bottom equation for impact speed $v$ ). Once a mean value $v_{\text {mean }}$ of the impact speed is determined, the particle mass $m$ can determined for each channel individually from the total charge $Q$ of the channel, from these up to three values the final mean value $m_{\text {mean }}$ is calculated.

According to Naumann, impact speed $v$ and particle mass $m$ can be determined by this analysis process with an accuracy of a factor of 2 and 5, respectively. 


\section{Signal screening}

Any analog sensor device, especially nonlinear devices like the MDC, not only produces wanted signals but also random noise that must be separated from the final data. Several methods are used by the MDC electronics to perform this and are described in this chapter. In paragraph 4.2, a routine used by the MDC onboard software to qualify all new-recorded signals and an upgrade that enables much better separation of noise data from impact data is described. In paragraph 4.3, a new method that enables even better separation of noise data based on artificial neural networks is described and evaluated.

\subsection{Basics and motivation}

The overall ratio of real impact signals in the downloaded MDC data files was lower than $1 \%$ for the whole MDC-NOZOMI mission. This has two main disadvantages for the operation of the MDC. First, the high amount of noise data must be downloaded to the Earth to be processed further, which requires large resources of the limited telemetry capacities of the NOZOMI spacecraft. Second, for a further analysis of impact data, all downloaded noise data must be separated from the impact data by manual signal screening by the experimenter. An automated identification of noise signals onboard the MDC would therefore disburden the NOZOMI telemetry as well as the human experimenter on ground. The MDC electronics and onboard software already provide some methods that reduce noise data or can be used for a further reduction of noise data. An optimization of one of these methods that enables significant reduction of noise data downloaded to the Earth is described in this chapter. On ground, with much more computing power available, a further automated screening of the remaining data becomes possible that disburdens the experimenter from browsing thousands of signals.

\subsection{Improvement of the onboard qualification}

The MDC electronics provides two methods that reduce noise and one method that can be used for further selection of real impact signals.

First, any signal must exceed a certain trigger level to be recorded by the MDC electronics at all. The implementation of such a trigger is necessary to protect the MDC electronics from overload, otherwise every interference would be recorded and stored by the MDC and would cause overload of the limited computing capacity. The level to which this trigger has to be set must be selected carefully. A very low trigger ensures that even small real signals can be recorded and analyzed, but with the price of high noise ratio. On the other hand, a high trigger level would give a low noise ratio, but would also prevent the sensor from recording of small real impact signals. Therefore, a compromise must be found.

The second method performed by the MDC to select real impacts from noise is a very crude analysis of any new-recorded signals, the so called KO-criteria, see Naumann [Nau2000] for 
details. A fast and straightforward analysis of certain criteria that have to be fulfilled by a signal is performed right after triggering and recording. If the signal fails these criteria, it is assumed to be noise and discarded.

A much more sophisticated method to analyze new signals is performed by the qualification routine described in this chapter. The qualification routine performs a more detailed analysis of recorded signals and as a result provides them with a numerical value, the quality key, which describes the quality of the signal, or the probability that it represents real data, respectively. The qualification routine takes about three seconds to run for every new signal; hence, the signals that have to be processed by this routine must be pre-selected by the other two methods, trigger and KO-criteria. In the basic version of the MDC, the quality keys are only used to determine the memory segment that will be overwritten if a new signal is recorded and no unused memory segment is available for storage. The new signal will then be stored in the memory segment that holds the signal with the lowest quality key. It would be a great benefit if a certain minimum quality key can be defined which separates noise from real impacts without losing any real impact signal but rejecting a high number of noise signals. This is discussed in the following paragraph. References for this paragraph are the monographies from Mitchell [Mit1998] and Heistermann [Hei1994] and the thesis work from Kyritsis [Kyr2003].

\subsubsection{The qualification routine}

In a first step, the qualification routine calculates a set of 7 criteria $v_{j, c h}$ for each channel $c h=$ IC, EC, NC, $j=0 \ldots 6\left(\max , \mu_{\text {pre }}, \mu_{\text {post }}, \Delta \mu, \Delta \max ; s_{\mathrm{c}}, \Delta \mathrm{t}_{\mathrm{n}} ; \mathrm{m}_{\mathrm{c}}, \Delta t_{\mathrm{i}}\right.$, see Fischer [Fis1998] for a detailed description of these values) that represent some characteristics of the signal shape, e.g. difference of mean pre trigger and mean post trigger value, maximum post trigger value etc. Out of these 21 values, a weighted sum $q u$ (the quality number) is calculated using the 21 qualification parameter set values $p_{j, c h}$, as follows (simplified):

$$
\begin{aligned}
& q u_{c h}=\sum_{j=0}^{6} p_{j, c h} \cdot v_{j, c h} ; \quad c h=\mathrm{IC}, \mathrm{EC}, \mathrm{NC} . \\
& q u=q u_{I C}+q u_{E C}+q u_{N C}
\end{aligned}
$$

The original parameter set $p_{j, c h}$ used for the calculation of the quality key $q u$ was determined by guess from the calibration impacts to give the higher values the better the signal is. It is obvious that this determination method of the qualification parameter set can only be a first pre-flight estimation since no optimization was performed and no information about the shape of noise signals was available, of course. Therefore, it can be expected that a systematic optimization of the qualification parameter set could improve the capabilities of selection of signals by the quality key as described above. Now, enough real impacts and noise signals are available to look on the qualification parameter set.

The intention is to find a parameter set that produces quality keys that separate real impact signals from noise signals in the best possible manner. This would allow the definition of a certain quality key threshold that determines the minimum quality key value a signal must 
have to be kept in memory and/or downloaded. All signals with quality keys below that threshold value are discarded or marked as free segments that can be overwritten, respectively.

The current parameter set does not allow an effective selection by the definition of a threshold value. The threshold value must be chosen in a manner that only a minimum of real impact signals with low quality number gets lost and a maximum number of noise signals can be discarded. Since the quality keys of real impacts and noise signals overlap in a very wide range, a threshold value would allow only about half of the noise signals to be discarded. An optimized parameter set will give a minimal overlap of quality keys.

Since there are 21 parameters $p_{j, c h}$ that are used for the quality key calculation, the optimal parameter set cannot be determined analytical at all. Other sophisticated methods are available and can be used to determine at least a local optimum of the parameter set concerning the intended results, e.g. genetic algorithms or simulated annealing. For the optimization of the qualification parameter set, genetic algorithms are used.

\subsubsection{Genetic algorithms}

The main problem finding the optimum of a function in a high dimensional space is that any straight forward method would require a huge amount of computing time, in certain cases this time could exceed the age of the universe even when using today's fastest available computers. For the MDC qualification routine, 21 parameters with a range of 150 give $150^{21}=5 \cdot 10^{45}$ possible different parameter sets that had to be tested; the age of the universe is $4.5 \cdot 10^{17}$ seconds. Genetic algorithms (also called evolutionary algorithms) provide a method for the determination of an at least local optimum which comes close to the then still unknown global optimum.

In biological science, evolution is understood as the process of formation of different species and their optimization with respect to their given environment. This process is characterized by recombination, mutation and selection. Each individual carries a set of attributes (the genes) that determines the individual's performance in its environment (e.g. its capability to compete with other species about a limited environmental resource like food, its ability to escape or hide from predators, and its performance in reproduction). The optimization of the species' performance is coupled to the optimization of the genetic code of its individuals (its genotype). Recombination of the genetic code of two different individuals produces new child individuals with a different performance that is close to that of its parents, while random mutation allows larger, random changes in genetic code and performance and may open up new biological niches that can be occupied by the new branch of the species. Most important for a target-oriented evolution process is the selection of high-performance individuals as preferred parents for new child generations. In nature, this selection process is carried out by the environment of the species itself, which gives high-performance individuals a slightly better chance to reproduce than low performance individuals. From generation to generation, the adaptation of the species to the properties of the given environment increases.

Genetic algorithms are based on similar principles. The genes of an individual are identified with numerical a set of parameters whose performance has to be optimized with respect to a performance goal that has to be reached by the optimization process. Starting with a 
population of individuals with random (or guessed) numerical sets of parameters, the performance (normally one numerical value) of each of these different individuals is determined with respect to the goal by a defined objective function. A set of highperformance individuals is then selected from the population by a selection function or operator (e.g. take only the best $10 \%$ of individuals). These selected individuals become the parents of the next generation; each member of this new child generation gets new parameter sets (or genes) that are combinations of the parameter sets of two individuals from the parental generation; a previously defined recombination operator defines the method of recombination (e.g. crossing over, a strategy where a random number of parameters are swapped between the two parents). In this stage, random mutation can be performed with a defined, but low probability to allow larger jumps in genotype. After the new generation is created, the genetic iteration process continues with testing the performance of the individuals and selecting the new parents for the next generation and so on. The optimization process continues until a predefined performance goal is reached or no significant improvement of performance can be achieved for a defined number of generations. Several different strategies of recombination, mutation, selection were described in literature, see Heistermann [Hei1994] for a discussion.

\subsubsection{Optimization of the qualification parameter set}

The optimization of the quality routine parameters was implemented in $M A T L A B^{\circledR}$. The quality key routine was rebuild in $M A T L A B^{\circledR}$ to compute the quality keys for any given parameter set. The input values $\left(\max , \mu_{\text {pre }}, \mu_{\text {post }}, \Delta \mu, \Delta \max ; s_{\mathrm{c}}, \Delta t_{\mathrm{n}} ; m_{\mathrm{c}}, \Delta t_{\mathrm{i}}\right)$ were determined once from the whole set of $\approx 20000$ signals. The optimization run was carried out as described in the following.

At first, a set of set of reference impact signals was defined that was required for the determination of the performance of the different qualification parameter sets. This reference set was buildt from the previously identified real impact signals from the reviewed 1998 data, plus the already identified real impact signals of the 1999 and later data, and thus represents a minimum set of wanted signals that must be identified as possible impact signals from the optimized qualification routine.

A simple, self-developed genetic algorithm was used for optimization of the quality routine parameter set. The 21 individual values of the parameter set $p_{j, c h}$ are the "genes" of the individual parameter set, which determine its "performance", that means, the capability of the individual parameter set for separation of noise signals from impact signals. To determine this performance, the quality numbers of the whole available MDC data (noise and possible impact signals) were calculated by the qualification routine using the parameter set to be tested. From the obtained list of quality numbers, a threshold value $Q$ was set to the $4^{\text {th }}$ but smallest quality key of the set of reference signals, to ensure that only a minimum number (four) of reference signals get lost by the separation. All signals with a higher quality key than this threshold value are then identified as possible impact signals. The performance of the given parameter set was determined as the ratio of the number of reference impact signals having quality key higher than the threshold value to the full number of signals passing the threshold value, $r_{i m p}=$ (No. of reference signals with quality $>Q$ )/(No. of all signals with quality $>Q$ ). A performance value of $r_{i m p}=0.25$ therefore means that $25 \%$ of all signals 
passing the threshold value are reference impact signals, while the rest is noise. The higher the value of $r_{i m p}$, the better the performance of the given parameter set is.

The optimization of the qualification routine parameter set was then carried out as follows. Starting with a single parameter set (the original one or a random one) a fixed number of $n=$ 1000 initial parental parameter sets was created by mutation only (random variation of the parent's parameter set). Now the optimization routine entered an endless loop. First, the performance of the individual parameter sets was tested (the values $r_{i m p}$ were calculated), and the members of the population were sorted according to their performance $r_{i m p}$. The worst $50 \%$ of parameter sets was then discarded from this generation. From the remaining members of this parental generation, an equal number of child parameter sets was created by combination of the parameters $p_{j, c h}$ (the "genes") of two randomly selected members of the parental generation in each case. The random selection of the two parents was designed to give parameter sets with high performance a greater chance to recombine than those with low performance. Recombination of parameters was carried out by building a new value from the two corresponding single parameters that was lying preferably closer to the value of one of its parents. Next, child and parental parameter sets were merged and used as new parental generation. This process was iterated until new generations did not show any more improvement in performance, Figure 4-1 shows the development of the performance $r_{i m p}$ of the best parameter set for each generation over the iteration process. The parameter set with the highest achieved value of $r_{i m p}$ was then chosen as the optimum parameter set. Since in most cases this optimum is one of a number of local optimums rather than the one and only global optimum, and may vary depending on the first parent used, this optimization must be performed several times using different random parent parameter sets.

The optimization process can be summarized as follows:

1. Create an initial parental generation of size $n=1000$ from the single starting parameter set by mutation only

2. Calculate the performance $r_{\text {imp }}$ of each member of the parental generation

3. Sort parental generation according to performance $r_{\text {imp }}$

4. Discard the worst $50 \%$ of parameter sets

5. Create child generation of equal size from the members of the remaining parental generation by recombination and mutation of the parental parameter sets

6. Merge parental and child generation

7. Proceed with step 2., with the merged population as new parental generation

No fixed stop criterion was defined, the iteration was stopped by the experimenter after no further increase in performance $r_{i m p}$ of the best parameter set of each generation could be observed over a number of generations. 


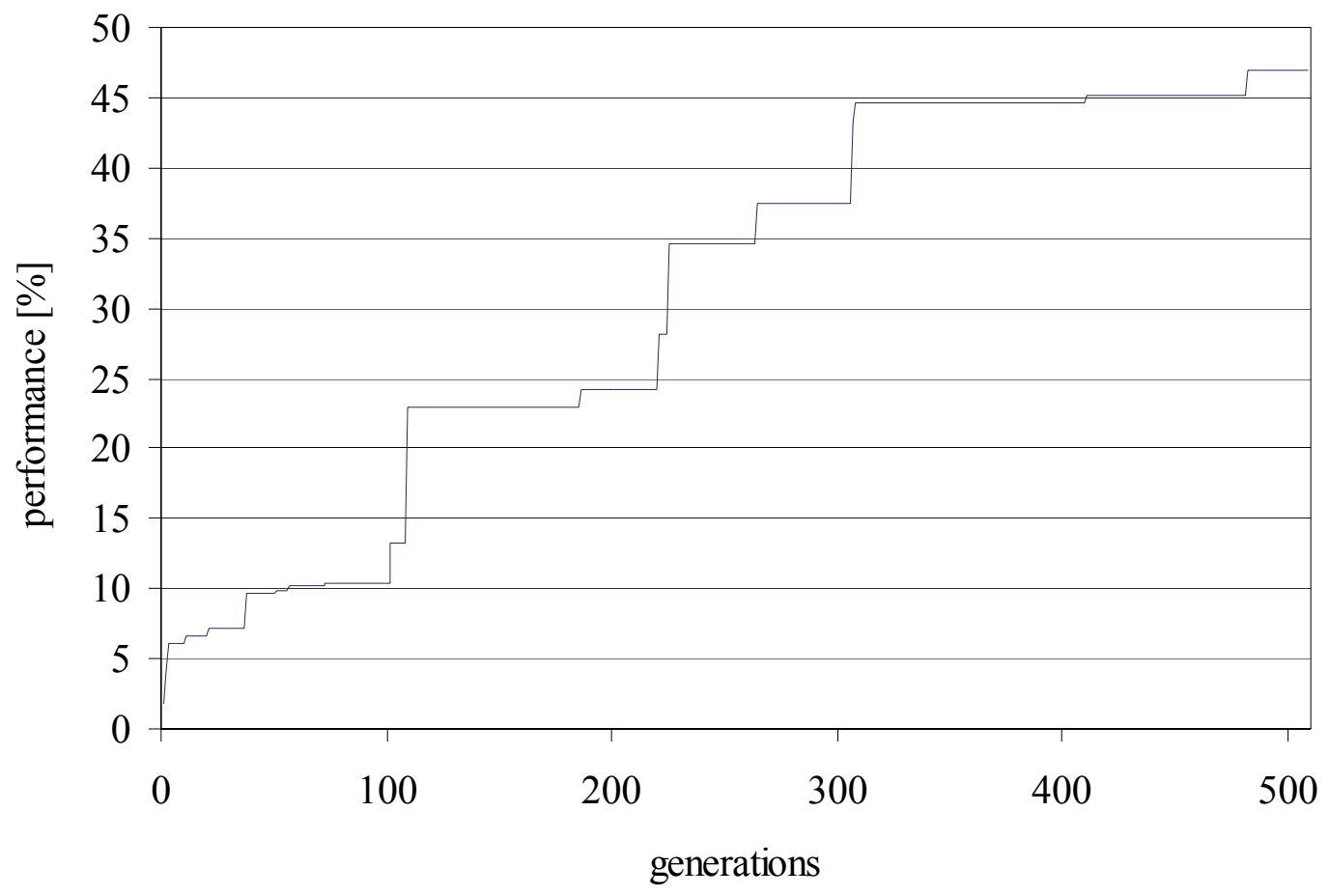

Figure 4-1: Evolution of the performance $r_{i m p}$ of the parameter sets.

Starting with the original parameter set with a performance of $3.51 \%$, the performance rises with increasing generations. The experimenter stopped the optimization after 500 generations; achieved performance was $46.9 \%$.

The results are shown in Figure 4-2 and Figure 4-3. The quality numbers of the full available MDC data were calculated from the original parameter set and from the best one found by the optimization routine. The frequence of these quality numbers is displayed over the full range, black bars represent reference signals, grey bars other signals. A threshold value was defined so that the four reference impact signals with the lowest quality key are rejected. The value $p_{Q}$ defines the ratio of signals that pass the threshold, while the value $r_{i m p}$ defines the ratio of real impact signals in the set of signals that have passed.

Figure 4-2 shows the result of the original quality routine parameter set. A threshold value set at $Q=102$ separates $87 \%$ of noise data from the whole set of data while $p_{\mathrm{q}}=13 \%$ pass. The ratio of real impact signals in the set of passed signals is $r_{i m p}=3.51 \%$. 


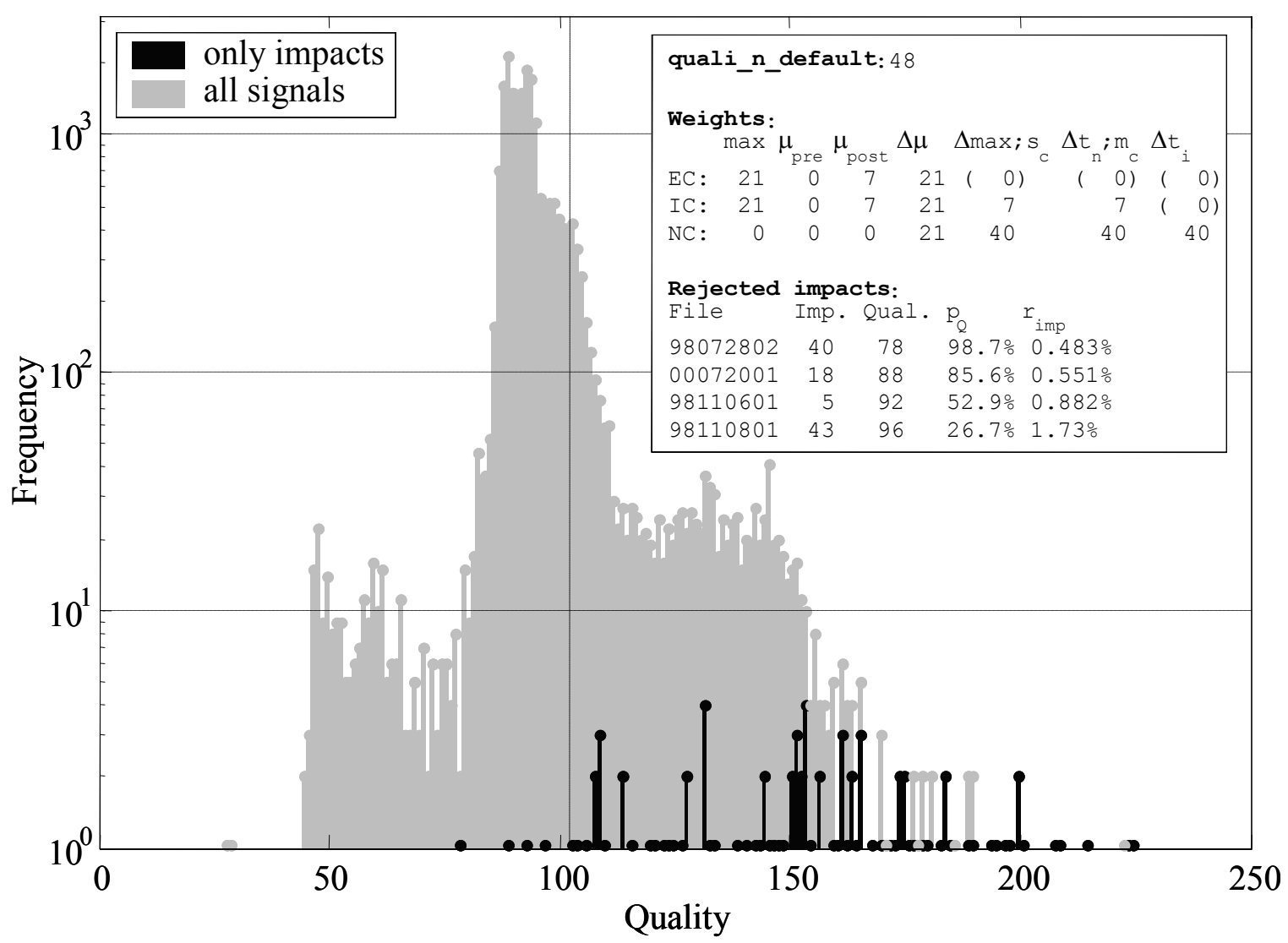

Figure 4-2: Separation capabilities of the original parameter set

Using the original parameter set for qualification, and a quality threshold of $\mathrm{Q}=102, p_{Q}=13 \%$ of all signals pass the threshold; the impact ratio within the signals with $\mathrm{Q}>102$ is $r_{i m p}=3.51 \%$.

The improvement that can be achieved by optimization of the quality routine parameter set is shown in Figure 4-3. Given a threshold value of $Q=117$, only $p_{Q}=0.903 \%$ of signals pass the threshold, and the ratio of real impact signals in this set of data rises to $r_{i m p}=50.5 \%$. 


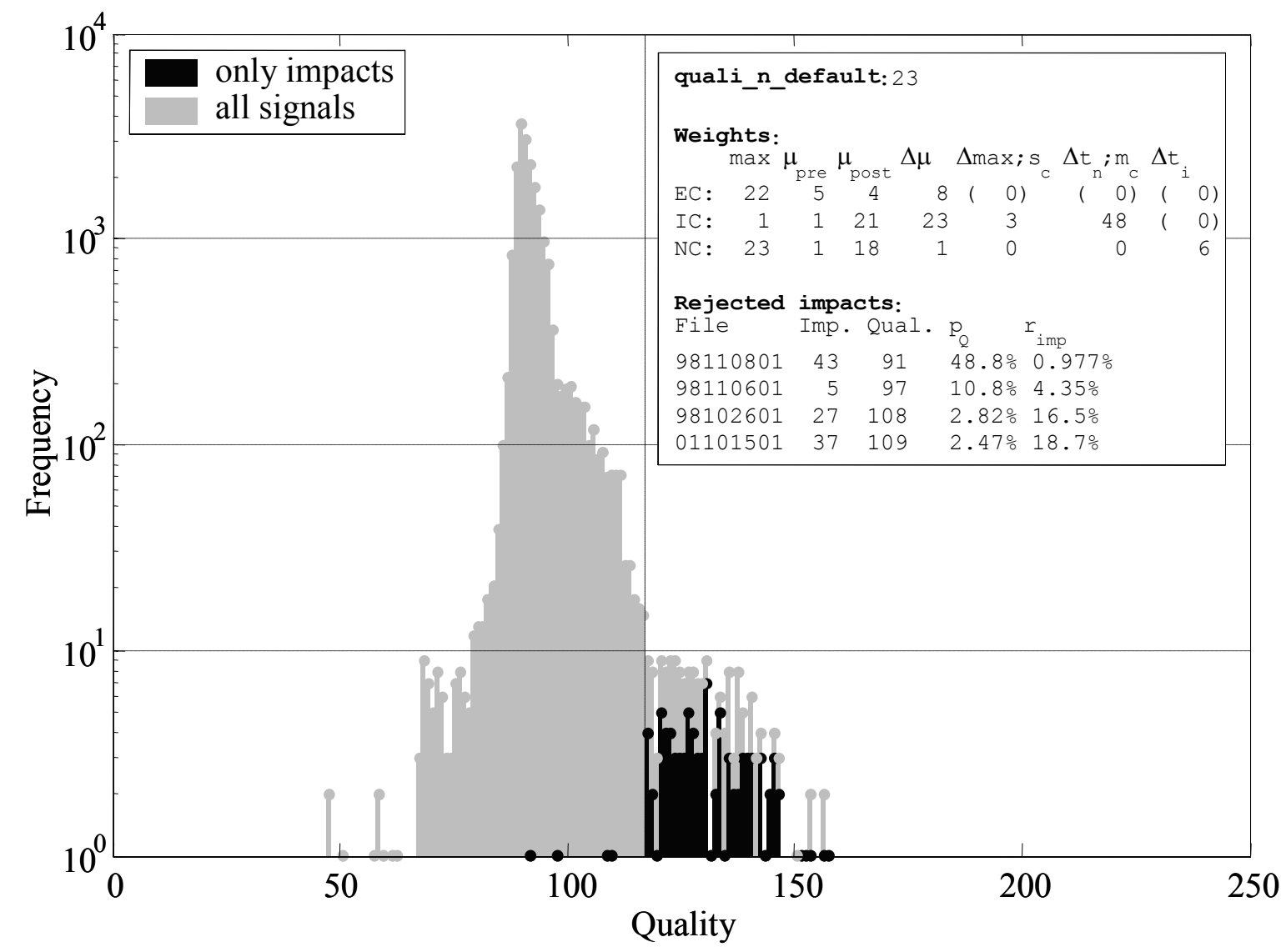

Figure 4-3: Separation capabilities of the best resulting parameter set

Using the original parameter set for qualification, and a quality threshold of $\mathrm{Q}=117$, only $p_{Q}=0.9 \%$ of all signals pass the threshold; the impact ratio within the signals with $\mathrm{Q}>117$ is $r_{i m p}=50.5 \%$.

Thus, a great reduction of the overall amount of data can be achieved by determining and uploading an optimized set of quality routine parameters. The results are summarized in Table 4-1.

Table 4-1: Results of the optimization of the quality routine parameter set

\begin{tabular}{llll} 
Parameter set & $p_{Q}$ & $r_{i m p}$ & reduction factor \\
\hline Original & $13 \%$ & $3.51 \%$ & $\approx 7.5$ \\
Best guess & $0.903 \%$ & $50.5 \%$ & $\approx 110$
\end{tabular}

In summary, an optimized quality routine parameter set could give a reduction of the amount of data to be downloaded to Earth by a factor of about 100 . To benefit from these capabilities, a modification of the memory readout routine must be implemented that finally carries out the separation by skipping all signals with quality numbers lower than the threshold. Even without a further modification of the memory readout routine, a reduction of data can be achieved by simply performing less frequent memory readouts. As described in paragraph 3.2.2, new-recorded signals are stored in the memory segment with the lowest quality key. A parameter set with good separation capabilities therefore gives much more security that real impact signals will not be overwritten during long periods where no readout is performed. 


\subsection{Signal screening by neural networks}

The following method of signal screening cannot be performed onboard the current MDC model since it requires more computing power and memory, but can be used in future models' onboard software or on ground to unburden the experimenter from time consuming work. A selection of signals from noise by an artificial neural network was implemented and tested. For long missions like the MDC, this would reduce the time consuming and troublesome selection of impact signals from a huge amount of (mostly noise) data by the experimenter and would enable some kind of automation of the whole data analysis process.

The first intention was to develop a method that is able to sort out real impact signals from noise signals automatically to create a basis for further automation of the whole data analysis process. Additionally, the experimenter can be unburdened from browsing several thousands of signals by hand, as it was necessary during the first year of the mission and would have been necessary if NOZOMI will enter Mars orbit with its full functionality. Finally, neural networks can replace the routines used in the MDC onboard software data processing in future experiments, when more computing power may be available.

References for the following paragraphs are the monographies from Scherer [Sch1997] and Zell [Zel1994], and the thesis work from Glas [Gla2003].

\subsubsection{History and basics}

First introduced in 1943 as a mathematical model that is able to solve any logical and arithmetical problem, neural networks soon caused a hype in mathematical science. After a backstroke in 1969, when an article challenged in principle their logical skills, neural networks became more popular again in the mid 1980s when computing power became broadly available and cheap. New techniques were developed and new fields of application were opened up. Today neural networks are used in a wide field of applications such as simulation of biological nervous systems, parallel information processing, control techniques, and pattern recognition. The latter application of neural networks is used here to create a procedure that recognizes real impact signals and separates them from noise signals produced by the MDC sensor device. Another possible application of neural networks could be a cluster analysis performed on the mass of noise signals to filter out certain patterns of reproducible noise signals that were recognized in the MDC data stream, see also paragraph 6.2.1.

In principle, neural networks consist out of a set of simple mathematical units, called neurons, which are interconnected in a special way. In these terms, the mathematical neural network corresponds to the biological nervous system that consists out of comparatively simple units (the cells) that are interconnected to a quite complex network, which shows much more abilities than the sum of the basic units does. The intelligence, or the mathematical and logical abilities, respectively, is represented in the connections between the neurons. The overall "shape" of a neural network, e.g. the number of neurons and the pattern of interconnection, is called the topology of the neural network. Neural networks are characterized by learning aptitude, parallelism, robustness and fault tolerance. 


\subsubsection{Components and functionality of neural networks}

A neural network like the one used here for pattern recognition can be seen as a system (a "black box") that is presented a certain pattern as input and, after processing the presented information, creates an output that is identified as the networks answer. The number of input parameters as well as the number of possible answers strongly depends on the problem that has to be solved with the help of the neural network.
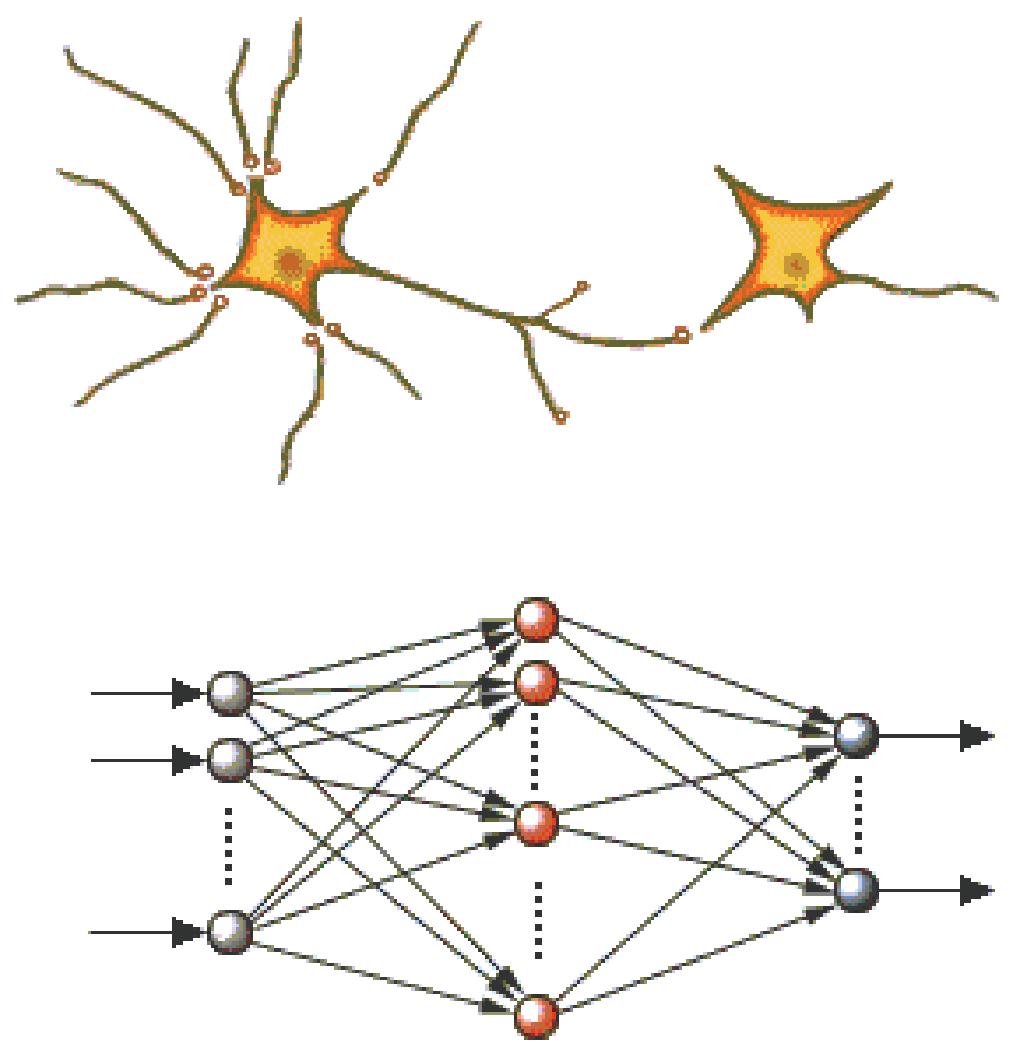

Figure 4-4: Biological and artificial neurons and related networks

The basic topology of biological neural networks (brains etc., top image) and artificial neural networks (bottom image) is very similar. Every neuron gets input from a number of other neurons, and creates one single output. The output is determined from the input, the network's knowledge and the current state of the neuron by rules that are implemented as functions inside every neuron. In artificial neural networks, the network's knowledge is represented in weights of the connections. These initially random weights have to be adjusted during the training of the neural network.

Here, the artificial neural network ought to be used to distinguish real impact signals from noise signals. Therefore, the input parameters must represent the overall characteristics of the signal in a unique way. To optimize the performance of the pattern recognition process, it is recommended to minimize the number of input parameters and avoid any redundancies. The desired output for the given problem reduces to a simple "yes/no" answer, either the presented signal is identified as a real impact signal or not. 
The neuron itself is represented as a quite simple mathematical unit. In principle, the neurons can be seen as local processing units that create one certain output from a random number of inputs. The inputs can either be identical with the input of the neural network itself (the neuron then belongs to the "input layer") or may be output from other neurons. The output of a certain neuron can already be part of the neural network's answer (the neuron then belongs to the "output layer") or become input of other neurons. If the neuron is connected to other neurons on the input and on the output side, it belongs to a "hidden layer" that has no connections to the outside of the network. Commonly, all neurons are combined in certain layers. Every network consist of one input layer, one or more (or, for simple applications, even no) hidden layers and one output layer. If the connections are directed and always point from one certain layer to the following one, the network is called a "feed forward" network.

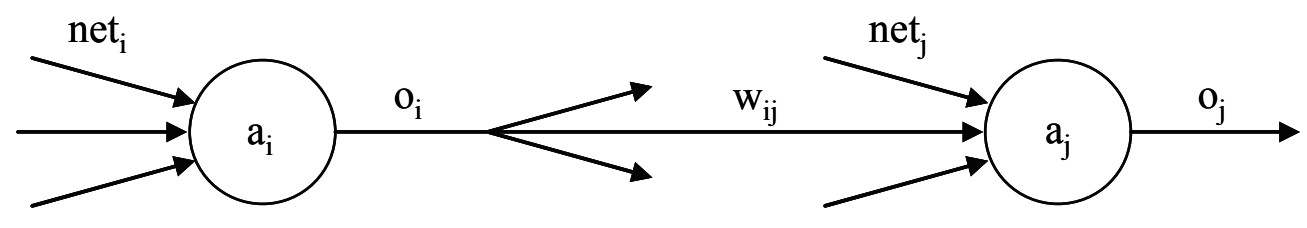

Figure 4-5: Input, attributes and output of neurons.

Attributes of single neurons (see text for details) and weight matrix $w_{i j}$

Any neuron holds the following attributes:

Propagation function $n e t_{j}$ that represents the neurons input and is buildt from the output $o_{i}$ of the previous neutrons and the weight matrix $w_{i j}$

$$
n e t_{j}(t)=\sum_{i} o_{i}(t) w_{i j}
$$

Activation state $a_{j}$ at time $t$

$$
a_{j}(t) \in R \text {. }
$$

Activation function $f_{\text {act }}$ that determines the new activation state $a_{j}(t+1)$ from the input net $t_{j}$

$$
a_{j}(t+1)=f_{\text {act }}\left(a_{j}(t), n e t_{j}(t)\right)
$$

Output function $f_{\text {out }}$ that determines the neurons output $o_{j}$ from its new activation state $a_{j}$

$$
o_{j}(t+1)=f_{\text {out }}\left(a_{j}(t+1)\right) \text {. }
$$

For neurons on the input layer, the input $n e t_{j}$ consist of a single input value from the pattern that is presented to the neural network for classification. The output $o_{j}$ of neurons in the output layer is one single value of the networks answer.

Every connection inside the neural network holds a certain "weight" $w_{i j}$, the weights of the connection structure are represented by the weight matrix $\mathbf{W}$. This weight matrix represents the "knowledge" of the neural network and must be determined by initial training of the neural network. The training procedure strongly determines the later capabilities of the neural 
network. Initially, all weights are set to random values and the network does not hold any kind of specific knowledge. The training itself is performed by presenting the network a set of training data that consists out of a representative part of the possible input data, and adjusting the weight matrix depending on the answers that are given by the untrained neural network. The adjustment of the weights is depending on the learning strategy used.

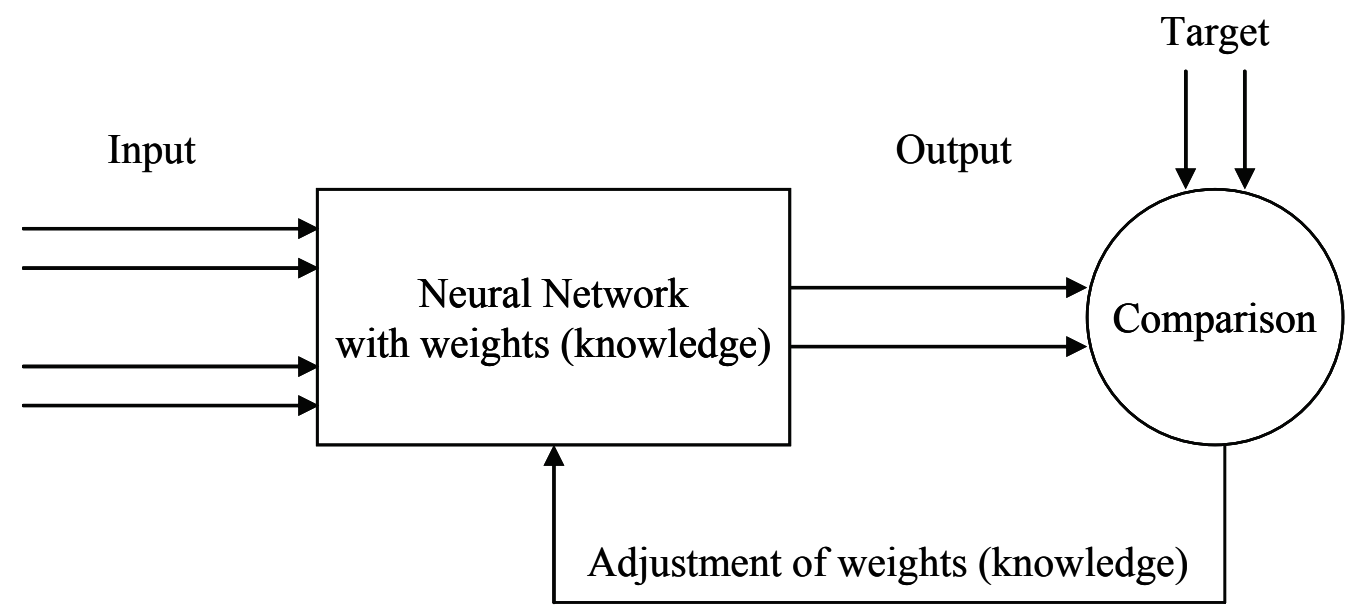

Figure 4-6: Illustration of the basic learning strategy

The training of a network is carried out iteratively by presenting the network a known input, comparing the network's output to the target (the true answer) and adjusting the weight factors (the knowledge) of the network depending on the difference of the output and the target.

Different kinds of learning strategies were developed and are described in literature. During the learning process of a neural network, the weight matrix $w_{i j}$ must be adjusted to increase the networks ability for giving correct answers. Most strategies are based on the general strategy introduced by Hebb in 1949:

$$
\Delta w_{i j}=\eta h\left(o_{i}, w_{i j}\right) g\left(o_{j}, t_{j}\right)
$$

whereas $\Delta w_{i j}$ is the change that is applied to the certain weight $w_{i j}$. The value $t_{j}$ is the target output value that is expected from the neuron, while the value $o_{j}$ is the actual output value. The functions $g$ and $h$ and the parameter $\eta$ determine the correction factor $\Delta w_{i j}$ from the difference $g()$ between target and actual output and the relation $h()$ of the output value to the actual weight $w_{i j}$, and differ depending on the specific learning strategy.

Networks that contain hidden layers require more sophisticated training methods. Since the teaching input can only be applied to the output layer because no teaching input is available for neurons in hidden layers, a rule must be defined to propagate this teaching input from the output layer back to the hidden layers. This strategy is called "back propagation" and is based on the following rules:

$$
\Delta w_{i j}=\eta o_{i} \delta_{j}
$$

whereas 


$$
\delta_{j}=\left\{\begin{array}{cc}
f_{j}^{\prime}\left(\text { net }_{j}\right)\left(t_{j}-o_{j}\right) & j \text { is an output neuron } \\
f_{j}^{\prime}\left(\text { net }_{j}\right) \sum_{k}\left(\delta_{k} w_{j k}\right) & j \text { is a hidden neuron }
\end{array} .\right.
$$

The function $f_{j}^{\prime}()$ is an analogon to the activation function. With this strategy, the teaching input is back propagated through the network and an adjustment of all weights $w_{i j}$ in the network becomes possible.

\subsubsection{Implementation}

The neural network that shall be implemented and tested for the classification of the MDC data must meet the following requirements:

- All segments that contain real impact signals must be recognized

- The number of segments that are falsely identified as real impacts should be minimal

- The network itself should be as slim as possible

- The number of input parameters should be as small as possible

The parameters of the neural network, namely number of input parameters, size of the network (number of neurons and hidden layers) and the teaching algorithm, are confined by the structure of the problem, but not defined strictly. Only the number of output neurons is fixed to two since the desired answer is a simple yes/no decision. The other parameters are subject to optimization that was performed by implementing different possible networks and evaluating their classification capabilities.

The answer of the neural network is read from the activation of the two output neurons $\mathrm{a}_{1}$ and $\mathrm{a}_{2}$ after a certain input was presented to the networks input layer. A signal is classified as a real impact signal if the activation of neuron $2\left(a_{2}\right)$ succeeds the activation of neuron $1\left(a_{1}\right)$

As input parameters, the signal itself or the whole set of digitized data, respectively, was not concerned as a practical approach since that would require more than 800 input neurons and lead to a very huge neural network. Instead, a set of 56 parameters that represent the characteristics of the signal was used as input parameters. These parameters include a reduced set of data points, calculated parameters like mean-pre-trigger or total amplitude of the signal, and environmental data.

In a first approach, the network was designed quite large, using all the 56 input parameters that describe the signal shape, two hidden layers with 14 and 12 neurons, respectively, and two output neurons $(56 \times 14 \times 12 \times 2)$. Although this network showed only a weak classification performance, it was used as a basis for the following optimizations. 


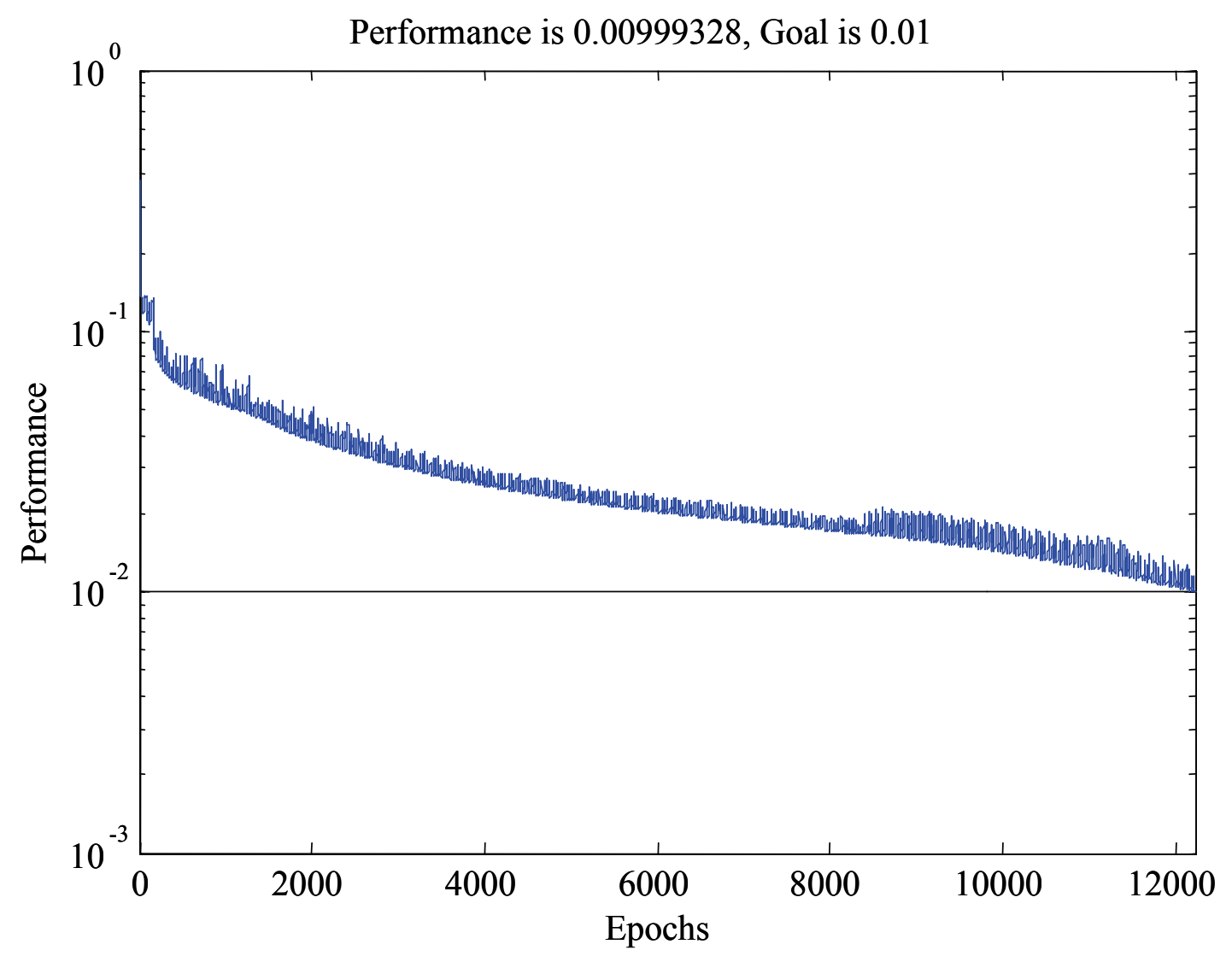

Figure 4-7: Training process of the neural network

This graph shows a typical training progression of a neural network containing two hidden layers with 15 and 11 neurons. Training goal of 0.01 (which means, an accuracy of higher than $99 \%$ in classification performance) was reached after 12225 epochs (training cycles).

\subsubsection{Reduction of the input parameters}

As mentioned before, the number of input parameters should be reduced to a minimum without losing classification performance. To carry out this reduction in a systematic way, the basic network described above was used to determine the significance of the input parameters. The numerical significance of each parameter can be calculated from the weight matrix of the trained network by the equation

$$
f_{s i g, i}= \begin{cases}\sum_{j}\left|w_{i j} f_{s i g, j}\right| & j \text { is a hidden neuron } \\ \sum_{j}\left|w_{i j}\right| & j \text { is an output neuron }\end{cases}
$$

It must be mentioned here that the significance of certain parameters differs widely for different instances of the network; therefore, the selection of parameters that are discarded must be carried out carefully. Also, parameters that represent the same characteristic value but are calculated from different channels do not show any correspondence in significance, as one 
would expect. Finally, the input parameters were reduced to a set of the 16 most significant parameters.

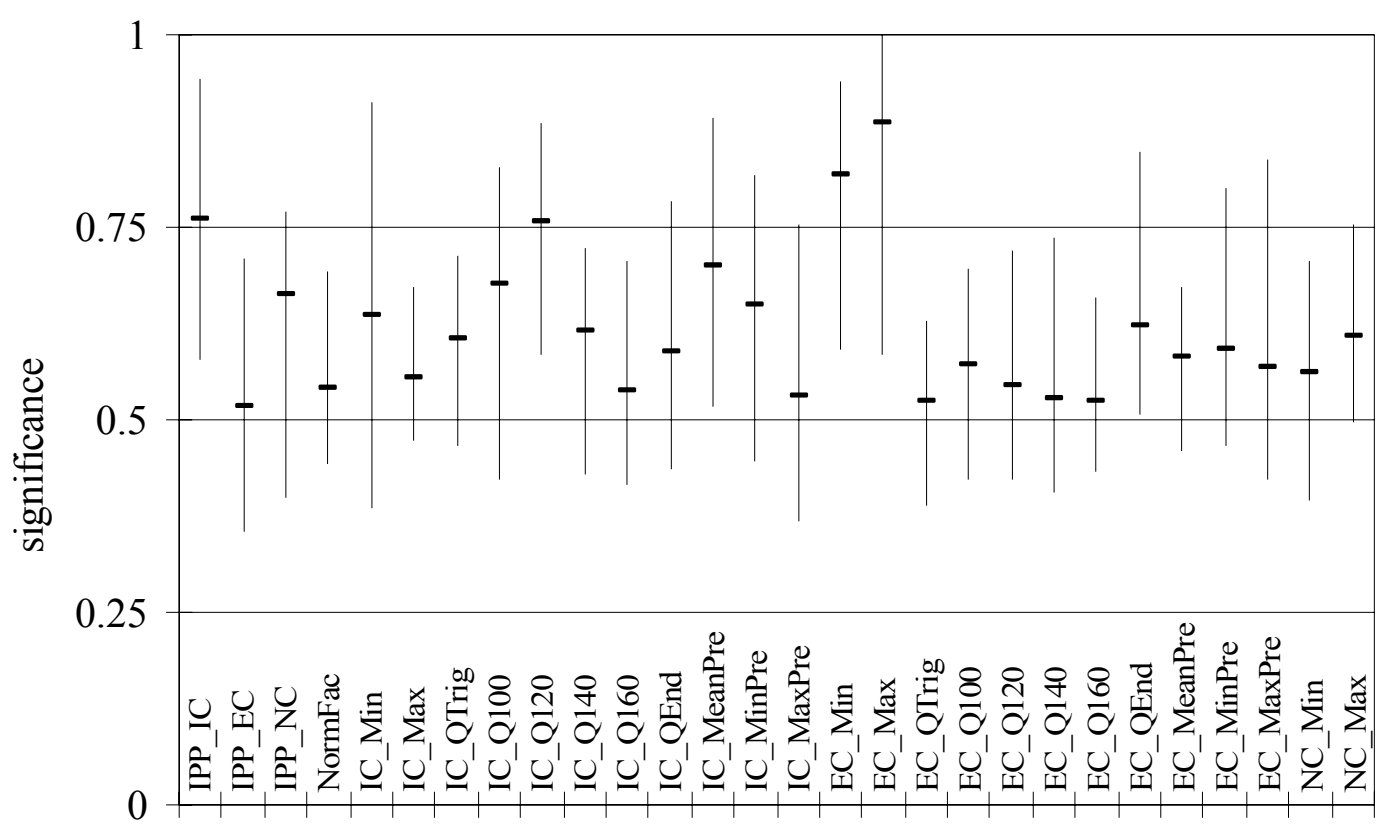

parameter

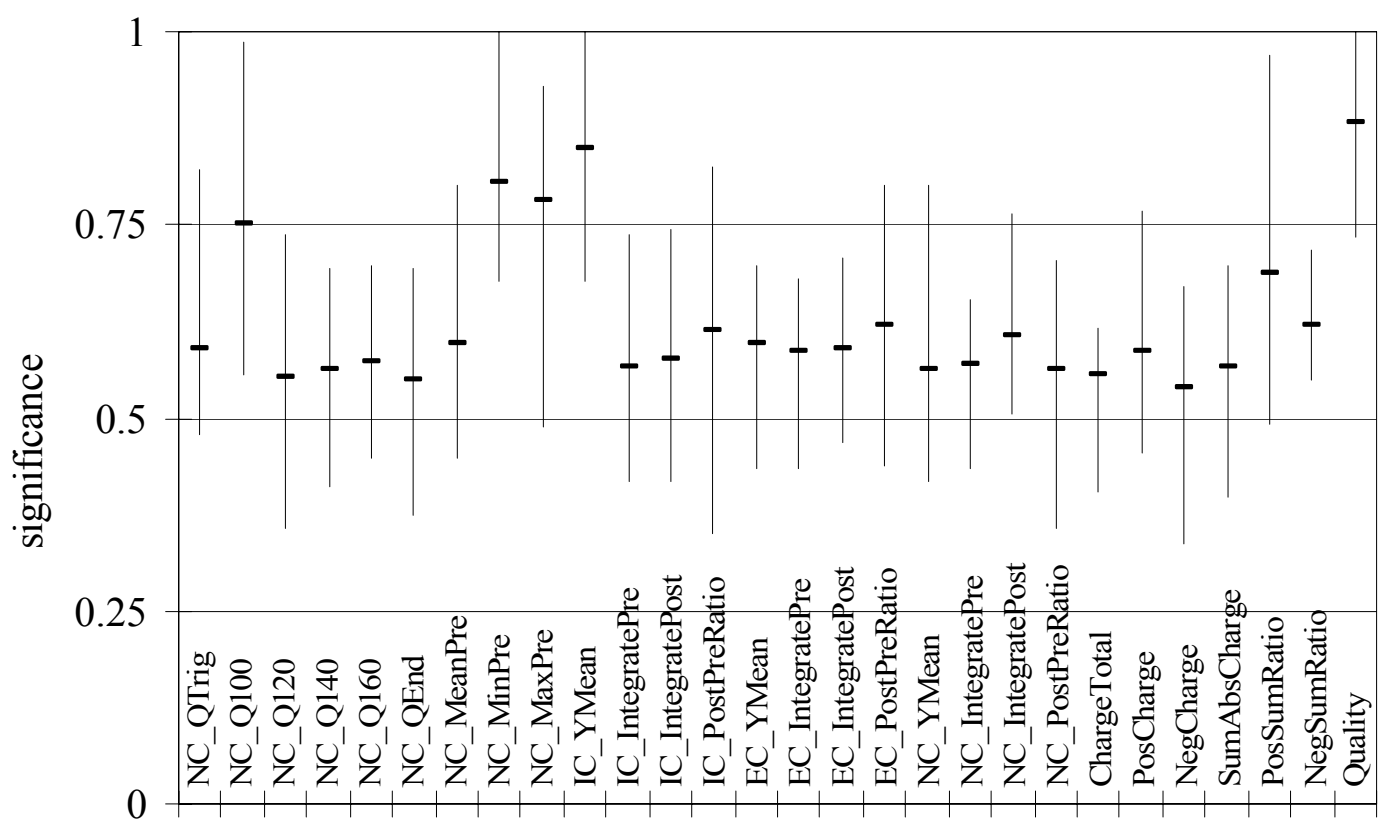

parameter

Figure 4-8: Significance of the input parameters

The significance of each input parameter calculated from the equation above is shown here. Values are mean values from 10 simulations; the bars represent the maximum and minimum value, respectively. 


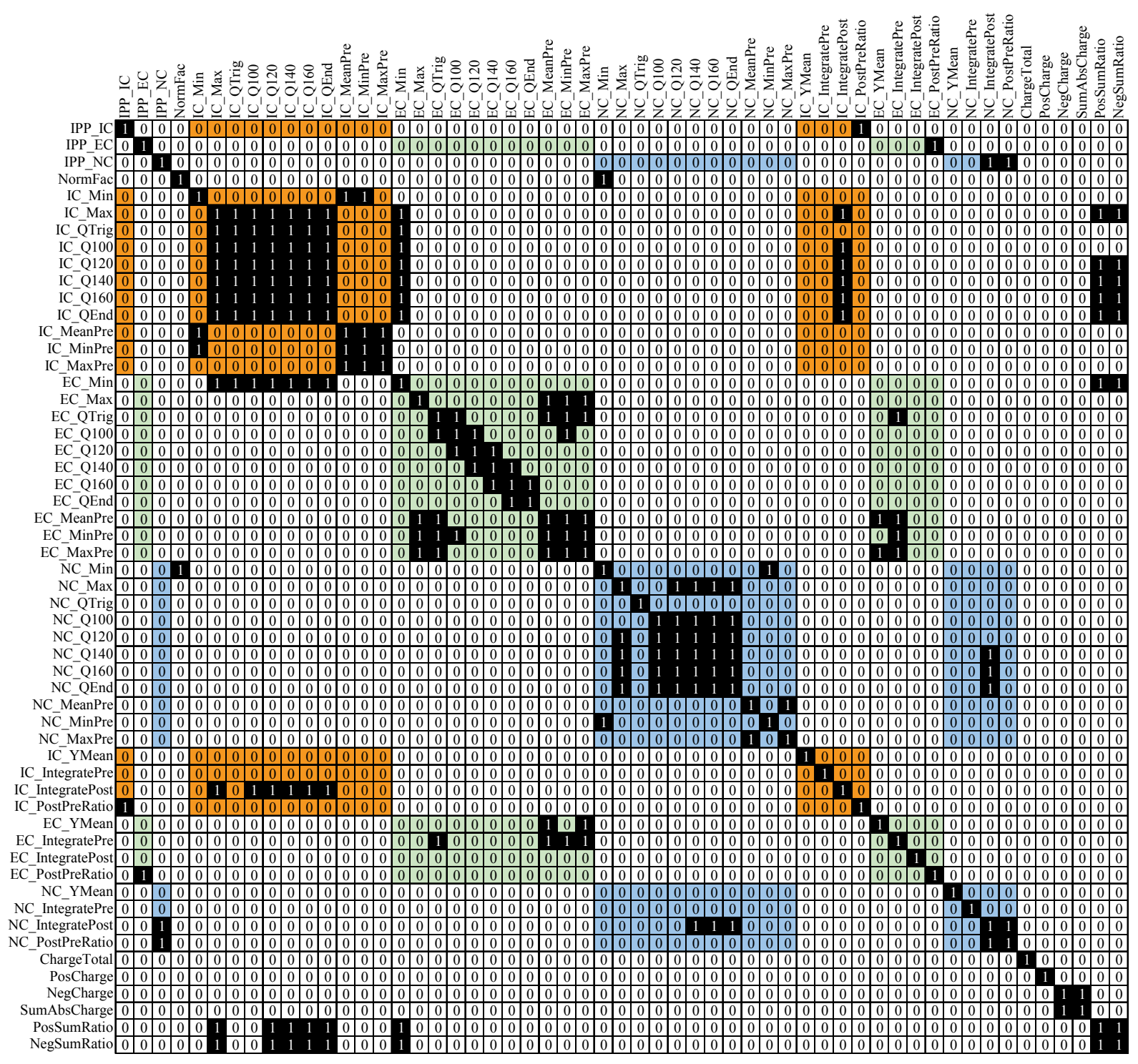

Figure 4-9: Correlations of the specific values

Correlations between the specific values. Correlations greater than 0.75 are displayed as 1 , correlations lower than 0.75 are displayed as 0 . Most correlations are present within values that correspond to the same channel.

In Figure 4-9, the correlations of the specific values that were used as input values for the neural network are shown. The most significant values from Figure 4-8 show only little correlation to other values.

\subsubsection{Determination of the optimal topology}

Given the boundary conditions of 16 input parameters and 2 output parameters, there are several different topologies of the neural network which can be conceived, as neither the number of hidden layers nor the number of neurons in each hidden layer are predetermined. To carry out a systematic determination of the best performing network topology, all possible and reasonable topologies were implemented, trained and validated as described in paragraph 
4.3.2. As the same network shows different performance for each new created instance, 5 runs for each topology were made to get a significant average. As reasonable topologies, networks with one hidden layer containing 3 to 17 neurons, and networks with 2 hidden layers containing $3 / 3$ to $17 / 17$ neurons were considered. Since the full test run of these configurations requires more than 24 hours on an average $800 \mathrm{MHz}$ desktop PC, larger configurations were not considered as reasonable.

Table 4-2: Performance of neural networks with $1 \mathrm{HL}$

\begin{tabular}{ccc}
\hline 1st HL & PI mean & DI mean \\
\hline 6 & 261 & 2.8 \\
11 & 265 & 2.4 \\
17 & 269 & 1.2 \\
10 & 272 & 1.6 \\
5 & 275 & 3.2 \\
8 & 279 & 2.6 \\
14 & 285 & 0.6 \\
16 & 289 & 0.8 \\
7 & 300 & 1.4 \\
13 & 302 & 1.6 \\
15 & 317 & 1.6 \\
9 & 319 & 0.6 \\
12 & 327 & 2.0 \\
4 & 361 & 1.2 \\
3 & 368 & 1.6 \\
\hline
\end{tabular}

1 st $\mathrm{HL}=$ number of neurons in hidden layer, PI = Possible Impacts, DI = Dropped Impacts Mean values from 5 evaluation runs, PI rounded 
Table 4-3: Performance of neural networks with 2 HL, best 15 topologies

\begin{tabular}{cccc}
\hline 1st HL & 2nd HL & PI mean & DI mean \\
\hline 4 & 12 & 243 & 0.2 \\
3 & 4 & 247 & 0.8 \\
17 & 10 & 252 & 0.2 \\
11 & 7 & 253 & 0.2 \\
6 & 13 & 260 & 0.2 \\
11 & 15 & 261 & 0.0 \\
13 & 14 & 262 & 0.2 \\
4 & 16 & 262 & 0.8 \\
4 & 14 & 263 & 0.8 \\
4 & 15 & 266 & 1.6 \\
5 & 9 & 267 & 0.2 \\
12 & 9 & 268 & 0.2 \\
8 & 11 & 269 & 0.2 \\
5 & 17 & 270 & 0.4 \\
9 & 4 & 272 & 0.4 \\
\hline
\end{tabular}

1st/2nd $\mathrm{HL}=$ number of neurons in 1st/2nd hidden layer,

PI $=$ Possible Impacts, DI = Dropped Impacts

Mean values from 5 evaluation runs, PI rounded

The results are shown in Table 4-2 for networks with one hidden layer, and in Table 4-3 for networks with two hidden layers. An optimal neural network will identify a low number of signals as possible impacts for best noise reduction (parameter PI), but also drop a low number of real impacts (parameter DI). Figure 4-10 shows the network with the best configuration for networks with one hidden layer.

As can be seen, the optimal topology comes out as a network with two hidden layers containing 11 and 15 neurons in the hidden layers. This network identifies 261 of the more than 20000 signals as impacts and drops none of the (at this time) 120 possible impacts identified by the experimenter (real impacts were later reduced to 98 after reviewing the data). 


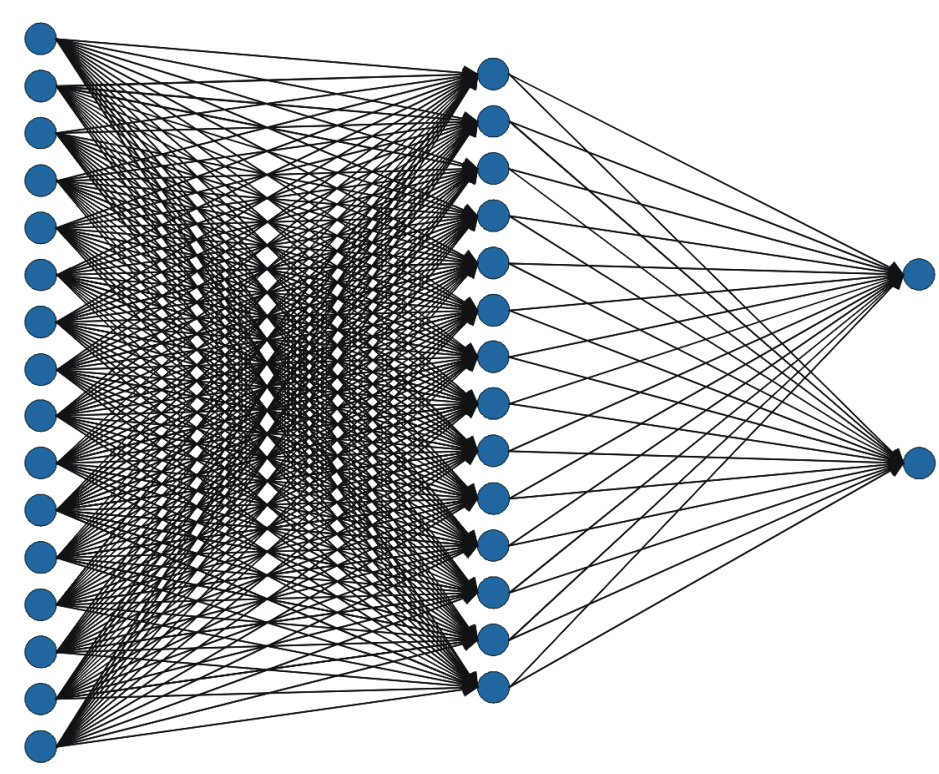

\section{input layer $\quad$ hidden layer output layer}

Figure 4-10: Final topology of the neural network with one hidden layer The best topology of a neural network that is used for the classification of MDC signals is a network with 16 input neurons, one hidden layer containing 14 neurons and 2 output neurons.

\subsubsection{Further potential applications}

In this chapter, it was proofen that neural networks are a powerful method for the classification of MDC signals. The classification efficiency (number of signals classified as possible impacts) of the neural networks described above is about in the same range as the classification capacity of the current qualification routine onboard the MDC (in case the new qualification parameter set is used), but neural networks show a much higher reliability with respect to the number of dropped impacts.

In this chapter, the neural network was used to recognize real impact signals in the MDC's data stream and separate them from unwanted noise signals. Further applications of neural networks on experiments like the MDC can be considered. The next step after identification of a real impact signal is the classification of the signal to determine the impact position inside the sensor box. Until now, this is done by a routine that simply compares the newly identified signal with several thousands of calibration signals with known impact positions, a method that requires much computing power and memory. A neural network that is designed and trained to determine the class of a new signal could reduce these expenses and even be implemented onboard a new designed experiment. 


\subsection{Summary and discussion}

Intelligent pattern recognition methods like neural networks evaluated in this chapter provide a good alternative to the existing routine that is used by the MDC for the onboard identification of noise signals and the separation of wanted signals. Although it was shown that the existing onboard routine (the qualification routine) could achieve much better results in separating noise data from wanted data after optimization of the used parameter set, the artificial neural network showed even better performance. While the neural network reduces noise data to around $50 \%$ of the whole data without dropping a wanted signal erroneously, the qualification routine can only compete with this result by dropping at least three impact signals.

If NOZOMI was recovered from its system failure on April 24, 2002 and had finally reached Mars orbit, the new determined parameter set of the qualification routine would have enabled much longer time spans between MDC memory dumps without the risk of losing real impact signals due to overwriting. Therefore, telemetry time, which would have been a strictly limited resource on NOZOMI, could be saved.

An artificial neural network like the one described and evaluated in this chapter can be used in future instruments for an even better separation of noise data from wanted data.

Both methods require some kind of knowledge about the properties of wanted signals and noise signals prior to be deployed. While the properties of wanted signals that will be recorded in space can be estimated from the signals recorded during the calibration experiments, the properties of possible noise signals cannot be estimated prior to flight. Deployment of methods that discard unwanted noise signals without the possibility of intervention by the experimenter must be done with care. Training and optimization of the methods described in this chapter can be done during the first mission phase using recorded and downloaded noise and impact data as training data, until a satisfying level of pattern recognition capability of the instrument is reached. It is, however, essential that the experimenter can still trust in the data delivered by the experiment. An increasing level of experiment autonomy in separation of noise data means a decreasing knowledge of the experimenter about the discarded signals. If the environment of the spacecraft remains unaltered for a long period of time, and a high level of trust is achieved for the given environment, a high grade of autonomy can be accepted. Every alteration of the experiments environmental conditions (e.g. significant change of the spacecraft's orbit or even injection into a planetary orbit with a different dust environment and probably new sources of noise) needs a reconsideration of the level of autonomy granted to the experiment, and possibly intervention by the experimenter and new training of the experiments pattern recognition methods.

The possibilities of a full automation of the MDC data analysis process was discussed by Naumann [Nau2000]. It was shown that the further steps of the analysis process, which are the classification of an impact signal, the determination of the required signal characteristics (rise time, charge amplitude) and the final determination of particle mass and impact speed can be performed by an automated system with only a little loss of accuracy of the determined values of mass and impact speed. The biggest challenge in automation of the MDC (or a future similar) experiment is still the separation of noise signals from impact signals. While 
possible errors in the further steps of the automated analysis process will produce deviations from the final values of particle mass and impact speed that are within the overall accuracy of the MDC experiment as shown by Naumann, a wrong qualification of noise signals as impact signals will falsify the overall results of the whole mission in an unacceptable manner. The good results of signal qualification by a simple artificial neural network as it was shown above are a basis for further investigation of this method to achieve reliable qualification results. It was shown by Heistermann [Hei1990] that a combination of conventional learning strategies and genetic algorithms as optimization technique for a neural networks knowledge gives an improved pattern recognition performance of the neural network for complex problems. Also, topology generation of neural networks by genetic algorithms provides better adaptation of a neural network to the given problem (see Scholz [Sch1995]) than simple straight-forward methods like the one used above for topology determination do. As both the optimization of the qualification routine parameter set by a genetic algorithm and qualification by an artificial neural network showed good performance, a further investigation of these technologies and their possible combination may allow full automation of future dust experiments. 



\section{Data compression onboard the MDC}

For space missions, the amount of data that can be transferred back to Earth is limited not only by the nominal capacity of the transponders and antennas, but also by other factors as visibility and the pointing direction of the antenna or the attitude of the satellite, respectively. All these three factors take effect for a planetary deep space mission like NOZOMI. In principle, if downlink is possible at all, the available downlink capacity is limited by the power of the available transponders and decreases with increasing distance from Earth. NOZOMI's maximum nominal data rates vary from 2048 bps to 32768 bps. Not later than after injection into Mars orbit, this data rates will only be available for strictly limited time spans since NOZOMI will be hidden behind Mars at regular intervals, and additionally the remaining high gain antenna will not point to Earth continuously due to the attitude requirements of the scientific instruments on board.

Consequently, the overall amount of data that has to be transferred to Earth must be restricted to the essential minimum. The actual version of the Mars Dust Counter does not implement any kind of data compression. Nevertheless, the basic MDC onboard software already provides different opportunities for the implementation of data reduction. One is already described in chapter 4 , a simple optimization of the parameter set of the qualification routine gives to the possibility to implement a significant reduction of noise data by setting a threshold value in quality number for data segments selected for download.

In the following chapter, a routine that implements a compression of data segments by a factor of 10 is introduced. References are the monographies from Debnath [Deb2001] and Louis et al. [Lou1998], the technical report from Fischer [Fis1999] and the thesis works from Kyritsis [Kyr2003] and Rott [Rot2004]. 


\subsection{Basics and motivation}

As described in paragraph 2.3.2, NOZOMI's downlink capabilities were reduced in 1999 due to failure of the omni-directional S-Band transceiver. The X-Band downlink requires pointing of the antenna and thus the NOZOMI spin axis to Earth, which will not be given all the time. As a tribute to the reduced data transmission capabilities, a reduction of the amount of data that has to be transferred to Earth for MDC data analysis to a minimum is aimed, as the downlink capacity available for the MDC after insertion into Mars orbit will be strongly limited due to activation of the other scientific instruments onboard. In addition, if recovery of NOZOMI's damaged power supply unit and thus Mars insertion and switch back to normal telemetry fails, only a very limited downlink capacity will be available.

To achieve these aims, two possible modes of data compression were elaborated:

- A compression of the digitized signals by a lossy compression algorithm,

- A partly data analysis onboard to achieve a maximum data reduction

In the first case, compression of the signals by MDC's onboard computer makes it possible to reconstruct the original shape of the signal after download by applying the corresponding decompression algorithm. When using lossy compression algorithms, the compression rate has to be adjusted to give a maximum data reduction without losing the signal's relevant information. Basically, the high frequencies parts of the signal get lost, which mainly represent noise but also may represent sharp edges which are relevant for data analysis. The low frequency parts of the signal are kept for reconstruction of the original signal's shape. As shown later, the Discrete Wavelet Compression allows a reduction of the amount of data to $1 / 16$ or less of the original data without losing relevant information.

In the second case, when performing a partial data analysis onboard, the original shape of the signal gets lost. Thus, this can only be seen as an emergency procedure for the case that recovery of NOZOMI's nominal telemetry fails and only the highly limited telemetry capabilities in ranging mode are available. The transmission of single data bits by the use of NOZOMI's beacon signals would be sufficient to download all of the collected data, as the dust flow in the interplanetary space was quite low during the first years of the mission. Indeed, a better qualification and thus pre-selection of data sets to be stored safely for transmission to Earth is required, as described in chapter 4. 


\subsection{Data compression}

In this paragraph, first the principles of lossy compression techniques are illustrated on the example Fourier Transformation, a widely used and well-known technology in communications engineering (paragraph 5.2.1). In paragraph 5.2.2, the Discrete Wavelet Transformation technique (DWT), its difference from the Fourier Transformation and its special advantages for the compression of the MDC signals is explained in detail.

\subsubsection{Lossy compression}

The original data of the MDC consists of a sequence of numerical values representing the shape of the signal recorded by the MDC electronics. Mathematically, this defines a discrete function $f(k)$ consisting of $N$ values, $k=0 \ldots N-1$, where $N$ is 413 for the electron channel and 213 and 212 for the ion channel and the neutral channel, respectively. Physically, this is the representation of the signal in the time domain.

In some cases, this representation is rather unsuitable for further analysis of the signal. To circumvent these restraints, a transformation of the signal to some other representation can be performed. Generally, a transformation of a function

$$
f(k), k=0 \ldots N-1
$$

gives a new function

$$
F[m], m=0 \ldots N-1,
$$

that represents the same data in a different way. As an example of a widely used transformation, the Discrete Cosine Transformation, a variety of the Discrete Fourier Transformation, will be mentioned here ${ }^{2}$.

Mathematically, the function or the signal is folded with a series of $N$ scaled basis functions, in the case of Cosine Transformation scaled cosine functions are used as basis functions. Strictly speaking, a $\mathrm{N}$-dimensional scalar product is calculated from the discrete signal and the $m^{\text {th }}$ basis function giving one single scalar value (in the case of continuous functions, the scalar product is calculated in the Hilbert space, a infinite dimensional vector space). All $N$ values $F[m]$ fully represent the original signal $f(k)$ in a new domain, called frequency domain when Fourier Transformation is used.

$$
F[m]=w_{m} \sum_{k=0}^{N-1} f[k] e^{j 2 \pi \frac{m k}{N}}, \quad w_{m}=\left\{\begin{array}{c}
\frac{1}{\sqrt{N}}, m=0 \\
\sqrt{\frac{2}{N}}, 1 \leq m \leq N-1
\end{array}\right.
$$

\footnotetext{
${ }^{2}$ In case of Fourier Transformation, which is based on periodical basis functions, the signal $f(k)$ has to be periodical as well to get proper results. Non-periodic signals can be periodically extended by defining a function $f^{*}\left(k^{*}\right), 0<k^{*}<2 N$ where $f^{*}(k+N)=f^{*}(k), 0<k<N$. Here, this will not be regarded further.
} 
By applying the appropriate inverse transformation, the original signal $f(k)$ can be restored exactly from the coefficients $F[m]$, as long as all $N$ coefficients are available for the inverse transformation. After transformation, certain manipulations and analyses of the signal are much easier using the $F[m]$ representation, in particular filtering of the signal.

Analogue signals of sensitive instruments like the MDC show natural noise, superposing the mainly low frequent wanted signal in a wide frequency range. To eliminate disturbing noise, in many cases a high pass filter may be useful for further processing of the signal. Transformation into the frequency domain makes it easy to apply such a filter, since the coefficients $F[\mathrm{~m}]$ of low order (small $\mathrm{m}$ ) represent the existence of low frequencies in the signal, and those of high order (high $m$ ) represent the existence of high frequencies. Simply setting of the coefficients of unwanted frequencies to zero will remove these frequencies in the retransformed signal.

In most cases, when removing high frequencies, the wanted signal is smoothed and better suitable for further analysis. In terms of data compression, lossless compression algorithms like LZW (Lempel-Ziv-Welch) or RLE (Run Length Encoding) will work much better (i.e., lead to better compression rates) when processing a smooth signal rather than a noisy one. Alternatively, the low order coefficients $F[m], 0<m<m_{\max }<N$ themselves can be used as a compressed representation of the signal, where $m_{\max }$ must be preset as a fixed value giving the fixed compression rate. This value must be chosen with respect to the required accuracy of the wanted signal. Signals that consist mainly from low frequency parts will not be substantially affected even if high compression is applied.

One drawback of compression techniques like those described above is that leaps and bounds occurring in the wanted signal are represented by high order coefficients in the frequency space, and thus will also be smoothed. In certain cases, like the signals produced by the MDC, this is not acceptable and sets strong boundaries to the achievable compression rates.

Advanced compression techniques like the Discrete Wavelet Transformation DWT described in the following paragraph are designed to avoid this behavior and thus are more suitable for certain applications, e.g. the compression of MDC signals.

\subsubsection{Discrete Wavelet Transformation (DWT)}

The Discrete Fourier Transformation and its variants described before are based on periodical basis functions. The transformation of the signal into the frequency domain provides information about which frequencies the signal comprises and how strong these frequencies are represented overall. As a tribute to the periodicity of the basis functions, information about the time at which a particular frequency is present or absent is missing in the frequency domain.

As mentioned before, leaps and bounds in the signal are represented by high frequencies and will be smoothed when the signal is compressed. There are some newer methods available, which avoid this drawback; one is the Discrete Wavelet Transformation. 


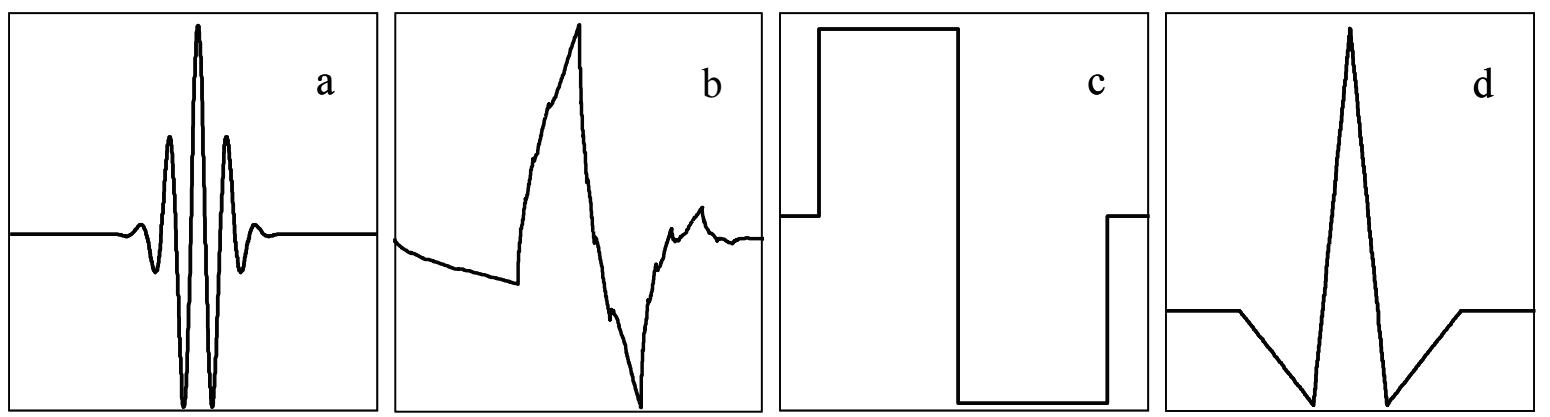

Figure 5-1: Some families of Wavelets

a) Morlet, b) Daubechies-4, c) Haar, d) Biorthogonal 2-2. Each family of Wavelets suites for different applications.

This method is quite similar to the Fourier Transformation, but is based on discontinuous basis functions rather than continuous ones. These basis functions are called Wavelets or Wavelet families, respectively. Many different wavelet families were developed which suite best for different applications like transformation and compression of speech, images etc. Some important Wavelet families are shown in Figure 5-1. For the transformation of the MDC signals, the Biorthogonal 2-2 was seen as best choice, because the simple construction of the Wavelet by a few straight lines allows fast integer addition, subtraction and left-shift operations which keep computing time short as it is required. First tests of transformation of MDC signals with the Biorthogonal 2-2 Wavelet family showed good results in achievable compression. Wavelet transformation is based on the following equation:

$$
W[m, n]=\frac{1}{\sqrt{2^{m}}} \sum_{k=0}^{N-1} f[k] \Psi\left(\frac{k-n}{2^{m}}\right),
$$

whereas the function $\Psi(x)$ describes the chosen Wavelet. Here, the scale is determined by the factor $m$, which is analogue to the frequency series used in the Fourier Transformation method, whereas the supplementary factor $n$ defines the shift of the discontinuous basis function on the time axis (this makes no sense if continuous basis functions are used). Since there are now two parameters rather than one, the resulting coefficients are no longer one series of coefficients, but a number of individual series of coefficients.

The transformation method used for the MDC signals is based on the so-called Multiresolutional Analysis method. The original signal is decomposed systematically into approximations and details. An additional scaling function $\Phi(x)$, which must be orthogonal to the wavelet function $\Psi(x)$, is needed. Wavelet and scaling function are correlated by

$$
\Phi(x)=2 \sum_{k} h_{k} \Psi(2 x-k) ; \quad \Psi(x)=2 \sum_{k} g_{k} \Phi(2 x-k),
$$

with the low-pass coefficients $h_{k}$ and high-pass coefficients $g_{k}$. For the transformation of the original signal, these low-pass and high-pass coefficients are needed. The transformation of a signal $f(k), k=1 \ldots N$ is then carried out by 


$$
\begin{array}{ll}
a^{\prime}(n)=\sum_{k} h_{k} f(n-k) \rightarrow a(m)=a^{\prime}(2 m+1) ; & 0<\mathrm{m}<\frac{\mathrm{N}}{2}-1 \\
d^{\prime}(n)=\sum_{k} g_{k} f(n-k) \rightarrow d(m)=d^{\prime}(2 m) ; & 0<\mathrm{m}<\frac{\mathrm{N}}{2}-1
\end{array},
$$

which gives the approximations $a(m)$ of size $N / 2$ and the details $d(m)$ of size $N / 2$. Approximations and details together have the same size $N$ as the original signal. Now, the detail part $d(m)$ is stored, and decomposition continues with the approximation part $a(m)$. This process is iterated until the size of the approximation and detail part becomes one single value (in case of the MDC signals, iteration was stopped when the size reached 4 values to save computing time). All approximation parts but the last one are discarded. The total size of approximations and details is then again $N$, which is the size of the original signal. The decomposition tree of a MDC neutral channel signal is shown in Figure 5-2.

After decomposition is finished, a compression of the signal can be carried out by removing (which means, setting to zero) transformation coefficients from the details parts obtained. This is described in the following paragraph.

Reconstruction of the original signal is carried out with the inverse transformation equations (not shown here, see Kyritsis [Kyr2003]). From the approximation part $\mathrm{a}_{6}$ and the detail part $\mathrm{d}_{6}$, the approximation part $\mathrm{a}_{5}$ can be reconstructed. This is iterated subsequently until the original signal is reconstructed from approximation part $\mathrm{a}_{1}$ and detail part $\mathrm{d}_{1}$, see Figure 5-2. 

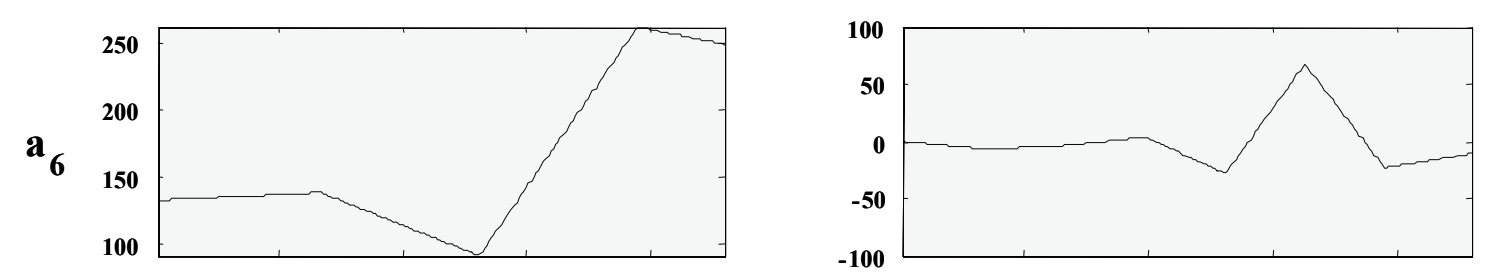

\section{$\mathbf{d}_{6}$}
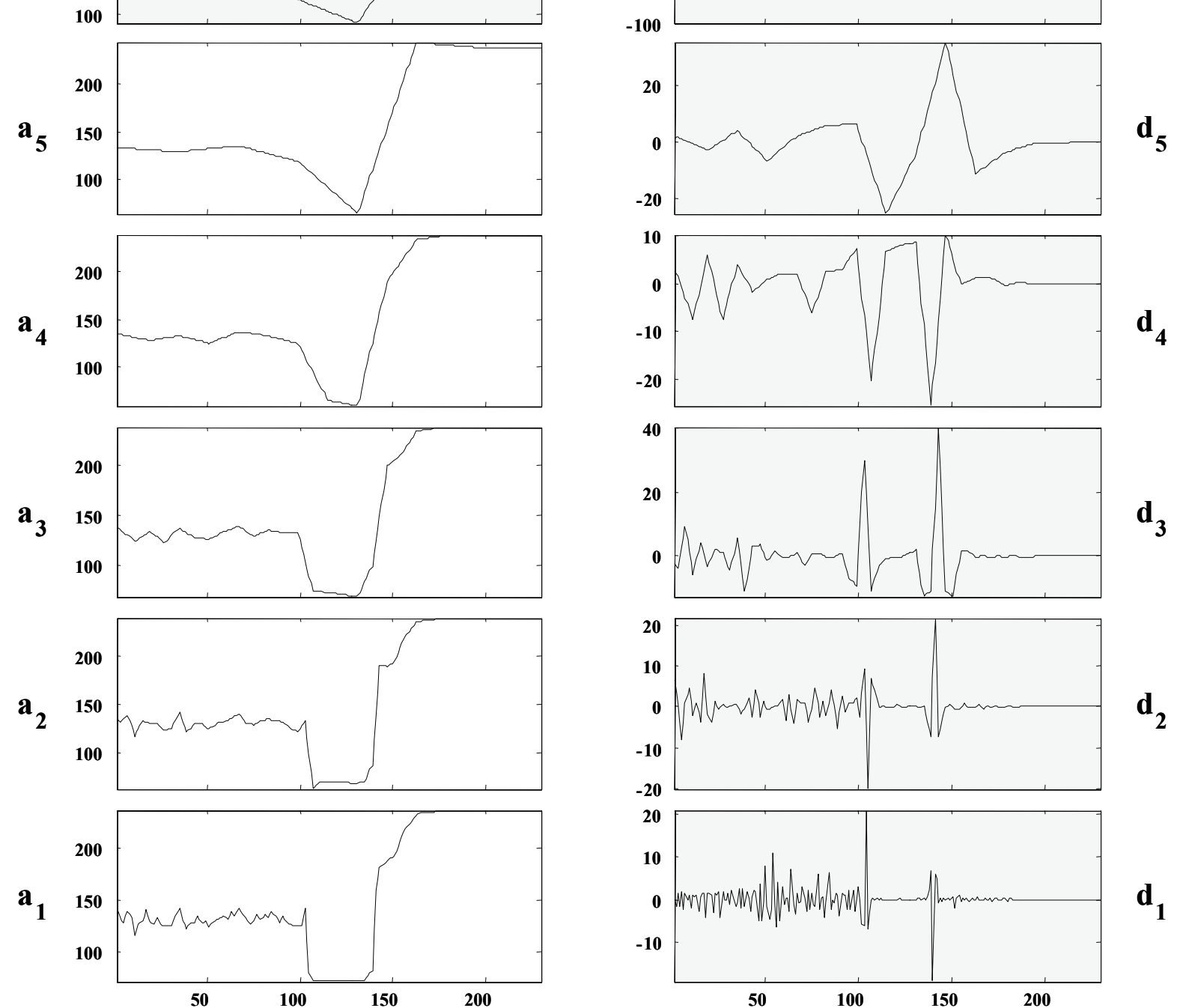

$\mathbf{d}_{5}$

$d_{4}$

$d_{3}$
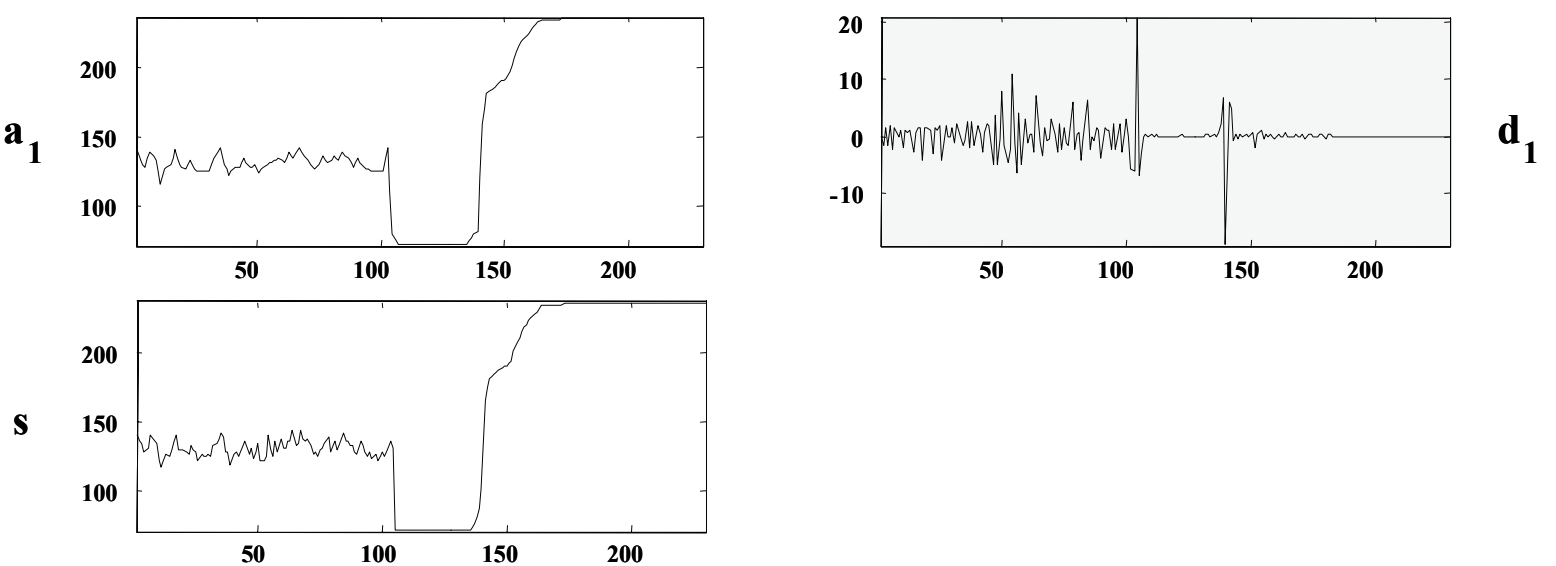

$-10$

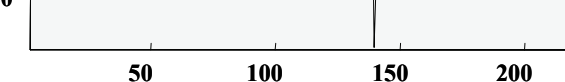

$\mathbf{d}_{2}$

$\mathbf{d}_{1}$

Figure 5-2: Decomposition tree of an MDC neutral channel signal

The original signal $(s)$ is decomposed step by step into approximations $\left(a_{1}\right.$ to $\left.a_{6}\right)$ and details $\left(d_{1}\right.$ to $\left.d_{6}\right)$. For a complete reconstruction of the original signal, all detail parts $d_{1}$ to $d_{6}$ and the last approximation part $a_{6}$ need to be stored. 


\subsubsection{Compression of MDC signals}

After transformation of the signal, the size of the data is still the same as before transformation. Now, coefficients that represent details that are not needed for the later analysis of the signal can be removed. To illustrate this, the coefficients of the detail parts of a transformed signal are shown in a two dimensional view in Figure 5-3 (approximation part $\mathrm{a}_{6}$ is not shown here). In Figure 5-3 a), the full set of coefficients is shown, the values of the coefficients are indicated by colors (white: zero, black: maximum value). As a first step, all detail coefficients that are located below a zero value detail coefficient are removed, based on the assumption that a detail located below a zero detail represents a single peak, which is classified as noise and could be deliberately removed. This leads to Figure 5-3 b). Now, all details left of the trigger are removed since they are not needed for further data analysis. The pre-trigger shape of the reconstructed signal is then represented only by the remaining approximation part $\mathrm{a}_{6}$. Figure 5-3 c) shows the remaining coefficients. Next, coefficients are removed subsequently beginning with the smallest, until the desired compression rate (or, number of remaining coefficients) is reached. Figure 5-3 d) shows the final coefficients of the compressed signal. The desired compression rate for the MDC is set to a factor of 16, which means that only $6.25 \%$ of non-zero coefficients remain for later reconstruction of the original signal.

Now, as many coefficients as possible were set to zero with respect to the requirements of the data analysis regarding the shape of the reconstructed signal. No real compression at all is performed until now. Compression of the data is finally carried out by Differential Pulse Code Modulation, a well-established method of lossless compression of data streams. The more zero values are represented in the data that has to be compressed, the better the DPCM compression works.

The now compressed data of the signal shape is merged with a subset of the MDC memory segments housekeeping data and stored in the MDC memory as described in paragraph 5.3.2. A MDC memory segment of 1 kByte size can now hold 10 compressed signals rather than one uncompressed.

Figure 5-4 shows the overall performance of the data compression routine. Reconstruction error $e$ (absolute difference of the reconstructed signal from the original signal, scaled by the signal range) is lower than $10 \%$ for $91 \%$ of all available MDC signals. Achieved compression factors are between 16 and $\approx 55$ for all available MDC signals. 


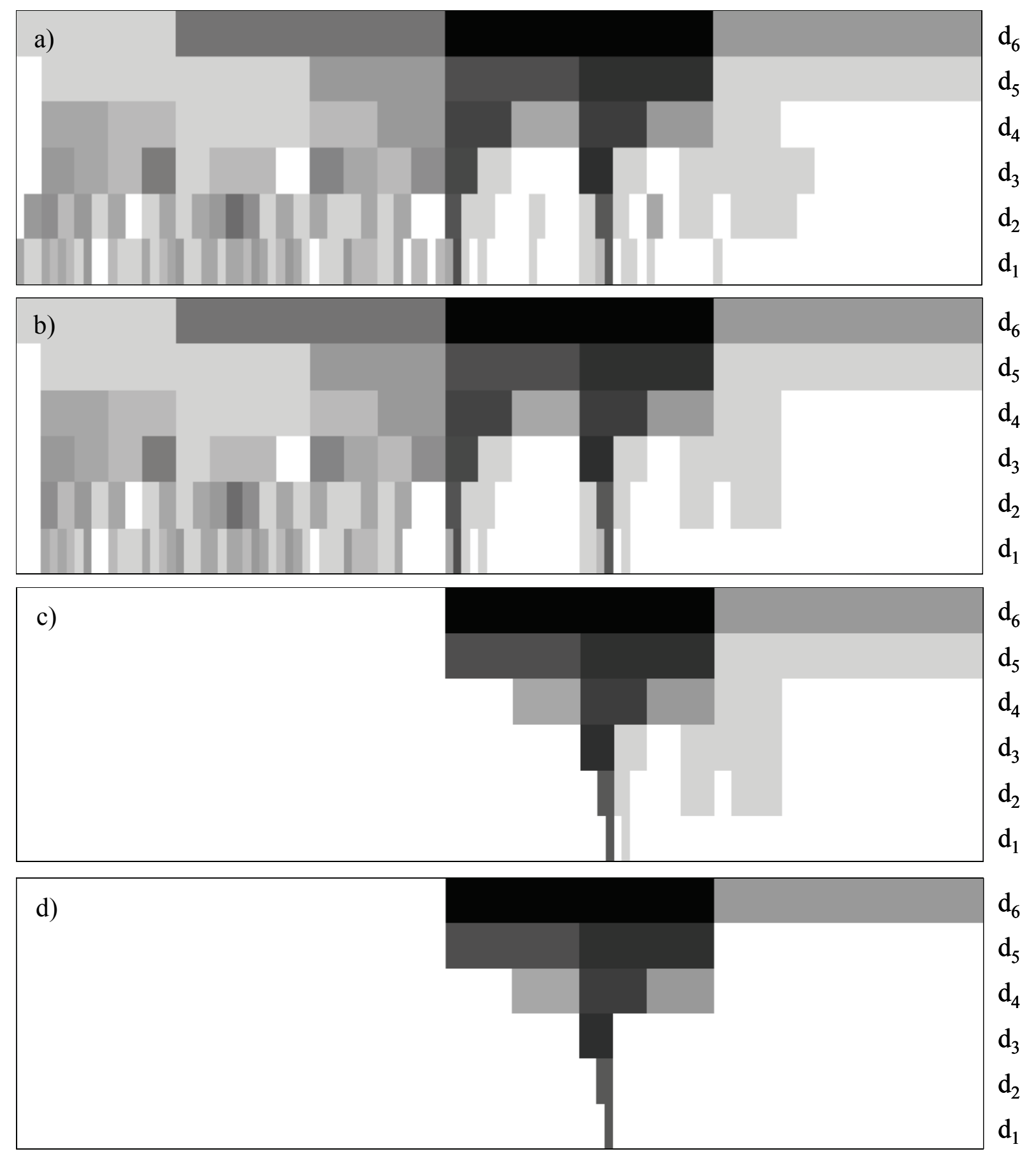

Figure 5-3: Compression of an MDC signal

a) Detail coefficients of the original signal; b) Detail coefficients after removal of small details without a large detail on top; c) Detail coefficients after removal of pre-trigger details; d) Detail coefficients after removal of small coefficients. Colors indicate the coefficients numerical value, white represents zero, black the maximum value of 56 . 

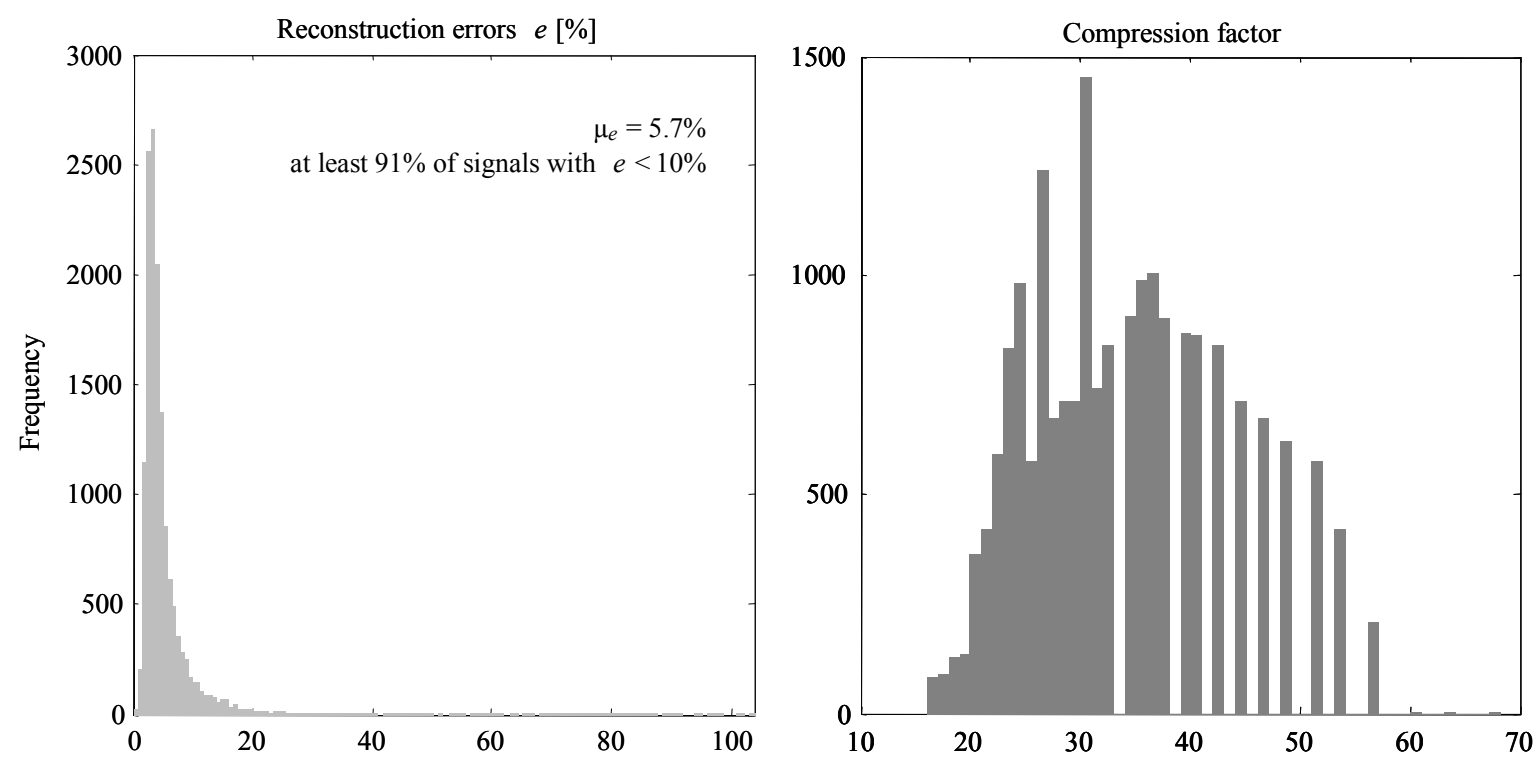

Figure 5-4: Performance of the data compression Frequency of reconstruction errors and compression factors for all MDC signals. 


\subsection{Implementation and integration of the MDC onboard software upgrade}

In this paragraph, the implementation and integration of the software upgrade that performs data compression onboard the MDC is described with respect to the limitations that are given by the MDC onboard data processing capabilities.

\subsubsection{Hardware and software restrictions}

Strong requirements have to be fulfilled when changing or upgrading a reliable and running system. The restrictions that have to be considered are discussed in this paragraph.

Due to the limited resources of the MDC onboard computer in memory and computing power (see paragraph 3.2.2), strict requirements have to be defined for a large software upgrade like the compression routine described here.

The limitations of the hardware enforce the following requirements:

- Any compression routine must be executable by the onboard processor in a time span of 1 to max. 3 seconds.

- All calculations must be implemented using integer arithmetic.

- The final code of the software upgrade must not require more than one KByte of RAM memory

The existing onboard software requires further restrictions regarding a software upgrade. During the MDC's operation since launch, the existing onboard software has proven to work stable and accurate. Therefore, it has to be ensured that the stability and functionality of the software will not be affected by the upgrade, even if the upgrade does not work properly or has to be removed.

In the case of the existing MDC software, the functionality of the existing memory management and the memory dump routines must not be affected by the software upgrade in any way. This is a strong requirement, since a possible deactivation of the upgrade caused by any case must not lead to any mismatch of memory segmentation. The following requirements must be fulfilled:

- The compression routine has to be integrated into the existing software without affecting the consisting operating sequence,

- The new memory management needed to store compressed data has to be implemented in a way that the existing memory management can operate as before and no adaptation is needed.

These requirements can be met by a smart exploitation of the provided extension options of the existing software. 


\subsubsection{Integration of the upgrade into the existing software}

The qualification routine of the existing software provides the ideal integration point for the new compression routine. Any new-recorded signal will be qualified by a routine running as a background task. In principle, the qualification routine and the compression routine each perform an extensive calculation task, which creates new data from the original signal data, a qualification number and the compressed data, respectively.

Now, the branch table of the MDC software can be manipulated to call the new compression routine instead of the qualification routine after all other tasks are completed. The operation sequence of the preceding tasks is not affected by this manipulation, as described above this is a fundamental requirement. Indeed, the existing software provides several points for the integration of new routines into the original operation sequence; fortunately, this was not necessary for the software upgrade described here.

The compression routine itself first calls the original qualification routine, and subsequently performs the compression of the original data and the storage of the results. This should work without problems as long as the computing time of the compression routine does not exceed 1 to 2 seconds. A longer computing time is not acceptable since during phases of high dust flux or strong interferences (which cause a high rate of new signals to be qualified, compressed and stored) a proper completion of qualification and compression may not be guaranteed any more, which could result in loss of data.

After compression, the signal data is reduced to about $6.25 \%$ of the original amount of data. Added segment and housekeeping information, every new data set requires $10 \%$ of one 1 kByte segment. These new data sets have to be stored in memory in a different way than the original data, since every old 1 kByte segment can now hold 10 new data sets instead of one original data set. As mentioned before, the original memory management working with the 1 kByte segments must not be affected.

To achieve this, a new type of memory segment was defined which is designed to store the new compressed data sets, but shows up as a conventional 1 kByte memory segment when processed by the old memory management. Only the data structure inside these new segments differs from the conventional segment structure, while the original memory management identifies these new segments as segments that must not be manipulated in any way. The software identifies segments by their quality number, which is stored in the first byte of each segment. Quality numbers from 0 to 245 identify segments which can be used to store uncompressed (original) signal data, while quality numbers higher than 245 represent segments used as calculation space, code or parameter segments. As code segments will never be manipulated by the old memory management, the new segments, which contain compressed data sets, are marked as code segments with quality number 253 . This enables the programmer to implement a new memory management handling the new compressed data sets without affecting the old memory management. The calculated quality number of a new data set is stored inside the data set, since the segment quality number of a new segment is fixed to be 253 .

The original software stores new signals after qualification either in an unused memory segment or, if there are no more unused segments, in the segment presenting the lowest 
quality number. This ensures that segments holding potentially good signals are relatively secured from being overwritten, if frequently memory readouts take place.

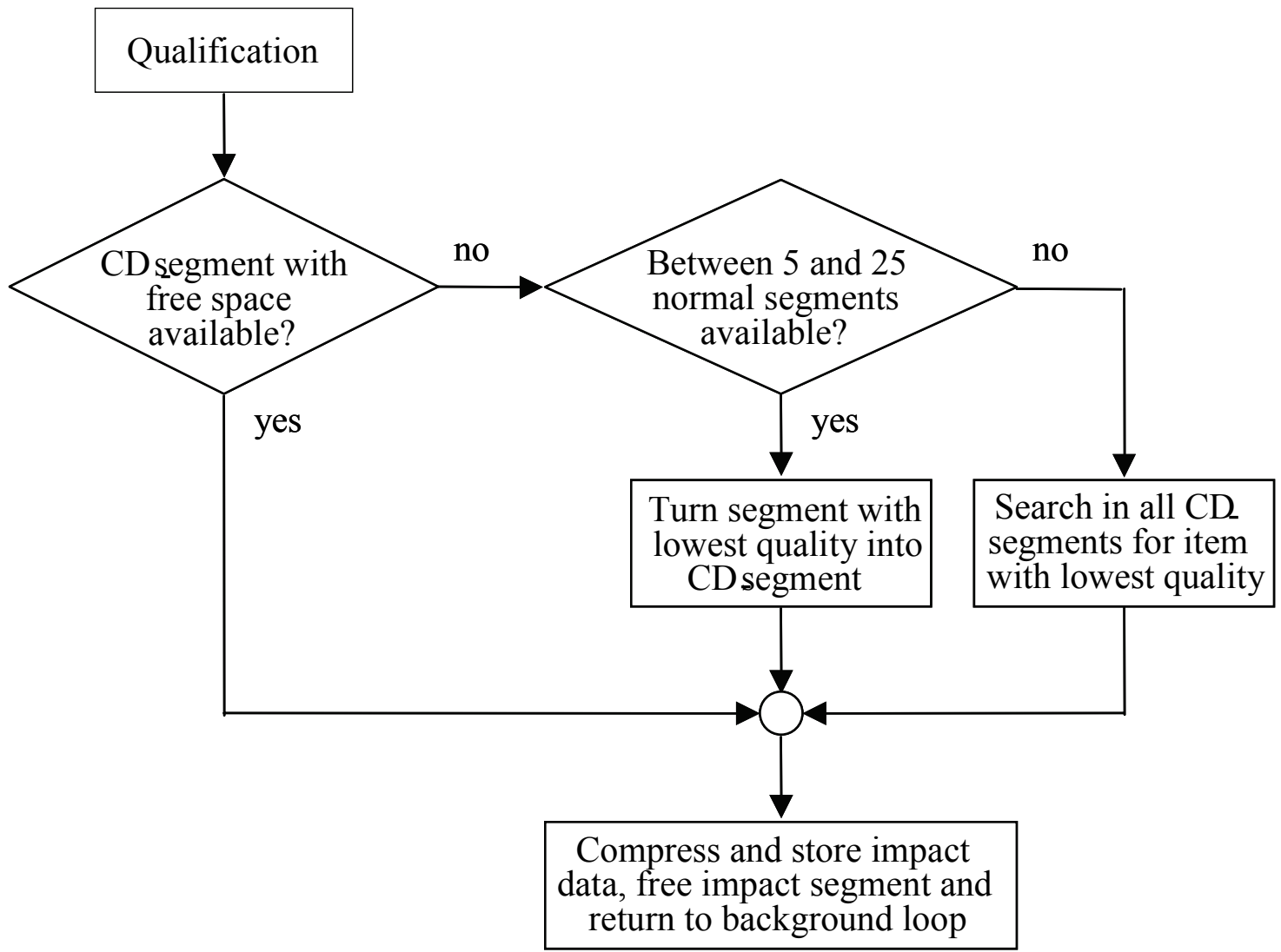

Figure 5-5: Flow chart of data processing of the new memory management After a new-recorded signal was processed by the qualification routine, the new MDC memory management looks for free space to store the compressed signal and calls the compression routine. $\mathrm{CD}$ segment $=$ Compressed data memory segment.

The upgrade is working in a quite similar way. Instead of searching for the best 1 kByte segment to store a new signal, free memory places are searched inside the existing new segments already containing compressed data. If there is no more memory available inside the existing new segments, a free $1 \mathrm{kByte}$ segment (or the one with the lowest quality number) is labeled and formatted as a new segment. The new compressed data set is then stored in the first memory place of this new segment. To ensure that enough conventional memory segments are available for the functionality of the old software, a limit of a maximum number of 35 to 40 new segments will be fixed ad hoc. 


\subsection{Verification of the new analysis process}

As mentioned before, the resulting signal shape of MDC data after compression/decompression differs from the original signal shape, see Figure 5-6 for a comparison between the original and the compressed/decompressed signal shape. Although the difference is only slight, one can say that the required characteristic points used as input for the calculation of mass and velocity of an identified impact will be set to different coordinates, both by a human experimenter and by an automated routine. In the bottom chart of Figure 5-6, this can be seen directly. For the NC channel (blue line), the maximum of the charge is reached a few $\mu$ s later on the compressed/decompressed curve than on the original one (thin black solid line next to the blue line). For the EC channel (green line), the maximum charge is higher on the compressed/decompressed curve than on the original curve (thin black solid line next to the green line). These differences influence the derived values of particle mass and impact speed, the magnitude of these induced errors in mass and impact speed is determined in the following.

To determine how much this difference of coordinates influences the final analysis results, test series were carried out. In principle, a set of original data that was analyzed previously using the uncompressed data was chosen, and the analysis was rerun using the same data sets after compression/decompression, giving new values for mass and impact speed for each data set.

Finally, the values resulting from the original analysis and the new analysis were compared and an overall deviation was calculated. 

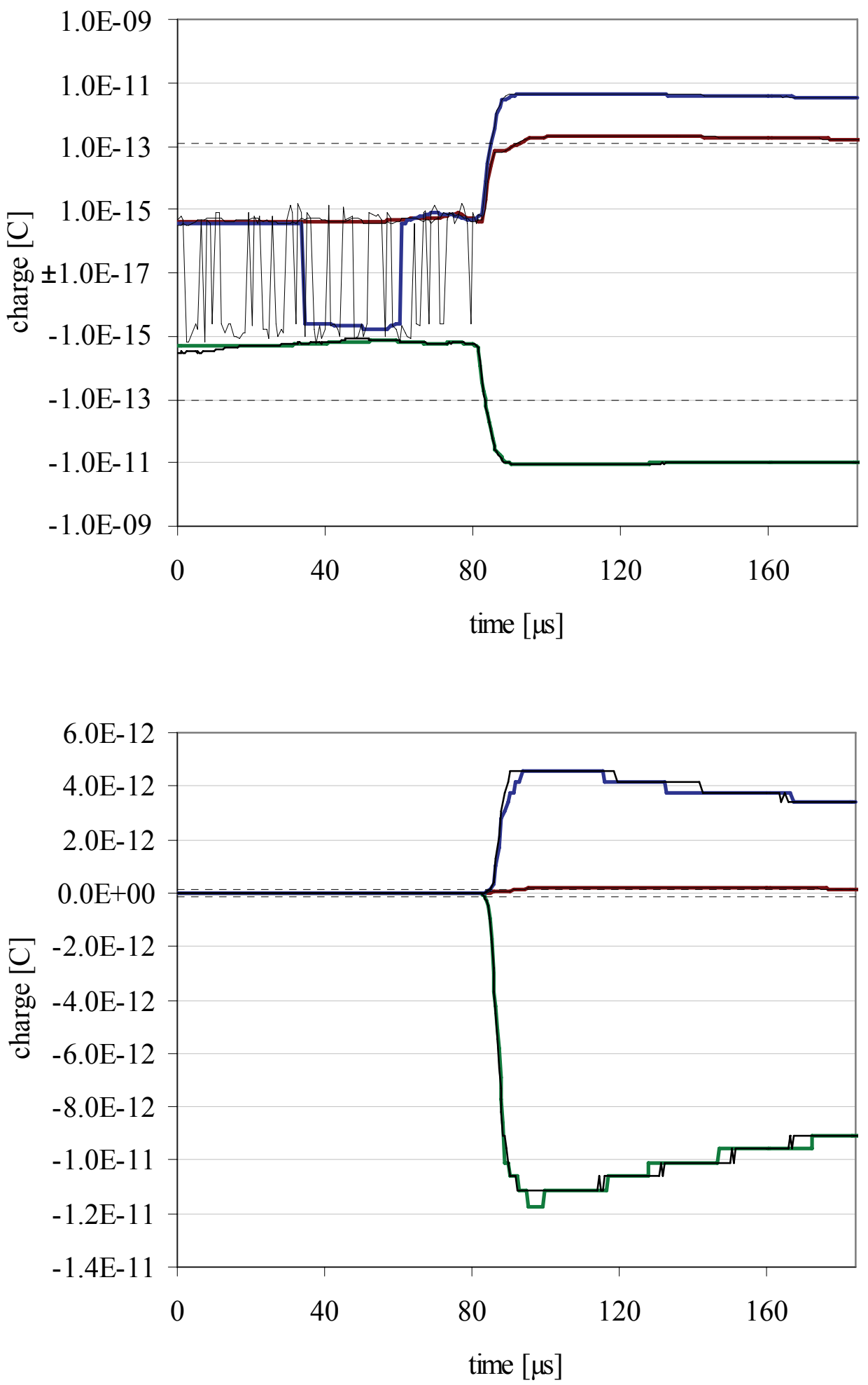

Figure 5-6: A typical MDC signal before and after compression

In this chart, a typical original MDC signal (solid black lines) is compared to the resulting signal after compression/decompression (solid colored lines), top: logarithmic view, bottom: linear view. As can be seen, the resulting signal is nearly identical to the original during the most important time period from $80 \mu \mathrm{s}$ to $120 \mu \mathrm{s}$. Most information is lost in the time period from $0 \mu \mathrm{s}$ to $80 \mu \mathrm{s}$, which is not relevant for MDC data analysis. 


\subsubsection{Analysis of MDC-NOZOMI data after compression/decompression}

The analysis of MDC-NOZOMI signals is carried out using the Microsoft ${ }^{\circledR}$ Windows $^{\circledR}$ MDCCDA program (Mars Dust Counter Control and Data Analysis) developed by Naumann [Nau2000] on the basis of the MS-DOS programs for MDC-HITEN and MDC-BREMSAT data analysis.

As the MDC data files that will be downloaded from NOZOMI after the software upgrade is installed will contain segments of compressed data, the MDCCDA program must be able to perform a decompression prior to the analysis of the data. Hence, the decompression routine was integrated into the existing MDCCDA. After decompression, the signals are analyzed in the same manner as before. To demonstrate that the analysis of compressed/decompressed signals gives accurate and unadulterated results, the compression routine used in the MDC software upgrade was also implemented into the MDCCDA. Thus, all the signals identified as impacts since launch of the NOZOMI mission and were already analyzed, can be compressed, filtered and decompressed and finally reanalyzed by the MDCCDA program. The algorithm used for compression onboard the MDC was mapped one-to-one from assembler to $\mathrm{C}++$ code to ensure identical compressed data sets.

\subsubsection{Analysis of MDC-HITEN data after compression/decompression}

To get an even more reliable verification of the integrity of the new data analysis process, the compression routine designed for the MDC-NOZOMI data was also applied to the data of MDC-HITEN, the predecessor of the MDC-NOZOMI. Configuration, electronics and, most important, signal shape differ slightly from the more complex MDC-NOZOMI. Thus, if this verification also gives satisfying results, the developed method can be proven as generally applicable to future experiments based on similar configurations like the MDCNOZOMI/MDC-HITEN configuration.

Unlike the MDC-NOZOMI, the compression/decompression was not integrated into the original data analysis software. Since this software was created parallel to the development process of the MDC-HITEN, and is now more than ten years old, it was decided that a reproduction of the analysis process using modern software can be performed much easier and more transparent than an extensive modification of the original software. Another reason for this decision was that the compression routines already existed in $M A T L A B^{\circledR}$, and only the final calculation process had to be reproduced.

Nevertheless, some slight modification of the original software was necessary to convert the MDC-HITEN data files from the original (binary) telemetry format to a format that can be handled by $M A T L A B^{\circledR}$, preferable text files. As a spin-off, the routines implemented for text export of the original data were used to create a universal XML (eXtensible Markup Language) representation of all MDC-HITEN data that can be easily used for future work, electronic publication and archiving (see chapter 7) 
After importing the data into $M A T L A B^{\circledR}$, the analysis was carried out in the following way:

The chosen data set was compressed, filtered and decompressed by the previously developed $M A T L A B^{\circledR}$ routines. The resulting signal was displayed by the integrated $M A T L A B^{\circledR}$ data handling functionality in a way that the coordinates of the required data points could be easily determined by the user.

The resulting values $\Delta t, \Delta q, q_{e}, q_{i}$ were carried over into a Microsoft ${ }^{\circledR}$ Excel $^{\circledR}$ sheet, where the final calculation of mass and velocity was performed using the appropriate equations and parameters required for the chosen type of signal.

It must be pointed out here that the determination of the coordinates of the data points was performed without any knowledge of the position of these points according to the analysis made previously, to make sure that the experimenter is free of undue influence. 


\subsection{Summary and discussion}

The results of the rerun analysis of MDC-HITEN data are summarized in the following figures, and in Table 5-1 and Table 5-2, broken down into the different signal types, see Münzenmayer [Mün1995] for a detailed description of the different MDC-HITEN signal types. In Figure 5-7, Figure 5-8, Figure 5-9 and Figure 5-10 mean values (solid red line) and standard deviation (dashed red lines) are visualized.

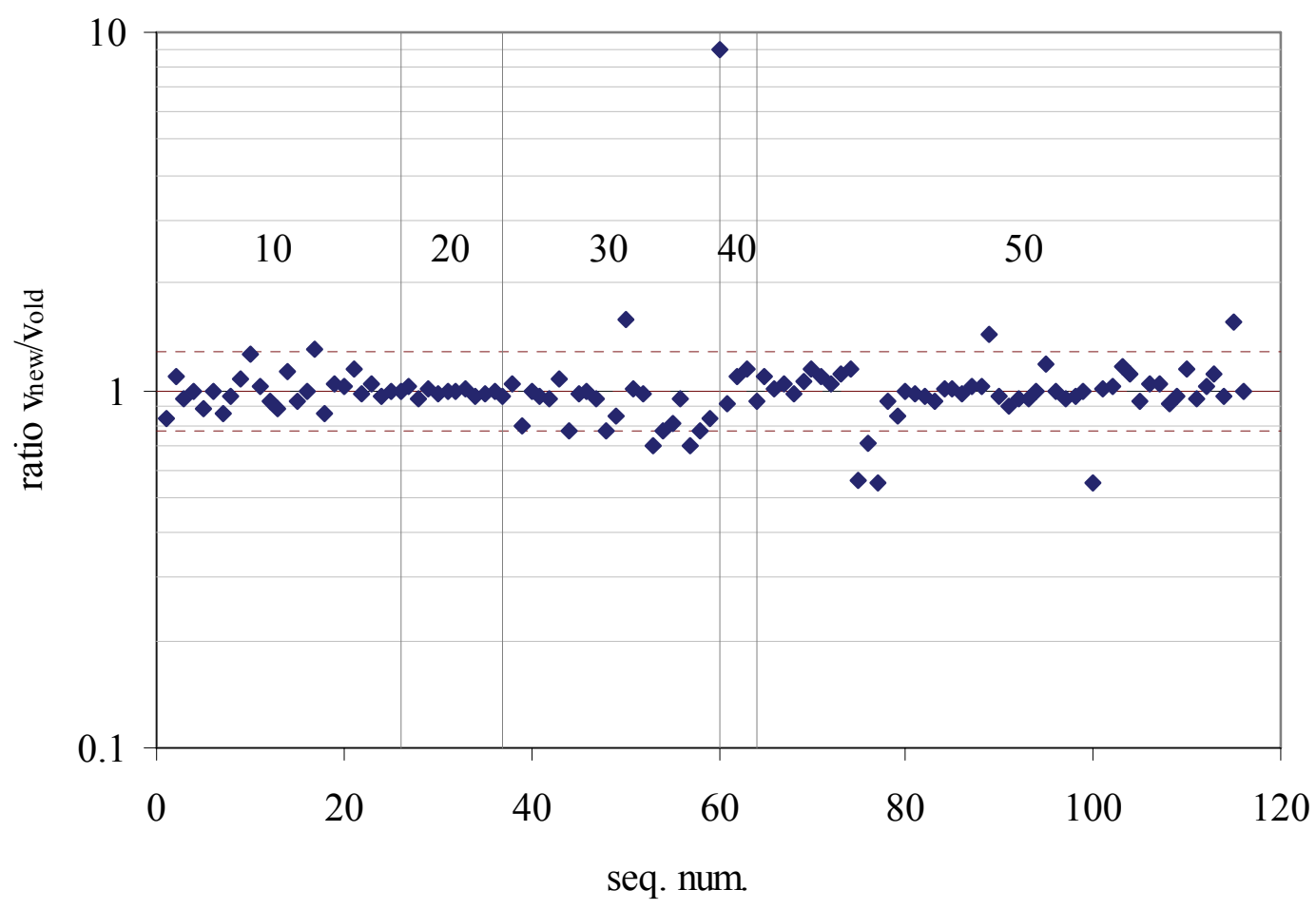

Figure 5-7: Impact speed ratio new/original analysis, MDC-HITEN

Impact speed ratio of the 115 values determined by the rerun analysis based on compressed/ decompressed signals to the values of the original analysis. Numbers 10 to 50 indicate the MDC-HITEN signal types. 


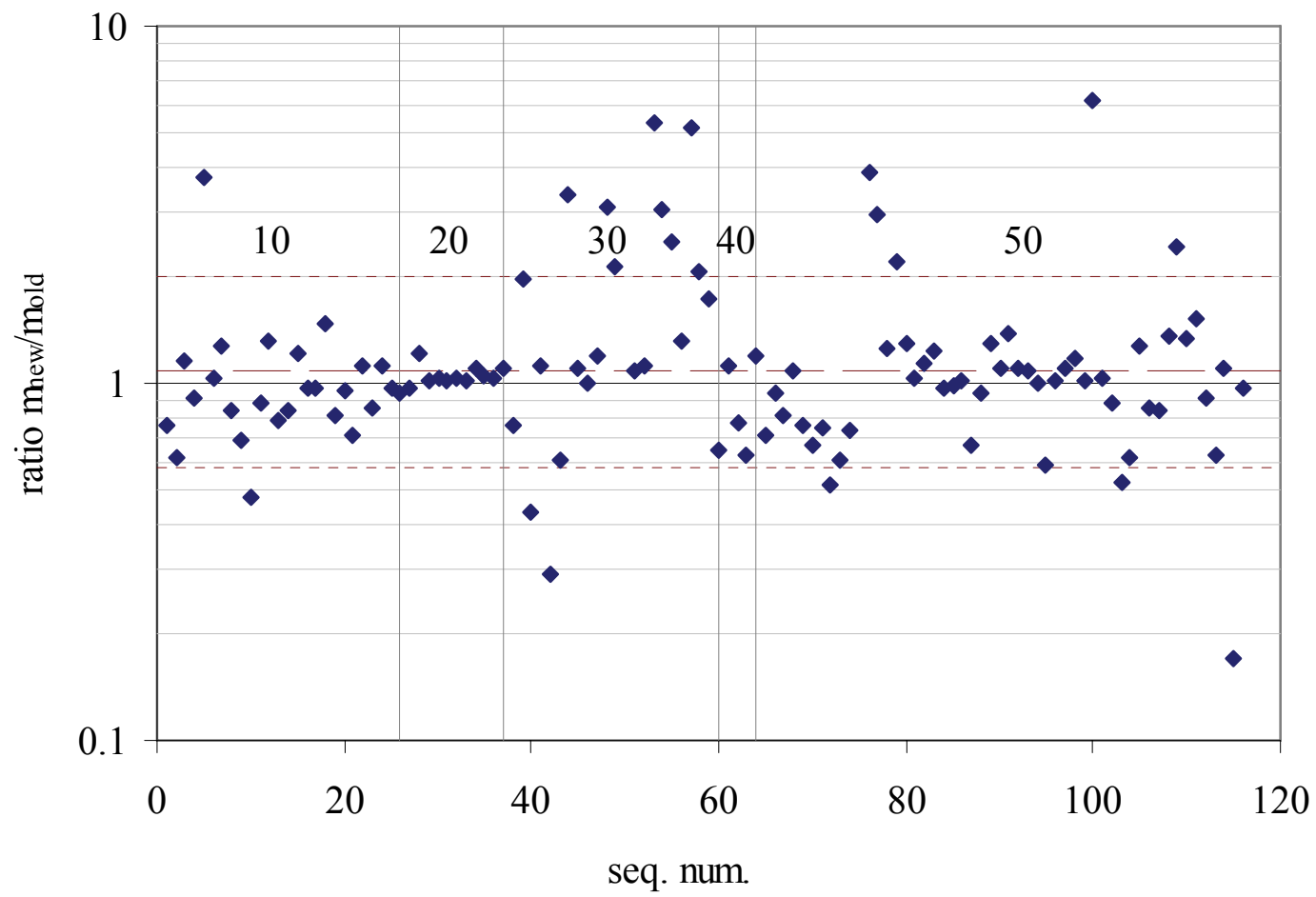

Figure 5-8: Particle mass ratio new/original analysis, MDC-HITEN

Particle mass ratio of the 115 values determined by the rerun analysis based on compressed/ decompressed signals to the values of the original analysis. Numbers 10 to 50 indicate the MDC-HITEN signal types.

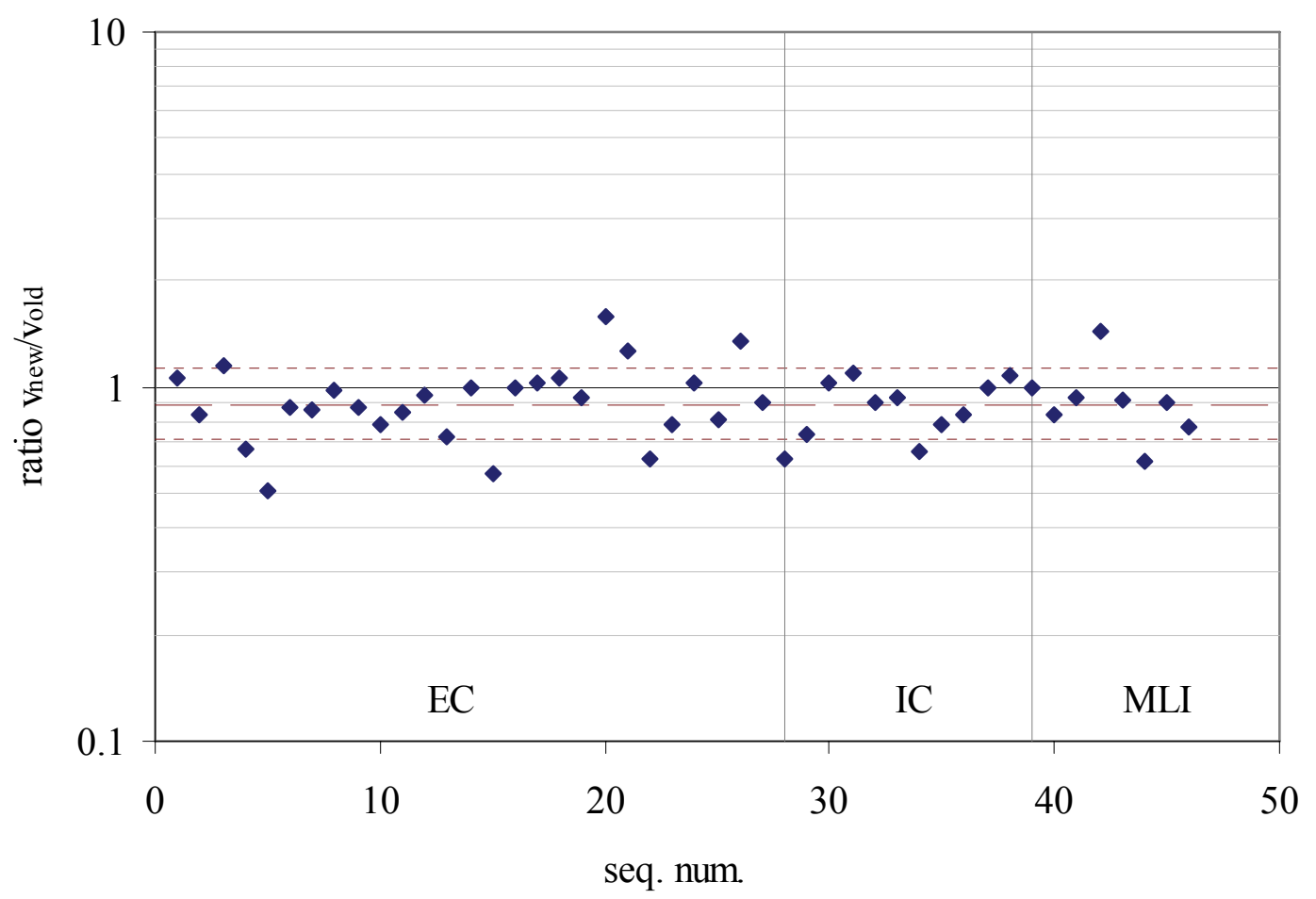

Figure 5-9: Impact speed ratio new/original analysis, MDC-NOZOMI Impact speed ratio of the 45 values determined by the rerun analysis based on compressed/ decompressed signals to the values of the original analysis. 


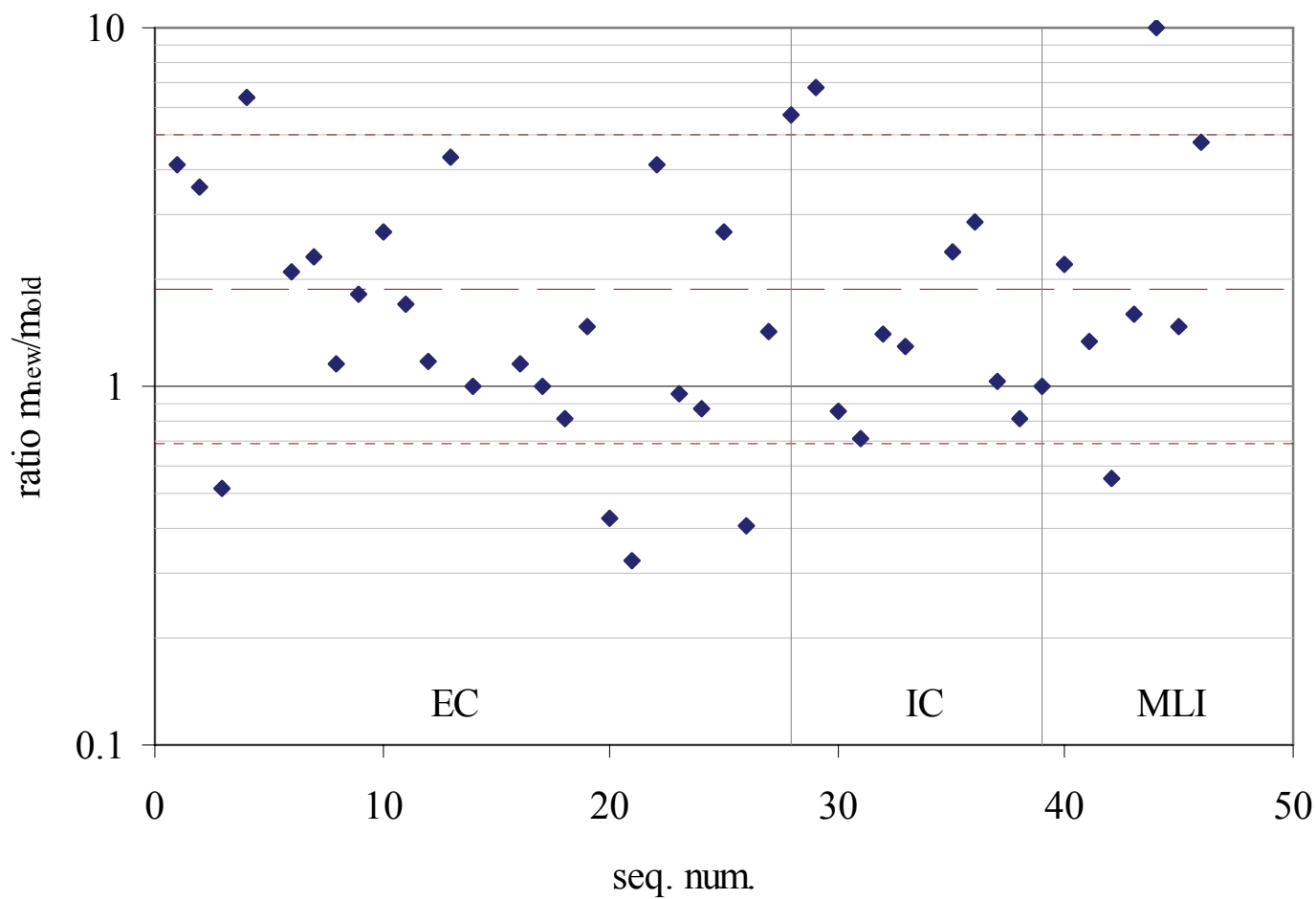

Figure 5-10: Particle mass ratio new/original analysis, MDC-NOZOMI Particle mass ratio of the 45 values determined by the rerun analysis based on compressed/ decompressed signals to the values of the original analysis.

The values shown in the following tables were calculated by the following equations:

$$
\begin{gathered}
\bar{m}=\exp \left(\frac{1}{N} \sum_{i=1}^{N} \log \left(\frac{m_{i, \text { new }}}{m_{i, \text { old }}}\right)\right) \\
\bar{v}=\exp \left(\frac{1}{N} \sum_{i=1}^{N} \log \left(\frac{v_{i, \text { new }}}{v_{i, \text { old }}}\right)\right) \\
\sigma_{m, \log }=\sqrt{\frac{1}{N} \sum_{i=1}^{N}\left(\log \left(\frac{m_{i, \text { new }}}{m_{i, \text { old }}}\right)-\log (\bar{m})\right)^{2}}, \bar{m}^{ \pm}=\exp \left(\log (\bar{m}) \pm \sigma_{m, \log }\right) \\
\sigma_{v, \log }=\sqrt{\frac{1}{N} \sum_{i=1}^{N}\left(\log \left(\frac{v_{i, \text { new }}}{v_{i, \text { old }}}\right)-\log (\bar{v})\right)^{2}}, \bar{v}^{ \pm}=\exp \left(\log (\bar{v}) \pm \sigma_{v, \log }\right)
\end{gathered}
$$

where $\bar{m}$ and $\bar{v}$ are the mean values, and $\bar{m}^{ \pm}$and $\bar{v}^{ \pm}$are the upper and lower boundaries of the standard deviation. 
Table 5-1: Validation of the compression on the MDC-HITEN data

Signals of type 10,26 signals analyzed

\begin{tabular}{llllll}
\hline $\bar{v}^{-}$ & $\bar{v}$ & $\bar{v}^{+}$ & $\bar{m}^{-}$ & $\bar{m}$ & $\bar{m}^{+}$ \\
0.90 & 1.00 & 1.12 & 0.67 & 0.97 & 1.41 \\
\hline
\end{tabular}

Signals of type 20,11 signals analyzed

\begin{tabular}{llllll}
\hline $\bar{v}^{-}$ & $\bar{v}$ & $\bar{v}^{+}$ & $\bar{m}^{-}$ & $\bar{m}$ & $\bar{m}^{+}$ \\
0.96 & 0.98 & 1.01 & 0.99 & 1.05 & 1.12 \\
\hline
\end{tabular}

Signals of type 30,23 signals analyzed

\begin{tabular}{llllll}
\hline $\bar{v}^{-}$ & $\bar{v}$ & $\bar{v}^{+}$ & $\bar{m}^{-}$ & $\bar{m}$ & $\bar{m}^{+}$ \\
0.60 & 1.00 & 1.66 & 0.50 & 1.28 & 3.26 \\
\hline
\end{tabular}

Signals of type 40,4 signals analyzed

\begin{tabular}{llllll}
\hline $\bar{v}^{-}$ & $\bar{v}$ & $\bar{v}^{+}$ & $\bar{m}^{-}$ & $\bar{m}$ & $\bar{m}^{+}$ \\
0.91 & 1.02 & 1.14 & 0.66 & 0.90 & 1.22 \\
\hline
\end{tabular}

Signals of type 50,52 signals analyzed

\begin{tabular}{llllll}
\hline $\bar{v}^{-}$ & $\bar{v}$ & $\bar{v}^{+}$ & $\bar{m}^{-}$ & $\bar{m}$ & $\bar{m}^{+}$ \\
0.82 & 0.98 & 1.18 & 0.58 & 1.07 & 1.99 \\
\hline \multicolumn{7}{c}{} & & & & \\
\multicolumn{7}{l}{ Overall results, } & 115 signals analyzed & & & \\
\hline$\overline{\bar{v}}^{-}$ & $\bar{v}$ & $\bar{v}^{+}$ & $\bar{m}^{-}$ & $\bar{m}$ & $\bar{m}^{+}$ \\
0.76 & 0.99 & 1.29 & 0.58 & 1.08 & 2.00 \\
\hline
\end{tabular}

Table 5-2: Validation of the compression on the MDC-NOZOMI data EC signals, 28 signals analyzed

\begin{tabular}{llllll}
\hline $\bar{v}^{-}$ & $\bar{v}$ & $\bar{v}^{+}$ & $\bar{m}^{-}$ & $\bar{m}$ & $\bar{m}^{+}$ \\
0.76 & 0.96 & 1.20 & 0.58 & 1.36 & 3.19 \\
\hline \multicolumn{7}{c}{ IC signals, } & 11 & signals analyzed & & & \\
\hline$\overline{\bar{v}}^{-}$ & $\bar{v}$ & $\bar{v}^{+}$ & $\bar{m}^{-}$ & $\bar{m}$ & $\bar{m}^{+}$ \\
0.60 & 0.77 & 0.97 & 1.07 & 3.67 & 12.59 \\
\hline
\end{tabular}

MLI signals, 6 signals analyzed

\begin{tabular}{llllll}
\hline $\bar{v}^{-}$ & $\bar{v}$ & $\bar{v}^{+}$ & $\bar{m}^{-}$ & $\bar{m}$ & $\bar{m}^{+}$ \\
0.77 & 0.85 & 0.93 & 1.46 & 2.19 & 3.27 \\
\hline \multicolumn{7}{c}{} & & & & \\
\multicolumn{2}{l}{ Overall results, } & 45 signals analyzed & & & \\
\hline $\bar{v}^{-}$ & $\bar{v}$ & $\bar{v}^{+}$ & $\bar{m}^{-}$ & $\bar{m}$ & $\bar{m}^{+}$ \\
0.70 & 0.89 & 1.12 & 0.69 & 1.87 & 5.04 \\
\hline
\end{tabular}


For the MDC-HITEN data, the overall mean values of the ratios between the single results of the old analysis and the new analysis show only very low deviation in velocity, which is about a factor 0.99 towards lower velocity, and a deviation in mass of about a factor 1.08 towards higher mass. Since the appreciated accuracy of the analysis overall is about a factor 2 in velocity and a factor 5 in mass, the deviation caused by the compression/decompression is far below the accuracy that can be achieved with the instrument in principle.

Analysis of the MDC-NOZOMI control data shows an analog behavior. Velocity determined from the compressed data sets shifts about a factor 0.89 towards lower impact speed, while particle mass shifts by a factor 1.87 towards higher mass. A small number of mavericks cause most of this large deviation, while all other signals show much lower deviation from the old data. Since the analysis process of the MDC-HITEN and MDC-NOZOMI are similar besides the third channel in the MDC-NOZOMI data, it is most likely that the three-channel based MDC-NOZOMI analysis is much more sensible to small changes in signal shape than the more robust MDC-HITEN analysis. Nevertheless, the deviation of the impact speed is by far within the instruments accuracy. For further analysis and interpretation of the particle properties (orbital elements etc.), only impact speed is required.

It must be emphasized here that the shown values demonstrate the accuracy of the new analysis in comparison to the old analysis. They must not be interpreted as an absolute assessment of the new analysis process in comparison to any known, $100 \%$ reliable data.

The MDC software upgrade was ready for upload by the end of the year 2002, and the decompression routine was already implemented in the MDC analysis software. The assembler code for wavelet transformation and compression takes 555 bytes RAM for the code, plus one 1 kByte buffer segment for computations. The transformation of all three channels of a MDC signal is performed within 510000 clock cycles, which corresponds to $290 \mathrm{~ms}$ computing time.

If NOZOMI had been recovered from the system failure on April 24, 2002, and finally entered Mars orbit with its full functionality, there would have been three options for the analysis of MDC dust data downloaded in compressed format:

1. Leave the analysis process as it is with the only difference that compressed signals are used for the determination of mass and impact speed of the particles. This method was used above to validate the software upgrade. Resulting mass and speed are shown to be within the instrument's accuracy.

2. Determine correction factors for mass and impact speed for every MDC impact type (EC, IC and MLI) to minimize the deviation of the analysis results derived from the compressed signals from the results derived from the original signals, based on the results shown above. This method would give more accurate analysis results with a minimum effort.

3. Perform a complete recalibration of the MDC. This means, compress and decompress all calibration signals that were used for the determination of the parameter sets of the analysis process, and determine new parameter sets from these compressed calibration impact signals. This method would give the highest possible accuracy, but with a high effort. 
For the MDC on NOZOMI, option 2 would have been the method of choice since it offers the best cost-value ratio.

For possible future dust experiments based on the MDC that require their own calibration anyway, option 3 is the method of choice. Integration of the compression and decompression routines into the calibration process, the onboard software and the data analysis process can be easily done during the development of the required software routines. The systematic changes in signal shape that are caused by the compression/decompression process are then integrated into the whole data handling process and do not have any effect on the final analysis results, as long as the required parameters (charges, rise time) can be determined from the resulting signal shapes, which is the case for signals compressed by the Wavelet compression routine discussed in this chapter. 



\section{Mission results}

In this chapter, the scientific results of the MDC mission are presented and discussed. At first, paragraph 6.2 gives a closer look to the instrument's and NOZOMI's flight data, and a brief look at the noise data that was recorded by the MDC. Both the detector limits and the unusual high amount of noise data (compared with the previous mission MDC-HITEN) affect or may affect the final interpretation of the MDC impact data and thus were examined carefully. Finally, interpretation and determination of NOZOMI flight data that is required for further data analysis is described.

Although NOZOMI and the MDC could not fulfill their primary mission goals (see paragraphs 2.3 and 2.3.3), at least the MDC's measurements of cosmic dust in the vicinity of Earth and Moon and later in the interplanetary space yield interesting scientific results. To make allowance for the different environmental conditions, the discussion is separated into two sections. In paragraph 6.3, the results of the mission's first 6 months are discussed where NOZOMI resided in the vicinity of Earth and Moon. In paragraph 6.4, the results of more than 3 years in the interplanetary space are discussed. A detailed description of NOZOMI's mission can be found in paragraph 2.3 .

Analysis of all data presented in the following paragraphs was performed as described in paragraph 3.3.2.

\subsection{Overview}

The detection capabilities of an instrument like the MDC as well as the interpretation of the measured dust data are strongly dependent not only on the instrument's principal properties, but also on the pointing directions of the instrument which in turn is determined by the attitude data of the hosting spacecraft. Very careful examination of the spacecraft's flight data (orbital position, attitude, flight direction) with special respect on the pointing directions of the MDC is required for correct interpretation of the measured dust data, to avoid wrong conclusions from observed patterns in impact data that are not of natural origin but are caused by the spacecraft's and the MDC's pointing direction. NOZOMI flight data and the vast amount of noise data recorded by the MDC are investigated prior to the final analysis of the impact data at the end of this chapter. 


\subsection{Noise signals and flight data interpretation}

For the further interpretation of the MDC impact data, the instrument's characteristics have to be discussed first. Since the measuring range of the MDC electronics (charge amplifiers and $\mathrm{A} / \mathrm{D}$ converters) is limited, every statistical analysis of the impact data must be related to the MDC detection capabilities. The MDC detection capabilities are already discussed in paragraph 3.2.4, but also the huge amount of noise data recorded by the MDC may influence the detection capabilities since every single signal triggered and processed by the MDC electronics causes some instrument dead time, and may therefore affect statistical analysis of dust flow. The effects and characteristics of the recorded noise rate and noise signals are discussed in paragraph 6.2.1. Interpretation of the absolute direction of the identified particles requires an exact determination of the MDC view axis at a given impact time, and a detailed examination of the spacecraft's attitude and thus spin-averaged sensor pointing directions for statistical analysis of particle flight directions. In paragraph 6.2.2, the calculation of the MDC view axis and the absolute particle velocity from the given NOZOMI flight data is described in detail, in paragraph 6.2.3 the NOZOMI flight data are examined in detail. 


\subsubsection{Noise signals and instrument dead time}

Unlike the previous experiment on the mission HITEN, the MDC-NOZOMI recorded a huge amount of noise data that either was discarded onboard by the KO-criteria and can only be recognized by the instrument's rapidly rising noise count $\mathrm{NC}$, or has passed the KO-criteria and can be seen in the downloaded raw data files.

Many of these noise signals are not shaped randomly as it could be expected for noise caused by random electromagnetic background from different possible sources like radiation in space or the satellite's electrical systems. Several nearly identical signal shapes were identified which occur in certain patterns.

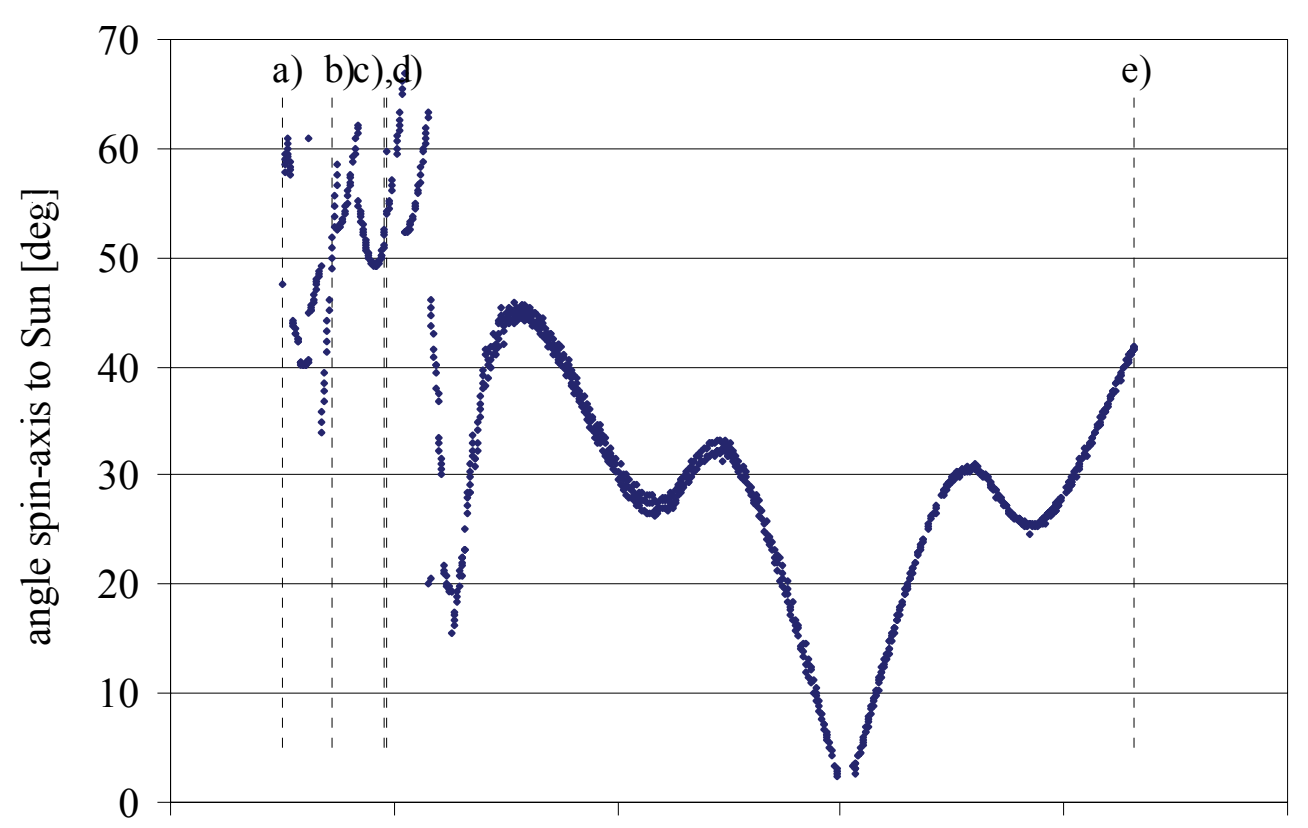

January-98 January-99 January-00 January-01 January-02 January-03

Figure 6-1: Angle of the NOZOMI spin-axis to the Sun over mission time For most of the mission time, the angle between the NOZOMI spin-axis to the Sun was lower than $45^{\circ}$, which was necessary to ensure sufficient power generation by the solar panels. For angles higher than $45^{\circ}$, like in 1998 and early 1999, direct sunlight was falling into the MDC sensor box due to NOZOMI's spatial configuration and may have produced noise signals due to photo-ionization of the inner sensor box surfaces. The double and even triple lines since March 1999 are a result of the frequently (every 3-4 days) conducted attitude maneuvers that were necessary to keep the NOZOMI spin-axis and thus high-gain antenna pointing to Earth.

Every noise signal triggered has to be processed by the MDC electronics as described in paragraph 3.2.2. As shown in Figure 3-3, noise signals triggered and discarded by the KOcriteria cause an instrument dead time of $65 \mathrm{~ms}$, while signals passing the KO-criteria cause an even higher instrument dead time of $120 \mathrm{~ms}$. During periods of high noise rates, the total 
instrument dead time must eventually be considered for further quantitative analysis and determination of dust flux.

Several periods of very high noise rates (steep rise of the noise count NC) were identified during the whole mission, as shown in Table 6-1. The origin of these sporadic interferences remains unclear. As one possible source, solar eruptions were taken into account since such events cause a very high proton flux and therefore may be recorded by the MDC as a series of noise events (as mentioned in paragraph 2.3.2, a solar eruption and the following very high proton flux was made responsible for the fatal NOZOMI system failure). In addition, the time span of the MDC high noise periods of several hours to a couple of days meets the usual time span of solar events. To verify this assumption, coincidences between scientifically proven solar eruptions observed by the SOHO spacecraft and periods of high noise recorded by the MDC were examined. This only makes sense for orbital configurations during which SOHO and NOZOMI are located within the same Sun centered solid angle interval, which is given e.g. during NOZOMI's first six months mission time where NOZOMI was in Earth orbit. SOHO is located around the Lagrange point between Earth and Sun. During this time span, none one of the five identified solar events observed by SOHO could be associated clearly to a certain period of high noise recorded by the MDC. Therefore, solar events must be excluded as a possible source of high noise recorded by the MDC. Other possible sources of high noise rates like the NOZOMI electrics and electronics itself were not examined due to missing information.

Table 6-1: Detector dead time

\begin{tabular}{cccc} 
Period & dNC & dEC & $\begin{array}{c}\text { max. / min. / av. } \\
\text { dead time [\%] }\end{array}$ \\
\hline $02 / 06 / 99-14 / 06 / 99$ & 83000 & 2156 & $5.6 / 0.7 / 3.2$ \\
$08 / 11 / 99-28 / 11 / 99$ & 572000 & 29405 & $14.5 / 1.3 / 5.3$ \\
$22 / 08 / 00-28 / 11 / 00$ & 727000 & 23562 & $7.1 / 0.3 / 2.7$ \\
$16 / 08 / 01$ & 9000 & 105 & $1.7 / 1.7 / 1.7$ \\
\hline
\end{tabular}

Nevertheless, the total detector dead time caused by the high noise rates was determined for the most significant high noise periods, for details see Hübner [Hüb2003]. The results of this analysis are shown in Table 6-1. The highest determined detector dead time during the whole mission is $14.5 \%$ and occurs only once on November 8, 1999 for a period of about six hours, while during the other analyzed high noise periods the detector dead time varies between less than $1 \%$ and nearly $6 \%$. For the latter analysis of dust flux, the detector dead time needs not to be considered in any way since the overall dead time was much lower than $1 \%$. 

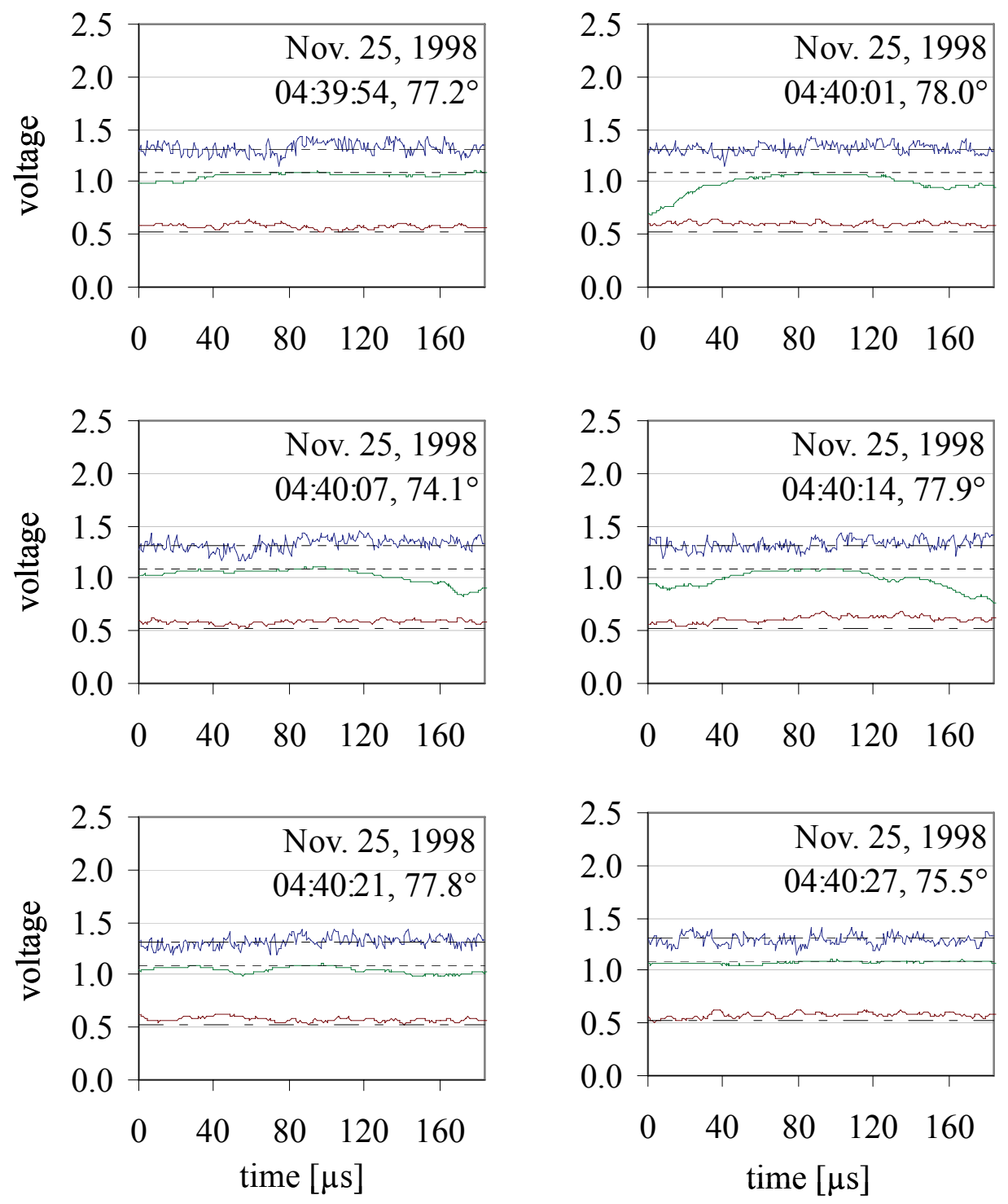

Figure 6-2: A series of noise signals with period $\approx$ NOZOMI spin-period Signal series like the one shown here with nearly equal signal shape and time intervals of one or more NOZOMI spin periods were identified throughout the NOZOMI mission time. Mean interval between the signals shown is $6.67 \mathrm{sec}$, NOZOMI spin-rate on Nov. 25, 1998 was $9.16 \mathrm{rpm}$ or $6.55 \mathrm{sec} /$ revolution, respectively, as derived from NOZOMI attitude data. Signal identifiers from top left to bottom right are 98112506-3 to 98112506-8. For all these signals, housekeeping data showed good experiment condition.

A first look at the noise data showed that many groups of noise signals with similar shape and at time intervals of about the NOZOMI spin rate can be identified, see Figure 6-2 for an example. Because of this observation, the spin dependency of the noise signals was investigated further.

In a first approach, all 20538 signals recorded by the MDC during the mission were sorted into one-degree spin angle intervals. The result is shown in Figure 6-4 (top chart). As can be seen, the noise signals are not equally distributed over the spin angle. Around spin angles of 
$0^{\circ}$ to $60^{\circ}$, a higher number of noise signals were recorded than around spin angles of $180^{\circ}$ to $240^{\circ}$. This behavior can ad hoc be explained as being caused by an interference of the MDC experiment with radiation or particle streams originating from the Sun, e.g. sunlight, since at a spin angle of $0^{\circ}$ the NOZOMI side panel \#8 where the MDC is mounted points towards the sun for most orbital configurations. From previous missions, it is known that direct sunlight causes noise events when entering the MDC sensor box. In contrast, the strong peak at about $128^{\circ}$ to $130^{\circ}$ degrees cannot be explained in this manner since at those spin angles the MDC experiment is protected from sunlight by the NOZOMI body.

To separate spin-dependent noise signals from spin-independent noise signals, a simple filter algorithm was applied on the whole set of noise signals that identifies all signals as spindependent that have temporal neighbors (up to the fifth neighbor) with time differences of whole-numbered multiples (up to five) of the actual NOZOMI spin rate within a $5^{\circ}$ interval. The result is shown in Figure 6-4 (middle and bottom chart). By this simple analysis, about a third of all noise signals are identified as being spin-dependent. While the principal distribution of the remaining spin-independent noise signals is constant over the whole angular range, the peak at $128^{\circ}$ to $130^{\circ}$ can not completely separated from the spinindependent noise data by this simple filtering method.

In Figure 6-3, the noise signals are again sorted into intervals of $1^{\circ}$ width in spin-angle, but were separated into time spans of half-years previously. This analysis shows that the peak at $128^{\circ}$ to $130^{\circ}$ is not present during the whole mission time but vanishes during the periods $\mathrm{I} / 2000, \mathrm{I} / 2001$ and II/2001.

With the available information, it can only be assumed that the peak originates from some reflections of sunlight on parts of the NOZOMI body. A detailed analysis would require a three dimensional model of the completely integrated NOZOMI body with unfolded solar panels, and a simulation of the reflections that can occur with respect to the real NOZOMI attitude. This was not performed for the MDC-NOZOMI mission since no detailed model including surface characteristics of the MLI was available for the NOZOMI body. Thus, the origin of the noise peak at $128^{\circ}$ to $130^{\circ}$ still remains unclear. 

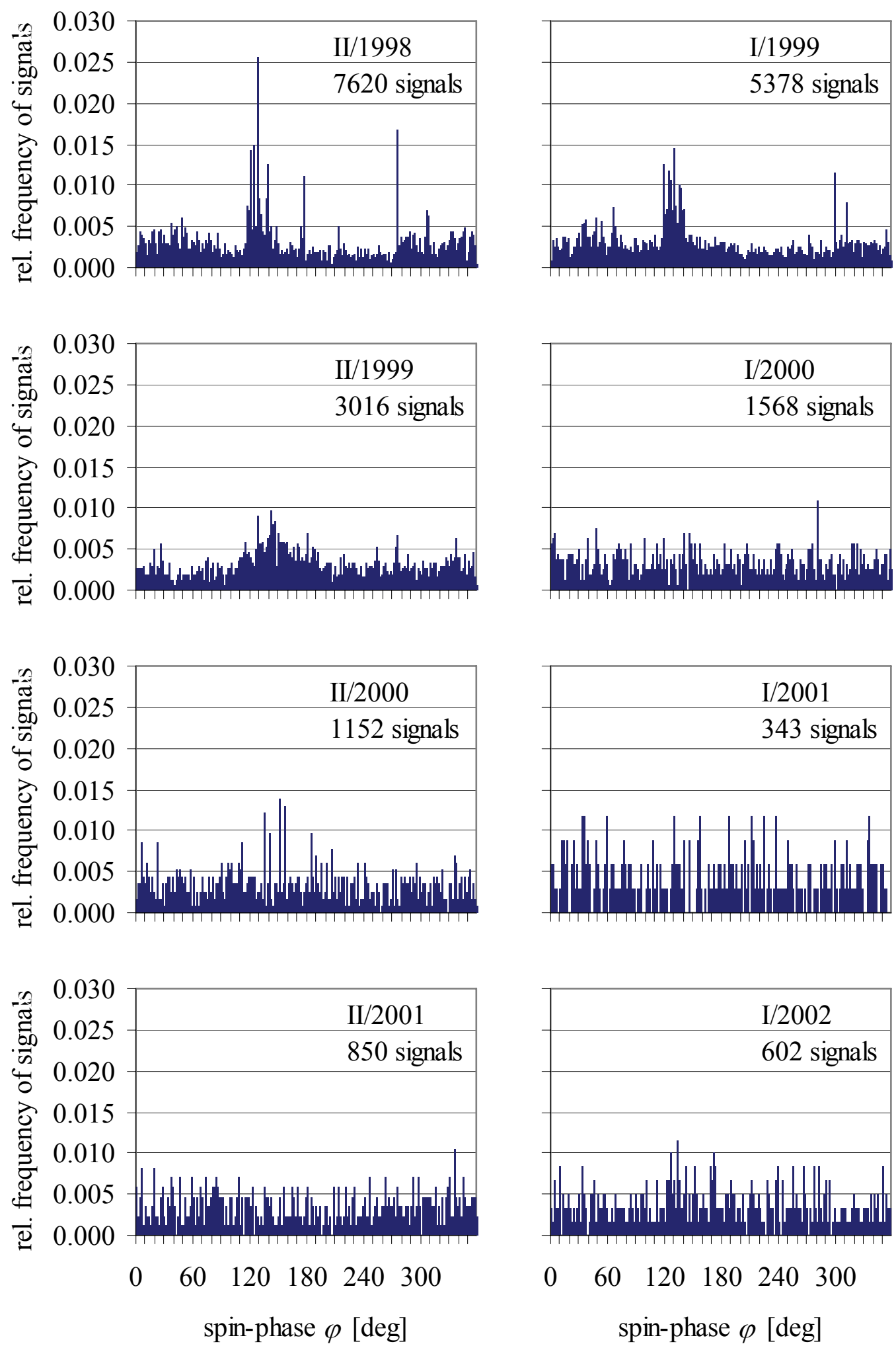

Figure 6-3: Distribution of noise signals over spin-phase, half years Relative frequency of noise signals over spin-angle, separated into time spans of half years. During 5 of these time spans, a peak of noise data can be identified at $128^{\circ}$ to $130^{\circ}$ spin angle. During time spans that lack this peak, the overall distribution of the noise signals is nearly constant. 

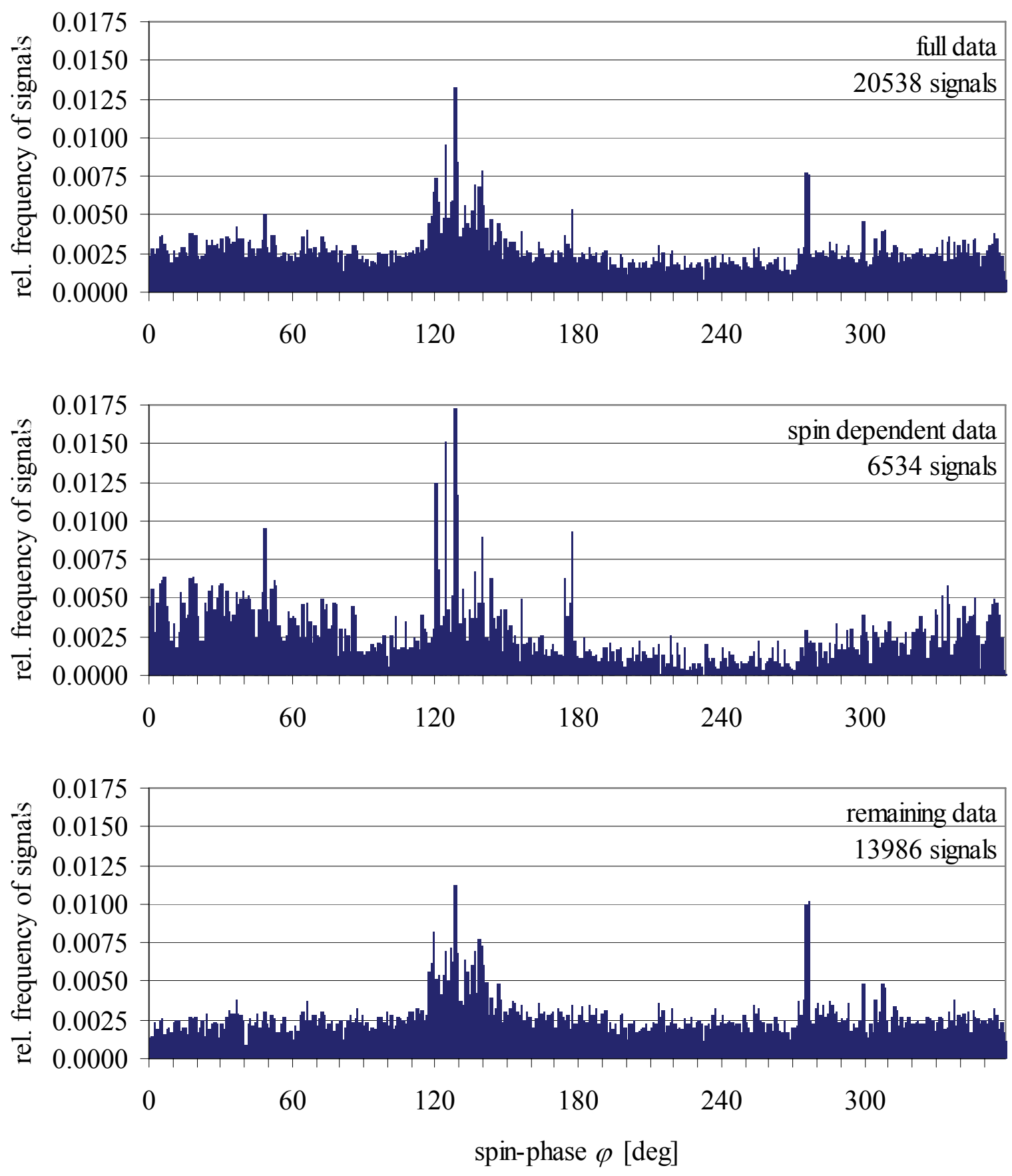

Figure 6-4: Distribution of noise signals over spin-phase, spin-dependency The top chart was created from the whole set of noise data, while the middle and bottom chart represent the spin-dependent (one third) and spin-independent (two third) component of the noise data as identified by a simple filtering algorithm (see text for details)

To get a deeper understanding of the patterns and the origin of the noise signals recorded by the MDC, a hierarchical cluster analysis was performed on the MDC noise signals. Cluster analysis is a powerful standard method to identify groups (or clusters, respectively) of data sets that belong together due to certain similarities in a large amount of data sets, see Deichsel 
\& Trampisch [Dei1985] for more information. While in a two- or three-dimensional space clusters can easily be identified by a human experimenter (see also Figure 6-5), this is impossible for higher dimensions due to the limits of visualization options and human imagination, thus an analytical method is required. As an example, in archaeological science cluster analysis is used to identify vessels or shards from archaeological excavation sites by associating them to clusters of already identified vessels whose cultural, temporal and regional origin is known (the author was involved in a research project on this subject that was carried out at the nuclear research reactor of the Technische Universität München in Garching, Germany). As specific properties of the shards, the chemical composition of the material was used, which was determined non-destructive by neutron activation analysis. The rates of selected chemical elements in the chemical composition of a certain shard were used to describe the properties of the shard and can be seen as a multi-dimensional state vector of the certain shard.

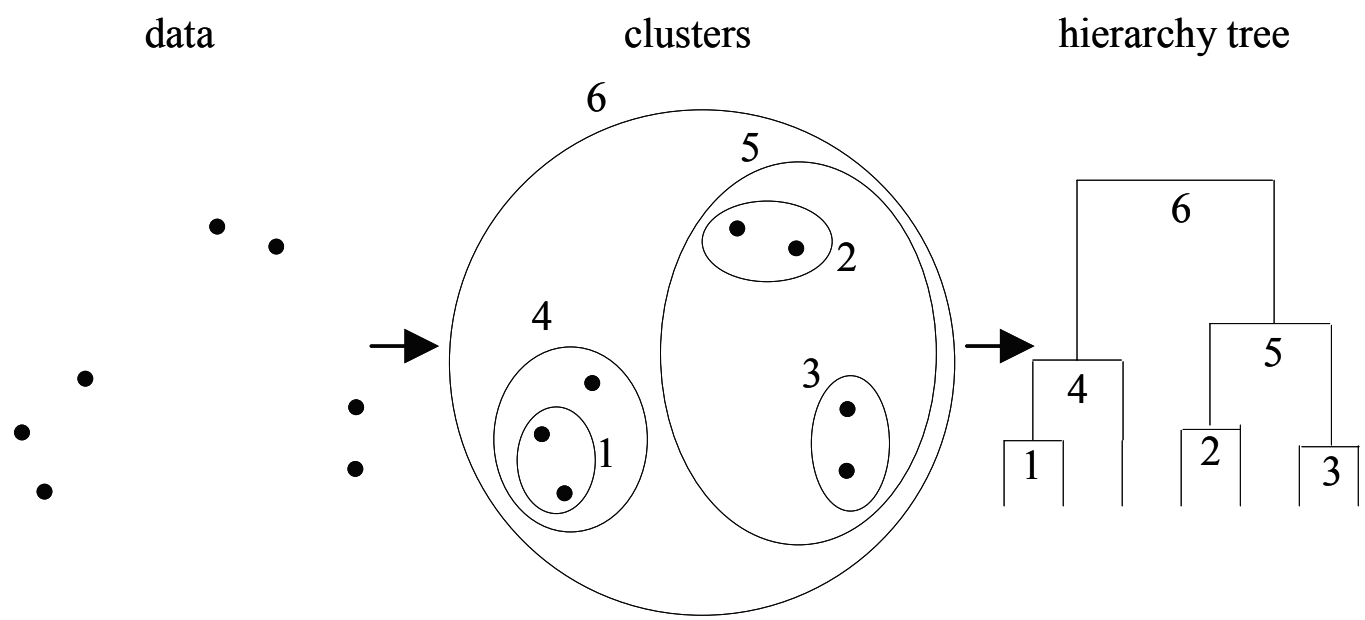

Figure 6-5: 2-Dimensional illustration of the principles of cluster analysis A two-dimensional set of data points (left) is successively combined to clusters of data points (middle) that are ordered in a certain hierarchy (right). The result depends on the distance metric used for calculating the distance of two data points or data clusters, respectively, and the linkage method that defines what coordinate is used for a cluster containing more than one data point, see text for details.

In case of the MDC noise signals, a set of 55 values that were determined from the signal shape is used to describe a certain signal's properties. These 55 values can be interpreted as the state vectors of the signals in a 55-dimensional space. Like in any space of any dimension, a metric can be defined that enables the calculation of distances between certain coordinate points in that space, see below for description of some metrics used in cluster analysis. Also, scalar products (but not vectorial products which are defined in 3-dimensional space only) can be defined, that are calculated similar to the 3-dimensional scalar product by

$$
\mathbf{a} \cdot \mathbf{b}=\sum_{i=1}^{N} a_{i} \cdot b_{i}
$$

whereas $N$ is the dimension of the coordinate system. 
Remark: In quantum mechanics, even infinite-dimensional state vectors in the infinite dimensional Hilbert space are used to describe the state of a physical particle or system, the scalar product is then given by

$$
\langle a \mid b\rangle=\int_{x=-\infty}^{\infty} a(x) \cdot b(x) d x .
$$

For cluster analysis, the distance of the coordinates or state vectors is required and can be calculated using different metrics.
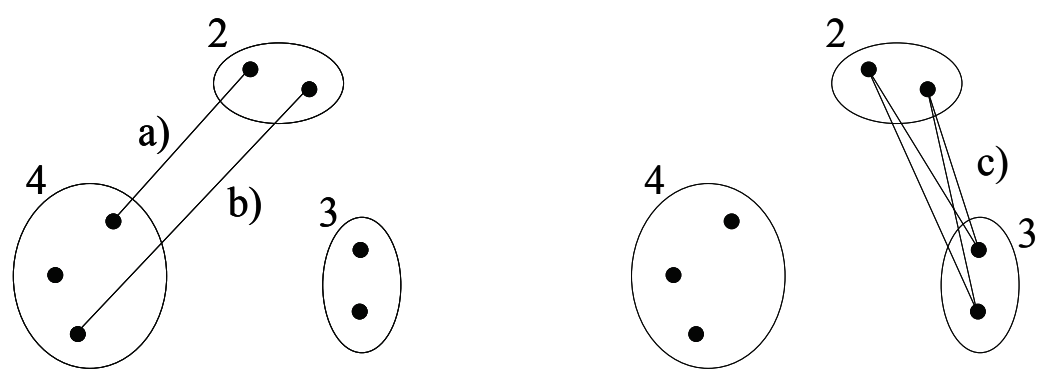

Figure 6-6: 2-Dimensional illustration of linkage methods

Three different linkage methods are available for the distance calculation of non-single clusters. a) Single linkage, the distance between the clusters \#2 and \#4 is the minimum distance of two data points. b) Complete linkage, the distance between the clusters \#2 and \#4 is the maximum distance of two data points. c) Average linkage, the distance between the clusters \#2 and \#4 is the mean distance of all data points.

For a given number $M$ of data sets, a distance matrix of size $M \times M$ is calculated that contains the distances of each data set to every other data set. The two data sets closest to each other are then linked together to give a new data set that replaces the two original data sets. Different methods are available to determine the new coordinates of such a merged data set (cluster), see below. Then, the distances of this new data set to the now remaining $M-2$ data sets are calculated with respect to the metric and the coordinate determination method. These steps are iterated until there is only one top data set (cluster) left that contains all smaller data sets (clusters) down to the original single data sets. This linkage builds a hierarchical tree that is then analyzed to identify a reasonable number of clusters of reasonable size, the decision of what is reasonable number and size of the clusters must be made with respect to the given problem.

The cluster analysis described in the following was performed using the MATLAB ${ }^{\circledR}$ statistics toolbox. First, a cluster analysis was performed on a subset of all MDC noise data (all signals contained in the telemetry data files that were downloaded in the first six months of the year 2000) to evaluate the best distance metric and linkage method (coordinate determination method for non-single clusters for the distance calculation) for the given problem. All 55 values of the noise data were rescaled to give a absolute sum of 1 for each row in the full noise data set (this was necessary since the scale of the different values ranges from magnitudes of $\approx 10^{-16}$ for charge values and of $\approx 1000$ for integrated values). From the available and established metrics and methods, three standard metrics (Euclidean, Minkowski and Chebychev) and three standard linkage methods (Single Linkage, Average Linkage and 
Complete Linkage, see Figure 6-6) were chosen for evaluation. Also, for testing the significance of the cluster analysis result, a control data set of same size and dimension like the evaluation noise data set, but containing random data, was created and undertaken the same cluster analysis.

The distance between two coordinate points is calculated in the three different metrics by

Euclidean distance: $\quad d_{i j}=\sqrt{\left(x_{i}^{1}-x_{j}^{1}\right)^{2}+\left(x_{i}^{2}-x_{j}^{2}\right)^{2}+\ldots+\left(x_{i}^{N}-x_{j}^{N}\right)^{2}}$,

Minkowski distance:

$$
d_{i j}=\left[\sum_{k=1}^{N}\left|x_{i}^{k}-x_{j}^{k}\right|^{p}\right]^{\frac{1}{p}},
$$

Chebychev distance: $\quad d_{i j}=\max \left(\left|x_{i}^{1}-x_{j}^{1}\right|,\left|x_{i}^{2}-x_{j}^{2}\right|, \ldots,\left|x_{i}^{N}-x_{j}^{N}\right|\right)$,

whereas $N$ is the dimension of the coordinate system. In fact, the Euclidean distance is a special case of the Minkowski distance with $p=2$, and the Chebychev distance is a special case of the Minkowski distance with $p \rightarrow \propto(p=1$ would give the so called City Block distance). In the following, $p=3$ is used as exponent for the Minkowski distance.

The linkage methods that determines which cluster coordinates are used for later distance calculations are defined as

- Single Linkage: The distance between two non-singular clusters is the minimum of all distances between the members of the two clusters.

- Average Linkage: The distance between two non-singular clusters is the average of all distances between the members of the two clusters.

- Complete Linkage: The distance between two non-singular clusters is the maximum of all distances between the members of the two clusters.

For singular clusters (clusters that contain only one data set), the three linkage methods become identical. Three metrics and three linkage methods give nine different cluster analyses that need to be evaluated. The results are shown in Figure 6-7 and Table 6-2.

There were three steps from the source data set to the final clustering result. First, the distance matrix has to be calculated by the MATLAB ${ }^{\circledR}$ command

Dist=pdist (SpecVal_norm, 'euclid');

whereas the $M \times 55$ matrix variable SpecVal_norm contains the scaled source data. Next, the leaf nodes (singular clusters) have to be connected to higher nodes to build the hierarchy tree by the command

Link=linkage (Dist, ' complete') ; 
Finally, the desired number of clusters is created by going down the hierarchy tree from the single top node until the maximum cluster number is reached, this is performed by the command

Clust=cluster (Link, 'maxclust', 100);

whereas the maximum number of clusters to be created is set to 100 in this example. Now, the vector variable Clust contains the cluster number each original signal was assigned to in the order of the original data set.
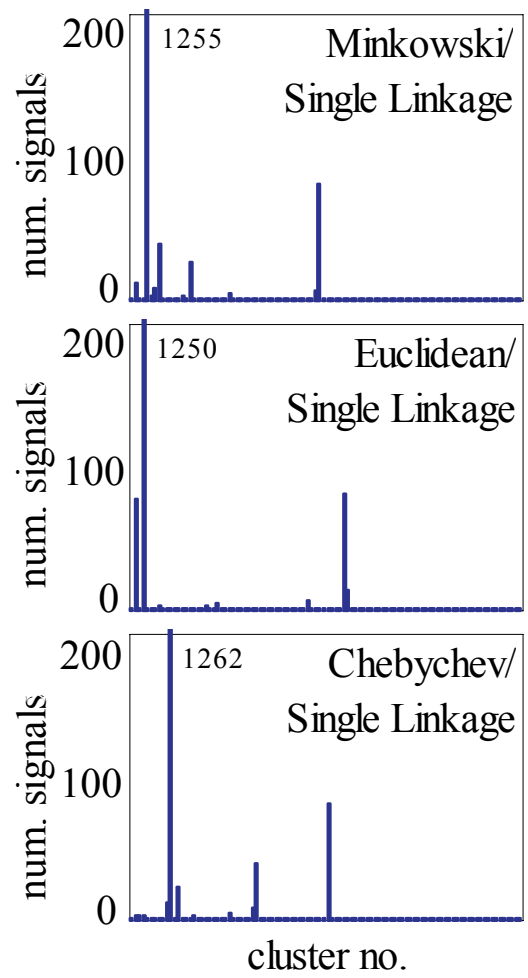
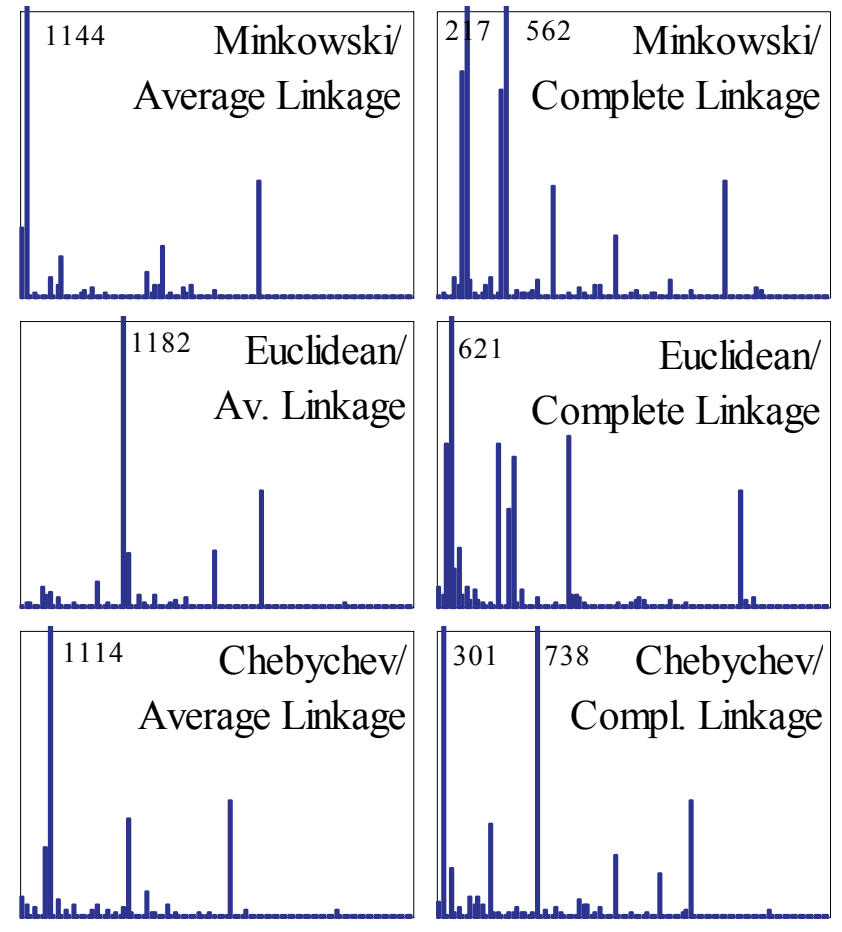

cluster no.

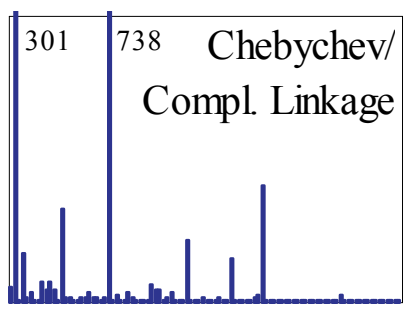

cluster no.

Figure 6-7: Results of the evaluation of metric and linkage method

For the given problem, the best choice of distance metric and linkage method is a combination that computes a large number of clusters that contain a large number of signals each.

Table 6-2: Results of the evaluation of metric and linkage method

\begin{tabular}{cccc} 
Metric $\backslash$ Linkage & Single Linkage & Average Linkage & Complete Linkage \\
\hline Euclidean Dist. & $6 / 1438$ & $13 / 1433$ & $27 / 1449$ \\
Minkowski Dist. & $8 / 1440$ & $15 / 1438$ & $23 / 1436$ \\
Chebychev Dist. & $7 / 1435$ & $18 / 1439$ & $22 / 1432$
\end{tabular}

values given as: number of clusters containing $\geq 5$ signals / number of signals in these clusters total number of signals: 1556

Reasonable cluster size for the given problem is about 5 signals per cluster to several hundred signals per cluster as can be assumed by manual investigation of MDC noise data. A 
reasonable number of identified clusters of the given reasonable size can be assumed to be 20 to 30 , since by (time consuming and unreliable) manual analysis, 16 clusters were identified in the noise data recorded during first half year of the mission, see Hübner [Hüb2003] (collegiate thesis work on the MDC noise data). It can be assumed that automated cluster analysis will identify some more clusters that may have been overseen during manual analysis.

As can be seen in Figure 6-7 and Table 6-2, the combination of Euclidean metric and Complete Linkage gives the best fitting cluster analysis result with respect to the mentioned cluster size and cluster number mentioned above. Based on these results and some intuition (which, as in life, may not give the best choice but at least a good one), this combination was used in the following for a cluster analysis on the complete MDC noise data.

The significance of the cluster analysis can be seen in Figure 6-8 and Figure 6-9 on the following pages. Both, the identified clusters and the hierarchy tree derived from MDC noise data differ significantly from those derived from the random control data set. 

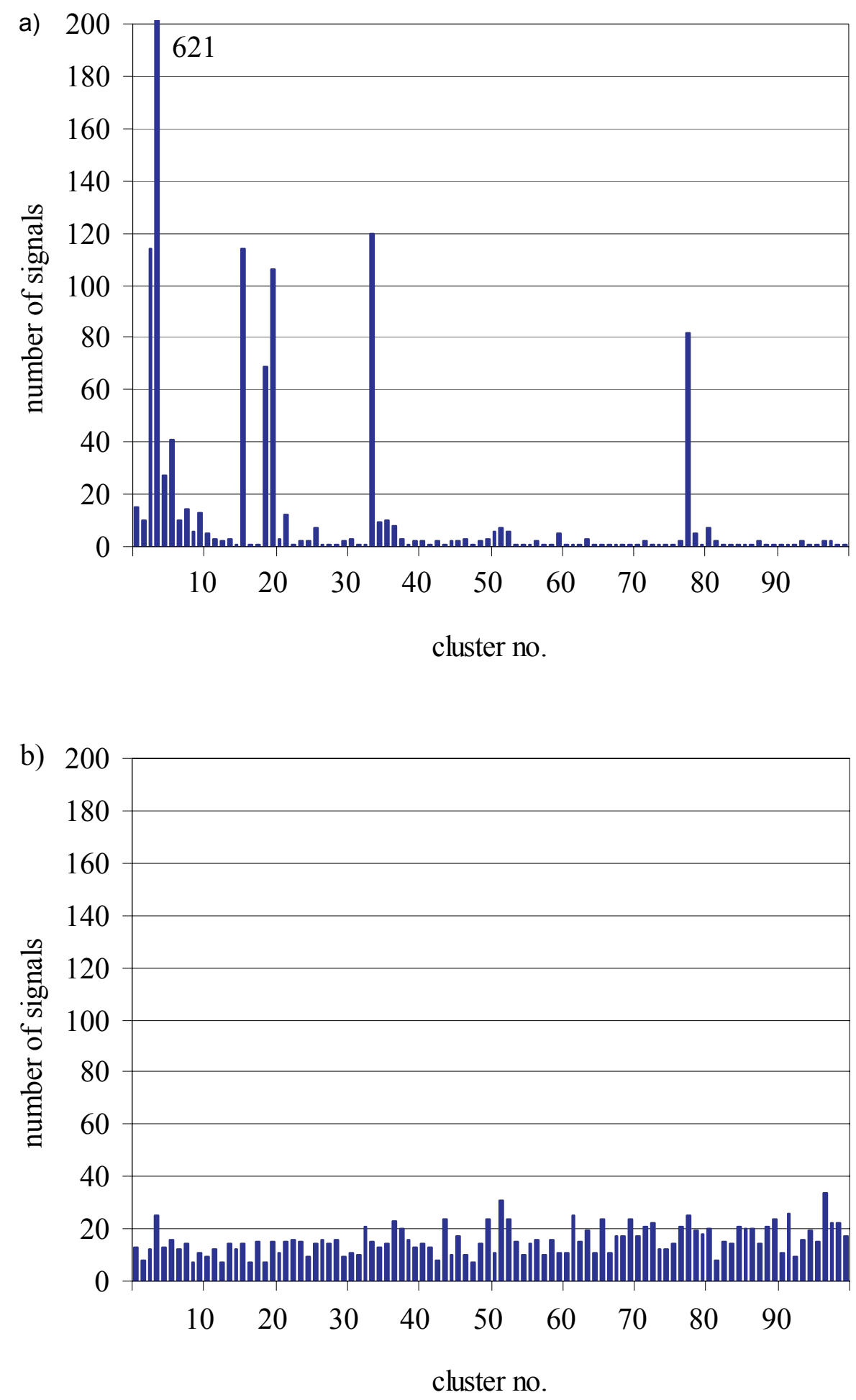

Figure 6-8: Cluster analysis result of MDC eval. noise data and control data While the random control data set $b$ ) does not show any pattern, the real data set a) shows a number of well defined clusters. 

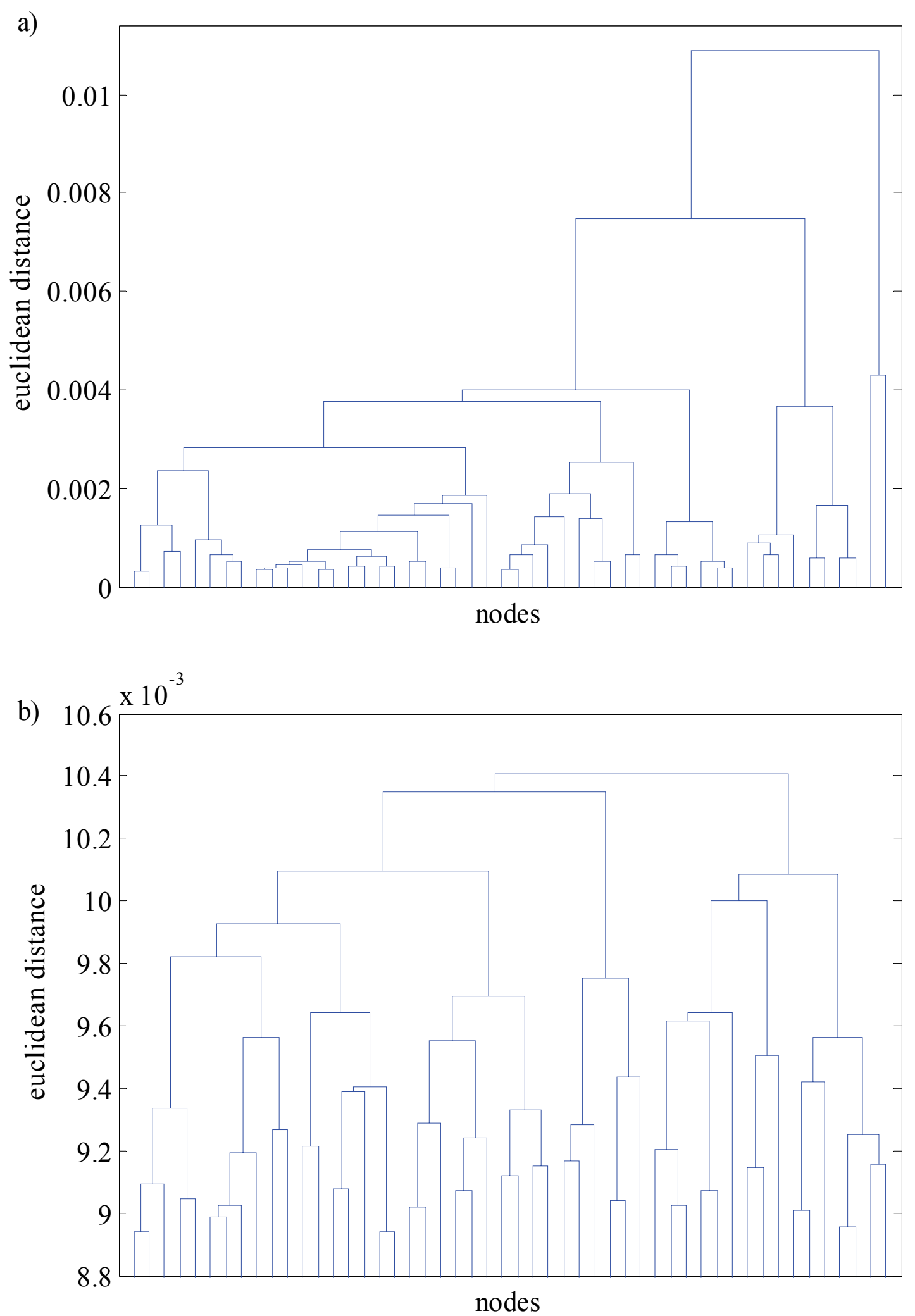

Figure 6-9: Hierarchy tree of MDC evaluation noise data and control data Also, the hierarchy tree of the control data set $b$ ) differs significantly from the hierarchy tree of the real noise data set a). 
Further analysis of the noise signals could give evidence of the origin of the noise signals that are combined into one certain cluster. This was not performed for the NOZOMI mission due to a lack of information about possible sources of noise (e.g. electromagnetic interferences caused by the NOZOMI electrics and electronics itself).

The primary result of the cluster analysis performed on the full MDC data of 20538 signals is shown in Figure 6-10 (due to the large number of signals, this analysis had to be performed on a high performance system at the Leibniz Rechenzentrum LRZ; hierarchy tree visualization could not be created from this large data set with the available computer systems). Most conspicuous result is that more than two third of all signals can be found in one single cluster (no. 42) containing 15683 signals. For further analysis of the data contained in this cluster, the data had to be extracted from the remaining data and clustered separately a second time, since a simple increase of the number of clusters did not give a better separation of the large cluster, but resulted in further disintegration of smaller clusters.

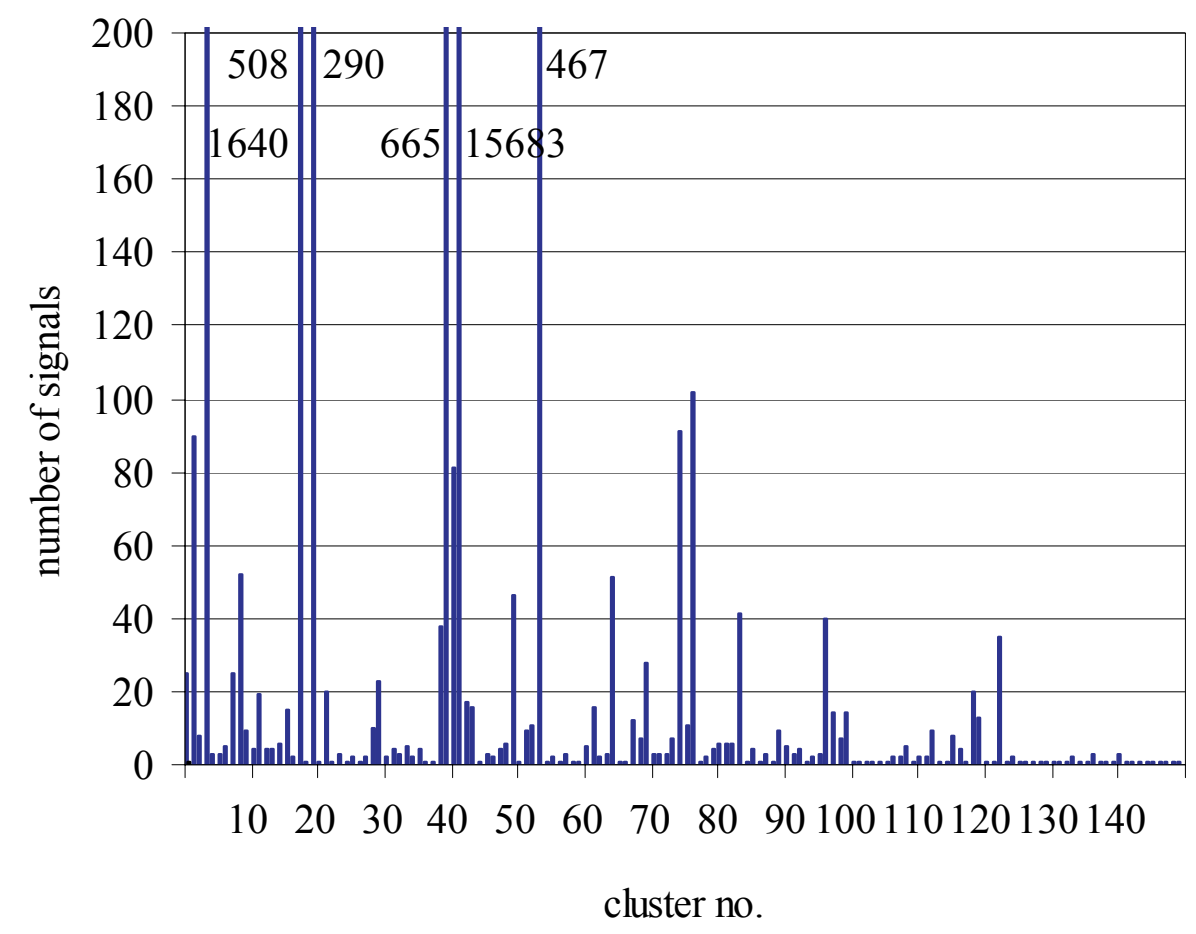

Figure 6-10: Cluster analysis result of the full MDC data

Clustering of the full MDC data gave the result shown above. The numbers next to the bars exceeding a value of 200 indicate the number of signals in the specific cluster. 15 clusters consist of 40 signals ore more. From the 20538 signals, about two third (15683 signals) are sorted into one single cluster. The data in this large cluster was extracted from the full data and clustered separately.

In Table 6-3, the identified impact signals during the year 1998 are crosschecked against the size of the clusters they were assigned to. Since a real impact signal is a result of a single, individual impact event, it can be assumed that real impact signals will be most likely found in clusters containing only a few signals. Most impact signals are indeed assigned to clusters 
containing 12 signals or less, from the eight remaining impact signals, three are assigned to the large cluster no. 42. In clusters no. 86 and 73, all signals are real impact signals.

Table 6-3: Clustering results, real impact signals

\begin{tabular}{lrr}
\hline \multicolumn{1}{c}{ Imp. No. } & Cluster No. & $\Sigma$ signals \\
\hline $98071101-26$ & 49 & 6 \\
$98071601-3$ & 40 & 665 \\
$98073001-5$ & 47 & 2 \\
$98073101-29$ & 86 & 4 \\
$98080701-3$ & 50 & 46 \\
$98082201-8$ & 68 & 12 \\
$98090501-14$ & 68 & 12 \\
$98092201-9$ & 73 & 3 \\
$98092201-23$ & 73 & 3 \\
$98101401-21$ & 113 & 9 \\
$98102701-37$ & 50 & 46 \\
$98110901-17$ & 42 & 15683 \\
$98110901-29$ & 73 & 3 \\
$98111001-1$ & 42 & 15683 \\
$98111701-8$ & 48 & 4 \\
$98111801-1$ & 125 & 2 \\
$98111801-6$ & 86 & 4 \\
$98112101-6$ & 86 & 4 \\
$98120201-17$ & 42 & 15683 \\
$98120301-9$ & 113 & 9 \\
$98121801-35$ & 86 & 4 \\
\hline
\end{tabular}

As an example, cluster no. 2 was investigated further. Table 6-4 shows an excerpt of the signals assigned to cluster no. 2. Although this cluster contains signals from the whole mission time, 73 out of 90 signals in this cluster are found in series of more than four signals that occur within a few hours. Altogether, eight of such series were identified in cluster no. 2, three of them are shown in Table 6-4. Except of signal no. 99110404-44, all signals were assigned quality keys from 90 to 96 . The spin angle of the signals shows a quite mysterious behavior within the series of signals, a nearly constant rise of the signal's spin angle can be observed from the first signal of a series to the last. Until now, this behavior could not be traced back to any known properties of the spacecraft/MDC system or its orbital attributes. In addition, no artifacts from data analysis could be identified that would explain this phenomenon. 
Table 6-4: Signals assigned to cluster no. 2, excerpt

\begin{tabular}{|c|c|c|c|c|c|}
\hline Imp. No. & Cluster No. & Date & Time & Quality & Spin Angle \\
\hline$\ldots$ & $\ldots$ & & & $\ldots$ & $\ldots$ \\
\hline $98112502-35$ & 2 & 25-Nov-98 & 04:09:31 & 95 & 8.8 \\
\hline $98112502-38$ & 2 & 25-Nov-98 & 04:09:38 & 93 & 15.1 \\
\hline $98112502-39$ & 2 & 25-Nov-98 & 04:09:44 & 93 & 6.5 \\
\hline $98112505-9$ & 2 & 25-Nov-98 & $04: 30: 24$ & 92 & 51.2 \\
\hline $98112505-24$ & 2 & 25-Nov-98 & $04: 31: 16$ & 90 & 59.1 \\
\hline $98112505-26$ & 2 & 25-Nov-98 & $04: 31: 23$ & 91 & 60.5 \\
\hline $98112505-28$ & 2 & 25-Nov-98 & $04: 31: 30$ & 93 & 60.6 \\
\hline $98112507-41$ & 2 & 25-Nov-98 & $04: 54: 13$ & 90 & 110 \\
\hline$\ldots$ & $\ldots$ & $\ldots$ & $\ldots$ & $\ldots$ & $\ldots$ \\
\hline $98120203-4$ & 2 & 2-Dec-98 & 05:06:07 & 92 & 25.1 \\
\hline 98120203-18 & 2 & 2-Dec-98 & $05: 06: 53$ & 91 & 26.9 \\
\hline $98120206-22$ & 2 & 2-Dec-98 & $05: 23: 17$ & 94 & 53.8 \\
\hline $98120206-27$ & 2 & 2-Dec-98 & $05: 23: 36$ & 92 & 54 \\
\hline $98120207-21$ & 2 & 2-Dec-98 & $05: 28: 31$ & 92 & 65.1 \\
\hline $98120210-4$ & 2 & 2-Dec-98 & $05: 53: 14$ & 90 & 139.5 \\
\hline $98120210-9$ & 2 & 2-Dec-98 & $05: 53: 33$ & 93 & 130.5 \\
\hline $98120212-12$ & 2 & 2-Dec-98 & $06: 27: 59$ & 91 & 243.9 \\
\hline $98120212-33$ & 2 & 2-Dec-98 & $06: 31: 03$ & 92 & 262.5 \\
\hline$\ldots$ & $\ldots$ & $\ldots$ & $\ldots$ & $\ldots$ & $\ldots$ \\
\hline $99110402-3$ & 2 & 4-Nov-99 & $07: 24: 12$ & 92 & 4.6 \\
\hline $99110402-10$ & 2 & 4-Nov-99 & $07: 24: 43$ & 91 & 0.3 \\
\hline $99110402-5$ & 2 & 4-Nov-99 & 07:43:09 & 91 & 91.2 \\
\hline 99110404-27 & 2 & 4-Nov-99 & 08:54:08 & 96 & 58.6 \\
\hline 99110404-36 & 2 & 4-Nov-99 & $08: 56: 15$ & 92 & 80.3 \\
\hline 99110404-40 & 2 & 4-Nov-99 & $08: 56: 31$ & 95 & 80.2 \\
\hline 99110404-44 & 2 & 4-Nov-99 & $08: 56: 47$ & 105 & 78.9 \\
\hline $99110404-45$ & 2 & 4-Nov-99 & $08: 56: 54$ & 90 & 74.8 \\
\hline $99110404-51$ & 2 & 4-Nov-99 & $08: 59: 25$ & 91 & 97.1 \\
\hline $99110405-7$ & 2 & 4-Nov-99 & 09:19:43 & 92 & 249.9 \\
\hline 99110405-19 & 2 & 4-Nov-99 & $09: 21: 34$ & 91 & 253.9 \\
\hline $99110405-21$ & 2 & 4-Nov-99 & 09:21:41 & 91 & 253.5 \\
\hline $99110405-26$ & 2 & 4-Nov-99 & $09: 23: 48$ & 90 & 282.7 \\
\hline $99110405-31$ & 2 & 4-Nov-99 & 09:28:41 & 94 & 323.4 \\
\hline $99110405-35$ & 2 & 4-Nov-99 & $09: 28: 57$ & 91 & 321.9 \\
\hline$\ldots$ & $\ldots$ & $\ldots$ & $\ldots$ & $\ldots$ & $\ldots$ \\
\hline
\end{tabular}

Two signals of each of the three series in Table 6-4 are shown in Figure 6-11. The similar signal shape can be seen easily. Housekeeping information for all these signals showed good experiment condition. 

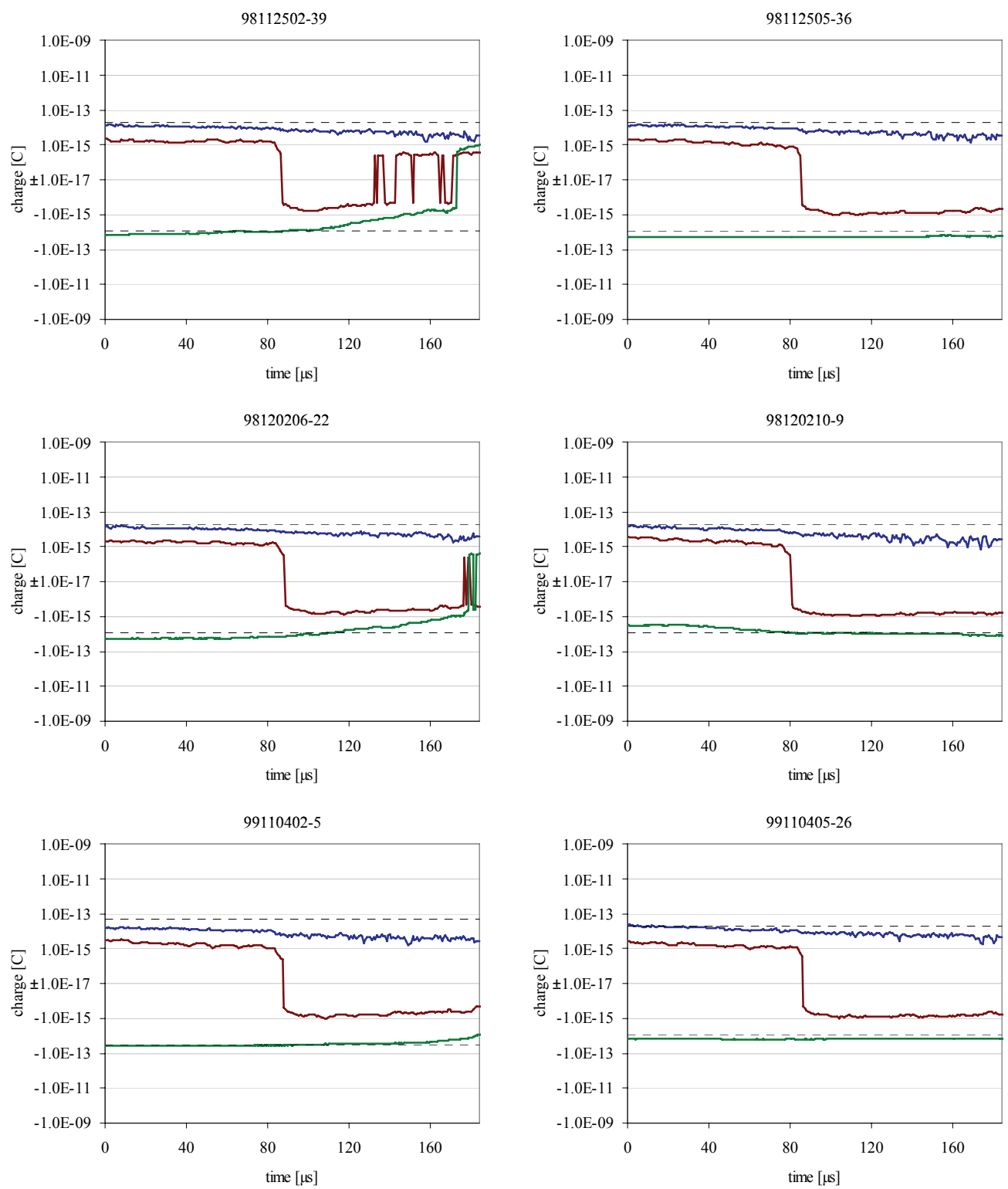

Figure 6-11: Signals assigned to cluster no. 2, signal shape

Signal shapes from cluster no. 2; top, middle and bottom pairs represent signals from the first, second and third series shown in Table 6-4, respectively. From each series, the third and the antepenultimate signal were chosen for this visualization.

Another attempt to classify noise data was made by Sauer [Sau2004] in her collegiate thesis work on the MDC noise data, where the noise signals were identified by a triple code (CIC, $\mathrm{CEC}, \mathrm{CNC}$ ) that describes the shape of the three signal channels and gives a unique identifier for clusters of signals of similar shape. Although this method could be automated by a selfdeveloped proprietary software solution, cluster analysis as a standard method was favored.

In addition, since cluster analysis showed good results when performed on the MDCNOZOMI noise data, clustering of the MDC-BremSat data can be assumed to give important help to understand the data and noise data produced by this MDC mission. As mentioned before, the MDC-BremSat mission suffered from a high amount of noise data that may have 
been caused by atmospheric or charged particle disturbances. See Kellerer [Kel2004] (collegiate thesis work on the MDC-BremSat data) for a review of the MDC-BremSat data. 


\subsubsection{MDC view-axis determination from NOZOMI attitude}

NOZOMI flight data were provided by ISAS/JAXA. Orbital data were given as Cartesian vectors $\mathbf{r}_{\text {nozomi }}=\left(r_{1}, r_{2}, r_{3}\right)^{\mathrm{T}}$ based on the Earth-equatorial reference system (indexed as "eeq" in the following, with the Earth as center body) from launch until January 6, 1999, several data per day at different times, and the Earth-ecliptic reference system (indexed as "eec" in the following, with the Sun as center body) from January 6, 1999, one data per day at midnight (the index "nozomi" is omitted in the following for NOZOMI orbital data). Attitude data were given as tuples $(\lambda, \beta)$ where $\lambda$ is longitude (mathematically positive rotation around the $\mathrm{z}$ axis) and $\beta$ latitude (mathematically negative rotation around the y-axis) of the NOZOMI spin-axis, also one data per day at different times and based on the Earth ecliptic reference system. Since NOZOMI is spin-stabilized, the spin-angle $\varphi$ is not given with the attitude data but, in case of the MDC, is provided by the satellite's AOCS as a parameter of the housekeeping data attached to the scientific data.

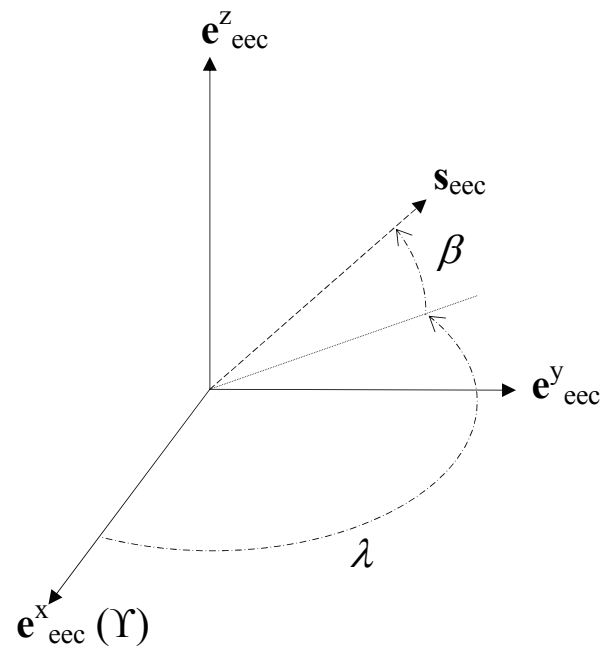

Figure 6-12: Illustration of NOZOMI attitude $(\lambda, \beta)$ and spin-axis vector $\mathrm{s}_{\mathrm{eec}}$ NOZOMI attitude is given as $(\lambda, \beta)$, whereas $\beta$ is measured from the positive $\mathrm{x}$-axis in mathematically negative direction (rotation around $\mathrm{y}$ axis) and $\lambda$ is measured from the positive $\mathrm{x}$-axis in mathematically positive direction (rotation around z-axis). The spin-axis vector $\mathbf{s}_{\mathrm{eec}}$ is calculated from $\lambda, \beta$ as described in equation (6-9).

To allow easier handling of NOZOMI orbital data, all orbital data that were given in the Earth equatorial reference system were transformed to the Earth ecliptic reference system first. This can be performed by a simple rotation of the Earth equatorial coordinates of NOZOMI orbital data by the mean angle of the Earth's spin axis to the ecliptic plane, given as $\varepsilon=23.5^{\circ}$, and vectorial addition of the current Earth orbital position $\mathbf{r}^{\text {Earth }}$, see Figure 6-13. Higher orders of $\varepsilon$ and further corrections that are caused by precession and nutation of the Earth's spin axis, and perturbations by other celestial bodies are not concerned here since the mean value is sufficient for the required accuracy. The transformation from Earth equatorial to Earth ecliptic reference system of orbital coordinates is given by 


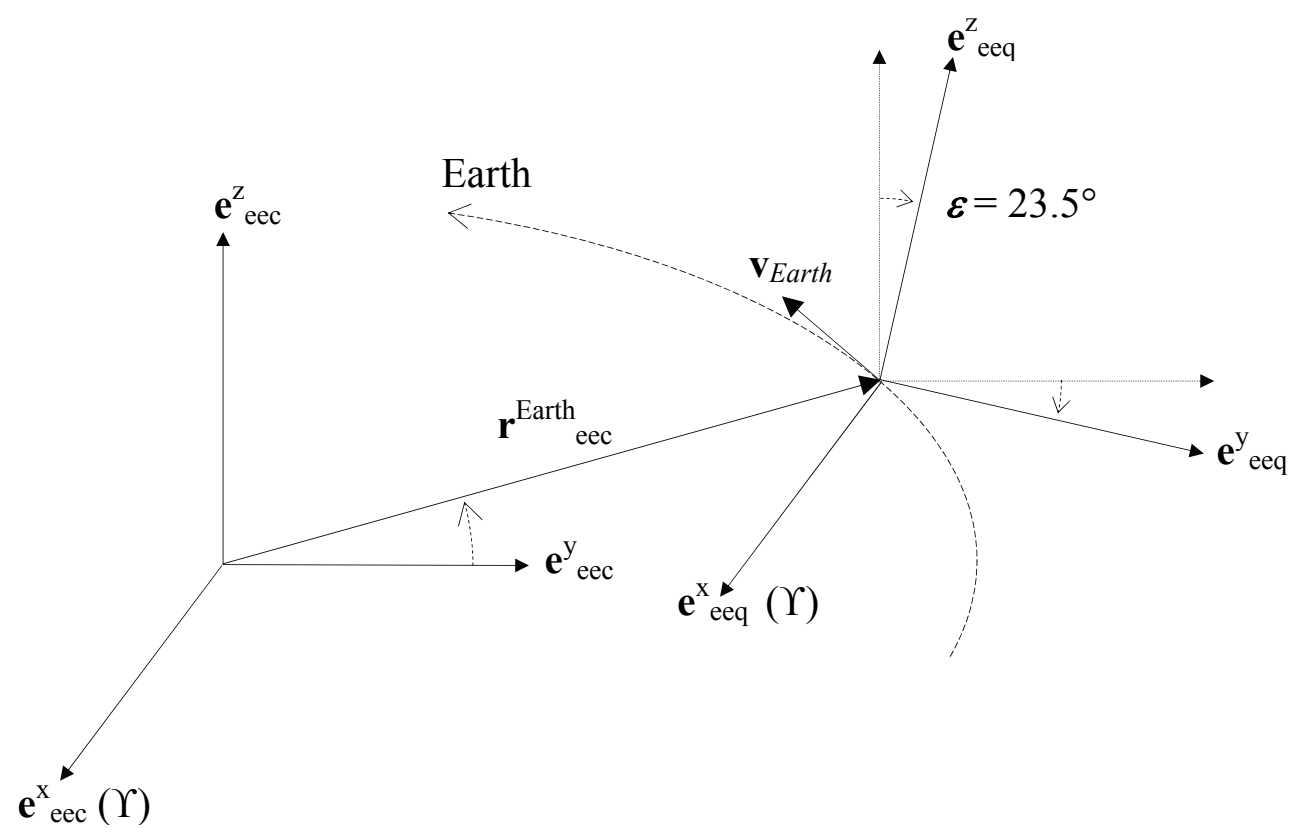

Figure 6-13: The Earth-ecliptic and the Earth-equatorial reference system The Earth-equatorial reference system is tilted by $\varepsilon=23.5^{\circ}$ against the Earth-ecliptic reference system, fixed to the center of the Earth and moving with the Earth. In both reference systems, the $\mathbf{e}^{\mathbf{x}}$-axis is pointing towards the vernal equinox (point of Aries $\Upsilon$ ).

whereas

$$
\mathbf{r}_{\text {eec }}=\mathbf{A}_{\text {eec,eeq }} \mathbf{r}_{\text {eeq }}+\mathbf{r}_{\text {eec }}^{\text {Earth }}
$$

$$
\mathbf{A}_{\text {eec,eeq }}=\left(\begin{array}{ccc}
1 & 0 & 0 \\
0 & \cos \varepsilon & \sin \varepsilon \\
0 & -\sin \varepsilon & \cos \varepsilon
\end{array}\right) .
$$

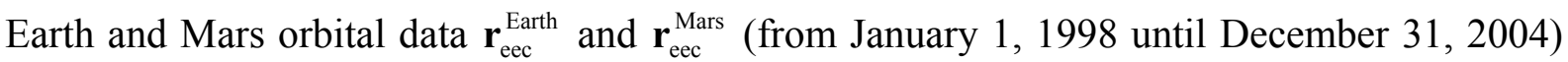
that were needed for this transformation and for latter visualization were derived from the jpleph410 ephemeris data, which were provided by NASA's Johnson Propulsion Laboratory (JPL).

The positions $\mathbf{r}_{\text {eec }}$, directions of the spin-axis $\mathbf{s}_{\mathrm{eec}}$ and velocities $\mathbf{v}_{\mathrm{eec}}$ of NOZOMI at a given impact time $t^{\mathrm{imp}}$ were then determined from the provided flight data by the linear interpolations

$$
\mathbf{r}_{\text {eec }}=\frac{\mathbf{r}_{\mathrm{eec}}^{\mathrm{f}}-\mathbf{r}_{\mathrm{eec}}^{\mathrm{p}}}{t^{\mathrm{f}}-t^{\mathrm{p}}}\left(t^{\mathrm{imp}}-t^{\mathrm{p}}\right)+\mathbf{r}_{\mathrm{eec}}^{\mathrm{p}}=\left(\begin{array}{l}
\overline{-} \\
\frac{r_{1}}{r_{2}} \\
r_{3}
\end{array}\right)=\left(\begin{array}{l}
\frac{r_{1}^{\mathrm{f}}-r_{1}^{\mathrm{p}}}{t_{f}-t_{p}}\left(t^{\mathrm{imp}}-t^{\mathrm{p}}\right)+r_{1}^{\mathrm{p}} \\
\frac{r_{2}^{\mathrm{f}}-r_{2}^{\mathrm{p}}}{t^{\mathrm{f}}-t^{\mathrm{p}}}\left(t^{\mathrm{imp}}-t^{\mathrm{p}}\right)+r_{2}^{\mathrm{p}} \\
\frac{r_{3}^{\mathrm{f}}-r_{3}^{\mathrm{p}}}{t^{\mathrm{f}}-t^{\mathrm{p}}}\left(t^{\mathrm{imp}}-t^{\mathrm{p}}\right)+r_{3}^{\mathrm{p}}
\end{array}\right),
$$




$$
\mathbf{s}_{\mathrm{eec}}=\left(\begin{array}{c}
\overline{s_{1}} \\
\overline{s_{2}} \\
\overline{s_{3}}
\end{array}\right)=\left(\begin{array}{c}
\cos \bar{\lambda} \cos \bar{\beta} \\
\sin \bar{\lambda} \cos \bar{\beta} \\
\sin \bar{\beta}
\end{array}\right), \quad\left(\frac{\bar{\lambda}}{\bar{\beta}}\right)=\left(\begin{array}{c}
\frac{\lambda^{\mathrm{f}}-\lambda^{\mathrm{p}}}{t^{\mathrm{f}}-t^{\mathrm{p}}}\left(t^{\mathrm{imp}}-t^{\mathrm{p}}\right)+\lambda^{\mathrm{p}} \\
\frac{\beta^{\mathrm{f}}-\beta^{\mathrm{p}}}{t^{\mathrm{f}}-t^{\mathrm{p}}}\left(t^{\mathrm{imp}}-t^{\mathrm{p}}\right)+\beta^{\mathrm{p}}
\end{array}\right),
$$

and

$$
\mathbf{v}_{\mathrm{eec}}=\frac{\mathbf{r}_{\mathrm{eec}}^{\mathrm{f}}-\mathbf{r}_{\mathrm{eec}}^{\mathrm{p}}}{t^{\mathrm{f}}-t^{\mathrm{p}}}
$$

whereas the indices $f$ and $p$ define the temporally following and previous orbital and attitude data point, respectively. With respect to the much higher inaccuracy of the MDC data analysis process, this linear interpolation gives sufficient accuracy of the NOZOMI flight data for further data interpretation (e.g. in case of a circular orbit, the maximum deviation from the exact orbital position is $\hat{r} / r=\cos (2 \pi / n)>0.9999$ with $n=500$ data points per orbit, where $\hat{r}$ and $r$ are the interpolated and real orbital radius, respectively). In most cases, the NOZOMI flight data around the impact time of the recorded and analyzed impacts show a low gradient, and linear interpolation will give reliable results. Only some impacts were recorded during or temporally near orbital maneuvers or a swing-by phase, both events lead to large gradients in orbital and attitude data and therefore unreliable interpolation results.

To determine the complete NOZOMI attitude at the given impact time $t^{\text {imp }}$, the directions of the NOZOMI x-axis, and y- or z-axis are needed. A reference system "noz" is defined that is fixed to the NOZOMI body, where the x-axis points in the direction of the NOZOMI spinaxis, and the $y$-axis points from the NOZOMI center of mass in the direction of the side panel \#8 where the SSAS (Spin-type Sun Aspect Sensor) and the MDC are located (this is different from the NOZOMI engineering reference system, where the z-axis points in the direction of the NOZOMI spin-axis). Spin-phase $\varphi=0$ is defined as the attitude when the Sun passes by the SSAS view-plane. The NOZOMI x-axis is given as $\mathbf{x}_{\mathrm{eec}}=\mathbf{s}_{\mathrm{eec}}$, while the direction of the $\mathrm{y}$ axis can be calculated from the orbit position $\mathbf{r}_{\mathrm{eec}}$ and the spin-axis $\mathbf{s}_{\mathrm{eec}}$ for spin phase $\varphi=0$ as

$$
\mathbf{y}_{\mathrm{eec}}=\left[\frac{\left(\mathbf{r}_{\mathrm{eec}} \times \mathbf{s}_{\mathrm{eec}}\right) \times \mathbf{s}_{\mathrm{eec}}}{\left|\mathbf{r}_{\mathrm{eec}}\right|}\right]_{\varphi=0} \text {. }
$$

For the determination of the flight direction of an impacting particle, the view axis of the MDC in the Earth ecliptic reference system must be calculated from the NOZOMI configuration, attitude data and orbital data. It is common sense in dust sciences that the flight direction of an impacting particle is defined as the opposite direction of a dust experiment's view axis. In the MDC instrument's reference system (indexed as "mdc" in the following), the flight direction of a particle is therefore given as $\mathbf{n}_{\text {mdc }}=(-1,0,0)^{\mathrm{T}}{ }_{\mathrm{mdc}}$ if the positive $\mathrm{x}$-axis is defined to be the view axis (the other axes need not to be defined here). First, this must be transformed to the NOZOMI fixed reference system "noz". In this system, the NOZOMI spinaxis is given as $\mathbf{s}_{\mathrm{noz}}=(1,0,0)^{\mathrm{T}}{ }_{\mathrm{noz}}$, and the MDC view axis is rotated by $\gamma=0^{\circ}$ around the NOZOMI x-axis (SSAS and MDC are having the same view axis), and $\delta=135^{\circ}$ around the NOZOMI z-axis. The opposite view direction of the MDC in this system is given by the transformation

$$
\mathbf{n}_{\text {noz }}=\mathbf{A}_{\gamma} \mathbf{A}_{\delta} \mathbf{n}_{\text {mdc }}, \text { or } \mathbf{n}_{\text {noz }}=\mathbf{A}_{\text {noz,mdc }} \mathbf{n}_{\text {mdc }},
$$


whereas $\mathbf{A}_{\text {noz,mdc }}$ is the transformation matrix from the MDC fixed reference system to the NOZOMI fixed reference system.

$$
\begin{gathered}
\mathbf{A}_{\gamma}=\left(\begin{array}{ccc}
1 & 0 & 0 \\
0 & \cos \gamma & \sin \gamma \\
0 & -\sin \gamma & \cos \gamma
\end{array}\right), \mathbf{A}_{\delta}=\left(\begin{array}{ccc}
\cos \delta & \sin \delta & 0 \\
-\sin \delta & \cos \delta & 0 \\
0 & 0 & 1
\end{array}\right), \\
\mathbf{A}_{\text {noz,mdc }}=\mathbf{A}_{\gamma} \mathbf{A}_{\delta}=\left(\begin{array}{ccc}
\cos \delta & \sin \delta & 0 \\
-\sin \delta \cos \gamma & \cos \delta \cos \gamma & \sin \gamma \\
\sin \delta \sin \gamma & -\cos \delta \sin \gamma & \cos \gamma
\end{array}\right) .
\end{gathered}
$$

Now the NOZOMI spin-phase must be considered. The spin-phase of NOZOMI is given as $\varphi=0 \ldots 359^{\circ}$, where $\varphi=0$ is defined as the rotational angle where the SSAS points towards the Sun. The transformation into the rotated reference system "noz $\varphi$ " is given as

$$
\mathbf{n}_{n o z \varphi}=\mathbf{A}_{\varphi} \mathbf{n}_{n o z}, \text { or } \mathbf{n}_{n o z \varphi}=\mathbf{A}_{n o z \varphi, n o z} \mathbf{n}_{n o z},
$$

with the transformation matrix

$$
A_{\text {noz } \varphi \text {, поz }}=\left(\begin{array}{ccc}
1 & 0 & 0 \\
0 & \cos \widetilde{\varphi} & \sin \widetilde{\varphi} \\
0 & -\sin \widetilde{\varphi} & \cos \widetilde{\varphi}
\end{array}\right) .
$$

The angle $\widetilde{\varphi}$ must be calculated from the spin-phase $\varphi$ with respect to the NOZOMI attitude and orbit position, respectively, as described in the following. 


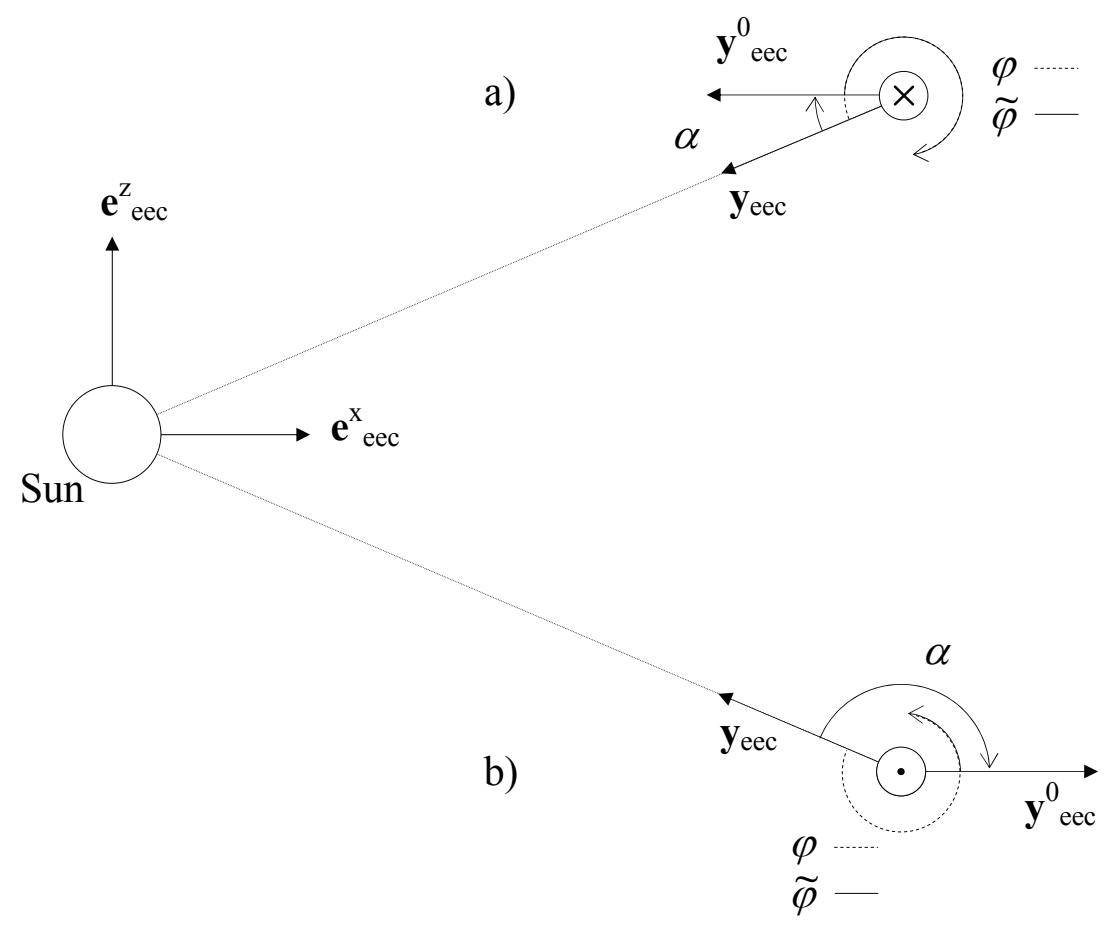

Figure 6-14: Illustration of correction angle $\alpha$ and spin-phase $\varphi$ and $\widetilde{\varphi}$

The determination of the correction angle $\alpha$ and the angles $\varphi$ and $\widetilde{\varphi}$ is shown for two different orbital configurations: a) NOZOMI is located north of the ecliptic plane, with the spin axis parallel to the positive y direction, and b) NOZOMI is located south of the ecliptic plane with the spin-axis parallel to the negative $\mathrm{y}$ direction. While the given spin-phase $\varphi$ is measured from the $\mathrm{y}_{\text {eec }}$ axis, the corrected spin-phase $\widetilde{\varphi}$ is measured from the $\mathbf{y}_{\text {eec }}^{0}$ axis. For configuration a) the angle $\widetilde{\varphi}$ must be calculated by $\widetilde{\varphi}=\varphi-\alpha$, for configuration b) by $\widetilde{\varphi}=\varphi-\left(360^{\circ}-\alpha\right)=\varphi+\alpha$, because the rotation of the axis $\mathrm{y}_{\text {eec }}$ is in mathematically negative direction for a) and in mathematically positive direction in b) as seen from the spin-axis.

The NOZOMI orbital plane is tilted against the Earth-ecliptic by about $\vartheta=2.94^{\circ}$ during most of the interplanetary cruising phase until December 21, 2002, by $\vartheta=6.21^{\circ}$ between the two Earth swing-by's on December 21, 2002 and June 19, 2003 and by $\vartheta=0.06^{\circ}$ after June 19, 2003 (see Figure 2-15). These values were not given by ISAS/JAXA, but calculated from NOZOMI orbital data by

$$
\vartheta=\frac{1}{n-(m-10)} \sum_{t_{n}}^{t_{m-10}} \arccos \left[\left(\frac{\mathbf{r}\left(t_{n}\right)}{\mid \mathbf{r}\left(t_{n)} \mid\right.} \times \frac{\mathbf{r}\left(t_{n+10}\right)}{\mid \mathbf{r}\left(t_{n+10)} \mid\right.}\right) \cdot \mathbf{e}^{z}\right],
$$

where the indices $n$ and $m$ define the first and last orbital data point for the desired period, respectively. These angles lead to a deviation of the direction of the $\mathbf{y}_{\text {eec }}$ axis from the ecliptic plane, and require an additional coordinate transformation by a rotation around NOZOMI's xaxis that can be performed by a correction of the spin-phase $\varphi$, since the spin-phase is measured from the $\mathbf{y}_{\text {eec }}$ axis. The direction of this axis is determined by $\varphi=0^{\circ}$ when the Sun crosses the view-plane of the SSAS. To determine the correction angle $\alpha$, first the NOZOMI $\mathrm{y}$-axis $\mathbf{y}_{\text {eec }}^{0}$ is determined from the given attitude data $(\bar{\lambda}, \bar{\beta})$ as 


$$
\mathbf{y}_{\mathrm{eec}}^{0}=\left(\begin{array}{ccc}
\cos \bar{\beta} \cos \bar{\lambda} & -\sin \bar{\lambda} & -\sin \bar{\beta} \cos \bar{\lambda} \\
\cos \bar{\beta} \sin \bar{\lambda} & \cos \bar{\lambda} & -\sin \bar{\beta} \sin \bar{\lambda} \\
\sin \bar{\beta} & 0 & \cos \bar{\beta}
\end{array}\right) \cdot \mathbf{e}_{\mathrm{eec}}^{\mathrm{y}}=\left(\begin{array}{c}
-\sin \bar{\lambda} \\
\cos \bar{\lambda} \\
0
\end{array}\right)_{\mathrm{eec}}
$$

The correction angle $\alpha$ is determined by

$$
\alpha=\arccos \left(\mathbf{y}_{\mathrm{eec}}^{0} \cdot \mathbf{y}_{\mathrm{eec}}\right) .
$$

The angle $\alpha$ already includes the possible discontinuous shift of the direction of the $\mathrm{y}_{\mathrm{eec}}$ axis by $180^{\circ}$ around $\mathbf{s}_{\text {eec }}$ (if $\mathbf{s}_{\text {eec }}$ is lying in the NOZOMI orbital plane), or fast move from $\approx 0^{\circ}$ to $\approx 180^{\circ}$ (if $\mathbf{s}_{\mathrm{eec}}$ is not lying in the NOZOMI orbital plane) when the Sun passes by NOZOMI's stern or bow in longitudinal direction (see Figure 6-15), but not the direction into which the correction of $\varphi$ has to take place.

b)

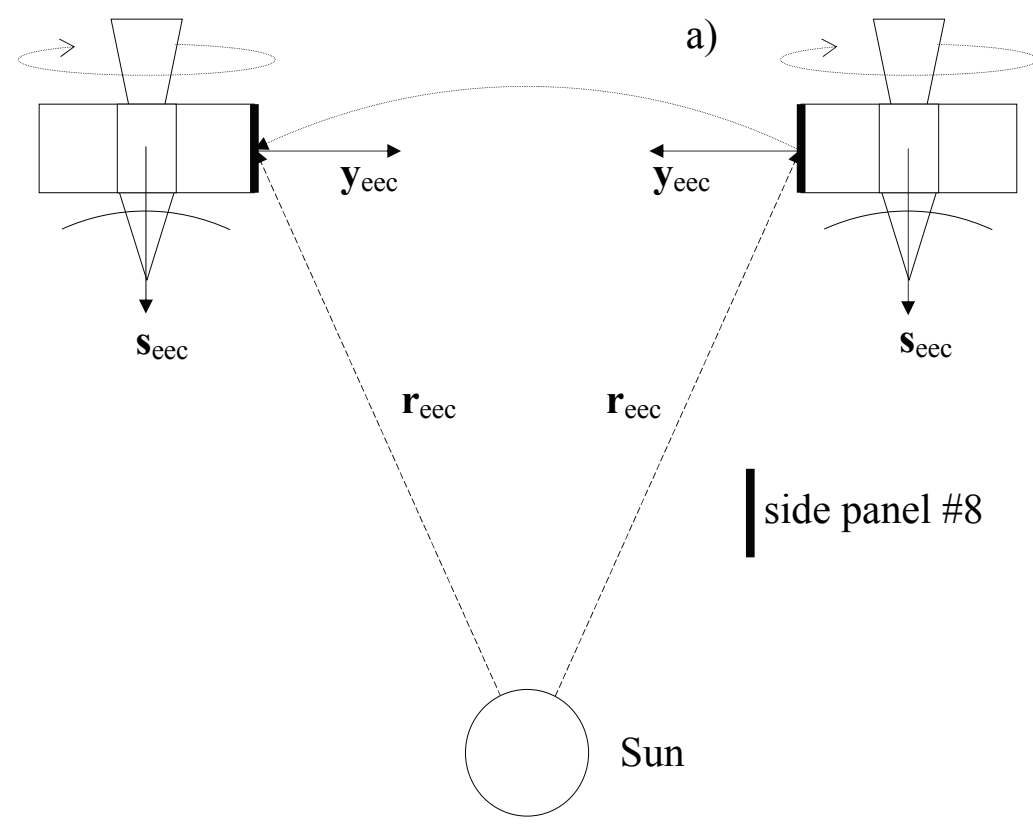

Figure 6-15: Illustration of the shift of the $\mathbf{y}_{\mathrm{eec}}$ axis and the spin-phase $\varphi$ Spin-phase $\varphi=0^{\circ}$ occurs when the Sun passes by the view plane of the SSAS, the SSAS is located on the side-panel \#8 like the MDC. If the Sun passes by NOZOMI's stern or bow, as it happens between positions a) and b), the $\mathbf{y}_{\text {eec }}$ axis defined by $\varphi=0^{\circ}$ shifts about $180^{\circ}$.

The direction can easily be determined if an auxiliary vector

$$
\mathbf{h}_{\mathrm{eec}}=\frac{\mathbf{y}_{\mathrm{eec}}^{0} \times \mathbf{y}_{\mathrm{eec}}}{\left|\mathbf{y}_{\mathrm{eec}}^{0} \times \mathbf{y}_{\mathrm{eec}}\right|}
$$

is defined, that is either parallel or anti-parallel to the spin-axis $\mathbf{s}_{\text {eec. }}$ Then the angle $\widetilde{\varphi}$ is finally given as 


$$
\widetilde{\varphi}=\varphi+\left(\mathbf{h}_{\mathrm{eec}} \cdot \mathbf{s}_{\mathrm{eec}}\right) \alpha,
$$

where the scalar product of $\mathbf{h}_{\mathrm{eec}}$ and $\mathbf{s}_{\mathrm{eec}}$ evaluates either to $+1\left(\mathbf{h}_{\mathrm{eec}}\right.$ parallel $\left.\mathbf{s}_{\mathrm{eec}}\right)$ or to $-1\left(\mathbf{h}_{\mathrm{eec}}\right.$ anti-parallel $\mathbf{s}_{\text {eec }}$ ). If the NOZOMI orbit and spin-axis were in the ecliptic plane, the angle $\alpha$ would always be exactly zero or $180^{\circ}$, respectively.

The last transformation is the transformation from NOZOMI's rotated reference system to the Earth ecliptic reference system, which is performed by

$$
\begin{gathered}
\mathbf{n}_{\mathrm{eec}}=\mathbf{A}_{-\bar{\lambda}} \mathbf{A}_{\bar{\beta}} \mathbf{n}_{\mathrm{noz} \varphi}=\mathbf{A}_{\mathrm{eec}, \mathrm{noz} \varphi \varphi} \mathbf{n}_{\mathrm{noz} \varphi}, \\
\mathbf{A}_{\bar{\beta}}=\left(\begin{array}{ccc}
\cos \bar{\beta} & 0 & -\sin \bar{\beta} \\
0 & 1 & 0 \\
\sin \bar{\beta} & 0 & \cos \bar{\beta}
\end{array}\right), \mathbf{A}_{-\bar{\lambda}}=\left(\begin{array}{ccc}
\cos \bar{\lambda} & -\sin \bar{\lambda} & 0 \\
\sin \bar{\lambda} & \cos \bar{\lambda} & 0 \\
0 & 0 & 1
\end{array}\right), \\
\mathbf{A}_{\text {eec,noz } \varphi}=\mathbf{A}_{-\bar{\lambda}} \mathbf{A}_{-\bar{\beta}}=\left(\begin{array}{ccc}
\cos \bar{\beta} \cos \bar{\lambda} & -\sin \bar{\lambda} & -\sin \bar{\beta} \cos \bar{\lambda} \\
\cos \bar{\beta} \sin \bar{\lambda} & \cos \bar{\lambda} & -\sin \bar{\beta} \sin \bar{\lambda} \\
\sin \bar{\beta} & 0 & \cos \bar{\beta}
\end{array}\right) .
\end{gathered}
$$

The different signs at $\lambda$ and $\beta$ result from the different mathematical directions in which $\lambda$ and $\beta$ are given. In summary, the opposite view axis direction of the MDC is calculated from the NOZOMI flight data at $t=t^{\text {imp }}\left(\mathbf{r}_{\text {eec }}, \bar{\lambda}, \bar{\beta}, \varphi\right)$ and spatial configuration $(\gamma, \delta)$ by the transformation

$$
\mathbf{n}_{\text {eec }}=\mathbf{A}_{\text {eec, noz } \varphi} \mathbf{A}_{\text {noz } \varphi \text {,noz }} \mathbf{A}_{\text {noz,mdc }} \mathbf{n}_{\text {mdc }} .
$$

Finally, the absolute particle velocity $\mathbf{u}_{\mathrm{eec}}$ is given by the vectorial subtraction

$$
\mathbf{u}_{\mathrm{eec}}=u^{\mathrm{imp}} \mathbf{n}_{\mathrm{eec}}-\mathbf{v}_{\mathrm{eec}},
$$

whereas $u^{\text {imp }}$ is the scalar impact speed of the particle as derived from the impact data analysis, and $\mathbf{v}_{\text {eec }}$ is the current speed vector of NOZOMI at the given impact time $t^{\text {imp }}$.

If not specified, all vectors (coordinates and velocities) are given in the Earth-ecliptic reference system in the following paragraphs, and the index "eec" is therefore omitted. MDC impact data during the Earth-Moon phase can easily be transformed back to the Earth equatorial reference system by the inverse of the transformation given in equation (6-6), and therefore can be analyzed and displayed in both reference systems. 

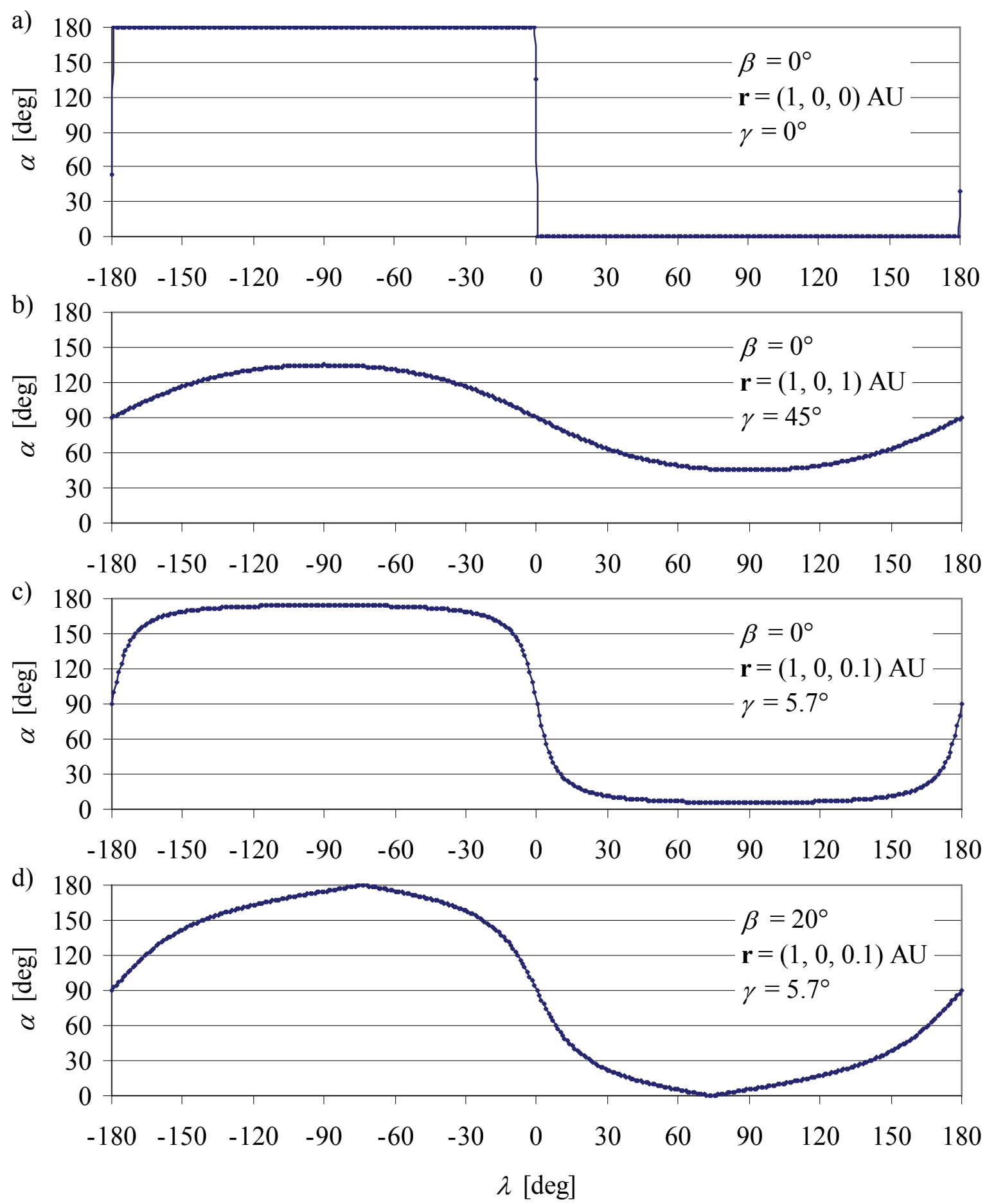

Figure 6-16: Spin-phase correction angle $\alpha$.for longitudes $\lambda=-180^{\circ} \ldots 180^{\circ}$ The correction angle $\alpha$ is displayed for two hypothetic a), b) (only for illustration) and two realistic c), d) orbital configurations over the NOZOMI spin-axis longitude $\lambda$. The NOZOMI orbital position $\mathbf{r}$ and spin-axis latitude $\beta$ are given for each configuration (the absolute value $r$ is not relevant), the angle $\gamma$ is the angle between the NOZOMI radius vector and the ecliptic plane and calculates from $\mathbf{r}$. As can be seen, the angle $\alpha$ varies between $0^{\circ}$ and $180^{\circ}$ with different shapes. For configuration a) and other thinkable configurations, there is a singularity at $\lambda=0^{\circ}$ or $\pm 180^{\circ}$. 


\subsubsection{NOZOMI flight data and sensor pointing directions}

For further interpretation of the primary analysis results of the MDC data, the characteristics of the spacecraft's flight data and the resulting sensor pointing directions over mission time must be taken into account. NOZOMI orbital data and attitude data were supplied by ISAS/JAXA as derived from the spacecraft's telemetry. Orbital position data and attitude data are shown and analyzed here with respect to the MDC instrument requirements.

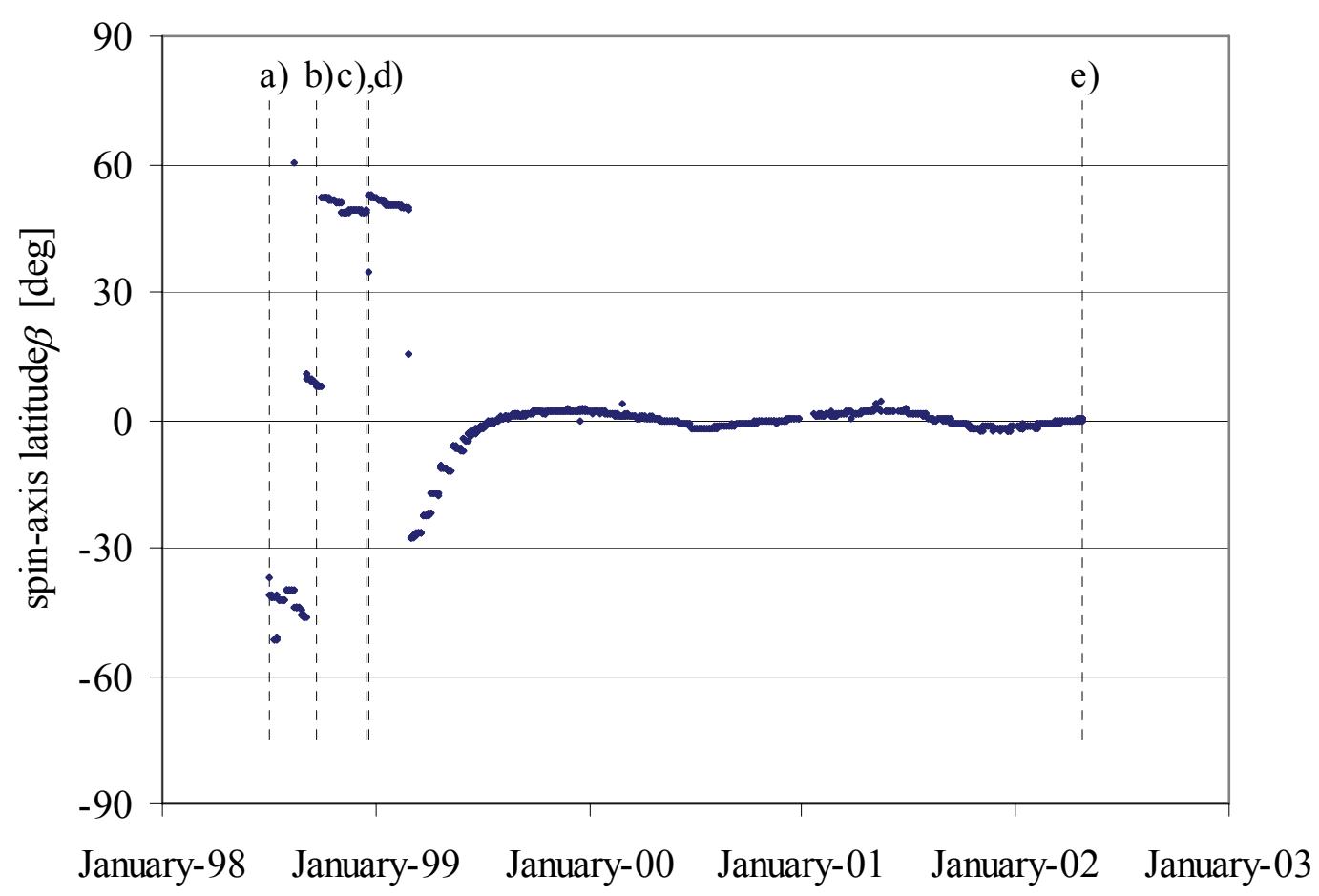

Figure 6-17: NOZOMI spin-axis latitude $\beta$ over mission time, 1998-2002 Mission events: a) Launch July 4, 1998, b) Moon swing-by \#1, September 24, 1998, c) Moon-swing-by \#2, December 18, 1998, d) Earth swing-by, December 20, 1998, e) System failure, April 24, 2002.

Critical for the latter determination of dust flux and the statistical interpretation of particle characteristics are both the orbital position of the spacecraft and the overall sensor pointing directions. It is obvious that the MDC cannot measure any particle flux from directions it never looked at.

Latitude and longitude of the NOZOMI spin axis pointing directions over mission time are shown in Figure 6-17 and Figure 6-18. Spin-axis latitude varies between $+45^{\circ}$ and $-45^{\circ}$ during the first months of the mission and stabilizes at $+-3^{\circ}$ for the rest of the mission time, while longitude covers the whole range from $0^{\circ}$ to $360^{\circ}$ for $2 \frac{1}{2}$ times. 


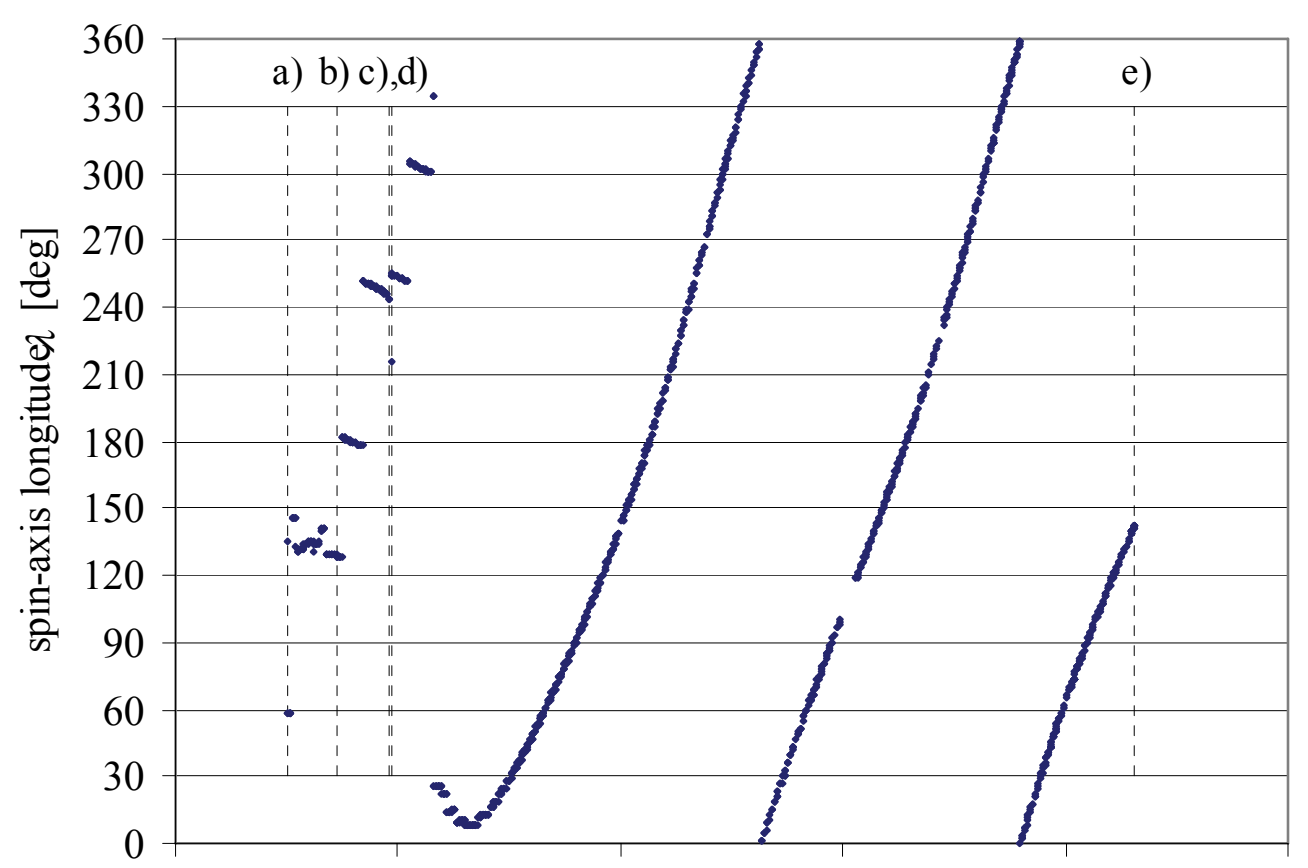

January-98 January-99 January-00 January-01 January-02 January-03

Figure 6-18: NOZOMI spin-axis longitude $\lambda$ over mission time, 1998-2002 Mission events: a) Launch July 4, 1998, b) Moon swing-by \#1, September 24, 1998, c) Moon-swing-by \#2, December 18, 1998, d) Earth swing-by, December 20, 1998, e) System failure, April 24, 2002.

Figure 6-19 shows the overall heliospheric coverage of the MDC field-of-view during the full mission time. The coverage of each solid angle element $\Delta \lambda \Delta \beta$ was calculated from the MDC spin-averaged sensitivity and the NOZOMI attitude data for each single day, integrated over the whole mission time and normalized to $100 \%$. Minimum value is $0.0011 \%$ for $\lambda=76^{\circ}$ and $\beta=41^{\circ}$, maximum value is $0.0021 \%$ for $\lambda=259^{\circ}$ and $\beta=-2^{\circ}$. Mean value is $0.0015 \%$ (which is $100 \% /(360 \times 180))$. In summary, the whole heliosphere was covered well by the MDC with maximum and minimum coverage deviating about $50 \%$ from the average value.

The coverage during the first six months in Earth orbit and the remaining mission time in the interplanetary space are shown separately in Figure 6-20 (lower left and lower right graphs, respectively). For illustration, the coverage of a single mission day (upper right graph) and a fictitious in-ecliptic spinning dust sensor (upper left graph) are shown. The shape of the coverage for a single mission day resembles the shape of the spin-averaged sensitivity of the MDC as shown in Figure 3-15. 


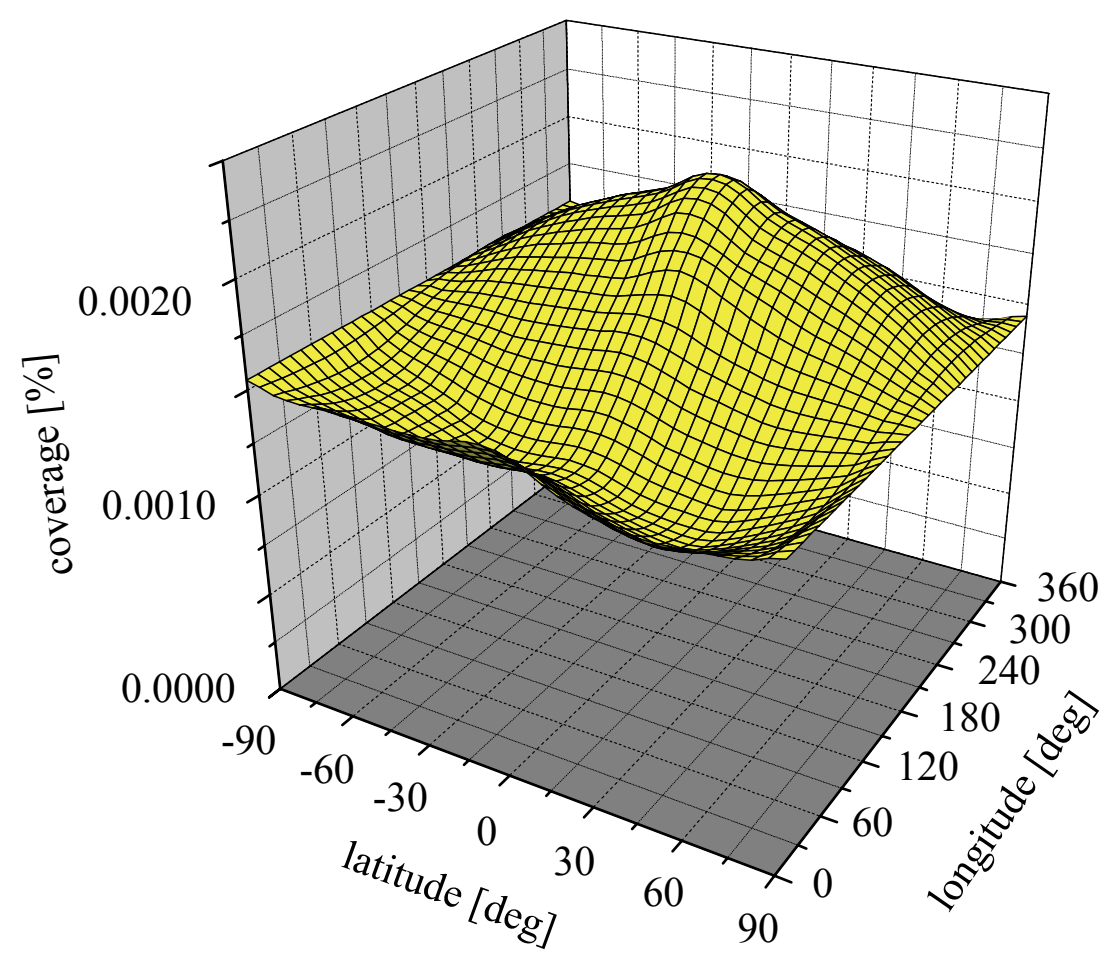

Figure 6-19: Overall heliospheric coverage of the MDC sensor view

Based on the spin-averaged sensitivity of the MDC on NOZOMI and the NOZOMI flight data, the overall coverage of the MDC sensor over longitude and latitude of the heliosphere is shown in this graph. Coverage is given in $\%$ per $\Delta \lambda \Delta \beta\left(1^{\circ}\right.$ step width each) interval and must be scaled to solid angle elements when used for further statistical analysis. 

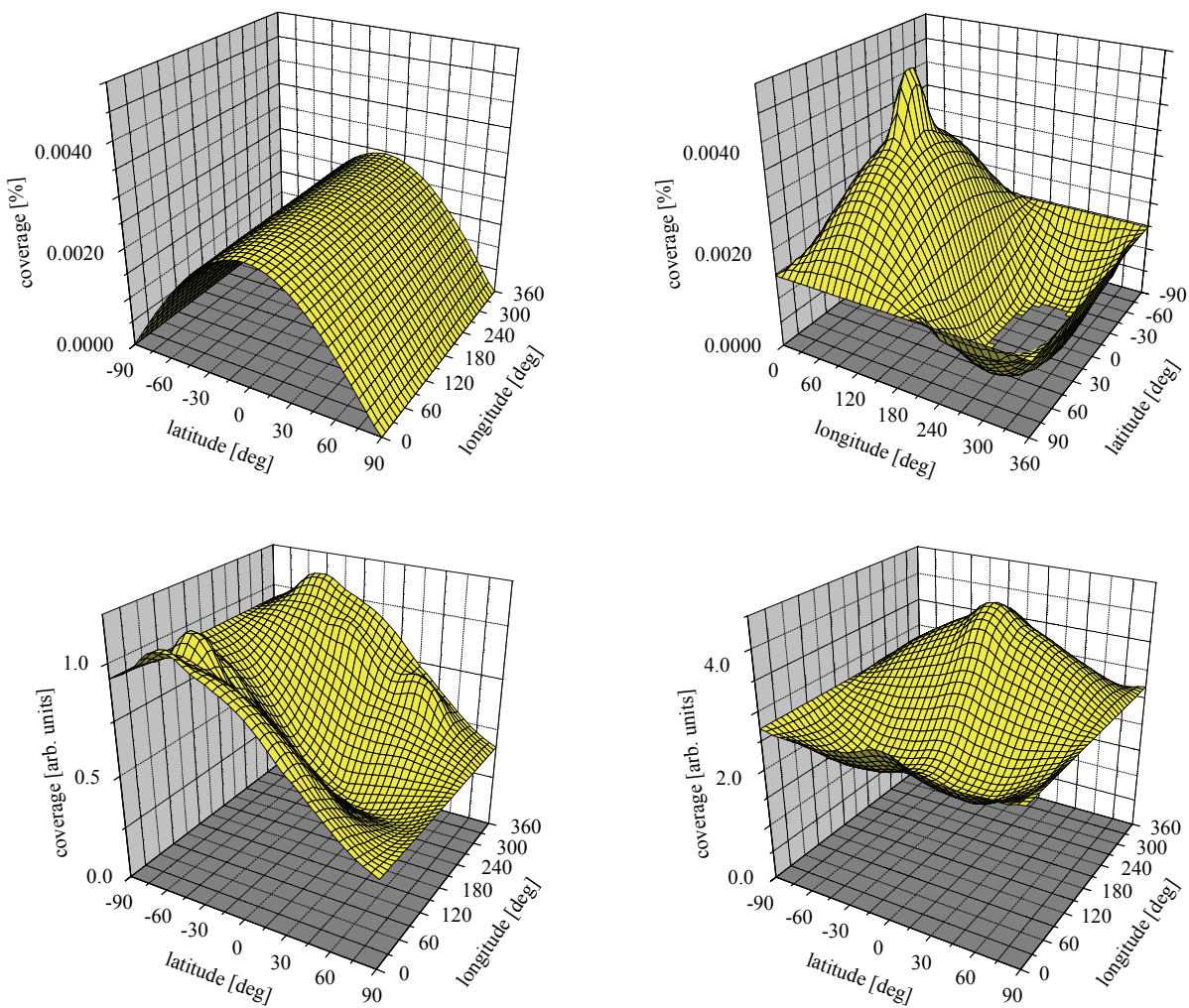

Figure 6-20: Heliospheric coverage, illustration

For illustration purpose, the heliospheric coverage of a flat, in-ecliptic spinning surface with solid angle $2 \pi \mathrm{sr}$ (upper left), the MDC sensor on NOZOMI for a fictitious single day with constant longitude $-90^{\circ}$ and latitude $0^{\circ}$ (upper right) and the MDC sensor during the Earth-Moon mission phase with latitude $\approx+45^{\circ}$ and $-45^{\circ}$ (lower left) and the interplanetary cruising phase with latitude $\approx 0^{\circ} \pm 2^{\circ}$ (lower right) is shown here. Coverage was not normalized for intermediate results. 
NOZOMI spin-rate over mission time is shown in Figure 6-21. Initially between 9 and $10.3 \mathrm{rpm}$, the spin rate was lowered to $7.7 \mathrm{rpm}$ after injection into the interplanetary orbit and later to 6.9 to $6.8 \mathrm{rpm}$. The NOZOMI spin rate does not affect the analysis of impact data (rotation speed of the MDC is much lower than the orbital speed of the spacecraft and the nominal impact speed of dust particles, and therefore negligible), but was used for the analysis of the MDC noise data as described in paragraph 6.2.1.

Figure 6-22 shows the angle of the NOZOMI spin axis to the NOZOMI orbital radius vector in the Sun centered eec system. After injection into the transfer orbit to Mars, the angle never falls below $135^{\circ}$, which means that the minimum angle of the MDC bore sight direction to the radius vector is always lower than $90^{\circ}$ and no direct sunlight was falling into the MDC sensor box (see also Figure 6-1 and paragraph 6.2.1 for a more detailed discussion).

Figure 6-23 shows the angle of the NOZOMI spin axis to the NOZOMI velocity vector in the Sun centered ecliptic coordinate system. During the mission period in the interplanetary space, the angle drops to a minimum of $45^{\circ}$ and rises continuously to finally nearly $135^{\circ}$. For angles below $90^{\circ}$, the MDC sensitivity is enhanced into the spacecraft's anti-apex direction, while for angles above $90^{\circ}$, MDC sensitivity is enhanced into the spacecraft's apex direction. This has consequences for the dust detection capabilities of the MDC and is discussed later in this paragraph.

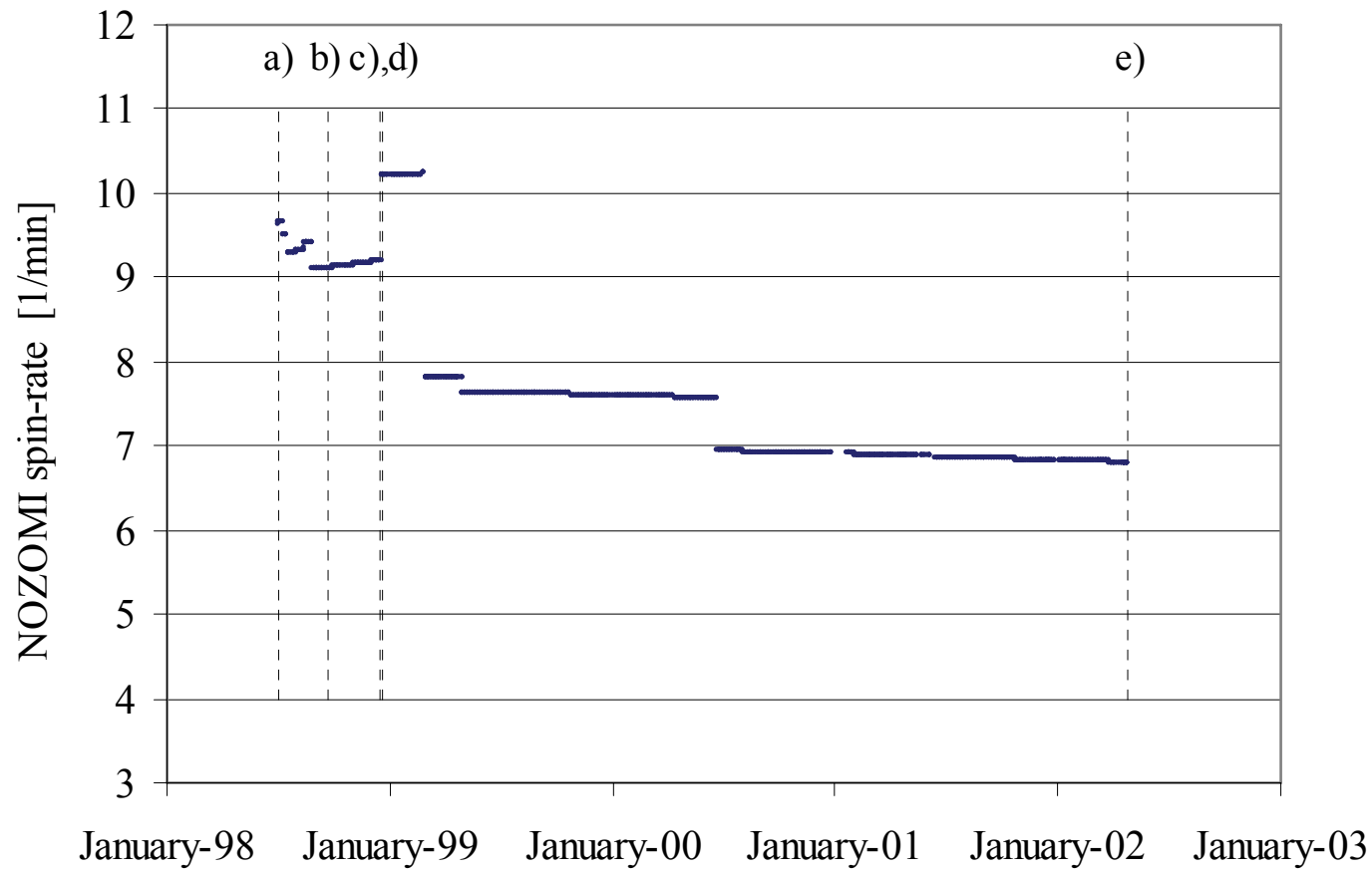

Figure 6-21: NOZOMI spin rate over mission time

Mission events: a) Launch July 4, 1998, b) Moon swing-by \#1, September 24, 1998, c) Moon-swing-by \#2, December 18, 1998, d) Earth swing-by, December 20, 1998, e) System failure, April 24, 2002. 


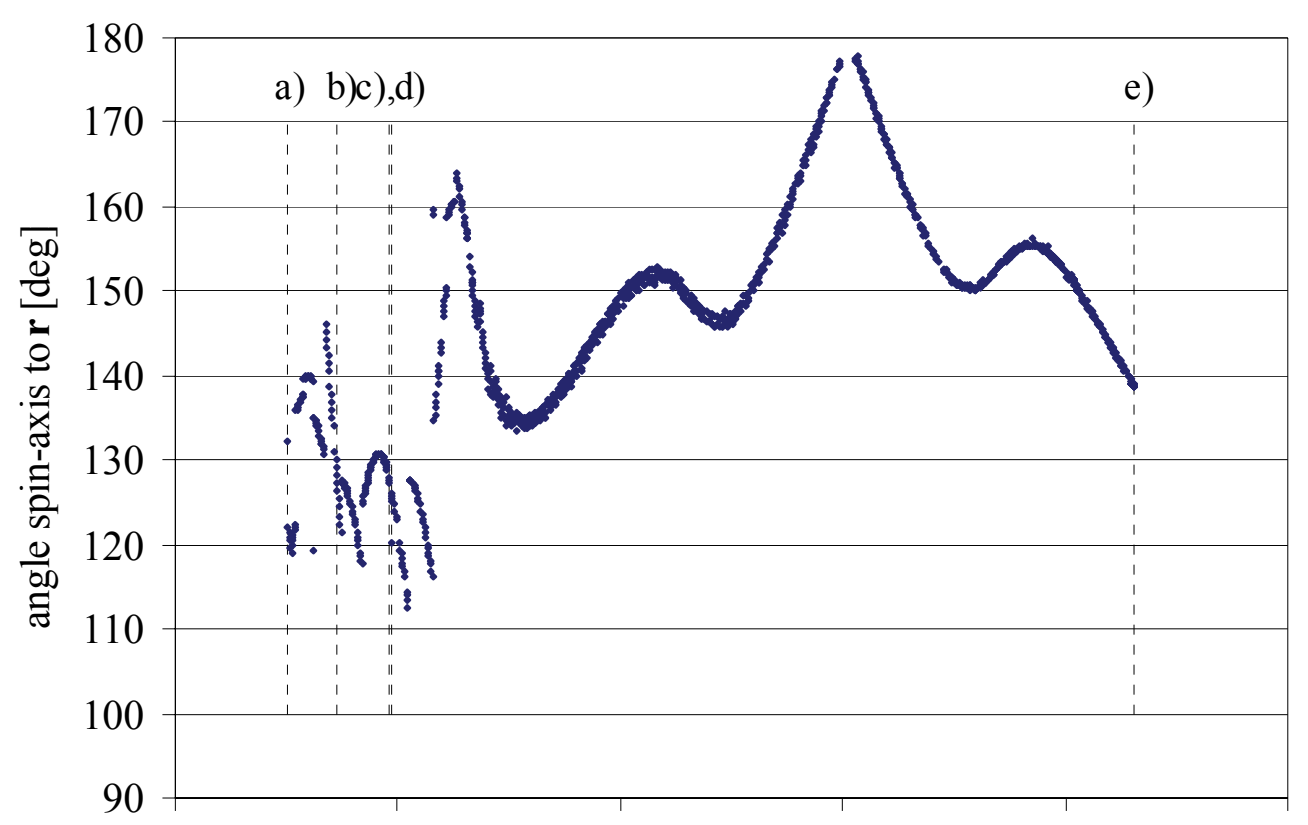

January-98 January-99 January-00 January-01 January-02 January-03

Figure 6-22: Angle of NOZOMI spin-axis to NOZOMI radius vector

Mission events: a) Launch July 4, 1998, b) Moon swing-by \#1, Sep. 24, 1998, c) Moon-swing-by \#2, Dec. 18, 1998, d) Earth swing-by, Dec. 20, 1998, e) System failure, April 24, 2002.

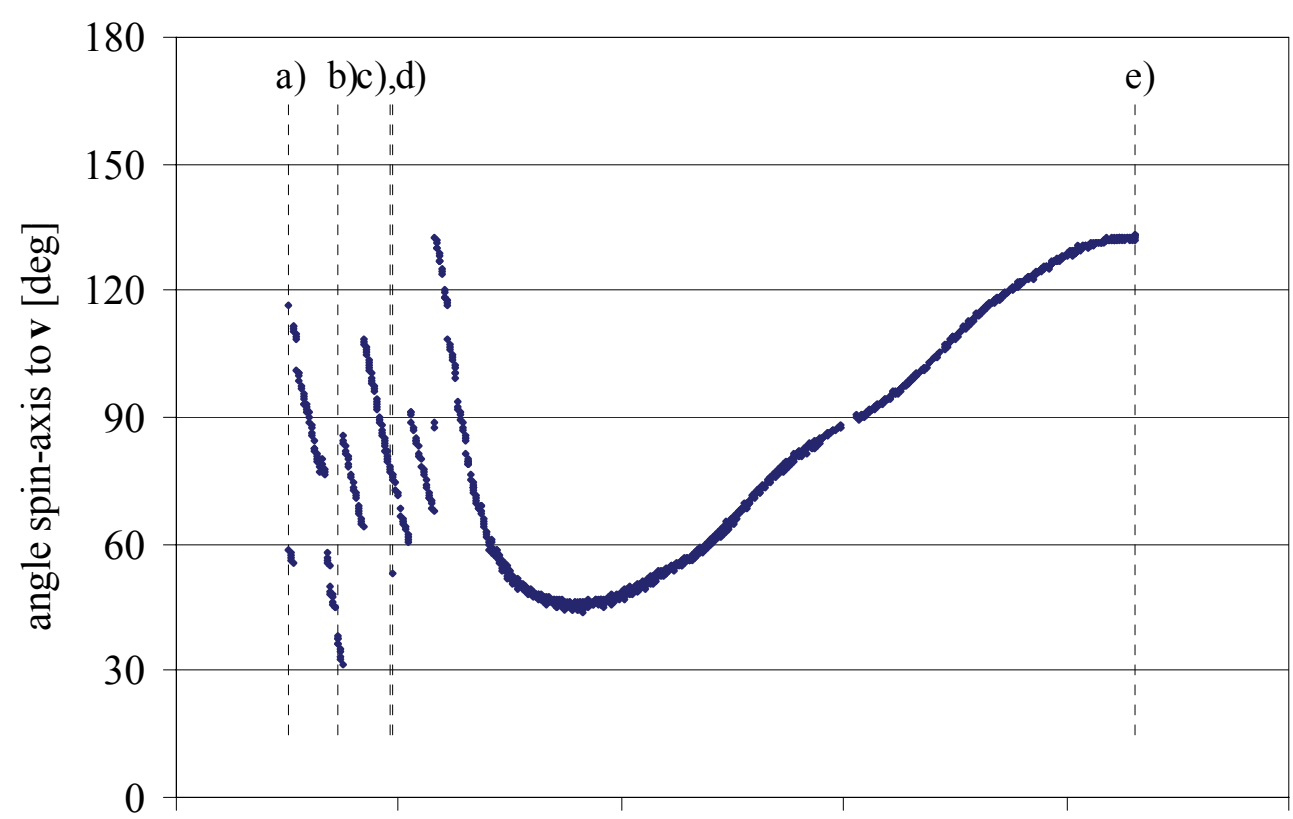

January-98 January-99 January-00 January-01 January-02 January-03

Figure 6-23: Angle of the NOZOMI spin-axis to the velocity vector Mission events: a) Launch July 4, 1998, b) Moon swing-by \#1, September 24, 1998, c) Moon-swing-by \#2, December 18, 1998, d) Earth swing-by, December 20, 1998, e) System failure, April 24, 2002. 
NOZOMI distance from the Earth during 1998 (see Figure 6-24) and from the Sun (see Figure 6-26) during the whole mission is shown on the following pages. From launch until the first Moon swing-by on September 24, 1998 NOZOMI was in a high elliptic orbit around the Earth with perigee at around $8000 \mathrm{~km}$ and apogee at around $420000 \mathrm{~km}$ to $515000 \mathrm{~km}$. Between the two Moon swing-bys, apogee was 1.7 million km.

After the Earth swing-by on December 20, 1998, NOZOMI was injected into an elliptic orbit around the Sun with perihelion at $0.98 \mathrm{AU}$ and aphelion at $1.45 \mathrm{AU}$.

In Figure 6-25 and Figure 6-27, NOZOMI's duration of stay in different radius intervals is shown. Due to the elliptic characteristics of the orbits, the duration of stay is significantly higher at apogee and aphelion than at perigee and perihelion, respectively. This must be considered in later interpretation of measured MDC dust data, especially for the determination of dust flux measured by the MDC. 


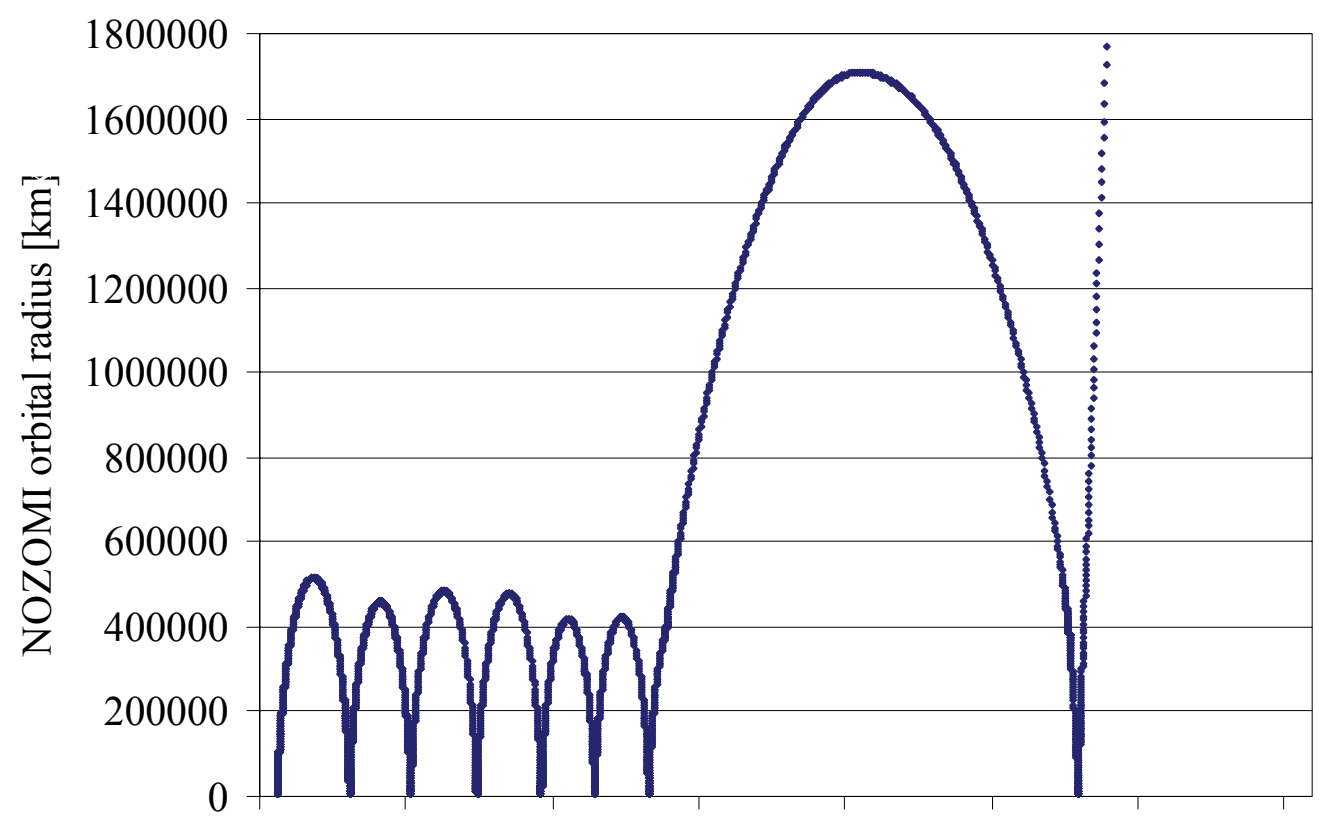

Jul-98 Aug-98 Sep-98 Oct-98 Nov-98 Dec-98 Jan-99 Feb-99

Figure 6-24: NOZOMI distance from the Earth 1998

After six high elliptical orbits with apogee beyond the Moon orbit (400000 to $500000 \mathrm{~km}$ ), NOZOMI performed two Earth swing-by's that finally brought it on it's trajectory to Mars.

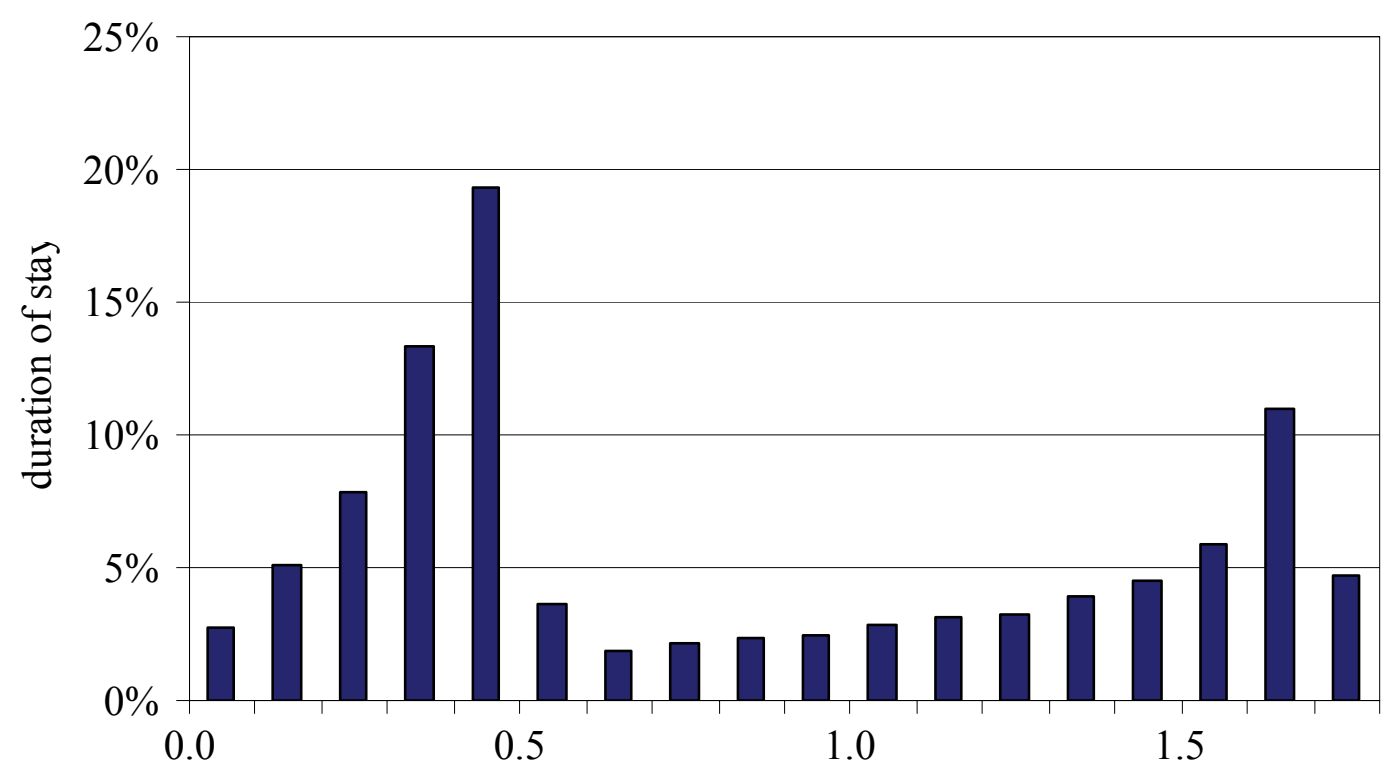

NOZOMI orbital radius [million $\mathrm{km}$ ]

Figure 6-25: NOZOMI distance from the Earth, duration of stay

For further statistical interpretation of particle impacts, the duration of stay of the NOZOMI spacecraft at the different radius intervals was determined. 


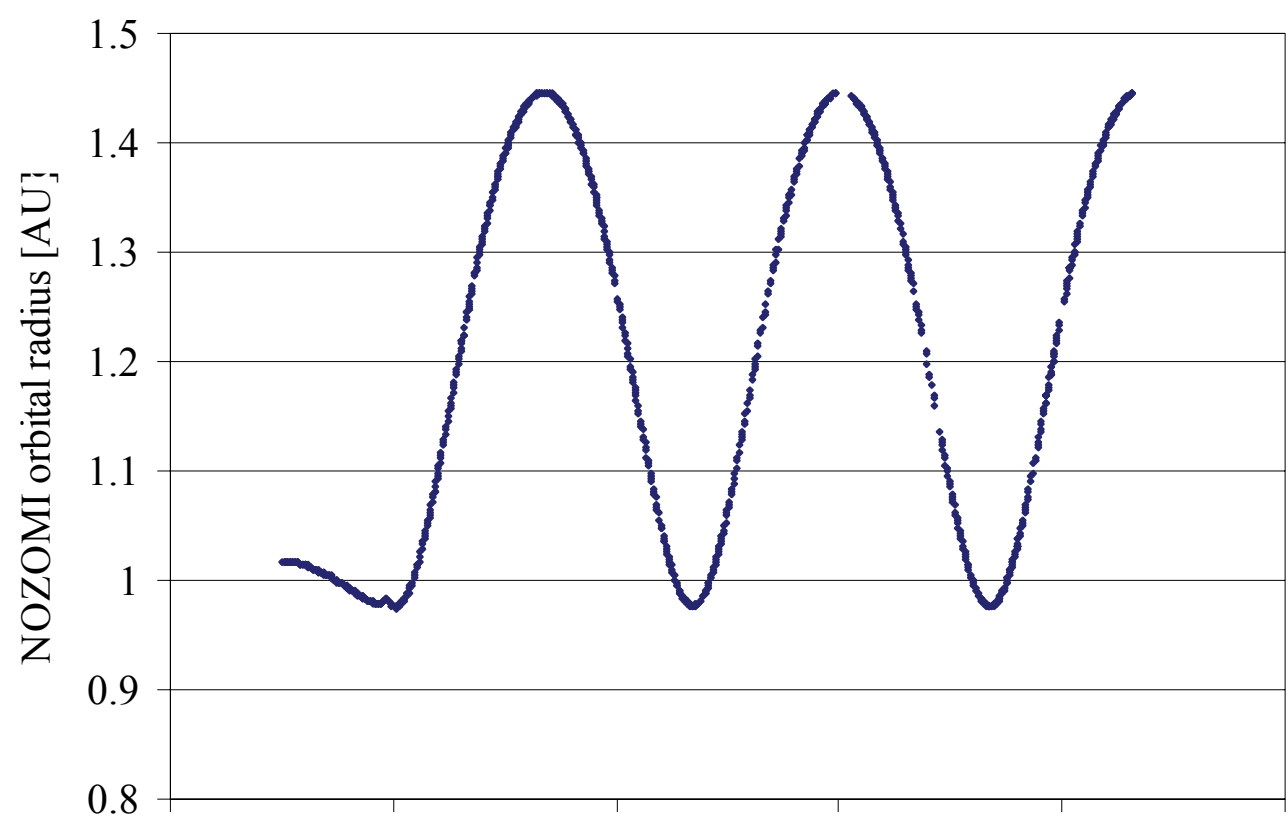

January-98 January-99 January-00 January-01 January-02 January-03

Figure 6-26: NOZOMI distance from the Sun

NOZOMI's distance from the Sun varies between 0.99 and $1.01 \mathrm{AU}$ in 1998 (orbit around the Earth), and between 0.98 and 1.45 AU during the interplanetary cruising phase.

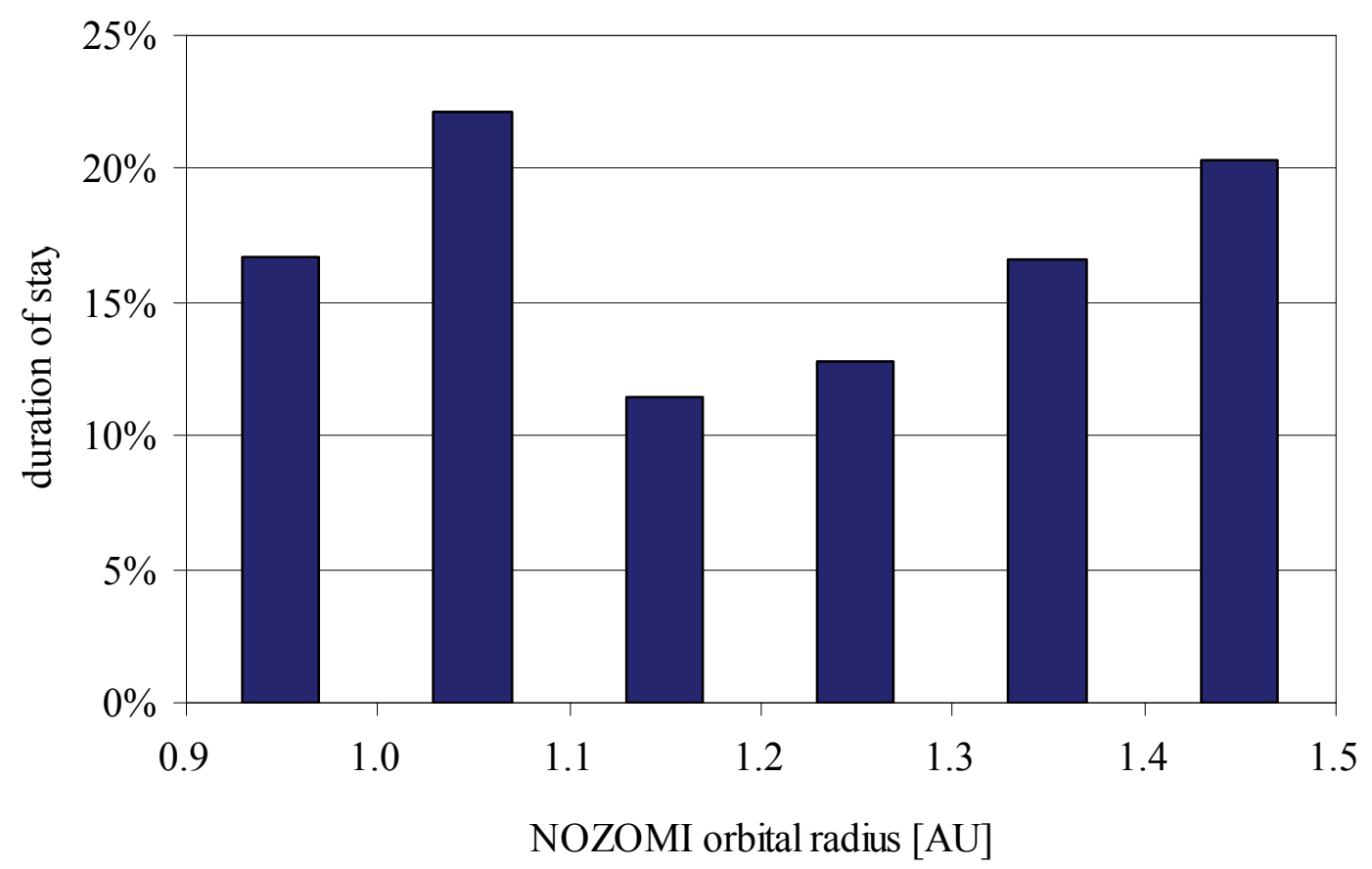

Figure 6-27: NOZOMI distance from the Sun, duration of stay

For further statistical interpretation of particle impacts, the duration of stay of the NOZOMI spacecraft at the different radius intervals was determined. 
Based on the NOZOMI flight data and the MDC spin-averaged sensitivity, the MDCNOZOMI detection capabilities for three specific populations of dust particles were estimated. The three populations investigated were apex-particles, $\beta$-meteoroids and interstellar dust grains.

The so called apex particles are a population of the interplanetary dust flux that are expected to orbit in nearly circular orbits with a lower circular speed than macroscopic objects due to the effect of radiation pressure, see paragraph 2.1.1. Because of this lower speed, such particles are expected to impact preferable from the apex direction of the spacecraft.

$\beta$-meteoroids are expected to travel on high-speed hyperbolic orbits that deviate only by a few degrees from the radial direction.

Interstellar dust grains are expected to have flight directions nearly similar to that of the interstellar gas flux. The interstellar gas flux is crossing the solar system at $\lambda=79^{\circ}$ and $\beta=-5^{\circ}$ downstream direction.

The MDC's sensitivity for these three populations of particles was determined from the NOZOMI orbital position, NOZOMI flight direction, NOZOMI attitude data and the MDC spin-averaged sensitivity for each day of the mission. The results are shown and discussed in the following.

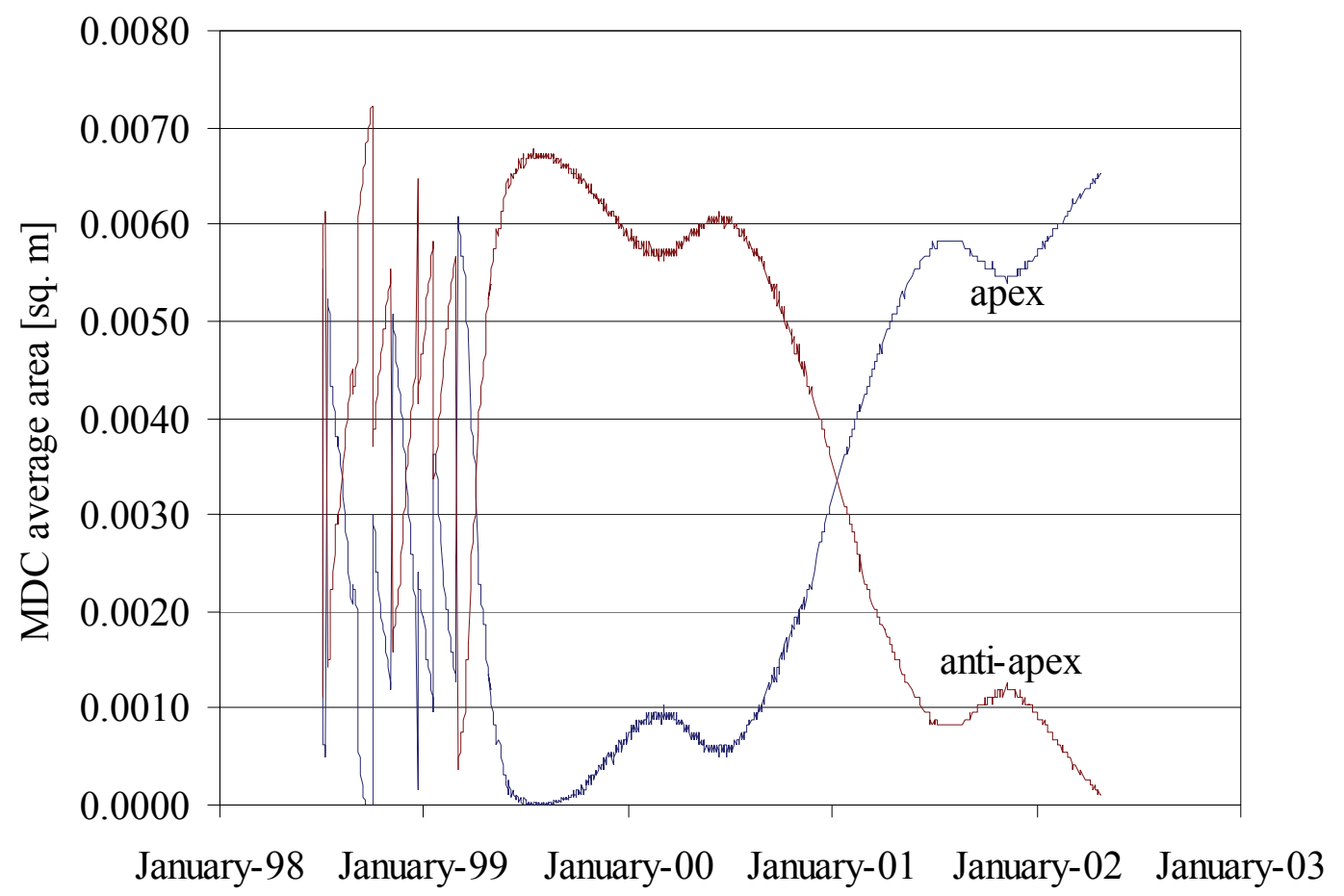

Figure 6-28: MDC sensitivity for apex-particles

Sensitivity of the MDC for apex (blue line) and anti-apex (red line) particles approaching exactly from the front of the spacecraft (apex) and at an angle of $0^{\circ}$ to the spacecraft's flight direction (anti-apex). 
In Figure 6-28, the average sensitive area of the MDC for apex (blue line) and anti-apex (red line) particles is shown. Sensitivity is fluctuating during the first months of the mission where the NOZOMI attitude was changed frequently due to orbital maneuvers, and Earth and Moon swing-by maneuvers. After NOZOMI spin-axis was set to Earth-pointing direction in March 1999, the sensitivity for apex particles was first falling to zero in July and August 1999 and afterwards rising discontinuously throughout the mission time with two local maxima. The average sensitive area for apex and anti-apex particles never reaches the maximum possible sensitive area of $0.01025 \mathrm{~m}^{2}$ since the angle of the NOZOMI spin-axis to the NOZOMI flight direction never was lower than $45^{\circ}$.

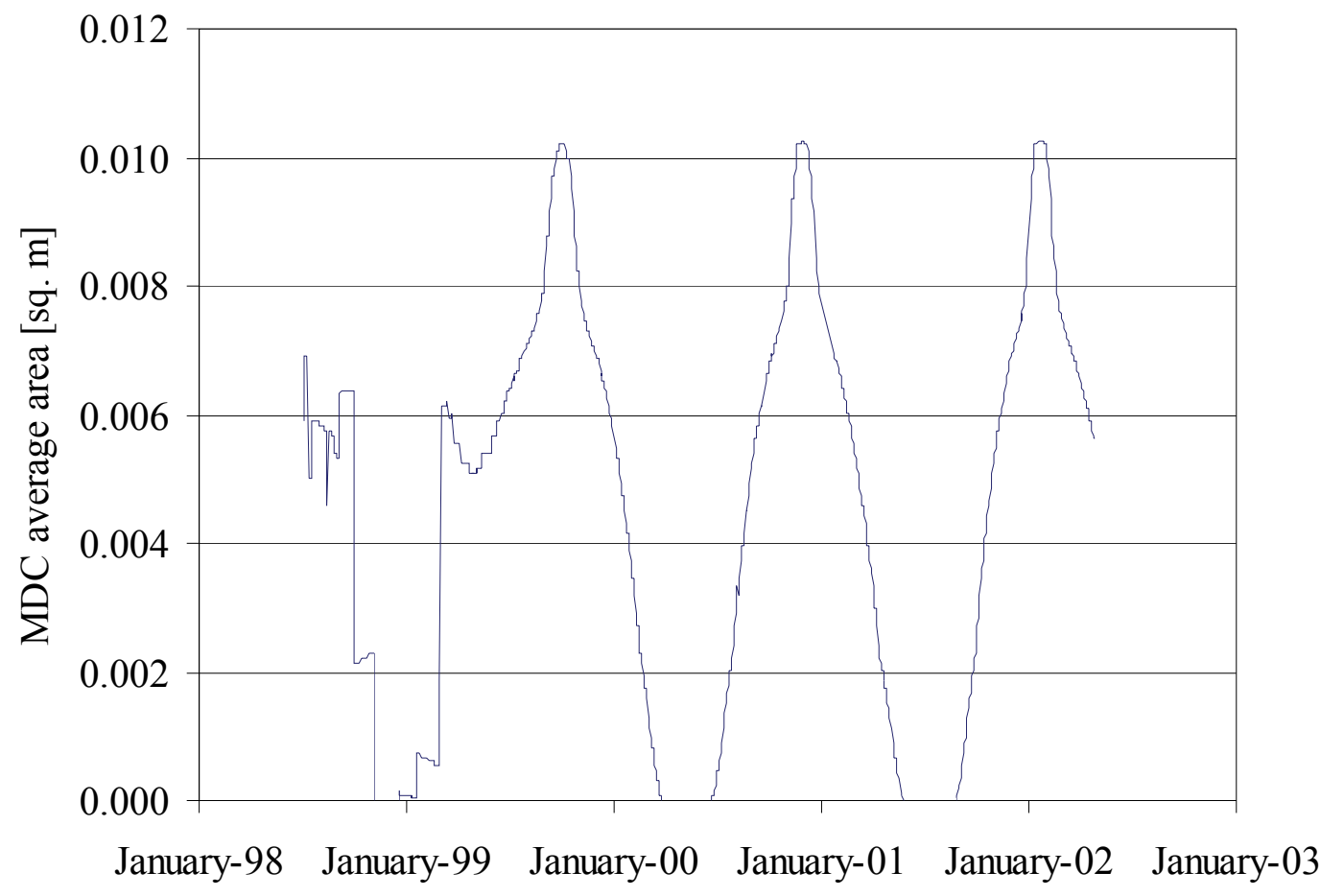

Figure 6-29: MDC sensitivity for ISD grains

Sensitivity of the MDC for particles arriving from the direction of the interstellar gas and dust flux.

In Figure 6-29, the average sensitive area for particles approaching from the direction of the interstellar gas and dust flow $\left(\lambda=79^{\circ}, \beta=-5^{\circ}\right.$ downstream direction) is shown. After a short period of medium sensitivity at the beginning of the mission, the sensitivity drops to zero until NOZOMI spin-axis was set to Earth-pointing direction in March 1999. Afterwards, the sensitivity fluctuates between a maximum value near the theoretical maximum of $0.01025 \mathrm{~m}^{2}$ and zero depending on the NOZOMI spin-axis longitude. Maximum sensitive area was given on Oct. 7, 1999, Dec. 7, 2000 and Jan. 26, 2002. 


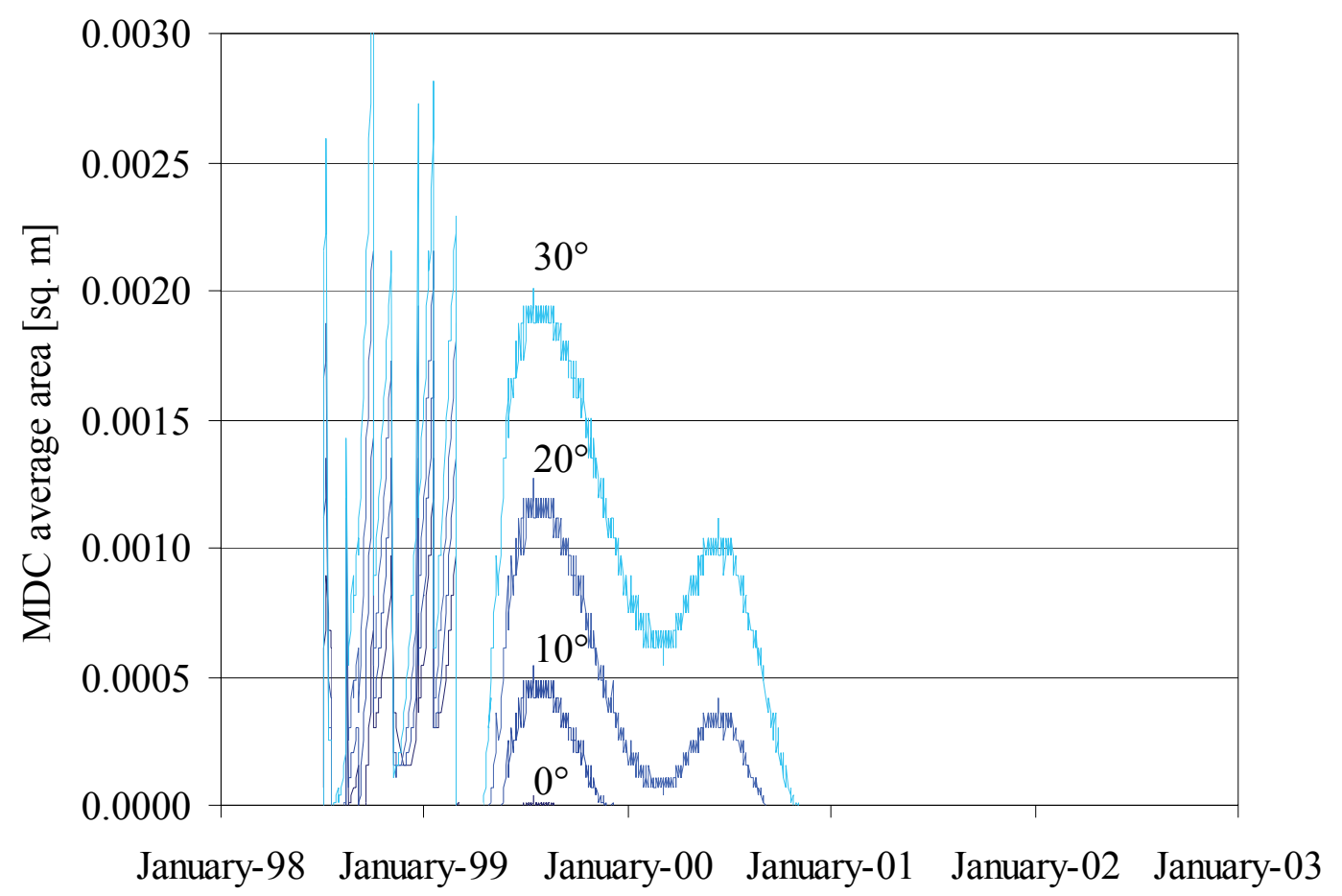

Figure 6-30: MDC sensitivity for $\beta$-meteoroids

Sensitivity of the MDC for particles traveling on a radial trajectory and on trajectories with different angles to the radial direction (in prograde direction).

In Figure 6-30, the MDC sensitive area for particles traveling on radial or near radial trajectories (with deviances of $10^{\circ}, 20^{\circ}$ and $30^{\circ}$ from the exact radial direction in prograde direction) is shown. Deviances in the magnitude of tens of degrees for $\beta$-meteoroids were derived from theoretical calculations by Wehry and Mann [Weh1999] ( $9^{\circ}$ to $24^{\circ}$ at $2.5 \mathrm{AU}$ for different conditions), and by Iglseder et al. [Ig11993b] from the MDC-HITEN data $\left(\approx 30^{\circ}\right.$ in average at $1 \mathrm{AU})$. During the first months of the mission, where NOZOMI attitude was changed several times, the MDC was indeed pointing to the sun and detection of $\beta$-meteoroids traveling on an exactly radial trajectory $\left(0^{\circ}\right.$ in the graph) was possible for a couple of days. For the rest of the mission, sensitivity for such $\beta$-meteoroids equals to zero with negligible non-zero values around July 16, 1999. For particles on trajectories with deviance from the exact radial direction, the MDC sensitive area has a maximum around July 16, 1999. The higher the deviance, the larger the maximum of the sensitive area is and the broader the time interval becomes during which the detection of such $\beta$-meteoroids becomes possible. The speed of the NOZOMI spacecraft was not considered in this estimation and will lower the shown sensitivities. The maximum sensitive area for particles with $30^{\circ}$ deviation from the radial flight direction is $0.0020 \mathrm{~m}^{2}$, which is one fifth of the maximum sensitive area for particles approaching exactly in the direction of the NOZOMI spin-axis (anti-apex).

One question raised by the integration of the MDC on NOZOMI and the orbital configuration (the MDC is mounted at an angle of $135^{\circ}$ to the NOZOMI spin-axis, the spin-axis of the NOZOMI spacecraft is mainly lying in the ecliptic plane) is: How would the distributions of orbital elements for special populations of dust particles look like? To get an impression about this, a simulation was run based on the true orbital data of the NOZOMI spacecraft (orbital 
positions, direction of the spin-axis) and a fictitious population of dust particles orbiting on circular orbits with an orbital speed that is determined by the gravitational force and the Sun's radiation pressure. A mass of $10^{-12} \mathrm{~g}$ and a resulting $\beta$-value of 0.4 were assumed for particles of this model population, hence the simulated particles have lower orbital speed than the NOZOMI spacecraft (as a macroscopic body that is not affected by radiation pressure) has. They are "overtaken" by the spacecraft and will impact from the apex direction of the spacecraft (so called "apex-particles"). Effects of the slightly inclined and elliptic shape of the NOZOMI orbit, and of the MDC entrance grid were neglected in this simple simulation. The simulation results are shown in Figure 6-31 and Figure 6-32, respectively. While the resulting distribution of inclination is close to the exact value of $i=0^{\circ}$ for all particles $(82 \%$ of the particles are assigned inclinations between $0^{\circ}$ and $10^{\circ}$ ), the resulting distribution of eccentricity differs significantly from the exact value of $e=0.0$ for all particles, and has a peak at $e=0.3$ to 0.4 . This is an effect of the out-of-ecliptic spinning of the MDC sensor, and the limited accuracy in the determination of the particles' flight direction (which is assumed perpendicular to the MDC sensor area, as best possible guess). Consequently, the distributions of the orbital elements derived from the real impact data recorded by the MDC (see paragraph 6.4) should be discussed with respect to the artifacts that are caused by the MDC's and NOZOMI's configuration as discussed here. Within the scope of this work, the distributions of orbital elements derived from the MDC impact data are discussed "as is", that means, without respect to the mentioned artifacts.

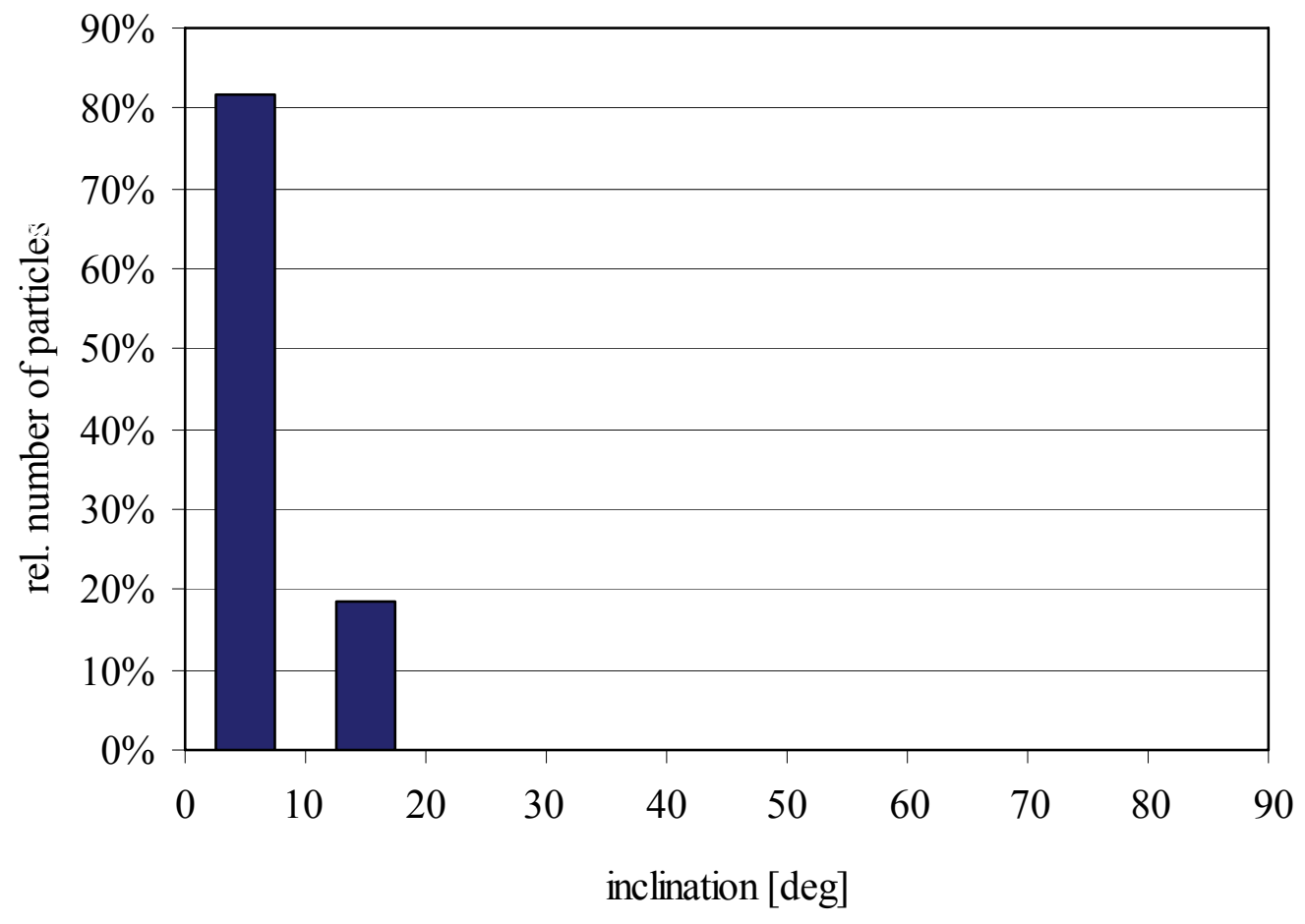

Figure 6-31: Simulation of inclination of a model particle population Inclination distribution as it is derived from the MDC on NOZOMI for a model population of apex-particles having exact inclination $i=0^{\circ}$. 


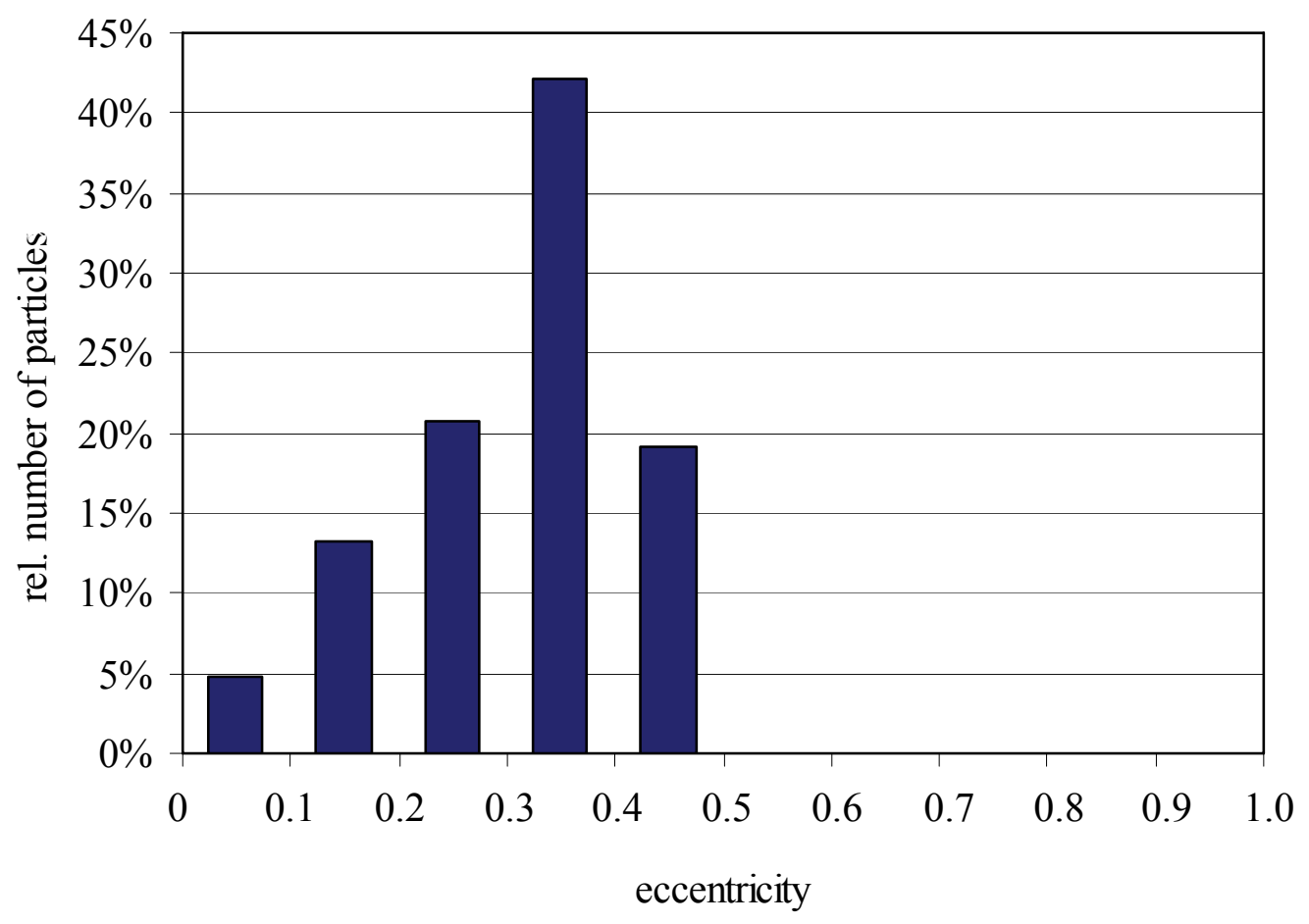

Figure 6-32: Simulation of eccentricity of a model particle population Eccentricity distribution as it is derived from the MDC on NOZOMI for a model population of apex-particles having exact eccentricity $e=0.0$. 


\subsubsection{MDC operating data}

The MDC operating data that includes information about the state of the experiment can be derived either from the housekeeping telemetry data that contains full sets of MDC operating data, or from the impact housekeeping information that contains a subset of the MDC operating data and is attached to each single recorded signal data set.

Unfortunately, the housekeeping telemetry data contains many erroneous data sets that complicate further analysis.

The following voltage and temperature charts were created from the much more reliable impact housekeeping information.

As can be seen in Figure 6-33, the $+5 \mathrm{~V}$ power supply voltage shows a very high stability around $5.0 \mathrm{~V} \pm 0.1 \mathrm{~V}$ during the whole mission time, while the $\pm 12 \mathrm{~V}$ power supply voltage rose rapidly from $12.0 \mathrm{~V}$ right after launch to a means of $13.0 \mathrm{~V}$ and $13.5 \mathrm{~V}$, respectively. The initial rise of these voltages and their fluctuation by $0.5 \mathrm{~V}$ in the order of months during the whole mission time are correlated well to the temperatures of the MDC electronics and sensor box as shown in Figure 6-34. These temperature fluctuations in turn are correlated well to the distance of the NOZOMI spacecraft from the sun (see Figure 6-26). 


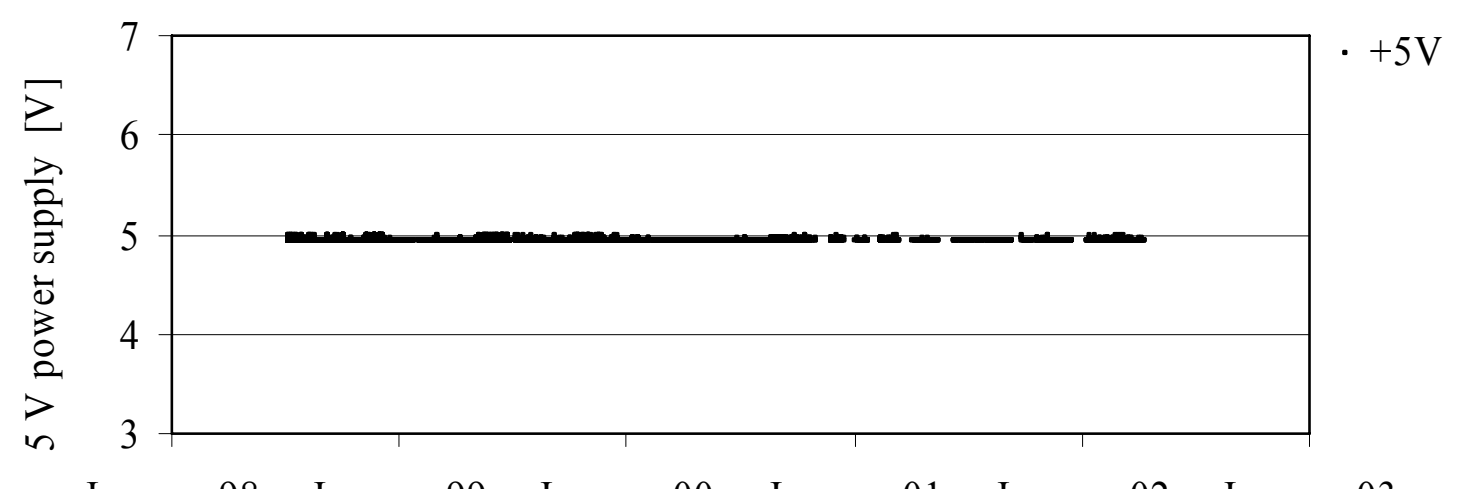

January-98 January-99 January-00 January-01 January-02 January-03

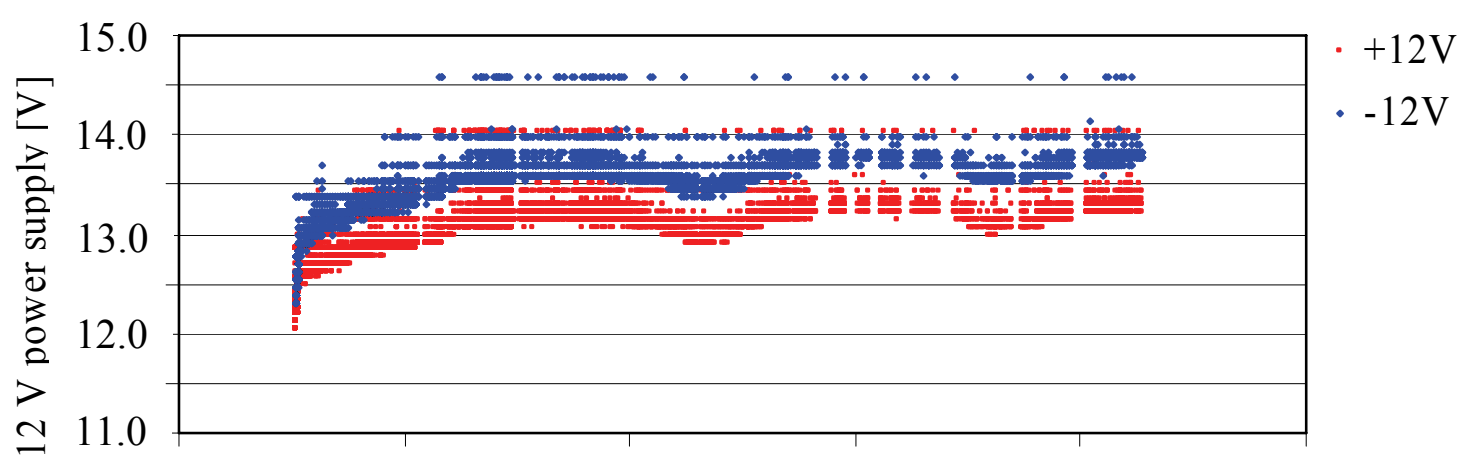

January-98 January-99 January-00 January-01 January-02 January-03

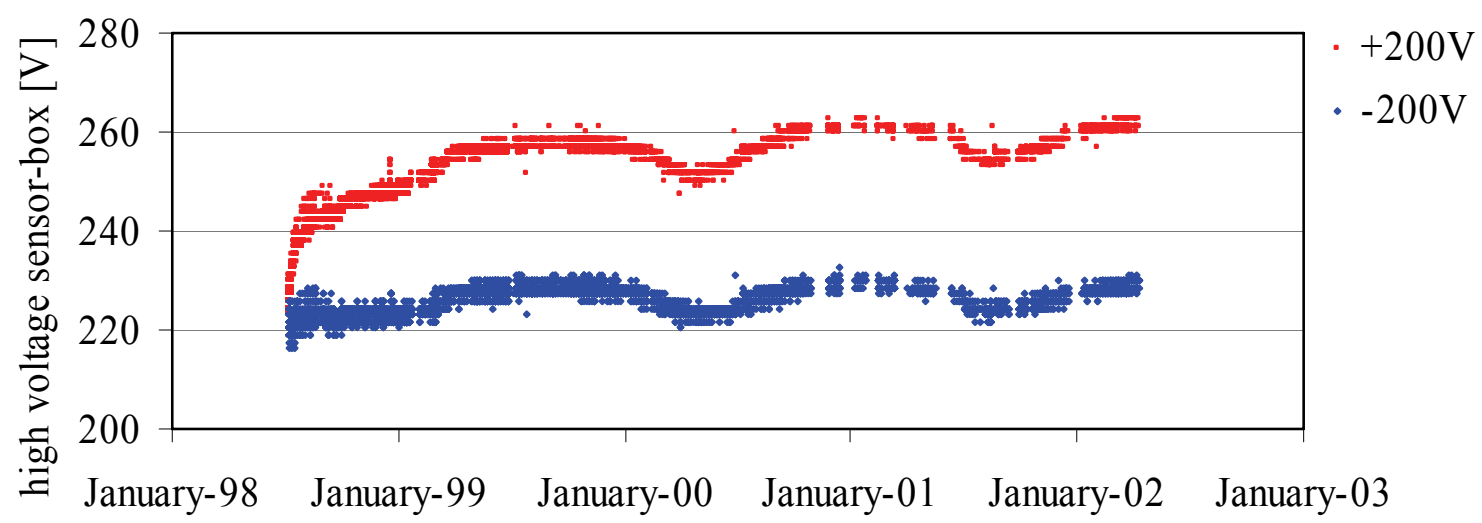

Figure 6-33: Power supply voltages and high-voltages

The power supply voltages of the MDC as derived from the impact housekeeping data. The MDC electronics box is powered by the $+5 \mathrm{~V}$ voltages, while the $\pm 12 \mathrm{~V}$ voltage powers the MDC sensor box high voltages. 


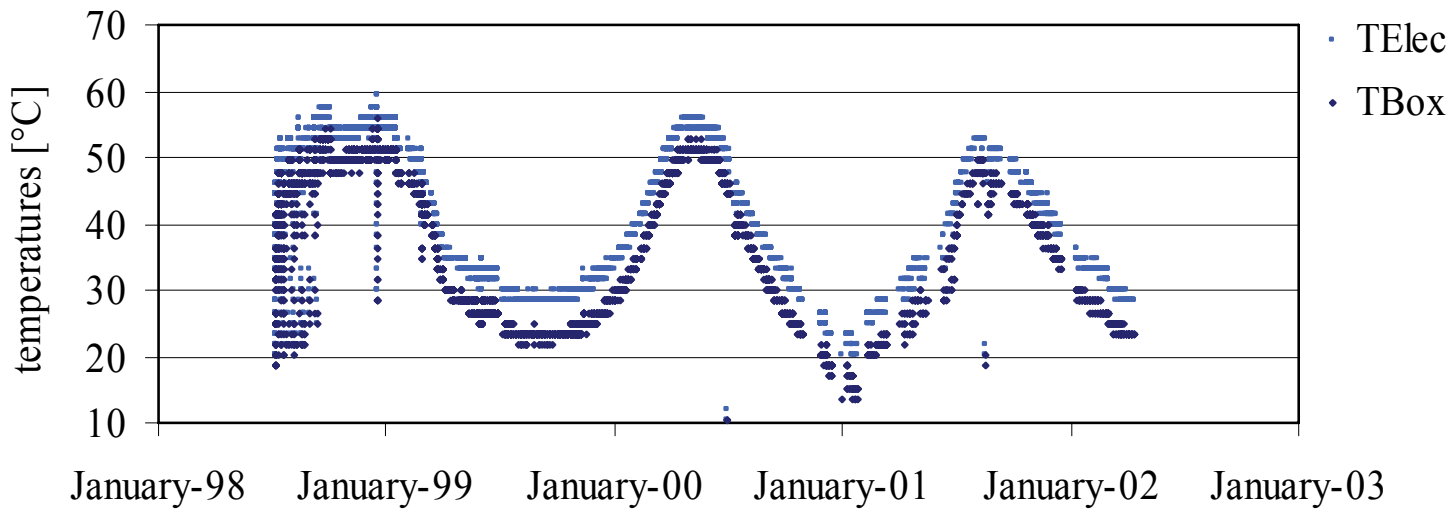

Figure 6-34: Temperatures of the MDC electronics- and sensor-box

The temperatures of the MDC experiment vary between $15^{\circ} \mathrm{C}$ and $60^{\circ} \mathrm{C}$ depending on the spacecraft's distance from the Sun.

Although the fluctuations of the $\pm 12 \mathrm{~V}$ power supply voltages cause even higher fluctuations of the sensor box high voltages and thus of the electrostatic field inside the box, these fluctuations have only little influence on the signal shape that is created by the charges an impacting particle produces. As it was mentioned in paragraph 3.1.2, the strength of the electrostatic field inside an impact ionization detector (and thus an MDC like sensor box) has measurable influence on the recorded signal until the high voltages reach $\approx 200 \mathrm{~V}$. A further rise of the voltages, as it can be observed in the MDC operating data, has only very little influence on the charge signal and thus on the further analysis results. The large difference between the $-200 \mathrm{~V}$ and the $+200 \mathrm{~V}$ values was caused by erroneous monitoring electronics, as it was proven soon after launch by measurements on the spare flight model by Naumann [Nau2000].

In summary, all parameters of the MDC experiment are within the ranges that ensure reliable operation and dust detection. The observed fluctuations of the power supply voltages and experiment temperatures are therefore not considered in the data analysis process and the scientific results that are presented in the following paragraphs.

Table 6-5 shows the MDC command log for the first day of the mission. Refer to Fischer [Fis 1998] for a detailed description of the different commands. From the MDC command log of the first 428 days of the mission, a total downtime of the MDC of 33 days was determined (by analyzing MDC_HV_OFF and MDC_HV_ON commands that switch the high voltage of the MDC sensor box), which gives a ratio of $7.7 \%$ overall downtime for this time span. During the interplanetary cruising phase from January 1999 to September 1999, downtime was $3 \%$. 
Table 6-5: MDC command log, first day of mission

\begin{tabular}{|c|c|c|c|c|c|c|}
\hline Date & ComCode & ComName & Transmit & Verifye & VerifyF & Verifys \\
\hline 10.07 .1998 & 80 & MDC_HV_ENA & $07: 22: 14$ & $07: 22: 19$ & $07: 22: 24$ & \#NV \\
\hline 10.07 .1998 & $\mathrm{CO}$ & $\mathrm{MDC} \_\mathrm{HV} \_\mathrm{ON}$ & $07: 22: 41$ & $07: 22: 46$ & $07: 22: 50$ & $\# \mathrm{NV}$ \\
\hline 10.07 .1998 & 01 & MDC_RST & $07: 23: 42$ & $07: 23: 47$ & $07: 23: 51$ & $\# N V$ \\
\hline 10.07 .1998 & 30 & MDC_CPU_RESET & $07: 24: 29$ & $07: 24: 34$ & $07: 24: 39$ & \#NV \\
\hline 10.07 .1998 & 30 & MDC_CLEAR_RAM & $07: 25: 06$ & $07: 25: 11$ & $07: 25: 16$ & $\# N V$ \\
\hline 10.07 .1998 & 30 & MDC_TEST_PULSE & $07: 25: 56$ & $07: 26: 01$ & $07: 26: 05$ & $\# N V$ \\
\hline 10.07 .1998 & 31 & MDC_TESTNCPOS & $07: 26: 44$ & $07: 26: 48$ & $07: 26: 53$ & \#NV \\
\hline 10.07 .1998 & 30 & MDC_TEST_PULSE & $07: 27: 19$ & $07: 27: 24$ & $07: 27: 29$ & $\# \mathrm{NV}$ \\
\hline 10.07 .1998 & 30 & MDC_TEST_PULSE & $07: 27: 46$ & $07: 27: 51$ & $07: 27: 56$ & $\# N V$ \\
\hline 10.07 .1998 & 30 & MDC_TEST_PULSE & $07: 28: 20$ & $07: 28: 25$ & $07: 28: 30$ & $\# N V$ \\
\hline 10.07 .1998 & 31 & MDC_TESTNC10P & $07: 28: 54$ & $07: 28: 59$ & $07: 29: 04$ & $\# N V$ \\
\hline 10.07 .1998 & 30 & MDC_TEST_PULSE & $07: 29: 17$ & $07: 29: 22$ & $07: 29: 26$ & $\# N V$ \\
\hline 10.07 .1998 & 30 & MDC_TEST_PULSE & $07: 29: 46$ & $07: 29: 50$ & $07: 29: 55$ & $\# \mathrm{NV}$ \\
\hline 10.07 .1998 & 30 & MDC_TEST_PULSE & $07: 30: 07$ & $07: 30: 12$ & $07: 30: 17$ & \#NV \\
\hline 10.07 .1998 & 31 & MDC_TESTNC10N & $07: 30: 47$ & $07: 30: 52$ & $07: 30: 56$ & $\# N V$ \\
\hline 10.07 .1998 & 30 & MDC_TEST_PULSE & $07: 31: 12$ & $07: 31: 16$ & $07: 31: 21$ & $\# N V$ \\
\hline 10.07 .1998 & 30 & MDC_TEST_PULSE & $07: 31: 38$ & $07: 31: 43$ & $07: 31: 48$ & \#NV \\
\hline 10.07 .1998 & 30 & MDC_TEST_PULSE & $07: 32: 01$ & $07: 32: 06$ & $07: 32: 11$ & $\# N V$ \\
\hline 10.07 .1998 & 31 & MDC_TESTNCNEG & $07: 32: 29$ & $07: 32: 34$ & $07: 32: 38$ & $\# N V$ \\
\hline 10.07 .1998 & 30 & MDC_TEST_PULSE & $07: 33: 05$ & $07: 33: 10$ & $07: 33: 14$ & $\# N V$ \\
\hline 10.07 .1998 & 30 & MDC_TEST_PULSE & $07: 33: 41$ & $07: 33: 45$ & $07: 33: 50$ & $\# N V$ \\
\hline 10.07 .1998 & 30 & MDC_TEST_PULSE & $07: 34: 07$ & $07: 34: 12$ & $07: 34: 17$ & $\# N V$ \\
\hline 10.07 .1998 & 30 & MDC_PWSON & $07: 34: 45$ & $07: 34: 50$ & $07: 34: 55$ & $\# N V$ \\
\hline 10.07 .1998 & 31 & MDC_SETTRM_12 & $07: 43: 31$ & $07: 43: 36$ & $07: 43: 41$ & $\# N V$ \\
\hline 10.07 .1998 & 31 & MDC_SETTRM_04 & $07: 47: 57$ & $07: 48: 02$ & $07: 48: 07$ & $\# N V$ \\
\hline 10.07 .1998 & 22 & DHU_MODE_CHNG & $07: 51: 27$ & $07: 51: 32$ & $07: 51: 37$ & $\# N V$ \\
\hline 10.07 .1998 & 30 & MDC_PWSOFF & $07: 54: 21$ & $07: 54: 26$ & $07: 54: 31$ & $\# N V$ \\
\hline 10.07 .1998 & 30 & MDC_PWSON & $08: 01: 32$ & $08: 01: 37$ & $08: 01: 42$ & $\# N V$ \\
\hline 10.07 .1998 & 31 & MDC_SETTRM_03 & $08: 02: 28$ & $08: 02: 32$ & $08: 02: 37$ & \#NV \\
\hline 10.07 .1998 & 30 & MDC_TEST_PULSE & $08: 03: 33$ & $08: 03: 38$ & $08: 03: 42$ & $\# N V$ \\
\hline 10.07 .1998 & 30 & MDC_TEST_PULSE & $08: 04: 04$ & $08: 04: 09$ & $08: 04: 14$ & $\# N V$ \\
\hline 10.07 .1998 & 31 & MDC_KO_CRITERIA_02 & $08: 04: 50$ & $08: 04: 55$ & $08: 05: 00$ & $\# \mathrm{NV}$ \\
\hline 10.07 .1998 & 31 & MDC_KO_CRITERIA_03 & $08: 10: 29$ & $08: 10: 33$ & $08: 10: 38$ & $\# N V$ \\
\hline 10.07 .1998 & 30 & MDC_TEST_PULSE & $08: 12: 45$ & $08: 12: 50$ & $08: 12: 55$ & $\# N V$ \\
\hline 10.07 .1998 & 22 & DHU_MODE_CHNG & $08: 13: 58$ & $08: 14: 03$ & $08: 14: 08$ & $\# N V$ \\
\hline 10.07 .1998 & 31 & MDC_SETTRM_04 & $08: 44: 02$ & $08: 44: 07$ & $08: 44: 12$ & $\# N V$ \\
\hline 10.07 .1998 & 30 & MDC_PWSOFF & $08: 44: 33$ & $08: 44: 38$ & $08: 44: 43$ & $\# \mathrm{NV}$ \\
\hline 10.07 .1998 & 31 & MDC_KO_CRITERIA_04 & $08: 45: 26$ & $08: 45: 31$ & $08: 45: 36$ & $\# N V$ \\
\hline 10.07 .1998 & 31 & MDC_CAL_TP_MIX & $08: 46: 01$ & $08: 46: 06$ & $08: 46: 11$ & $\# N V$ \\
\hline 10.07 .1998 & 31 & MDC_LIMIT $12 \mathrm{~V}$ & $08: 46: 36$ & $08: 46: 41$ & $08: 46: 46$ & $\# N V$ \\
\hline 10.07 .1998 & 22 & DHU_MODE_CHNG & $08: 47: 06$ & $08: 47: 11$ & $08: 47: 16$ & $\# N V$ \\
\hline 10.07 .1998 & 31 & MDC_KO_CRITERIA_02 & $08: 48: 47$ & $08: 48: 52$ & $08: 48: 57$ & $\# \mathrm{NV}$ \\
\hline 10.07 .1998 & 40 & $\mathrm{MDC} \_\mathrm{HV} \_\mathrm{OFF}$ & $10: 54: 24$ & $10: 54: 29$ & $10: 54: 33$ & \#NV \\
\hline 10.07 .1998 & 31 & MDC_SETTRM_13 & $10: 55: 13$ & $10: 55: 17$ & $10: 55: 22$ & $\# \mathrm{NV}$ \\
\hline 10.07 .1998 & 22 & DHU_MODE_CHNG & $11: 05: 59$ & $11: 06: 04$ & $11: 06: 08$ & $\# N V$ \\
\hline 10.07 .1998 & 80 & MDC_HV_ENA & $11: 07: 32$ & $11: 07: 37$ & $11: 07: 42$ & $\# N V$ \\
\hline 10.07 .1998 & $\mathrm{CO}$ & $\mathrm{MDC}_{-}^{-} \mathrm{HV}_{-} \mathrm{ON}$ & $11: 07: 55$ & $11: 08: 00$ & $11: 08: 04$ & \#NV \\
\hline 10.07 .1998 & 31 & MDC_SETTRM_04 & $13: 10: 37$ & $13: 10: 42$ & $13: 10: 46$ & $\# N V$ \\
\hline 10.07 .1998 & 22 & DHU_MODE_CHNG & $13: 10: 54$ & $13: 10: 59$ & $13: 11: 04$ & $\# \mathrm{NV}$ \\
\hline 10.07 .1998 & 30 & MDC_PWSON & $13: 12: 24$ & $13: 12: 29$ & $13: 12: 33$ & \#NV \\
\hline 10.07 .1998 & 31 & MDC_SETTRM_03 & $13: 12: 50$ & $13: 12: 55$ & $13: 13: 00$ & $\# \mathrm{NV}$ \\
\hline 10.07 .1998 & 22 & DHU_MODE_CHNG & $13: 21: 15$ & $13: 21: 20$ & $13: 21: 25$ & $\# N V$ \\
\hline 10.07 .1998 & 31 & MDC_KO_CRITERIA_05 & $13: 33: 49$ & $13: 33: 54$ & $13: 33: 59$ & $\# \mathrm{NV}$ \\
\hline 10.07 .1998 & 31 & MDC_SETTRM_13 & $13: 34: 46$ & $13: 34: 51$ & $13: 34: 56$ & $\# N V$ \\
\hline 10.07 .1998 & 22 & DHU_MODE_CHNG & $13: 35: 18$ & $13: 35: 22$ & $13: 35: 27$ & $\# N V$ \\
\hline 10.07 .1998 & 30 & $\mathrm{MDC}{ }_{-} \mathrm{PWSOF}$ & $13: 37: 23$ & $13: 37: 28$ & $13: 37: 33$ & $\# N V$ \\
\hline 10.07 .1998 & 30 & MDC_PWSON & $13: 39: 13$ & $13: 39: 18$ & $13: 39: 22$ & \#NV \\
\hline 10.07 .1998 & 22 & DHU_MODE_CHNG & $13: 39: 51$ & $13: 39: 56$ & $13: 40: 00$ & $\# N V$ \\
\hline 10.07 .1998 & 31 & MDC_KO_CRITERIA_04 & $13: 42: 32$ & $13: 42: 36$ & $13: 42: 41$ & \#NV \\
\hline
\end{tabular}




\subsection{Particles detected in the vicinity of Earth and Moon}

During the first half year of the mission, NOZOMI was in a high elliptic orbit around Earth with perigee at around $8000 \mathrm{~km}$ and apogee beyond the orbital radius of the Moon. Therefore, it was expected that the MDC would detect interplanetary dust grains encountering the Earth as well as dust grains and space debris particles that are on bound orbits around the Earth. For this reason, the particles detected during the first half year of the mission were investigated separately from the particles that were detected during NOZOMI's interplanetary cruising phase. The results are presented and discussed in this paragraph.

The orbital elements inclination and eccentricity of the detected particles were calculated with respect to the gravitational force only, although most particles discussed in this paragraph experience additional forces like radiation pressure due to their size.

\subsubsection{Overview}

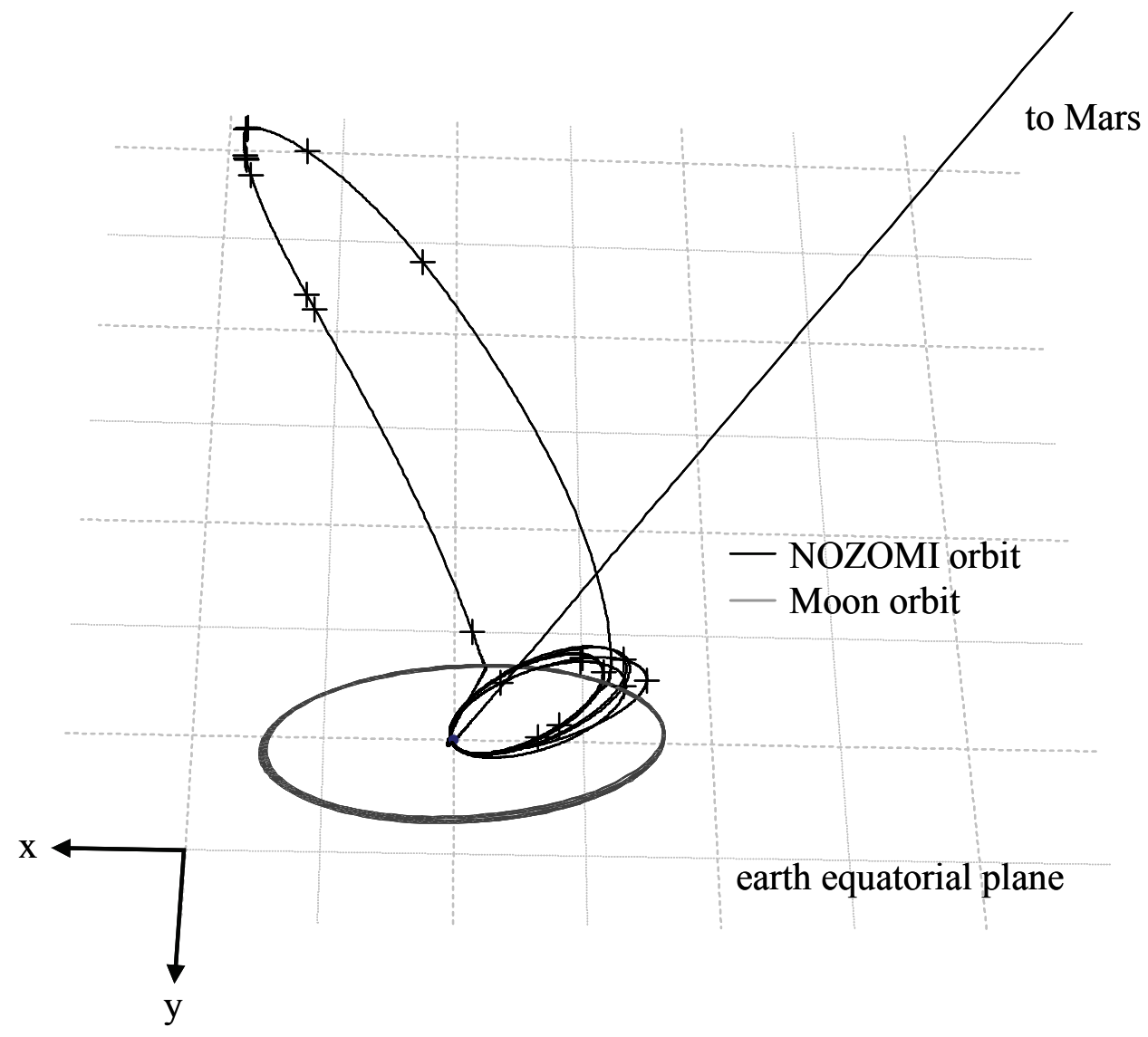

Figure 6-35: Spatial distribution of the particles in the Earth-Moon system

Plus signs indicate the orbital positions of the particles detected in the year 1998 when NOZOMI was in orbit around Earth.

NOZOMI's first mission phase lasted from July 1998 until January 1999. About 10000 signals were triggered, recorded, qualified and downloaded during this time. Manual 
screening of these bulk signals was applied to separate noise signals from potential impact signals. 21 good signals were identified as impacts, 20 were analyzed and are discussed here, see Table 6-6. One signal (98111001-1) was identified as impact, but the electron channel EC was out of range, hence a correct determination of rise time and charge amplitude, both needed for further analysis, was not possible.

In Figure 6-35, the NOZOMI orbit during the first half-year and the orbital positions of the detected particles are shown. As can already be seen in this view, all particles were detected far away from Earth near the apogee of the NOZOMI orbits. This is discussed later in this paragraph.

Figure 6-36 shows mass vs. impact speed of the detected particles. Lowest impact speed was $7.7 \mathrm{~km} / \mathrm{sec}$, highest was $50.9 \mathrm{~km} / \mathrm{sec}$. The detector limits shown in this graph are mean values from all impact positions inside the MDC sensor box.

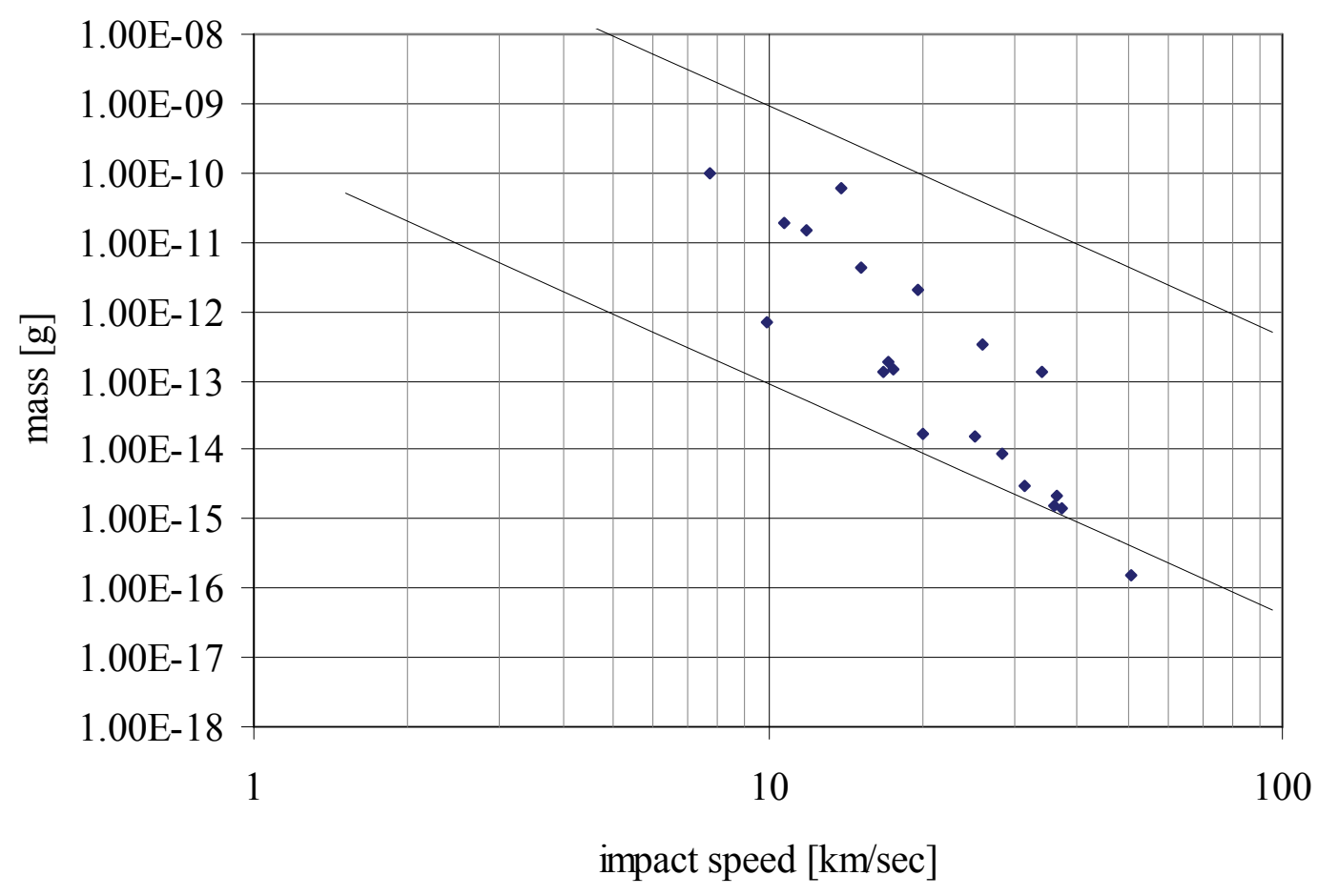

Figure 6-36: Mass vs. imp. speed of the particles in the Earth-Moon system The two lines show the detector limits. The upper limit is defined by the maximum charge that can be processed by the charge amplifiers and the A/D converters without overload; the lower limit is defined by the sensitivity of the instrument that is set by a certain trigger level. . 
Table 6-6: Detected particles in the vicinity of Earth and Moon

\begin{tabular}{|c|c|c|c|c|c|c|c|}
\hline File & Imp. No. & Date [UT] & Time $[\mathrm{UT}]$ & Pos. $\mathrm{X}[\mathrm{km}]$ & Pos. Y [km] & Pos. Z [km] & Orb. Rad. [km] \\
\hline Sp. $X[\mathrm{~km} / \mathrm{s}]$ & Sp. Y $[\mathrm{km} / \mathrm{s}]$ & Sp. Z [km/s] & Abs. Sp. $[\mathrm{km} / \mathrm{s}]$ & Imp. Sp. $[\mathrm{km} / \mathrm{s}]$ & Mass [g] & Incl. [deg] & Ecc. \\
\hline 98071101 & 26 & $\begin{array}{l}1998-07-10 \\
\end{array}$ & $15: 08: 21$ & 47071711 & -144794138 & 28784 & $\overline{152253372}$ \\
\hline 54.31 & 12.79 & -16.65 & 58.23 & 31.57 & 3.0E-15 & 16.67 & 2.88 \\
\hline 98071601 & 3 & 1998-07-16 & 03:52:01 & 60278018 & -139746168 & 63454 & 152192098 \\
\hline 12.70 & 15.17 & -2.74 & 19.97 & 15.24 & 4.4E-12 & 8.81 & 0.66 \\
\hline 98073001 & 5 & 1998-07-28 & $23: 23: 54$ & 88381925 & -123617666 & 59061 & 151962809 \\
\hline 22.49 & 24.85 & -0.53 & 33.53 & 7.69 & 1.0E-10 & 0.92 & 0.35 \\
\hline 98073101 & 29 & 1998-07-31 & $06: 37: 46$ & 93191670 & -119958656 & 49421 & 151903815 \\
\hline 30.52 & 47.47 & -18.68 & 59.45 & 34.14 & $1.3 \mathrm{E}-13$ & 19.33 & 2.91 \\
\hline 98080701 & 3 & 1998-08-05 & $11: 14: 40$ & 102884667 & -111488040 & 27355 & 151706423 \\
\hline 23.22 & 25.17 & -19.06 & 39.19 & 20.04 & $1.7 \mathrm{E}-14$ & 29.18 & 0.76 \\
\hline 98082201 & 8 & 1998-08-22 & $00: 48: 36$ & 129049547 & -78765918 & 55978 & $\overline{151188156}$ \\
\hline 13.97 & 24.87 & -25.21 & 38.07 & 25.34 & $1.6 \mathrm{E}-14$ & 41.49 & 0.65 \\
\hline 98090501 & 14 & 1998-09-02 & $21: 28: 35$ & 141712211 & -51326819 & 44935 & 150720918 \\
\hline 7.19 & 25.25 & -9.09 & 27.78 & 9.89 & $6.7 \mathrm{E}-13$ & 19.14 & 0.14 \\
\hline 98092201 & 9 & 1998-09-21 & $10: 30: 04$ & 149955432 & -4648768 & -13433 & 150027474 \\
\hline-32.72 & 40.19 & -15.35 & 54.05 & 37.41 & $1.4 \mathrm{E}-15$ & 21.41 & 1.90 \\
\hline 98092201 & 23 & 1998-09-22 & $00: 23: 40$ & 149929104 & -3207880 & -4758 & 149963418 \\
\hline-1.13 & 57.53 & 21.78 & 61.52 & 35.85 & $1.6 \mathrm{E}-15$ & 20.75 & 3.27 \\
\hline 98101401 & 21 & 1998-10-13 & $03: 45: 06$ & 140661832 & 48872548 & 470810 & 148911042 \\
\hline-9.00 & 14.04 & 33.99 & 37.86 & 36.46 & $2.1 \mathrm{E}-15$ & 64.51 & 0.61 \\
\hline 98102701 & 37 & 1998-10-26 & $10: 07: 48$ & 125317767 & 79079706 & 597923 & $\overline{148184007}$ \\
\hline-14.26 & 18.65 & 27.95 & 36.50 & 28.64 & $8.5 \mathrm{E}-15$ & 50.09 & 0.49 \\
\hline 98110901 & 17 & 1998-11-08 & $23: 02: 03$ & 102744228 & 105734878 & 623866 & 147433476 \\
\hline-13.78 & 15.62 & 14.26 & 25.24 & 17.15 & $1.9 \mathrm{E}-13$ & 34.45 & 0.30 \\
\hline 98110901 & 29 & 1998-11-08 & $11: 38: 43$ & 103639158 & 104886737 & 624512 & 147454036 \\
\hline-16.60 & 18.92 & 15.88 & 29.76 & 16.82 & $1.3 \mathrm{E}-13$ & 32.30 & 0.06 \\
\hline 98111001 & 1 & 1998-11-09 & $10: 35: 00$ & 101823105 & 106580827 & 623077 & 147403547 \\
\hline N/A & $\mathrm{N} / \mathrm{A}$ & N/A & N/A & $\mathrm{N} / \mathrm{A}$ & $\mathrm{N} / \mathrm{A}$ & N/A & N/A \\
\hline 98111701 & 8 & 1998-11-17 & $04: 23: 19$ & 86086726 & 119183754 & 591866 & 147023950 \\
\hline-33.15 & 8.81 & 1.77 & 34.34 & 11.86 & $1.5 \mathrm{E}-11$ & 3.26 & 0.46 \\
\hline 98111801 & 1 & 1998-11-17 & $17: 02: 59$ & 84951085 & 119965169 & 588452 & 146998894 \\
\hline-33.33 & 17.81 & 10.99 & 39.36 & 13.92 & $6.0 \mathrm{E}-11$ & 16.37 & 0.72 \\
\hline 98111801 & 6 & 1998-11-18 & $00: 35: 19$ & 84273081 & 120428155 & 586334 & 146987198 \\
\hline-44.83 & 0.58 & 5.17 & 45.13 & 26.09 & $3.3 \mathrm{E}-13$ & 8.10 & 1.18 \\
\hline 98112101 & 6 & 1998-11-20 & $10: 39: 41$ & 78952092 & 123855597 & 568035 & 146880783 \\
\hline-20.16 & 10.53 & 7.40 & 23.92 & 10.80 & $1.9 \mathrm{E}-11$ & 18.10 & 0.37 \\
\hline 98120201 & 17 & 1998-12-01 & $18: 58: 50$ & 52215696 & 136913926 & 431437 & 146533574 \\
\hline-33.56 & 13.15 & 16.36 & 39.58 & 17.46 & $1.5 \mathrm{E}-13$ & 24.42 & 0.73 \\
\hline 98120301 & 9 & 1998-12-02 & $18: 41: 39$ & 49768815 & 137804201 & 415244 & 146516570 \\
\hline-13.20 & 4.69 & 47.96 & 49.96 & 50.90 & $1.6 \mathrm{E}-16$ & 73.72 & 1.76 \\
\hline 98121801 & 35 & 1998-12-17 & 10:17:34 & 12054841 & 146232969 & 65009 & 146729018 \\
\hline-30.69 & 4.83 & 19.20 & 36.52 & 19.65 & $2.0 \mathrm{E}-12$ & 31.78 & 0.48 \\
\hline
\end{tabular}

Table 6-6 shows the complete data of the 21 identified particles during the mission period from July to December 1998. More details for each data set/particle including signal shape and housekeeping information can be found in Appendix C.

In Figure 6-37 and Figure 6-38, mass vs. absolute speed of the detected particles in the eeq and eec system are shown. Absolute speed was derived from the particles' impact speed and NOZOMI orbital and attitude data as described in paragraph 6.2.3. Since the speed of the NOZOMI spacecraft in the eeq system (several km/sec down to $0.1 \mathrm{~km} / \mathrm{sec}$ ) is far below the impact speed of the particles (several $\mathrm{km} / \mathrm{sec}$ up to $90 \mathrm{~km} / \mathrm{sec}$ ), Figure 6-37 differs only slightly from Figure 6-36. 


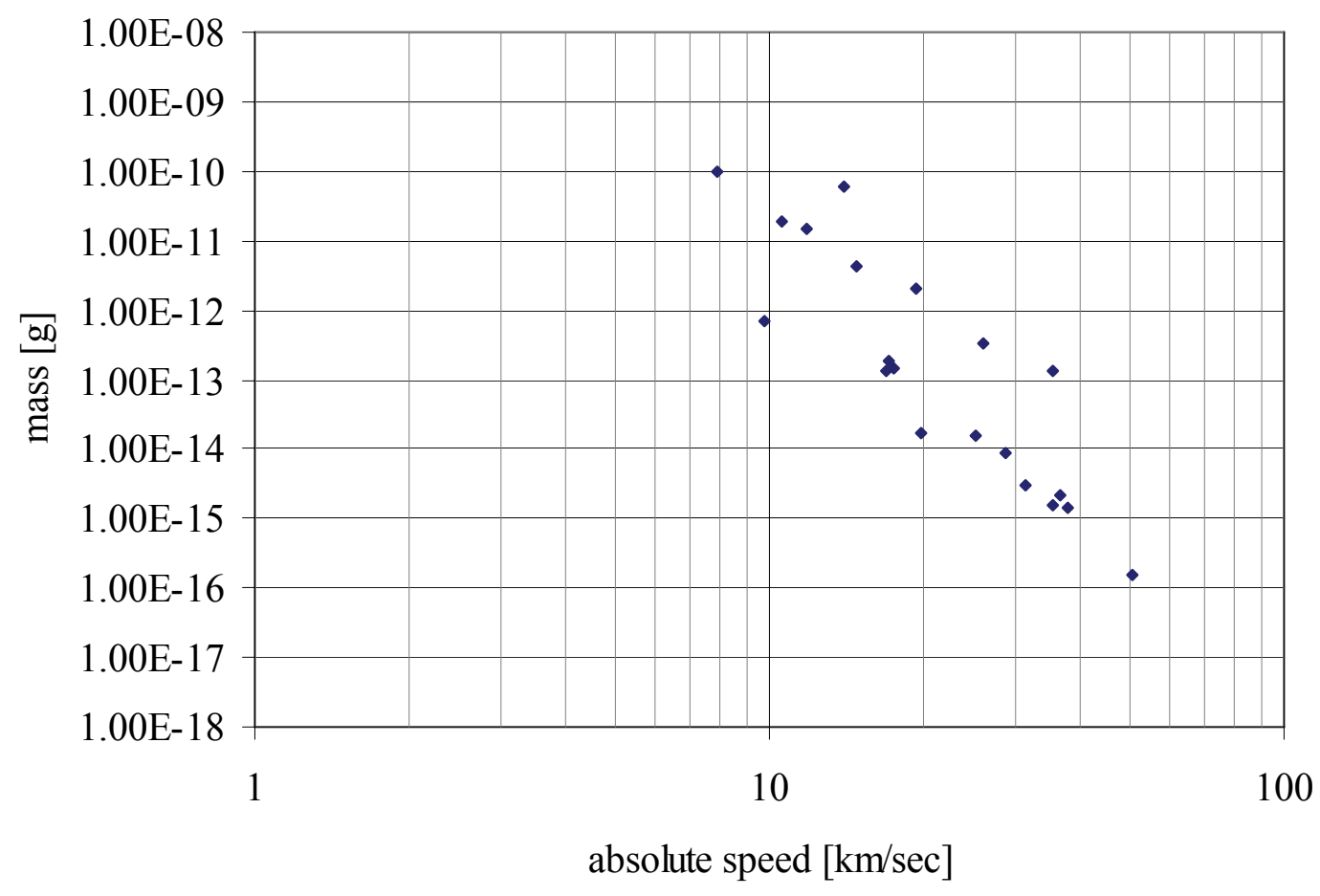

Figure 6-37: Mass vs. abs. speed of the particles in the Earth-Moon system The absolute speed vector of the particles was determined from the flight direction of the particles, impact speed and the NOZOMI attitude.

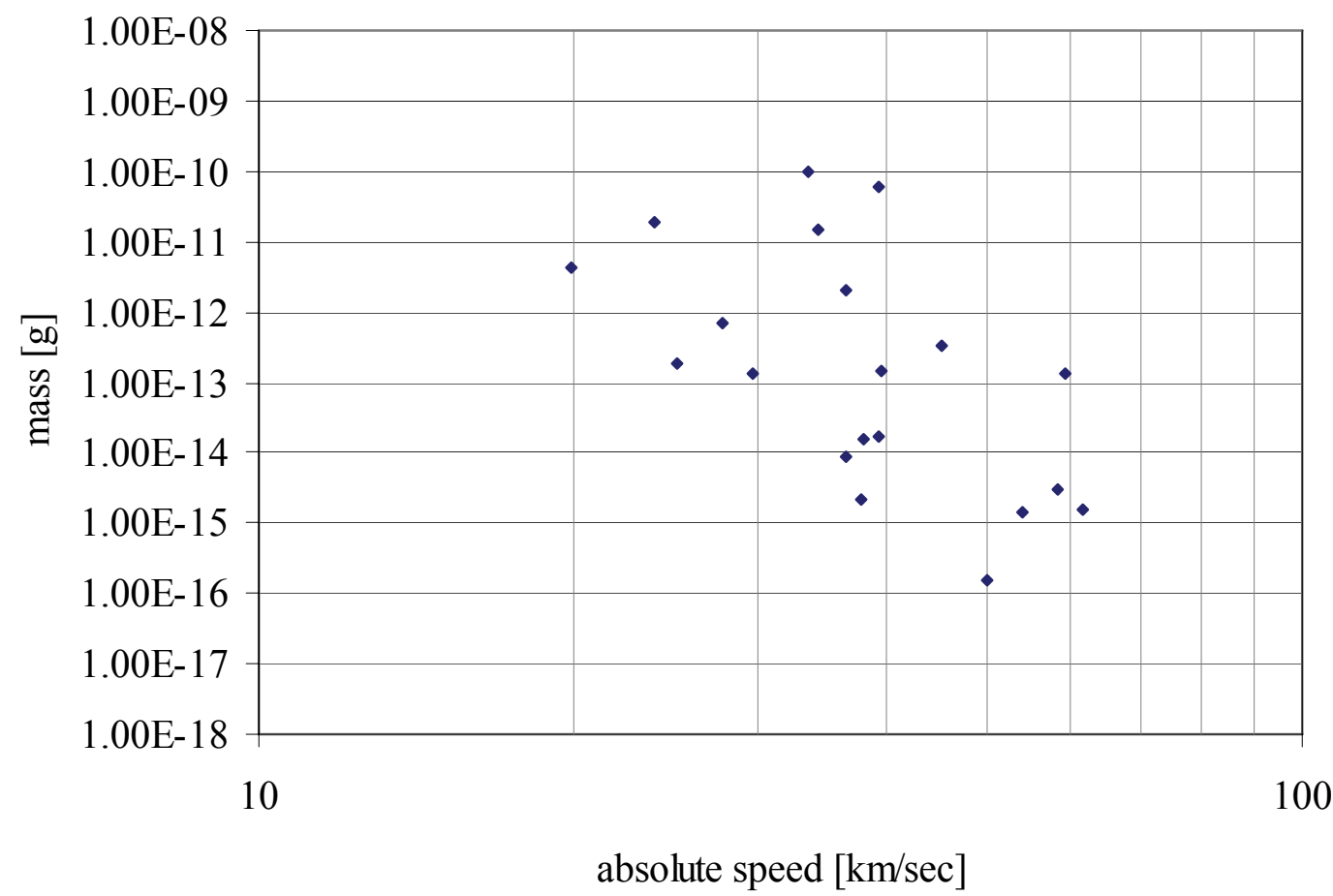

Figure 6-38: Mass vs. abs. speed of the particles in the eec system The absolute speed vector of the particles was determined from the flight direction of the particles, impact speed and NOZOMI attitude. 


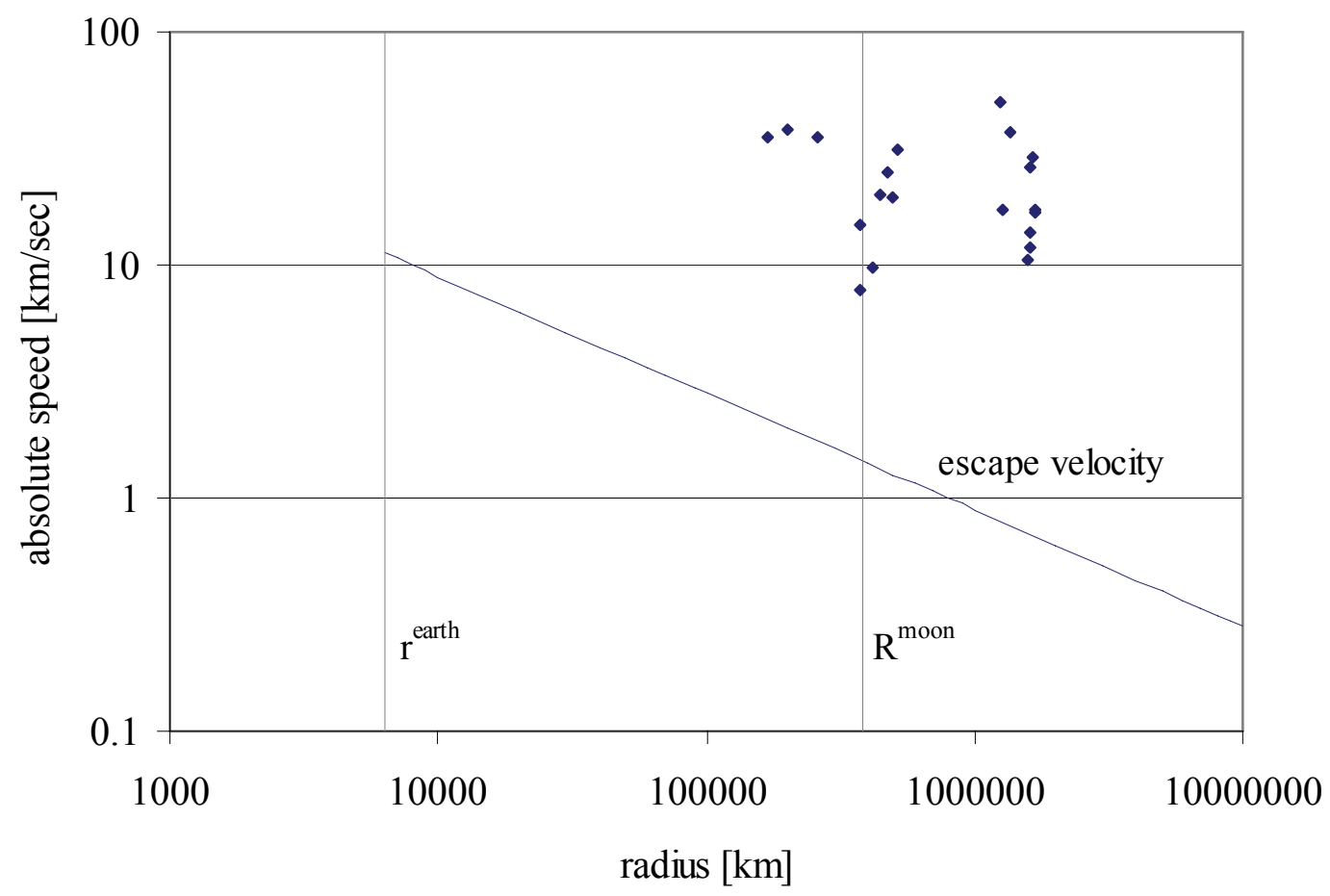

Figure 6-39: Orbital radius vs. abs. speed of the particles in the eeq system The absolute speed of all particles is far beyond the escape speed at the given distance of the particles.

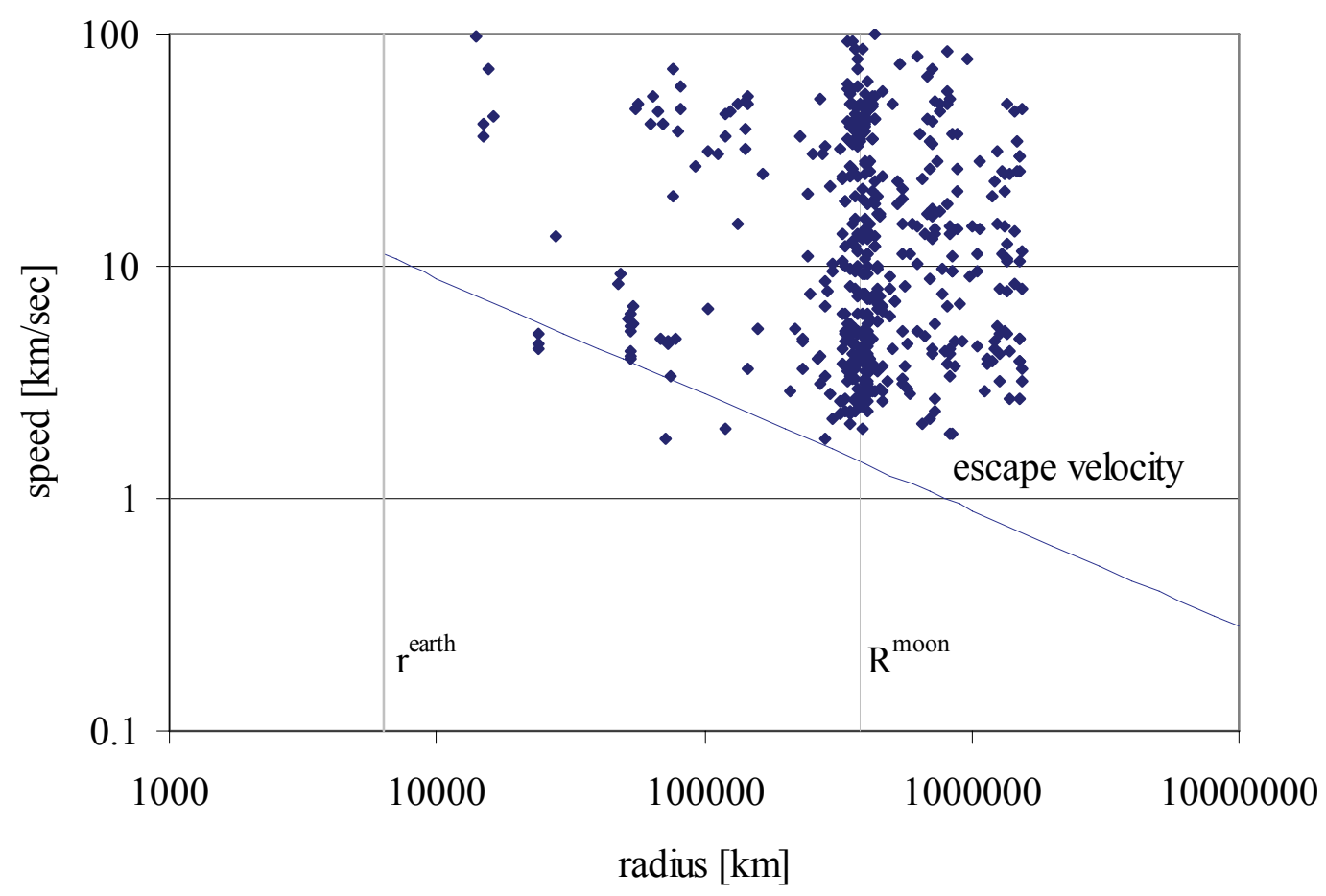

Figure 6-40: Orbital radius vs. abs. speed of the HITEN particles The speed of most particles detected by the MDC-HITEN is also beyond the escape speed at the given distance of the particles. 
As can be seen in Figure 6-37, absolute speed in the eeq system is higher than $8 \mathrm{~km} / \mathrm{sec}$ for all particles, and exceeds the escape speed from the Earth orbit for NOZOMI's all orbital distances from Earth. The absolute speed of the particles and the escape speed are compared in Figure 6-39. All particles' speed is far beyond the escape speed at the given orbital radius. For this reason, all particles detected during the first half year of the mission are of interplanetary or interstellar origin. Also, most particles detected by the MDC-HITEN during its three year mission in the Earth-Moon system are of interplanetary or interstellar origin, see Figure 6-403.

Figure 6-41 shows the number of detected particles per radius interval during the first half year of the mission. All particles were detected at orbital radii between $100000 \mathrm{~km}$ and $600000 \mathrm{~km}$, or between 1 million $\mathrm{km}$ and 2 million $\mathrm{km}$, respectively. Although the accumulation of orbital radii of the particles around the mean orbital distance of the Moon (which is $384000 \mathrm{~km}$ ) may suggest that there is some correlation of these dust particles with a possible dust ring around the Earth in the distance of the Moon, this cannot be the case because of the absolute speed of the particles discussed above. In addition, no increase of detection rate was registered during the two Moon swing-by maneuvers. The particles detected by the MDC-HITEN show a similar distribution due to comparable orbital characteristics, although the MDC-HITEN detected particles in nearly all radius intervals due to its much longer mission time in the Earth-Moon system, see Figure 6-42.

The explanation of the accumulated impact rates at the two orbital radius intervals is the duration of stay of NOZOMI in these specific intervals, see Figure 6-25. The duration of stay of NOZOMI in the radius interval from $100000 \mathrm{~km}$ to $600000 \mathrm{~km}$ is $49 \%$ of the mission time, and $39 \%$ in the interval from 1 million $\mathrm{km}$ to 2 million $\mathrm{km}$. The duration of stay was only $3 \%$ of the mission time below $100000 \mathrm{~km}$, and $8 \%$ of the mission time between $600000 \mathrm{~km}$ and 1 million $\mathrm{km}$.

\footnotetext{
${ }^{3}$ Speed is given as relative speed, since no absolute speed data in the Earth centered coordinate system was available for MDC-HITEN data. Absolute speed differs by the speed of the HITEN satellite at the utmost, which is $1.12 \mathrm{~km} / \mathrm{sec}$ in average.
} 


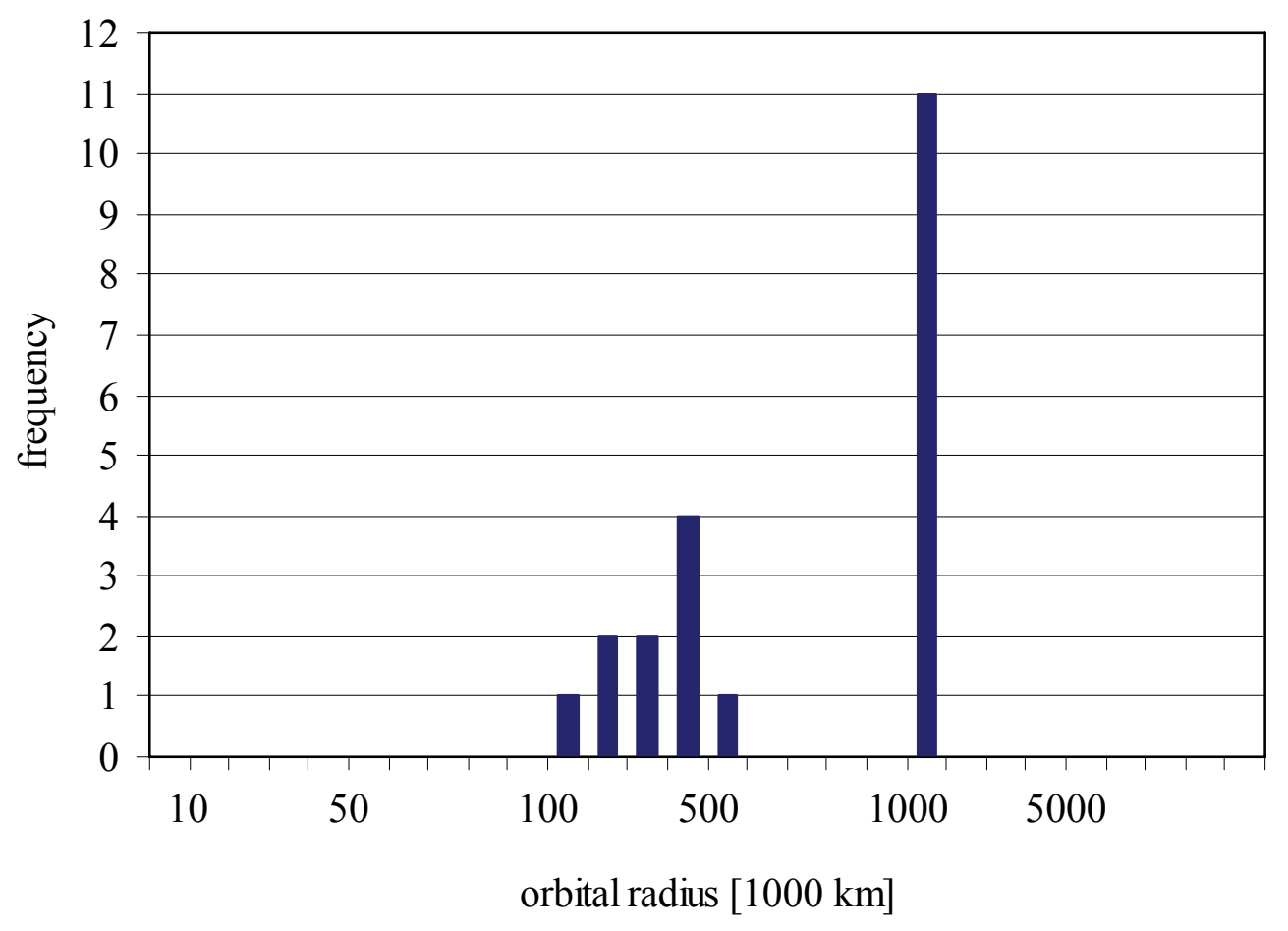

Figure 6-41: Number of particles per radius interval All detected particles are located within two radius intervals, $100000 \mathrm{~km}$ to $600000 \mathrm{~km}$ and $1000000 \mathrm{~km}$ to $2000000 \mathrm{~km}$, respectively.

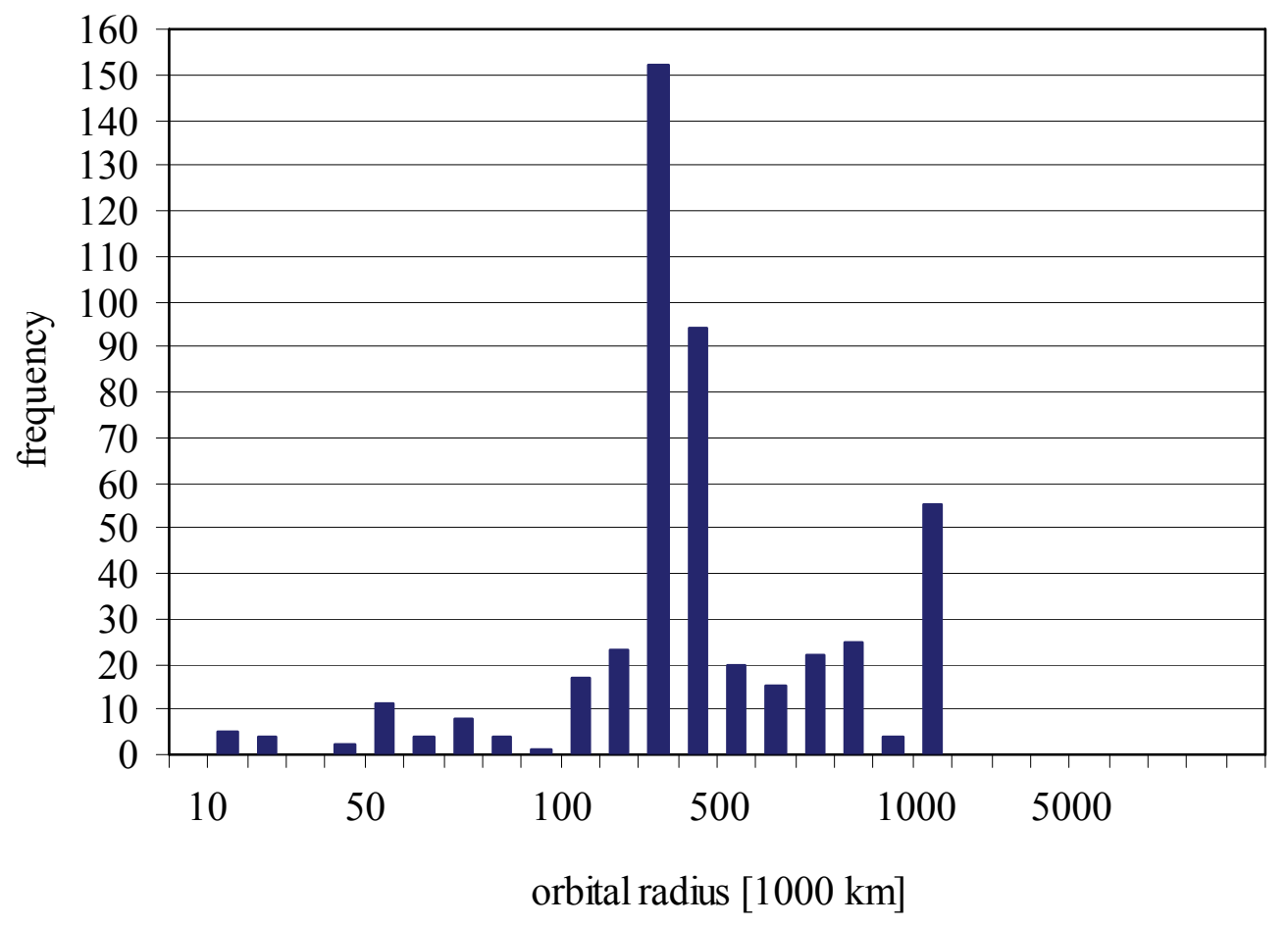

Figure 6-42: Number of particles per radius interval, HITEN

The number of particles detected by the MDC-HITEN has peaks at orbital radii from 300000 to $500000 \mathrm{~km}$, and at 1000000 to $2000000 \mathrm{~km}$. 
In summary, all particles detected during the first half year of the mission where NOZOMI was in an orbit around the Earth must be of interplanetary or interstellar origin. Therefore, these particles are merged with the particles that were detected in the interplanetary space and that are discussed in paragraph 6.4.

The MDC on NOZOMI did neither detect dust particles of natural origin that are bound to the Earth, nor space debris.

\subsubsection{Dust flux determination}

Although the MDC-NOZOMI did not detect particles that are bound to the Earth or may originate from the Earth-Moon system, the overall dust flux measured by the MDC during the mission time in the Earth-Moon system was determined and compared to previous flux measurements by the MDC-HITEN.

Between July 10, 1998 and January 6, 1999, the MDC-NOZOMI detected 21 particles during $\Delta T=180$ days measuring time. From these 21 particles, $N=18$ can be used for dust flux determination (two MLI impacts outside the sensor box and one GRID impact were ignored). This gives a total rate of 0.12 particles per day. With the given values of the MDC-NOZOMI sensor area $\left(A=0.145 \mathrm{~m}^{2}\right)$ and effective solidangle $(\Omega=0.81 \pi \mathrm{sr})$ the total flux calculates to

$$
\Phi=\frac{\pi}{A \cdot \Omega} \cdot \frac{N}{\Delta T}, \Phi_{\mathrm{MDC}}^{1998}=\frac{\pi}{0.0145 \mathrm{~m}^{2} \cdot 0.81 \pi} \cdot \frac{18}{180 \mathrm{~d}}=8.51 \frac{1}{\mathrm{~m}^{2} \cdot \mathrm{d}}
$$

Since the detection capabilities of the MDC-NOZOMI are strongly limited for small particles, dust flux was also determined for particles with mass heavier than $10^{-12} \mathrm{~g}$, which calculates to 2.84 particles $/\left(\mathrm{m}^{2} \cdot\right.$ day $)$.

For the MDC-HITEN, a total dust flux of up to $5.5 \cdot 10^{-4}$ particles $/\left(\mathrm{m}^{2} \cdot \mathrm{sec}\right)$ is given in literature, which is 48 particles $/\left(\mathrm{m}^{2} \cdot\right.$ day $)$, see Iglseder et al. [Igl1993a]. This value corresponds to $N=498$ detected particles during $\Delta T=1142$ days measuring time, with a sensor area of $A=0.1 \mathrm{~m}^{2}$ and an effective solid angle of $0.91 \pi \mathrm{sr}$ (calculated from $\beta=145^{\circ}$ aperture angle). In a later review of the MDC-HITEN data by Münzenmayer [Mün1995], 231 of the 498 particles were identified as most likely noise rather than impacts. From the remaining 267 particles, the overall dust flux calculates to $25.7 \mathrm{particles} /\left(\mathrm{m}^{2} \cdot\right.$ day $)$. Dust flux for particles heavier than $10^{-12} \mathrm{~g}$ calculates to 16.7 particles $/\left(\mathrm{m}^{2} \cdot\right.$ day $)$.

The results of dust flux measurements of MDC-HITEN and MDC-NOZOMI in the EarthMoon system are summarized in Table 6-7. 
Table 6-7: Dust flux measurements by MDC-HITEN and MDC-NOZOMI

\begin{tabular}{lll} 
Particle mass & all & $\mathrm{m}>10^{-12}$ \\
\hline Flux MDC-NOZOMI & 8.51 & 2.84 \\
Flux MDC-HITEN $^{1}$ & 48.0 & 33.5 \\
Flux MDC-HITEN & & 16.7 \\
\hline Flux values given as particles $/\left(\mathrm{m}^{2} \cdot\right.$ day $)$ & & \\
1 values by Iglseder & & \\
${ }^{2}$ values by Münzenmayer & &
\end{tabular}

Although the MDC-HITEN and MDC-NOZOMI instrument capabilities and measuring range are comparable, as well as the orbital trajectories of HITEN and NOZOMI with respect to the distances from the Earth, the dust flux determined from the MDC-HITEN data is significantly higher than the flux determined from the MDC-NOZOMI data. Even the more conservative values from Münzenmayer give a three to six time higher flux measured by MDC-HITEN than the values measured by MDC-NOZOMI. The sensitivity of the instruments is nearly equal, the trigger levels varied only slightly during the missions, for MDC-NOZOMI it was nearly constant around $1 \cdot 10^{-13} \mathrm{C}$, for the MDC-HITEN it was set from $2.3 \cdot 10^{-13} \mathrm{C}$ to $4.0 \cdot 10^{-13} \mathrm{C}$ after the first year in space. The mass distribution of the particles detected by the MDC-HITEN and the MDC-NOZOMI is also nearly similar to each other. The significant difference in dust flux can thus not be explained exclusively by the instrument capabilities.

There are two significant differences in the configurations of the satellites HITEN and NOZOMI that may be responsible for the difference in dust flux measurements. First, HITEN performed several Moon swing-by maneuvers and finally entered Moon orbit, while NOZOMI only performed two Moon and one Earth swing-by. Second, as already described in paragraphs 2.2.2 and 2.3.2, HITEN was a spin-stabilized satellite with spin axis perpendicular to the ecliptic plane, while NOZOMI was a spin-stabilized satellite with spin axis nearly parallel to the ecliptic plane. While the MDC-HITEN thus was scanning the whole latitude range of the ecliptic each revolution of the satellite, the MDC-NOZOMI scanning directions were determined mainly by the satellites attitude that was changed only a few times during the first half year of the mission. Within this work, it can not be proven if this difference in configuration can fully explain the difference in dust flux, since detailed information of the dust distribution in and around the Earth-Moon system would be necessary. 


\subsubsection{Characteristics of the detected particles}

On Nov. 18, 1998, NOZOMI encountered the Leonid particle stream. The Leonid particles originate from the comet 55P/Tempel-Tuttle and, as viewed from Earth, seem to come from the Leo constellation $\left(\alpha=153.67^{\circ}, \delta=21.70^{\circ}\right)$. The particles can be viewed as meteors from Nov. 14 to Nov 21 every year, with a maximum on Nov. 17. Leonid particles are expected to travel at high speed of around $70 \mathrm{~km} / \mathrm{sec}$, the average flight direction is longitude $\lambda=327^{\circ}$ and latitude $\beta=-10^{\circ}$ in Sun centered ecliptic coordinates, width of the particle stream is around $35,000 \mathrm{~km}$. NOZOMI crossed the Leonid particle stream about 1 day after the maximum flux was observed on Earth.

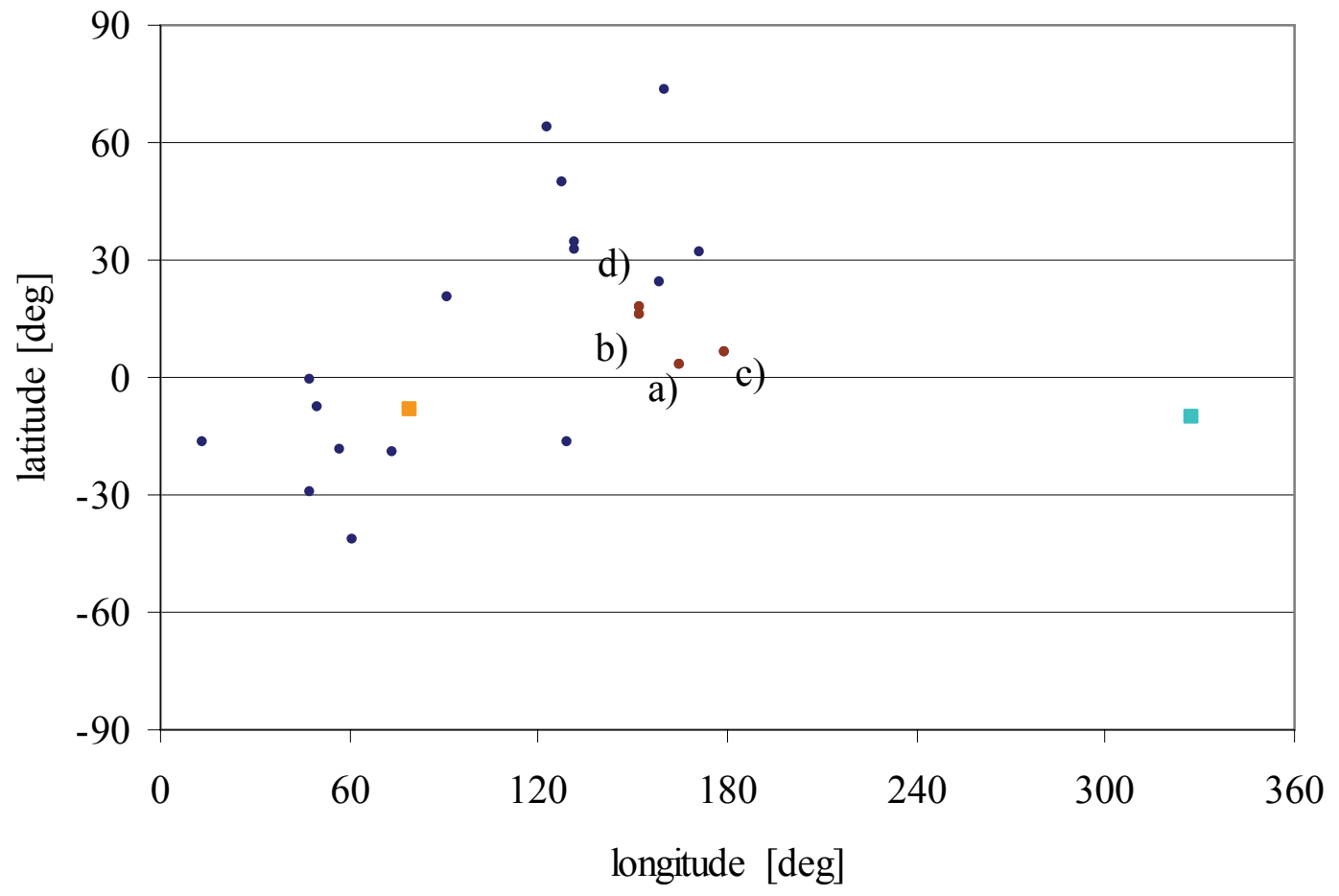

Figure 6-43: Flight direction of 1998 particles

Blue dots: Flight direction of particles detected in the first six months of MDC operation. Red dots: Flight direction of four particles detected during Leonid dust stream encounter on Nov. 18, 1998. Orange square: Mean flight direction of interstellar dust cloud particles. Turquoise square: Flight direction of Leonid dust stream particles.

Although the MDC high voltage was switched off during the encounter on Nov. 18, 4:00 UT to avoid possible damage to the experiment, a significant high number of particles were detected in Nov. 1998 that even show a correlation in flight direction, see Figure 6-43. 
Table 6-8: Particles detected around Nov. 18, 1998

\begin{tabular}{lcccrrrr}
\hline Label & File & Imp. No. & $\begin{array}{c}\text { Date } \\
{[\mathrm{UT}]}\end{array}$ & $\begin{array}{r}\text { Abs. Speed } \\
{[\mathrm{km} / \mathrm{sec}]}\end{array}$ & $\begin{array}{r}\text { Mass } \\
{[\mathrm{g}]}\end{array}$ & $\begin{array}{r}\text { Latitude } \\
{[\mathrm{deg}]}\end{array}$ & $\begin{array}{r}\text { Longitude } \\
{[\mathrm{deg}]}\end{array}$ \\
\hline a) & 98111701 & 8 & $17-$-Nov-98 & 11.78 & $1.5 \mathrm{E}-11$ & 2.96 & 165.12 \\
b) & 98111801 & 1 & $17-$ Nov-98 & 13.89 & $6.0 \mathrm{E}-11$ & 16.22 & 151.88 \\
c) & 98111801 & 6 & $18-$-Nov-98 & 26.01 & $3.3 \mathrm{E}-13$ & 6.58 & 179.26 \\
d) & 98112101 & 6 & 20-Nov-98 & 10.61 & $1.9 \mathrm{E}-11$ & 18.02 & 152.43 \\
\hline
\end{tabular}

However, for all four particles detected by the MDC around Nov. 18, 1998 (see Table 6-8) neither traveling speed nor flight direction fit to the Leonid dust particle stream, see Figure 6-43. In contrary, the detected particles nearly show anti-Leonid direction, and thus cannot be identified as Leonid dust particles. Nevertheless, a significant influence of the Leonid particle stream on the lunar sodium tail during the 1998 encounter was observed [Smi1999]. This gives evidence that the significant high number of particles detected by the MDC may result from other environmental influences such as collisions of larger particles of the Leonid dust stream with the Moon that produce secondary dust particles, see Sasaki [Sas2002]. The orbital configuration of Earth, Moon and NOZOMI, where NOZOMI was located behind the Moon as seen in the direction of the Leonid particle stream and the Earth-Moon system was moving nearly against the direction of the Leonid particle stream, though makes this explanation unlikely. 


\subsection{Particles detected in the interplanetary space}

After leaving Earth orbit on December 21, 1998, NOZOMI was in an orbit around the sun with perigee at 1.0 AU and apogee at $1.44 \mathrm{AU}$. The MDC was in operation during the whole mission time in the interplanetary space, and was expected to detect interplanetary and interstellar dust particles. Until the system failure on April 24, 2002, NOZOMI completed 3 1/2 orbits.

\subsubsection{Overview}

NOZOMI's interplanetary cruising phase lasted from January 1999 until April 2002 (in fact, NOZOMI continued its interplanetary orbit, but in April 2002, telemetry was lost and data transmission stopped, see chapter 2.3). About 11000 signals were triggered, recorded, qualified and downloaded.

To separate noise signals from potential impact signals, a neural network as described in chapter 4.3 was used. The chosen topology was a network with two hidden layers containing 11 and 15 neurons, as it came out as optimal topology in the evaluation process described in chapter 4.3. As training data for this neural network, all during data review manually identified impacts from the year 1998, a number of signals identified as impacts during the first data analysis by Münzenmayer and Naumann from the 1999 and 2000 data, and a number of signals that could not be identified clearly as impact or noise signals were used (the latter signals were included into the training data to ensure that no potential impact signals are lost by the screening process). An equal number of noise signals required for training of the network was randomly chosen from the MDC noise data. In this configuration, the neural network identified 288 of the more than 11000 signals that were downloaded from the MDC during the years 1999 to 2002 as possible impacts. Since all training data signals from the years 1999 and 2000 were included in these 288 possible impact signals identified by the neural network, it can be assumed that these 288 signals contain all possible impact signals from the unreviewed 1999 and 2000 data and the previously not analyzed 2001 and 2002 data. Also, manual random examination and cross-checking of a number of downloaded data sets did not give any new possible impacts identified by the experimenter that were not included in the 288 possible impacts identified by the neural network. Finally, the 288 possible impact signals were examined carefully by the experimenter, and a remainder of 77 good signals was identified doubtlessly as impact signals.

Altogether 98 impacts signals (77 good signals plus all 21 signals detected during the EarthMoon cruising phase which are all assumed to be of interplanetary origin due to the high distances from Earth and orbital speeds exceeding the escape speed from the Earth by far) were identified and analyzed and are discussed here. From these 98 signals, 13 were identified as impacts onto the MDC sensor box shielding grid (for these signals, no mass and speed determination was possible due to a lack of calibration data), and 5 were not analyzed due to charge overload in one or more channels. Particle mass and impact speed were determined for the remaining 80 signals. The results are summarized in Table 6-9, Table 6-10 and Table 6-11, see also Table 6-6 for the particles detected during the first half year. 
All orbital coordinates and elements in the following paragraphs are given in the Sun centered Earth ecliptic (eec) coordinate system.

Again, the orbital elements inclination, eccentricity and semi major axis of the detected particles were calculated with respect to the gravitational force only, although most particles discussed in this paragraph experience additional forces like radiation pressure due to their size. The distributions of the orbital elements shown in this chapter are discussed without respect to the simulations shown in paragraph 6.2.3.

In Figure 6-44, the NOZOMI orbit during the interplanetary cruising phase and the orbital positions of the detected particles are shown. The higher number of particles detected north of the ecliptic plane results from the fact that the northern part of the orbit was flown through three times and the southern part only two times until data transmission stopped on April 24, 2002.

Figure 6-45 shows mass vs. impact speed of all detected particles including those from the first half year. Lowest impact speed was $3.0 \mathrm{~km} / \mathrm{sec}$, highest was $69.6 \mathrm{~km} / \mathrm{sec}$. The detector limits shown in this graph are mean values from all impact positions inside the MDC sensor box.

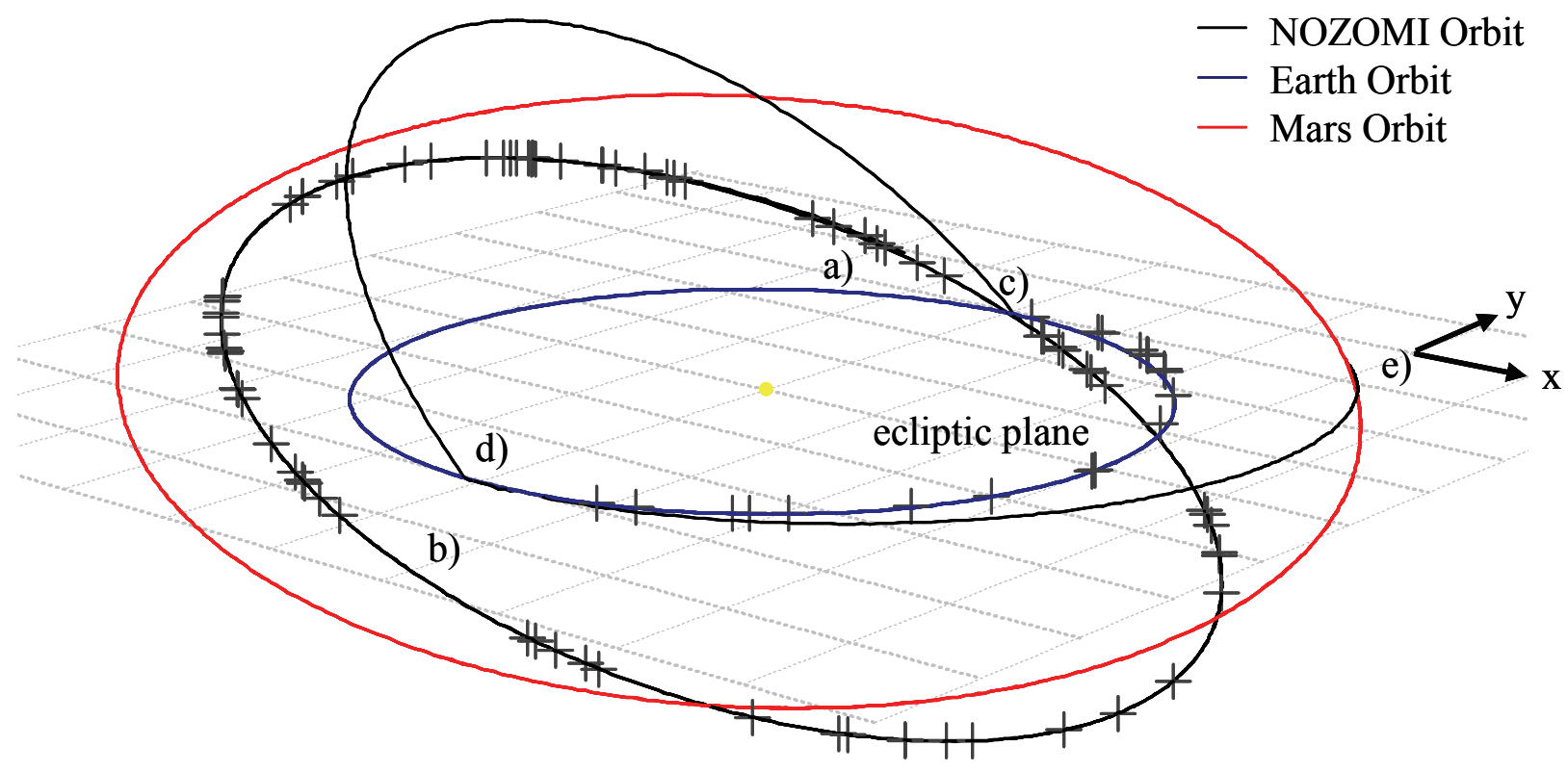

Figure 6-44: Spatial distribution of the particles in the interplanetary space

Mission events: a) Injection into Sun orbit, b) System failure (end of data transmission), c) $1^{\text {st }}$ Earth swing-by, d) $2^{\text {nd }}$ Earth swing-by, e) Mars fly-by. 


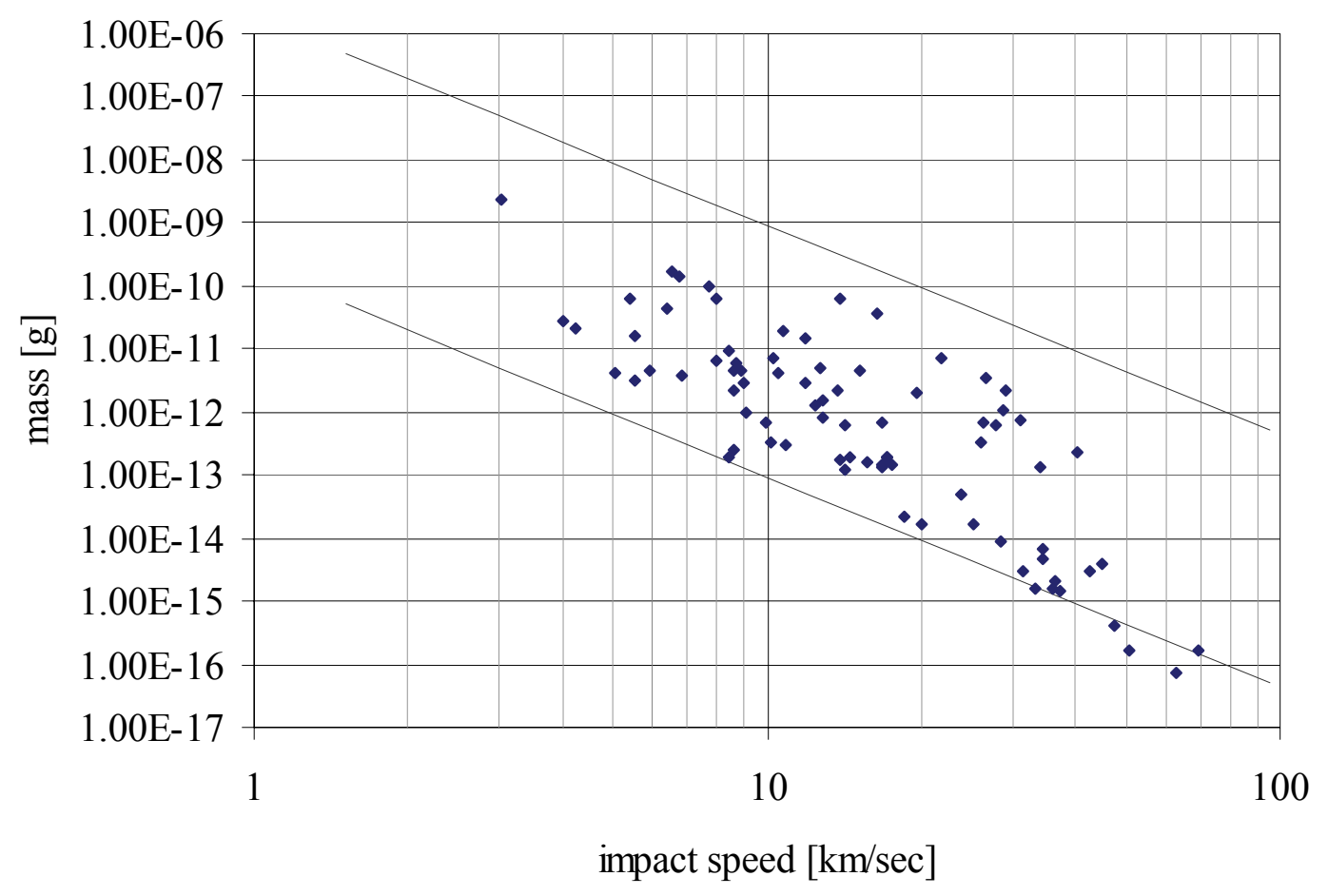

Figure 6-45: Mass vs. impact speed of the particles in the int. space The two lines show the detector limits. The upper limit is defined by the maximum charge that can be processed by the charge amplifiers and the A/D converters without overload; the lower limit is defined by the sensitivity of the instrument that is set by a certain trigger level. 
Table 6-9: Detected particles in the interplanetary space, Part 1

\begin{tabular}{|c|c|c|c|c|c|c|c|}
\hline File & Imp. No. & Date [UT] & Time [UT] & Pos. X [km] & Pos. Y [km] & Pos. Z [km] & Orb. Rad. [km] \\
\hline Sp. X $[\mathrm{km} / \mathrm{s}]$ & Sp. Y $[\mathrm{km} / \mathrm{s}]$ & Sp. Z [km/s] & Abs. Sp. $[\mathrm{km} / \mathrm{s}]$ & Imp. Sp. $[\mathrm{km} / \mathrm{s}]$ & Mass [g] & Incl. [deg] & Ecc. \\
\hline 99011201 & 43 & 1999-01-11 & $17: 36: 43$ & -60090702 & 133262786 & 3301958 & 146221631 \\
\hline-25.75 & -19.00 & 5.91 & 32.54 & 8.89 & 4.6E-12 & 11.03 & 0.26 \\
\hline 99021401 & 20 & 1999-02-12 & 03:47:27 & -129195358 & 80347667 & 6705510 & 152289698 \\
\hline-14.13 & -26.58 & 2.91 & 30.24 & 5.88 & 4.7E-12 & 6.22 & 0.08 \\
\hline 99030104 & 29 & 1999-03-01 & $09: 40: 43$ & -152913439 & 40469814 & 7845675 & 158372600 \\
\hline-68.94 & 4.25 & 24.34 & 73.23 & 69.58 & $1.6 \mathrm{E}-16$ & 57.20 & 2.07 \\
\hline 99030901 & 3 & 1999-03-05 & 22:01:49 & -157266956 & 29376881 & 8067958 & 160190476 \\
\hline 31.34 & -11.02 & 14.82 & 36.37 & 47.41 & 4.1E-16 & 72.88 & 0.93 \\
\hline 99030901 & 31 & 1999-03-08 & $19: 07: 38$ & -159641635 & 22221957 & 8188819 & 161388735 \\
\hline-7.80 & -24.77 & -6.26 & 26.71 & 7.95 & $6.8 \mathrm{E}-12$ & 14.46 & 0.20 \\
\hline 99042001 & 29 & 1999-04-20 & 09:14:18 & -160847494 & -81802403 & 8235959 & 180641580 \\
\hline 15.15 & -20.76 & 3.25 & 25.91 & 10.18 & $3.3 \mathrm{E}-13$ & 8.13 & 0.17 \\
\hline 99052701 & 39 & 1999-05-27 & $00: 50: 57$ & -121929404 & -153506611 & 6228099 & 196137320 \\
\hline 27.58 & -20.13 & -13.39 & 36.68 & 16.78 & $1.5 \mathrm{E}-13$ & 21.43 & 0.99 \\
\hline 99060201 & 15 & 1999-06-01 & $18: 55: 49$ & -113496858 & -162463142 & 5794150 & 198265936 \\
\hline 19.41 & -14.17 & -2.78 & 24.19 & 4.23 & $2.1 \mathrm{E}-11$ & 6.83 & 0.13 \\
\hline 99071201 & 29 & 1999-07-11 & 08:00:29 & -45209701 & -205043548 & 2284434 & 209980933 \\
\hline 26.48 & 1.79 & 5.83 & 27.18 & 12.45 & $1.3 \mathrm{E}-12$ & 13.09 & 0.32 \\
\hline 99072401 & 34 & 1999-07-21 & $16: 10: 04$ & -25529578 & -210559673 & 1273877 & 212105535 \\
\hline 26.26 & -2.93 & -5.64 & 27.02 & 6.38 & 4.2E-11 & 12.05 & 0.17 \\
\hline 99091201 & 12 & 1999-09-11 & $13: 53: 33$ & 73154780 & -203522168 & -3790628 & 216303637 \\
\hline 37.73 & 14.96 & -3.48 & 40.74 & 18.55 & $2.1 \mathrm{E}-14$ & 5.03 & 1.70 \\
\hline 99092401 & 7 & 1999-09-15 & $05: 27: 15$ & 79662563 & -200929557 & -4124835 & 216184702 \\
\hline 20.13 & 13.83 & 3.32 & 24.65 & 6.76 & $1.4 \mathrm{E}-10$ & 7.77 & 0.22 \\
\hline 99101901 & 45 & 1999-10-18 & 09:37:27 & 132995420 & -165734631 & -6863033 & 212609620 \\
\hline 1.00 & 48.32 & -27.55 & 55.63 & 44.83 & $3.8 \mathrm{E}-15$ & 42.84 & 3.08 \\
\hline 99112001 & 13 & $1999-11-20$ & $03: 51: 16$ & 170868118 & -112651281 & -8803966 & 204850517 \\
\hline 9.71 & 23.82 & -2.49 & 25.84 & 3.04 & 2.3E-09 & 6.59 & 0.19 \\
\hline 99112501 & 3 & 1999-11-24 & $23: 36: 37$ & 174817334 & -103541015 & -9005852 & 203378828 \\
\hline-15.37 & 31.53 & 5.02 & 35.43 & 26.72 & $3.6 \mathrm{E}-12$ & 11.19 & 0.98 \\
\hline 00040501 & 19 & 2000-04-02 & $15: 23: 17$ & 50731385 & 142029583 & -2592619 & 150840305 \\
\hline-48.18 & 8.82 & 1.96 & 49.02 & 17.18 & 1.9E-13 & 2.38 & 1.72 \\
\hline 00041101 & 7 & $2000-04-05$ & $16: 51: 25$ & 42452439 & 143886305 & -2166598 & 150033904 \\
\hline N/A & N/A & N/A & N/A & N/A & N/A & N/A & N/A \\
\hline 00041601 & 8 & $2000-04-12$ & 01:28:10 & 24907116 & 146475307 & -1264008 & 148583235 \\
\hline-29.54 & -31.05 & -3.49 & 42.99 & 34.44 & 4.5E-15 & 9.02 & 1.02 \\
\hline 00052301 & 29 & $2000-05-20$ & $04: 29: 12$ & -78913331 & 123954539 & 4072224 & 146998723 \\
\hline-24.52 & -20.40 & 2.54 & 32.00 & 5.55 & 1.6E-11 & 5.04 & 0.18 \\
\hline 00052301 & 36 & $2000-05-17$ & $07: 38: 15$ & -71787710 & 127846302 & 3706283 & 146669318 \\
\hline-39.43 & -31.41 & -12.65 & 51.97 & 23.97 & $5.1 \mathrm{E}-14$ & 14.11 & 1.97 \\
\hline 00061301 & 19 & 2000-06-09 & $20: 39: 49$ & -123385600 & 87376625 & 6354660 & 151324362 \\
\hline N/A & N/A & N/A & N/A & $\mathrm{N} / \mathrm{A}$ & N/A & N/A & N/A \\
\hline 00062101 & 4 & 2000-06-19 & $11: 51: 26$ & -139206255 & 66309094 & 7165354 & 154358737 \\
\hline-7.96 & -30.02 & 5.73 & 31.58 & 10.88 & $3.1 \mathrm{E}-13$ & 11.41 & 0.23 \\
\hline 00062101 & 37 & 2000-06-19 & $08: 42: 39$ & -139013539 & 66608656 & 7155521 & 154313572 \\
\hline-12.12 & -27.67 & 1.39 & 30.24 & 5.07 & $4.0 \mathrm{E}-12$ & 3.80 & 0.07 \\
\hline 00062101 & 42 & $2000-06-17$ & $07: 46: 12$ & -135938575 & 71223534 & 6998044 & 153626367 \\
\hline $\mathrm{N} / \mathrm{A}$ & N/A & N/A & N/A & N/A & N/A & N/A & N/A \\
\hline 00070701 & 22 & 2000-06-30 & $05: 52: 23$ & -152766946 & 40869640 & 7859392 & 158334574 \\
\hline-1.49 & -30.94 & 6.98 & 31.75 & 12.71 & $5.1 \mathrm{E}-12$ & 13.84 & 0.28 \\
\hline 00070701 & 26 & 2000-06-30 & $18: 13: 38$ & -153307441 & 39615177 & 7886932 & 158539388 \\
\hline-3.62 & -28.61 & -0.34 & 28.84 & 8.60 & $2.6 \mathrm{E}-13$ & 2.87 & 0.13 \\
\hline
\end{tabular}


Table 6-10: Detected particles in the interplanetary space, Part 2

\begin{tabular}{|c|c|c|c|c|c|c|c|}
\hline File & Imp. No. & Date [UT] & Time [UT] & Pos. X [km] & Pos. Y [km] & Pos. Z [km] & Orb. Rad. [km] \\
\hline Sp. X $[\mathrm{km} / \mathrm{s}]$ & Sp. Y $[\mathrm{km} / \mathrm{s}]$ & Sp. Z $[\mathrm{km} / \mathrm{s}]$ & Abs. Sp. $[\mathrm{km} / \mathrm{s}]$ & Imp. Sp. $[\mathrm{km} / \mathrm{s}]$ & Mass [g] & Incl. [deg] & Ecc. \\
\hline 00070701 & 29 & $2000-07-03$ & $19: 07: 25$ & -156281040 & 32162643 & 8038908 & 159758640 \\
\hline-4.06 & -31.92 & -6.72 & 32.88 & 10.46 & 4.3E-12 & 11.96 & 0.31 \\
\hline 00072601 & 17 & $2000-07-22$ & $12: 16: 06$ & -167002683 & -14687530 & 8584081 & 167866930 \\
\hline 5.54 & -26.32 & -2.07 & 26.97 & 9.02 & $2.8 \mathrm{E}-12$ & 5.02 & 0.15 \\
\hline 00081301 & 3 & $2000-08-06$ & $12: 18: 13$ & -166716711 & -51676975 & 8564725 & 174752184 \\
\hline 9.95 & -23.56 & -2.06 & 25.66 & 8.39 & $2.0 \mathrm{E}-13$ & 5.18 & 0.17 \\
\hline 00081801 & 37 & $2000-08-15$ & $18: 50: 25$ & -163003944 & -73553807 & 8370791 & 179026586 \\
\hline 11.00 & -22.61 & -1.57 & 25.19 & 6.49 & $1.7 \mathrm{E}-10$ & 4.40 & 0.15 \\
\hline 00092901 & 3 & $2000-09-25$ & $15: 04: 09$ & -121162129 & -154420890 & 6208188 & 196378752 \\
\hline 20.83 & -2.97 & 1.41 & 21.08 & 16.39 & 3.5E-11 & 5.75 & 0.58 \\
\hline 00101201 & 16 & $2000-10-07$ & $17: 52: 39$ & -102758167 & -172353058 & 5259521 & 200729868 \\
\hline 21.82 & 4.72 & 5.76 & 23.05 & 21.79 & 7.3E-12 & 20.73 & 0.67 \\
\hline 00101501 & 37 & $2000-10-13$ & $14: 04: 38$ & -93199460 & -179901458 & 4766798 & 202665725 \\
\hline 21.72 & -7.86 & 4.29 & 23.49 & 8.62 & 2.3E-12 & 10.87 & 0.20 \\
\hline 00103001 & 13 & $2000-10-27$ & $00: 45: 25$ & -69838166 & -194489332 & 3563473 & 206678900 \\
\hline 19.99 & -5.41 & -2.27 & 20.84 & 5.55 & 3. $2 \mathrm{E}-12$ & 6.28 & 0.33 \\
\hline 00103001 & 22 & $2000-10-24$ & $14: 48: 28$ & -74153607 & -192156991 & 3785745 & 206003394 \\
\hline 20.10 & -5.18 & -3.36 & 21.03 & 6.81 & 3.7E-12 & 9.19 & 0.33 \\
\hline 01012501 & 8 & 2001-01-09 & $06: 44: 50$ & 70699447 & -204417263 & -3665361 & 216329064 \\
\hline 21.30 & 10.61 & 1.42 & 23.84 & 3.99 & 2.7E-11 & 3.45 & 0.15 \\
\hline 01012501 & 19 & 2001-01-19 & $13: 17: 01$ & 88861867 & -196719539 & -4598394 & 215907743 \\
\hline-3.31 & 46.49 & 43.78 & 63.94 & 62.40 & 7.4E-17 & 69.39 & 4.10 \\
\hline 01012501 & 29 & $2001-01-21$ & $19: 41: 04$ & 92751876 & -194750035 & -4798212 & 215762623 \\
\hline 24.30 & 34.16 & -11.27 & 43.41 & 26.36 & $6.5 \mathrm{E}-13$ & 17.79 & 1.89 \\
\hline 01030701 & 32 & 2001-03-02 & $17: 23: 13$ & 151659347 & -144638115 & -7821226 & 209718653 \\
\hline-4.70 & 45.71 & -27.42 & 53.51 & 42.48 & $3.1 \mathrm{E}-15$ & 43.93 & 2.80 \\
\hline 01030701 & 42 & 2001-03-04 & $04: 39: 46$ & 153407310 & -142285263 & -7910657 & 209383565 \\
\hline 2.37 & 16.94 & -4.25 & 17.62 & 11.86 & $2.8 \mathrm{E}-12$ & 18.35 & 0.69 \\
\hline 01031401 & 28 & 2001-03-14 & $03: 56: 34$ & 164272640 & -125429220 & -8467062 & 206856667 \\
\hline 5.26 & 29.21 & -7.72 & 30.67 & 12.86 & $1.6 \mathrm{E}-12$ & 17.54 & 0.60 \\
\hline 01031401 & 30 & $2001-03-14$ & $04: 32: 27$ & 164297309 & -125385114 & -8468330 & 206849571 \\
\hline N/A & N/A & $\mathrm{N} / \mathrm{A}$ & N/A & N/A & N/A & N/A & N/A \\
\hline 01042801 & 43 & $2001-04-25$ & $18: 20: 58$ & 187835874 & -39757497 & -9667764 & 192240577 \\
\hline N/A & N/A & N/A & N/A & N/A & N/A & N/A & N/A \\
\hline 01042801 & 49 & 2001-04-13 & $05: 21: 54$ & 185210475 & -66819656 & -9536246 & 197126169 \\
\hline N/A & N/A & $\mathrm{N} / \mathrm{A}$ & N/A & N/A & N/A & N/A & N/A \\
\hline 01051701 & 6 & $2001-05-12$ & $03: 53: 34$ & 185013498 & -3160239 & -9517613 & 185285096 \\
\hline-20.86 & -8.85 & 12.60 & 25.93 & 40.38 & $2.3 \mathrm{E}-13$ & 128.56 & 0.82 \\
\hline 01070201 & 1 & 2001-06-29 & $09: 35: 55$ & 130348109 & 98285898 & -6692786 & 163387700 \\
\hline-22.57 & 13.19 & 8.33 & 27.44 & 10.29 & 7.0E-12 & 18.30 & 0.39 \\
\hline 01070201 & 6 & 2001-06-11 & $06: 08: 30$ & 159342208 & 63034534 & -8188596 & 171552747 \\
\hline-16.68 & 17.61 & -3.57 & 24.52 & 7.93 & $6.0 \mathrm{E}-11$ & 10.43 & 0.42 \\
\hline 01070201 & 40 & $2001-06-22$ & 08:06:52 & 142835370 & 85253451 & -7336723 & 166505019 \\
\hline N/A & N/A & N/A & N/A & N/A & N/A & N/A & N/A \\
\hline 01070201 & 50 & $2001-06-21$ & $12: 59: 31$ & 144147630 & 83721769 & -7404466 & 166861320 \\
\hline N/A & N/A & N/A & N/A & N/A & N/A & N/A & N/A \\
\hline 01071101 & 3 & 2001-07-02 & $05: 38: 10$ & 124917369 & 103219225 & -6412911 & 162171770 \\
\hline-21.18 & 12.28 & -3.16 & 24.69 & 8.72 & 6.2E-12 & 8.93 & 0.42 \\
\hline 01071101 & 14 & 2001-07-03 & $08: 32: 53$ & 122701224 & 105114802 & -6298786 & 161692259 \\
\hline N/A & N/A & $\mathrm{N} / \mathrm{A}$ & N/A & N/A & N/A & N/A & N/A \\
\hline 01080701 & 35 & 2001-08-06 & $13: 30: 43$ & 40249764 & 144297129 & -2053286 & 149819628 \\
\hline N/A & N/A & N/A & N/A & N/A & N/A & N/A & N/A \\
\hline
\end{tabular}


Table 6-11: Detected particles in the interplanetary space, Part 3

\begin{tabular}{|c|c|c|c|c|c|c|c|}
\hline File & Imp. No. & Date [UT] & Time [UT] & Pos. X [km] & Pos. Y [km] & Pos. Z [km] & Orb. Rad. [km] \\
\hline Sp. $X[\mathrm{~km} / \mathrm{s}]$ & Sp. Y [km/s] & Sp. Z [km/s] & Abs. Sp. $[\mathrm{km} / \mathrm{s}]$ & Imp. Sp. $[\mathrm{km} / \mathrm{s}]$ & Mass [g] & Incl. [deg] & Ecc. \\
\hline 01081601 & 8 & 2001-08-14 & $17: 42: 07$ & 17568823 & 147008013 & -886524 & 148056764 \\
\hline-31.95 & -10.70 & -9.37 & 34.97 & 16.72 & 7.1E-13 & 17.25 & 0.53 \\
\hline 01081601 & 14 & 2001-08-14 & $23: 33: 07$ & 16885348 & 147047013 & -851363 & 148015755 \\
\hline-29.44 & -22.47 & -21.12 & 42.63 & 33.47 & $1.6 \mathrm{E}-15$ & 38.56 & 1.02 \\
\hline 01081601 & 39 & 2001-08-11 & $06: 49: 33$ & 27219203 & 146222479 & -1382973 & 148740751 \\
\hline-25.03 & -0.60 & -0.88 & 25.05 & 8.59 & 4.7E-12 & 2.22 & 0.36 \\
\hline 01091201 & 4 & 2001-09-04 & $04: 37: 18$ & -39704522 & 140557870 & 2058017 & 146072583 \\
\hline N/A & N/A & $\mathrm{N} / \mathrm{A}$ & N/A & N/A & $\mathrm{N} / \mathrm{A}$ & N/A & N/A \\
\hline 01091201 & 24 & 2001-09-08 & $22: 14: 27$ & -52485293 & 136371100 & 2714743 & 146147708 \\
\hline-25.69 & -11.09 & 0.19 & 27.98 & 5.40 & 6.4E-11 & 1.15 & 0.14 \\
\hline 01091201 & 33 & $2001-08-31$ & $01: 13: 26$ & -28290410 & 143408893 & 1471372 & 146180104 \\
\hline-25.41 & -14.07 & -7.99 & 30.13 & 13.66 & 2.2E-12 & 15.94 & 0.30 \\
\hline 01091201 & 35 & 2001-09-10 & $04: 43: 43$ & -55854359 & 135073123 & 2887877 & 146194384 \\
\hline N/A & N/A & N/A & N/A & N/A & N/A & N/A & N/A \\
\hline 01101501 & 15 & $2001-10-07$ & $06: 07: 38$ & -118445072 & 92784313 & 6100892 & 150583481 \\
\hline 0.11 & -17.25 & -16.57 & 23.92 & 28.96 & $1.1 \mathrm{E}-12$ & 50.08 & 0.57 \\
\hline 01101501 & 37 & $2001-10-08$ & $20: 27: 08$ & -121445047 & 89542857 & 6254968 & 151016381 \\
\hline-10.17 & -16.33 & -1.93 & 19.33 & 13.84 & $1.7 \mathrm{E}-13$ & 6.05 & 0.58 \\
\hline 01102601 & 47 & $2001-10-25$ & $20: 22: 45$ & -147692924 & 51559764 & 7599835 & 156618538 \\
\hline-3.70 & -29.54 & 9.99 & 31.40 & 14.26 & 1.2E-13 & 19.65 & 0.24 \\
\hline 01110301 & 8 & 2001-11-01 & $18: 22: 40$ & -155260426 & 34800106 & 7986757 & 159313011 \\
\hline N/A & N/A & N/A & N/A & N/A & N/A & N/A & N/A \\
\hline 01110301 & 43 & $2001-10-29$ & $16: 13: 37$ & -152106974 & 42349726 & 7825425 & 158086268 \\
\hline-7.44 & -23.83 & -4.51 & 25.37 & 8.45 & $9.2 \mathrm{E}-12$ & 10.70 & 0.23 \\
\hline 01110301 & 50 & $2001-10-30$ & $06: 59: 05$ & -152762671 & 40854760 & 7858999 & 158326590 \\
\hline 7.91 & -1.62 & -9.16 & 12.20 & 34.64 & $6.5 \mathrm{E}-15$ & 93.10 & 0.91 \\
\hline 01112101 & 3 & $2001-11-16$ & $12: 16: 52$ & -165364919 & -2030892 & 8501495 & 165595762 \\
\hline N/A & N/A & $\mathrm{N} / \mathrm{A}$ & N/A & $\mathrm{N} / \mathrm{A}$ & N/A & $\mathrm{N} / \mathrm{A}$ & N/A \\
\hline 01120901 & 40 & $2001-12-02$ & $22: 06: 35$ & -167460239 & -42873428 & 8603767 & 173075380 \\
\hline N/A & N/A & $\mathrm{N} / \mathrm{A}$ & $\mathrm{N} / \mathrm{A}$ & N/A & N/A & N/A & N/A \\
\hline 01121801 & 31 & $2001-12-15$ & $17: 18: 10$ & -163047897 & -73317671 & 8373146 & 178969850 \\
\hline 7.11 & -1.35 & 11.18 & 13.32 & 27.87 & $6.0 \mathrm{E}-13$ & 70.08 & 0.81 \\
\hline 02020501 & 2 & $2002-01-26$ & $20: 50: 42$ & -119420615 & -156340981 & 6118480 & 196827898 \\
\hline 8.61 & -7.41 & -4.43 & 12.20 & 14.15 & $6.4 \mathrm{E}-13$ & 21.46 & 0.78 \\
\hline 02020501 & 30 & 2002-02-01 & 04:10:21 & -111524003 & -164433827 & 5711383 & 198767972 \\
\hline 9.78 & -6.04 & 4.54 & 12.35 & 14.51 & 1.9E-13 & 21.68 & 0.77 \\
\hline 02021501 & 14 & $2002-02-12$ & $00: 27: 34$ & -94200049 & -179153738 & 4818354 & 202467103 \\
\hline 22.52 & -3.10 & -11.50 & 25.47 & 15.72 & 1.6E-13 & 27.92 & 0.31 \\
\hline 02021501 & 31 & $2002-02-10$ & 19:09:15 & -96222343 & -177623575 & 4922522 & 202072029 \\
\hline 1.55 & 4.60 & -13.95 & 14.77 & 29.10 & 2.2E-12 & 93.42 & 0.72 \\
\hline 02022501 & 25 & $2002-02-23$ & $05: 15: 40$ & -74917678 & -191727021 & 3825042 & 205879916 \\
\hline 8.84 & 8.41 & -22.29 & 25.41 & 31.35 & 7.4E-13 & 76.82 & 0.45 \\
\hline 02032301 & 8 & 2002-03-19 & $17: 27: 36$ & -29441565 & -209681925 & 1483941 & 211743990 \\
\hline N/A & N/A & N/A & N/A & N/A & N/A & N/A & N/A \\
\hline 02032301 & 12 & $2002-03-22$ & $20: 37: 50$ & -23430977 & -211023843 & 1174685 & 212323933 \\
\hline N/A & N/A & N/A & N/A & N/A & N/A & N/A & N/A \\
\hline 02033101 & 7 & $2002-03-27$ & $05: 49: 45$ & -14980509 & -212550436 & 739853 & 213078978 \\
\hline 14.11 & -1.18 & -4.31 & 14.80 & 9.11 & $9.5 \mathrm{E}-13$ & 16.92 & 0.65 \\
\hline 02041401 & 34 & 2002-04-01 & $13: 17: 15$ & -4703522 & -213835193 & 211179 & 213887020 \\
\hline 12.48 & 1.60 & -8.34 & 15.10 & 12.84 & 8.4E-13 & 33.82 & 0.64 \\
\hline
\end{tabular}

Table 6-9, Table 6-10 and Table 6-11 show the complete data of the particles detected during the interplanetary cruising period from January 1999 to April 2002. More details for each data set/particle can be found in Appendix C. 


\subsubsection{Determination of interplanetary dust flux}

During the whole mission time, 98 particles were detected by the MDC. From these, 17 particles were identified as MLI impacts outside the sensor box, 13 particles were identified as grid impacts and were not analyzed due to lacking calibration data, and 5 particles were identified as impacts but were not analyzed due to charge overload. In the following, only the remaining 63 particles were used for the determination of interplanetary dust flux. Between July 10, 1998 and April 14, 2002 (last memory dump sent to Earth), the MDC was recording data for altogether 1374 days. This gives a total rate of 0.047 particles per day, or one particle every 3 weeks. For the determination of the instrument independent total dust flux, the instruments characteristics must be taken into account. Commonly, dust flux is given as particles per area per time interval, as in Table $6-12$ as particles $/\left(\mathrm{m}^{2} \cdot\right.$ day $)$. Hence, the calculated rate of 0.046 particles per day must be corrected by the instruments sensor area $\left(A=0.0145 \mathrm{~m}^{2}\right)$ and effective solid angle $(\Omega=0.81 \pi \mathrm{sr})$, these characteristics are discussed in chapter 3.2.3. Also, the instruments measuring range must be regarded carefully to get reliable results for dust flux. The total dust flux $\Phi$ is therefore given by

$$
\Phi=\frac{\pi}{A \cdot \Omega} \cdot \frac{N}{\Delta T}, \Phi_{\mathrm{MDC}}^{\mathrm{int}}=\frac{\pi}{0.0145 \mathrm{~m}^{2} \cdot 0.81 \pi} \cdot \frac{63}{1374 \mathrm{~d}}=3.90 \frac{1}{\mathrm{~m}^{2} \cdot \mathrm{d}}
$$

whereas $N$ is the number of particles detected during the time interval $\Delta T$.

Applying the instrument characteristics, the total flux of interplanetary particles detected by the MDC calculates to 3.90 particles $/\left(\mathrm{m}^{2} \cdot\right.$ day $)$, the cumulated flux of particles heavier than $10^{-12} \mathrm{~g}$ (29 particles) calculates to 1.80 particles $/\left(\mathrm{m}^{2} \cdot\right.$ day $)$, for particles heavier than $10^{-9} \mathrm{~g}$ (1 particle) to 0.06 particles $/\left(\mathrm{m}^{2} \cdot\right.$ day $)$. These results are summarized and compared to the model by Grün et al. [Grü1985] in Table 6-12.

Table 6-12: Comparison of the MDC results and the Grün model.

\begin{tabular}{llll} 
Particle mass & $\mathrm{m}>10^{-15} \mathrm{~g}$ & $\mathrm{~m}>10^{-12} \mathrm{~g}$ & $\mathrm{~m}>10^{-9} \mathrm{~g}$ \\
\hline Flux MDC & 3.72 & 1.80 & 0.06 \\
Flux Grün model & 92 & 2.98 & 0.26 \\
\hline
\end{tabular}

Flux values given as particles $/\left(\mathrm{m}^{2} \cdot\right.$ day $)$

The cumulated dust flux measured by the MDC lies far below the predictions of the Grün model for particle masses greater than $m=10^{-9} \mathrm{~g}$ and $m=10^{-15} \mathrm{~g}$, while the cumulated flux for particle masses greater than $m=10^{-12} \mathrm{~g}$, which is 1.80 particles $/\left(\mathrm{m}^{2} \cdot\right.$ day $)$, meets the predicted value of 2.98 particles $/\left(\mathrm{m}^{2} \cdot\right.$ day $)$ by a factor of $\approx 1.5$. Since the measuring range of the MDC allows a reliable dust flux determination only for particle mass around $10^{-11} \mathrm{~g}$ to $10^{-12} \mathrm{~g}$, this good consistency confirms the reliability of the MDC dust data measurements.

Unlike it is presumed by the model, the MDC sensor area's normal is not rotating in the ecliptic plane, this was not taken into account for the calculations. Detector dead time caused by processing noise signals was neglected. Also, detector down time, which can be assumed to a few percent of mission time (see paragraph 6.2.4), was not considered in the determination of dust flux. The calculated values are mean values over the whole mission time. A further breakup into smaller time intervals (months or half years) is possible but was 
not regarded to give meaningful results, due to the small number of detected particles that are available for flux determination and the varying sensitivities of the MDC caused by the attitude of the NOZOMI spacecraft (see paragraph 6.2.3).

\subsubsection{Characteristics of the detected particles}

In this paragraph, the characteristics of the detected particles are discussed. First, some statistical analyzes of mass, speed and orbital elements of the particles are presented. Subsequent, patterns were identified within the whole set of detected particles and discussed separately.

The orbital elements eccentricity $e$, inclination $i$ and semi-major axis $a$ discussed in this paragraph were calculated from the radius vector $\mathbf{r}$ and the speed vector $\mathbf{v}$ of a particle as follows (see Steiner \& Schagerl [Ste2004]):

$$
\begin{aligned}
& i=\arccos \left(\frac{\mathbf{r} \times \mathbf{v}}{|\mathbf{r} \times \mathbf{v}|} \mathbf{e}_{z}^{e e c}\right) \\
& e=\frac{|\mathbf{q}|}{\mu} ; \quad \mathbf{q}=\mathbf{v} \times(\mathbf{r} \times \mathbf{v})-\mu \frac{\mathbf{r}}{|\mathbf{r}|} ; \mu=G M_{\text {Sun }} \\
& a=\frac{(\mathbf{r} \times \mathbf{v})^{2}}{\mu\left(1-e^{2}\right)}
\end{aligned}
$$

whereas $G$ is the gravitational constant and $M_{\text {Sun }}$ the mass of the Sun.

In Figure 6-46, mass vs. absolute speed of the detected particles is show. A clear tendency towards smaller mass with increasing orbital speed can be seen for the speed range of $20 \mathrm{~km} / \mathrm{s}$ upwards. A number of medium sized particles from $10^{-11} \mathrm{~g}$ to $10^{-14} \mathrm{~g}$ does not show this behavior and has low orbital speed between 10 and $20 \mathrm{~km} / \mathrm{s}$. 16 particles have orbital speed higher than $40 \mathrm{~km} / \mathrm{s}$, which is about the mean escape speed at the orbital radius range from 1.0 AU to 1.4 AU.

The absolute speed of the particles in relation to the escape speed is shown in Figure 6-47. Escape speed (with respect only to the gravitational force) decreases from $44 \mathrm{~km} / \mathrm{s}$ at $0.9 \mathrm{AU}$ to $35 \mathrm{~km} / \mathrm{s}$ at $1.5 \mathrm{AU}$. Again, 16 particles have orbital speed greater than the escape speed, which is shown as a dashed line. The solid line represents the orbital speed of bodies on a circular orbit (again with respect only to the gravitational force). A majority of the detected particles has orbital speed below this circular speed, which indicates that these particles are either on elliptical orbits and were detected near the aphelion of their orbits, or, if they are on circular orbits, are affected by the Sun's radiation pressure (which decreases the circular orbital speed of such particles). Most particles detected by the MDC are within the mass (and therefore size) range of particles that have $\beta$-values greater than zero and thus are affected by solar radiation pressure.

As can also be seen in Figure 6-47, a high number of particles were detected at orbital radii of around 1.0 AU and 1.4 AU, while in between the number of detected particles is significantly 
lower. Since the duration of stay of NOZOMI (see Figure 6-27) also is higher at 1.0 AU and 1.4 AU due to the orbital configuration, the detected number of particles was scaled by the duration of stay. Since the high detection rate during the first six months where NOZOMI was in an orbit around the Earth may be a result of some effects caused by the Earth (e.g. gravitational focusing) or the Moon, all 1998 particles were removed from the data for this analysis. During the remaining time span, NOZOMI never encountered Earth or Mars. The result is shown in Figure 6-48. As can be seen, there is still a significantly higher detection rate at around 1.0 AU and 1.4 AU than in between.

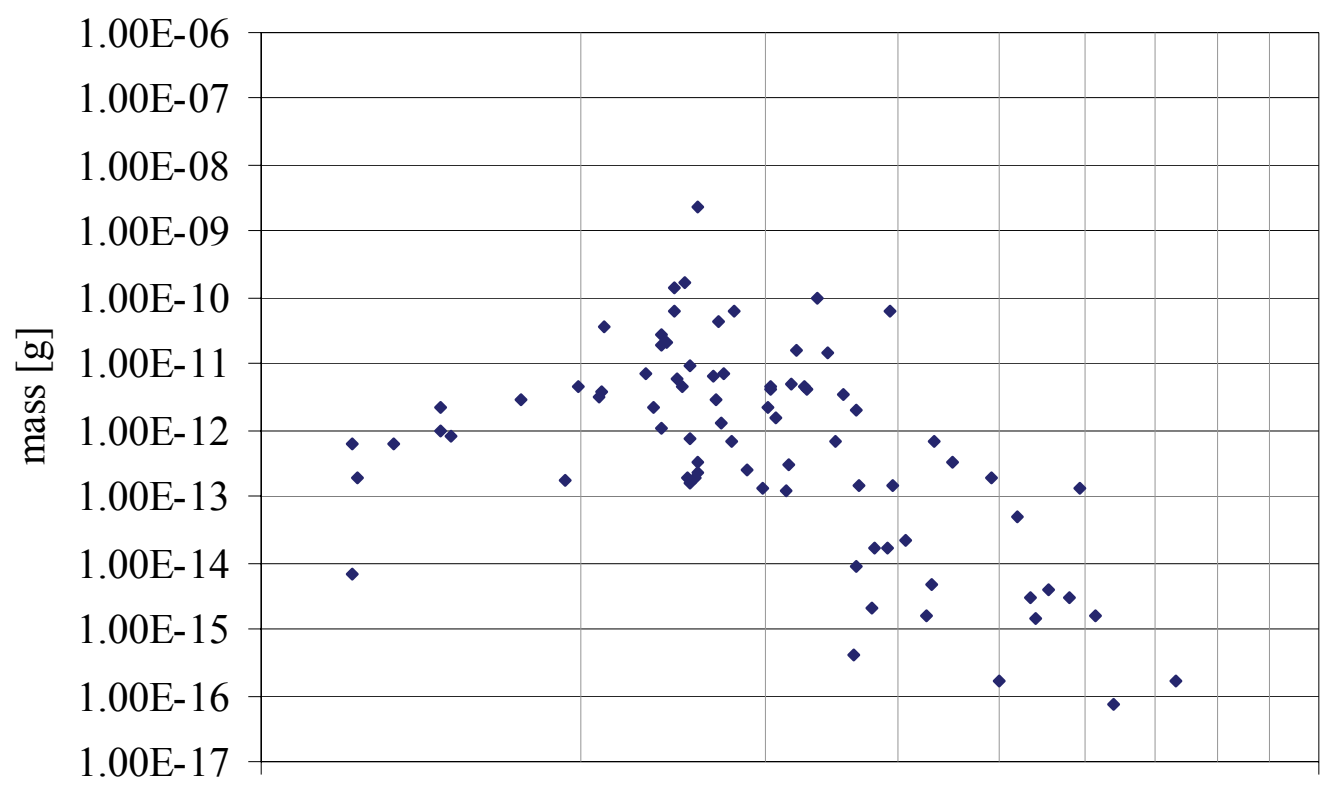

10

absolute speed $[\mathrm{km} / \mathrm{sec}]$

Figure 6-46: Mass vs. absolute speed of the detected particles The absolute speed vector of the particles was determined from the impact speed and the NOZOMI orbital position and attitude. 


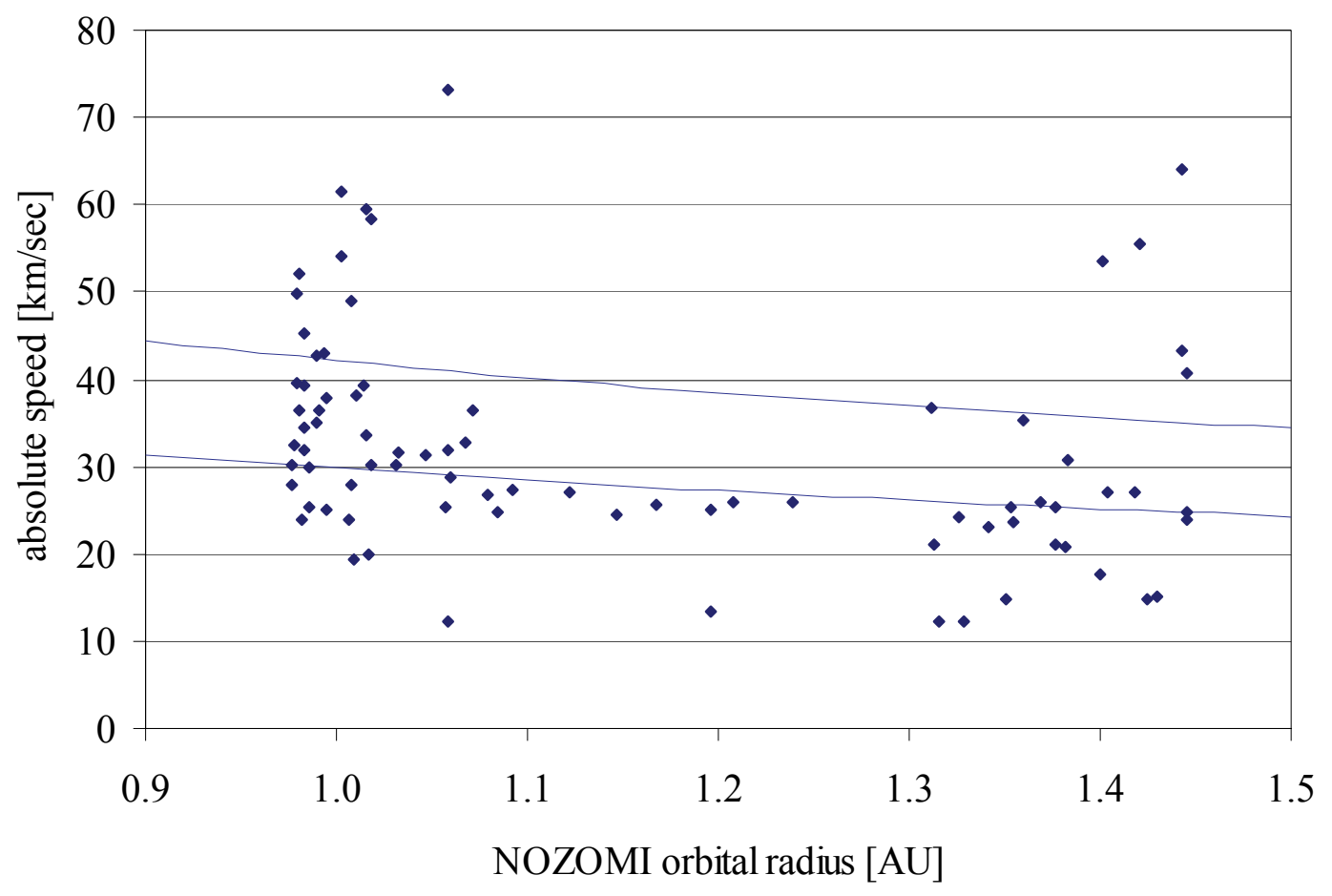

Figure 6-47: Orbital radius vs. abs. speed of the particles

The two lines represent the circular orbital speed (lower, solid line), and the escape speed (upper, dashed line).

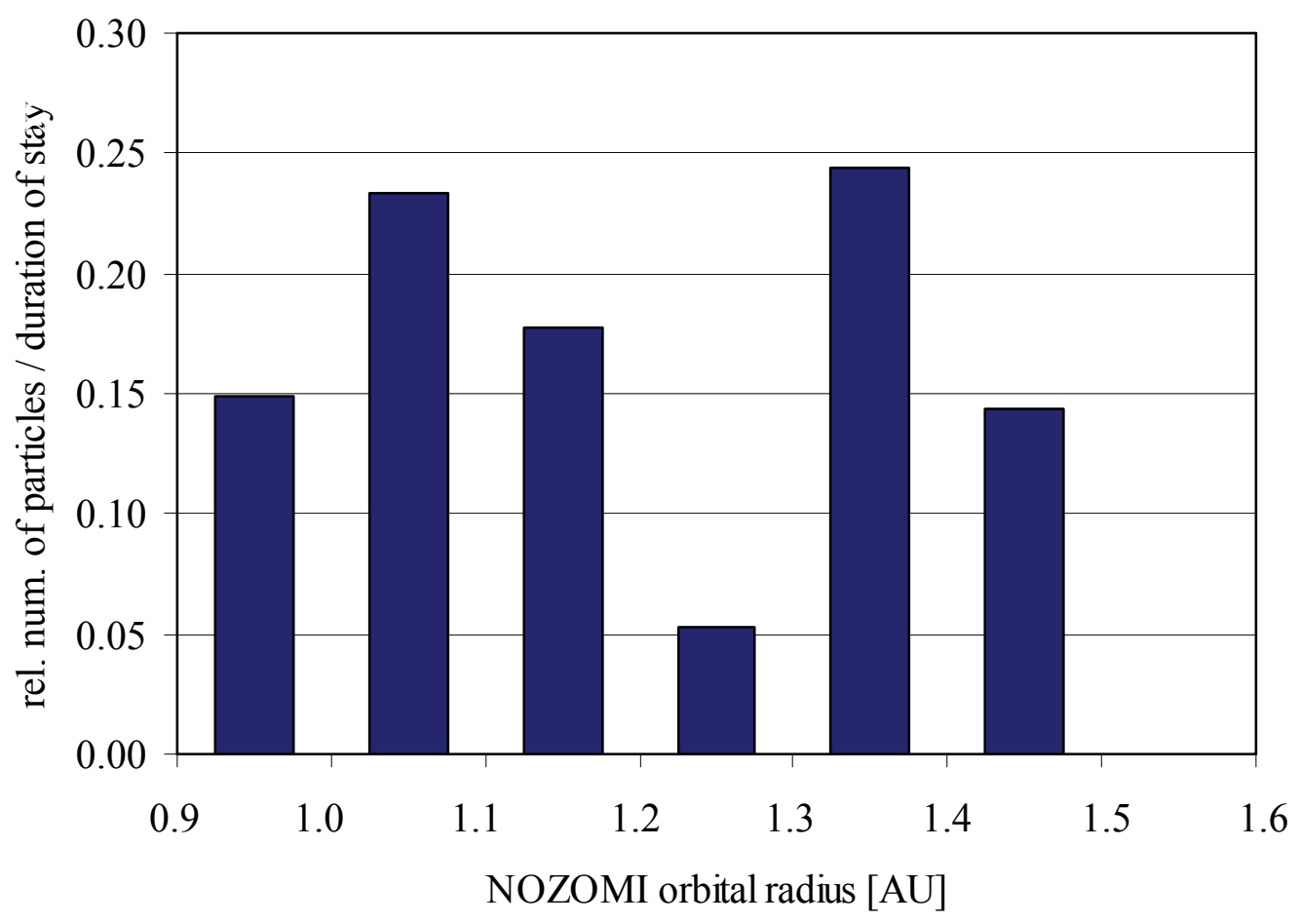

Figure 6-48: Number of particles per radius interval, corrected The number of particles detected around 1.0 AU $\left(R^{\text {Earth }}\right)$ and 1.4 $\mathrm{AU}\left(R^{\text {Mars }}\right)$ after correction by the duration of stay of the NOZOMI spacecraft (see Figure 6-27) is still higher than between the orbits of Mars and Earth. 
On the following pages, the distributions of mass and orbital speed and of the orbital elements inclination and eccentricity of the detected particles are shown.

The distribution of orbital speed of the particles detected by the MDC is shown in Figure 6-49. Orbital speed ranges from $12.2 \mathrm{~km} / \mathrm{s}$ to $73 \mathrm{~km} / \mathrm{s}$ with a strong peak at $25 \mathrm{~km} / \mathrm{s}$ to $30 \mathrm{~km} / \mathrm{s}$. $67 \%$ of all particles have an orbital speed in the range from $20 \mathrm{~km} / \mathrm{s}$ to $40 \mathrm{~km} / \mathrm{s}$.

The distribution of mass of the particles detected by the MDC is shown in Figure 6-50. Particle mass ranges from $2.3 \cdot 10^{-9} \mathrm{~g}$ down to $7.4 \cdot 10^{-17} \mathrm{~g}$ with a peak at $10^{-11} \mathrm{~g}$ to $10^{-13} \mathrm{~g}$.

The shown distributions of speed and mass of the detected particles are determined mainly by the MDC measuring range, see Figure 3-17. They must not be interpreted as absolute speed and mass distributions of dust particles in the interplanetary space. To get information about the real distributions of mass and speed of the dust particles, similar data from several in-situ and remote sensing instruments with different measuring ranges must be combined, as described in paragraph 2.1.2.

The distributions of inclination and eccentricity of the detected particles are shown in Figure 6-51 and Figure 6-52, respectively. The inclination distribution of the detected particles has a strong peak to $0^{\circ}$ to $20^{\circ}$, and falls rapidly for higher inclination (one retrograde particle with inclination $128^{\circ}$ is not included in this graph). The eccentricity distribution of the detected particles has a strong peak at 0.1 to 0.2 , and falls for rising eccentricity until $\varepsilon \approx 1$. There are two populations of unbound particles with eccentricities from 1.7 to 2.1 and from 2.7 to 3 .

The number of detected particles per month over the whole mission time is shown in Figure 6-53. After a period of high detection rate during the first half year of the mission, where 21 particles were detected (extrapolated $\approx 42$ particles/year), the detection rate falls down to 15 particles/year during 1999 and rises to 20 particles/year in the year 2000 and 33 particles/year in the year 2001. In the year 2002, 9 particles were detected from January to March, which gives an extrapolated value of $\approx 36$ particles/year. These detection rates correspond well with the calculated sensitivity of the MDC for apex particles (which represent the main constituent of the interplanetary dust cloud) as shown in Figure 6-28. None of the strong peaks in June 2000, June 2001, August 2001 and October 2001 can be associated with a particle stream since the detected particles during these months do not show any similarities in speed or flight direction. The November 1998 peak is discussed in paragraph 6.3.3. The mentioned peaks and the gaps from December 1999 to February 2000 and in November/December 2000 can only be explained by statistical fluctuations of the detection rate. 


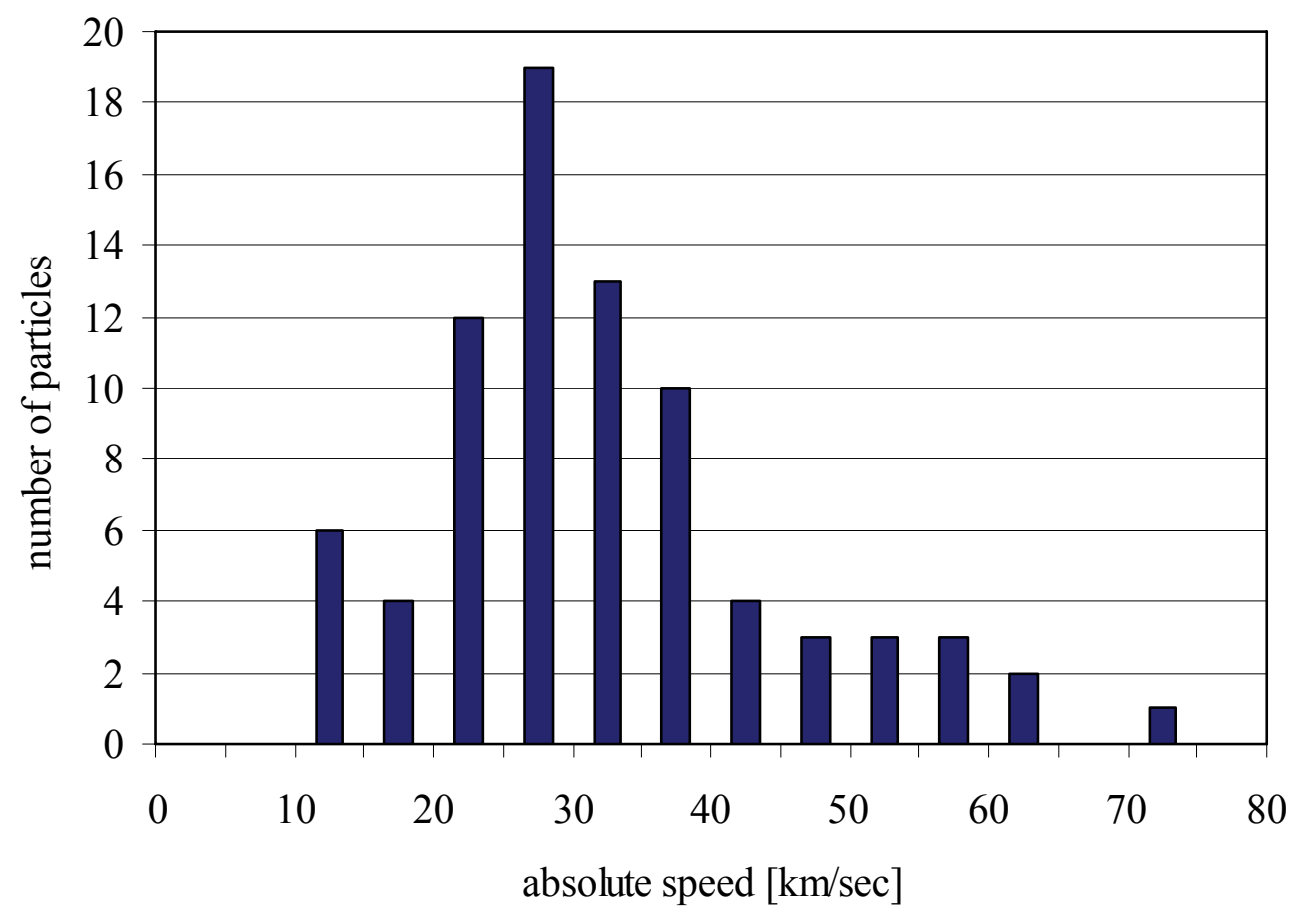

Figure 6-49: Number of particles vs. absolute speed

The absolute speed of the detected particles ranges from $10 \mathrm{~km} / \mathrm{sec}$ up to 75 $\mathrm{km} / \mathrm{sec}$, with a peak at 25 to $30 \mathrm{~km} / \mathrm{sec}$.

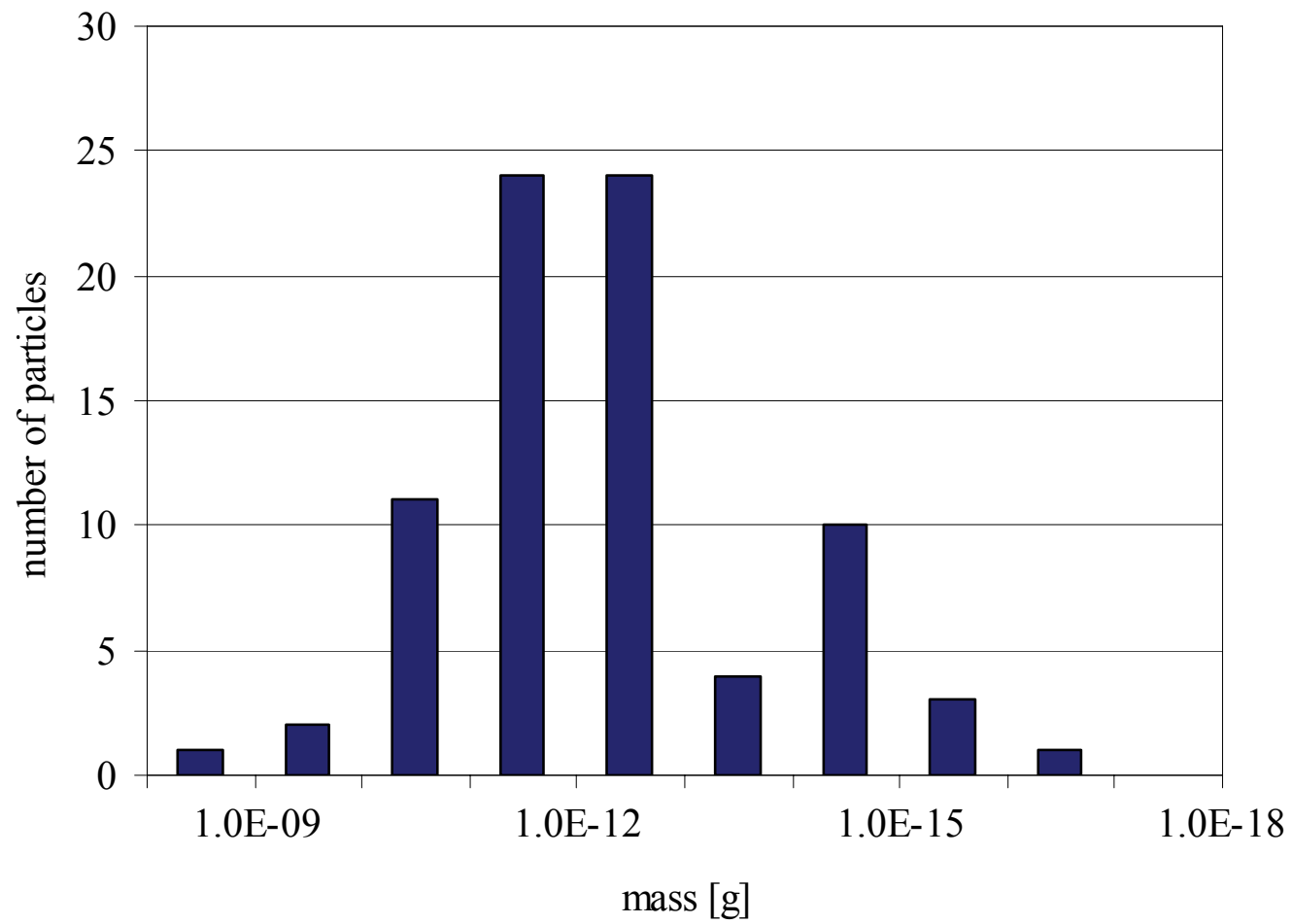

Figure 6-50: Number of particles vs. mass

The mass of the detected particles ranges from $10^{-8} \mathrm{~g}$ to $10^{-17} \mathrm{~g}$, with peaks at $10^{-11}$ to $10^{-13} \mathrm{~g}$. 


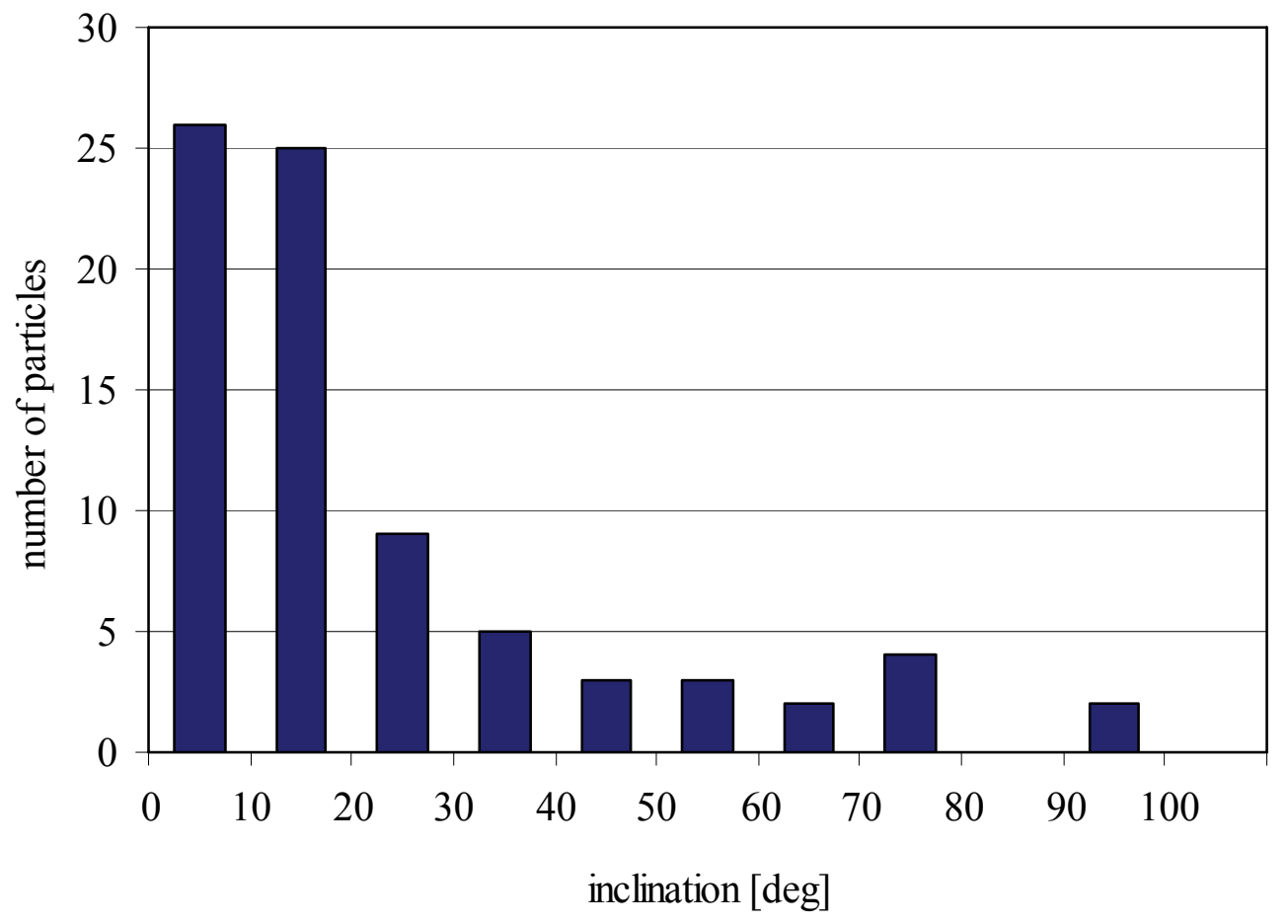

Figure 6-51: Number of particles vs. inclination Inclination of the detected particles ranges from $0^{\circ}$ up to $93^{\circ}$ (with one retrograde particle, $i=128^{\circ}$, not included), with maximum at $0^{\circ}$ to $20^{\circ}$.

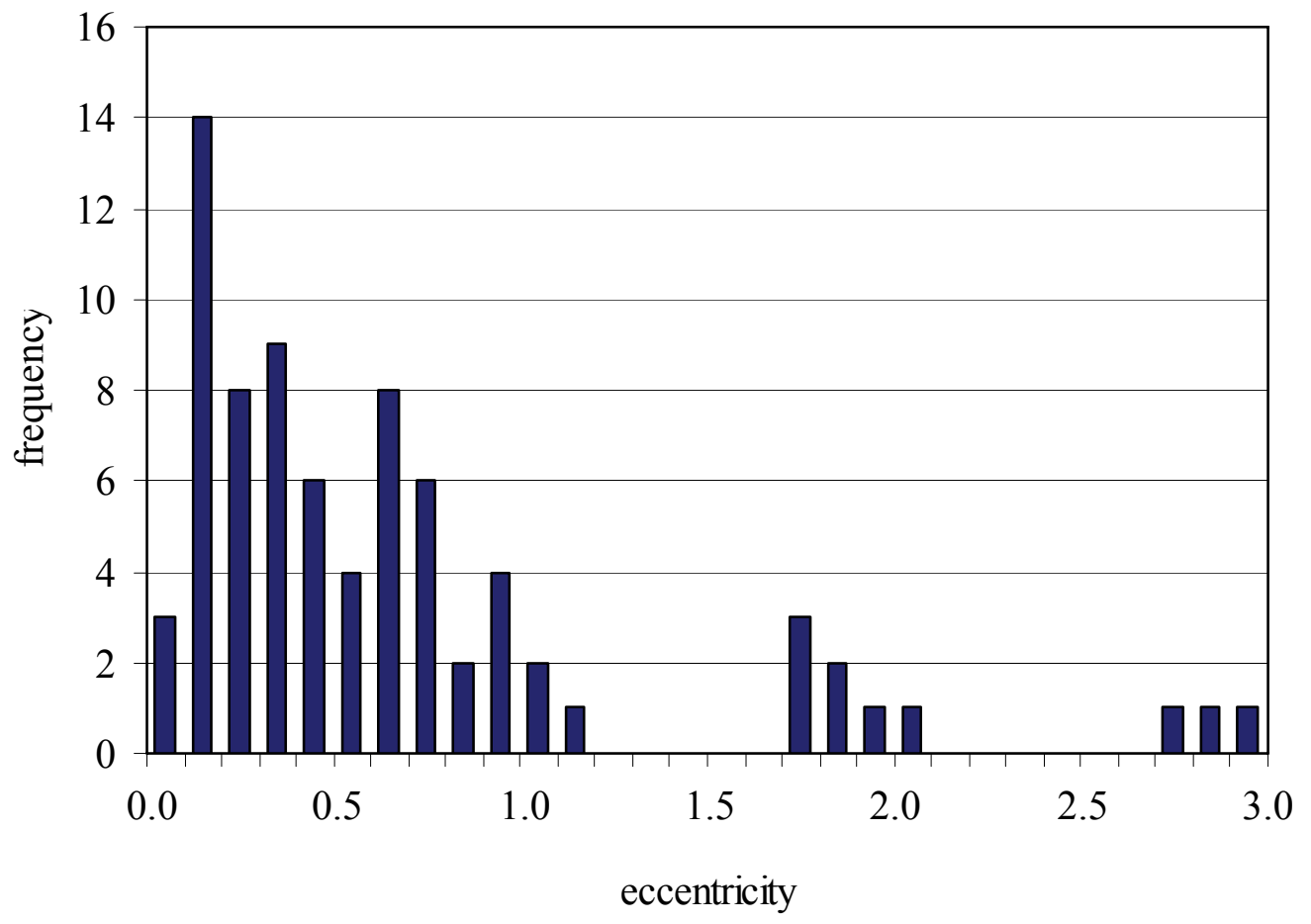

Figure 6-52: Number of particles vs. eccentricity

The eccentricity of the detected particles splits into one elliptical and two hyperbolical populations. Elliptical eccentricities have a peak at 0.1 to 0.2. 


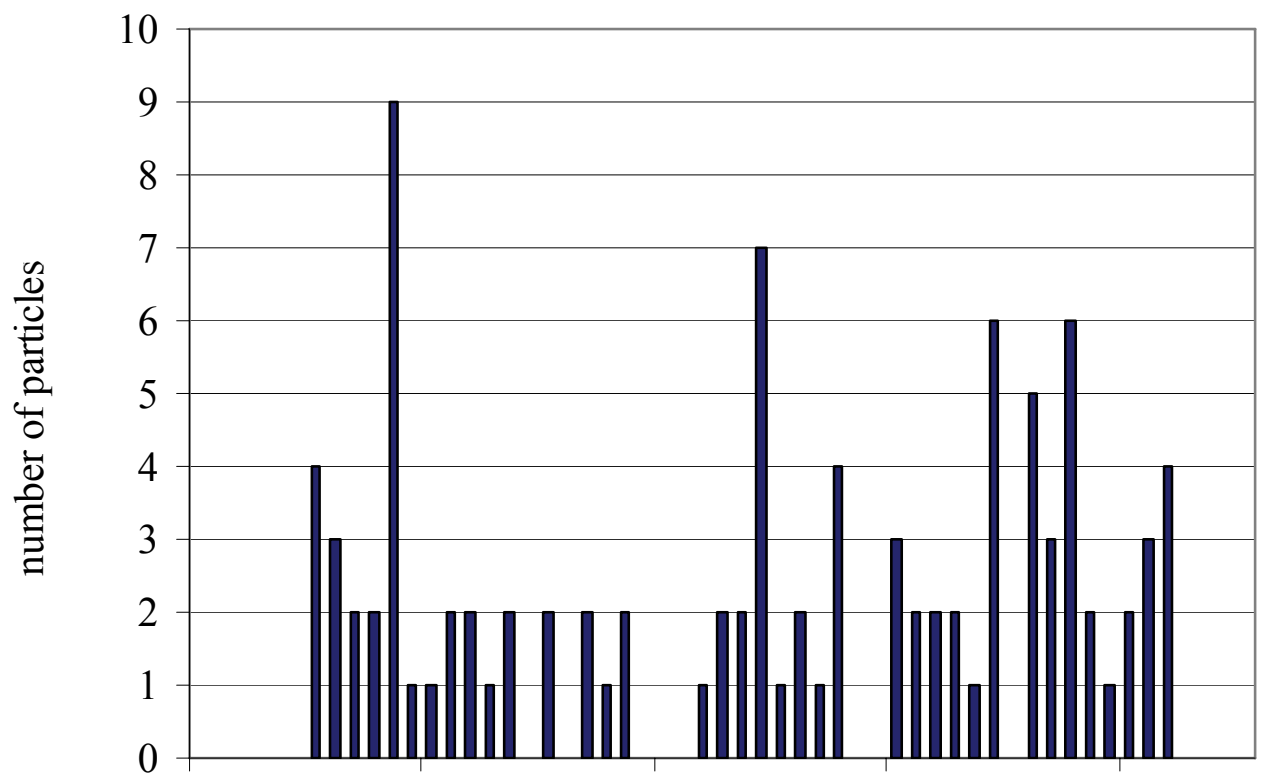

January-98 January-99 January-00 January-01 January-02

time $[\mathrm{UT}]$

Figure 6-53: Number of particles over mission time

Distribution of the number of detected particles per month over mission time. Peaks and gaps are not assigned to particle streams or caused by orbital configuration. 
To identify possible separable populations of particles in the measured MDC data, a cluster analysis was performed on the individual characteristic attributes of the particles: Mass $m$, eccentricity $e$ and inclination $i$. Again, a standard cluster analysis method as described in paragraph 6.2.1 was used (with the parameters Complete Linkage, Euclidean distances). Based on the three populations of bound particles identified by Grün et al. [Grü1997], a fixed number of nine clusters was chosen to have sufficient space for all possible populations of bound and unbound particle populations, and also mavericks (like one previously identified retrograde particle). The results of this analysis are shown and discussed in the following.

Table 6-13: Dust populations according to Grün et al.

\begin{tabular}{|c|c|c|c|c|c|}
\hline $\begin{array}{l}\text { Population } \\
\text { mass distribution } \\
\text { maximum }\end{array}$ & $\begin{array}{c}\text { Asteroidal } \\
10^{-3} \mathrm{~g} \\
\end{array}$ & $\begin{array}{l}\text { Core } \\
10^{-5} \mathrm{~g} \\
\end{array}$ & $\begin{array}{c}\mathrm{A} \\
10^{-11} \mathrm{~g} \\
\end{array}$ & $\begin{array}{c}\mathrm{B} \\
10^{-13} \mathrm{~g} \\
\end{array}$ & $\begin{array}{c}\mathrm{C} \\
5 \times 10^{-15} \mathrm{~g} \\
\end{array}$ \\
\hline \multicolumn{6}{|l|}{ Eccentricity } \\
\hline 0.00 & 0.572 & 0.572 & 1.628 & 0.975 & 0.627 \\
\hline 0.10 & 2.078 & 2.078 & 1.864 & 0.975 & 0.266 \\
\hline 0.25 & 1.753 & 1.753 & 1.791 & 0.878 & 0.140 \\
\hline 0.45 & 1.110 & 1.110 & 0.225 & 1.244 & 0.063 \\
\hline 0.65 & 0.898 & 0.898 & 0.011 & 0.078 & 0.063 \\
\hline 0.80 & 0.155 & 0.155 & 0.326 & 0.780 & 1.198 \\
\hline 0.98 & 0.000 & 0.000 & 3.016 & 5.384 & 7.678 \\
\hline \multicolumn{6}{|l|}{ Inclination } \\
\hline $0^{\circ}$ & 2.809 & 2.809 & 1.684 & 0.525 & 0.513 \\
\hline $10^{\circ}$ & 2.809 & 2.809 & 2.020 & 2.707 & 1.566 \\
\hline $20^{\circ}$ & 1.039 & 1.039 & 1.347 & 1.998 & 2.278 \\
\hline $30^{\circ}$ & 0.286 & 0.286 & 0.673 & 0.608 & 1.109 \\
\hline $45^{\circ}$ & 0.073 & 0.073 & 0.337 & 0.112 & 0.154 \\
\hline $60^{\circ}$ & 0.000 & 0.000 & 0.002 & 0.001 & 0.026 \\
\hline $90^{\circ}$ & 0.000 & 0.000 & 0.000 & 0.000 & 0.001 \\
\hline
\end{tabular}

Altogether five populations of bound particles were identified, with different but overlapping mass range, and different distributions in eccentricity and inclination. These populations were named E1, E2, E3, E4 and E5 ("E" for bound populations on "e"lliptic orbits). "Population" E5 contains only one particle on a bound, but retrograde orbit.

Also, four populations of unbound particles were found, also with different mass range, inclination and eccentricity, named $\mathrm{H} 1, \mathrm{H} 2, \mathrm{H} 3$ and $\mathrm{H} 4$ ("H" for unbound populations on "h"yperbolic orbits). Populations H3 and H4 contain only two and one particle of very low mass, respectively.

On the following pages, the different characteristics of the identified populations are analyzed statistically. In Figure 6-54 and Figure 6-55, the eccentricities and inclinations of the larger populations E1, E2, E3, H1 and H2 are shown; all populations are displayed in a different color. Statistical analyses of the larger populations are shown subsequently. 
Population E1 (blue dots) consists of particles with mean mass around $1 \cdot 10^{-10} \mathrm{~g}$ on low inclination, low eccentricity orbits.

Population E2 (green dots) consists of lighter particles with mean mass around $7 \cdot 10^{-13} \mathrm{~g}$ on low inclined orbits and a different, bimodal distribution in eccentricity.

Population E3 (turquoise dots) consists of light particles with mean mass around $6 \cdot 10^{-15} \mathrm{~g}$, on high inclined orbits and with high eccentricity $(>0.5$ and $<1.0)$.

Population E4 (not shown) consists of three particles with mean mass around $1.2 \cdot 10^{-12} \mathrm{~g}$, on very high inclined orbits and with medium eccentricity.

Population E5 (not shown) consists of one particle with mass $2.3 \cdot 10^{-13} \mathrm{~g}$, on a retrograde, high inclined orbit and with high eccentricity 0.82 (single value).

Population H1 (red dots) consists of particles with mean mass around $1.8 \cdot 10^{-13} \mathrm{~g}$, on low inclined orbits with mean eccentricity around 1.6 (mean value).

Population H2 (orange dots) also consists of light particles with mean mass around $2.9 \cdot 10^{-14} \mathrm{~g}$, with higher inclination and very high eccentricity around 3.0 (mean value).

Population H3 (not shown) consists of two very light particles with mass $1.6 \cdot 10^{-16}$ on high inclined orbits with eccentricity around 1.9 (mean value).

Population H4 (not shown) consists of one very light particles with mass $7.4 \cdot 10^{-17}$ on a high inclined orbit with eccentricity 4.1 (single value). 


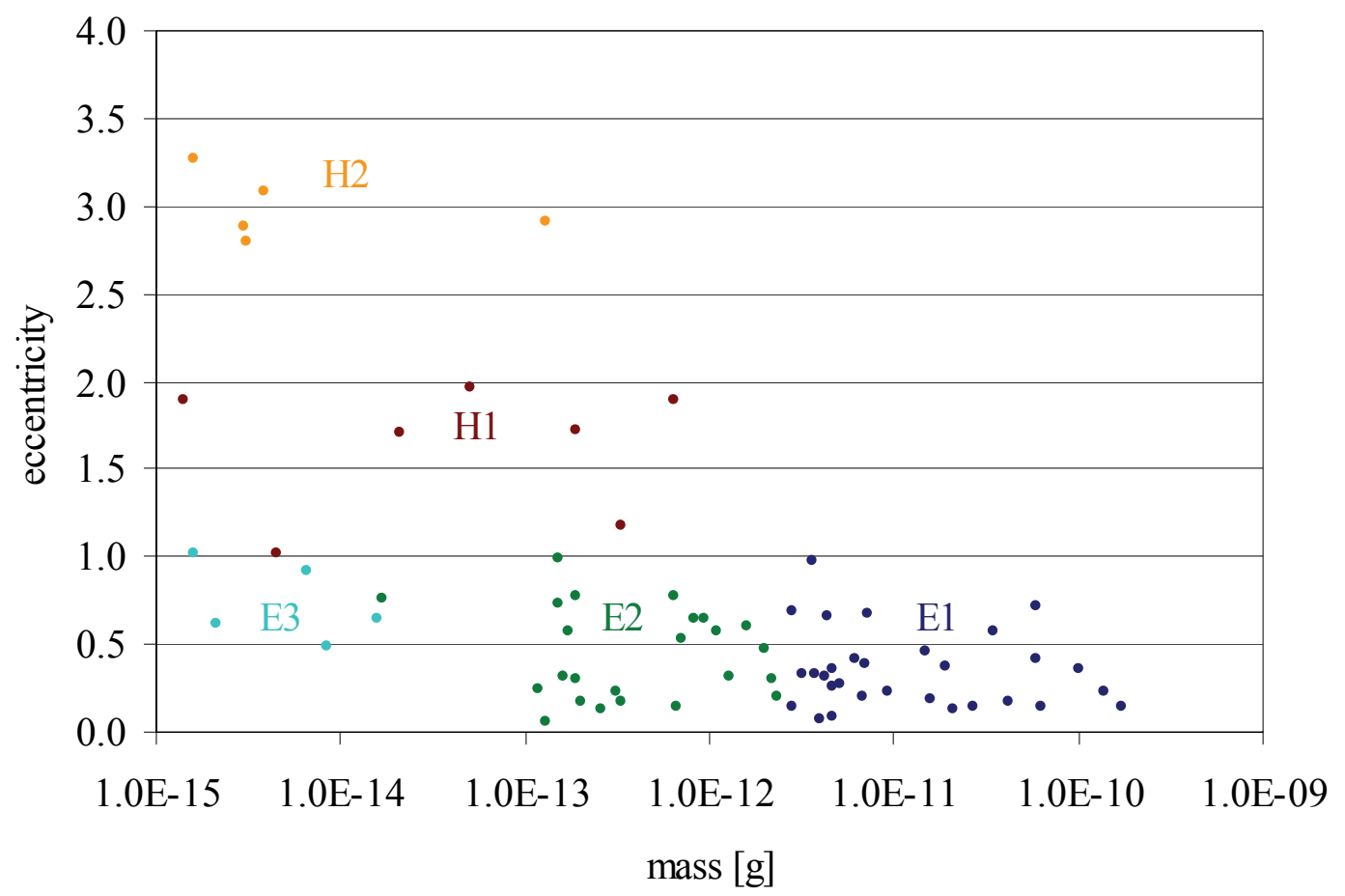

Figure 6-54: Eccentricity over mass, identified populations Population E1, blue dots; Population E2, green dots; Population E3, turquoise dots; Population H1, red dots; Population H2, orange dots.

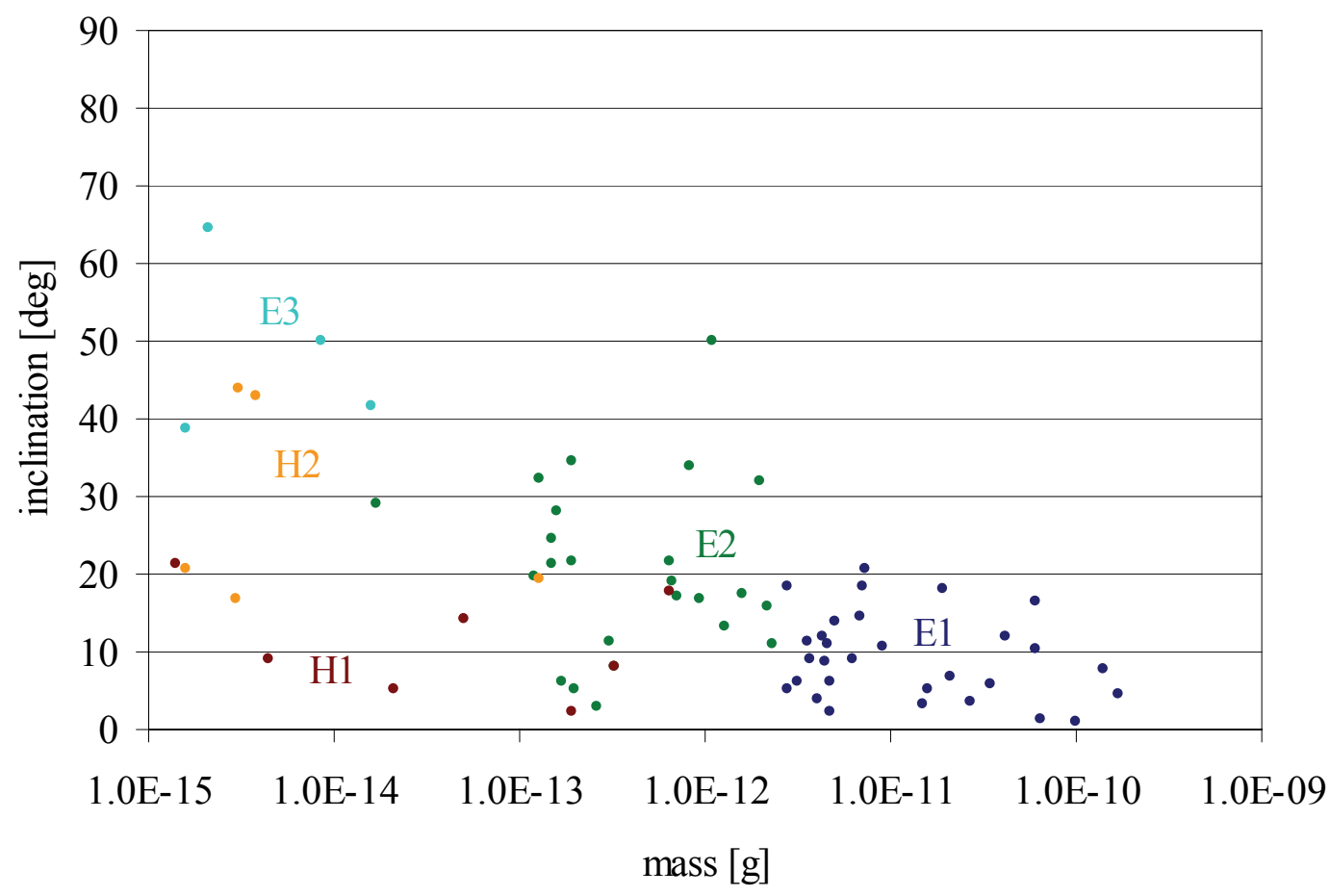

Figure 6-55: Inclination over mass, identified populations

Population E1, blue dots; Population E2, green dots; Population E3, turquoise dots; Population H1, red dots; Population H2, orange dots. 
The main properties of the 31 population E1 particles are summarized in Table 6-14. Flight directions of the particles are shown in Figure 6-56.

Population E1 particles show some similarities to population A particles described by Grün et al. The mass distribution of population E1 particles is shown in Figure 6-57. The mean mass of population E1 particles of $1.02 \cdot 10^{-10} \mathrm{~g}$ fits to the maximum of the mass distribution of Grün's population A $\left(1 \cdot 10^{-10} \mathrm{~g}\right)$. Also, the distributions of eccentricity and inclination of population E1 (see Figure 6-59 and Figure 6-60) fit well to the corresponding distributions of population A, besides that the distribution of eccentricity does not show the second peak at high eccentricities near 1 and the distribution of inclination has a maximum at $0^{\circ}$ to $10^{\circ}$ instead of $10^{\circ}$ for Grün's population A particles.

Population E1 particles preferable impact from the apex direction of the spacecraft (see Figure 6-58). This is the expected behavior for particles of non-negligible $\beta$-value orbiting in nearly circular orbits, since the circular orbit speed for particles experiencing radiation pressure is lower than the circular orbit speed with respect only to gravitational force.

Table 6-14: Properties of the 31 population E1 particles

\begin{tabular}{ccccc}
\hline Pop. E1 & Mass [g] & Eccentricity & Incl. [deg] & Sm. ax. [AU] \\
\hline Max & $2.30 E-09$ & 0.98 & 20.73 & 3.45 \\
Min & $2.80 E-12$ & 0.07 & 0.92 & 0.66 \\
Mean & $\mathbf{1 . 0 2 E}-10$ & $\mathbf{0 . 3 4}$ & $\mathbf{9 . 1 3}$ & $\mathbf{1 . 1 8}$ \\
\hline
\end{tabular}

One maverick value omitted im semimajor axis mean value

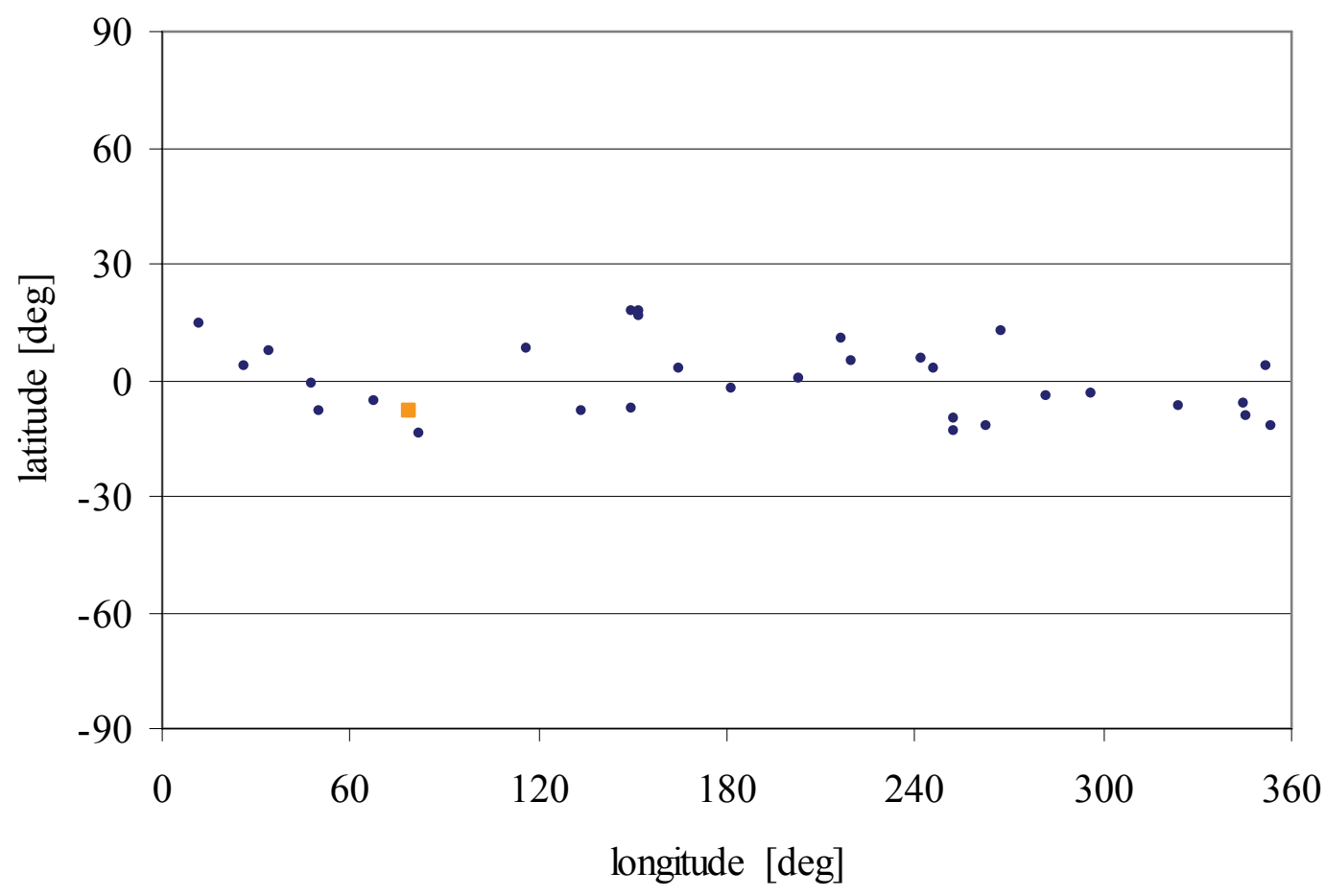

Figure 6-56: Latitude vs. longitude of population E1 particles

Population E1 particles do not show a preferred flight direction. The orange square marks the downstream direction of interstellar dust flux. 


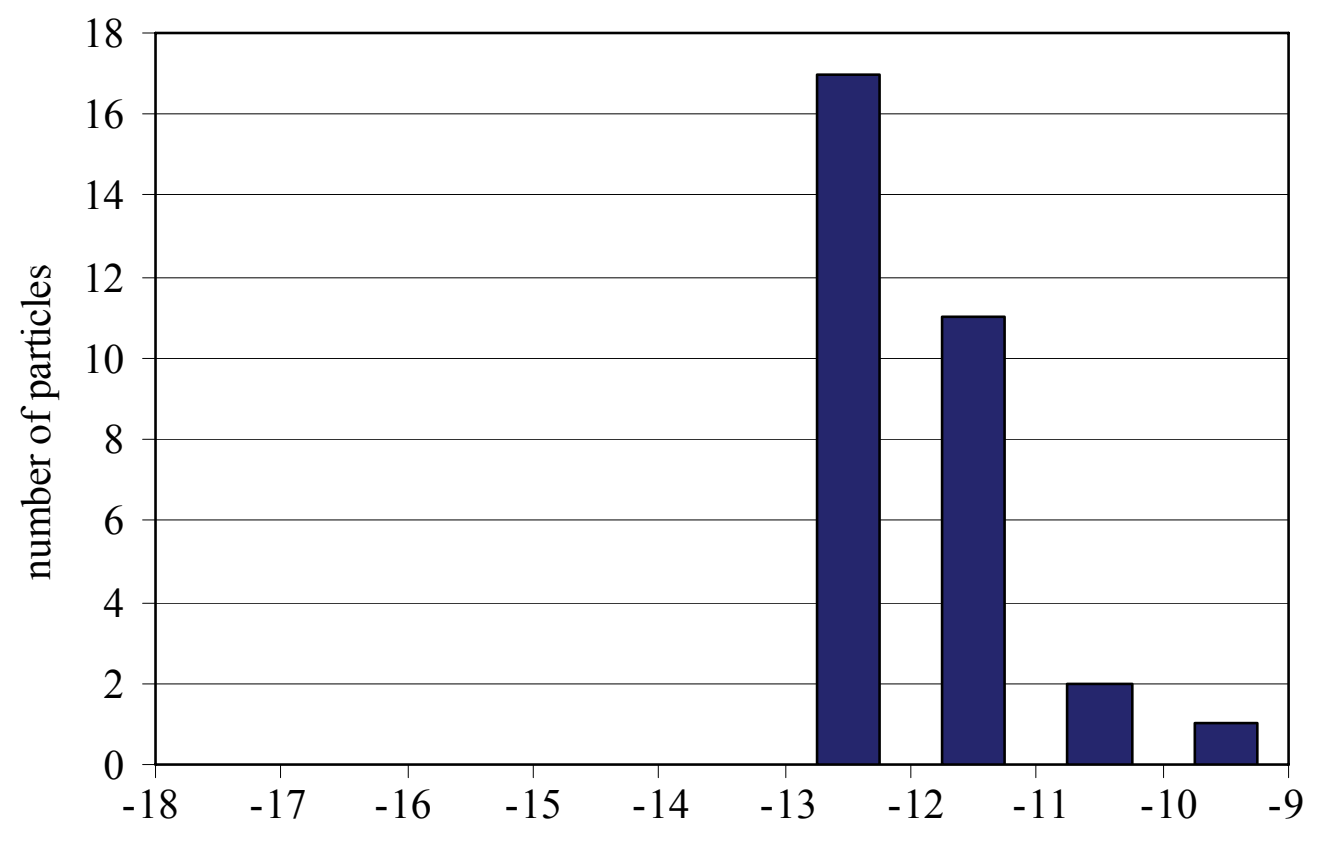

$\log$ mass $[\mathrm{g}]$

Figure 6-57: Mass distribution of population E1 particles

Population E2 particles have high mass from $10^{-9} \mathrm{~g}$ to $10^{-13} \mathrm{~g}$, with a peak at $10^{-13} \mathrm{~g}$.

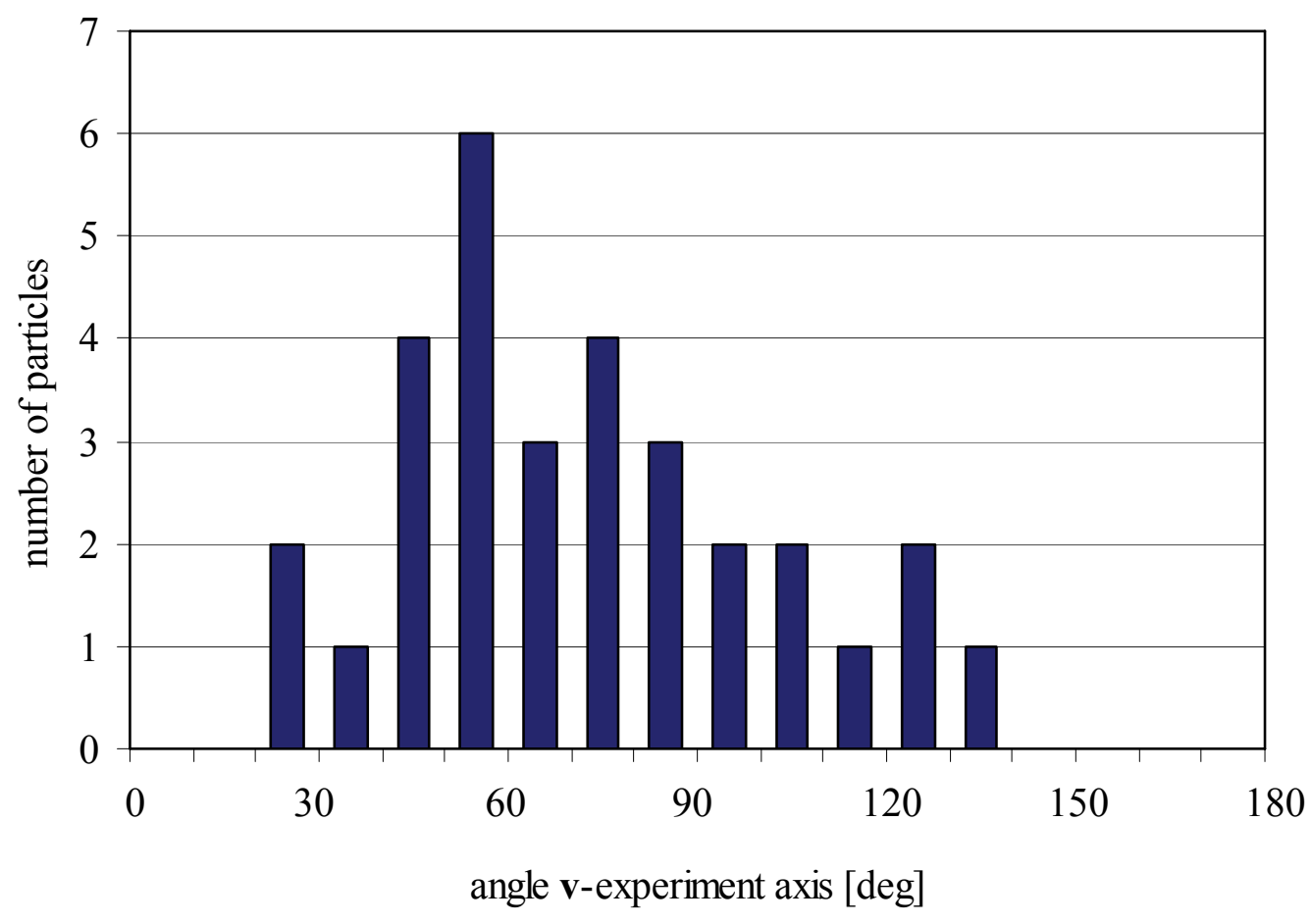

Figure 6-58: Impact direction distribution population E1 particles Population E1 particles mainly arrive from the spacecrafts apex direction (angle experiment axis - flight direction $<90^{\circ}$ ). 


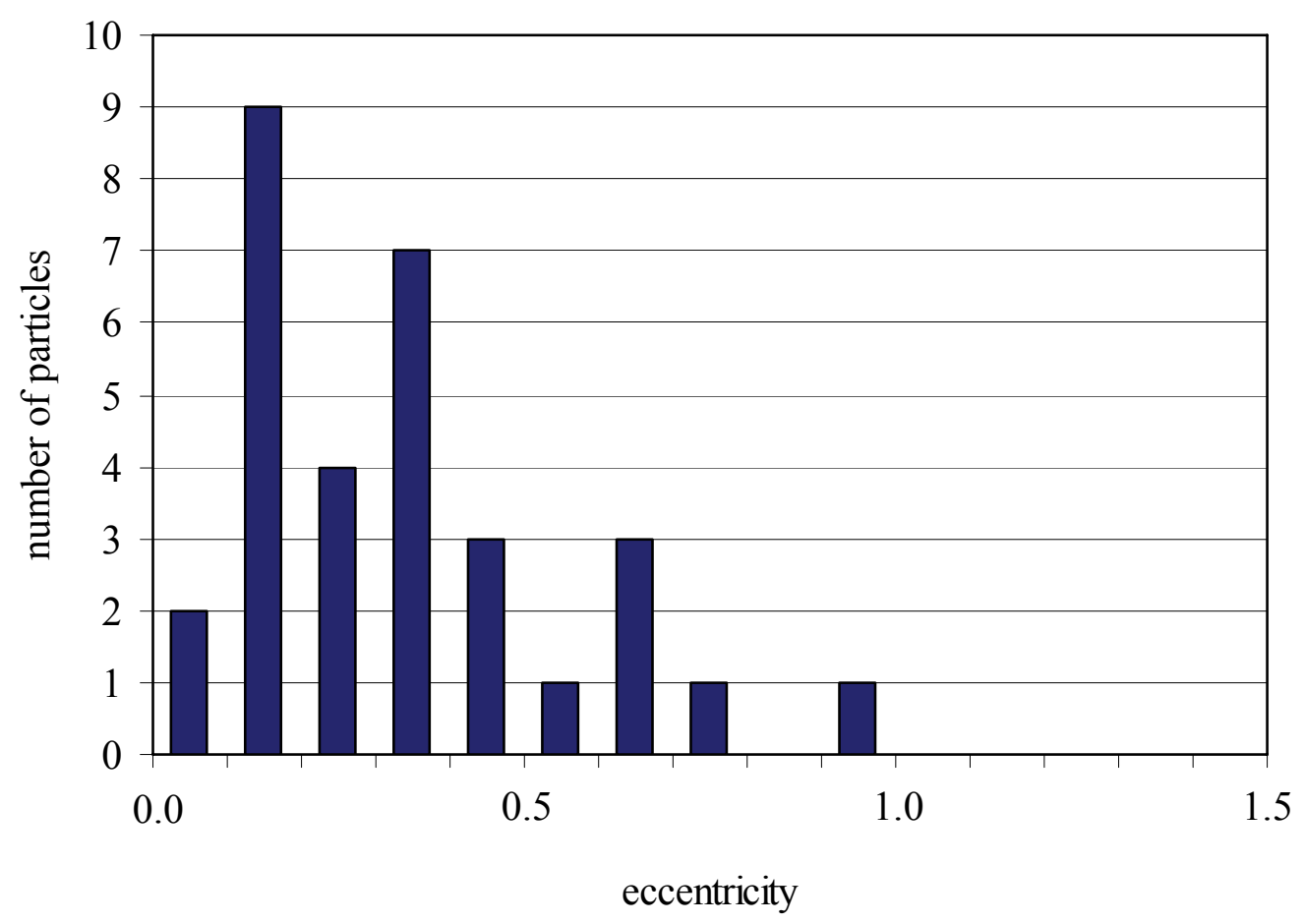

Figure 6-59: Eccentricity distribution population E1 particles Population E1 particles mainly have low eccentricities with a peak at $10^{\circ}$ to $20^{\circ}$.

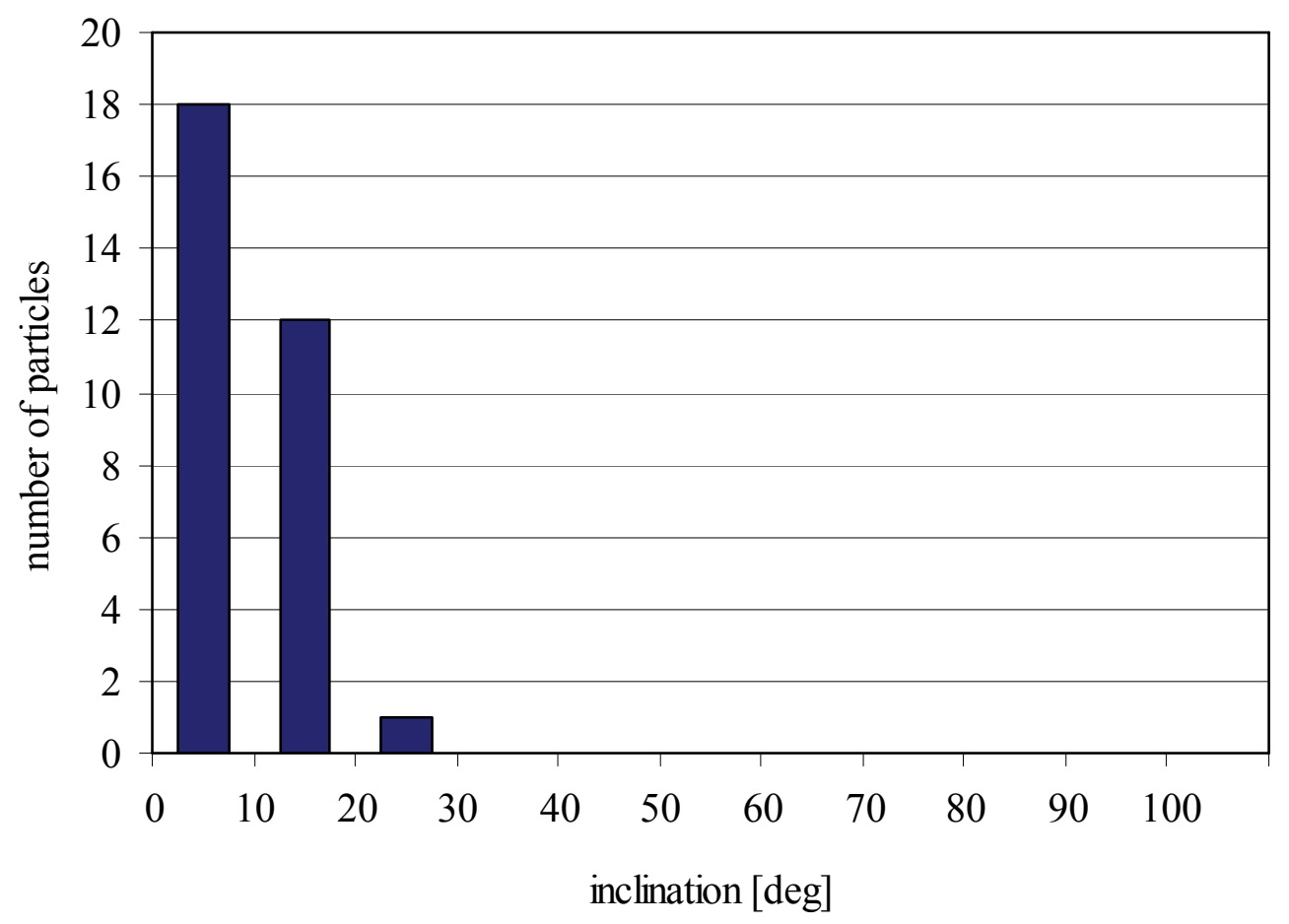

Figure 6-60: Inclination distribution population E1 particles Population E1 particles have low inclinations $\left(<30^{\circ}\right)$ with a peak at $0^{\circ}$ to $10^{\circ}$. 
The main properties of the 24 population E2 particles are summarized in Table 6-15. Flight directions of the particles are shown in Figure 6-61.

Population E2 particles have lower mass, but broader distributions in eccentricity and inclination towards higher eccentric and higher inclined orbits. In addition, population E2 particles show some similarities to Grün's model. The mass distribution of population E2 particles is shown in Figure 6-62 and has a mean value of $6.95 \cdot 10^{-13} \mathrm{~g}$, which fits to the maximum of the mass distribution of Grün's population B $\left(1 \cdot 10^{-13} \mathrm{~g}\right)$ within the limits of the accuracy in mass determination. Also, the distributions of eccentricity and inclination of population E2 (see Figure 6-59 and Figure 6-60) fit well to the corresponding distributions of population $\mathrm{B}$, the distribution of eccentricities of population E2 particles even shows the bimodal characteristic like Grün's population B particles do, but with the second peak at lower eccentricity than population B particles.

Like population E1 particles, population E2 particles preferable impact from the apex direction of the spacecraft (see Figure 6-63).

Table 6-15: Properties of the 24 population E2 particles

\begin{tabular}{ccccc}
\hline Pop. E2 & Mass [g] & Eccentricity & Incl. [deg] & Sm. ax. [AU] \\
\hline Max & $2.30 E-12$ & 0.99 & 50.08 & 4.15 \\
Min & $1.70 E-14$ & 0.06 & 2.87 & 0.64 \\
Mean & $\mathbf{6 . 9 5 E}-13$ & $\mathbf{0 . 4 4}$ & $\mathbf{2 0 . 5 2}$ & $\mathbf{1 . 3 9}$ \\
\hline
\end{tabular}

One maverick value omitted im semimajor axis mean value

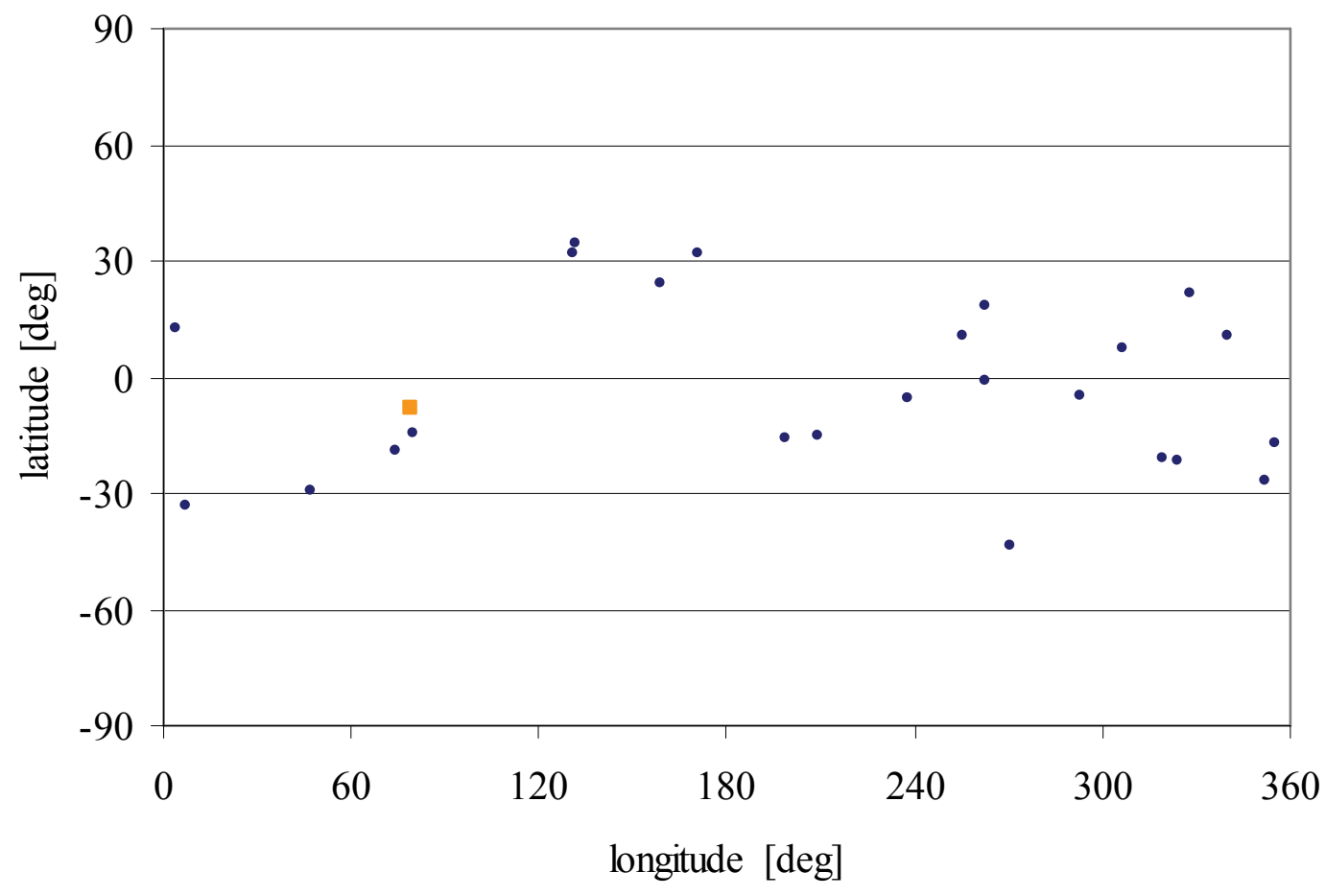

Figure 6-61: Latitude vs. longitude of population E2 particles

Population E2 particles do not show a preferred flight direction. The orange square marks the downstream direction of interstellar dust flux. 


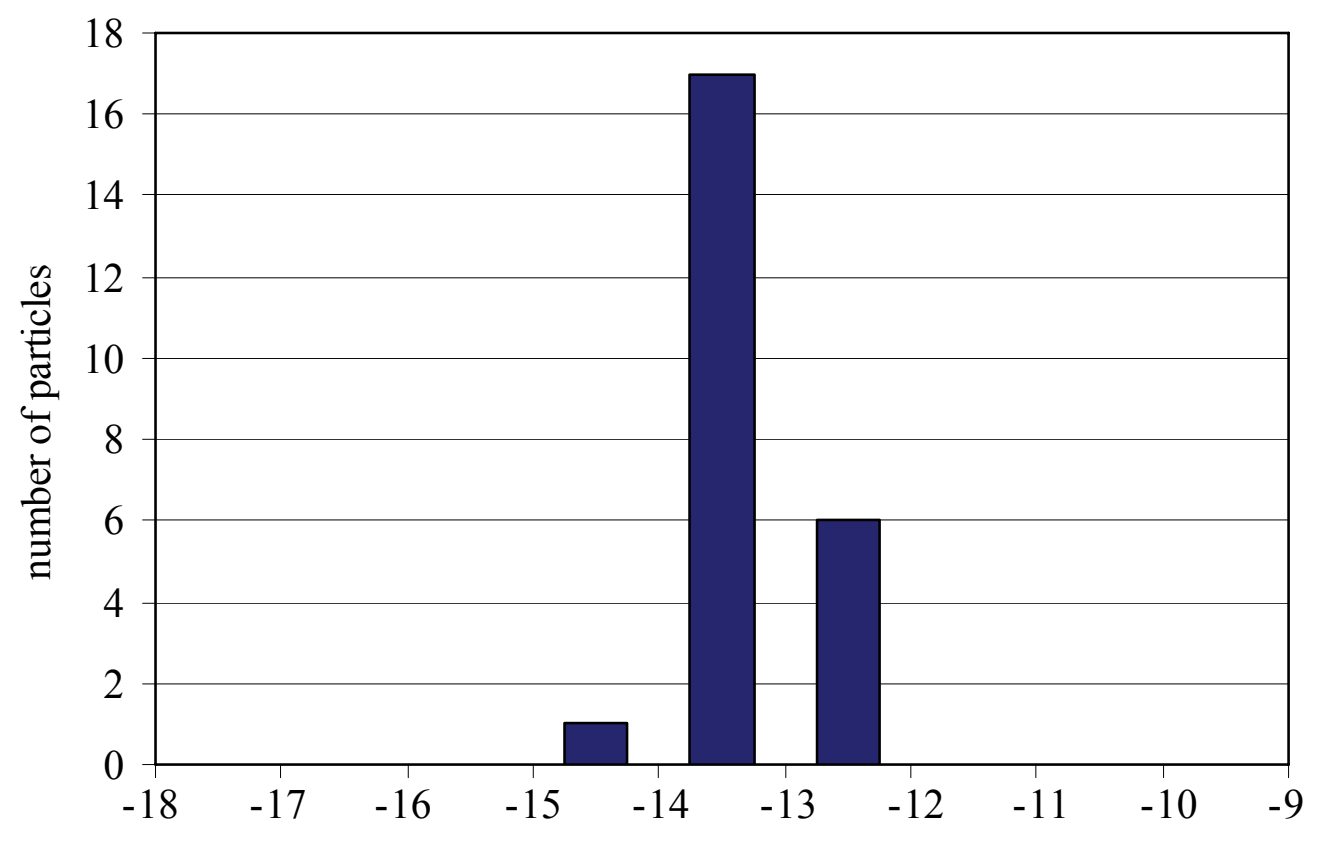

$\log$ mass $[\mathrm{g}]$

Figure 6-62: Mass distribution of population E2 particles

Population E2 particles have low mass from $10^{-12} \mathrm{~g}$ to $10^{-15} \mathrm{~g}$, with a peak at $10^{-13}$ to $10^{-14} \mathrm{~g}$.

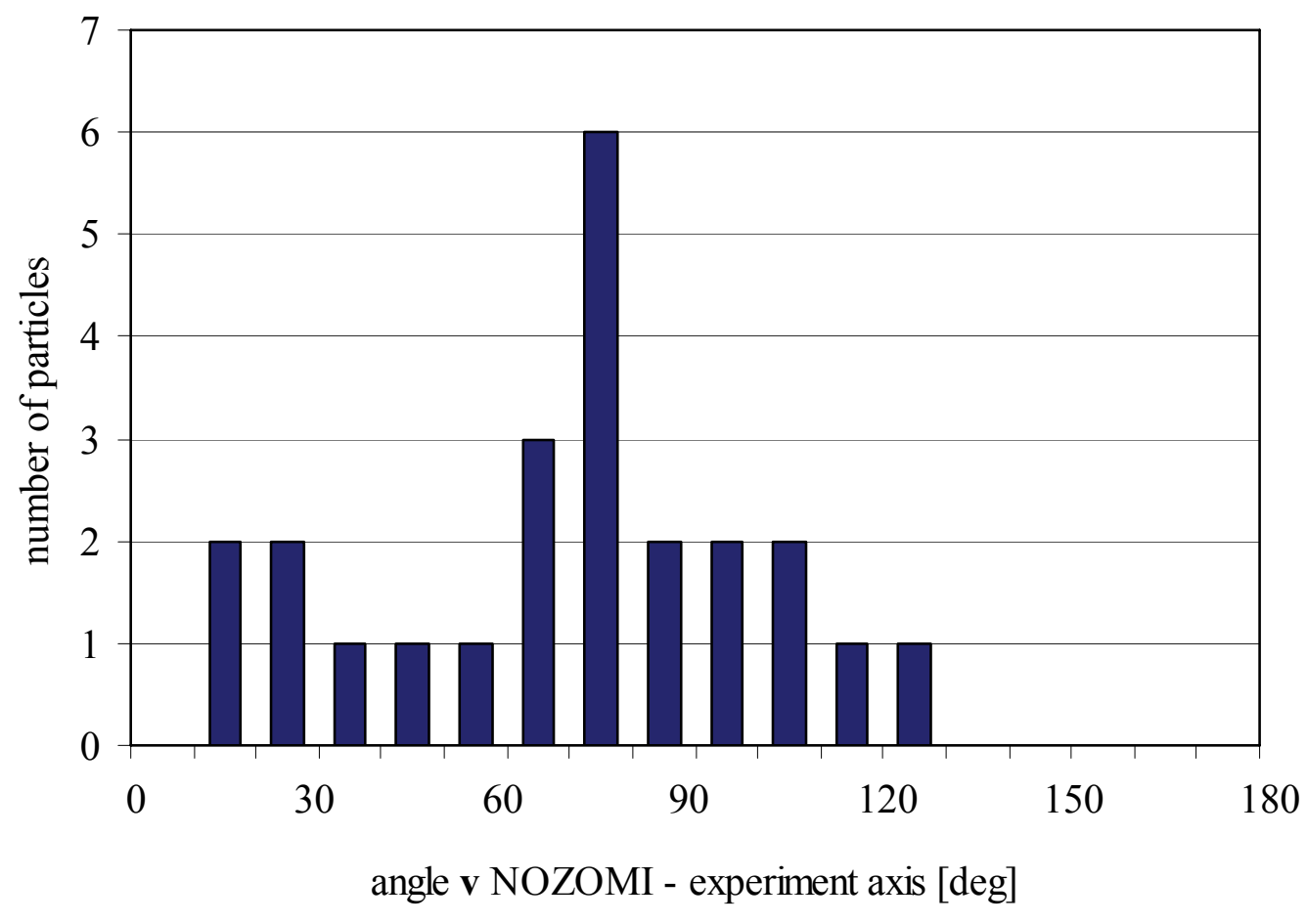

Figure 6-63: Impact direction distribution population E2 particles Population E2 particles mainly arrive from the spacecrafts apex direction (angle experiment axis - flight direction $<90^{\circ}$ ). 


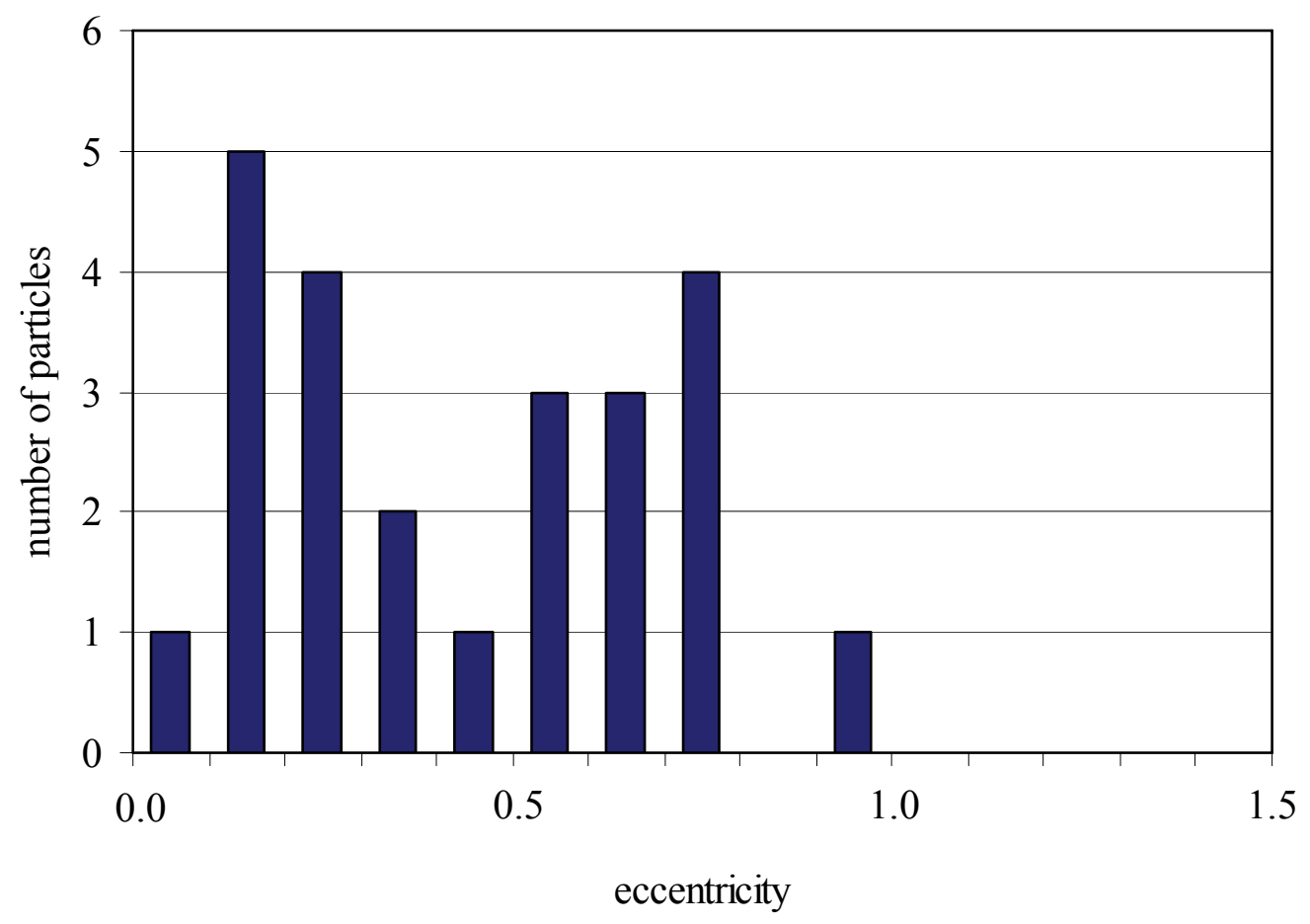

Figure 6-64: Eccentricity distribution population E2 particles Population E2 particles have a bimodal distribution in eccentricity with a peaks at 0.1 to 0.2 and at 0.7 to 0.8 .

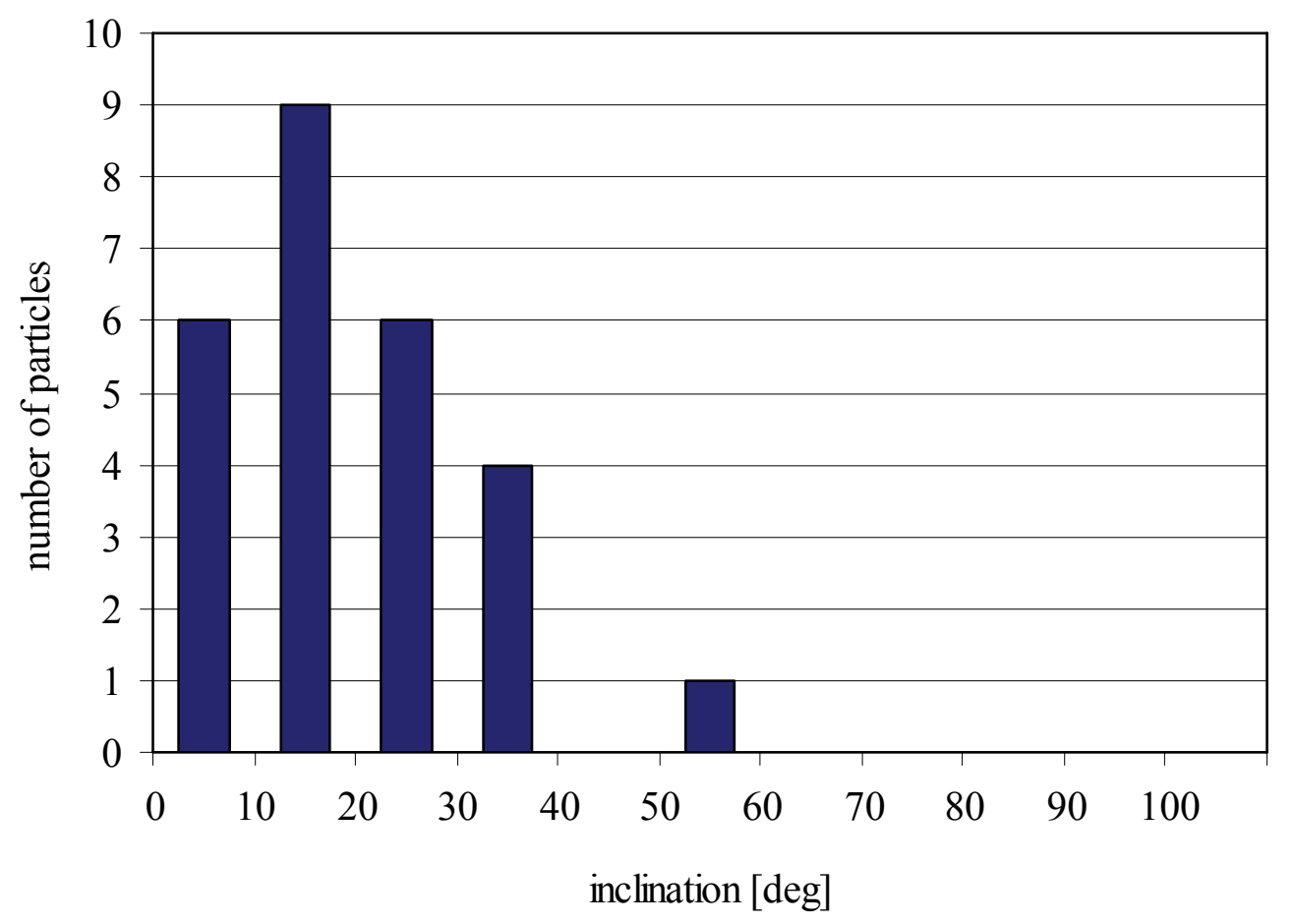

Figure 6-65: Inclination distribution population E2 particles

Population E2 particles have medium inclination (up to $60^{\circ}$ ) with a peak at $10^{\circ}$ to $20^{\circ}$. 
Populations E3 and E4 contain only 6 and 3 particles, respectively. Because of this low number of particles, no distributions in orbital elements or further statistical analyses were made. Particles of both populations have high inclinations with mean values of $60^{\circ}(\mathrm{E} 3)$ and $80^{\circ}$ (E4), and medium eccentricity of 0.77 (E3) and 0.66 (E4). While population E4 particles are of medium mass with a mean value of $1.18 \cdot 10^{-12} \mathrm{~g}$, population E3 particles are very light with a mean mass of $5.85 \cdot 10^{-15} \mathrm{~g}$.

All particles of both populations were impacting from the apex direction of the spacecraft.

Table 6-16: Properties of the 6 population E3 particles

\begin{tabular}{ccccc}
\hline Pop. E3 & Mass [g] & Eccentricity & Incl. [deg] & Sm. ax. [AU] \\
\hline Max & $1.60 \mathrm{E}-14$ & 1.02 & 93.10 & 2.89 \\
Min & $4.10 \mathrm{E}-16$ & 0.49 & 38.56 & 0.58 \\
Mean & $\mathbf{5 . 8 5 E}-15$ & $\mathbf{0 . 7 7}$ & $\mathbf{6 0 . 1 0}$ & $\mathbf{2 . 1 2}$ \\
\hline
\end{tabular}

One hyperbolic value omitted im semimajor axis mean value

Table 6-17: Properties of the 3 population E4 particles

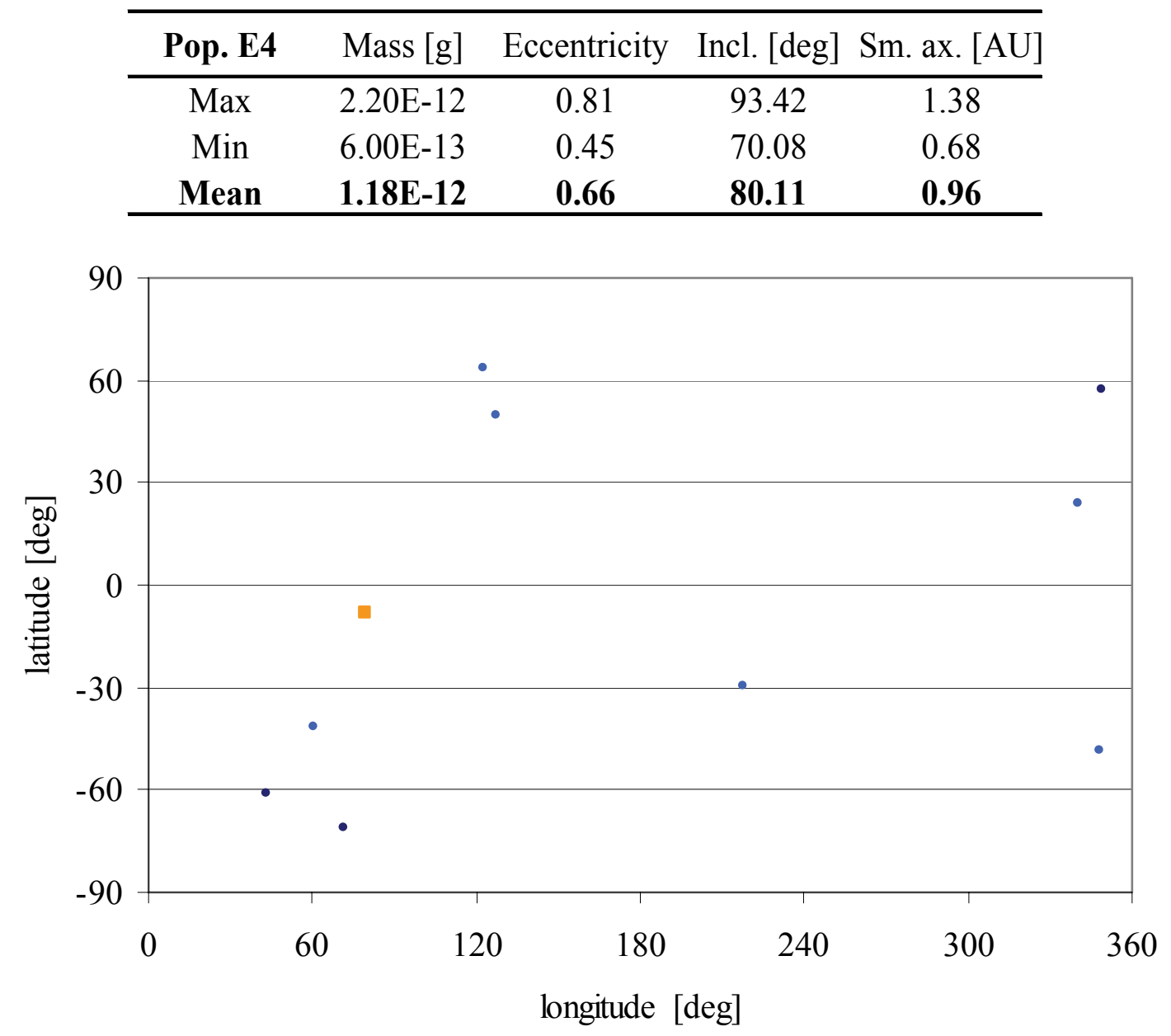

Figure 6-66: Latitude vs. longitude of population E3 and E4 particles Population E3 particles (light blue dots) and population E4 (dark blue dots) do not show a preferred flight direction. 
Population H1 particles have medium mass with a mean value of $1.78 \cdot 10^{-13} \mathrm{~g}$, hyperbolic eccentricity with a mean value of 1.62 and low inclinations with a mean value of $11.12^{\circ}$.

All but one of the 7 population $\mathrm{H} 1$ particles were impacting from the anti-apex direction of the spacecraft.

From the hyperbolic characteristics of the orbits, the strongly preferred anti-apex impact direction, and the low inclinations of population $\mathrm{H} 1$ particles, it can be supposed that population $\mathrm{H} 1$ may (at least partially) consist of $\beta$-meteoroids. It is indeed true that from the 7 population $\mathrm{H} 1$ particles, two were registered during mission times where the detector sensitivity for particles traveling on nearly radial trajectories was nearly maximal, and four were registered at a time where this detector sensitivity was in medium range (see Figure 6-30 for the detector sensitivities). In addition, population $\mathrm{H} 1$ particles do not show a preferred flight direction (see Figure 6-67) and thus do not belong to a particle stream of any origin. However, a more detailed analysis of this assumption cannot be done within the scope of this work.

Table 6-18: Properties of the 7 population $\mathrm{H} 1$ particles

\begin{tabular}{cccc}
\hline Pop. H1 & Mass [g] & Eccentricity & Incl. [deg] \\
\hline Max & $6.50 \mathrm{E}-13$ & 1.97 & 21.41 \\
Min & $1.40 \mathrm{E}-15$ & 1.02 & 2.38 \\
Mean & $\mathbf{1 . 7 8 E}-13$ & $\mathbf{1 . 6 2}$ & $\mathbf{1 1 . 1 2}$ \\
\hline
\end{tabular}

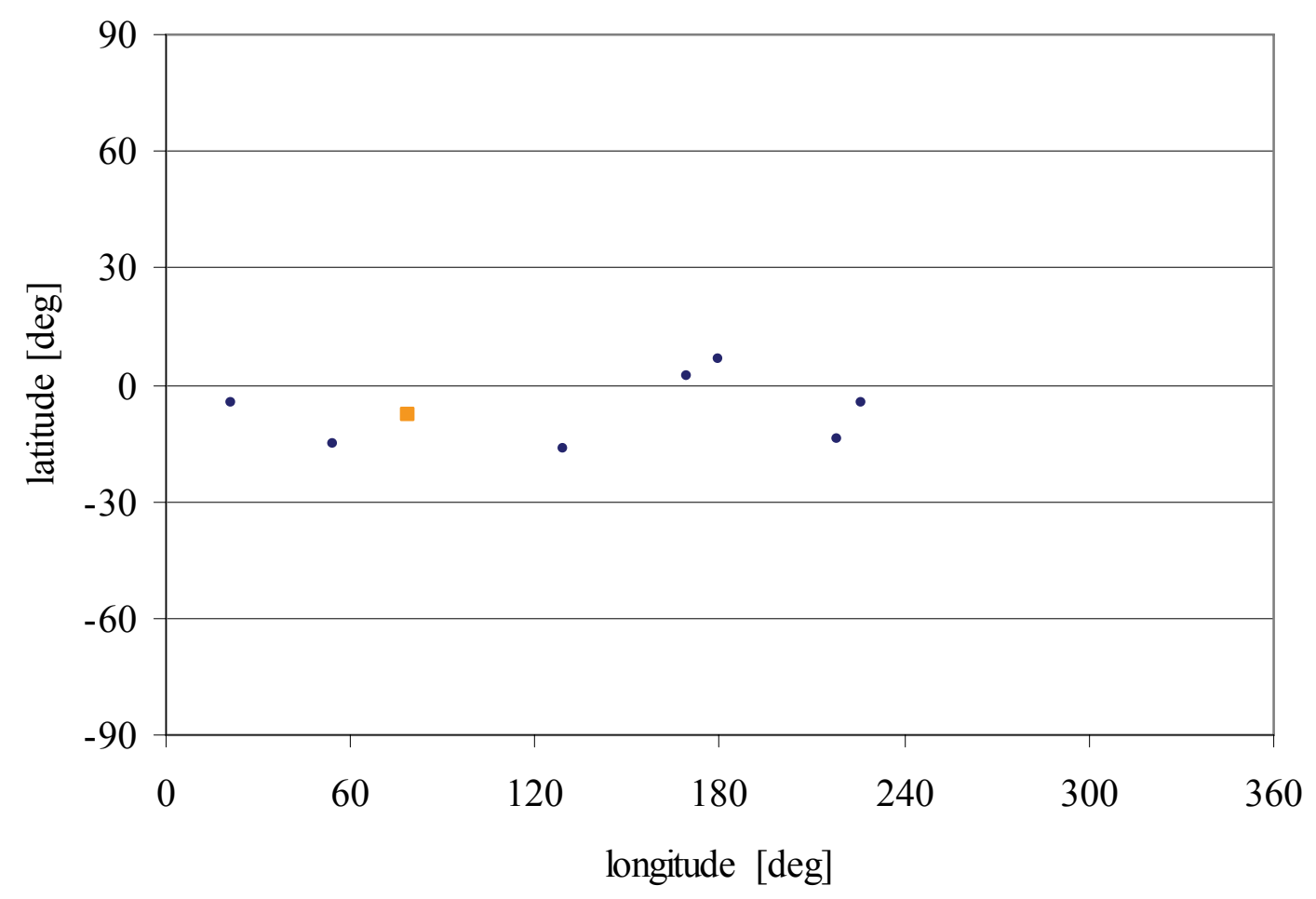

Figure 6-67: Latitude vs. longitude of population H1 particles

Population $\mathrm{H} 1$ particles do not show a preferred flight direction. The orange square marks the downstream direction of interstellar dust flux. 
Population H2 particles have small mass with a mean value of $2.83 \cdot 10^{-14} \mathrm{~g}$, hyperbolic eccentricity with a mean value of 2.99 and medium inclinations with a mean value of $28.70^{\circ}$.

All of the 5 population $\mathrm{H} 1$ particles were impacting from the anti-apex direction of the spacecraft.

Population $\mathrm{H} 2$ particles show a correlation in flight direction to the downstream direction of the interstellar gas and dust flux that is within the accuracy of the determination of particle flight direction, see Figure 6-68. The high speed of the particles and the correlation to the direction of the interstellar gas and dust flux suggests that population $\mathrm{H} 2$ particles may be of interstellar origin. This possibility must be discussed with respect to the known properties of ISD grains and their dynamics in the heliosphere.

Table 6-19: Properties of the 5 population H2 particles

\begin{tabular}{cccc}
\hline Pop. H2 & Mass [g] & Eccentricity & Incl. [deg] \\
\hline Max & $1.30 \mathrm{E}-13$ & 3.27 & 43.93 \\
Min & $1.60 \mathrm{E}-15$ & 2.80 & 16.67 \\
Mean & $\mathbf{2 . 8 3 E - 1 4}$ & $\mathbf{2 . 9 9}$ & $\mathbf{2 8 . 7 0}$ \\
\hline
\end{tabular}

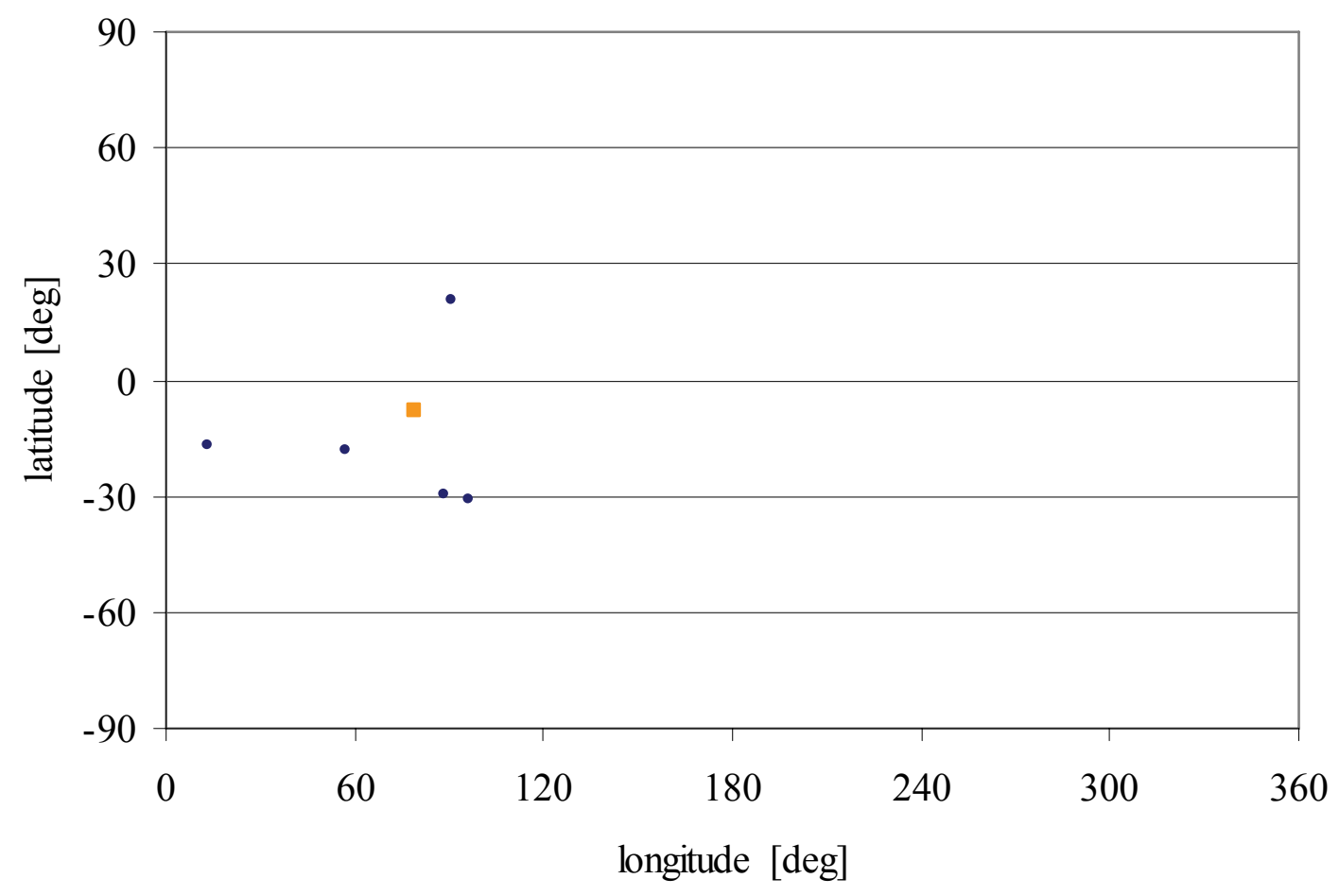

Figure 6-68: Latitude vs. longitude of population $\mathrm{H} 2$ particles

Population $\mathrm{H} 2$ particles show a preferred flight direction that is correlated with the downstream direction of the interstellar gas and dust flux. 


\subsection{Summary}

In this chapter, the scientific results of the MDC on NOZOMI were presented and discussed. For the first time, the full dust data recorded by the MDC were analyzed in a consistent way and with respect to the instrument's and the spacecraft's properties. In advance, the characteristics of the NOZOMI flight data, the noise signals recorded by the MDC during the mission, the MDC detection capabilities and the instrument conditions throughout the mission were discussed.

At first, the huge amount of noise signals recorded by the MDC was examined. It was shown that about a third of all noise signals recorded is likely caused by solar radiation that influences the MDC sensor electronics indirectly. A number of clusters of noise signals of very similar shape were identified within the whole noise data. The origins of these signals, which are assumed the same within each single cluster, could not be assigned to any known source of noise (no information about the NOZOMI spacecraft's internal operations that may be a source of noise recorded by the MDC was available). Several periods of highly increased noise rates were identified during the whole mission time, each lasting hours to weeks, which are surrounded by periods with very low noise rates. A temporal correspondence of these high noise periods to solar eruptions detected by the SOHO satellite was not approved. In summary, the origin of most of the high amount of noise recorded by the MDC and the observed patterns within the noise data could not be clarified with the available information. However, the detector dead time caused by the processing of recorded noise signals was shown to be negligible, since only during some short periods a significant value of a few percent of time was reached.

The NOZOMI flight data and the MDC sensor pointing directions were examined in detail. The determination of the MDC view axis at a given impact time from the NOZOMI attitude and orbit data is required for the later analysis of impact data and was carried out thoroughly. For a later statistical interpretation of the impact data recorded by the MDC, knowledge about the pointing directions and detection capabilities of the MDC over the mission time is required, both strongly dependent on the NOZOMI attitude data and its orbit positions. The NOZOMI orbit and attitude data was analyzed and discussed. Detection capabilities of the MDC were determined and discussed with respect to the NOZOMI attitude and orbit data.

The MDC operating data as derived from the telemetry data was analyzed and discussed. The MDC was in good condition throughout the mission. Fluctuations of the power supply voltage and the high voltage inside the sensor box are correlated with the MDC instruments temperatures, which in turn are correlated to the NOZOMI distance from the Sun. Influences of the high voltage fluctuations on the results of the primary data analysis process (which are mass and impact speed of the detected particles) were not considered. From the MDC command log, a detector down time of a few percent of the total mission time can be determined that should be considered for later dust flux determinations, but was neglected due to missing command data, which prevented an exact down time determination for the time after September 1999.

From the results of the primary analysis of the detected particles (particle mass and impact speed), the MDC pointing direction at the moment of impact, and the NOZOMI flight data (attitude, orbital position and flight speed), a full set of data (orbital position $\mathbf{r}$ and speed 
vector v) was derived that describes the particle's orbits. From this, the orbital elements inclination, eccentricity and semi major axis, which are more convenient for further interpretation, were calculated. The analysis of the impacts that were detected by the MDC was split into two parts with respect to the different environmental conditions during the first half year of the mission when NOZOMI was in an orbit around the Earth, and the rest of the mission time when NOZOMI was in the interplanetary space. Unlike it was expected, the MDC did neither detect dust particles on orbits bound to the Earth, nor space debris. All particles detected during the first half year were of interplanetary or interstellar origin and were discussed together with the particles detected in the interplanetary space. From the whole set of particles detected, a number of populations were identified that showed similarities in mass, inclination and eccentricity, four (plus one small) bound populations and two (plus two small) hyperbolic populations. From the four bound populations identified, two showed significant similarities in the distributions of mass, inclination and eccentricity to populations described in literature that are part of a model of the interplanetary dust cloud derived from dust data of optical observations and other in-situ experiments. Two of the four hyperbolic populations may consist of ISD grains or $\beta$-meteoroides, respectively. These assumptions need further discussion.

All data discussed in this chapter will be available in the MDC file database, see chapter 7 for more information. 



\section{MDC online application and file database}

In this chapter, some problems that occurred during the project lifetime are described that were caused by proprietary data formats and software. A solution based on modern information technology methods was developed to prototype status and is discussed here.

\subsection{Basics and motivation}

The analysis of the MDC data was subject of intense discussion among the involved scientists and engineers. This, and the fact that scientists and engineers are distributed to several different institutes in Europe and Japan as well as the long running project (designed project life was 3 years initially) and thus several personnel changes at the LRT have lead to several problems regarding information flow and consistency of different existing copies of data files. In this chapter, a solution started in the year 2002 and based on a central project database and a standard user interface that is able to avoid these problems in future projects and additionally is a good platform for the publication and distribution of the MDC-NOZOMI data is presented.

An analog, but much more general approach is made by the DLR Neustrehlitz by developing the DLR-VirtualLab, "a repository of online executable scientific software", see Ernst et al. [Ern2003] (also http://vl.nz.dlr.de/VL, hyperlink valid on October 27, 2005). The main idea of this project is to collect software solutions developed by scientists for usage in their individual projects, and connect these software packages to the internet via a web-browser based standardized user interface. This shall enable scientists all over the world to profit from their colleagues work in a much easier way than it is possible until today.

\subsection{Description}

The proprietary data formats, the data storage and the analysis process that is based on a proprietary single user application described in [Nau2000] lead to some serious trouble during the MDC project. One of the main problems was that inconsistencies occurred due to redundant copies of the data files that contain the final data analysis results, and there was no more chance to reproduce the validity of the different copies due to the ineluctably information loss during the handovers of the project from one scientist to the next. The disadvantages of the current data storage and data processing can be summarized as follows:

- No centralized data storage

- No consistent storage of additional information (comments etc.)

- Distribution of analysis results without intermediate results and information

- Data storage based on proprietary, platform dependent data formats 
To avoid these disadvantages, a solution that satisfies the requirements of a distributed scientific community, especially concerning data access, data processing and consistent documentation of analysis tasks and discussions, was designed and developed with respect to the requirements of the MDC project's IT structure. The improvements can be summarized as follows:

- Raw data, intermediate analysis results, final results and additional information are stored in a central database

- Decentral data access for all users by platform independent standard software with adapted user rights

- Efficient data browsing, searching and comparing

The database system, the corresponding user interface and the server architecture are described in the following paragraphs.

\subsubsection{Online application}

The basic technologies used for the implementation of the MDC online database are HTML for the user interface, PostGreSQL as database engine and Java ${ }^{\mathrm{TM}}$ as programming language of the online application. Furthermore, all data that is imported into the database was converted from proprietary formats to the standardized XML document description language. As server applications, Apache as standard internet server and Jakarta-Tomcat for processing the Java ${ }^{\mathrm{TM}}$ applets that build the online application are being used. For the development of the $\mathrm{Java}^{\mathrm{TM}}$ applets, the developer software Eclipse was used which is also available as open source.

The use of these standard technologies ensures that not only access to the database but also the database and the online application themselves are platform independent. The open source character of all used technologies and server applications ensures that future use and upgrading of the online application can easily be done due to unlimited availability of the required developer software and documentation.

The online application is based on the MVC architecture (Model - View - Controller), see e.g. Buschmann et al. [Bus1996]. The separation of the tasks into three independent layers eases future improvements due to modular structure and central definition of variables. The different tasks are distributed as follows to the three layers:

- View layer: Creates HTML code for the user interface, based on JSP with CSS stylesheets.

- Model layer: Event dispatcher with subclasses for the different required tasks (e.g. LoginManager for Login processing etc.) and other supporting classes.

- Controller-layer: Management of user requests and access control. 
Access control, managed by the controller-layer, supports and defines different user roles with corresponding user rights. This gives the opportunity to open the database for public and press as well as for scientists that are not involved in the project itself but may be interested in the mission results.

Central instance of the database is a single MDC memory segment that contains signal and basic housekeeping information. All other information, e.g. satellite flight data, further HSK data, intermediate analysis results, final analysis results, comments and links to calibration data are grouped around this central instance.

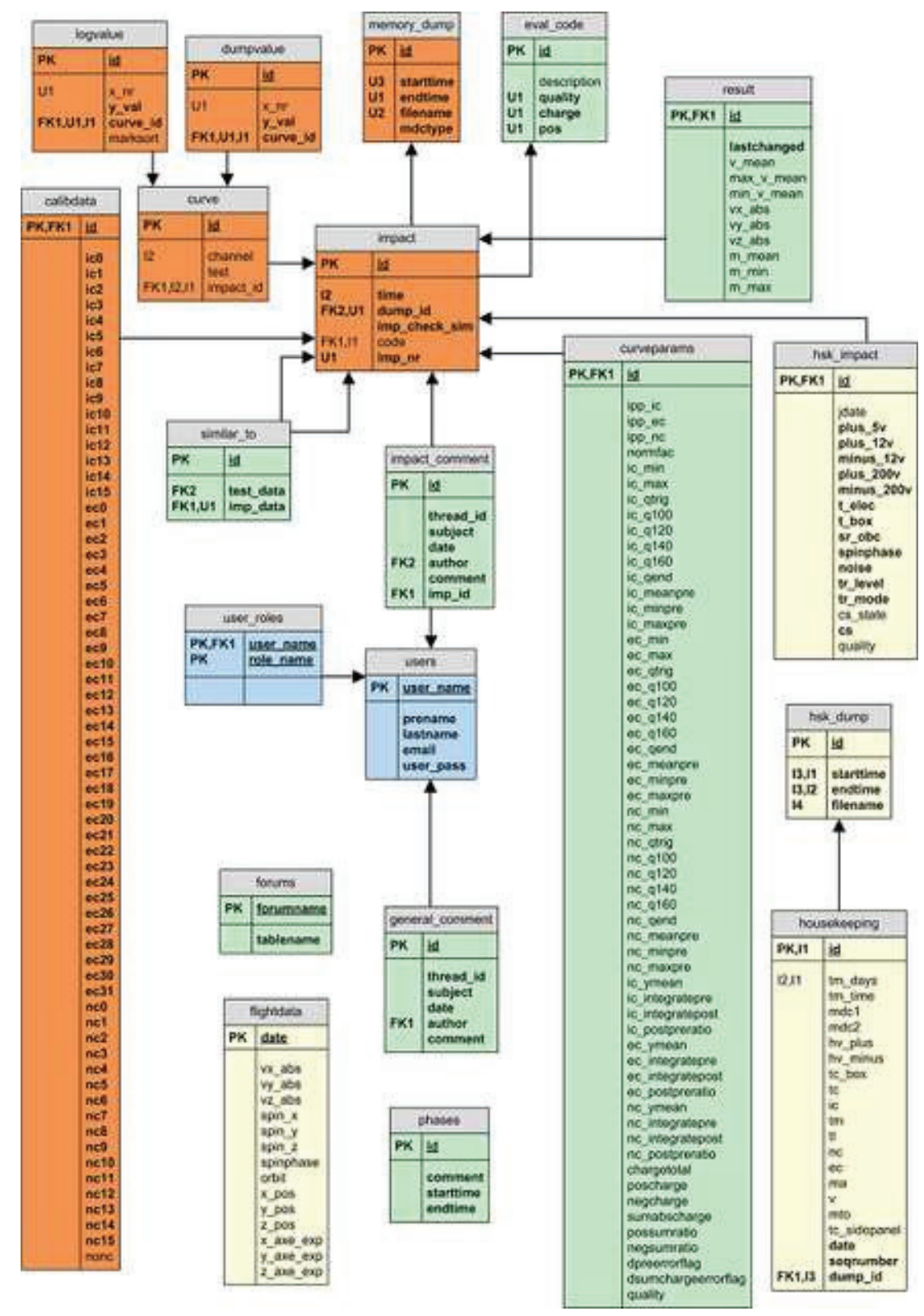

Figure 7-1: Table structure of the MDC online database

Central element is a single MDC signal (impact). All other tables are grouped around this element. 
The user interface is based on pure HTML code and can be viewed and used with every standard internet browser.

Depending on the user role, the user interface offers the following functions:

1. Viewing, browsing and searching options on the full MDC signal data

2. Adding comments about single MDC signals/impacts

3. Adding classification parameters and analysis results to single MDC signals/impacts

4. Adding new raw data files

\subsubsection{File database}

At the end of the Mars Dust Counter project, all appropriate files were reorganized in a consistent directory structure. Here, the directory structure and the content of the final MDC data Volumes \#1 to \#8 are documented.

All essential data files of the MDC project were stored in platform independent data format where possible, either as formatted text or as XML data files (see also Appendix D for the structure of the MDC raw data XML files). Microsoft ${ }^{\circledR}$ Office files are stored in Office 2002 or earlier data format. All thesis works on Volume \#7 were developed by undergraduate students under supervision of the author of this work; parts of the results were used in this work. All files and the MDC Online Database will be published on the LRT WWW-Server after completion of the MDC-NOZOMI project.

Every directory and subdirectory contains a special file 0000 readme.txt, that describes the content and structure of the files stored in the specific directory. For all files that are stored in proprietary data formats, the specific proprietary software and version number is given to ensure access to the files' content.

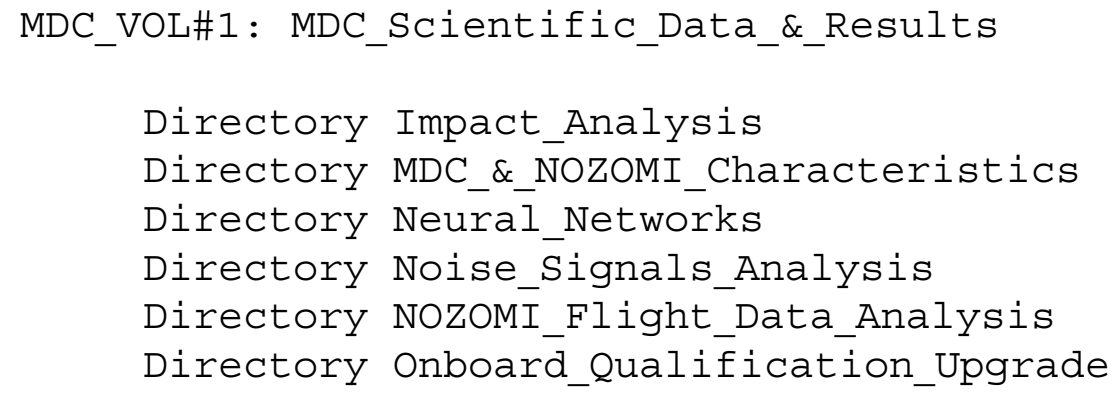

MDC_VOL\#2: MDC_Design_\&_Technical_Documents

Directory Design

Directory Images 
Directory Onboard_Software

Directory Technical_Documents

MDC_VOL\#3 : Raw_Data_\&_Calibration_Data

Directory Calibration_Data_BIN

Directory Calibration_Data_XML

Directory Housekeeping_Data_BIN

Directory Housekeeping_Data_TXT

Directory Housekeeping_Data_XML

Directory Telemetry_Data_BI $\bar{N}$

Directory Telemetry_Data_XML

MDC_VOL\#4 : MDC-HITEN_\&_MDC-BremSat

Directory MDC_Bremsat

Directory MDC_HITEN

MDC_VOL\#5: Publications_\&_Talks

Directory Publications

Directory Talks

MDC_VOL\#6: Doctoral_Thesis_Works

Directory Dissertation_Robert_Senger

MDC_VOL\#7: Collegiate_Thesis_Works

Directory DA_03_15_Dominic_Kyritsis

Directory IDP_Melanie_Dietz

Directory SA_03_06_Martin_Glas

Directory SA_03_07_Anna_Hübner

Directory SA_04_15_Ariane_Sauer

Directory SA_04_17_Christian_Rott

Directory SA_04_18_Johannes_Kellerer

MDC_VOL\#8: MDC_Online_Database_\&_WWW-Pages

Directory MDC_Online_Database

Directory MDC_WWW_Pages 
Decentral access to the whole MDC file database via Internet can be realized in different ways. The simplest one, based on standard Microsoft ${ }^{\circledR}$ Windows ${ }^{\circledR}$ file sharing capabilities and server access via Virtual Private Network (VPN) from remote clients, was realized in the year 2001 and was used by the author of this work and contributing students throughout the project. However, since this requires proprietary clients with special system setups, it is planned to integrate the MDC file database into the MDC online application to enable platform independent access to the file database content via standard HTML browser software. Standard solutions like WebDAV (Web-based Distributed Authoring and Versioning) can be easily installed and configured on the LRT servers to enable browsing and downloading (and uploading if required) of project files from any standard remote client. More sophisticated solutions that enable enhanced file version control like CVS (Concurrent Versions System), a standard solution mainly used for Open Source software projects, can be used for a subset of files (e.g. software source code), but again require a special setup of the remote clients.

\subsection{Summary and future prospects}

The main intention of the decision to develop the MDC online database was to create a platform that provides central data storage and a corresponding user interface for data access to avoid the previously mentioned problems, namely the inconsistencies that occurred regarding the analysis results and their validity during the long running project time.

In it's current version, the MDC online database provides useful features for data overview like searching, browsing, annotating and evaluating signals and add final analysis values to signals identified as impacts. In addition, a basic discussion forum was implemented that gives the scientific community the chance to discuss issues regarding MDC signal analysis in an easy and effective way.

Many future extensions of this basic functionality can be imagined. Until now, the whole data analysis process is still based on proprietary software that was developed at the LRT and runs as a single user application. As a first and most important step, the analysis software could be connected to the new MDC online database. Since the online database possesses standard database interfaces this can be made with little effort and would lead to a consistent and comprehensible data analysis process. The network based database structure would enable every user of the analysis software, independently of his or her location, to access the always up-to-date data and analysis results. As a perfection of this integration, the whole analysis process can be implemented as part of the online application using JSP or Java ${ }^{\mathrm{TM}}$ applets rather than proprietary single user software. Further extensions like the integration of all satellite flight data, technical documentation, and interpretation of the analyzed impact data displayed by dynamically created graphics or charts based on the actual analysis results can be implemented and integrated into the existing database and user interface. For future dust science projects that may be developed at the LRT, the author recommends the use of an adapted database system and online application as described in this chapter.

The consequent use of methods that enable simple and decentral access to project data, as they are described in this chapter, may open new possibilities for future science projects like the MDC project. Against the background of the great success of Open Source software projects, an "Open Science" project based on the technologies described above and supervised 
by the project scientist may open up a new pool of brainpower that can contribute to the scientific success of small and concise projects like the MDC project.

One of the main activities of the European Planetology Network (EuroPlaNet, founded in January 2005, a network of research institutes form 17 European countries including the LRT and funded by the European Commission under the $6^{\text {th }}$ Framework Programme), activity N7, is dedicated to the development of the so called Integrated and Distributed Information Service (IDIS) and its evolution into a European Virtual Planetary Observatory. The IDIS platform is described in the EuroPlaNet Green Paper for Activity N7 [Eur2006]:

"IDIS will provide a general platform for exchanging and accessing data and information, integrating and linking relevant data centres, data bases and information systems, providing a more unified access to the information, [...] and to the benefit of the dissemination of data produced in Europe."

Currently, a demo version of the IDIS platform based on the same technologies used for the MDC online application (Java ${ }^{\mathrm{TM}}$, Jakarta-Tomcat) is in development. This demo version is intended to allow access to sample contents coming from the CASSINI-HUYGENS mission. The possibility of an integration of the final MDC dust data into a forthcoming state-of-the-art IDIS platform should be considered and clarified. 



\section{Summary and discussion}

In this work, the scientific data of the Mars Dust Counter mission on NOZOMI were analyzed and interpreted. The signals recorded by the MDC were investigated carefully to separate noise data from impact data. Impact data was reviewed and particle mass and impact speed were derived. The MDC detected 98 particles of interplanetary and interstellar origin during its 4-year journey through the solar system. Neither space debris nor planetary dust bound to the Earth were detected. The characteristics of the MDC experiment and the MDC/NOZOMI system were investigated thoroughly to allow further analysis of the derived dust data. The NOZOMI flight data was investigated and visualized, dust data must be interpreted with respect to the flight data and the sensor pointing directions during the flight. The MDC dust data was interpreted with respect to the derived characteristics of the MDC experiment and the MDC/NOZOMI system, and the NOZOMI flight data. Interpretation of the MDC dust data showed good correlation to the known properties of interplanetary dust particles. The dust flux derived from the MDC dust data correlates well with dust flux models from literature that were developed from dust data of various other sources. From the whole amount of particles, separate populations of dust particles were identified. The characteristics of some of these populations found in the MDC dust data fit to particle populations published in literature that were introduced to match dust data derived from other in-situ dust detection instruments.

With respect to the technical troubles the spacecraft NOZOMI suffered from, a new data compression method for the onboard data was developed and evaluated. The data compression method is based on Wavelet transformation of the original signal and subsequent compression of the transformed data set. Compression factors of 16 to 50 were achieved with this method, without losing relevant information for the latter analysis of the signals. This was proved by studies carried out with the existing MDC-NOZOMI and MDC-HITEN data. The data compression method was therefore suitable for the MDC-NOZOMI if it had reached Mars orbit. It can be used in future instruments to reduce the amount of data that has to be transferred to Earth. The routine that performs the compression onboard the MDC was ready for upload, but this was not possible any more due to the system failure on NOZOMI that occurred on April 24, 2002.

Also, an optimized parameter set for signal qualification onboard was determined. The qualification routine onboard the MDC can be used for a primitive distinction of impact signals from noise signals. With the introduction of a threshold value that separates all data into good and bad signals, and a simple upgrade of the memory readout routine, a reduction of data by a factor of 100 could be achieved. Optimization of the parameter set that is used by the qualification routine was carried out by a genetic algorithm.

These two improvements of the onboard data processing would enable the MDC to transmit its data back to earth despite the restricted NOZOMI telemetry capacities. For future missions, an even better signal qualification method based on neural networks was developed and tested on the existing MDC-NOZOMI data. It was proven by Hofschuster [Hof2002] in laboratory experiments that instruments based on the MDC experiment architecture can be equipped with a time-of-flight mass spectrometer. This would give such future experiments new capabilities for the additional determination of the chemical composition of cosmic dust 
particles. For such instruments, efficient methods for onboard processing of the data become even more important.

All MDC-NOZOMI data was stored in a new developed database system that is easily accessible for public and interested scientists. Further data that is not included in the database is stored in standard XML format, or proprietary data formats where no conversion was possible. This ensures that the project data will be accessible in the future for further investigations. All data that is presented in this work is available electronically from the LRT. 


\section{Appendix A: Bibliography}

[Alt2003] Altobelli, N., Kempf, S., Landgraf, M., Srama, R., Dikarev, V., Krüger, H., Moragas-Klostermeyer, G., Grün, E., "CASSINI between Venus and Earth: Detection of interstellar dust”, J. Geophys. Res., Vol. 108, No. A10, 8032, 2003

[Alt2005] Altobelli, N., Kempf, S., Krüger, H., Landgraf, M., Roy, M., Grün, E., "Interstellar dust flux measurements by the GALILEO dust instrument between the orbits of Venus and Mars“, J. Geophys. Res., Vol. 110, A07102, 2005

[Alt2006] Altobelli, N., Grün, E., Landgraf, M., "A new look into the HELIOS dust experiment data: presence of interstellar dust inside the Earth's orbit", Astron. Astroph., Vol. 448, pp. 243-252, 2006

[Aue2002] Auer, S., Grün, E., Srama, R., Kempf, S., Auer, R., "The charge and velocity detector of the cosmic dust analyzer on CASSINI", Planet. Space Sci., Vol. 50, pp. 773-779, 2002

[Bag1995] Baguhl, M., Grün, E., Hamilton, D.P., Linkert, G., Riemann, R., Staubach, P., Zook, H.A., "The flux of interstellar dust observed by ULYSSES and GALILEO”, Space Sci. Rev., Vol. 72, pp. 471-476, 1995

[Bag1996] Baguhl, M., Grün, E., Landgraf, M., "In-situ measurements of interstellar dust with the ULYSSES and GALILEO spaceprobes“, Space Sci. Rev., Vol. 78, pp. 165-172, 1996

[Ban1991] Banaszkiewicz, M., Ip, W.H., "A statistical study of impact ejecta distribution around Phobos and Deimos", Icarus, Vol. 90, pp. 237-253, 1991

[Ban1994] Banaszkiewicz, M., Fahr, H.J., Scherer, K., "Evolution of dust particle orbits under the influence of solar wind outflow asymmetries and the formation of the zodiacal dust cloud“", Icarus, Vol. 107, pp. 358-374, 1994

[Bau1996] Baumgärtel, K., Sauer, K., Bogdanov, A., Dubinin, E., Dougherty, M., "'Phobos events': signatures of solar wind dust interactions", Planet. Space Sci., Vol. 44, No. 6, pp. 589-601, 1996

[Bro1996] Brownlee, D.E., Burnett, D., Clark, B., Hanner, M.S., Horz, F., Kissel, J., Newburn, R., Sandford, S., Sekanina, Z., Tsou, P., Zolensky, M., "STARDUST: Comet and interstellar dust sample return mission", in Phys. Chem. and Dyn. of Interplanetary Dust, ASP Conf. Series 104 (eds. B.A.S. Gustafson and M.S. Hanner), pp. 223-226, 1996 
[Bur1979] Burns, J.A., Lamy, P.L., Soter, S., "Radiation forces on small particles in the solar system", Icarus, Vol. 40, pp. 1-48, 1979

[Bur2002] Burchell, M.J., Willis, M.J., Armes, S.P., Khan, M.A., Percy, M.J., Perruchot, C., "Impact ionization experiments with low density conducting polymer-based micro-particles as analogues of solar system dusts", Planet. Space Sci., Vol. 50, pp. 1025-1035, 2002

[Bus1996] Buschmann, F., Meunier, R., Rohnert, H., Sommerlad, P., Stal, M., "Pattern-Oriented Software Architecture", John Wiley and Sons, 1996

[Cas1699] Cassini, G.D., "Découverte de la lumière celeste qui paroist dans le zodiaque", Mémoires de l'Académie Royale des Sciences depuis 1666 jusqu'a 1699, Vol. VIII, pp. 119-209, 1699

[Cer1995] Cermák, I., Grün, E., Švestka, J., "New results in studies of electric charging of dust particles“, Adv. Space Res., Vol. 15, No. 10, pp. (10)59(10)64, 1995

[Che2003] Chesley, S.R., Ostro, S.J., Vokrouhlický, D., Čapek, D., Giorgini, J.D., Nolan, M.C., Margot, J.-L., Hine, A.A., Benner, L.A.M., Chamberlin, A.B., "Direct detection of the Yarkovsky effect via radar ranging to asteroid 6489 Golevka”, Science, Vol. 302, pp. 1739-1742, 2003

[Con1980] Consolmagno, G.J., "Influence of the interplanetary magnetic field on cometary and primordial dust orbits: Applications of Lorentz scattering", Icarus, Vol. 43, pp. 203-214, 1980

[Dal1977] Dalmann, B.K., Grün, E., Kissel, J., Dietzel, H., “The ion composition of the plasma produced by impacts of fast dust particles", Planet. Space Sci., Vol. 25, pp. 135-147, 1977

[Dav1981] Davis, D.R., Housen, K.R., Greenberg, R., "The unusual dynamical environment of Phobos and Deimos", Icarus, Vol. 47, pp. 220-233, 1981

[Deb2001] Debnath, L., "Wavelet Transforms and Time-Frequency Signal Analysis", Birkhauser, 2001

[Dei1985] Deichsel, G., Trampisch, H.J., "Clusteranalyse und Diskriminanzanalyse", Gustav Fischer Verlag, Stuttgart, 1985

[Die1972] Dietzel, H., Neukum, G., Rauser, P., "Micrometeoroid simulation studies on metal targets", J. Geophys. Res., Vol. 77, No. 8, p. 1375, 1972

[Dik2002] Dikarev, V., Jehn, R., Grün, E., "Towards a new model of the interplanetary meteoroid environment", Adv. Space Res., Vol. 29, No. 8, pp. 1171-1175, 2002 
[Dik2005] Dikarev, V., Grün, E., Baggaley, J., Galligan, D., Landgraf, M., Jehn, R., "The new ESA meteoroid model", Adv. Space Res,. Vol. 35, pp. 12821289,2005

[Div1993] Divine, N., "Five poulations of interplanetary meteoroids", J. Geophys. Res., Vol. 98, No. E9, pp. 17029-17048, 1993

[Dob1980] Dobrovolskis, A.R., Burns, J.A., "Life near the Roche limit: Behavior of ejecta from satellites close to planets”, Icarus, Vol. 42, No. 3, pp. 422-441, 1980

[Dra1974] Drapatz, S., Michel, K.W., "Theory of shock-wave ionization upon highvelocity impact of micrometeorites", Zeitschrift für Naturforschung, Vol. 29a, pp. 870-879, 1974

[Dra1984] Draine, B.T., Lee, H.M., "Optical properties of interstellar graphite and silicate grains", Astrophys. J., Vol. 285, pp. 89-108, 1984

[Dub1991] Dubinin, E.M., Pissarenko, N.F., Barabash, S.V., Zacharov, A.V., Lundin, R., Pellinen, R., Schwingenschuh, K., Yeroshenko, Ye.G., "Plasma and magnetic field effects associated with Phobos and Deimos tori", Planet. Space Sci., Vol. 39, No. 1/2, pp. 113-121, 1991

[Eic1975] Eichhorn, G., "Measurements of the light flash produced by high velocity particle impact”, Planet. Space Sci., Vol. 23, pp. 1519-1522, 1975

[Eic1976] Eichhorn, G., "Analysis of the hypervelocity impact process from impact flash measurements”, Planet. Space Sci., Vol. 24, pp. 771-781, 1976

[Ern2003] Ernst, Th, et al., "DLR's VirtualLab: Scientific software just a mouse click away", Computing in Science and Engineering, January/February 2003

[ESA1999] ESA, "ESA Space Debris Mitigation Handbook", Release 1.0, April 7, 1999

[Eur2006] European Planetology Network (EuroPlaNet), "Green Paper for Activity N7: Integrated and Distributed Information Service", $3{ }^{\text {rd }}$ draft, February 15,2006

[Fis1998] Fischer, F.G., "Mars Dust Counter on Planet-B: On-Board Software Internals", Laboratory for Process Control and Real-Time Systems, Technische Universität München, Germany, 1998

[Fis 1999] Fischer, F.G., “On-Board Software 'Autotrigger' Extension: Closed Loop MDC Sensitivity Control”, Laboratory for Process Control and Real-Time Systems, Technische Universität München, 1999

[Fri1963] Friichtenicht, J.F., Slattery, J.C., "Ionisation associated with hypervelocity impact”, NASA TN D-2091, 1963 
[Gla2003] Glas, M., "Signalklassifikation mit Neuronalen Netzen", Semesterarbeit RT SA 03/06, Lehrstuhl für Raumfahrttechnik, Technische Universität München, 2003

[Gö11988] Göller, J.R., "Kalibrationsmessungen an Mikrometeoritendetektoren für die Missionen ULYSSES, GALILEO und GIOTTO“, Dissertation, Ruprecht-Karls-Universität Heidelberg, 1988

[Gö11989] Göller, J.R., Grün, E., "Calibration of the GALILEO/ULYSSES dust detectors with different projectile materials and at varying impact angles", Planet. Space Sci., Vol. 37, No. 10, pp. 1197-1206, 1989

[Gol2002] Goldsworthy, B.J., Burchell, M.J., Cole, M.J., Green, S.F., Leese, M.R., McBride, N., McDonnell, J.A.M., Müller, M., Grün, E., Srama, R., Armes, S.P., Khan, M.A., "Laboratory calibration of the CASSINI cosmic dust analyser (CDA) using new, low density projectiles", Adv. Space Res., Vol. 29, No. 8, pp. 1139-1144, 2002

[Gri1997] Grinberg, E.I., Grigoryev, B.V., Nikolaev, V.S., Sokolov, N.A., "Interaction of space debris with liquid metal circuit of RORSAT satellites", Proc. $2^{\text {nd }}$ European Conf. Space Debris, pp. 273-277, 1997

[Grj1964] Grjebine, T., Lalou, C., Ros, J., Capitant, M., "Study of magnetic spherules in three cores of the occidential basin of the mediterranean sea", Ann. N.Y. Acad. Sci., Vol. 119, Art. 1, pp. 143-165, 1964

[Grü1985] Grün, E., Zook, H.A., Fechtig, H., Giese, R.H., "Collisional balance of the meteoritic complex“, Icarus, Vol. 62, pp. 244-272, 1985

[Grü1992a] E. Grün, H. Fechtig, R.H. Giese, J. Kissel, D. Linkert, D. Maas, J.A.M. McDonnell, G.E. Morfill, G. Schwehm, H.A. Zook, "The Ulysses dust experiment", Astron. Astrophys. Supp. Ser. 92, 411-423, 1992

[Grü1992b] E. Grün, H. Fechtig, M.S. Hanner, J. Kissel, B.-A. Lindblad, D. Linkert, D. Maas, G.E. Morfill, H.A. Zook, "The Galileo dust detector", Space Science Reviews 60, 317-340, 1992

[Grü1994] Grün, E., Gustafson, B., Mann, I., Baguhl, M., Morfill, G.E., Staubach, P., Taylor, A., Zook, H.A., "Interstellar dust in the heliosphere", Astron. Astrophys., Vol. 286, pp. 915-924, 1994

[Grü1995a] Grün, E., Baguhl, M., Hamilton, D.P., Kissel, J., Linkert, D., Linkert, G., Riemann, R., "Reduction of GALILEO and ULYSSES dust data", Planet. Space Sci., Vol. 43, No. 8, pp. 941-951, 1995

[Grü1995b] Grün, E., Baguhl, M., Divine, N., Fechtig, H., Hamilton, D.P., Hanner, M.S., Kissel, J., Lindblad, B.-A., Linkert, D., Linkert, G., Mann, I., McDonnel, J.A.M., Morfill, G.E., Polanskey, C., Riemann, R., Schwehm, G., Siddique, N., Staubach, P., Zook, H.A., "Three years of GALILEO dust data", Planet. Space Sci., Vol. 43, No. 8, pp. 953-969, 1995 
[Grü1995c] Grün, E., Baguhl, M., Divine, N., Fechtig, H., Hamilton, D.P., Hanner, M.S., Kissel, J., Lindblad, B.-A., Linkert, D., Linkert, G., Mann, I., McDonnell, J.A.M., Morfill, G.E., Polanskey, C., Riemann, R., Schwehm, G., Siddique, N., Staubach, P., Zook, H.A., "Two years of ULYSSES dust data", Planet. Space Sci., Vol. 43, No. 8, pp. 971-999, 1995

[Grü1997] Grün, E., Staubach, P., Baguhl, M., Hamilton, D.P., Zook, H.A., Dermott, S., Gustafson, B.A., Fechtig, H., Kissel, J., Linkert, D., Linkert, G., Srama, R., Hanner, M.S., Polanskey, C., Horányi, M., Lindblad, B.A., Mann, I., McDonnell, J.A.M., Morfill, G.E., Schwehm, G., "South-north and radial traverses through the interplanetary dust cloud", Icarus, Vol. 129, pp. 270288, 1997

[Grü2001] Grün, E., Landgraf, M., "Fast dust in the heliosphere", Space Sci. Rev., Vol. 99, pp. 151-164, 2001

[Grü2001b] Grün, E., Baguhl, M., Svedhem, H., Zook, H.A., "In situ measurements of cosmic dust", in Interplanetary Dust (eds. E. Grün, B.A.S. Gustafson, S.F. Dermott, H. Fechtig), Springer-Verlag Berlin Heidelberg, 2001

[Gus1986] Gustafson, B.A.S., Misconi, N.Y., "Interplanetary dust dynamics: I. Longterm gravitational effects of the inner planets on zodiacal dust", Icarus, Vol. 66, pp. 280-287, 1986

[Gus1987a] Gustafson, B.A.S., Misconi, N.Y., Rusk, E.T., "Interplanetary dust dynamics: II. Poynting-Robertson drag and planetary perturbations on cometary dust”, Icarus, Vol. 72, pp. 568-581, 1987

[Gus1994] Gustafson, B.A.S., "Physics of zodiacal dust", Ann. Rev. Earth Planet. Sci., Vol. 22, pp. 553-595, 1994

[Ham1994] Hamilton, D.P., “A comparison of Lorentz, planetary gravitational, and satellite gravitational resonances”, Icarus, Vol. 109, pp. 221-240, 1994

[Ham1996] Hamilton, D.P., "The asymmetric time-variable rings of Mars", Icarus, Vol. 119, pp. 153-172, 1996

[Har1976] Hartung, J.B., "Lunar microcraters and interplanetary dust fluxes", Lecture Notes in Physics, Vol. 48, Interplanetary Dust and Zodiacal Light, pp. 209-226, 1976

[Hei1990] Heistermann, J., "Learning in neural nets by genetic algorithms", Parallel Processing in Neural Systems and Compilers, Elsevier Science Publishers North-Holland, 1990

[Hei1994] Heistermann, J., "Genetische Algorithmen", B.G. Teubner Verlagsgesellschaft, Stuttgart, 1994 
[Hem1964] Hemenway, C.L., Linscott, I., Secretan, L., Dubin, M., "Preliminary study of possible cosmic dust impacts on project Mercury vehicle periscopic lenses", Ann. N.Y. Acad. Sci., Vol. 119, Art. 1, pp. 106-115, 1964

[Hof1971] Hoffmann, H.-J., "Entwicklung eines Detektors zur Massen- und Geschwindigkeitsanalyse von kosmischen Staubteilchen", Dissertation, Ruprecht-Karls-Universität, Heidelberg, 1971

[Hof2002] Hofschuster, G., "Hochgeschwindigkeitseinschläge auf teildurchlässige Oberflächen", Dissertation, Fachgebiet Raumfahrttechnik, Technische Universität München, 2002

[Hor1981] Hornung, K., Drapatz, S., "Residual ionization after impact of large dust particles“, ESA SP-155, pp. 23-37, 1981

[Hor1996a] Hornung, K., Malama, Yu. G., Thoma, K., "Modeling of the very high velocity impact process with respect to in-situ ionization measurements", Adv. Space Res., Vol. 17, No. 12, pp. (12)77-(12)86, 1996

[Hor1996b] Horányi, M., "Charged dust dynamics in the solar system", Annu. Rev. Astron. Astrophys, Vol. 34, pp. 383-418, 1996

[Hüb2003] Hübner, A. "Charakterisierung des Rauschverhaltens am Mars Dust Counter”, Semesterarbeit RT-SA 03/07, Technische Universität München, Lehrstuhl für Raumfahrttechnik, 2003

[Ige1998] Igenbergs, E., Sasaki, S., Münzenmayer, R., Ohasi, H., Färber, G., Fischer, F., Fujiwara, A., Glasmachers, A., Grün, E., Hamabe, Y., Iglseder, H., Klinge, D., Miyamoto, H., Mukai, T., Naumann, W., Nogami, K.-I., Schwehm, G., Svedhem, H., Yamakoshi, K., "Mars Dust Counter", Earth Planets Space, Vol. 50, pp. 241-245, 1998

[Ig11993a] Iglseder, H., Münzenmayer, R., Svedhem, H., Grün, E., “Cosmic dust and space debris measurements with the Munich Dust Counter on board the satellites HITEN and BremSat", Adv. Space Res., Vol. 13, No. 8, pp. (8)129-(8)132, 1993

[Igl1993b] Iglseder, H., Grün, E., Münzenmayer, R., Svedhem, H., “Analysis of the results of two-year operations of the Munich Dust Counter - A cosmic dust experiment on board the satellite HITEN", in "Meteoroids and their parent bodies", (eds. J. Štohl and I.P. Williams), Astronomical Inst., Slovak. Acad. Sci., Bratislava, 1993

[Igl1996] Iglseder, H., Uesugi, K., Svedhem, H., "Cosmic dust measurements in lunar orbit”, Adv. Space Res., Vol. 17, No. 12, pp. (12)177-(12)182, 1996

[Ina1999] Inada, A., Kawamata, M., Sumikawa, S., Mukai, T., "Planned observation of Phobos/Deimos by Mars Imaging Camera (MIC) on NOZOMI", Adv. Space Res., Vol. 23, No. 11, pp. 1911-1914, 1999 
[ISA1998] ISAS, "Planet-B Prelaunch Report", ISAS SES Data Center, SES-TD-98002, 1998

[Ish1994] Ishimoto, H., Mukai, T., "Phobos dust rings", Planet. Space Sci., Vol. 42, No. 8, pp. 691-697, 1994

[Ish1996] Ishimoto, H., "Formation of Phobos/Deimos dust rings", Icarus, Vol. 122, pp. $153-165,1996$

[Ish1997a] Ishimoto, H., Kimura, H., Nakagawa, N., Mukai, T., "Planned observation of Phobos/Deimos dust rings by PLANET-B”, Adv. Space Res., Vol. 19, No. 1, pp. (1)123-(1)126, 1997

[Jac1997] Jackson, A., Eichler, P., Reynolds, R., Potter, A., Johnson, N., "The historical contribution of solid rocket motors to the one centimeter debris population", Proc. $2^{\text {nd }}$ European Conf. Space Debris, pp. 279-284, 1997

[Jac2001] Jackson, A.A., "The capture of interstellar dust: the pure PoyntingRobertson case", Planet. Space Sci., Vol. 49, pp. 417-424, 2001

[Kaw1995] Kawaguchi, J., Yamakawa, H., Uesugi, T., Matsuo, H., "On making use of lunar and solar gravity assists in LUNAR-A, PLANET-B missions", Acta Astronautica, Vol. 35, No. 9-11, pp. 633-642, 1995

[Kaw2003] Kawaguchi, J., Nakatani, I., Uesugi, T., Tsuruda, K., "Synthesis of an alternative flight trajectory for Mars explorer NOZOMI", Acta Astronautica, Vol. 52, pp. 189-195, 2003

[Kel2004] Kellerer, J., "MDC BremSat Datenanalyse”, Semesterarbeit RT-SA 04/18, Technische Universität München, Lehrstuhl für Raumfahrttechnik, 2004

[Kho1993] Kholshevnikov, K.V., Krivov, A.V., Sokolov, L.L., Titov, V.B., "The dust torus around Phobos orbit”, Icarus, Vol. 105, pp. 351-362, 1993

[Kis1987] Kissel, J., Krüger, F.R., "Ion formation by impact of fast dust particles and comparison with related techniques", Appl. Phys. A, Vol. 42, pp. 69-85, 1987

[Kli1997] Klinkrad, H., Bendisch, J., Sdunnus, H., Wegener, P., Westerkamp, R., "An introduction to the 1997 ESA-MASTER model", Proc. $2^{\text {nd }}$ European Conf. Space Debris, pp. 217-224, 1997

[Kna1980] Knabe, W., "Untersuchungen am Einschlags-Ionisationsdetektor für die Galileo- und ISP-Mission", Diplomarbeit, MPI für Kernphysik, Heidelberg, 1980

[Koc2003] Kocifaj, M., Klačka, J., "The capture of interstellar dust: the pure electromagnetic case”, Planet. Space Sci., Vol. 51, pp. 617-626, 2003 
[Koc2004] Kocifaj, M., Klačka, J., "The capture of interstellar dust: the Lorentz force case”, Planet. Space Sci., Vol. 52, pp. 839-847, 2004

[Kri1997] Krivov, A., Hamilton, D.P., "Martian dust belts: waiting for discovery", Icarus, Vol. 128, pp. 335-353, 1997

[Kri1999] Krivov, A., Jurewicz, A., "The ethereal dust envelopes of the Martian moons”, Planet. Space Sci, Vol. 47, pp. 45-56, 1999

[Krü1999b] Krüger, H., Grün, E., Hamilton, D.P., Baguhl, M., Dermott, S., Fechtig, H., Gustafson, B.A., Hanner, M.S., Horányi, M., Kissel, J., Lindblad, B.A., Linkert, D., Linkert, G., Mann, I., McDonnell, J.A.M., Morfill, G.E., Polanskey, C., Riemann, R., Schwehm, G., Srama, R., Zook, H.A., “Three years of GALILEO dust data II: 1993-1995”, Planet. Space Sci., Vol. 47, pp. 85-106, 1999

[Krü1999c] Krüger, H., Grün, E., Landgraf, M., Baguhl, M., Dermott, S., Fechtig, H., Gustafson, B.A., Hamilton, D.P., Hanner, M.S., Horányi, M., Kissel, J., Lindblad, B.A., Linkert, D., Linkert, G., Mann, I., McDonnell, J.A.M., Morfill, G.E., Polanskey, C., Schwehm, G., Srama, R., Zook, H.A., “Three years of ULYSSES data: 1993-1995”, Planet. Space Sci., Vol. 47, pp. 363383, 1999

[Krü2001a] Krüger, H., Grün, E., Graps, A., Bindschadler, D., Dermott, S., Fechtig, H., Gustafson, B.A., Hamilton, D.P., Hanner, M.S., Horányi, M., Kissel, J., Lindblad, B.A., Linkert, D., Linkert, G., Mann, I., McDonnell, J.A.M., Morfill, G.E., Polanskey, C., Schwehm, G., Srama, R., Zook, H.A., "One year of Galileo dust data from the Jovian system: 1996", Planet. Space Sci., Vol. 49, pp. 1285-1301, 2001

[Krü2001b] Krüger, H., Grün, E., Landgraf, M., Dermott, S., Fechtig, H., Gustafson, B.A., Hamilton, D.P., Hanner, M.S., Horányi, M., Kissel, J., Lindblad, B.A., Linkert, D., Linkert, G., Mann, I., McDonnell, J.A.M., Morfill, G.E., Polanskey, C., Schwehm, G., Srama, R., Zook, H.A., "Four years of ULYSSES data: 1996-1999”, Planet. Space Sci., Vol. 49, pp. 1303-1324, 2001

[Kry1992] Krymskii, A.M., Breus, T.K., Dougherty, M.K., Southwood, D.J., Axford, W.I., "The electromagnetic effects of the solar wind interaction with the Phobos neutral gas halo and dust torus", Planet. Space Sci., Vol. 40, No. 8, pp. 1033-1041, 1992

[Kyr2003] Kyritsis, D., "On-Board Software Upgrade of the Space Experiment Mars Dust Counter", Diplomarbeit RT SA 03/15, Lehrstuhl für Raumfahrttechnik, Technische Universität München, 2003

[Lan2002] Landgraf, M., Liou, J.-C., Zook, H.A., Grün, E., “Origins of solar system dust beyond Jupiter”, The Astron. J., Vol. 123, pp. 2857-2861, 2002 
[Lev1990] Levasseur-Regourd, A.C., Dumont, R., "IRAS observations and local properties of interplanetary dust", Adv. Space Res., Vol. 10, No. 3-4, pp. (3)163-(3)170, 1990

[Lin2000] Linde, T. J., Gombosi, T. I., "Interstellar dust filtration at the heliospheric interface”, J. Geophys. Res., Vol. 105, No. A5, p. 10411, 2000

[Lio1995a] Liou, J.-C., Zook, H.A., "Radiation pressure, Poynting-Robertson drag, and solar wind drag in the restricted three-body problem", Icarus, Vol. 116, pp. 186-201, 1995

[Lio1995b] Liou, J.C., Dermott, S.F., Xu, Y.L., "The contribution of cometary dust to the zodiacal cloud”, Planet. Space Sci., Vol. 43, No. 6, pp. 717-722, 1995

[Lio1996a] Liou, J.-C., Zook, H.A., Dermott, S.F., "Kuiper Belt dust grains as a source of interplanetary dust particles“, Icarus, Vol. 124, pp. 429-440, 1996

[Lio1996b] Liou, J.-C., Zook, H.A., "Comets as a source of low eccentricity and low inclination interplanetary dust particles", Icarus, Vol. 123, pp. 491-502, 1996

[Lio1997] Liou, J.-C., Zook, H.A., "Evolution of interplanetary dust particles in mean motion resonances with planets", Icarus, Vol. 128, pp. 354-367, 1997

[Lio1999] Liou, J.-C., Zook, H.A., Jackson, A.A., "Orbital evolution of retrograde interplanetary dust particles and their distribution in the solar system", Icarus, Vol. 141, 13-28, 1999

[Lou1998] Louis, A.K., Maaß, P., Rieder, A., "Wavelets. Theorie und Anwendungen", Teubner-Verlag, 1998

[Lov1994] Love, S.G., Joswiak, D.J., Brownlee, D.E., "Densities of stratospheric micrometeorites", Icarus, Vol. 111, pp. 227-236, 1994

[Man1991] Mann, I., Kneißel, B., "Out-of-ecliptic distribution of interplanetary dust derived from near earth flux“, Adv. Space Res., Vol. 11, No. 12, pp. (12)123-(12)126, 1991

[Man1996a] Mann, I., Grün, E., Wilck, M., "The contribution of asteroid dust to the interplanetary dust cloud: The impact of ULYSSES results on the understanding of dust production in the asteroid belt and on the formation of the IRAS dust bands“, Icarus, Vol. 120, pp. 399-407, 1996

[Mar1994] Marzari, F., Vanzani, V., "Orbital evolution of dust particles near mean motion resonances with the Earth", Planet. Space Sci., Vol. 42, No. 2, pp. 101-107, 1994 
[McD2000] McDonnell, J.A.M., Burchell, M.J., Green, S.F., McBride, N., Vaughan, A.M., Zarnecki, J.C., Tsou, P., Hanner, M.S., Tuzzolino, A.J., DiDonna, F., Brownlee, D.E., Clark, B., "The STARDUST dust flux monitor", Adv. Space Res., Vol. 25, No. 2, pp. 335-338, 2000

[Mel1964] Melton, C.E., "Atmospheric aerosol collection near 40,000 feet with DC8", Ann. N.Y. Acad. Sci., Vol. 119, Art. 1, pp. 116-125, 1964

[Mit1998] Mitchell, M., "An Introduction to Genetic Algorithms (Complex Adaptive Systems)", The MIT Press, 1998

[Mou2005] Moussi, A., Drolshagen, G., McDonnell, J.A.M., Mandeville, J.-C., Kearsley, A.T., Ludwig, H., "Hypervelocity impacts on HST solar arrays and the debris and meteoroids population", Adv. Space Res., Vol. 35, pp. $1243-1253,2005$

[Mün1995] Münzenmayer, R., "Beiträge zur experimentellen Erforschung des Staubes im Weltall", Dissertation, Lehrstuhl für Raumfahrttechnik, Technische Universität München, 1995

[Mün1997] Münzenmayer, R., Igenbergs, E., Iglseder, H., Svedhem, H., “The Munich Dust Counter on board the MUSES-A mission: Calibration of impacts inside and in front of the detector", Adv. Space Res., Vol. 20, No. 8, pp. 1485-1488, 1997

[Nak1995] Nakatani, I., Tsuruda, K., Yamamoto, T., "PLANET-B: Mars mission with small spacecraft but potentially with large scale science reward", Acta Astronautica, Vol. 35, pp. 337-344, 1995

[Nau2000] Naumann, W, "Rechnergestützte Automatisierung eines Experiments im interplanetaren Raum", Dissertation, Lehrstuhl für Raumfahrttechnik, Technische Universität München, 2000

[Naz1997] Nazzario, R.C., Hyde, T.W., "The dust bands of the planet Mars", Adv. Space Res., Vol. 20, No. 8, pp. 1535-1538, 1997

[Neu1984] Neugebauer, G.H., Habing, H., van Diunen, R., Aumann, H., Baud, B., Beichmann, C., Beintema, D., Boggess, N., Clegg, P., de Jong, T., Emerson, J., Gautier, T.N., Gillett, F., Harris, S., Hauser, M., Houck, J., Jennings, R., Low, F., Marsden, P., Miley, G., Olnon, F., Pottasch, S., Raimond, E., Rowan-Robinson, M., Soifer, B., Walker, R., Wesselius, P., Young, E., "The Infrared Astronomical Satellite (IRAS) mission", Astroph. J., Vol. 278, pp. L1-L6, 1984

[Poy1903] Poynting, J. H., "Radiation in the solar system: its effect on temperature and its pressure on small bodies", Philosophical Transactions of the Royal Society of London, Series A. 202, pp. 525-552, 1903 
[Rat1996] Ratcliff, P.R., Gogu, F., Grün, E., Srama, R., "Plasma production by secondary impacts: Implications for velocity measurements by in-situ dust detectors", Adv. Space Res., Vol. 17, No. 12, pp. (12)111-(12)115, 1996

[Rat1997] Ratcliff, P.R., Reber, M., Cole, M.J., Murphy, T.W., Tsembelis, K., "Velocity thresholds for impact plasma production", Adv. Space Res. Vol. 20, No. 8, pp. 1471-1476, 1997

[Rob1937] Robertson, H. P., "Dynamical effects of radiation in the solar system", Monthly Notices of the Royal Astronomical Society, Vol. 97, pp. 423-438, 1937

[Rot2004] Rott, C., "Validation of the Software Update on MDC NOZOMI with the MDC HITEN Data", Semesterarbeit RT SA 04/17, Technische Universität München, Lehrstuhl für Raumfahrttechnik, 2004

[Sas 1999] Sasaki, S., "Dust ring/torus around mars, waiting for detection by NOZOMI”, Adv. Space Res., Vol. 23, No. 11, pp. 1907-1910, 1999

[Sas2002] Sasaki, S., Igenbergs, E., Ohashi, H., Münzenmayer, R., Naumann, W., Hofschuster, G., Born, M., Färber, G., Fischer, F., Fujiwara, A., Glasmachers, A., Grün, E., Hamabe, Y., Iglseder, H., Kawamura, T., Miyamoto, H., Morishige, K., Mukai, T., Naoi, T., Nogami, K., Schwehm, G., Svedhem, H., "Observation of interplanetary and interstellar dust particles by Mars Dust Counter (MDC) on NOZOMI", Adv. Space Res., Vol. 29. No. 8, pp. 1145-1153, 2002

[Sau2004] Sauer, A., “Analyse der Housekeepingdaten im Jahr 1998 des Weltraumexperiments MDC auf der japanischen Mission NOZOMI“, Semesterarbeit RT-SA 04/15, Technische Universität München, Lehrstuhl für Raumfahrttechnik, 2004

[Sch1964] Schmidt, R.A., "Microscopic extraterrestrial particles from the antarctic peninsula", Ann. N.Y. Acad. Sci., Vol. 119, Art. 1, pp. 186-204, 1964

[Sch1995] Scholz, M., “Topologiegenerierung für Neuronale Netzte durch Genetische Algorithmen", Dissertation, Fachbereich 13 Informatik, Technische Universität Berlin, 1995

[Sch1996] Schobert, D., "Aluminiumoxid-Belastung niedriger Erdumlaufbahnen durch feststoffgestützte Platzierung geostationärer Satelliten", Dissertation, Fachgebiet Raumfahrttechnik, Technische Universität München, 1996

[Sch1997] Scherer, A., "Neuronale Netze. Grundlagen und Anwendungen", Friedr. Vieweg \& Sohn Verlagsgesellschaft $\mathrm{mbH}$, Braunschweig/Wiesbaden, 1997 
[Smi1999] Smith, S.M., Wilson, J.K., Baumgardner, J., Mendillo, M., "Discovery of the Distant Lunar Sodium Tail and its Enhancement Following the Leonid Meteor Shower of 1998", Geophys. Res. Letters Vol. 26, pp. 1649-1652, 1999

[Sot1971] Soter, S., "The dust belts of Mars", Report of Center for Radiophysics and Space Research, No. 462, Cornell University, Ithaca, NY, 1971

[Sra1997] Srama, R., Grün, E., "The dust sensor for CASSINI”, Adv. Space Res., Vol. 20, No. 8, pp. 1467-1470, 1997

[Sra2004] Srama, R., Ahrens, T.J., Altobelli, N., Auer, S., Bradley, J.G., Burton, M., Dikarev, V.V., Economou, T., Fechtig, H., Görlich, M., Grande, M., Graps, A., Grün, E., Havnes, O., Helfert, S., Horányi, M., Igenbergs, E., Jessberger, E.K., Johnson, T.V., Kempf, S., Krivov, A.V., Krüger, H., Mocker-Ahlreep, A., Moragas-Klostermeyer, G., Lamy, P., Landgraf, M., Linkert, D., Linkert, G., Lura, F., McDonnell, J.A.M., Möhlmann, D., Morfill, G.E., Müller, M., Roy, M., Schäfer, G., Schlotzhauer, G., Schwehm, G.H., Spahn, F., Stübig, M., Svestka, J., Tschernjawski, V., Tuzzolino, A.J., Wäsch, R., Zook, H.A., "The CASSISI cosmic dust analyzer”, Space Sci. Rev., Vol. 114, pp. 465-518, 2004

[Sri1999] Srikanth, R., "Physical interpretation of the Poynting-Robertson effect", Icarus, Vol. 140, pp. 231-234, 1999

[Sta1993] Staubach, P., Divine, N., Grün, E., "Temperatures of zodiacal dust", Planet. Space Sci., Vol. 41, No. 11/12, pp. 1099-1108, 1993

[Sta1995] Staubach, P., Grün, E., "Development of an upgraded meteoroid model", Adv. Space Res., Vol. 16, No. 11, pp. (11)103-(11)106, 1995

[Sta1997] Stark, J., Nombro, A., Walker, R., Crowther, R., "A model for the generation of micro-debris resulting from atomic oxygen impact", Proc. $2^{\text {nd }}$ European Conf. Space Debris, pp. 285-288, 1997

[Ste2004] Steiner, W., Schagerl, M., "Raumflugmechanik. Dynamik und Steuerung von Raumfahrzeugen", Springer Verlag, Berlin, Heidelberg, New York, 2004

[Str1986] Strazzulla, G., "'Primitive' galactic dust in the solar system?", Icarus, Vol. 67, pp. 63-70, 1986

[Stü2001] Stübig, M., Schäfer, G., Ho, T., Srama, R., Grün, E., "Laboratory simulation improvements for hypervelocity micrometeorite impacts with new dust particle source", Planet. Space Sci., Vol. 49, pp. 853-858, 2001

[Sve1992] Svedhem, H., Pedersen, A., "Behaviour of ejecta particles and generated plasma at hypervelocity impacts", in "Hypervelocity Impacts in Space", (ed. J.A.M. McDonnell), University of Kent at Canterbury, 1992 
[Syk1992] Sykes, M.V., Walker, R.G., "Cometary dust trails I: Survey", Icarus, Vol. 95, pp. 180-210, 1992

[Tsu1996] Tsuruda, K., Nakatani, I., Yamamoto, T., "PLANET-B mission to Mars 1998”, Adv. Space Res., Vol. 17, No. 12, pp. (12)21-(12)29, 1996

[Ues1991] Uesugi, K., Matsuo, H., Kawaguchi, J., Hayashi, T., "Japanese first double lunar swingby mission 'HITEN", Acta Astronautica, Vol. 25, Issue 7, pp. $347-355,1991$

[Ues1996] Uesugi, K., "Results of the MUSES-A 'HITEN' mission", Adv. Space Res., Vol. 18, No. 11, pp. (11)69-(11)72, 1996

[Weh1999] Wehry, A., Mann, I., "Identification of $\beta$-meteoroids from measurements of the dust detector onboard the Ulysses spacecraft", Astron. Astrophys., Vol. 341, pp. 296-303, 1999

[Wen1989] Wenzel, K.-P., Marsden, R.G., Page, D.E., Smith, E.J., "ULYSSES: The first high-latitude heliospheric mission", Adv. Space Res., Vol. 9, No. 4, pp. (4)25-(4)29, 1998

[Wil1996] Wilck, M., Mann, I., "Radiation pressure forces on 'typical' interplanetary dust grains", Planet. Space Sci., Vol. 44, No. 5, pp. 493-499, 1996

[Wi12004] Willis, M.J., Burchell, M.J., Cole, M.J., McDonnell, J.A.M., "Influence of impact ionisation methods on determination of dust particle flux in space", Planet. Space Sci., Vol. 52, pp. 711-725, 2004

[Zel1994] Zell, A., "Simulation Neuronaler Netze”, Addison-Wesley, Stuttgart, 1994 



\section{Appendix B: Picture credits}

Figure 2-1

Figure 2-2

Figure 2-4

Figure 2-5

Figure 2-6

Figure 2-7

Figure 2-8

Figure 2-9

Figure 2-10

Figure 2-13

Figure 2-16

Figure 3-1

Figure 3-2

Figure 3-3

Figure 3-4

Figure 3-5

Figure 3-7

Figure 3-18

Figure 3-19

Figure 3-20

Figure 4-2

Figure 4-3

Figure 4-4

Figure 4-10

Figure 5-1

Figure 5-2

Figure 5-3

Figure 5-4

Figure 7-1
Dominic Cantin, Quebec, Canada, September 2, 2003

MPI-K, Heidelberg, Webpage http://www.mpi-hd.mpg.de/dustgroup/ [Ham1996]

NASA/JPL

NASA/JPL

NASA/JPL

NASA/JPL

MPI-K, Heidelberg

ISAS/JAXA

ISAS/JAXA

ISAS/JAXA

LRT

LRT

LRT

LRT

LRT

ISAS/JAXA

LRT

LRT

LRT

[Kyr2003]

[Kyr2003]

[Gla2003]

[Gla2003]

[Kyr2003]

[Kyr2003]

[Kyr2003]

[Kyr2003]

LRT 


\section{Appendix C: Tables}

\section{C.1 Signals, HK data and analysis results of the identified impacts}

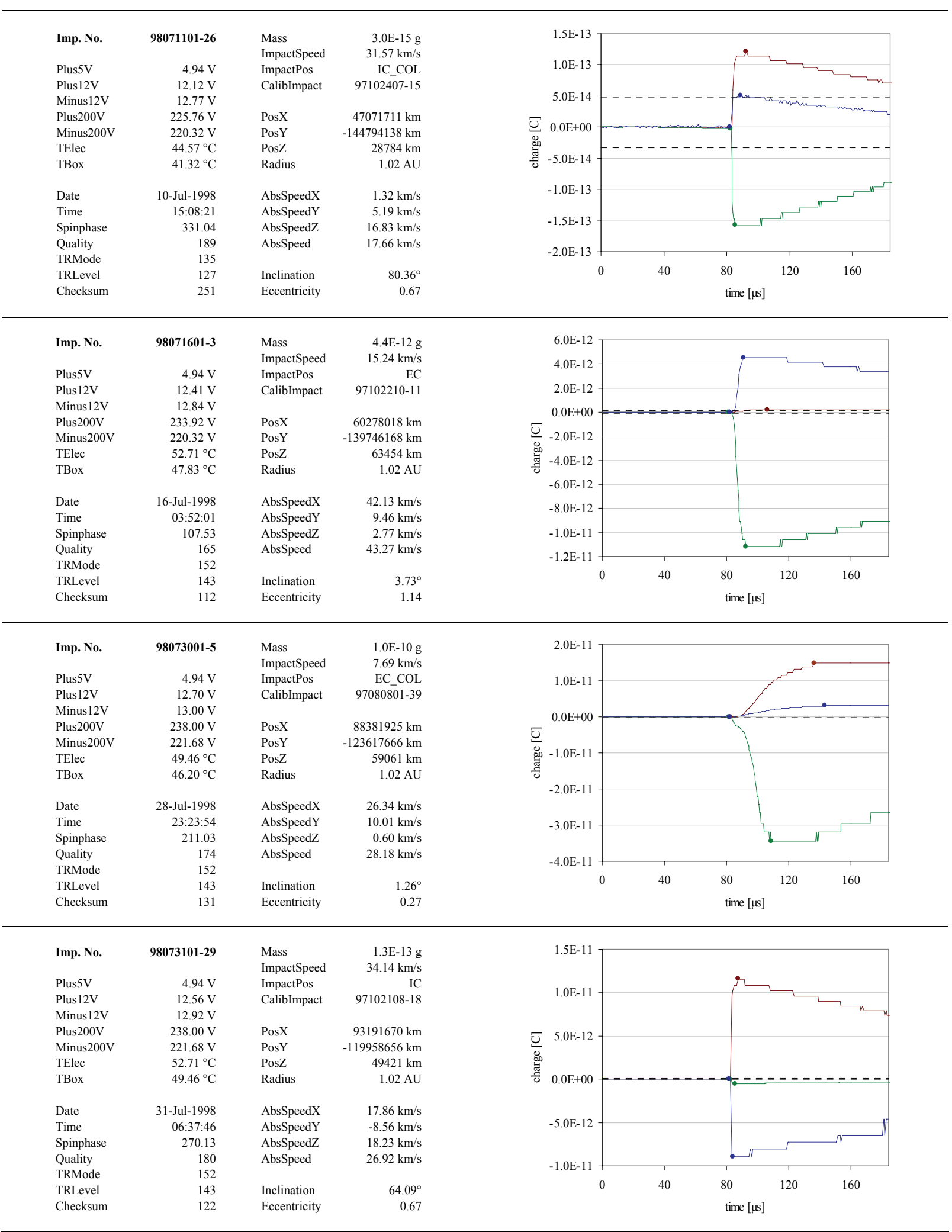




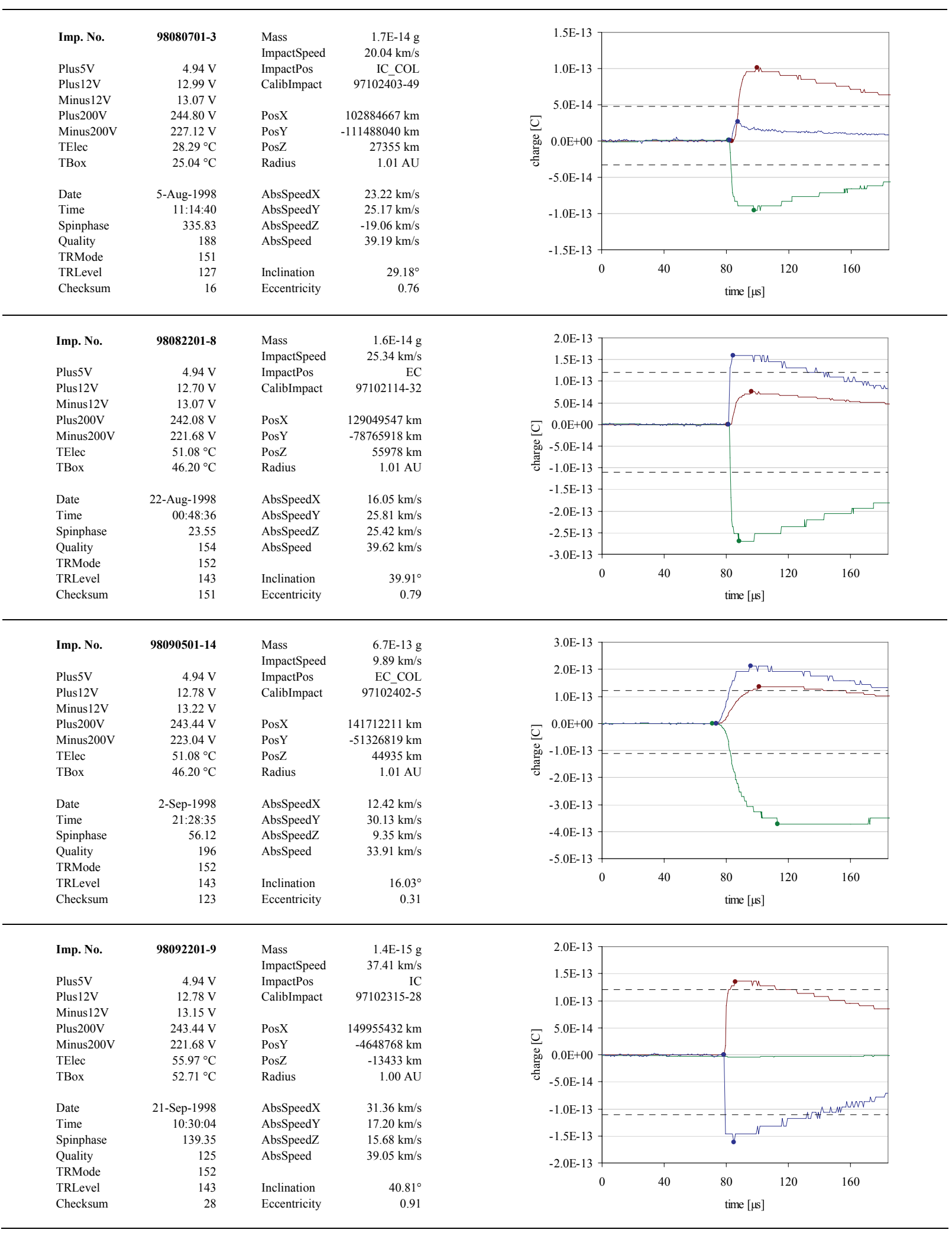




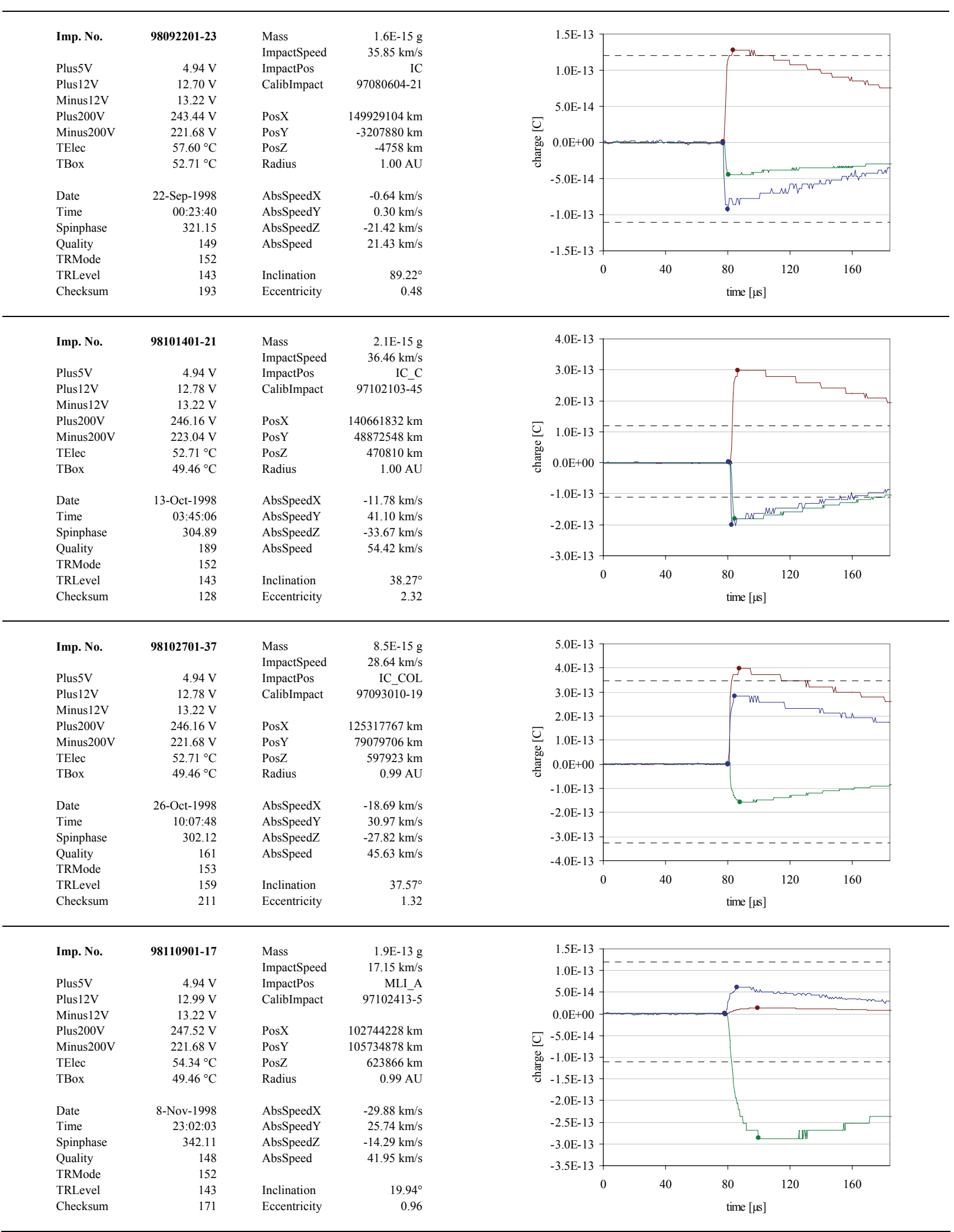




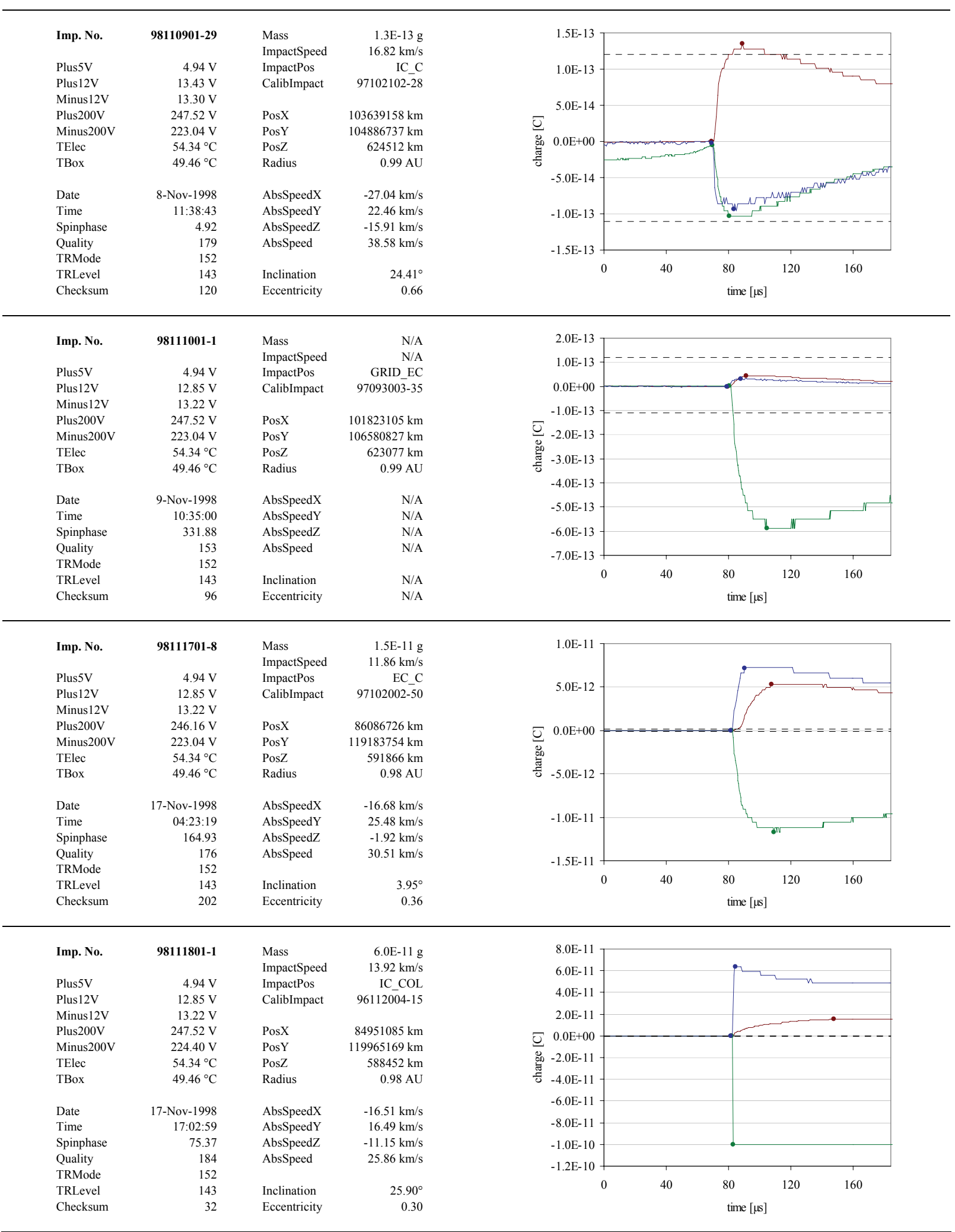




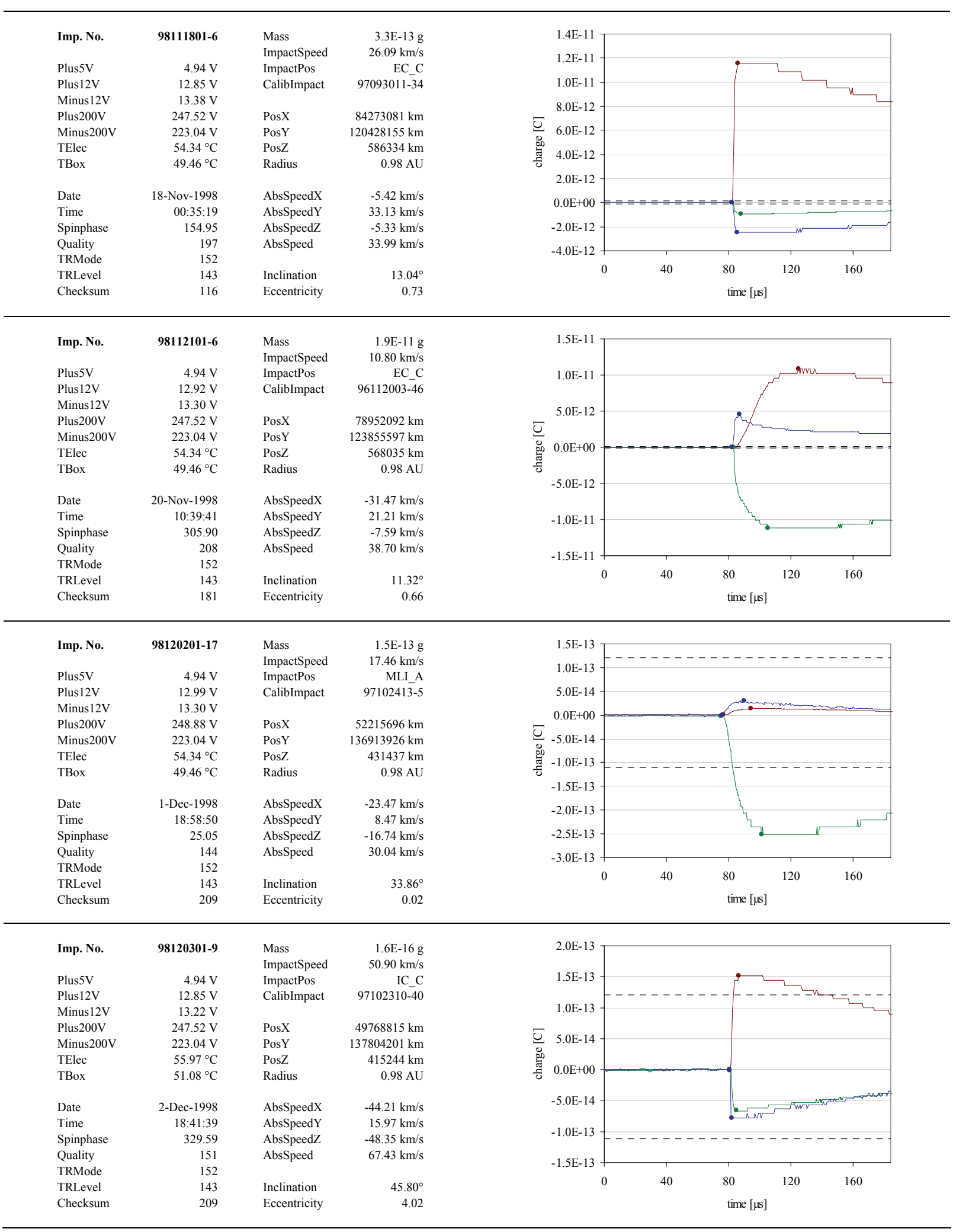




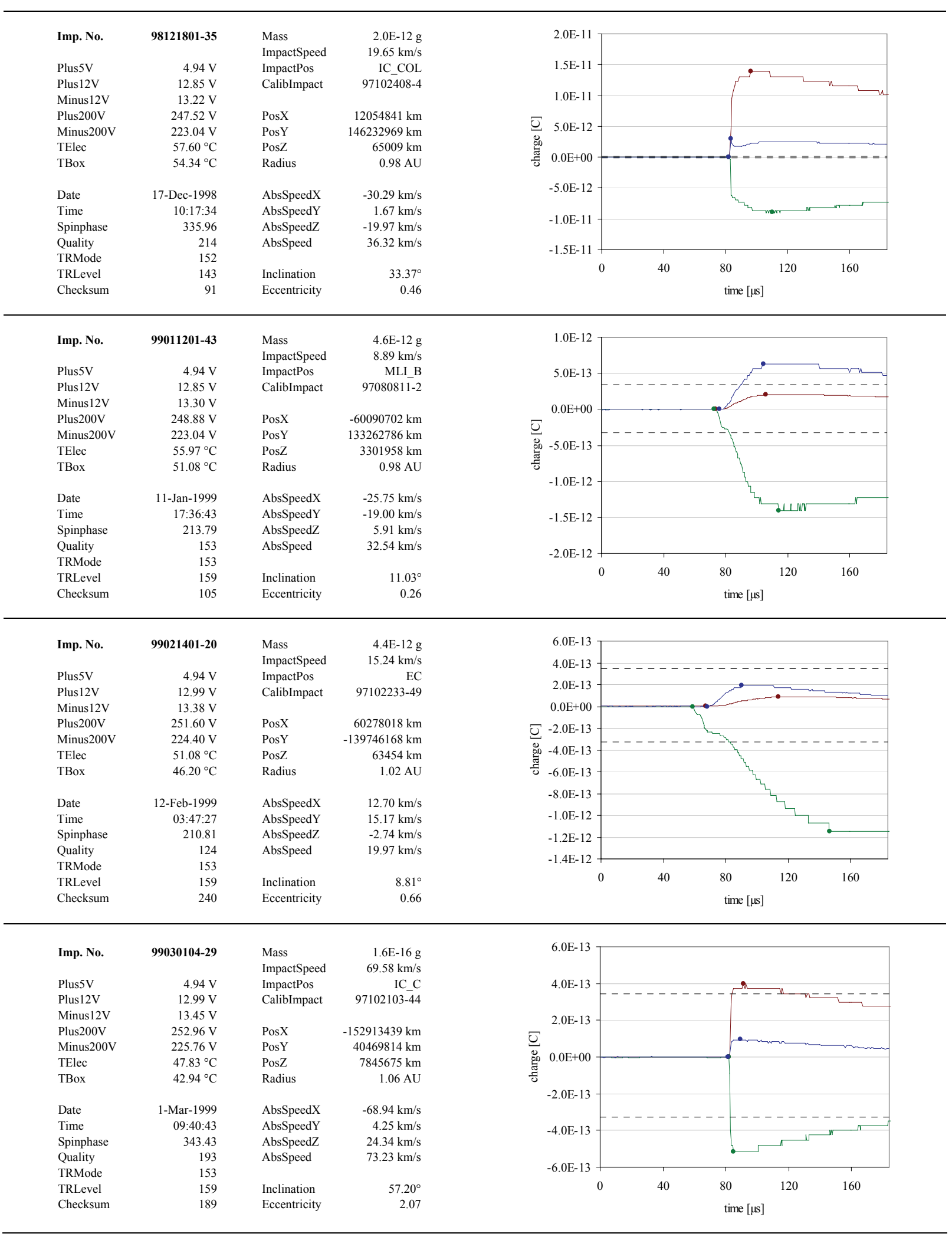




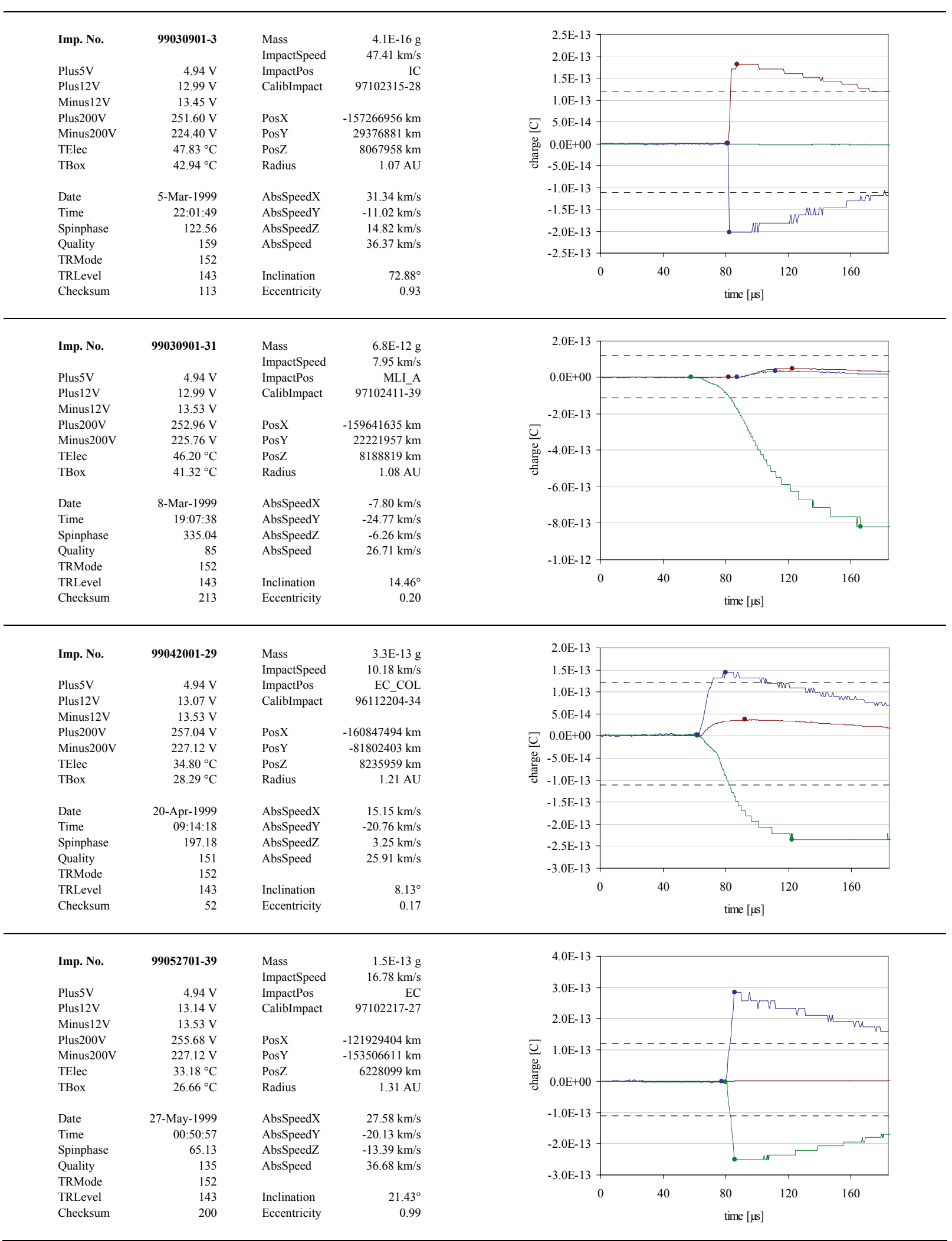




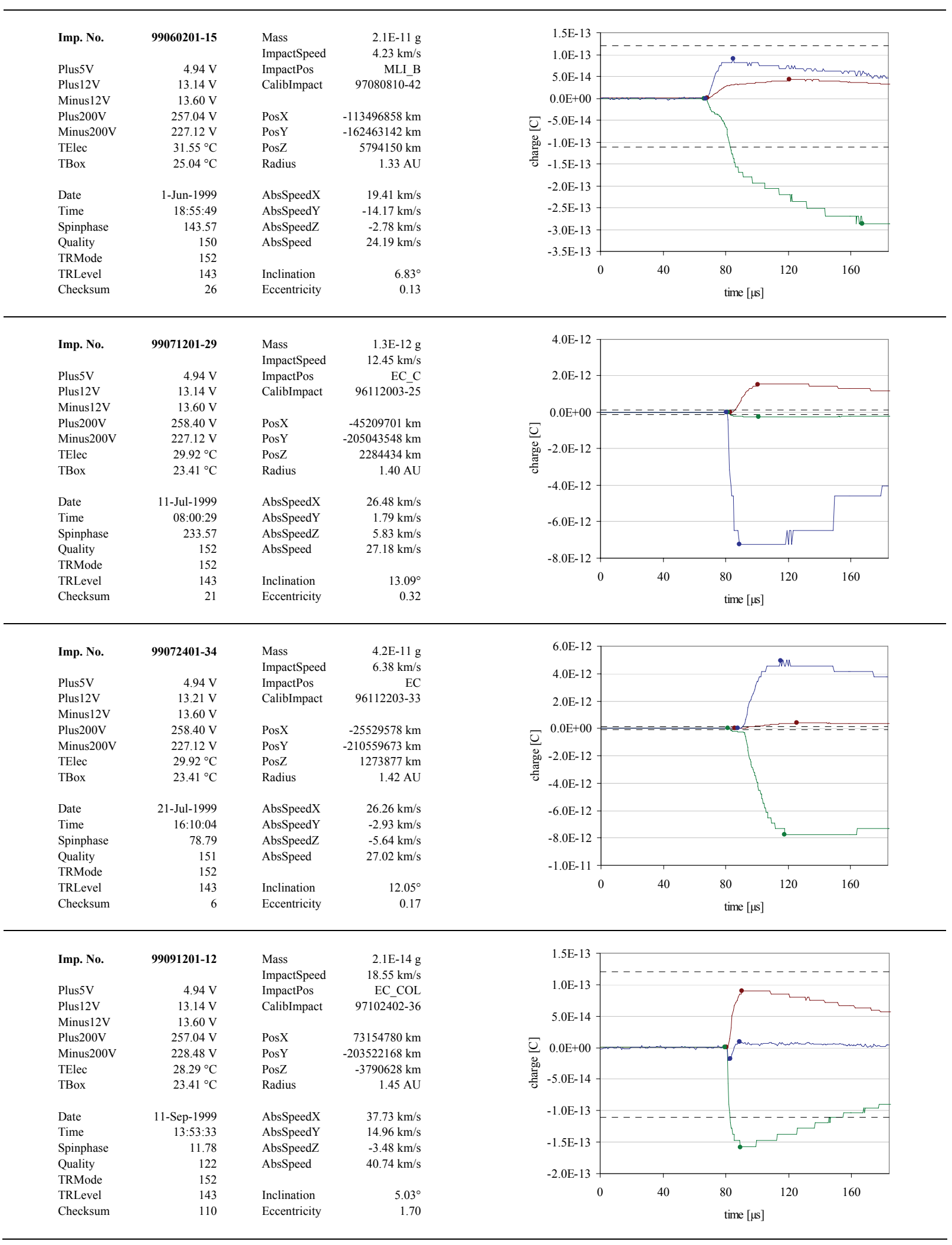




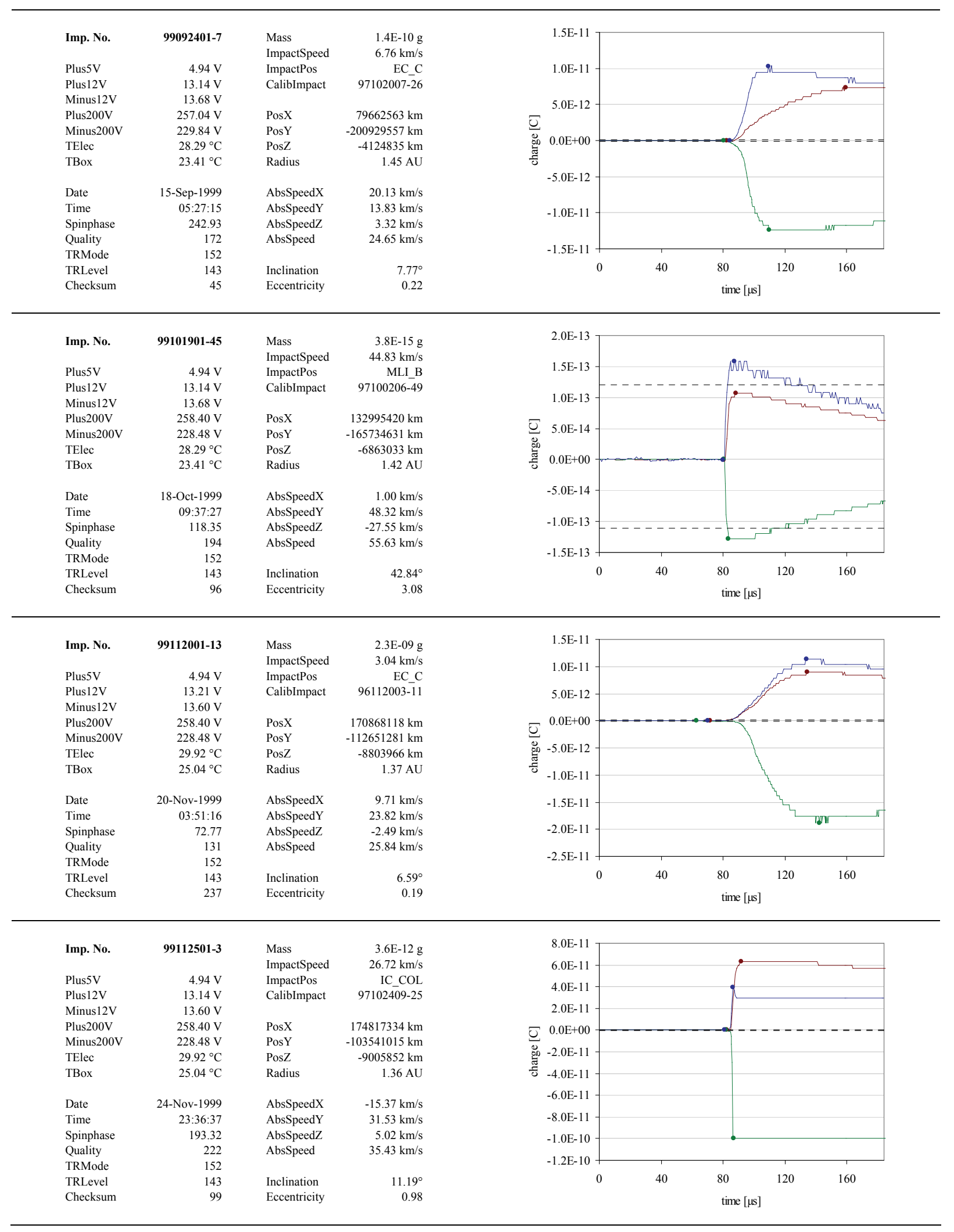




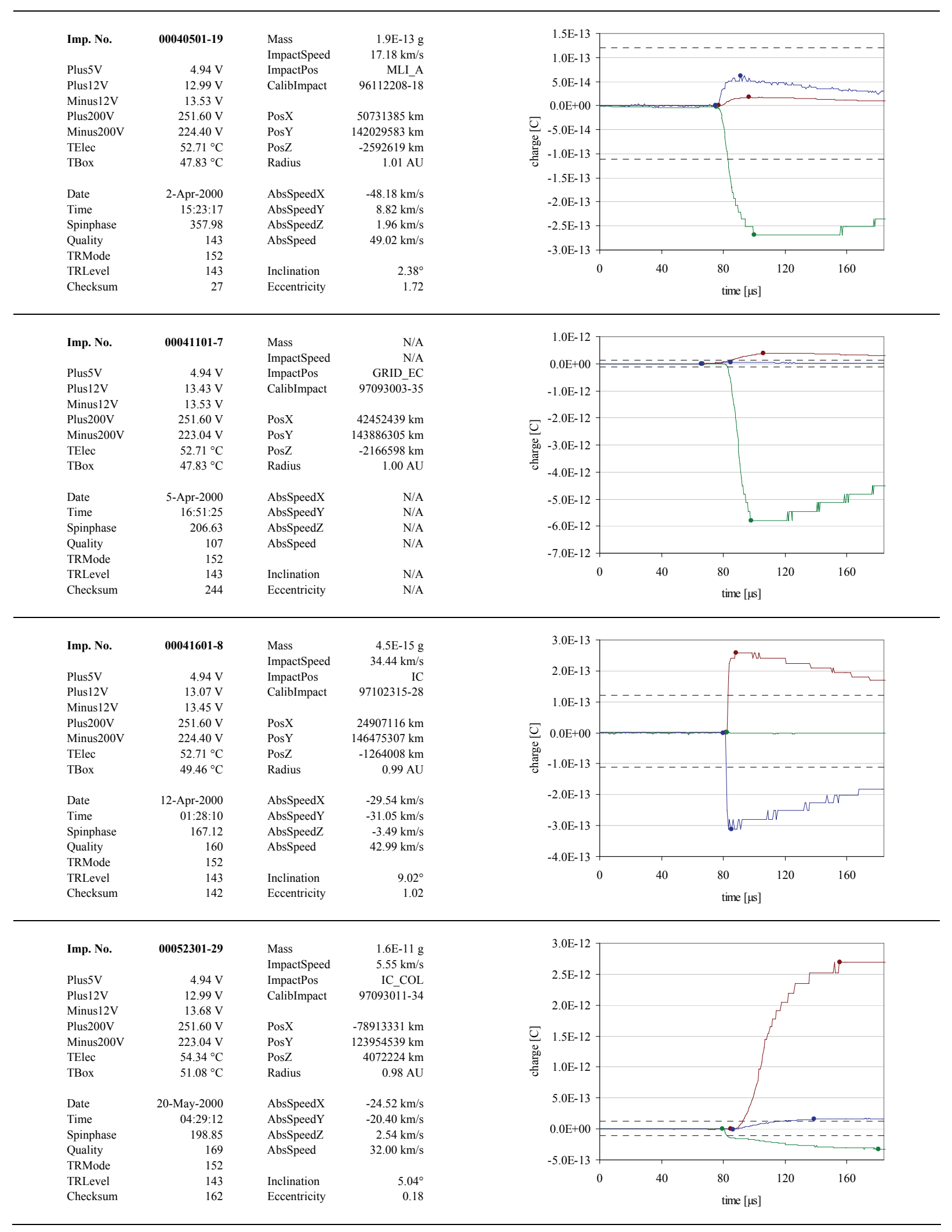




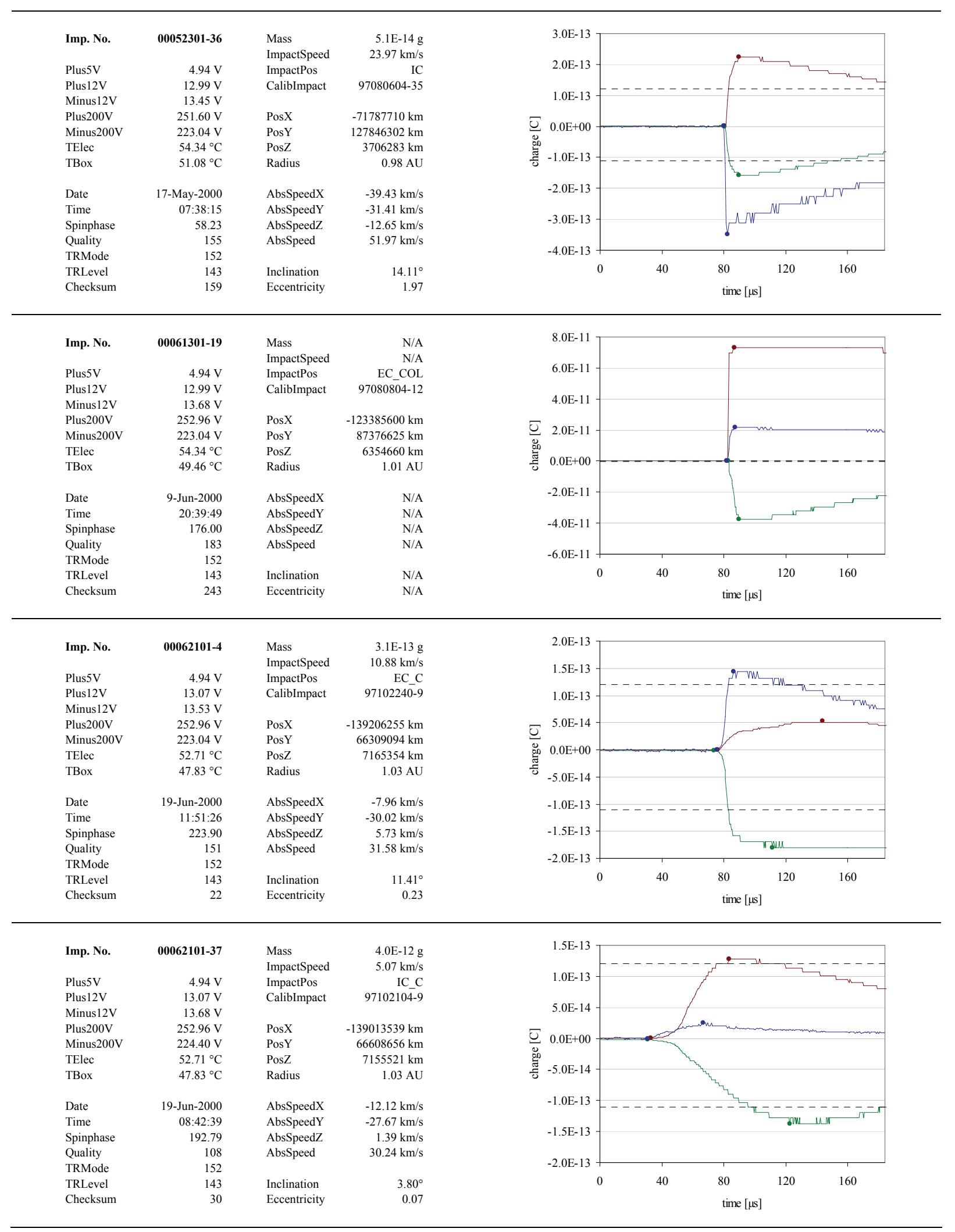




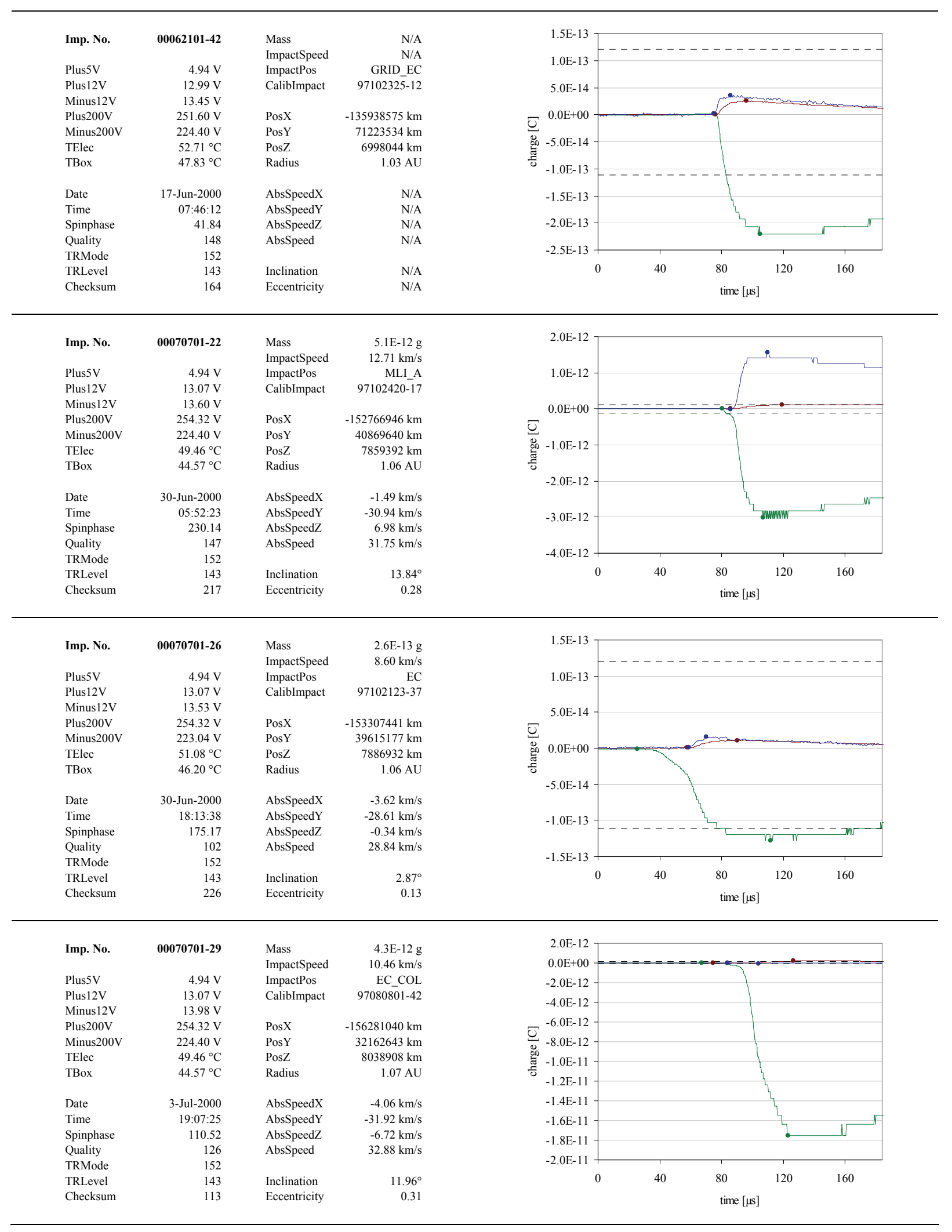




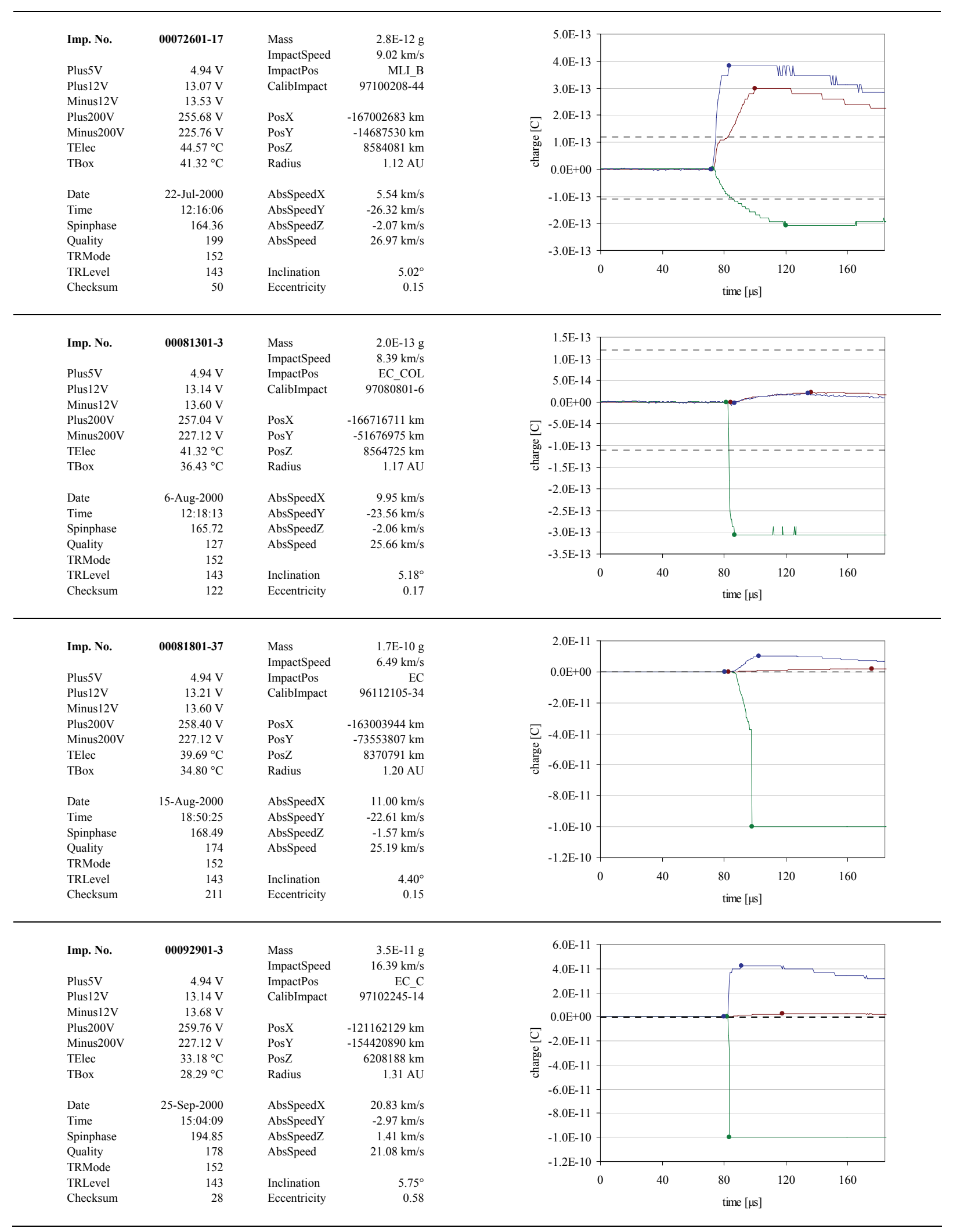




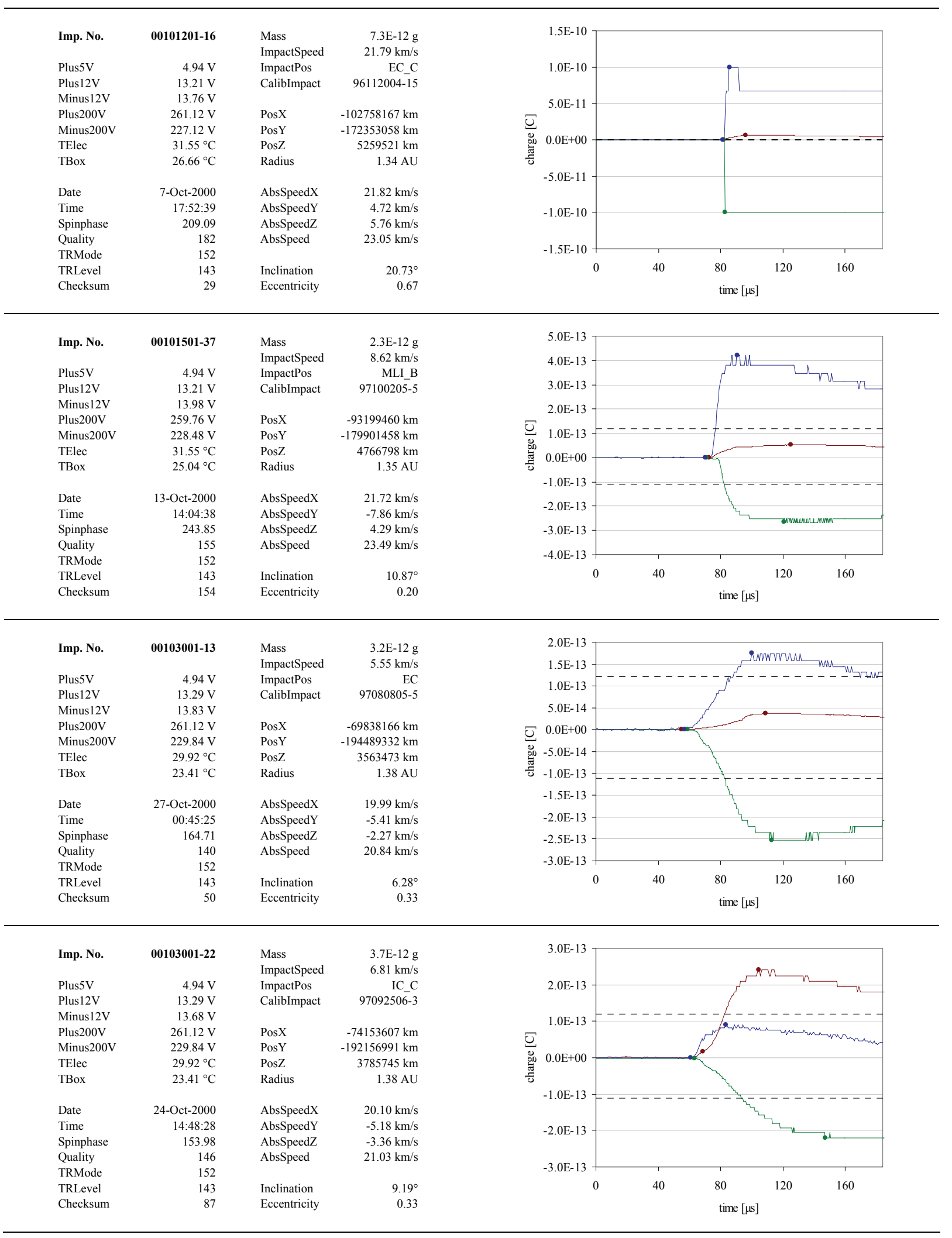




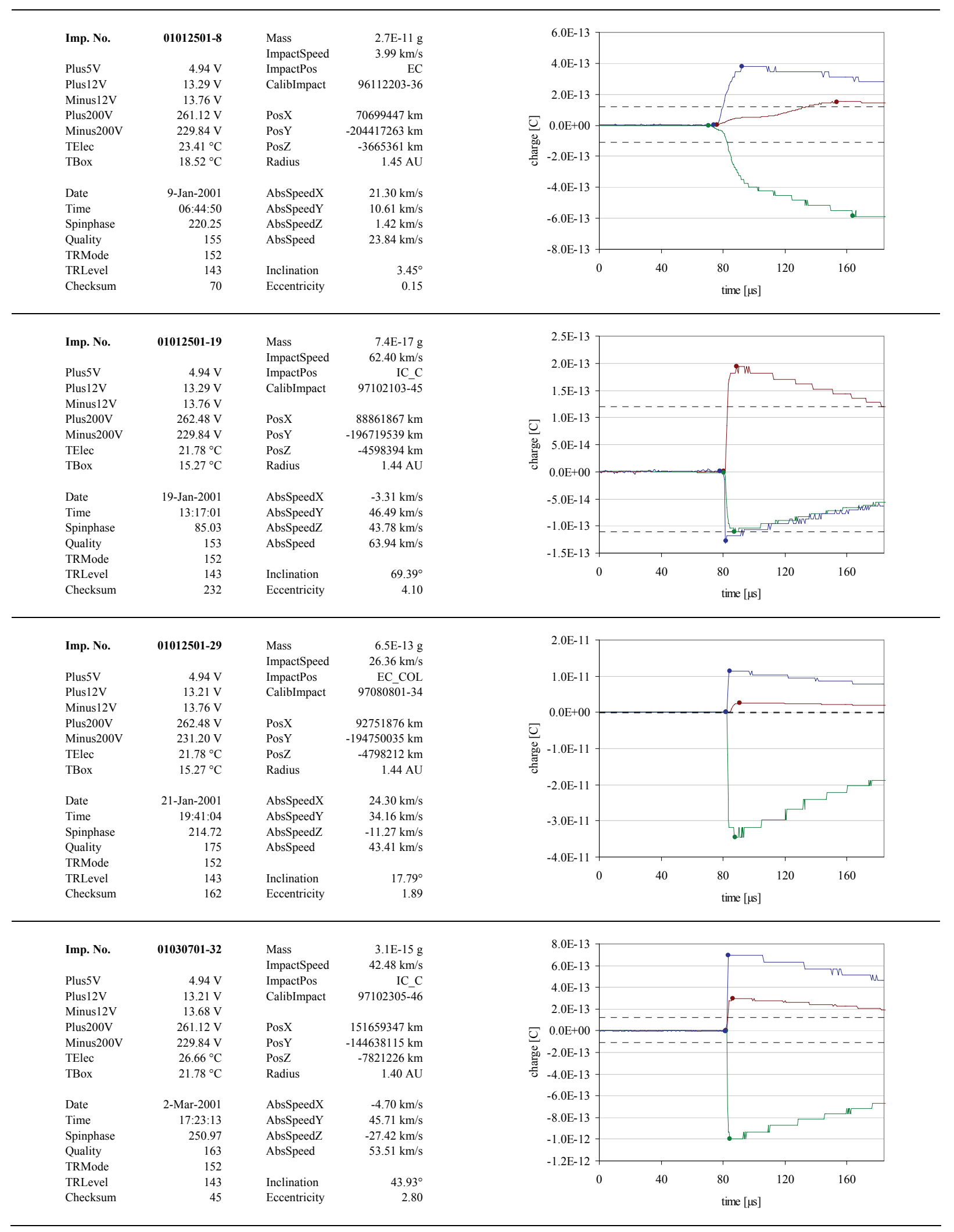




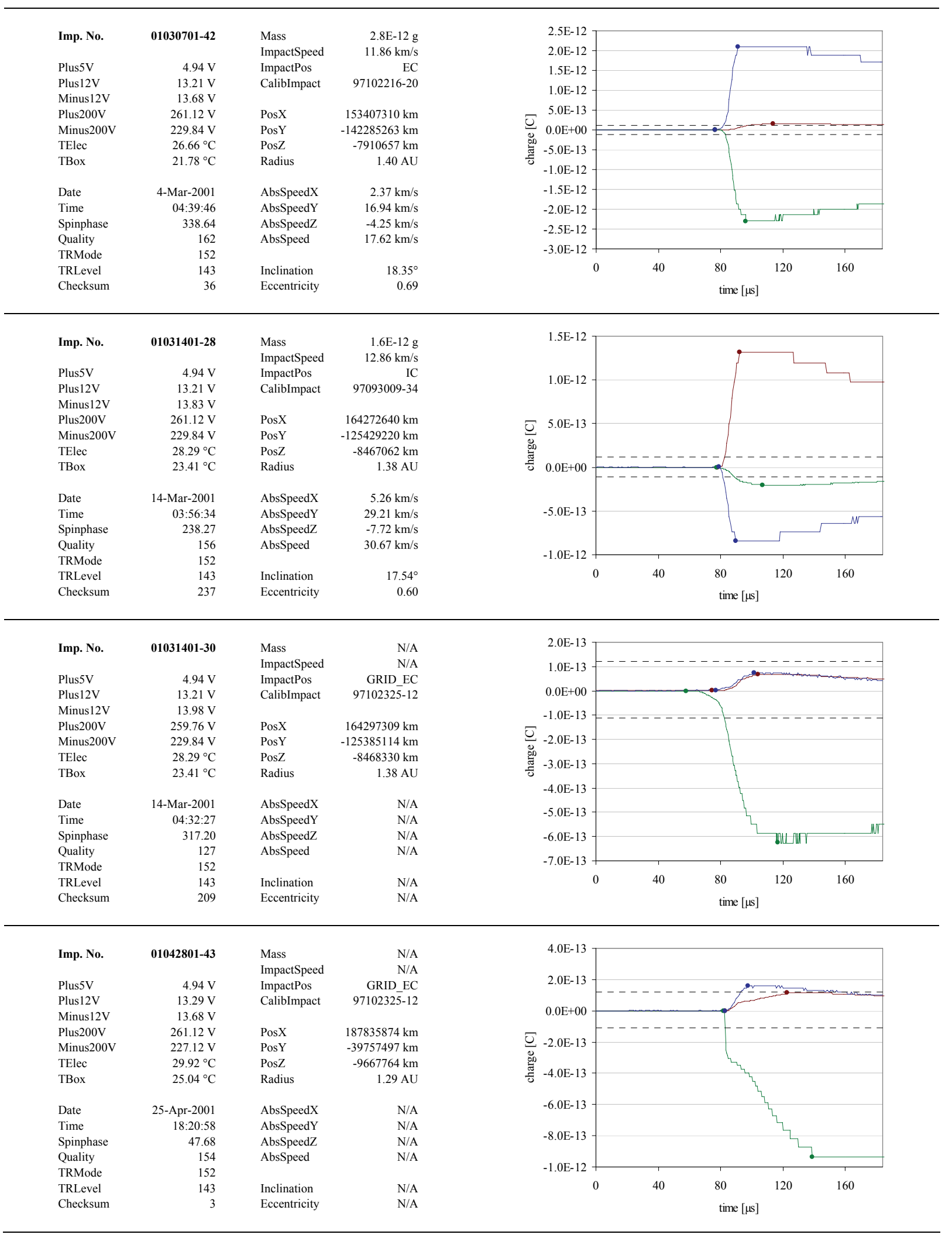




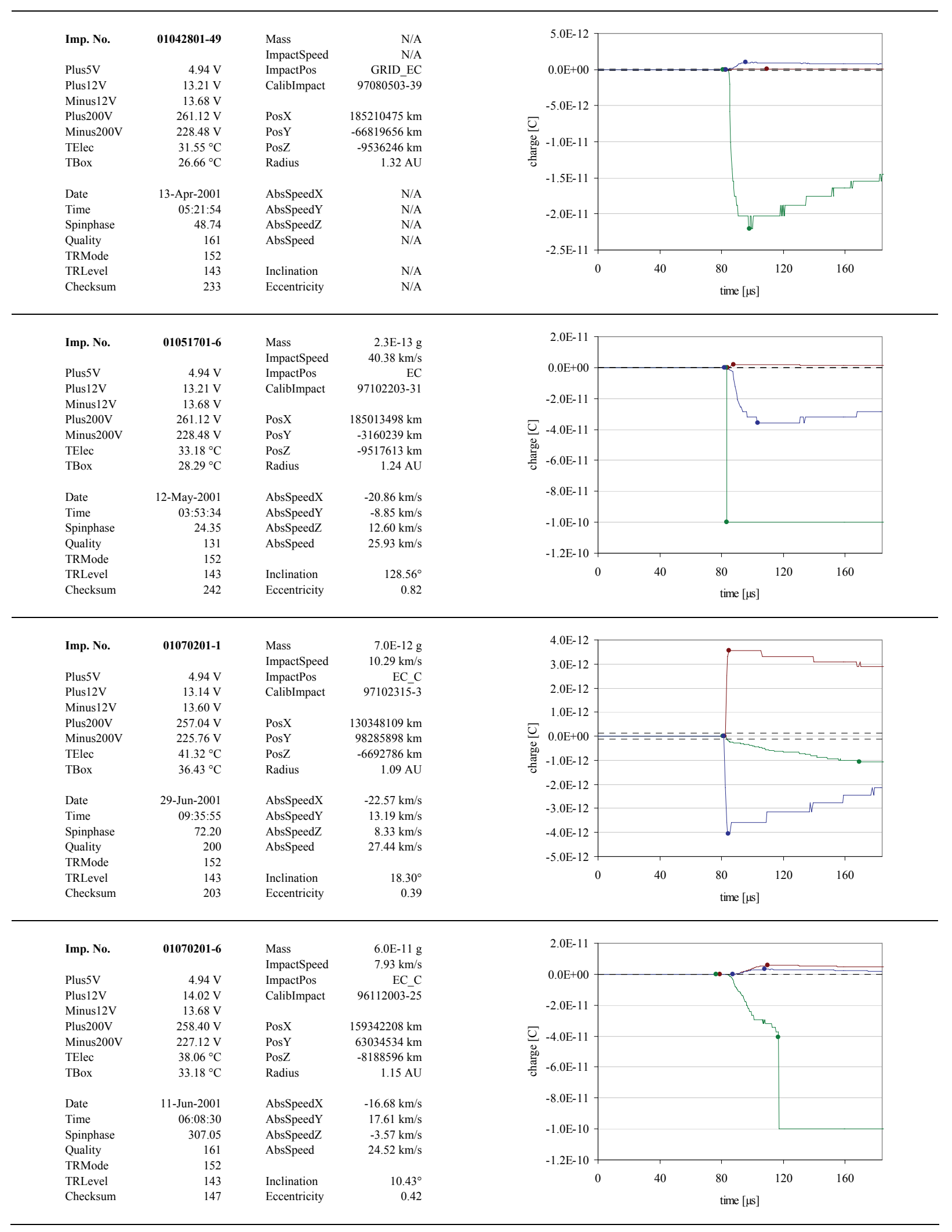




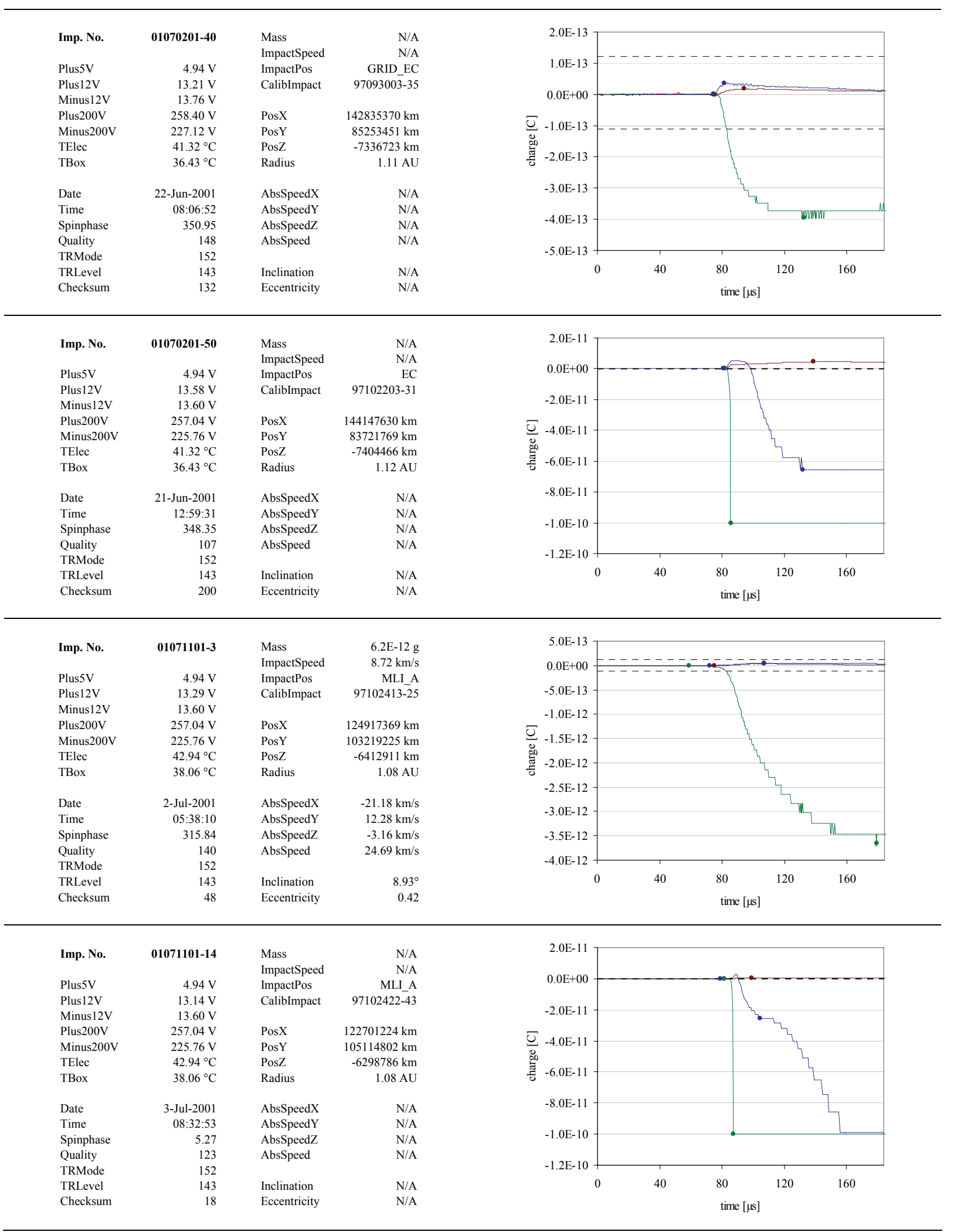




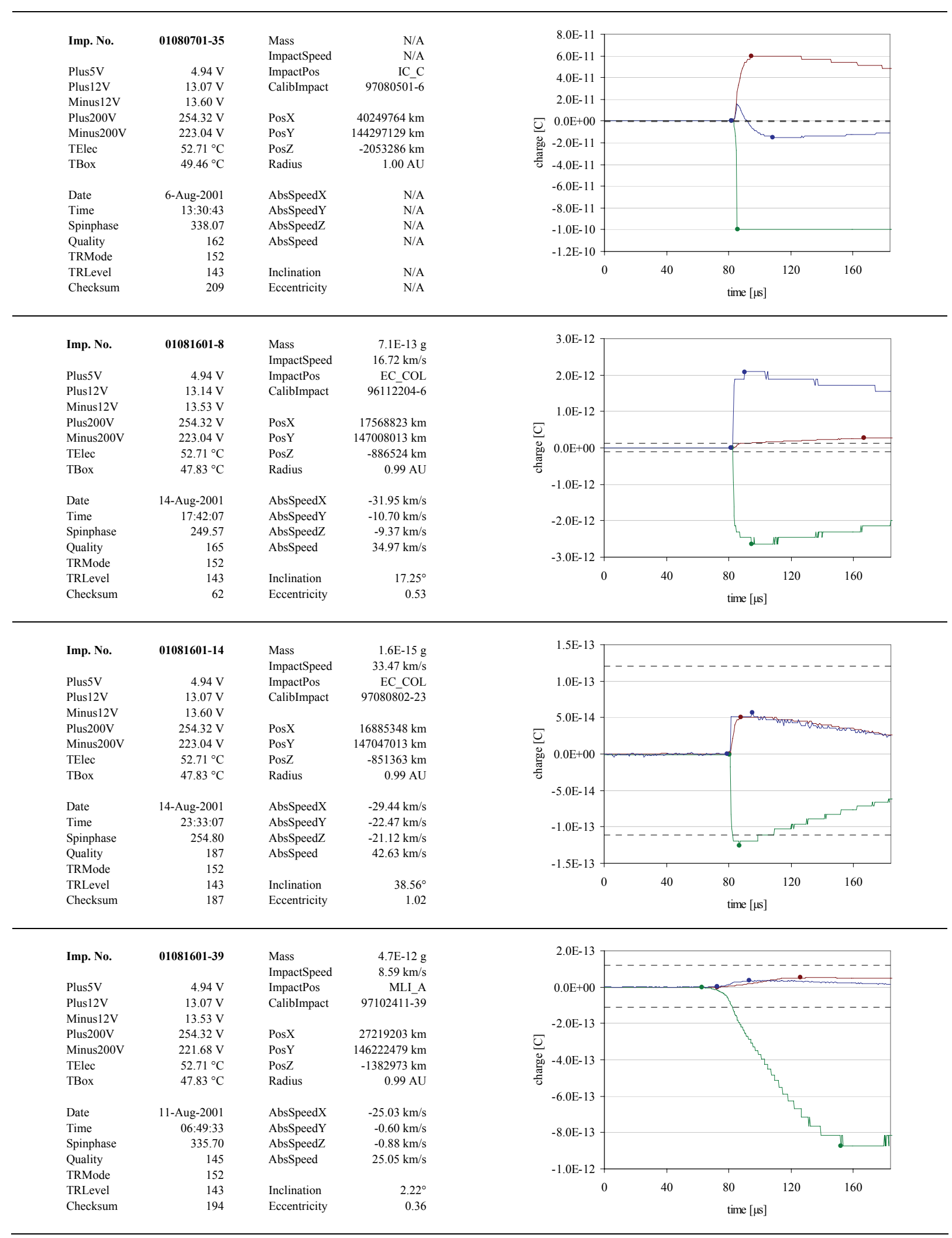




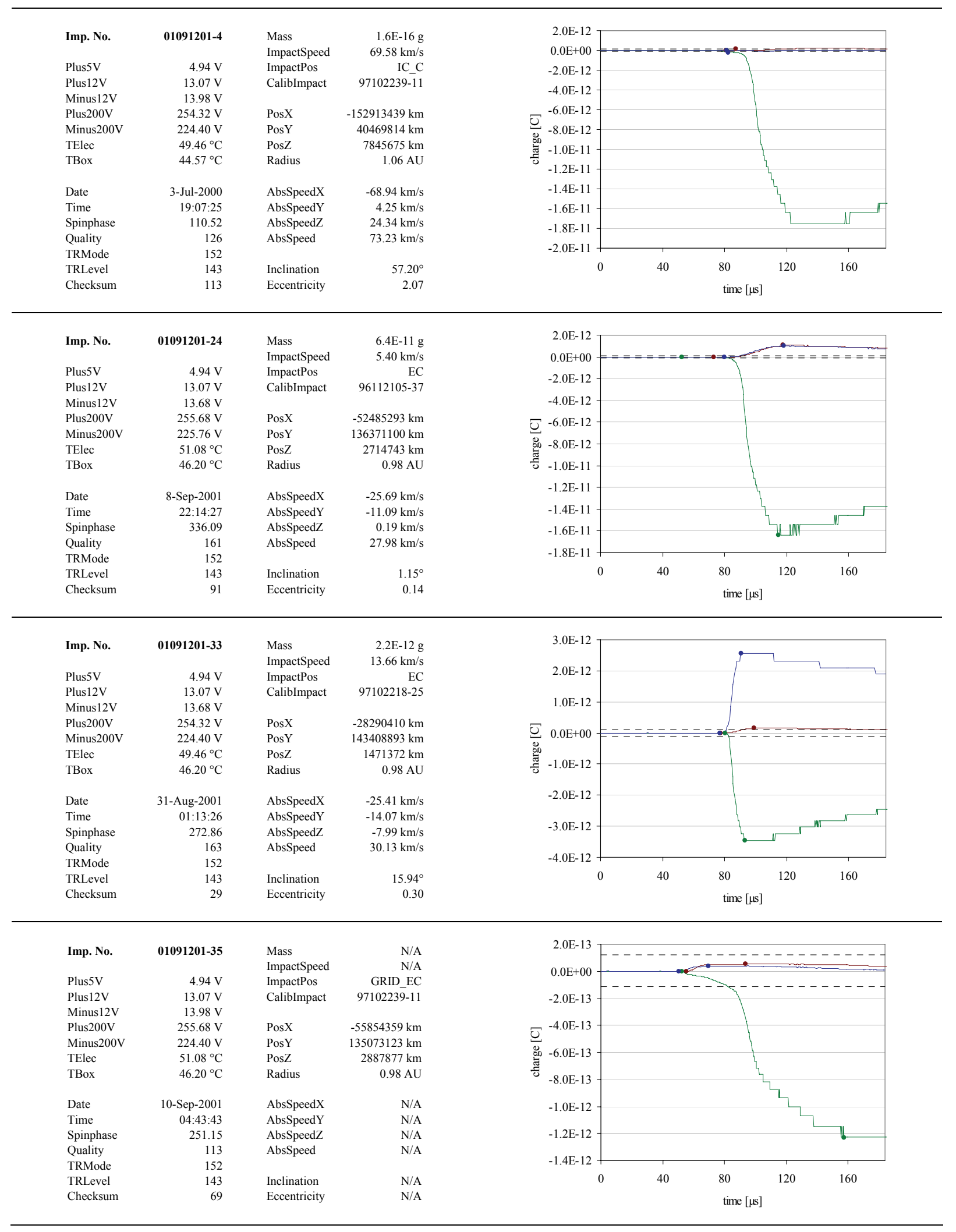




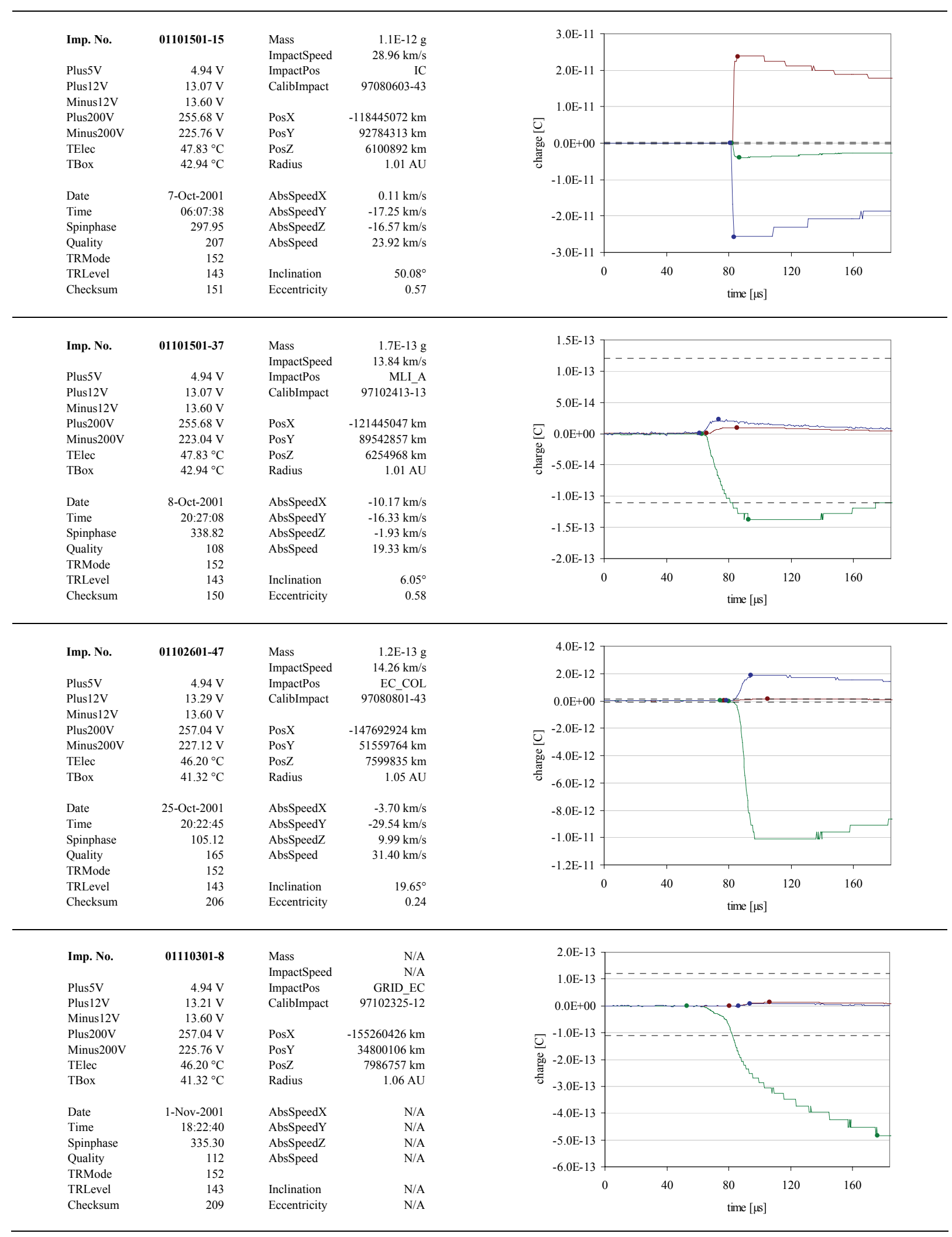




\begin{tabular}{|c|c|c|c|c|c|c|c|c|c|}
\hline \multirow[t]{2}{*}{ Imp. No. } & \multirow[t]{2}{*}{ 01110301-43 } & Mass & $9.2 \mathrm{E}-12 \mathrm{~g}$ & \multirow{2}{*}{\multicolumn{2}{|c|}{$5.0 \mathrm{E}-13$}} & \multirow{2}{*}{\multicolumn{4}{|c|}{$----------------\ldots$}} \\
\hline & & ImpactSpeed & $8.45 \mathrm{~km} / \mathrm{s}$ & & & & & & \\
\hline Plus5V & $4.94 \mathrm{~V}$ & ImpactPos & MLI_A & \multicolumn{2}{|r|}{$0.0 \mathrm{E}+00$} & ----- & & & \\
\hline Plus12V & $13.29 \mathrm{~V}$ & CalibImpact & $97102413-25$ & \multirow{2}{*}{\multicolumn{2}{|c|}{$-5.0 \mathrm{E}-13$}} & & & & \\
\hline Minus12V & $13.60 \mathrm{~V}$ & & & & & & & & \\
\hline Plus200V & $257.04 \mathrm{~V}$ & PosX & $-152106974 \mathrm{~km}$ & \multirow{2}{*}{\multicolumn{2}{|c|}{$\frac{\sigma}{\sigma}-1.0 \mathrm{E}-12$}} & & & & \\
\hline Minus200V & $225.76 \mathrm{~V}$ & PosY & $42349726 \mathrm{~km}$ & & & & & & \\
\hline TElec & $46.20^{\circ} \mathrm{C}$ & PosZ & $7825425 \mathrm{~km}$ & \multirow{2}{*}{\multicolumn{2}{|c|}{ 氜 $-1.5 \mathrm{E}-12$}} & & & & \\
\hline TBox & $41.32{ }^{\circ} \mathrm{C}$ & Radius & $1.06 \mathrm{AU}$ & & & & & & \\
\hline Date & 29-Oct-2001 & AbsSpeedX & $-7.44 \mathrm{~km} / \mathrm{s}$ & \multirow{4}{*}{\multicolumn{2}{|c|}{$\begin{array}{l}-2.0 \mathrm{E}-12 \\
-2.5 \mathrm{E}-12\end{array}$}} & & & & \\
\hline Time & $16: 13: 37$ & AbsSpeedY & $-23.83 \mathrm{~km} / \mathrm{s}$ & & & & & & \\
\hline Spinphase & 302.39 & AbsSpeedZ & $-4.51 \mathrm{~km} / \mathrm{s}$ & & & & & & \\
\hline Quality & 113 & AbsSpeed & $25.37 \mathrm{~km} / \mathrm{s}$ & & & & & - & (1) \\
\hline TRMode & 152 & & & \multirow{2}{*}{\multicolumn{2}{|c|}{$\begin{array}{r}-3.0 \mathrm{E}-12+ \\
0\end{array}$}} & & & & \\
\hline TRLevel & 143 & Inclination & $10.70^{\circ}$ & & & 40 & 80 & 120 & 160 \\
\hline Checksum & 174 & Eccentricity & 0.23 & \multicolumn{6}{|c|}{ time $[\mu \mathrm{s}]$} \\
\hline \multirow[t]{2}{*}{ Imp. No. } & 01110301-50 & Mass & $6.5 \mathrm{E}-15 \mathrm{~g}$ & & $2.0 \mathrm{E}-12$ & & & & \\
\hline & & ImpactSpeed & $34.64 \mathrm{~km} / \mathrm{s}$ & & & & & & \\
\hline Plus5V & $4.94 \mathrm{~V}$ & ImpactPos & IC_C & & $1.5 \mathrm{E}-12$ & & & & \\
\hline Plus12V & $13.14 \mathrm{~V}$ & CalibImpact & $97102310-31$ & & $1 . J \mathrm{~L}^{-12}$ & & & & th \\
\hline Minus12V & $13.60 \mathrm{~V}$ & & & & & & & & \\
\hline Plus200V & $257.04 \mathrm{~V}$ & $\operatorname{Pos} \mathrm{X}$ & $-152762671 \mathrm{~km}$ & $\sigma$ & $1.0 \mathrm{E}-12$ & & & & \\
\hline Minus200V & $224.40 \mathrm{~V}$ & PosY & $40854760 \mathrm{~km}$ & 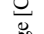 & $1.0 \mathrm{~L}-12$ & & & & \\
\hline TElec & $46.20^{\circ} \mathrm{C}$ & PosZ & $7858999 \mathrm{~km}$ & 哿 & & & & & \\
\hline TBox & $41.32^{\circ} \mathrm{C}$ & Radius & $1.06 \mathrm{AU}$ & & $5.0 \mathrm{E}-13$ & & & & \\
\hline Date & $30-O c t-2001$ & AbsSpeedX & $7.91 \mathrm{~km} / \mathrm{s}$ & & & ---- & $--\bullet$ & $-{ }_{-1}$ & \\
\hline Time & 06:59:05 & AbsSpeedY & $-1.62 \mathrm{~km} / \mathrm{s}$ & & $0.0 \mathrm{E}+00$ & $-\ldots$ & $\longrightarrow$ & & \\
\hline Spinphase & 336.93 & AbsSpeedZ & $-9.16 \mathrm{~km} / \mathrm{s}$ & & & & & & \\
\hline Quality & 144 & AbsSpeed & $12.20 \mathrm{~km} / \mathrm{s}$ & & & & & & \\
\hline TRMode & 152 & & & & $-5.0 \mathrm{E}-13+$ & & & & \\
\hline TRLevel & 143 & Inclination & $93.10^{\circ}$ & & & 40 & 80 & 120 & 160 \\
\hline Checksum & 68 & Eccentricity & 0.91 & & & & & & \\
\hline Imp. No. & 01112101-3 & Mass & N/A & & $5.0 \mathrm{E}-13$ & & & & \\
\hline & & ImpactSpeed & N/A & & $0.0 \mathrm{E}+00$ & 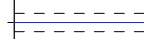 & $-\div$ & En & $-\ldots$ \\
\hline Plus5V & $4.94 \mathrm{~V}$ & ImpactPos & GRID_EC & & $-5.0 \mathrm{E}-13-$ & & & & \\
\hline Plus12V & $13.29 \mathrm{~V}$ & CalibImpact & 97093003-35 & & -3.0L-13 & & & & \\
\hline Minus12V & $13.60 \mathrm{~V}$ & & & & $-1.0 \mathrm{E}-12$ & & & & \\
\hline Plus200V & $258.40 \mathrm{~V}$ & PosX & $-165364919 \mathrm{~km}$ & & $-1.5 \mathrm{E}-12$ & & & & \\
\hline Minus200V & $227.12 \mathrm{~V}$ & PosY & $-2030892 \mathrm{~km}$ & $\overrightarrow{0}$ & $-2.0 \mathrm{E}-12$ & & & & \\
\hline TElec & $44.57^{\circ} \mathrm{C}$ & PosZ & $8501495 \mathrm{~km}$ & 望 & $\begin{array}{l}-2.0 \mathrm{E}-12 \\
-25 \mathrm{E}-12\end{array}$ & & & & \\
\hline TBox & $39.69^{\circ} \mathrm{C}$ & Radius & $1.11 \mathrm{AU}$ & & $-2.5 \mathrm{E}-12$ & & & & \\
\hline Date & 16-Nov-2001 & AbsSpeedX & N/A & & $-3.0 \mathrm{E}-12$ & & & & \\
\hline Time & 12:16:52 & AbsSpeedY & N/A & & $-3.5 \mathrm{E}-12$ & & & & \\
\hline Spinphase & 263.72 & AbsSpeedZ & N/A & & $-4.0 \mathrm{E}-12$ & & & & \\
\hline Quality & 93 & AbsSpeed & N/A & & $-4.5 \mathrm{E}-12$ & & & & \\
\hline TRMode & 152 & & & & & 0 & 80 & 120 & 160 \\
\hline TRLevel & 143 & Inclination & N/A & & & 40 & 80 & 120 & 100 \\
\hline Checksum & 173 & Eccentricity & N/A & & & & & & \\
\hline Imp. No. & 01120901-40 & Mass & $\mathrm{N} / \mathrm{A}$ & & $5.0 \mathrm{E}-13$ & & & & \\
\hline & & ImpactSpeed & N/A & & & & & & \\
\hline Plus5V & $4.94 \mathrm{~V}$ & ImpactPos & GRID_EC & & $0.0 \mathrm{E}+00$ & & & & \\
\hline Plus12V & $13.14 \mathrm{~V}$ & CalibImpact & $97102239-11$ & & & & & & \\
\hline Minus12V & $13.76 \mathrm{~V}$ & & & & $-5.0 \mathrm{E}-13$ & & & & \\
\hline Plus200V & $258.40 \mathrm{~V}$ & $\operatorname{Pos} \mathrm{X}$ & $-167460239 \mathrm{~km}$ & $\sigma$ & & & & & \\
\hline Minus200V & $225.76 \mathrm{~V}$ & PosY & $-42873428 \mathrm{~km}$ & 光 & & & & & \\
\hline TElec & $41.32{ }^{\circ} \mathrm{C}$ & PosZ & 8603767 km & 旌 & $-1.0 \mathrm{E}-12$ & & & & \\
\hline TBox & $36.43^{\circ} \mathrm{C}$ & Radius & $1.16 \mathrm{AU}$ & है & & & & & \\
\hline & & & & & $-1.5 \mathrm{E}-12$ & & & & \\
\hline $\begin{array}{l}\text { Date } \\
\text { Time }\end{array}$ & $\begin{array}{r}\text { 2-Dec-2001 } \\
\text { 22:06:35 }\end{array}$ & $\begin{array}{l}\text { AbsSpeedX } \\
\text { AbsSpeedY }\end{array}$ & $\begin{array}{l}\mathrm{N} / \mathrm{A} \\
\mathrm{N} / \mathrm{A}\end{array}$ & & & & & & 1 \\
\hline Spinphase & 346.16 & AbsSpeedZ & $\mathrm{N} / \mathrm{A}$ & & $-2.0 \mathrm{E}-12$ & & & & \\
\hline Quality & 143 & AbsSpeed & $\mathrm{N} / \mathrm{A}$ & & & & & & \\
\hline TRMode & 152 & & & & $-2.5 \mathrm{E}-12+$ & & & & \\
\hline TRLevel & 143 & Inclination & N/A & & & 40 & 80 & 120 & 160 \\
\hline Checksum & 126 & Eccentricity & N/A & & & & & & \\
\hline
\end{tabular}




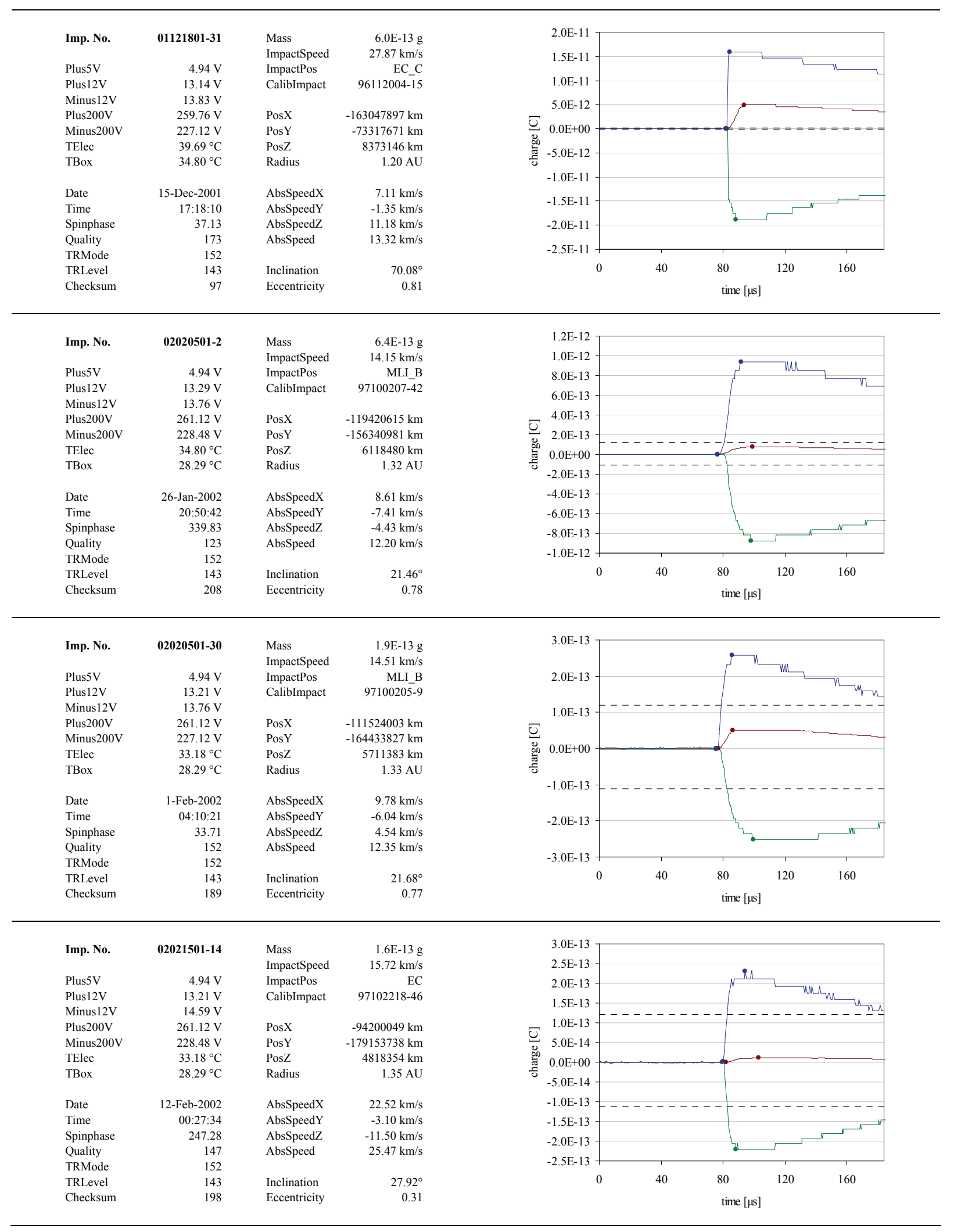




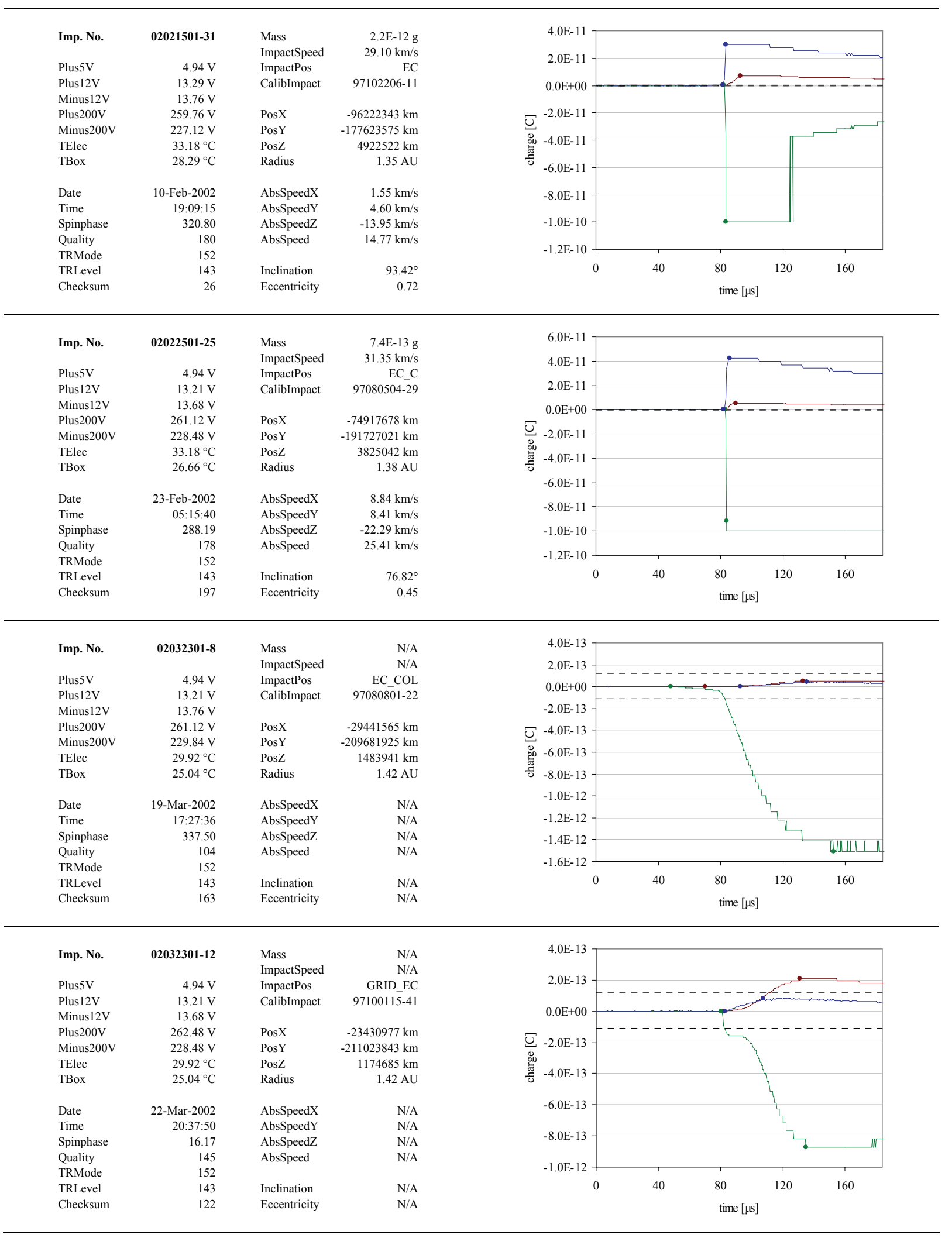




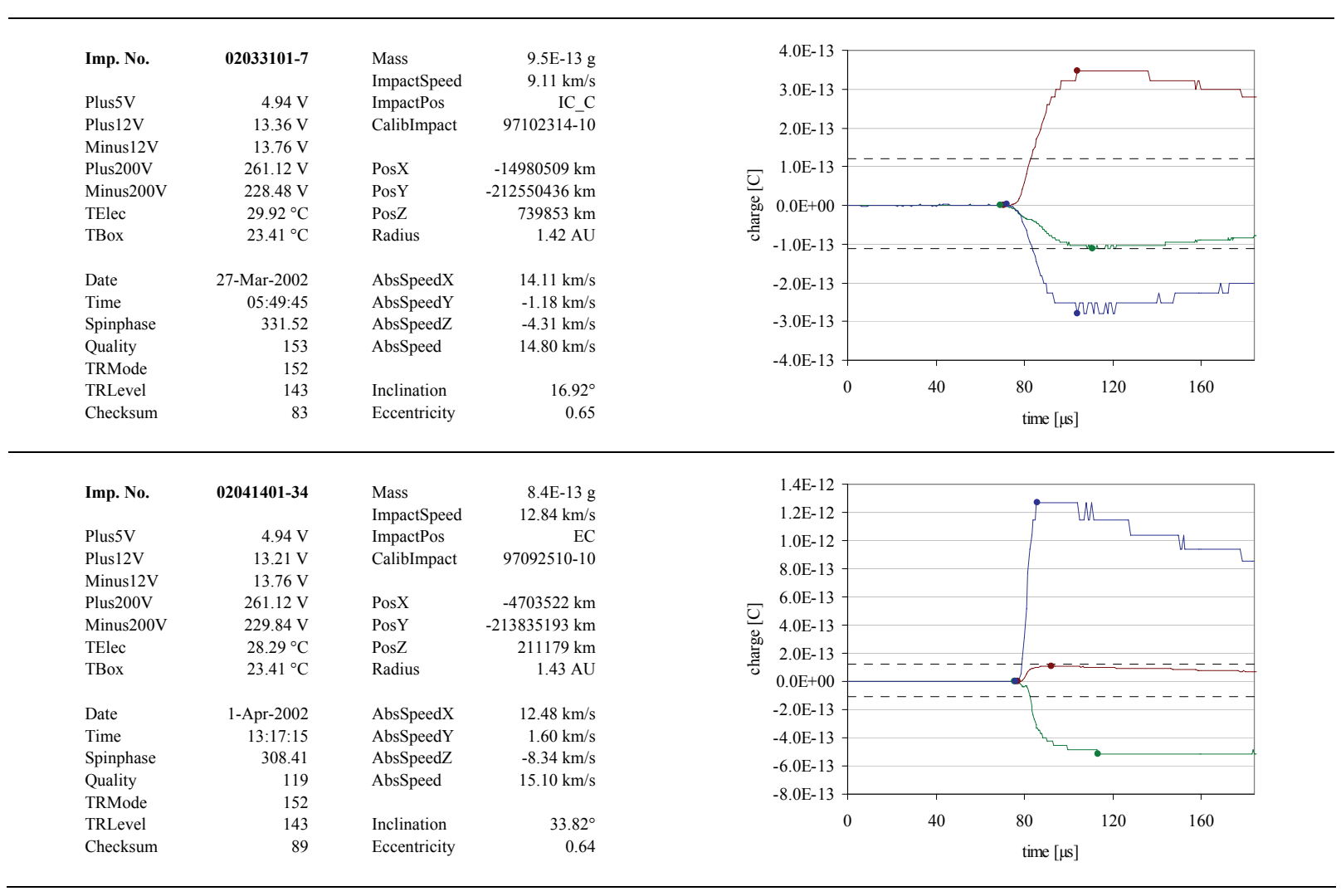




\section{Appendix D: XML files Document Type Descriptions}

File telemetry.dtd used for document type description of MDC telemetry files (downloaded data files from NOZOMI and calibration data files).

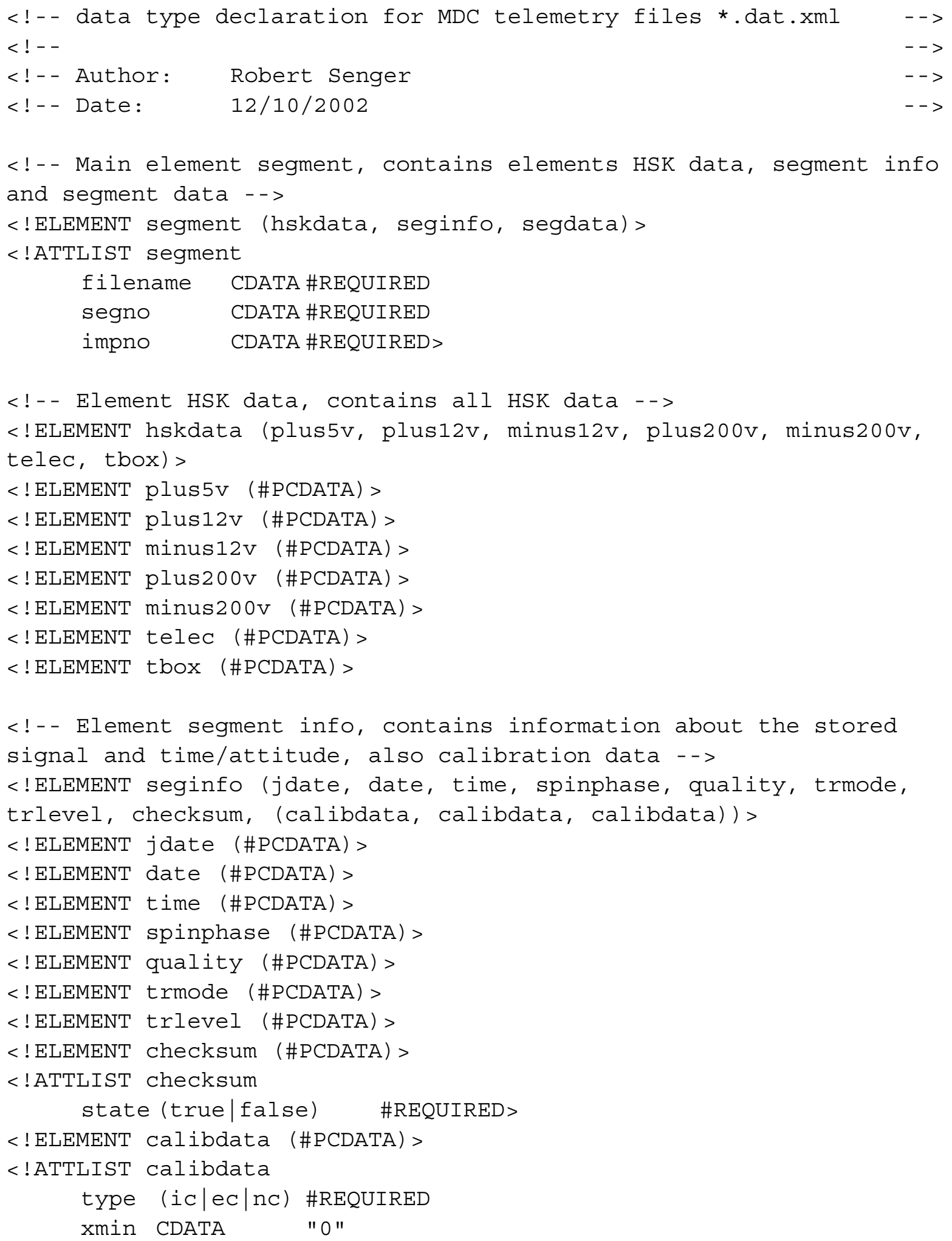




$\begin{array}{llc}\text { xmax } & \text { CDATA } & \text { "0" } \\ \text { ymin } & \text { CDATA } & \text { "0" } \\ \text { ymax } & \text { CDATA } & \text { "0" > }\end{array}$

<!-- Element segment data, contains the data of the three channels of the stored signal -->

$<$ !ELEMENT segdata ((chdata, chdata, chdata) +) >

$<$ !ELEMENT chdata (pdata) >

$<$ !ATTLIST chdata

type (ic|ec|nc) \#REQUIRED

format (dump|charge) \#REQUIRED>

$<$ ! ELEMENT pdata (\#PCDATA) > 
File housekeeping. dtd used for document type description of MDC housekeeping files (downloaded files from NOZOMI).

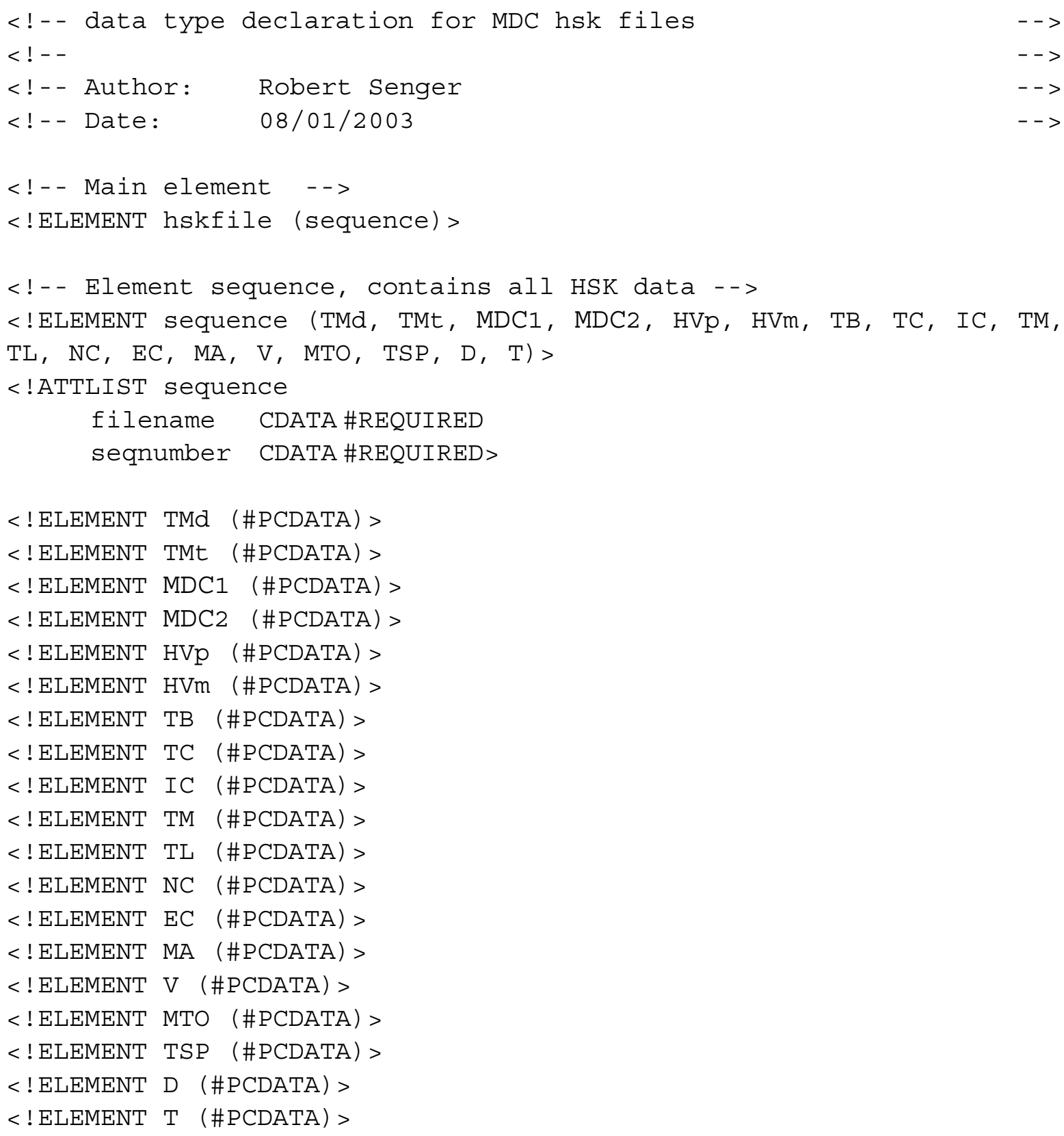




\section{Appendix E: MDC-NOZOMI Publications}

Igenbergs, E., Sasaki, S., Faber, G., Fischer, F., Münzenmayer, R., Fujiwara, A., Iglseder, H., Glasmachers, A., Grün, E., Mukai, T., Ohashi, H., Schwehm, G., Svedhem, H., Yamakoshi, K., "Mars Dust Counter on Board ISAS PLANET-B", in Physics, Chemistry, and Dynamics of Interplanetary Dust (ed. B.A.S. Gustafson and M.S. Hanner), ASP Conf. Series, Vol. 104, pp. 233-236, 1996

Igenbergs, E., Münzenmayer, R., Sasaki, S., Faber, G., Fischer, F., Fujiwara, A., Iglseder, H., Glasmachers, A., Grün, E., Mukai, T., Ohashi, H., Schwehm,G., Svedhem, H., Yamakoshi, K., "Mars Dust Counter on Board ISAS PLANET-B", in Proceedings of $20^{\text {th }}$ International Symposium on Space Technology and Space, Gifu, Japan, pp. 1222-1229, 1996

Igenbergs, E., Sasaki, S., Muenzenmayer, R., Ohashi, H., Faber, G., Fischer, F., Fujiwara, A., Hamabe, Y., Iglseder, H., Klinge, D., Miyamoto, H., Glasmachers, A., Gruen, E., Mukai, T., Naumann, W., Nogami, K., Schwehm,G., Svedhem, H., Yamakoshi, K., "Mars Dust Counter", Earth Planets Space, Vol. 50, pp. 241-245, 1998

Sasaki, S., Igenbergs, E., Münzenmayer, R., Naumann, W., Ohashi, H., Born, M., Faber, G., Fischer, F., Fujiwara, A., Glasmachers, A., Grün, E., Hamabe, Y., Iglseder, H., Miyamoto, H., Morishige, K., Mukai, T., Nogami, K.-I., Schwehm, G., Svedhem, H., Yamakoshi, K., "MDC (Mars Dust Counter) on Board NOZOMI: Launch and Initial Operations", Proc. $31^{\text {st }}$ ISAS Lunar Planet. Symp. (eds. H. Mizutani and M. Kato), pp.243-246, 1998

Sasaki, S., Igenbergs, E., Ohashi, H., Münzenmayer, R., Naumann, W., Born, M., Faber, G., Fischer, F., Fujiwara, A., Glasmachers, A., Grün, E., Hamabe, Y., Hofschuster, G., Iglseder, H., Miyamoto, H., Morishige, K., Mukai, T., Nogami, K.-I., Schwehm, G., Svedhem, H., Yamakoshi, K., "Initial results of Mars Dust Counter (MDC) on board NOZOMI: Leonids encounter", Lunar Planet. Sci., XXX 1581, 1999

Sasaki, S., Igenbergs, E., Münzenmayer, R., Ohashi, H., Hofschuster, G., Naumann, W., Faber, G., Fischer, F., Fujiwara, A., Glasmachers, A., Grün, E., Hamabe, Y., Iglseder, H., Miyamoto, H., Mukai, T., Nogami, K., Schwehm,G., Svedhem, H., Born, M., Kawamura, T., Klinge, D., Morishige, K., Naoi, T., Peeks, R., Yano, H., Yamakoshi, K., "Mars Dust Counter on board NOZOMI: First year results", Proceedings of $22^{\text {nd }}$ International Symposium on Space Technology and Science, Vol. 00-j-21, pp. 1664-1667, 2000

Sasaki, S., Igenbergs, E., Ohashi, H., Hofschuster, G., Münzenmayer, R., Naumann, W., Senger, R., Fischer, F., Fujiwara, A., Grün, E., Hamabe, Y., Kawamura, T., Mann, I., Miyamoto, H., Nogami, K., Svedhem, H., "Interplanetary dust observation in the Earth-Mars region by Mars Dust Counter (MDC) on board NOZOMI: Three-year results", Proceedings of the Meteoroids 2001 Conference, ESA SP-495, pp. 595-599, 2001 
Sasaki, S., Igenbergs, E., Ohashi, H., Münzenmayer, R., Naumann, W., Hofschuster, G., Born, M., Faber, G., Fischer, F., Fujiwara, A., Glasmachers, A., Grün, E., Hamabe, Y., Iglseder, H., Kawamura, T., Miyamoto, H., Morishige, K., Mukai, T., Naoi, T., Nogami, K., Schwehm, G., Svedhem, H., "Observation of interplanetary and interstellar dust particles by Mars Dust Counter (MDC) on board NOZOMI”, Adv. Space Res., Vol. 29, pp. 1145-1153, 2002

Sasaki, S., Igenbergs, E., Münzenmayer, R., Ohashi, H., Hofschuster, G., Naumann, W., Faber, G., Fischer, F., Fujiwara, A., Glasmachers, A., Grün, E., Hamabe, Y., Iglseder, H., Miyamoto, H., Mukai, T., Nogami, K., Schwehm, G., Svedhem, H., Born, M., Kawamura, T., Klinge, D., Morishige, K., Naoi, T., Peeks, R., Yano, H., Yamakoshi, K., "Mars Dust Counter (MDC) on Board NOZOMI: Initial Results", Proceedings of IAU Colloquium 181, Dust in the solar System and Other Planetary Systems (edited by S.F. Green, I. Williams, T. McDonnell \& N. McBride), pp.176-180, 2002

Sasaki, S., Igenbergs, E., Ohashi, H., Senger, R., Hofschuster, G., Münzenmayer, R., Naumann, W., Grün, E., Hamabe, Y., Kawamura, T., Mann, I., Miyamoto, H., Nogami, K.I., Shoji, S., Svedhem, H., "Mars Dust Counter (MDC) on board NOZOMI: Three-year results", Proceedings of $23^{\text {rd }}$ International Symposium on Space Technology and Science, 2002-k-17, 2002

Sasaki, S., Igenbergs, E., Ohashi, H., Senger, R., Hofschuster, G., Münzenmayer, R., Naumann, W., Grün, E., Fujiwara, A., Hamabe, Y., Mann, I., Miyamoto, H., Mukai, T., Nogami, K., Shoji, S., Svedhem, H., "Interplanetary and interstellar dust observation by Mars Dust Counter on board NOZOMI: Four-year operation" Proceedings of Asteroids, Comets, Meteors (ACM2002), ESA-SP-500, pp. 79-82, 2002 


\section{Appendix F: Curriculum vitae}

Feb. 9, 1970

$1976-1980$

1980-1989

June 1989

Nov. 1989 - Feb. 1998

Nov. 1994 - Oct. 1995

June 1995 - July 1995

Feb. 4, 1998

June 1999 - July 2004 born in Munich, Germany

Primary School, Munich

Luitpold Gymnasium, Munich

High-school degree (Abitur)

Physics studies at the Technische Universität München

Diploma thesis at the Lehrstuhl für Biophysik at Technische Universität München, Prof. Dr. E. Sackmann

Visiting undergraduate scientist at the University of Kent at Canterbury (UK), Research School of Biosciences, Prof. Dr. M. F. Tuite

University degree (Diploma)

Research assistant at LRT, Technische Universität München 


\section{Appendix G: Legal information}

Microsoft, Excel, Visual Basic, Visual C and Windows are either registered trademarks or trademarks of Microsoft Corporation in the United States and/or other countries.

$M A T L A B$ is a registered trademark of The MathWorks, Inc.

Intel and Pentium are trademarks or registered trademarks of Intel Corporation or its subsidiaries in the United States and other countries.

Kapton and Nomex are registered trademarks of E.I. du Pont de Nemours and Company.

Java is a trademark or registered trademark of Sun Microsystems, Inc. in the U.S. or other countries. 
